American Journal of Pharmaceutical Education 2018; 82 (5) Article 7158.

\title{
ABSTRACTS
}

\section{9th Annual Meeting of the American Association of Colleges of Pharmacy, Boston, Massachusetts, July 21-25, 2018}

\author{
Administrative Services \\ Completed Research
}

A Model to Predict NAPLEX Outcomes and Identify Remediation Candidates. Sachin Shah, Texas Tech University Health Sciences Center, Iverlyn Peng, Texas Tech University Health Sciences Center, Charles F. Seifert, Texas Tech University Health Sciences Center. Objectives: To develop a NAPLEX outcome predictive model that could be practical, measurable, and reliable. Method: The study consisted of students who graduated from the TTUHSC-SOP. The eligible students had taken NAPLEX and their first-attempt examination score were available to the SOP. Primary outcome of the study was to identify student specific factors that may help predict the NAPLEX examination outcome. Students were considered to have poor performance in NAPLEX examination if they received an overall score of less than or equal to 82 . Linear and logistic regression analysis were utilized to identify independent predictors. Results: There were 70 out of 433 students identified to be poor performers in the NAPLEX examination. Independent factors identified to be associated with a poor outcome on the NAPLEX examination were: age $>28$ years of age at graduation, PCAT scaled score $<74$, High Risk Drug Knowledge Assessment score $<90$, P3 PCOA scaled score $<349$, and grades of less than 74 in $>3$ courses. These predictors were utilized to stratify students into four risk groups of ShahTech model. The mean NAPLEX score for the Low, intermediate-1, Intermediate-2, and High risk groups were 106.4, 97.4, 87.1, and 75.1, respectively. Students in the High risk and Intermediate- 2 groups were identified to be at $78 \%$ and $35 \%$ risk of poor performance in the NAPLEX examination. Implications: The model could be used as a practical tool to identify students early who need remediation for the NAPLEX examination.

A Simple Method for Using Quality Indicator Data to Rank Colleges and Schools of Pharmacy. Lisa Lebovitz, University of Maryland. Objectives: The objective of this project was to create a pharmacy school ranking system by defining various indicators of quality, exploring available data, and devising a methodology. Method: US News \& World Report ranks pharmacy schools solely on a one-question general peer perception survey, while rankings of medical schools and many other programs utilize data as well as perception. Four indicators of quality for PharmD programs were defined as selectivity (number of applications/number of admitted P1 class), student:faculty ratio (number of PharmD enrolled/number of full time faculty), NAPLEX annual pass rate, and number matched for PGY1 residency. Four indicators of quality for research were defined as total research funding dollars, funding per funded faculty, number of PhDs conferred, and peer assessment score. All eight indicators were combined for institutional rankings. Data for 2016 were located from AACP, ASHP, NABP and US News \& World Report. Each category was ranked from highest to lowest, except that PharmD student: faculty ratio was ranked from lowest to highest. Categories were weighted proportionately, and only schools with data in all categories were ranked. Category ranks were summed, then sums were ranked for an overall ranking. The lowest overall sum determined the highest rank. Results: The Lebovitz Institutional Standardized Assessments, or LISA Rankings, moderately correlate to US News: institutional $=0.85$, research $=0.82$, and PharmD program $=0.42$ correlations. Implications: These rankings may be useful for PharmD and $\mathrm{PhD}$ applicant decision making, and for pharmacy schools to identify true and aspirational peers for strategic initiatives.

Assessing First-Year Student Pharmacists' Perceptions Regarding Usefulness of the APhA Career Pathways Tool for Career Exploration. Curtis G. Jefferson, University of Washington, Stanley S. Weber, University of Washington, Michaelene Kedzierski, University of Washington. Objectives: To understand incoming first-year pharmacy students' (PY1) perceptions of the usefulness of the APhA Career Pathways for Pharmacy Professionals as a tool to explore pharmacy careers and specializations. Method: All PY1 students $(\mathrm{N}=105)$ completed the Career Pathways assessment as part of pre-work for the introductory Profession of Pharmacy Course in autumn 2017 prior to arriving on campus for orientation. Students were asked to complete a brief survey after reviewing their results; 97 students (92\%) responded. A mid-quarter workshop to discuss assessment results was held for all students, following the close of the survey. Results: Most respondents (86\%) agreed that the Career Pathways assessment was a useful tool for thinking about careers in pharmacy. The majority of respondents $(77 \%)$ indicated they were surprised by one or more of their results; the same number also agreed that they could see how their results matched their stated preferences. When asked whether they expect to use their results to explore pharmacy career opportunities while in pharmacy school, $87 \%$ of respondents agreed. Implications: Students' initial reactions to the Career Pathways tool, process, and results indicates that students found the assessment useful and expect to make use of it as they explore pharmacy careers. Some students (8\%) also specifically cited Career Pathways as a significant learning activity in their post-course reflection. This supports the continued use of the tool with first-year students, and may suggest value in continuing to engage students with reviewing their results or even retaking the assessment at various points in their academic program.

Assessing the Effectiveness of Alpha Ranking System in Pharmacy Admissions. Natasha Baloch, University of South Florida, Nazach Rodriguez-Snapp, University of South Florida, Kevin B. Sneed, University of South Florida. Objectives: This analysis examines the effectiveness of the Alpha Ranking System in addition to other pre admission factors in successfully identifying student's at risk of progression issues in a Doctor of Pharmacy Program. The research examines students who had an interruption in PY1 vs students with no academic interruption. Method: The Alpha Ranking is utilized during the admissions process with each complete applicant file being assigned a letter rubric of A, B or C. The Admissions Director assigns an Alpha Ranking utilizing a standardized rubric set forth by the Admissions Committee. The rubric takes into consideration 


\section{American Journal of Pharmaceutical Education 2018; 82 (5) Article 7158.}

overall GPA, PCAT composite score, past academic, professional and criminal infractions. Descriptive statistics, and independent ttests were conducted to explore relationships between alpha ranking and other pre admission variables for the two groups of students. Results: All students with academic interruption $(n=9)$ in PY1 had an alpha ranking of $\mathrm{C}$. Results also show statistically significant $(\mathrm{p}<0.01)$ differences with lower performance in other pre admission variables including PCAT scores, prior university GPA and overall PY1 GPA for students with interruption vs students with no academic interruption. Implications: The findings provide support for the alpha ranking system in identifying students who may be at risk of progression issues in the first year. Specifically, along with other well established pre admission measures affecting student performance, alpha ranking may be utilized to aid the admissions process. Pre matriculation enrichment programs can be developed in order to prevent student interruption in the first year.

Determination of Predictors Impacting Performance on the Pharmacy Curriculum Outcomes Assessment (PCOA) at a HBCU. Muhammad J. Habib, Howard University, Salome B. Weaver, Howard University, LaMarcus T. Wingate, Howard University, Youness R. Karodeh, Howard University, Michael A. Marcus, Howard University, Earl Ettienne, Howard University, Mary Maneno, Howard University. Objectives: To determine the impact of pre-admissions and pharmacy school variables on PCOA performance at a Historically Black College of Pharmacy. Method: A retrospective analysis was conducted using data from three cohorts of students that took the PCOA in their third professional year from 2015 to 2017. Descriptive statistics were conducted in addition to correlational analysis and multivariate linear regression models were developed to determine which variables had a significant effect on PCOA score. Results: There were 182 students that took the PCOA during the study period, and the mean PCOA among these individuals was 62.7. Approximately two-thirds of (67.0\%) students were black, and over half $(54.9 \%)$ were female. The PCOA score during the 3rd year was significantly correlated with the PCAT score, the GPA during the fall semester of the third professional year, and whether or not an individual was on probation during their first semester in pharmacy school. After adjusting for other factors, the cumulative GPA during the P3 fall semester $(\mathrm{P}<0.001)$ and PCAT scores $(\mathrm{p}<0.001)$ were predictive of student's P3 PCOA scores. Implications: The GPA during pharmacy school and PCAT score are important factors in helping to predict PCOA scores among 3rd year professional students at an HBCU.

Ensuring Academic Integrity in a Remotely Proctored Online Exam Environment. Jason M. Brunner, University of Colorado, Lisha R. Bustos, University of Colorado, Kevin Lawanto, University of Colorado. Objectives: Electronic exams are common practice in pharmacy schools, whereby, most schools require students take their exams in the classroom. Advances in technology created the opportunity to offer remotely proctored online exams. The University of Colorado School of Pharmacy implemented a remotely proctored online exam program in 2016 for all required courses. Due to the variability of exam environments, the school developed methods to ensure academic integrity. This poster will address the methods by which academic integrity was ensured and assessed. Method: The school instituted the use of a remote proctoring service that uses a combination of technology and human proctors. Students provide proof of identity at the start of an exam and then are remotely monitored. Based on school identified expectations (i.e., head and eye movement, talking, leaving the exam environment), the software monitors for suspicious behaviors. A proctor intervenes when alerted by the software. The proctoring service provides documentation of concerning students and exam recordings to the school for additional review. Results: The school assessed the efficacy of this program by comparing three years of exam data for significant changes in year-over-year scores. Significant variation in individual student exam scores were also assessed. No significant differences were found in either of these datasets. Implications: The results of this research suggest that moving to a remotely proctored online exams does not put at risk the academic integrity of examinations. Additionally, students indicated a preference for online exams as it provides them the flexibility to take exams when and where they want.

Extending Pharmacy's Educational Reach to New Undergraduate Audiences. Nicole Kwiek, The Ohio State University, Katherine Watkins, The Ohio State University, Mary Higginbotham, The Ohio State University, Justin Habash, The Ohio State University, Henry J. Mann, The Ohio State University. Objectives: To strategically develop curricula and coursework to broadly reach undergraduate students pursuing biomedical careers and those majoring in non-science disciplines. Method: Beginning in 2014, The Ohio State University (OSU) College of Pharmacy has systematically overhauled and enhanced its undergraduate-targeted programming, including the Bachelor of Science in Pharmaceutical Sciences (BSPS) major, the Pharmaceutical Sciences (PS) minor, and the liberal arts-based General Education (GE) coursework. Faculty, staff, students, and community stakeholders collaboratively used the Backward Design process to create a BSPS curriculum of high appeal to students pursuing clinical and research-based careers. New opportunities to bring highly relevant Pharmacy topics to any student were subsequently developed and resulted in a flexible PS minor and new GE courses. Results: As of Spring 2018, the newly designed BSPS curriculum has been fully implemented. Growth of the program is strong as indicated by increases in overall major enrollees as well as applicants to our PharmD Early Admission Pathway. In only 2 years, PS minor enrollment is approaching 100 students. All sections of the college's GE courses are routinely filled and wait-listed. Since 2013, undergraduate enrolled credit hours for the college have increased over 105\%, reaching targeted student audiences. Implications: In a challenging admissions environment nationwide, Colleges of Pharmacy must look at novel avenues to attract and engage future PharmD students and other student audiences on campus. The OSU programs demonstrate a successful growth model that can be replicated at other institutions.

Identifying Optimal Selection Criteria for the Direct Admit Program. Candace M. Gonzalez, University of the Incarnate Word, Amy Diepenbrock, University of the Incarnate Word, Scott E. Smith, University of the Incarnate Word, Marcos Oliveira, University of the Incarnate Word, Kimberly A.B. Cauthon, University of the Incarnate Word. Objectives: To determine if the Feik School of Pharmacy Direct Admit (DA) program is using the best criteria in identifying students who successfully progress through the PharmD program. The hypothesis is DA students perform significantly better than traditionally-admitted pharmacy students. Method: Three student cohorts from the DA program have matriculated through the P1 year. DA students' grades were compared to Traditional students' grades from three P1 courses using R software. DA students' grades were used to fit linear models that assume the grades to be functions of the students' SAT/ACT Math scores, and high school math/ science GPAs, which are parameters for DA program acceptance. Results: This study analyzed $38 \mathrm{DA}$ and 215 Traditional students. DA student grades were higher than those of Traditional students; however no statistically significant differences were found in the average scores: Anatomy and Physiology $(p=0.444)$, Biochemistry $(p=0.274)$, Pharmaceutics $(p=0.0551)$. Using a logistic regression model that assumes the log odds of passing all three courses as a linear function of Math/Science GPA and 


\section{American Journal of Pharmaceutical Education 2018; 82 (5) Article 7158.}

SAT Math scores, it was found, while the GPA has a significant relationship with the probability of passing all three courses $(p=0.0283)$, SAT is not shown to have a significant relationship with the probability $(p=0.3843)$. Implications: Relaxing or eliminating SAT Math criteria could result in an increase in DA students without affecting the students' progression through the $\mathrm{P} 1$ year.

Impact of a New Grading Scheme on Course Grades in a PharmD Program Using Team-Based Learning. Osama A. Shoair, The University of Texas at Tyler. Objectives: Some PharmD programs that use team-based learning (TBL) require students to obtain a certain percentage on individual activities before earning grades from team activities leading to 1) increased complexity of grading schemes, 2) increased number of students who fail or need remediation, and 3) inflation of grades for some students. The objective of this study was to evaluate whether a new grading scheme will reduce the percentage of students who fail or need remediation and decrease grade inflation. Method: Our new grading scheme has 15\% greater weight on individual activities compared to old scheme. However, it allows students to earn all course grades without obtaining a specific percentage on individual activities. A retrospective comparison of grades using the new versus old scheme was performed on a random sample of three courses. Results: The distribution of grades using the new versus old scheme was: $\mathrm{P} 1$ course $(\mathrm{N}=87$, A: $24 \%$ vs. $32 \%$, B: $49 \%$ vs. $57 \%$, C: $25 \%$ vs. $5 \%$, D: $1 \%$ vs. $3 \%$, F: $0 \%$ vs. $2 \%$, respectively), P2 course ( $\mathrm{N}=98$, A: $19 \%$ vs. $42 \%$, B: $71 \%$ vs. $56 \%$, C: $9 \%$ vs. $0 \%$, D: $0 \%$ vs. $2 \%$, F: $0 \%$ vs. $0 \%$, respectively), and $\mathrm{P} 3$ course ( $\mathrm{N}=69$, A: $3 \%$ vs. $6 \%$, B: $54 \%$ vs. $71 \%$, C: $39 \%$ vs. $12 \%$, D: $4 \%$ vs. $6 \%$, F: $0 \%$ vs. $6 \%$, respectively). Implications: The new grading scheme reduces the percentage of students who fail or need remediation and decreases grade inflation. It offers a fair grading system for students in pharmacy programs using TBL, while maintaining students' individual accountability.

Implementation and Assessment of a Naloxone-Training Program for First-Year Student Pharmacists. Amanda Schartel, University of Maryland, Allison B. Lardieri, University of Maryland, Ashlee Mattingly, University of Maryland, Agnes A. Feemster, University of Maryland. Objectives: To develop a naloxone training activity and assess the impact on student-pharmacist knowledge and confidence to counsel about management of opioid overdose and naloxone administration. Method: First-year students participated in a naloxone training activity in a required abilities laboratory course. They completed a pre-lab reading and questions, received a brief lecture about responding to opioid overdose, and practiced counseling/administering intranasal and intramuscular naloxone. An objective structured clinical examination (OSCE) was conducted to assess their ability to counsel about the use of intranasal naloxone. Students also completed self-assessments about their confidence in counseling about management of opioid overdose and naloxone administration following the OSCE and at the end of the course. Results: One hundred fifty-eight students participated, and the average OSCE score was $82 \%$. In the post-encounter self-assessment, 93\% agreed or completely agreed that the OSCE improved their confidence in counseling about management of opioid overdose and intranasal naloxone administration. Fifty-nine students completed the end-of-course survey, and over $90 \%$ reported they were somewhat or very confident in their ability to administer intranasal or intramuscular naloxone, recognize the symptoms of opioid overdose, and counsel about the use of intranasal naloxone. Confidence in counseling about the use of intramuscular naloxone was slightly lower. Implications: A need for further study of training programs aimed to increase future healthcare professionals' ability to respond to opioid overdoses exists. This assessment demonstrates that incorporating a short training activity can increase student pharmacists' knowledge and confidence in counseling patients about opioid overdose and naloxone administration.

Implementation of an Early Intervention and Remediation Process Targeting Progression Standards. Laurel A. Sampognaro, The University of Louisiana at Monroe, David J. Caldwell, University of Arkansas for Medical Sciences, Elizabeth M. Lafitte, The University of Louisiana at Monroe. Objectives: To become compliant with ACPE standards related to student pharmacist progression, specifically a delayed graduation threshold of $<15 \%$, the School of Pharmacy created and implemented early intervention (EI) and remediation procedures beginning in the fall of 2015. Method: Through a faculty-led process, EI and remediation procedures were modified and approved by faculty and administration. For each course, students scoring $<70 \%$ on any exam resulting in an exam average $<70 \%$ are required to review missed item reports with a course coordinator and submit written responses to lecture objectives for instructor feedback one week before the next exam. Qualifying P1 students are also required to meet with the Director of Progression for academic coaching. Compliance with all requirements is a prerequisite of remediation. Only students with final averages $>65 \%$ that complied with EI requirements are eligible for a targeted remediation, which occurs during winter break or Maymester. If $>7 \%$ of enrolled students fail to earn a final grade of "C" or better, then remediation is allowed for any student earning a non-progressing grade. $70 \%$ must be earned on the remediation exam to successfully progress. Results: Delayed graduation rates for incoming classes have improved with the implementation of this process: $12.9 \%$ (2012), 15.5\% (2013), 10.2\% (2014), 6\% (2015), $1.2 \%$ (2016), 2.3\% (2017). The number of P1 students failing to progress has also decreased: 13 (2014), 0 (2015), 1 (2016), 2 (2017). Implications: Other schools of pharmacy could use this model to improve progression throughout the professional program.

Leading Student Pharmacists to "Drink the Waters" of Academic Support in an Accelerated Pharmacy Program. Diane W. Morel, South College. Objectives: The main objective is to reduce the approximately $30 \%$ higher intensity factor, defined as the ratio of didactic weeks to calendar weeks for delivery of pre-APPE curriculum, for student pharmacists in an accelerated, quarter-based Doctor of Pharmacy program by providing learning support that meets students where they are in personal and professional development. Method: A comprehensive needs assessment was performed via convergence of academic services usage data for an exisiting voluntary academic support program with data collected from informal focus groups and a newly designed survey of current students focused on student engagement with and perceptions of the value of and reasons for nonengagement with various academic support services. Results: Usage data indicated student reluctance to react promptly to academic challenges (failed exams) or respond to academic outreach. The most common reasons given was pride, and no convenient time to pursue given their busy schedule. Many indicated they just had to spend more time studying without consideration of different or more effective strategies. Implications: Convergence of utilization data, student reasons for non-engagement, and increased dismissals/attrition led to a pilot re-branding focused on student learning, with an academic mandate for students deemed 'at risk'. Success was indexed by reduced failed exams and re-exams, increased attendance and peer tutoring, and perseverance. The practice continues, now embedded in a Center for Student Learning, with co-curricular 'credit' for professionalism (tutees), leadership and service (tutors). 


\section{American Journal of Pharmaceutical Education 2018; 82 (5) Article 7158.}

Motivators and Barriers to Improve the Response Rate of Student Evaluations of Teaching. Velliyur Viswesh, Roseman University of Health Sciences, Catherine Cone, Roseman University of Health Sciences, Vasudha Gupta, Roseman University of Health Sciences, Elizabeth J. Unni, Roseman University of Health Sciences. Objectives: Student evaluation of teaching (SET) is used at nearly all US pharmacy schools. For Roseman University College of Pharmacy, the average SET response rate was $24 \%$ for academic year 2015-2016. The first aim of the study was to identify motivators and barriers to SET response rate and to identify strategies to increase the response rate. The second aim was to determine if the implemented strategies improved response rates to SETs. Method: The study used a mixed methods design using modified Delphi technique across four phases. In phases 1 and 2, motivators and barriers for completing SETs and potential remediation strategies were identified from students and consensus was formed. Phase 3 used Assessment Committee and phase 4 used faculty to refine and reach consensus with all the identified motivators, barriers, and potential strategies. All the strategies were implemented in 2015-16 academic year and response rates to SETs before and after implementation was analyzed using t-tests to determine if there was a difference in the response rates. Results: The strategies implemented based on the identified barriers were decreasing the length of the survey and rating scale, using validated SET items to decrease ambiguity, educating students and faculty on SETs, and incentivizing for completion of SET. The overall response rate improved significantly from an average of $24 \%$ to $66 \%(\mathrm{p}<0.001)$ after the changes. Implications: Engaging students and faculty resulted in a significant improvement in SET response rates which can be adopted by other schools struggling with low SET response rates.

Perceived Stress Comparison of Student Pharmacists With or Without a Bachelor's Degree. Miriam C. Purnell, University of Maryland Eastern Shore, Frederick R. Tejada, University of Maryland Eastern Shore, Terence N. Nkunbuma, University of Maryland Eastern Shore, Vu Nguyen, University of Maryland Eastern Shore. Objectives: To investigate and compare the perceived stress of student pharmacists (SP1, SP2, and SP3) who earned or did not earn a bachelor degree prior to enrollment in a 3-year program. A secondary objective was to determine the nature of coping strategies employed by pharmacy students to alleviate stress. Method: A link to an online survey was sent to 160 pharmacy students. The 13 question online survey included the validated 10-item Perceived Stress Scale, demographic information, degree status, assessment performance, and major stressors and coping mechanisms. SP1 and SP2 students were given time to complete the survey in class. SP3 students were invited to participate via email and completed the survey remotely. The survey was kept open for 5 days. Statistical analyses were completed using Minitab. Results: A total of $136(82.5 \%)$ responses were analyzed. The students at UMES School of Pharmacy had more perceived stress than the norm for the 18-29 age group. There was no statistically significant difference between students with or without a Bachelor degree in terms of perceived stress levels $(p=0.83)$. The students' ways to cope with stress included sleeping, personal time alone, watching TV, and exercising. Implications: With a rigorous 3-year concentrated pharmacy program, UMES-SOP should find ways to support and encourage stress relief amongst students.

Pharmacy College Admission Test Utilization and Minimum Composite Scores in Pharmacy Programs 2016 to 2018. Mark A. Gales, Southwestern Oklahoma State University, Donald K. Woodward, Rutgers, The State University of New Jersey. Objectives: To analyze Pharmacy College Admission Test (PCAT) usage and minimum composite score requirement trends for U.S. Doctor of Pharmacy Programs. Method: The PharmCAS website was reviewed for PCAT exam requirements and minimum composite scores considered for all U.S. Doctor of Pharmacy programs for the 2016 and 2018 admission cycles. Utilization trends for programs requiring PCAT for both the 2016 and 2018 were evaluated. Results: In 2016, 77\% of programs required PCAT versus $73 \%$ in 2018. Only 31\% of PCAT-requiring programs listed a minimum composite (range 20-60). Approximately $1 / 3$ of PCAT-requiring institutions provided no response or N/A to the minimum composite field. The remaining programs recommended preferred scores or gave guidance on competitive scores. Public institutions required the PCAT more frequently and were more likely to require minimum scores while private institutions include more preference or guidance statements. A total of 30 programs altered PCAT requirements between 2016 and 2018. Twenty-four lowered PCAT requirements; 10 programs stopped requiring the exam, 5 lowered minimum scores, 3 changed a minimum to a preference given and 3 programs removed a preference statement and 3 removed a minimum score. Six programs raised requirements; 2 added the exam, 3 added minimum scores, and 1 provided a guidance statement. Public and private institutions made PCAT requirement changes at a similar rate of $21 \%$. Implications: Admission PCAT requirements from 2016 to 2018 varied. Programs making changes were four times more likely to lower requirements (no longer require the exam or lower/eliminate minimum scores) thus expanding their qualified admission pool.

Pharmacy Training, Confidence, and Career Goals in Underrepresented Minority Students. Ami D. Patel, University of North Carolina at Chapel Hill, Jacqueline McLaughlin, University of North Carolina at Chapel Hill, Antonio A. Bush, University of North Carolina at Chapel Hill, Kate Zhang, University of North Carolina at Chapel Hill, Anita Jackson, University of North Carolina at Chapel Hill, Kimberly P. Lewis, Campbell University. Objectives: This study examined pharmacy training, confidence, and career goals in students identifying as underrepresented racial minorities (URM) currently enrolled in a Doctor of Pharmacy (PharmD) program. Method: PharmD students at the business session of 2017 Student National Pharmaceutical Association annual meeting were invited to complete a survey about pharmacy experience, confidence, and career goals. Data were analyzed using descriptive statistics, independent t-tests, and chi-square. Results: A total of 147 participants completed the survey. The average participant was a third year $(n=63,42.9 \%)$ African American $(n=56,38.1 \%)$ female $(n=114,77.6 \%)$. Sixty-nine $(46.9 \%)$ of the participants identified as URM (ie, African American, Hispanic/Latino, American Indian/Alaska Native, or Native Hawaiian/Pacific Islander. The frequency with which URM students engaged in professional activities during PharmD training in various pharmacy settings (e.g. community, hospital, research, industry) did not differ from nonURM students. On a scale of 1-(not confident at all) to 10-(highly confident), URM students indicated statistically higher confidence (mean \pm standard deviation) than non-URMs in ability to obtain a job in community retail $(9.4 \pm 1.0$ vs $8.3 \pm 2.0)$, hospital pharmacy $(7.6+1.7$ vs $6.5+2.2)$, academic research $(5.1+2.2$ vs $4.1+2.3)$, and pharmaceutical industry $(4.9+2.5$ vs $3.7+2.4)$. URM students comprised $43 \%(n=15)$ of all participants indicating career goals in community retail, $47 \%(n=41)$ in hospital, $4 \%(n=1)$ in academic research, $42 \%(n=5)$ in academic teaching, and $60 \%(\mathrm{n}=12)$ in industry. Implications: There were differences in the confidence levels and career goals of URM when compared to their counterparts. The results of this study may be useful for informing career and professional development opportunities. 


\section{American Journal of Pharmaceutical Education 2018; 82 (5) Article 7158.}

\begin{abstract}
Students Exploring Pharmacy (STEP): Developing \& Assessing a Pharmacy Career Exploration Program for High School Students. Curtis G. Jefferson, University of Washington, Andrew Brusletten, University of Washington, Cherelyn Espina, University of Washington, Allison M. Stephens, University of Washington, Peggy S. Odegard, University of Washington. Objectives: To engage high school students in hands-on activities that provide an introduction to pharmacy practice, inspire exploration of pharmacy and health care as a career option, and provide perspective on the ways pharmacists contribute to improving healthcare outcomes through a day camp model. Method: A four-day summer program, Students Exploring Pharmacy (STEP), employing informational and active learning sessions, such as hands-on skill exploration, problem-based learning activities, and group presentations was developed. A patient case activity was threaded through the program to better illustrate the various ways pharmacists work with patients and their families and to contextualize what students were learning. Results: The majority of student participants $(64 \%)$ reported their interest in a career in pharmacy increased through their participation in STEP. Of those who indicated their interest stayed the same (29\%), over half $(60 \%)$ reported their interest in pursuing a career in health care in general increased. Students found the active learning components most beneficial and suggested finding ways to increase active learning in future iterations of the program. Implications: Students report their participation in STEP helped them to better understand pharmacy and health care practice and to increase their interest in pursuing a pharmacy and/or health care career. Active learning strategies were effective in engaging high school student participants and opportunities to expand active learning should be explored for future years. The four-day program model is adaptable and transferable to other schools of pharmacy and may also be modified for other target audiences (community colleges, local undergraduates, etc.).
\end{abstract}

Students' Perception of Computer-Based Testing Using ExamSoft. Gauri Sabnis, West Coast University, Amber Verdell, West Coast University, Jennica Zaro, West Coast University, Mohammed A. Islam, West Coast University. Objectives: In Fall 2017, West Coast University School of Pharmacy implemented ExamSoft for testing. Three courses in each didactic year employed ExamSoft. Prior to this, courses had Scantron-based written exams. In response to this change, students expressed concerns about their grades being lowered. Furthermore, some students seemed to find no usefulness in computerbased testing. We hypothesized this was due to their inherent bias toward technology and its use in exams. Method: To assess this hypothesis, we conducted a survey of all students. The survey contained questions about comfort with technology and four questions on students' perceptions of ExamSoft and its effect. The survey responses were stratified according to the preference of respondents towards technology and its use in exams. Respondents were stratified into 3 groups: tech-embracers (answered - I love trying new technology, and I prefer to save paper and read on a screen) or tech-skeptics (answered - I hate having to learn new technology, and I prefer paper over electronics for any type of reading) or neutral. Results: Results showed that respondents classified as tech-skeptics tended to have a more negative view of ExamSoft and its perceived impact on their grades (59\% of tech-skeptics said their grades were slightly/ significantly reduced) than students who identified themselves as tech-embracers $(29 \%)$ or neutral $(27.7 \%)$. $52 \%$ of tech-skeptics agreed/strongly agreed that computer-based testing helps them prepare for licensure exams, while technology-embracers and neutral respondents ( $87 \%$ and $88 \%$ respectively) believed that ExamSoft would benefit them. Implications: Students' perception of ExamSoft is based on their inherent bias towards use of technology.

\section{Theoretical Models}

A Method to Streamline Management of Excused Absences. Shannon R. Tucker, University of Maryland, Chris Klimas, University of Maryland, Andrew Coop, University of Maryland, Cherokee Layson-Wolf, University of Maryland. Objectives: Improve assessment of the impact of student absences on program delivery, and responsiveness to students at risk of academic difficulty due to external issues such as illness. Ensure that policies related to student absences are enforced consistently. Method: The University of Maryland School of Pharmacy has long had a policy regarding student absences. Implementation of this policy, however, was the responsibility of individual instructors. Because of this, it was difficult to assess the impact of student absences both on a programmatic and individual basis. A policy was enacted creating a centralized process in which students submit excused absence requests to the Offices of Academic Affairs and Student Affairs. The two offices decided to track data related to requests in a dedicated web-based system instead of email or paper records. Results: A web-based form and associated tracking system was created to manage absence requests. Once submitted, the web system facilitates private discussion of requests by the associate deans of the two offices, communication with the student requestor, and notifications to instructors once a decision is made. The system also allows for reporting on aggregate data, such as type of request and length of absence. It also can help identify students who may be inappropriately using requested absences. Implications: Centralizing excused absence request handling in an administrative office can be accomplished with a relatively low amount of initial effort. In addition to increasing aggregate analytic capabilities as outlined above, recording the history of student requests in a dedicated space improves institutional memory.

Development and Implementation of Co-Curricular Requirements in a School of Pharmacy. Aida Oganesyan, West Coast University, Jessica Louie, West Coast University, Sarian A. Bangura, West Coast University, Anna Babakhanyan, West Coast University, Kyle Sousa, West Coast University. Objectives: To design, implement, and test the feasibility of co-curriculum requirements that enhance students' development of the knowledge, skills, abilities, and attitudes for self-awareness, leadership, innovation, entrepreneurship, and professionalism. Method: A Co-Curriculum Committee comprised of faculty and student representatives was formed to develop cocurricular learning outcomes, co-curricular requirements, and assessment criteria for a four-year Doctor of Pharmacy program. Results: The co-curriculum relates to activities that complement the academic learning experience and includes activities that engage students with opportunities to learn and refine technical, affective, and cognitive skills. Here, we describe the design of co-curricular requirements that articulate the minimum number of activities and reflections students must complete across the core categories of leadership, selfawareness, professional development, and innovation/ entrepreneurship. Attendance and co-curricular activity assessments are documented in RxPortfolio, assessed by faculty advisors, and tied to student progression through a pass/fail zero-credit course. Implications: The ACPE Standards 2016 require Schools of Pharmacy to implement a co-curriculum program. Additionally, the current job market values the qualities developed through the co-curriculum. The co-curriculum described herein, may be leveraged and modified by Schools/Colleges of Pharmacy as they seek to execute their respective co-curricula. 


\section{American Journal of Pharmaceutical Education 2018; 82 (5) Article 7158.}

Help Wanted: Trends in the Pharmacist Workforce and Pharmacy Education. Lisa Lebovitz, University of Maryland, Natalie D. Eddington, University of Maryland. Objectives: The objective is to review seismic shifts in pharmacist supply and demand, and urge immediate action. Method: Pharmacy education and pharmacist occupation data were compiled and compared. Results: US Bureau of Labor Statistics (BLS) ten-year growth projections for the pharmacist occupation have plummeted from 30.1\% projected growth between 2002-2012 (more than double the national employment growth projection) to only $5.6 \%$ between 2016-2026 (25\% below the national employment growth projection). The Pharmacist Demand Index (PDI) also indicates declining demand. Pharmacist employers are restructuring employment to save on labor costs instead of implementing more value-based business models; pharmacist salaries are plateauing and part-time positions are increasing. Pharmacy school applications have declined steadily since 2010, and yet pharmacy schools continue to increase supply. At current rates over the next ten years, $\sim 145,000$ new pharmacists will be looking to replace nearly half of the 312,500 currently-practicing pharmacists. Pharmacy student debt increased $30 \%$ in the last five years, and $2000+$ first-time testers failed the NAPLEX in 2016. Implications: Meanwhile, pharmacist patient care is expanding and transitioning from fee-for-service to value-based care should make sense to employers. Retail settings must accelerate changes in their current business model to seize upon new patient-focused revenue opportunities. Schools must accept only the highest quality applicants to train for these new practice models and maintain the profession's public trust. Together the schools, associations, and employers can change the trajectories of supply and demand if they take immediate and significant actions to tip the balance in favor of pharmacists of the future.

Streamlining Exam Administration Through Continual Stakeholder Engagement and Training. George Anagnostou, University of Maryland, Shannon R. Tucker, University of Maryland, Jenna Goldberg, University of Maryland, Andrew Coop, University of Maryland. Objectives: The objective of this project was to use a multi-phased training approach to improve examination administration and user preparedness. Method: Using comprehensive guides, one page sheets, in person trainings, and practical application of skills, the School developed a model for effective training and support. Training consisted of two separate phases of examination administration. The first phase focused on high level concepts, such as examination policy, and the second on the development of practical skills. To develop and refine skills, trainees completed a skills assessment within the ExamSoft portal receiving feedback throughout the process. Results: This multi-phased approach resulted in trainees having a better grasp of examination policy as well as an increased proficiency with the ExamSoft portal. The completion of a skills assessment provided trainers with insight into each individual's abilities as well as an awareness of common trouble areas. This informed the creation of targeted communications, refined training approaches, and proactive consultations to improve the exam administration abilities of faculty, staff, and teaching assistants. Implications: The success of this methodology has helped establish a repeatable approach to exam administration training that can improve exam reliability and reduce support requirements.

The Development of an Academic Success Center With the Implementation of an Early Alert Process. Joseph V. Etzel, St. John's University, Russell DiGate, St. John's University, Diana J. Patino, St. John's University. Objectives: The College's Academic Success Center (ASC) was developed to provide academic support and tutoring for all students enrolled in the College pertaining to their professional coursework. Additionally, a multifaceted Early Alert process was put into place to identify at-risk students to ensure identification and intervention Method: The center was developed to provide tutoring and workshops for pharmacy students in their professional years. Doctoral fellows, peer pharmacy (P4) graduate assistants, and faculty provide individual, group tutoring, and academic workshops focused on course specific topics, general study skills, and other topics. At-risk students can be identify through a multifaceted Early Alert Process, monitoring of in-class assessments and an electronic faculty driven notification system that allows for appropriate intervention which can include tutoring or workshops. Student are able to self-enroll in any of the ASC programs. Results: In 2016-17, 136 students visited the ASC for an excess of 1000 hours of tutoring. This past semester the Center served a total of 156 students receiving 619 hours of tutoring. Eight workshops were held this semester servicing 188 students. A student survey demonstrated $93 \%$ were either satisfied or very satisfied with their experience. Since implementations of these services, the program's graduation rate and on-time graduation rate has increased by $6 \%$ and $17 \%$, respectively. Implications: The implementation of these student centered services system was well received and proved to be beneficial. Continued growth of the services provided by the ASC will likely assist in assuring a higher rate of academic success for our students.

The Development of a Student Congress to Improve Communication, Promote Collaboration and Foster Interprofessional Activities. Diana J. Patino, St. John's University, Francis A. Ferraiuolo, St. John's University, Russell DiGate, St. John's University, Joseph V. Etzel, St. John's University. Objectives: The College of Pharmacy and Health Sciences developed a Student Congress to improve the communication and coordination of student-related activities and events between students, student organizations, faculty and the administration of the college as well as develop interprofessional activities. Method: The Student Congress membership includes the Presidents and Vice Presidents of 16 Pharmacy student organization, 1 Toxicology organization, 1 physician assistant organization, 1 Clinical Laboratory Sciences organization, and 1 Radiologic Sciences organization. In addition, 1 elected representative from each professional year for each of the above majors serves in the congress. Members of the student congress have formal bi-weekly meetings to discuss pertinent student concerns, discuss event strategies and the members are responsible for communicating back to the student body. In addition, a shared calendar was developed for all faculty, administrators, staff and students of the college that lists all student events and interprofessional activities. Members of the student congress were provide with additional opportunities to build on their own leadership skills. Results: In its first year, 40 co-sponsored events were developed and promoted by the student congress. These include: fundraising events, Giving Tuesday, community services, interprofessional clinical challenges, leadership panels, careers panels, residency and fellowship showcases. Implications: The development of the student congress enhanced communication and cooperation amongst the student organization within the college. Additionally, the student congress served to facilitate improved communication between the students, faculty and administrations of the student congress. Continued efforts will help the college in meeting the 2016 AACP standards of professionalism, interprofessional education and co-curricular activities.

\section{BIOLOGICAL SCIENCES}

Completed Research

A Combination Regimen for Overcoming Acquired-Resistance in Pancreatic Cancer Using Piperlongumine as Adjunctive Therapy. Terrick A. Andey, MCPHS University-Worcester/Manchester, Yasser 


\section{American Journal of Pharmaceutical Education 2018; 82 (5) Article 7158.}

Alharbi, MCPHS University-Worcester/Manchester, Wonwoo Chung, MCPHS University-Worcester/Manchester, Hyeyeun Heacock, MCPHS University-Worcester/Manchester. Objectives: An estimated 55,440 new pancreatic cancer cases and 44,330 deaths will occur in the United States by the end of 2018. Gemcitabine, erlotinib and gefitinib are approved for treating pancreatic cancer, however, acquired-resistance limits their long-term clinical efficacy. We investigated the anticancer efficacy of these therapies in a two-drug combination regimen with piperlongumine, a natural plant extract as a therapeutic strategy for overcoming acquired-resistance in pancreatic cancer. Method: Piperlongumine was investigated across a panel of pancreatic cancer cell lines (Capan-1, Panc-1 and MIA-PaCa-2) using the Resazurin dye-uptake assay for cell viability at $72 \mathrm{~h}$. Combination regimen of piperlongumine with either gemcitabine, erlotinib or gefitinib were tested in MIA$\mathrm{PaCa}-2$ cells for cell viability by fixing the concentration of piperlogumine $(0.001 \mu \mathrm{M}$ and $0.1 \mu \mathrm{M})$ and varying the concentration of the other drug, or vice versa. Result were analyzed and presented as concentration-response plots, and as the mean drug concentration that inhibits $50 \%$ of cell population (IC50). Results: Piperlongumine had an IC50 of $10.69 \pm 1.08 \mu \mathrm{M}, 6.62 \pm 0.14 \mu \mathrm{M}$ and $8.02 \pm 0.88 \mu \mathrm{M}$ in Capan-1, Panc-1 and MIA-PaCa-2 cells respectively. Piperlongumine was more potent compared to gemcitabine (IC50: $20.39 \pm 4.42 \mu \mathrm{M}$ ), erlotinib (IC50: $19.38 \pm 2.52 \mu \mathrm{M}$ ) and gefitinib (IC50: $11.88 \pm 1.28 \mu \mathrm{M}$ ) against MIA-PaCa-2 cells. Significantly, low-dose gemcitabine, erlotinib or gefitinib in combination piperlongumine was more effective in inhibiting MIA-PaCa-2 cell viability compared to single treatments. Implications: Piperlongumine is a potent natural plant extract that may be useful in adjunctive treatment of drug resistant pancreatic cancer.

Antihypertensive Effect of Thymoquinone in Rats: Role of Arachidonic Acid Metabolism. Fawzy A. Elbarbry, Pacific University Oregon, Aimy Ung, Pacific University Oregon, Saleh Batroukh, Pacific University Oregon. Objectives: We investigated the effects of thymoquinone (TQ), a Quinone phytochemical in the plant Nigella Sativa, on Arachidonic acid (AA) metabolism in the kidney and its effect on arterial blood pressure, using spontaneously hypertensive rats (SHR) as our animal model system. Method: Rats were treated for 7 weeks with TQ $(10,20$, or $40 \mathrm{mg} / \mathrm{kg})$ added to their drinking water; drinking water alone was used as our control. Mean arterial pressure (MAP) was measured at 7-day intervals throughout the study. At the end of the treatment, rats were euthanized, followed by preparation of kidney microsomes to measure the activity (using LC/UV and fluorescence assay) and expression (using immunoblotting) of enzymes involved in regulation of vasoactive metabolites: CYP4A, the key enzyme in the formation of 20-hydroxyeicosatetraenoic acid, and the soluble epoxide hydrolase $(\mathrm{sEH})$, which is responsible for the degradation of the vasodilator metabolites such as epoxyeicosatetraenoic acids. Results: We found that treatment with TQ leads to significant reductions in both the expression and activity of renal CYP4A isozyme. Only the high dose TQ resulted in a significant reduction in the expression and activity of renal $\mathrm{sEH}$. Consistent with these data, we have found that treatment with TQ resisted the progressive rise in MAP in the developing SHR in a dose-dependent manner. Implications: This is the first demonstration that TQ modulates the metabolism of AA by both kidney P450 enzymes and sEH in SHR rats. This may represent a novel mechanism by which TQ protects SHR rats against the progressive rise in blood pressure.

Design and Development of a Translational Research Elective Curriculum for Pharmacy Students. Paramita Basu, Touro College of Pharmacy-New York, Batoul Senhaji-Tomza, Touro College of Pharmacy-New York, Bupendra Shah, Touro College of Pharmacy-New
York, Zvi Loewy, Touro College of Pharmacy-New York. Objectives: A research-based sequence of electives was designed to provide pharmacy students with training in research methodology and scientific presentation to prepare them for research focused career opportunities. Method: A 3-course elective sequence was designed with a focus on translational research and develops research, analytical and scientific presentation skills. The first course provides training in the fundamental methods required for translational research. The second course applies the research methods to a short term scientific research project. The third course teaches the art of presenting and communicating scientific concepts and articulating the significance of the outcomes in both oral and written formats. Results: 16 PY2 and PY3 Students participated in the first course performing a number of hands-on experiments that included quantitative and qualitative analysis of macromolecules as well as application of cell culture and microbiology experimental approaches for different research questions. Students were evaluated on their knowledge of experimental design, scientific calculations, accuracy of technique, results, productivity and professionalism. Assessments included laboratory reports and examinations. Students evaluated the course at the end of the semester using a course evaluation survey. Students completing the course demonstrated elevated levels of knowledge of laboratory practices and technical skills, and increased interest in pursuing research careers within the pharmaceutical or biotechnology industry. Implications: A 3-course research sequence was successfully designed and implemented that allows PharmD students in the traditional 4-year program to participate in biological and pharmaceutical sciences research. Students report that the elective sequence enhanced their critical-thinking and problemsolving skills and helped them develop as independent learners.

Differential Expression of Nanoparticle-Induced High Mobility Group Box-1 in Human Hepatocyte Cell Line HC04. Christina Petrelis, University of Saint Joseph, Maria Valanca, University of Saint Joseph, Sangamesh Kumbar, University of Connecticut, Swetha Rudraiah, University of Saint Joseph. Objectives: Numerous products that are already on the market claim to have used nanotechnology in manufacturing processes. Nanoparticles can be toxic because of their size and exposure through air, food and the cosmetics/clothing. There is an increasing concern regarding their potential health hazards. Nanoparticles that enter the circulation end up in the liver and are trapped in the reticulo endothelial cells. Their potential toxic effects in hepatocytes are unknown. High mobility group box-1 (HMGB1) is a nonhistone nucleoprotein that regulates transcription. It plays an important role in many cellular processes including inflammation, cell differentiation and tumorcell migration. It is also considered a mechanistic marker of liver injury. Here, we report the effects of multiwalled carbon nanotubes (MWCNT) and titanium dioxide (TiO2) on HMGB1 expression in hepatocytes using a hepatocyte cell line (HC-04). Method: The acute cytotoxicity was quantified by measuring the LDH leakage into the media. In addition, hepatocellular HMGB1 protein expression following a single or repeated treatment was measured by western blotting. Results: A single MWCNT treatment did not significantly increase LDH leakage both at 24 and $48 \mathrm{~h}$. However, the cellular expression of HMGB1 protein increased significantly, $72 \mathrm{~h}$ after MWCNT treatment. No secreted HMGB1 was detected in the media. Similar results were seen with $\mathrm{TiO} 2$ treatment. Repeated MWCNT treatment for two weeks also increased HMGB1 protein expression significantly. Implications: Taken together, increased HMGB1 expression following MWCNT or TiO2 exposure is suggestive of mild hepatocellular injury. The functional consequences of HMGB1 expression after MWCNT expression in hepatocytes is unknown and is currently being investigated. 


\section{American Journal of Pharmaceutical Education 2018; 82 (5) Article 7158.}

Essentials of Pharmacogenomics for a PharmD Curriculum: A Method for Prioritizing Content. J. Shawn Jones, The University of Texas at Tyler, David F. Kisor, Manchester University, David R. Bright, Ferris State University, Heather Sibal, The University of Texas at Tyler. Objectives: Although ACPE requires inclusion of pharmacogenomics (PGx) into every PharmD curriculum, little guidance exists for the specific content. PGx coverage varies between institutions from minimal inclusion to stand-alone courses. The goal of this article is to provide faculty with a method for prioritizing the multitude of available PGx information that they may use to custom fit the information into the allocated time for PGx education in their PharmD curriculum. Method: The FDA Table of Pharmacogenomic Biomarkers in Drug Labels, Sigler's Top 200 Drug List and published guidelines from the Clinical Pharmacogenetic Implementation Consortium (CPIC) were analyzed to prioritize pharmacogenomic responses into a tier system. Results: Drugs were categorized into 4 tiers of importance for inclusion into a PharmD curriculum. The top tier consists of 11 drugs meeting 3 criteria: 1) FDA requires inclusion of PGx information in their drug labels; 2) they are included in Sigler's Top 200 Drug List for most prescribed drugs; and 3) they have published guidelines from CPIC that instruct pharmacists in how to translate a patient's genotype into an appropriate clinical decision for recommending adjustments to therapy. Implications: Institutions with limited time dedicated to PGx education could use the tier system as a guide for including PGx education into their curriculum, ensuring that their PharmD students will, at a minimum, receive education on the most commonly prescribed drugs with FDA-mandated PGx drug labeling and published guidelines for clinical decisions.

Forensics for Pharmacy Students, Really? Arturo G. Marti-Ortiz, University of Puerto Rico. Objectives: To offer an elective course on principles of forensic sciences that enhance the knowledge of pharmacy students (P3); to obtain student perceptions as to relevance of forensic topics to their pharmacy education. Method: Academic activities demanded active-participation. A reflection of each activity was required. The course commenced with an analysis for scientific correctness of a TV episode of CSI. Classroom lectures included: methods of analysis, drug variations depending on body collection sites, post-mortem redistribution (PMR), cause of death (COD), and expert testimony. Autopsy reports were analyzed by the class. Each student presented three forensic research papers. Marijuana legalization was discussed in an adversarial scenario. State forensic laboratories and Drug Court were visited. Class project was the development of a 21 st Century Drug Policy. Results: Students perceptions and reflections overwhelmingly favored inclusion of such a course or components into the pharmacy curriculum. Representative student reflections: the topics presented were useful to our professional development; the course offered a new perspective of pharmacy that I did not think possible; the subject matter is not covered in courses such as pharmacology or therapeutics; I will recommend this course to future students; I learned in detail about opioids, legal and illegal use and consequences; I plan to apply concepts learned to my pharmacy practice; the course experience has been unique showing me areas in forensics were a pharmacist can have influence. Implications: In the increasing complicated paradigm of drug use, knowledge of forensic concepts augments the possibility of new roles for pharmacists.

Impact of a Stepwise Approach to Re-Design Pre-APPE Assessment on Student Performance. Ligia Westrich, Fairleigh Dickinson University, Shreya Patel, Fairleigh Dickinson University, Lillian Rozaklis, Fairleigh Dickinson University, Anastasia M. Rivkin, Fairleigh Dickinson University. Objectives: To re-design pre-Advanced Pharmacy Practice
Experience (APPE) assessment on Identification of Drug Related Problems (IDRP) using a stepwise approach to improve student performance. Method: Readiness for APPE is evaluated using 11 skilland/or knowledge-based pre-APPE assessments embedded in didactic and Introductory Pharmacy Practice Experience (IPPE) courses and patient care laboratories. During review of trended data from two cohorts, the assessment committee identified a decline in the first attempt pass rate for IDRP assessment. A working group was formed to further analyze the current state of IDRP assessment and propose an action plan to remedy the decline in pass rate. Working group followed a stepwise approach to re-design the assessment, incorporating: pre-specified guidelines and checklists for creating patient vignettes (assessment instrument), a process for rigorous and in-depth review of cases, integration of practice-based patient cases into didactic curriculum, and a priori review session. Results: First attempt pass rate for current IDRP pre-APPE assessment was compared to trended data from prior administrations to evaluate impact of re-design. Cumulative first time pass rates increased from $76 \%$ to $80 \%$. The number of students who performed as strong (answered 3 out of 3 cases correctly) increased from $15.5 \%$ to $26.6 \%$. A similar trend of improved performance is predicted with future cohorts. Implications: Comprehensive evaluation of student performance longitudinally has informed important changes to the development and administration of IDRP pre-APPE assessment. Lessons learned from this approach are transferable for on-going improvement of all pre-APPE skills and knowledge-based assessments.

Inhibition of Akt-Survivin Pathway Synergizes the Cell Death Caused by Alpha-Santalol in Human Prostate Cancer Cells. Ajay K. Bommareddy, Wilkes University, Adam VanWert, Wilkes University, Chandradhar Dwivedi, South Dakota State University. Objectives: The present study was undertaken to investigate the mechanistic details associated with the induction of apoptosis by $\alpha$-santalol in cultured human prostate cancer cells (LNCaP and PC-3). The main objectives were (1) to examine the relationship between survivin and PI3K-Akt pathway in the induction of apoptosis by $\alpha$-santalol in prostate cancer cells. (2) to investigate the effect of PI3k/Akt pathway inhibition by pharmacological inhibitor Ly294002 on apoptosis induced by $\alpha$-santalol. Method: Expressions of major proteins studied were determined using standard Western blot protocol and analyzed by LICOR-Odyssey infra-red scanner. Levels of survivin was confirmed employing survivin ELISA kit. The cell viability was determined by trypan blue dye exclusion assay and caspase- 3 activity was confirmed using caspase-3 (active) ELISA kit. Results: Treatment of prostate cancer cells with alpha-santalol $(20,40 \mu \mathrm{M})$ resulted in the down regulation of survivin, XIAP and p-AKT (s-473) expressiion. Alpha-santalol also significantly reduced the levels of survivin as determined by survivin ELISA assay. Inhibition of PI3K-Akt pathway by pharmacological inhibitor LY294002 synergized the apoptotic cell death induced by $\alpha$-santalol as examined by cell viability, active caspase-3 activity and expression of cleaved PARP, caspase-3 levels. Implications: The study revealed that $\alpha$-santalol downregulates levels and expression of survivin and that inhibition of PI3K/Akt pathway synergizes the apoptotic cell death in human prostate cancer cells and warrants preclinical studies employing relevant in vivo models.

Inhibition of Protein Disulfide Isomerases Suppresses Mast Cell Activation and Function During Food Allergy. Stephanie Polukort, Western New England University, Dylan Krajewski, Western New England University, Edwin Kaczenski, Western New England University, Jeffrey Rovatti, Western New England University, Daniel R. Kennedy, Western New England University, Clinton Mathias, Western 


\section{American Journal of Pharmaceutical Education 2018; 82 (5) Article 7158.}

New England University. Objectives: Mast cell activation is a crucial step in the induction of allergic responses, resulting in the release of numerous proinflammatory cytokines. Thiol isomerases such as the oxidoreductase-protein disulfide isomerase (PDI) play critical roles during protein folding and also act as molecular chaperones during immune responses. We therefore hypothesized that mast cell activation and cytokine production will be attenuated in the absence of PDI induction, leading to the suppression of mast cell-mediated allergic responses. Method: Bone marrow-derived mast cells (BMMCs) were treated with the known PDI inhibitors bacitracin, rutin and PACMA-31 and mast cell activation was assessed. Similarly, wildtype mice were sensitized and challenged with ovalbumin and treated with PACMA-31 to determine whether PDI inhibition can modulate the development of food allergy. Results: Activation of BMMCS via $\mathrm{IgE}$ and antigen resulted in the enhanced expression and secretion of IL-4, IL-6, TNF-alpha and IL-13. In contrast, pretreatment with the PDI inhibitors resulted in decreased expression and secretion of these cytokines. Furthermore, treatment of wild-type mice with PACMA-31 in a model of ovalbumin-induced food allergy resulted in a significant attenuation in the development of food allergy symptoms including decreases in allergic diarrhea, mast cell activation and allergenspecific IgE. Implications: These data therefore suggest that mast cell activation during allergic responses may be regulated by PDI induction, and that blocking PDI function may prove to be of therapeutic benefit in allergic patients.

Metacognitive Awareness in Entering P1 Students. Margaret A. Weck, St. Louis College of Pharmacy, Chris N. Thai, St. Louis College of Pharmacy, Claude Gaebelein, St. Louis College of Pharmacy, George Vineyard, St. Louis College of Pharmacy. Objectives: To examine the potential relationship between metacognitive ability and academic success at the St. Louis College of Pharmacy. Method: Schraw \& Denison's Metacognitive Awareness Inventory (MAI) (1994) was administered in the fall of 2014 to incoming undergraduate students $(\mathrm{N}=92)$. The survey was then administered to all incoming P1 students early in fall $2017(\mathrm{~N}=252)$. Grade data was compiled by the VP for Institutional Research. Approval for data collection in 2014 was obtained [STLCOP IRB \# 2014-15] and renewed in 2017. Results: Data presented here are from the 93 students (48\% of 2014 Freshman class) comprising the "test-retest" group completing the MAI twice. There was no statistically sig. change in MAI scores between the first administration (ave. MAI score 4.03) and re-test (ave. MAI score 3.75) $p>0.05$. But small and statistically significant $(p<0.05)$ correlations were shown to exist between the 2017 MAI score and GPAs of the two preceding semesters; Fall 2016 GPA $(r=0.428)$ and Spring 2017 GPA $(r=0.291)$. This relationship was also seen for the knowledge of cognition and regulation of cognition sub-scores of the MAI in both semesters. Implications: Previously we have shown that the MAI scores were not correlated with the ACT scores of 2014 incoming freshmen, nor earned GPAs in their Freshman year ( $\mathrm{r}=0.013)$. But for this cohort there is an interaction between MAI scores and grade performance in the Junior year. Explicitly developing metacognition may help increase student success and persistence in degree programs.

Pharmacy Student Attitudes Towards Substance Use: An Analysis of Attitude Change Following an Elective Course Intervention. Wenola Tauro, Western New England University, Emily T. Cramer, Western New England University, Allissa M. Long, Western New England University, Jilla Sabeti, Western New England University. Objectives: This study implemented a standardized Substance Abuse Attitude Survey (SAAS) to measure explicit attitudes of early-level pharmacy students towards social drug use and substance users. The main question investigated was whether exposure to a semester-long drug abuse pharmacology course was sufficient to alter substance use attitudes towards alcohol, marijuana, and other substances. Method: Participants were pharmacy student volunteers $(\mathrm{N}=47)$ either enrolled (intervention group) or not enrolled (control group) in a 15-week elective course on drug abuse pharmacology. A previously validated SAAS instrument with 49 Likert-type questions was administered online on the first (baseline) and final week and data collected anonymously in both groups. Parametric mean scores were analyzed for statistical significance using the Sidak corrected t-tests. Results: At baseline, preexisting attitudes between the two groups were similar with $20-35 \%$ of students reporting highly permissive attitudes toward social drug use. At semester's end, a marked attitude shift took shape such that students completing the elective course showed significantly less tolerant attitudes towards social drug use, both in those experimenting and those with a substance use disorder. A decreased acceptance towards marijuana use was seen in the intervention group, whereas the control group reported increased openness towards cannabis use by semester's end. Stereotyped attitudes towards drug users, however, were not altered substantially in either group. Implications: These data suggest that even brief exposure to didactic content on drug abuse pharmacology can be of value in inducing attitude change, while certain stereotyped attitudes towards substance users are more resistant to change.

Polyphenol Ellagic Acid Exerts Anti-Inflammatory Actions by Disrupting Store-Operated Calcium Entry Pathway Activators and Coupling Mediators. Matthew Murphy, Western New England University, Xia Quin, Clinton Mathias, Western New England University, Diptiman Bose, Western New England University. Objectives: Pharmacological agents that can modulate calcium homeostasis are being extensively explored as potential anti-inflammatory agents. Ellagic acid (EA), a polyphenolic compound found in many fruits and plant extracts, has been known to possess anti-inflammatory properties. However, the mechanism of action behind its anti-inflammatory action is unclear. EA is thought to modulate calcium homeostasis. However effects of EA on calcium signaling is not clearly understood. We studied the impact of EA on calcium handling, in particular, storeoperated calcium entry (SOCE), a process critical to proper $\mathrm{T}$ cell function. Method: Jurkat T cells were loaded with calcium sensitive dye Fluo-4AM to measure changes in intracellular calcium. HEK293 cells were transfected using lipofectatmine 2000 according to the manufacturer's protocol. Results: Addition of EA produced calcium release $(\mathrm{EC} 50=63 \mu \mathrm{M})$ in Jurkat $\mathrm{T}$ cells in a dose-dependent manner. Incubating Jurkat T cells with EA (12h) decreased the ATP-induced calcium release and diminished the SOCE-mediated calcium influx in a dose-dependent manner $(\mathrm{IC} 50=23 \mu \mathrm{M})$. EA attenuated the ATP-induced calcium influx in EA-treated HEK293 cells transiently transfected with SOCE channel Orai1-myc and ER-sensor stromal interaction molecule (STIM1) (HEKSTIM/Orai). Furthermore, EA interfered with the Orail and STIM1 coupling by disrupting STIM1 puncta formation in the HEKSTIM/Orai cells. EA treatment also reduced cytokine secretion and nuclear factor of activated T-cell transcriptional activity in stimulated $\mathrm{T}$ cells. Hence, by inhibiting SOCE mediated calcium influx, EA decreased downstream activation of proinflammatory mediators. Implications: These observations suggest a novel target to EA-mediated effects and provide insight into the mechanisms underlying EA mediated anti-inflammatory effects.

Reactivation and Retention of Foundational Knowledge in a Third Professional Year Infectious Disease Course. Amanda M. Munson, Shenandoah University, Katrina Petrov, Shenandoah University, Mark S. Johnson, Shenandoah University. Objectives: To assess vertical and 


\section{American Journal of Pharmaceutical Education 2018; 82 (5) Article 7158.}

horizontal retention of foundational microbiology material in a required third-year infectious diseases integrated therapeutics course. Method: Students in a third-year infectious disease pharmacy course were administered a nine-question pre-quiz for bonus points at the start of the course to permit students to self-assess their retention of foundational microbiology material from their first professional year. Students were then provided with review material. The same assessment was administered two weeks later as a quiz and at the end of the course as a post-quiz for bonus points to allow students to self-assess retention of material. Results from nine similar questions on the first-year microbiology exam and the three third year quizzes were analyzed using paired $\mathrm{t}$-tests and ANOVA. Results: There was a significant decrease $(\mathrm{p}<0.01)$ in retention of knowledge from the first-year exam to the third-year prequiz, but a significant increase $(\mathrm{p}<0.01)$ from the third-year pre-quiz to the quiz, and no significant change $(p=0.58)$ in results from the quiz to the post-quiz. Further, there was no significant difference between performance on the first-year exam, the third-year quiz, or the third-year post-quiz $(p=0.50)$. Implications: Reactivation of knowledge from first professional year was achieved with an out-of-class review. Retention of this material persisted to the end of the infectious disease course demonstrating that a brief, student-driven review of material was sufficient to help students recall and retain knowledge from their first to their third professional year.

Student Faculty Partnership in Developing a Leveling Course. Manas Mandal, Roseman University of Health Sciences, Erik Jorvig, Roseman University of Health Sciences, Vijay Kale, Roseman University of Health Sciences, Jeffery Talbot, Roseman University of Health Sciences, Rebecca Keel-Jayakumar, Roseman University of Health Sciences, Helen C. Park, Roseman University of Health Sciences. Objectives: To develop an introductory primer course for incoming P1 students leveling the gap in knowledge base for successful completion of basic biomedical sciences blocks in an accelerated pharmacy program. Method: Focus group discussion with students and faculty led to the approach, identification of the problem and development of the course structure. Under-performing basic biomedical sciences blocks were identified through analysis of nine different assessment data for last three years by a group of P2 students. Voluntary faculty participation created six short learning modules with audio covering the identified areas with pretest and test questions. The modules were released to the incoming P1students prior to attending the class for two weeks. Course use activity and student satisfaction were monitored. Post-use experience was discussed at a P1 focus group. Results: Focus group discussion and assessment data analysis identified the areas of weaknesses in biochemistry and pharmacology with scores at $<60 \%,<30 \%$ or discrepancy in scores $\geq 50 \%$ between upper and lower $27 \%$. Our passing standard is $90 \%$. Course use data shows highest use $(66.16 \mathrm{hr})$ on the day of release that gradually declined to $23.4 \mathrm{hr}$ on day seven with a total use time of $307.29 \mathrm{hr}$ with average $0.72 \mathrm{hr} /$ user. Student satisfaction indicated a high and increasing trend (mean 3.9 and 4.1in Likert scale) for two consecutive years. Implications: Early intervention can be an effective measure to ensure satisfactory academic progression. A properly designed levelling course can bridge the gap in knowledge base leading to academic success with reduction in attrition rates.

Synthesis of Imidooxy Derivatives as Potential Anticancer Agents. Katherine Lee, University of Saint Joseph, Hillary Kuzaro, University of Saint Joseph, Mark A. Sweezy, University of Saint Joseph, Stacy Lin, Cedarville University, Samson Amos, Cedarville University, Ivan O. Edafiogho, University of Saint Joseph. Objectives: The objectives were to synthesize imidooxy compounds and evaluate them in different cancer cell lines at National Cancer institute and at one of our laboratories. Preliminary results from our laboratory indicate that certain imidooxy compounds possess promising anticancer activities against breast, lung, colon, prostate and brain cancer. The most potent compound, E49 (methyl N-phthalimidooxy-2-methylacrylate) has a potential anticancer activity in different cell lines at concentrations ranging from 10 -100 nanomolar. Method: The reaction of $\mathrm{N}$-hydroxyphthalimide with 2-bromomethylacrylate in triethylamine yielded the typical imidooxy analog E49. Using similar synthetic schemes, imidooxy formates, acetates, propionates, and methylacrylates were prepared from N-hydroxynaphthalimide, N-hydroxyphthalimide, $\mathrm{N}$-hydroxysuccinimide, and endo-N-hydroxy-5-norbornene. Eleven compounds AA1, E1, E3, E16, E29, E33, E38, E49, E62, GF1 and KL1 were evaluated for anticancer activity in different cell lines up to 48 hours of exposure. We determined the structural activity relationship with different ring sizes, side chain extensions, and bioisosteric substitutions on the anticancer activity of the imidooxy compounds. Results: In our laboratories, the imidooxies had anticancer activity in T98, U251, and U1242 glioblastoma cancer cell lines at 2.5 to 5 micromolar concentrations. Generally, the phthalimidooxy and naphthalimidooxy compounds afforded the more potent anticancer agents. Our initial in vitro evaluations indicate that E49 does not function as a DNA alkylator or DNA intercalator but it acts to disrupt cell cycle progression at the G1/S boundary. Implications: The potential advantages of the imidooxy anticancer agents include better toleration, reduced resistance during therapy, and synergistic effect when used with other anticancer agents.

Systems-Level Analyses to Elucidate Key Stress Response Candidates in Leishmania. Nicola Carter, Pacific University Oregon, Phillip A. Yates, Oregon Health \& Science University, Emily A. Weissgerber, Pacific University Oregon, Skyler Suchovsky, Pacific University Oregon, Phillip A. Wilmarth, Oregon Health \& Science University, Larry L. David, Oregon Health \& Science University. Objectives: Leishmania is a human pathogen that afflicts nearly 12 million worldwide. Current drug regimens for leishmaniasis are limited, and lack both specificity and efficacy. The goal of our research is to identify molecular targets within Leishmania to advance drug discovery efforts. Stress response pathways are a particularly attractive paradigm, since they protect parasites from fluctuations in the host environment and promote survival. Previously, we identified a mutant, $\Delta$ gmpr $\Delta$ impdh, in which parasite survival during purine limitation (a type of nutrient stress) may be manipulated. In this study, we used this feature of $\Delta \mathrm{gmpr} \Delta \mathrm{impdh}$ cells to identify key stress response candidates involved in the pro-survival response to purine limitation. Method: Quantitative proteomics and phosphoproteomics were used to query the proteome of $\Delta \mathrm{gmpr} \Delta \mathrm{impdh}$ cells cultured under normal growth conditions, as well as under three different purine limitation conditions with differing survival outcomes. Results: Across all four treatments, 4735 proteins (59\% coverage of the predicted leishmanial proteome) and 12405 phosphopeptides (which mapped to 3039 unique proteins) were identified, highlighting the quality and depth of the dataset. A large number of candidates were significantly altered in abundance in response to purine limitation, and of these, 206 proteins and 611 phosphopeptides were altered only in those stress conditions triggering a pro-survival outcome. Implications: These analyses highlight the utility of the $\Delta$ gmpr $\Delta$ impdh cell line and systems-level approaches for revealing key stress response mechanisms in Leishmania and have identified several candidates with a putative role in parasite adaptation and survival during nutrient stress.

The Grass Might Be Greener for Pharmacy Students and Faculty at Schools With Branch Campuses. Timothy J. Bloom, Shenandoah University, Nina Pavuluri, Lake Erie College of Osteopathic Medicine, Lisa Lebovitz, University of Maryland, Manas Mandal, Roseman 


\section{American Journal of Pharmaceutical Education 2018; 82 (5) Article 7158.}

University of Health Sciences, Veronica P. Shuford, Virginia Commonwealth University. Objectives: This retrospective study analyzed American Association of Colleges of Pharmacy (AACP) curriculum quality surveys to study perceptions of faculty and graduating students at one-campus colleges/schools of pharmacy (C/SOPs) compared to those at $\mathrm{C} / \mathrm{SOPs}$ with satellite or sequential campuses. Method: Branch campuses were identified using Accreditation Council for Pharmacy Education designations. Branch type was defined as satellite (content originating at one campus is shared with another; students are assigned to one campus for their entire program) or sequential (students complete didactic coursework at one main campus, then disperse to another campus for APPEs). The 2016 and 2017 AACP faculty and graduating student survey results were requested from the AACP Office of Institutional Research and Effectiveness, and separated into campus categories: one campus, satellite campus and sequential campus. The total percentage of respondents who selected "Strongly Agree" or "Agree" on the Likert-type scale for each item was calculated and perceptions on the three types of C/SOP campuses were analyzed using Chi square comparison of proportions. Results: Of the $32 \mathrm{C} / \mathrm{SOPs}$ with branch campuses, 21 were categorized as satellite and 11 as sequential. Students from one-campus C/SOPs indicated significantly lower satisfaction $(\mathrm{p}<0.05)$ in most areas when compared with satellite campus students. One-campus faculty also indicated significantly less satisfaction $(\mathrm{p}<0.05)$, particularly in faculty development, infrastructure, and research time when compared with faculty at satellite C/SOPs. Implications: This study provides insight into pathway comparability and potential drivers to improve satisfaction for students and faculty at different models of pharmacy schools.

The Role of Polyamines for Proliferation, Survival, and Infectivity of the Protozoan Parasite Leishmania donovani. Sigrid C. Roberts, Pacific University Oregon, Jasmine Perdeh, Pacific University Oregon, Irene Abuan, Pacific University Oregon, Linh Le, Pacific University Oregon, Yvette Leizorek, Pacific University Oregon, Nicole LoGiudice, Pacific University Oregon, Quintin Love, Pacific University Oregon, Kristen Turcu, Pacific University Oregon. Objectives: A better understanding of parasite biology and host-parasite interactions is critical for the development of much needed therapeutic strategies against the neglected tropical disease leishmaniasis. Recent studies have highlighted the critical nature of polyamines in parasites. The polyamine biosynthetic pathway in Leishmania consists of four enzymes: arginase (ARG), ornithine decarboxylase (ODC), spermidine synthase (SPD), and S-adenosylmethionine decarboxylase (ADOMETDC). These enzymes sequentially generate ornithine, putrescine, and spermidine. We have generated a complete set of gene deletion mutants ( $\Delta$ arg, $\Delta$ odc, $\Delta$ spd, and $\Delta$ adometdc), in order to study the functions of polyamines and to evaluate the polyamine biosynthetic enzymes as therapeutic targets. Method: Proliferation and replication rates of polyamine-depleted and supplemented parasites were established in vitro by counting parasites on a hemocytometer and analyzing bromodeoxyuridine (BrdU) incorporation, respectively. Parasite survival was assessed with qualitative rescue assays. The ability of gene deletion mutants to establish infections was investigated in macrophages and mice. Results: Depletion of both putrescine and spermidine leads to imminent cell death, while the presence of putrescine or spermidine alone allows parasites to enter a quiescent-like state. In vivo studies revealed surprising differences in infectivity phenotypes, with the $\Delta$ odc deletion exhibiting the most profound reduction in infectivity. Implications: The observed discrepancies in infectivity phenotypes suggest a model in which the polyamine precursor ornithine is readily salvaged by intracellular parasites, whereas the polyamines putrescine and spermidine are severely limited. Polyamine depletion causes a profound growth and survival defect in vitro and in vivo, validating the polyamine biosynthetic enzymes as potential therapeutic targets.

Utilizing PCOA, Milestone Assessments, and Online Practice Licensure Exams as Predictors of NAPLEX Outcomes. Vera C. Campbell, Hampton University, Monzurul A. Roni, Hampton University, Yogesh M. Kulkarni, Hampton University, Justin C. Wilder, Hampton University, Ronald Lyon, Hampton University, Patricia Richards-Spruill, Hampton University, Travonia Brown-Hughes, Hampton University, Angela Berry, Hampton University, Danielle Irby, Hampton University, Rhea Angeles, Hampton University. Objectives: To compare between Pharmacy Curriculum Outcomes Assessment (PCOA), Online Practice Licensure Exam and HUSOP Competency Exam (Milestone Assessment) results as early predictors of North American Pharmacy Licensure Examination (NAPLEX) outcome. Method: Third year pharmacy students were administered the PCOA (low-stakes), HUSOP Competency Exam (mid-stakes), and an Online Practice Licensure Exam (high-stakes). NAPLEX outcomes of the cohort were correlated with the assessments. Correlations, regression analysis and t-tests were performed; $\mathrm{p}$ value $<0.05$ was considered significant. Results: Correlation and regression analysis showed the strongest correlation existed between NAPLEX and the Exam Master-based Online Practice Exam $(\mathrm{R}=0.77, \mathrm{R} 2=0.59)$, followed by the Competency Exam $(\mathrm{R}=0.74, \mathrm{R} 2=0.54)$, and PCOA Scaled Score $(\mathrm{R}=0.72, \mathrm{R} 2=0.52)$. The PCOA strongly correlated with the Online Practice Licensure Exam $(\mathrm{R}=0.84, \mathrm{R} 2=0.72)$, followed by the Competency Exam $(\mathrm{R}=0.79, \mathrm{R} 2=0.63)$, and PCAT percentile score $(\mathrm{R}=0.73, \mathrm{R} 2=0.53)$. A comparison of students who failed and passed the NAPLEX indicated that students who passed had significantly higher Online Practice Licensure Exam scores (13\% higher, $p=1 \times 10-8)$, Competency Exam scores $(10.7 \%$ higher, $\mathrm{p}=5.6 \times 10-8)$, and PCOA Scaled Scores $(67$ points higher, $\mathrm{p}=3.7 \times 10-7)$. Implications: This pilot study provides evidence that the PCOA, Competency Exam (Milestone Assessment), and Online Practice Licensure Exams are strong predictors for identifying students who are at risk of failing the NAPLEX. At risk students identified with this method will receive addition support and remediation during $\mathrm{P} 4$ year.

Walking Beyond the Podium: Assessing the Use of Educational Games in Pharmacy Curriculum. Gurkishan S. Chadha, University of New England, Pierre Hanna, University of New England, Lindsey N. Parmelee, University of New England. Objectives: Although PowerPoint presentations are integral in conveying content to students, they offer limited opportunities for students to engage with one another. Game applications can incorporate fun activities, increase peer interactions and promote active learning. The aim of this study was to evaluate the impact of a mobile application, Quizlet towards enhancing student content comprehension and improving performance in course assessments. Method: Class material was uploaded into Quizlet. Students $(n=68)$ enrolled in Pathophysiology class at UNE College of Pharmacy were provided course content, either without including use of Quizlet or with use of Quizlet. Student performance in 4 assessments was compared (2 each with and without Quizlet). Additionally student's perception of Quizlet was examined using a survey instrument. Results: A Repeated Measures ANOVA was used to analyze differences in class performances. The analysis showed that there was a significant improvement in assessment scores that incorporated Quizlet compared to assessments that did not include use of Quizlet. With Quizlet, class mean score in assessments increased by more than $7.5 \%$ Furthermore, more than $81 \%$ of students felt that use of Quizlet provided them better access to content, made material more 


\section{American Journal of Pharmaceutical Education 2018; 82 (5) Article 7158.}

interesting, and easier to comprehend. About $90 \%$ of student's felt that Quizlet enhanced their ability in studying Pathophysiology, and more than $97 \%$ of student's felt that Quizlet should also be incorporated into other courses. Implications: Quizlet as a novel tool can help to enhance retention of massive amounts of information that students need to learn, and help them perform better in their classes.

\section{Theoretical Models}

Guidelines for Vitamin D Supplementation: A State of Ambivalence for Researchers and Pharmacists. Subrata Deb, Larkin University. Objectives: Epidemiological studies have shown that lower serum levels of 25(OH)D3 are associated with increased risk of cancer, immune disorder and osteoporosis. The purpose of the present work was to analyze the guidelines from various American health agencies and organizations for desired vitamin D levels in humans and recommended supplementation of vitamin D. Due to the inconsistencies between guidelines and their implications on pharmacist patient counseling and researchers, new recommendations are proposed. Method: The criteria for vitamin D deficiency, insufficiency, optimum and toxic were identified by analyzing the guidelines from NIH Office of Dietary Supplements/Institute of Medicine (IOM), The Endocrine Society, National Osteoporosis Foundation, American Cancer Society, National Cancer Institute and Vitamin D Council. Results: Our data suggest that there are essentially two different prototypes of $25(\mathrm{OH})$ D3 levels and daily supplementation recommendations. The IOM indicates that levels of $<12 \mathrm{ng} / \mathrm{mL}$ are deficient, $>20 \mathrm{ng} / \mathrm{mL}$ are sufficient and $>50$ $\mathrm{ng} / \mathrm{mL}$ are potentially toxic. In contrast, The Endocrine Society recognizes significantly higher levels for those categories: $<20 \mathrm{ng} / \mathrm{ml}$ is deficient and $21-29 \mathrm{ng} / \mathrm{ml}$ is insufficient. Similarly, lower daily supplementation (600 IU/day Vs 1500-2000 IU/day) was recommended by IOM. The guidelines of other agencies analyzed in this study fall in between these prototypes. Implications: Overall, there are inconsistencies in guidelines on vitamin D3 needs between different agencies and modulation of vitamin D3 levels by comorbidities or comedications confounds the interpretations. A model has been formulated that takes the pathophysiology and comedications into consideration and will propel future research on identification of diseasebased vitamin D level requirement.

Virtual Experimental Pharmacology Elective Course in a Pharmacy School Program. Paul Kaplita, MCPHS University-Worcester/ Manchester, Alok Sharma, MCPHS University-Worcester/Manchester. Objectives: The objective of the poster is to describe the design and experiences with a virtual experimental pharmacology course that allows the students to: * directly observe the action of representative drugs at the organ system or intact animal level, * gain a qualitative and quantitative understanding of the dose-response relationship of drug responses, * gain an understanding of receptor-specific vs. non-specific drug responses, and, * be able to perform and interpret graphical analysis of pharmacological data. Method: Students use two Windows-based computer software: BIOSOFT ${ }^{\circledR}$ CardioLab and BIOSOFT ${ }^{\circledR}$ Ileum (Stapleford Cambridge UK) to perform virtual classical in vivo and in vitro pharmacology experiments. These programs mimic the responses of heart rate and blood pressure in Cardiolab, and smooth muscle contractions in Ileum. The output from the software is a mock polygraph chart trace, and the drug-elicited deflections in the trace are measured and analyzed by students. Results: Preliminary evaluation of the course from the students' perspective has been positive. Future measurement of the impact of this elective course will be to correlate student performances in the virtual laboratory with their performances in the respective didactic Pharmacology courses. Implications: Virtual laboratory animal experiments can be designed and performed in a short amount of time with minimal cost and infrastructure. This allows the course to be easily scheduled into the curriculum and minimizes expenses associated with an animal facility. Computer simulations are therefore a useful platform to introduce students to in vitro and in vivo pharmacological methods.

\section{CHEMISTRY}

Completed Research

Characterization of a Novel Dopamine D4 Receptor Ligands Reveals Structure-Activity Relationships for Selective Partial Agonism. Comfort A. Boateng, High Point University. Objectives: To understand the Dopamine D4 Receptor (D4R) subtype-mediated signaling is essential to understanding and treating D4R-associated disorders, including substance abuse disorders. Method: We created a small next-generation compound library using computational modelling to design D4R ligands based on the parental scaffold of the prototypical D4R partial agonist A-412997 (2-(4-(pyridin-2-yl)piperidin1-yl)-N-(m-tolyl)acetamide). A series of novel A-412997 analogue ligands were synthesized by using $\mathrm{m}$-toluidine intermediate, and then coupling with 2-chloroacetyl chloride to give the intermediate 2-chloro$\mathrm{N}$-(m-tolyl)acetamide. Followed by coupling with substituted or unsubstituted piperidine and pyridine moieties. Their binding affinities were determined using both agonist and antagonist by competitive inhibition of OH-DPAT and $[3 \mathrm{H}] \mathrm{N}$-methylspiperone radioligand binding assays respectively in HEK293 cells expressing dopamine D2-like receptors. Followed by functional signaling analysis studies using $\beta$-arrestin recruitment and cAMP inhibition assays. Results: We found that substitutions on the piperidine and pyridine moieties, as well as modifications in linker length, led to several novel lead compounds with improved selectivity and potency, and varying degrees of agonist efficacy. Compounds were identified that displayed high binding affinity $(<100 \mathrm{nM})$ for the D4R coupled with a $>1000$-fold selectivity vs. the D3R and D2R. Implications: These ligands displayed a range of agonist efficacies from highly efficacious partial agonists to full antagonists of the D4R. Interestingly, some compounds with similar binding affinities showed divergent functional efficacies, allowing for the generation of a preliminary structure-activity relationship underlying functional efficacy. The studies have further extended our understanding of ligand-receptor interactions and the relationship between the orthosteric binding pocket and extended binding pocket.

Detection and Monitoring Diabetes Via Breath Acetone. Ronny Priefer, Western New England University, Michael Rust, New England Breath Technologies. Objectives: Current "triggers" to send patients for a diagnostic test of diabetes may miss years of potential preventative interventions. A correlation with breath acetone and $\mathrm{A} 1 \mathrm{C}$ levels as well as with blood glucose is known. Thus, a non-invasive method of testing breath acetone would provide immediate information regarding the diabetic-level of an individual as well as possibly supplant the current self-monitoring of blood glucose for those with diabetes. We have developed and tested a technology that can be used as both a screener and a monitoring device. Method: Our Stage 1 Clinical study was performed on 50 individuals: 26 non-diabetic and 24 diabetic individuals. Our Stage 2 Clinical study, with six volunteers, was done to monitor the blood glucose to breath acetone correlation over a 3 hrs time span. Results: Our Stage 1 Clinical study demonstrated that there was a clear correlation of blood glucose and breath acetone $(\mathrm{R}=0.99, \mathrm{P}<0.001)$ with breath acetone levels being noticeably higher for patients with diabetes. For the Stage 2 Clinical study, the directionality of the readings was clearly demonstrated (from a low of 58 to high of $370 \mathrm{mg} / \mathrm{dL}$ ), with a correlation of breath acetone to blood glucose ranging from $\mathrm{R}=0.72$ to 0.95 . Implications: We have been 


\section{American Journal of Pharmaceutical Education 2018; 82 (5) Article 7158.}

able to demonstrate that by analyzing for breath acetone it is possible to both screen for, and monitor diabetes. Therefore, the development of a portable, hand-held, non-invasive screening and monitoring device for diabetes is highly feasible.

Hydrolytic Property Studies of Nucleotide Analogs: pH-Dependent Degradation Pathways of Nucleoside Boranodiphosphates. Zhihong $\mathrm{Xu}$, South College. Objectives: Nucleoside diphosphates are important intermediates in various anabolic and catabolic cellular processes, and the study of bioactive nucleoside boranodiphosphate ((d)NDP $\alpha \mathrm{B})$ analogs has been an active area in nucleoside/nucleotide chemistry. Biological property studies of modified (d)NDP $\alpha \mathrm{B}$ stereoisomers require pure compounds, which is quite challenging for this type of compounds, as degradation products were often identified from the earlier purified isomers. Therefore, it is important to study the processes which contribute to the generation of the impurities. Method: Here, uridine/thymidine boranodiphosphate $\mathrm{Rp}$ and $\mathrm{Sp}$ isomers were chosen as representative compounds, and their hydrolytic properties were studied by HPLC, LC-MS and 31P-NMR in buffers of different $\mathrm{pH}$ at different temperature. Results: Based on the 31P-NMR data and HPLC analysis, four major hydrolysis/degradation products were identified, which were compared with chemically synthesized or commercially available standards, and further supported by LC-MS. The experimental data showed that lower $\mathrm{pH}$ buffers and higher temperature facilitated the title compound hydrolysis/degradation. Implications: With respect to the proposed different hydrolysis/degradation pathways of the tested isomers under acidic, neutral and basic conditions, we believe that these observations have applications for caution on related analog handling, new drug design and controlled drug release.

IPPE Simulation Assesses Sterile Compounding Competency. Susan L. Mercer, Lipscomb University, Matthew Vergne, Lipscomb University, James W. Torr, Lipscomb University. Objectives: The safe and accurate preparation of compounded sterile preparations (CSPs) is a fundamental responsibility of pharmacy practice. A pilot IPPE Simulation was developed to expose PY2 student pharmacists to clinical and distributive functions of pharmacist delivered care in an institutional setting. Objectives of the IPPE Simulation included assessing retention of sterile compounding competencies: 1) accuracy of a CSP and 2) demonstration of aseptic technique. Method: Students applied the pharmacist patient care process to a simulated patient case involving intravenous vancomycin therapy. Students calculated a patient specific vancomycin dose and prepared an aseptic formulation. HPLC analysis was used to determine the actual vancomycin dose in the student formulations using a linear calibration curve with five calibrants. Students completed gloved-fingertip and media-fill testing procedures to verify competency with aseptic processes. Results: Utilizing a $+/-10 \%$ acceptable range, the following results were determined. Preparations outside of the acceptable range were deemed "failures." The failure rates were $35 \%$ in cohort $1(n=23), 17 \%$ in cohort $2(n=24)$, and $0 \%$ in cohort $3(n=19)$. The average percent difference correlated to the calculated dose was $8.354 \%$, $4.313 \%$, and $1.932 \%$, respectively. Student retention of knowledge and skills for accurate CSP preparation is not adequately retained after 12 months. Aggregate results $(n=66)$ demonstrated success rates of $100 \%$ on media-fill and $93.9 \%$ on gloved-fingertip assessments suggesting retention of aseptic processing skills. Implications: IPPEs signify an opportunity to integrate and reinforce sterile compounding competencies throughout the curriculum. Such curriculum can fill training gaps in entry-level sterile compounding competency.

N-Arylpiperazines as Key Intermediates Towards Developing Bi-Functional Serotonergic Ligands to Reduce Repetitive Behaviors in Autism. Ola A. Ghoneim, University of Saint Joseph, Victoria
M. Gancarczyk, University of Saint Joseph, Lydia Tran, University of Saint Joseph, Dillon H. Keefe, University of Saint Joseph. Objectives: Communication deficits, and repetitive behaviors are two main domains defining autism. The frequency/severity of these behaviors vary and negatively affect the individual's quality of life. Increasing serotonin level at the synaptic cleft modulates repetitive behaviors via unknown mechanism. Level of serotonin can be increased by blocking the serotonin uptake via Selective Serotonin Reuptake Inhibitors (SSRIs). However, the elevated serotonin level stimulates inhibitory autoreceptors, exerting a negative feedback mechanism, contradicting SSRIs function. Our objective is developing bi-functional serotonergic ligands that inhibit serotonin uptake and inhibit serotonin autoreceptors simultaneously to achieve rapid/consistent increase in serotonin level and a corresponding quick control over the disruptive behaviors. Method: Our design utilized the tethering technique (i.e) linking the chemical structural component(s) responsible for the two desired pharmacological profiles via a metabolizable linker. As common serotonergic motifs, all our design includes n-arylpiperazines as key intermediates. Synthesis was accomplished via Williamson Ether synthesis of trifluoromethylphenol and methyl-bromophenylacetate, followed by hydrolysis. Then coupling the resulted (substituted)phenoxyacetic acid with various n-aryl(substituted)piperazines. Biological screening was conducted at Eurofins/Cerep, France. Results: Twenty-five novel compounds (VG, LG and KG series) have been synthesized and screened. Two compounds displayed $100 \%$ inhibition at both serotonergic targets with IC50 in nanomolar range. The synthetic scheme, and the effect of the various amines on the inhibition of the 5-HT uptake and serotonin autoreceptors will be presented. Implications: Phenoxyacetic acid analogs with n-arylpiperazine motif are potential lead compounds for developing bi-functional serotonergic ligands for repetitive behaviors modulation in autism.

The Impact of Course Scheduling on Student Exam Performance. Marcy Hernick, Appalachian College of Pharmacy. Objectives: To determine the effect of course scheduling on student exam performance. Method: Courses with various biochemistry and medicinal chemistry/pharmacology topics in a 3-year Pharm.D. program were taught with 3-6 hours of lecture per day and met 12-30 hours per week. Student learning was assessed using course quizzes and/or examinations. Results: We had previously shown that students performed best on examinations when topics were taught on 3 hour days, or on 6 hour days for courses not exceeding 12 hours per week (Schedule A, mean $=79.5$ ) compared to when topics were taught in courses scheduled to meet 4-6 hour days and 18-30 hours per week (Schedule B, mean 71.6). In light of these findings, the scheduling of an Infectious Disease Medicinal Chemistry/Pharmacology course was changed from meeting on 6 hour days $\mathrm{x} 4$ days per week to 3 hour days $\mathrm{x} 4$ days per week. Overall, the average student performance on topics increased by $\sim 6 \%$ points with a larger average improvement observed for questions related to SAR/pharmacophore recognition $(59.6 \%$ to $72.7 \%$ ) Importantly, student performance on matched examination questions for the Medicinal Chemistry topics improved from 59.3\% to $69.4 \%$ on multiple-choice questions (A-type, Select all that apply) and from $67.4 \%$ to $77.8 \%$ on matching questions. Implications: Course scheduling has a significant impact on student exam performance. To maximize student learning, courses should be designed to limit the number of direct science hours to not more than 3 hours per day or combined 15 hours per week.

The Search for Nicotinic Probes Through Structure Activity Relationship Development. Stephen R. Slauson, University of Saint Joseph, Zamzama Gafar, University of Saint Joseph, Melissa Kemp, 


\section{American Journal of Pharmaceutical Education 2018; 82 (5) Article 7158.}

University of Saint Joseph, Natalia Echeverry, University of Saint Joseph, Samantha Copeland, University of Saint Joseph, Alessandra Stoj, University of Saint Joseph, Hanh Vuong, University of Saint Joseph, Maria Simon, University of Saint Joseph. Objectives: The addictive potential of opioids has largely been ignored due to their therapeutic utility. Since this moral dilemma has reached epidemic status, alternatives for the treatment of severe pain are desperately needed. The natural product epibatidine introduced nicotinic receptor agonists as one possibility nearly three decades ago. Despite substantial research and extraordinary strides taken towards its development, the field remains in need of a robust structure activity relationship model in regards to nicotinic subtype specificity. The objective of this project is to develop this model through careful substitution of the analogous [2.2.2] scaffold in order to develop probe compounds for enhanced characterization of the nicotinic subtypes. Method: This framework avoids the equipotent nature of the [2.2.1] enantiomers by breaking the symmetry of the ring system, affording a more lucid understanding of the receptor binding constraints in relation to position substituted. In addition, the scaffold is easily prepared through a highyielding, microwave accelerated Diels-Alder reaction of dihydropyridines with the corresponding dienophile containing the aryl moiety. This method allows systematic substitution of each position of the bicyclic core. Results: Adaptation of literature methods combined with development of the microwave conditions have allowed the synthesis of initial analogs that insert a methyl group at each carbon of the [2.2.2] scaffold. The relative binding affinity of each at the nicotinic subtypes will direct further synthesis to develop a robust model. Implications: Once refined, this model will expedite insight of the desired receptor subtype profile and facilitate the development of these much needed alternative analgesic agents.

Utility of Chemotype Analysis in Differentiation of Medical Marijuana (Cannabis) Drug Products. Christopher T. Howard, MCPHS University-Worcester/Manchester, Christopher A. Boutari, MCPHS University-Worcester/Manchester, Katelyn Pimental, MCPHS University-Worcester/Manchester, Quang M. Bui, MCPHS UniversityWorcester/Manchester, Joseph D. Pirani, MCPHS University-Worcester/ Manchester, Joseph F. Biga, MCPHS University-Worcester/Manchester, Algis Domeika, Prime Wellness of Connecticut, George K. AcquaahMensah, MCPHS University-Worcester/Manchester, Matthew D. Metcalf, MCPHS University-Worcester/Manchester. Objectives: Cannabis drug products are typically discriminated by morphological classification systems and limited to "strain" data. The clinical utility of such classification systems of products sold as medical marijuana is tentative at best. Differences in relative cannabinoid and terpene content (chemotypes) of Cannabis products is postulated to be a better predictor of the pharmacological effects of the drug product and serve as a more clinically relevant classification system. In this study we sought to determine if Cannabis chemotypes can be used as a reliable discriminatory tool to differentiate Cannabis drug products. Method: Chemical profiles were obtained from state required certificates of analysis of all Cannabis products sold at a Connecticut medical marijuana dispensary during a 12 month time frame. Principal component analysis (PCA) was used to determine key chemical constituents used to perform the analysis. Three machine learning algorithms were used analyze various Cannabis products descriptors including: Type, Subtype, Strain, and Method. Results: Using a 6 month train- 6 month test set, the J48 machine learning approach correctly predicted Method $89.79 \%$ of the time. Type and Subtype were also predicted with the high success rates of $99.58 \%$ and $87.29 \%$ respectively. Month as a negative control, was poorly predicted $15.55 \%$. Implications: The results suggest that chemotype of Cannabis products can reliably predict useful discriminatory variables in a diverse set of Cannabis products. Chemotype classification systems provide clinical relevant discrimination of Cannabis drug products and ultimately could be used to match product chemotypes with disease states to identify potential treatments.

\section{Theoretical Models}

Demonstrating the Broader Impact of Chemistry With Altmetrics. Andrew Coop, University of Maryland, Shannon R. Tucker, University of Maryland, Lisa Lebovitz, University of Maryland. Objectives: The objectives are to describe the advantages of documenting the impact of chemistry with altmetrics, and contrast with existing tools to assess impact. Method: Understanding the broad impact of research with existing impact measurement tools limits the ability of researchers to show their research presence in an online environment. As a freely available tool, altmetrics provides researchers with instant access to publication analytics in social media. An evaluation of the altmetrics compared with current impact measures, focusing on the advantages, scope, and limitations for researchers was conducted. Results: Performing an altmetrics (though Altmetric.com's Bookmarklet) evaluation on one manuscript on a potential opioid lacking dependence (ACS Neuroscience $2013,4,1256$ ) the number of citations in the peer-reviewed literature was a low 22 , but the altmetrics score was 184 , placing it among the top 5\% of cited articles due to citations in the mainstream media (Washington Post, The Atlantic, The Scientist, Yahoo!). When comparing results for this, and other manuscripts, altmetrics showed the wider impact of chemistry on society, beyond the impact factor of peerreviewed publications. Implications: The impact of our activities as pharmacy chemistry faculty have traditionally been measured through citations in peer-reviewed journals. Although extremely important, this approach does not account for impact through social media and other non-peer reviewed avenues (such as the mainstream media, as well as Twitter) that reach far more of the general population. To capture such impact, there is a growing interest in altmetrics that captures references from such arenas.

Promoting Audience Appropriate Communication Through a Professional Communication Strategy Elective Bootcamp. Andrew Coop, University of Maryland, Shannon R. Tucker, University of Maryland, Cherokee Layson-Wolf, University of Maryland. Objectives: To provide PharmD students an elective experience supporting the development of professional communication strategies for use with any audience or setting. Method: Using the National Academies of Sciences, Engineering, and Medicine's framework for effective communication of Chemistry in informal environments, the following course objectives were developed: 1) Evaluate the format, context, and audience for a presentation to develop concise communication goals. 2) Appraise the causes of personal communication anxiety and plan appropriate approaches to manage them. 3) Discuss the impact of story/narrative as persuasive presentation elements. 4) Demonstrate an understanding of the FAIR USE/Digital Millennium Copyright Act and how they apply to presentations. 5) Develop an effective presentation given a context and format. 6. Provide constructive critique of presentations. Results: The resulting week long course was designed to longitudinally build in complexity as new skills were introduced. The course progression included: 1) individual 3-minute ice breaker presentations, 2) mock airplane conversation with unknown volunteers (staff), 3) elevator pitch development/delivery, 4) mock networking event (with real food!) with the goal of talking to the unfamiliar guests, 5) two-minute on-the-spot presentation of a randomly selected topic, and 6) a capstone individual PowerPoint presentation. After each 


\section{American Journal of Pharmaceutical Education 2018; 82 (5) Article 7158.}

exercise, peer-reflection was facilitated with the class. Implications: Communication is essential for health care professionals, yet many students are reluctant due to anxiety with public speaking. This course offered a "low-threshold" range of experiences that introduced the students to various situations they will encounter in their professional lives to build confidence.

\section{CONTINUING PROFESSIONAL DEVELOPMENT Completed Research}

Assessing Self-Directed Lifelong Learning Approaches at Colleges of Pharmacy: The Continuing Professional Development (CPD) Initiative. James Wheeler, The University of Tennessee, Beth A. Martin, University of Wisconsin-Madison, Teresa M. Elsobky, Shenandoah University, Jennifer M. Malinowski, Wilkes University. Objectives: To quantify and analyze self-directed lifelong learning approaches at colleges and schools of pharmacy across the United States. Method: Academic deans or faculty with assessment responsibilities from ACPE accredited institutions and associated distance campuses were electronically surveyed via Qualtrics. Prior to distribution, the survey was peer validated by four academic deans. Results: Responses were received from $38(n=38 / 140)$ colleges of pharmacy. All responding programs had learning outcomes aimed at self-directed lifelong learning. Twenty-two programs intentionally sequenced their CPD activities. CPD concepts were measured in didactic courses $(n=18)$, IPPE $(n=16)$, APPE $(n=17)$, and co-curricular activities $(n=25)$. Six respondents $(n=6 / 33)$ noted that faculty received training on self-directed learning. Most programs used validated learning inventories such as Strengthsfinder $(n=19)$ or the Pharmacist Inventory of Learning Styles (PILS) $(n=8)$. The most commonly cited barriers were concerns about faculty time to evaluate student activities $(n=21)$, faculty buy in $(n=15)$, and lack of a clear definition of responsible parties and their specific roles in CPD $(n=17)$. Implications: All respondents noted the importance of self-directed lifelong learning through learning outcomes, but few programs offered training for faculty. Most programs cited a large number of barriers to overcome for quality student self-directed lifelong learning. Future educational offerings and focused mapping with analysis of CPD initiatives could help overcome barriers and assist in producing "CPD ready" graduates.

A Statewide Initiative Preparing Post-Doctoral Pharmacy Trainees as Future Experiential Educators. Yekaterina Opsha, Rutgers, The State University of New Jersey, Anita Siu, Rutgers, The State University of New Jersey, Michael Toscani, Rutgers, The State University of New Jersey, Donna M. Feudo, Rutgers, The State University of New Jersey, Janice Allunario, Rutgers, The State University of New Jersey, Joseph A. Barone, Rutgers, The State University of New Jersey. Objectives: To evaluate the Ernest Mario School of Pharmacy (EMSOP) Teaching and Learning Certificate (TLC) program at enhancing both IPPE and APPE rotation experiences for pharmacy students. Method: In 2012, a TLC program in New Jersey was developed to prepare post-doctoral pharmacy trainees for their future role as preceptors. This nonprofit program emphasizes the important qualities and core skills consistent with the AACP criteria for preceptor excellence. All PGY-1, PGY-2 residents and pharmaceutical industry fellows can participate. It builds foundational knowledge in preceptor excellence for trainees to enhance their preparedness in offering students an exceptional learning experience. In addition, the program provides exposure to academic opportunities and in-depth training of becoming future experiential educators. Results: The qualities for preceptor excellence are emphasized in a 9-module seminar series which includes creating a practice site for IPPE or APPE experiences. At the end of the longitudinal coursework, participant precepts a pharmacy student with their program director. Completion of the precepting experience is a unique attribute of the program. The program is currently in its sixth year with 276 graduates to date. Since its inception, the participants provided students with 154 unique IPPE and 53 APPE rotation experiences and occasionally can co-precept students with each other. Implications: The EMSOP TLC program brought forth many opportunities for post-doctoral trainees. New rotations were created and many of our past trainees have remained preceptors for our Experiential Education program and this network continues to grow. This program also continues to provide great peer to peer networking opportunities.

Co-Curricular Plan Development and Assessment. Kathryn A. Schott, Drake University, Cheryl L. Clarke, Drake University, Charles R. Phillips, Drake University. Objectives: To describe the development and assessment strategies related to co-curricular programming that support Standards 2016. Method: Faculty, staff and students representing experiential, student affairs, and assessment offices collaborated to design the formalization and assessment of the co-curriculum. Co-curricular activities primarily include those developed by the college and student organizations, but also include those developed by other pharmacy professional organizations and non-pharmacy activities that support Standards 3 and 4. A reporting and assessment system was created within the E-Value electronic portfolio where students document co-curricular activities, link activities to objectives supporting Standards 3 and 4 through reflection, and develop a plan for future co-curricular engagement. A rubric was developed for advisors to provide feedback and assess student progress in their continuing professional development. Results: In the first semester of implementation, first-year student pharmacists documented 7975 hours of co-curricular activity, $46 \%$ of which were pharmacyrelated. Twenty-seven (27) percent of the pharmacy-related hours were identified as spent in a leadership role versus participant or other role. Students connected $64 \%, 68 \%, 35 \%$, and $66 \%$ of total hours completed to their development related to self-awareness, professionalism, innovation/entrepreneurship, and leadership, respectively. The first assessment by faculty advisors will take place in the spring of 2018 and will utilize the rubric. Those results will also be shared. Implications: The integration of the co-curriculum and its assessment into the professional program allows for both tracking activities and their associated effects on the affective domains of standard 3 and 4.

Development, Implementation and Lessons Learned From Professional Development Required Longitudinal Course Series Over 5 Years. Hoai-An Truong, University of Maryland Eastern Shore, Marie-Therese Oyalowo, University of Maryland Eastern Shore, Lana Sherr, University of Maryland Eastern Shore. Objectives: The Center for the Advancement of Pharmacy Education (CAPE) educational outcomes specifies personal and professional development as one of four domains for pharmacy graduate's competency. The objective is to describe the development, implementation and lessons learned from a required Professional Development (PD) longitudinal course series over 5 years. Method: A PD course series was created when the School initiated its new curriculum. This originated after mapping PD course objectives to CAPE 2004 then recently revised to match CAPE 2013 outcomes. This course series structure consists of bi-weekly didactic classes with complementary PD seminars from invited local and national speakers. The PD course series comprises four 1-credit courses spanning over first and second year of the curriculum. Teaching-learning strategies 


\section{American Journal of Pharmaceutical Education 2018; 82 (5) Article 7158.}

include classroom lectures, application of the continuing professional development model, interprofessional activities and seminars, service-learning, and participation at professional meetings and annual Legislative Day. These activities are assessed via students' written reflections, presentations, classroom participation, and impromptu speeches. Results: The PD course series have been offered to about 300 students over five years. Approximately 9,000 hours of servicelearning were provided by all the students to local and regional communities. Students documented required attendance and written reflections at approximately 1,400 local, state, and national professional meetings. They also had about 1,440 encounters with legislators or their staff. Students performed well on PD course series and reflected positive impact on their career planning. Implications: PD required longitudinal course series provided students with the knowledge and skills for their career planning and prepared them for selfdirected life-long learning.

Development of a Continuing Professional Development Course Series. Kathryn A. Schott, Drake University, Cheryl L. Clarke, Drake University, Charles R. Phillips, Drake University. Objectives: To describe the development and ongoing implementation of a six-semester continuing professional development (CPD) course series designed to meet Standards 2016 related to 1) co-curricular involvement; 2) interprofessional education (IPE); 3) introductory pharmacy practice experience (IPPE); and 4) personal and professional development. Method: A task force of faculty, staff, and students determined the best process to meet the Standards 2016 requirements related to personal and professional development. The resulting six-semester course series added a net, one credit hour to the curriculum while realigning the IPE and IPPE components of the curriculum, moving information from an existing two credit Introduction of Pharmaceutical Care course, and combining a two-semester pre-APPE preparatory course series. Learning objectives and assessment processes were developed across the series. Standard assignments were created across the series, which are housed in electronic student portfolios. Sample assignments include curriculum vitae, professional learning goals, self-awareness assessments, personal learning plans, faculty mentor meeting reflections, and professional development assessments. Results: The course series was implemented Fall 2017. Following the first semester, students rated their confidence related to 15 course-related objectives. The highest confidence areas were Meeting IPPE Expectations, Working with Your Faculty Mentor, and Reflecting on Academic Progress. Areas with less confidence included Writing SMART Goals, Planning Personal Development Activities, and Developing Your Personal Brand. Implications: A six-semester CPD course series was developed and implemented to meet Standards 2016 requirements. Students are gaining confidence in course-related objectives.

Effectiveness of a Pharmacy Teaching Certificate Program Offered to Practicing Pharmacists. Jaclyn Cole, University of South Florida, Melissa Ruble, University of South Florida, Gwendolyn Wantuch, University of South Florida, Kamila A. Dell, University of South Florida, Erini S. Serag-Bolos, University of South Florida, Carol Fox, University of South Florida, Janelle Perkins, University of South Florida. Objectives: To evaluate the effect of a universityaffiliated pharmacy teaching certificate program on participant knowledge and perceived confidence in areas of academic pharmacy and preceptor development. Method: A 10-month program consisting of 4-live teaching modules with associated continuing education (CE) credit was offered to pharmacy residents and practicing pharmacists. Content modules were delineated as: $1=$ Introduction to Teaching, $2=$ Publications, $3=$ Advanced Teaching Principles, $4=$ Precepting.
The program format included didactic and active learning strategies with "at home" assignments to reinforce material presented. Content knowledge was measured with written summative assessments provided at the start and end of each module. Perceived confidence was assessed through online surveys provided at the start and end of the entire program. All results were evaluated using paired t-tests. Results: Data were obtained from 32 participants over two cohort years. For each of the four modules, there was a significant difference in pre- and post- knowledge assessments (Module $1 \mathrm{p}=0.046$; Module $2 \mathrm{p}<0.001$; Module $3 \mathrm{p}<0.001$; Module $4 \mathrm{p}<0.001)$. The largest improvement from pre- to post- results was seen in Modules 2 and 3. Confidence data was significantly increased for each module $(p<0.001$ for all 4 modules). The smallest average difference in participant confidence was reported with Module 2. Implications: Knowledge assessments and perception surveys suggest that this university-affiliated teaching certificate program improved both knowledge and perceived confidence for program participants. There may be a need for additional training in scholarly work based on improved knowledge scores with sustained lower confidence reports.

Impact of a Non-Credit Pharmacy Readiness Course on Students' Academic Performance. Melinda Verdone, Midwestern University/ Downers Grove, Jacob P. Gettig, Midwestern University/Downers Grove, Medha D. Joshi, Midwestern University/Downers Grove, Thomas M. Bodenstine, Midwestern University/Downers Grove, Jacalyn M. Green, Midwestern University/Downers Grove, Sean M. Lynch, Midwestern University/Downers Grove, Nancy Fjortoft, Midwestern University/Downers Grove. Objectives: Students arrive at colleges of pharmacy with varying levels of academic preparedness for a rigorous pharmacy curriculum. It is important to identify students who may struggle early in their first year and provide interventions that support their learning throughout the program. In response to this need, a bridging course was developed to assist all students with this transition. The objective of this study is to describe and evaluate the shortterm effectiveness of this bridging course. Method: This Pharmacy Readiness course consisted of ten online, self-guided modules on physiology, biochemistry, math, and medical terminology. A comprehensive assessment of these topics was administered to all first-year pharmacy students during orientation (T1), after completion of the modules (T2), and at the beginning of the following quarter (T3). Students who scored $<70 \%$ at $\mathrm{T} 1$ took the T2 assessment, and all students took the T3 assessment. Results: A total of $180(100 \%)$, $138(77 \%)$ and $166(92 \%)$ students completed the T1, T2 and T3 assessments, respectively. Student who participated in the modules significantly improved assessment scores from T1 to T2 (54\% vs. $76 \%, \mathrm{p}<0.0001)$. Students who scored $<70 \%$ at T2 were contacted for follow-up intervention. All students demonstrated a significant improvement in assessment scores between T1 and T3; however, Pharmacy Readiness participants showed a greater proportional increase in their T3 scores (T1 53\% vs. T3 73\%, p <0.0001) over non-participants (T1 76\% vs. T3 81\%, p=0.004). Implications: The Pharmacy Readiness course proved successful as a system of early identification and intervention for students who needed additional academic support.

Pharmacy Pearl: Integrating Pharmacy Into an Interprofessional Master Science in Palliative Care (MSPC) Program. Shaun E. Gleason, University of Colorado, Kristin Speer, University of Colorado, Regina M. Fink, University of Colorado, F. Amos M. Bailey, University of Colorado. Objectives: To assess the educational "pearl" in strengthening pharmacy's role in an interprofessional palliative care program. Method: Our 36 credit-hour/ 12 course MSPC program is delivered primarily online to nurses, pharmacists, physicians, physician-assistants, 


\section{American Journal of Pharmaceutical Education 2018; 82 (5) Article 7158.}

psychologists, social-workers, and spiritual-care providers. Course content is provided by all disciplines. In response to previously reported data on students' perceived palliative care skills (lowest on medication-related issues), we increased pharmacy's role. Our primary method has been through the educational "pearl," brief recorded mini-lessons addressing pertinent pharmacy-related questions. For program quality assurance, we assessed "pearls" by percent inclusion in module topics, pearl topic categories, and identification of number and topic areas where further pharmacy opportunities exist. Success was assessed by course and instructor evaluations. Results: Pharmacy pearls are included in 27/65 (42.5\%) of biomedical topics in 9 courses. Pearl topic categories include: therapeutic use $=8$, drug choice $=5$, drug review $=4$, dosing $=3$, medication administration routes $=2$, pharmacy administration $=2$, adverse effects/drugdrug interactions $=2$, pharmacology $/$ pharmacokinetics $=1$, and patient education $=1$ (overlap). Opportunities for further pharmacy inclusion exist as new pharmacy information $(n=13)$ and contributions beyond pearls $(n=17)$ of 62 modules. Identified potential topic areas include druginformation $=20$, patient/family/provider education $=16$, pharmacy administration $=9.2017$ course evaluation scores: mean 4.72/5 $(\mathrm{n}=18$ questions); instructor evaluation scores: mean $=4.91 / 5$ ( $n=9$ questions); all 3-8 respondents of 16-18; 5=highly rated, 4 courses. Implications: Pharmacy pearls have been a successful method to establish pharmacy's role and provide education in our interprofessional curriculum. Pharmacy topics most often requested are drug-information. Areas where pharmacists can add further value, potentially beyond pearls, include additional drug information, pharmacy administrative topics (e.g. cost) and patient/ family/provider education.

Tracking Longitudinal Progression of Accreditation Standards for Personal and Professional Development Using Individual Development Plans. Margaret Schulte, Touro University California, Deanna Gee, Touro University California, Rae R. Matsumoto, Touro University California. Objectives: To begin tracking the longitudinal progression of students' awareness and achievement of key elements of Personal and Professional Development using Individual Development Plans (IDPs). Method: IDPs were completed by the Doctor of Pharmacy Class of $2020(\mathrm{~N}=85)$ during Fall of the first- (P1) and second-professional-year (P2) to guide them in defining career goals, self-evaluating skills, and developing individualized action plans. Study investigators rated whether four key elements (Self-Awareness, Leadership, Innovation/Entrepreneurship, Professionalism) were addressed as attained or needing development, with data summarized using descriptive and non-parametric statistics. Results: Professionalism and SelfAwareness were addressed in $100 \%$ of IDPs in the P1 and P2 years. Leadership was addressed as attained or needing development in $55.2 \%$ $(\mathrm{P} 1)$ and $62.4 \%(\mathrm{P} 2)$ of IDPs, with a significant increase $(\mathrm{P}<0.0001)$ in the proportion of students documenting attainment of leadership skills in the P1 (3.5\%) vs P2 (47.1\%) year. There appears to be underreporting of skills because only $76.5 \%$ of the 51 individuals elected to leadership positions addressed leadership in their IDPs. There was also a significant increase $(\mathrm{P}<0.005)$ in the proportion of students addressing Innovation/ Entrepreneurship as attained or needing development during the P1 $(22.4 \%)$ vs $\mathrm{P} 2(44.7 \%)$ year, with significant $(\mathrm{P}<0.001)$ growth for those documenting attainment of those skills in the P1 (1.2\%) vs P2 (16.5\%) year. Implications: IDPs can track progression of personal and professional development in pharmacy students. Most students enter pharmacy school recognizing the importance of Professionalism and Self-Awareness, but require additional guidance to instill awareness of the importance of Leadership and Innovation/Entrepreneurship.

Utilization of an "Escape Room" Activity in Continuing Education Programming for Pharmacists and Pharmacy Technicians. Jaclyn
Cole, University of South Florida, Melissa Ruble, University of South Florida. Objectives: The objective of this study was to evaluate the utilization and execution of an "escape room" as part of a continuing education (CE) program for pharmacists and pharmacy technicians. Method: This was a cohort study of participants enrolled in a pharmacy CE program. Study investigators provided a 2-hour Medication Error CE presentation, utilizing the first hour for lecture and the second hour for application through an "escape room" activity. Two escape rooms were run parallel. Solving each of the 8 puzzles in the room provided a piece of a root cause analysis for the fishbone diagram to be evaluated for a solution. An optional, online anonymous Qualtrics ${ }^{\circledR}$ survey was sent via email to attendees to gather feedback. Results: A total of 40 pharmacists and 7 technicians responded to the perception survey. Most felt this activity emphasized critical thinking (87\%) and was appropriate for the topic of Medication Errors (83\%). The majority agreed that this activity helped them to better understand a Root Cause Analysis (63\%). Only 48\% felt that the group sizes were appropriate and $76 \%$ felt instructions were clear. Over $75 \%$ would like to see this type of activity utilized in another CE program. Implications: This activity was well received by participants. This activity may emphasize critical thinking by participants and their understanding of topics presented. There is opportunity for improvement in terms of group sizes and clarity of instructions for participants.

\section{EXPERIENTIAL EDUCATION} Completed Research

A Case Study in Assessing APPE Assessments: Three Strategies for Gathering Evidence of Validity. Eric H. Gilliam, University of Colorado, Jason M. Brunner, University of Colorado. Objectives: Use of meaningful and valid measurements of student performance in advanced pharmacy practice experiences (APPES) is critical to competency-based pharmacy education, yet validating assessments can be challenging. This content validity study was designed to gather evidence of valid student assessment occurring in APPE practice settings as a result of newly designed setting-specific assessment tools. Method: Evidence of validity was gathered from three sources. Active preceptors completed a job-task analysis of proposed assessment items, evaluating each item in terms of criticality and frequency, during tool development. Once implemented, each tool was assessed for applicability to its respective practice setting by determining the rate of successful measurement. An a priori rate of $85 \%$ was established for each item. A blinded two-step summative grading process was developed allowing preceptors to recommend grades prior to receiving the tool's calculated score. A statistical comparison of preceptorrecommended and tool-generated grades was conducted. An acceptable target rate of agreement was established as $80 \%$ or higher. Results: Of 128 eligible preceptors, 52\% completed the job-task analysis, indicating all evaluative items were at least moderately valuable within their aligned practice settings. All student assessments completed during the first year of implication $(n=1,190)$ were reviewed. The mean rate of applicability for all items was $98 \%$ across all APPE setting types. The mean rate of agreement between tool-generated and preceptor-recommended scores for all assessments was $96 \%$. Each setting-specific APPE tool also achieved individual performance benchmarks. Implications: This study indicated valid tool design using strategies available within any pharmacy program.

A Multi-Perspective Evaluation of an Interprofessional Introductory Pharmacy Practice Experience Integrated in a FederallyQualified Health Center. Benjamin Chavez, University of Colorado, Jason M. Brunner, University of Colorado, Eric H. Gilliam, University 


\section{American Journal of Pharmaceutical Education 2018; 82 (5) Article 7158.}

of Colorado. Objectives: To evaluate an innovative interprofessional (IP) introductory pharmacy practice experience (IPPE), including the impact of pharmacy students on patients and prescribers, in an underserved setting. Method: Third year pharmacy students completed a 20-hour IP-IPPE with a licensed prescriber who is not a pharmacist. An innovative model was created at a federally-qualified health center (FQHC). This model allows a prescriber to be directly responsible for experiential training, while immersing and integrating the student into the prescriber's clinical workflow. Students are in direct contact with patients and other health care providers during the IP-IPPE. Student-patient encounters were tracked to assess impact on patient care. Prescribers assessed students' ability to collaborate within teams using a standardized tool, and described the value of integrating students into their practice. Students self-assessed professional growth in interprofessional collaboration related competencies. Results: Thirty-two students completed the IP-IPPE at this FQHC over the summer. Eleven percent of patients $(n=516)$ seen at the clinic over this time period had contact with a pharmacy student. Tasks conducted by students included medication reconciliation, patient education, and drug information services. Prescriber surveys indicated students had a positive impact on their day-to-day activities. Students reported that the experience contributed to their development of interprofessional collaboration related competencies. Implications: This innovative experiential model gave students a unique learning experience while providing a valuable service to the clinic. This successful model has engaged three cohorts of students. Similar programs have been developed based on this model at three other FHQC sites.

An Evaluation of Interprofessional Activity in an Advanced Pharmacy Practice Experience Program. Cheryl L. Clarke, Drake University, Da Won Choi, Drake University. Objectives: To evaluate the interprofessional experience of advanced pharmacy practice experience (APPE) students at one college of pharmacy. Objectives were to 1) Describe the interprofessional experience; 2) Compare findings to ACPE accreditation standards; and 3) Identify site characteristics associated with interprofessional activity. Method: Data were evaluated from one APPE year of site evaluations, which were completed by students following each APPE. These evaluations documented interprofessional activities including participating professionals, methods, and frequency of communication. Interactions with healthcare professionals and healthcare professional students were reported. In addition, students participated in a voluntary online survey at the end of the APPE year to confirm site evaluation findings and identify site characteristics associated with interprofessional activity. Results: Students reported 91\% of patient care APPEs involved interprofessional interactions with physicians. Face-toface interaction and daily communication were most commonly reported. Similarly, interactions with medical students occurred at $40 \%$ of APPEs, most frequently on a daily and face-to-face basis. Survey findings showed $87 \%$ of students were satisfied or very satisfied with their interprofessional experiences, and meaningful physician and medical student interactions were reported. Site characteristics associated with higher level interprofessional experiences included faculty preceptor presence, general medicine/acute care APPE, use of collaborative practice agreements, and medical residency affiliation. Implications: APPE students reported interprofessional activity in compliance with ACPE requirements for prescriber and prescriber student interactions via face-to-face communication. Survey results confirmed the site evaluation results and suggested APPE sites with faculty preceptors, collaborative practice agreements, and medical residencies provided meaningful interprofessional activity.

Assessing Medication Name Pronunciation on Introductory Pharmacy Practice Experiences. Courtney R. Caimano, Albany College of Pharmacy and Health Sciences, Alyssa J. Smith, Albany College of Pharmacy and Health Sciences, Monique R. Bidell, Albany College of Pharmacy and Health Sciences. Objectives: To assess acceptable pronunciation of common medications as part of an Advanced Pharmacy Practice Experience (APPE)-readiness initiative. Method: Pharmacy students in their first (P1) and second professional year (P2) were required to complete a medication pronunciation activity during either their 2017 Community or Institutional Introductory Pharmacy Practice Experience (IPPE). Students self-recorded pronunciation of 20 (Community) or 16 (Institutional) look alike-sound alike medications using Panopto $^{\mathrm{TM}}$ software. Recordings were evaluated by two faculty, 1 each for Community and Institutional, using a standardized qualitative rubric (exemplary, acceptable, or unacceptable). Students who received an "unacceptable" were required to submit a revised recording. Results: A total of 421 students (201 Community, 220 Institutional) completed the assignment. For the Community activity, 55.7\% (112) of students scored “acceptable," while 10.4\% (21) and 28.9\% (58) scored "exemplary" and "unacceptable," respectively. For the Institutional activity, most students scored "exemplary" $(62.3 \% ; 137)$, while $14.1 \%$ (31) and $23.6 \%$ (52) scored "acceptable" and "unacceptable," respectively. All "unacceptable" students who revised the assignment (110) received an "acceptable" score. Implications: Findings suggest that the majority of P2 students are able to correctly pronounce common medications in community and inpatient settings. However, approximately one quarter of students required revision due to unacceptable pronunciation. Future assessments will include progression of acceptable pronunciation among the same student cohort from one IPPE to the next (ie, community to institutional) as a continued effort to support APPE-readiness.

Assessing Professionalism in a Large IPPE/Seminar Course. Jane Shtaynberg, Long Island University, Anna Nogid, Long Island University. Objectives: To assess student professionalism in a large experiential/seminar course Method: LIU Pharmacy begins professional development the first semester with the Introductory Pharmacy Practice Experience; a combination of practice visits and on-campus seminars. The average class size is 200 students. Using the APhA-ASP's The 10 Traits of a Professional, a rubric was designed to evaluate 7 of the 10 traits applicable in this course on a 3 point Likert scale. Student assessment relied on multiple items: attendance, assignments, active engagement via audience response system, and documentation of incidents/violations as reported by course faculty. A passing professionalism grade resulted from receiving "Meets Expectations" (3) and/ or "Needs Development" (2) on each trait. A failing professionalism grade resulted from "Unacceptable" (1) in any of the components which could lead to course failure. Results: Of the 201 students evaluated, $192(96 \%)$ received a passing professionalism grade. Of these, 137 (68\%) received a " 3 " in all components and 55 (27\%) received a combination of " 3 " and " 2 ". The greatest area needing improvement was "Accountability for his/her work" which relied on punctuality and meeting deadlines (23\%), followed by "Knowledge and Skills of a Profession" which relied on participation in course activities and assignment completion (8\%), and "Commitment to Self-Improvement of Skills and Knowledge" which relied on assignment resubmissions where applicable (5\%). Implications: This type of evaluation, which can be done in a large class setting, can be used to document professionalism in a specific course or as a tool to longitudinally track professional development and growth throughout the PharmD program.

Assessment of APhA Career Pathways Program for Pharmacy Students in Developing Self and Career Awareness. Jennifer Prisco, MCPHS University-Boston, Catherine Taglieri, MCPHS UniversityBoston, Steven J. Crosby, MCPHS University-Boston, Rita Morelli, 


\section{American Journal of Pharmaceutical Education 2018; 82 (5) Article 7158.}

MCPHS University-Boston. Objectives: To assess perceptions of the APhA Career Pathway Evaluation Program for Pharmacy Professionals in increasing career pathway recognition, in guiding APPE selection, and in enhancing student self-awareness (2016 ACPE Standards Key Element 4.1). Method: PY3 students were required to complete Pathways prior to choosing their APPE rotations. A Qualtrics survey was developed and distributed to students after the program completion. Items were primarily assessed via Likert scale responses. Data were analyzed via one-sample signed rank tests and Wilcoxon signed rank tests (SigmaPlot software). This research study was reviewed and approved by the University Institutional Review Board. Results: The survey was sent to 307 students, and 189 responded (62\% response rate). Of the respondents, $79.9 \%$ found the program beneficial $(\mathrm{p}<0.001)$. Prior to completing the program, $32.3 \%$ reported recognition of 7 or more career paths; following completion, $76.2 \%$ reported recognition of 7 or more career paths $(\mathrm{p}<0.001)$. Completing the APhA program aided respondents to select APPE rotation sites that matched to Pathway results $(p<0.001)$ and helped students choose elective rotations $(\mathrm{p}<0.001)$. The majority of respondents $(\mathrm{N}=132)$ agreed that the program made them think about their personal career path $(\mathrm{p}<0.001)$, and agreed that the critical factors within the program correctly identified their personality and goals $(\mathrm{N}=107, \mathrm{p}<0.001)$. Implications: The APhA Career Pathway Evaluation Program is a useful tool to augment self-awareness and to guide APPE rotation selection among PY3 students. Further, the program may be considered as a useful component of a student learning portfolio for development of self-awareness and professionalism.

Assessment of Interprofessional Collaboration During Required APPEs. Kayla Johanna Bardzel, Wilkes University, Michelle HoltMacey, Wilkes University, Edward F. Foote, Wilkes University. Objectives: To evaluate the extent of interprofessional interactions on required APPE rotations. Method: Interprofessional opportunities are required throughout the pharmacy curriculum but are particularly challenging to assess during APPEs. After every 5-week rotation, P4 students completed a survey that measured patient demographics and interprofessional involvement. Results: Over the first five rotation periods, all students $(n=72)$ completed at least one required rotation. There were 175 required rotations completed. Four students had all four required rotations, 29 had three, 33 had two and 6 had only one required rotation. In $91 \%$ of the rotations, students agreed that to that they were able to collaborate as part of an interprofessional team. When asked about frequency of interprofessional interactions, 59\% of rotations were deemed high frequency (daily or almost daily), 25\% were moderate (1-3 times per week), 13\% were low (rare encounters) and just $2 \%$ of the rotations had no interprofessional interactions. Interactions were most common with physicians (or their students) (153 rotations). Interactions with nurses were recorded in 151 rotations and physician extenders in 88 rotations. Implications: Post rotation surveys are an easy and efficient way to measure the extent of interprofessional interactions on APPE. In their required rotations, our students reported a high occurrence of interactions with other healthcare providers. This data can be used to ensure sites are appropriate for APPE rotations and will also facilitate necessary site development. The quality and impact of these interactions need further study.

Assessment of Interprofessional Practice, Education and Collaboration During APPE. Diane M. McClaskey, University of MissouriKansas City, Valerie L. Ruehter, University of Missouri-Kansas City, Angela Brownfield, University of Missouri-Kansas City, Bradley J. Erich, University of Missouri-Kansas City. Objectives: Our first objective was to determine pharmacy students' confidence in interprofessional practice and education (IPE) and collaboration through a survey completed before and after Advanced Pharmacy Practice Experiences (APPE). Our second objective sought to determine the depth, breadth, and impact of IPE through reflection assignments during each required APPE. Method: Students were assigned to complete a modified Interprofessional Collaborative Competency Attainment Survey (ICCAS) to determine their confidence before and after completion of the APPE rotation year. An open-ended reflection assignment regarding IPE was assigned for each required APPE rotation to determine the type of healthcare providers encountered, as well as the frequency, nature, and impact of interaction with other healthcare providers. Results: Of 121 students surveyed, statistical significance was found in all domains including communication, collaboration, roles/responsibilities, patient/family-centered approach, conflict management/resolution, and team functioning $(\mathrm{p}<0.001)$. Acute care rotations were most impactful in knowledge gained and skill development, allowing students to confidently contribute to collaborative patient-centered care. Advanced community rotations were least impactful in this regard. Descriptive statistics from each domain and qualitative comments from reflection assignments will be included in discussion. Implications: Standard 11 of the Accreditation Standards of 2016 calls for tailoring of the IPE approach based on insight provided by evolving research within this important area. These results support the efficacy of IPE in experiential education by preparing practice-ready graduates. Results will also be utilized to make modifications in programmatic APPE learning objectives.

Assessment of Practice Site Readiness for Intentional Interprofessional Experiential Education. Whitney Maxwell, University of South Carolina, Omar Jamjoom, Kimberly N. Bucher, University of South Carolina, Jennifer L. Baker, University of South Carolina, Matt Walker, Medical University of South Carolina, Cathy L. Worrall, Medical University of South Carolina. Objectives: In a recent report from the AACP Experiential Education Task Force on Intentional Interprofesional Education (IEE), one of the recommendations for implementing IEE is determining readiness of practice sites using an intentional approach. The objective of this study was to use regression analysis to identify rotation-related variables that were significantly associated with student perceptions of high-level Interprofessional (IP) interaction and IP team integration, which were used as markers for IEE readiness. Method: To identify pharmacy student rotation sites that are IEE-ready, comments from post-rotation student reflections completed over one academic year $(n=1,449)$ were collected, coded, and analyzed. Results: Sixty-two percent of student comments indicated high-level IP interaction and $74.2 \%$ of comments indicated high-level IP team integration. Within our dataset, introductory pharmacy practice experience (IPPE) rotations were not associated with high levels of IP interaction or team integration. All advanced pharmacy practice experience (APPE) rotation types except community were associated with high-level IP interactions and team integration. The constituents of the healthcare team significantly associated with high-level IP interactions and team integration were: registered nurses, respiratory therapists, physical therapists, dietician/nutritionists, mental health professionals, social workers, case managers, and medical students. Students from other disciplines (nurse practitioner, physician assistant, nursing), chaplains, medical assistants, respiratory therapy technicians, case workers, speech therapists, occupational therapists, and staff from outpatient medical clinics were not associated with high-level IP interactions and team integration. Implications: Noncommunity APPE rotations with practitioners and students from certain disciplines are associated with high-level IP interactions and team integration, and therefore likely IEE-readiness. 


\section{American Journal of Pharmaceutical Education 2018; 82 (5) Article 7158.}

Community IPPEs: An Analysis of Cost and Time Spent Training Students at Practice Sites. Catherine L. Oswald, Roseman University of Health Sciences, Phillip Lawrence, Roseman University of Health Sciences, Erin L. Johanson, Roseman University of Health Sciences, Darla Zarley, Roseman University of Health Sciences. Objectives: The purpose of this study was to estimate the amount of time and money spent training students during a community-based IPPE rotation for two activities: processing new prescriptions and administering immunizations. Method: Data submitted by second year community IPPE pharmacy students via an electronic form were retrospectively analyzed. Students estimated, and preceptors confirmed, the amount of time spent with technicians and pharmacists training on 2 tasks: processing new prescriptions and administering immunizations. An average time to train a student for each activity was calculated. A cost impact was calculated by multiplying the average time for training by the Bureau of Labor Statistics 2017 average hourly wages for the corresponding personnel. Results: One hundred seventy eight forms were submitted by students ( $76 \%$ response rate). Data was excluded for 72 submissions due to a lack of preceptor confirmation or the student failing to list the designation of personnel that assisted with training ( $\mathrm{n}=106$ accepted submissions). Results showed it took an average of 34 minutes to train students to process a new prescription ( $71.7 \%$ of time spent with technicians) and 27 minutes to administer an immunization (100\% of time spent with pharmacist). The cost per student associated with training is $\$ 15.37$ (process prescription) and \$25.80 (immunizations). Implications: By quantifying the amount of time and money spent training student-pharmacists for their IPPE community rotations the authors will be able to compare this data to previously published cost savings these same students provide, once trained to do so, and the financial value of precepting students can be estimated.

Creation of Experiential Success Plan Initiative to Address Experiential Performance Deficiencies. Laurie L. Briceland, Albany College of Pharmacy and Health Sciences, Courtney R. Caimano, Albany College of Pharmacy and Health Sciences, Sandra W. Rosa, Albany College of Pharmacy and Health Sciences, Cindy Jablanski, Albany College of Pharmacy and Health Sciences, Megan Veselov, Albany College of Pharmacy and Health Sciences. Objectives: ACPE Standard 17.2 encourages systematic early detection and resolution of academic/ behavioral issues. The purpose of this initiative was to develop a formal process for identifying experiential deficiencies and engaging students in reflection as to improve future performance. Method: An Experiential Success Plan (ESP) policy was developed and applied to the 2017-18 APPE cycle. At final evaluation, and in cases where there is midpoint failure or preceptor request for assistance with a struggling student, preceptors assessed each performance competency as per usual procedure, assigning exceeds or meets competency; needs improvement (NI) or significant deficiency (SD). Criteria for student eligibility for ESP included: $>2$ $\mathrm{NI}$; $>1 \mathrm{SD}$; Final grade of $\mathrm{D}$ or $\mathrm{F}$ (which also requires course repeat); case-by-case preceptor comments. Upon eligibility, a studentspecific ESP is provided to the student, who then completes three essays: reasons for deficiency; corrective action plan to improve future performance; and a rubric-assessed critical reflection on how implementing the action plan will enhance personal/professional development. ESPs are approved by EE personnel. Results: To date, 44 ESPs ( $4.3 \%$ of cohort) have been generated; average \# deficiencies per student ESP was 5.8, spanning patient care process, communications and professionalism. All but 2 students provided critical ESP reflection. ESPs represented all core and elective APPE classifications, with inpatient (16) occurring most frequently, followed by community (10). Implications: Through this initiative, students with experiential performance deficiencies have been identified, created plans for future performance improvement, and understand the value of implementing such corrective actions, as evidenced by critical reflection.

Design and Evaluation of an Interprofessional Education Teaching Certificate Program for Faculty and Clinicians. Melissa E. Rotz, Temple University, Ina Lee S. Calligaro, Temple University, John Duffy, Temple University. Objectives: To describe the design and evaluation of interprofessional education (IPE) teaching certificate program for faculty and clinicians at one institution. Method: An IPE teaching certificate program was designed to train interprofessional faculty and clinicians to facilitate IPE activities at Temple University. The program consisted of three 2.5-hour modules (core principles of IPE, small group facilitation, assessment, and debriefing simulations). Each module incorporated pre-reading and online learning modules and utilized active learning strategies (e.g. team building games, video demonstrations, and role playing). Participants completed a survey evaluation at the end of each module. Results: Thirty-three participants (14 pharmacists; 5 nurses; 3 physical therapists; 2 athletic trainers; 2 dentists; 2 physicians; 2 occupational therapists; 1 certified therapeutic recreation specialist; 1 social worker; 1 podiatrist) completed the program. A majority of participants indicated that the learning objectives were achieved for each module. Thematic analysis demonstrated a majority of participants could articulate the key concepts from each of the activities. Of the participants who completed the program, $82 \%$ volunteered to be facilitators for subsequent IPE workshops which is a component of the IPE curriculum that is delivered to dental, nursing, medical, pharmacy, PT, OT, podiatry students. In aggregate, student evaluations of facilitators improved after implementation of this program. Notably, scores increased for the item "facilitators in this workshop were positive role models for interprofessional collaboration," which was a goal of the program. Implications: After completing this program, participants learned important skills for facilitating interprofessional groups which is critical to delivering a successful IPE curriculum.

Design and Impact of an Advanced Pharmacy Practice Experience Rotation in a Dental Clinic. Kalin L. Johnson, Creighton University, Karen O'Brien, Creighton University, Shana Castillo, Creighton University, Kimberley J. Begley, Creighton University. Objectives: Pharmacy students must complete advanced pharmacy practice experiences (APPEs) during their last year of pharmacy school while participating in interprofessional care teams. Dental patients often have multiple comorbid conditions and many medications that can impact their dental treatment and prescribed medications. The objective of this interprofessional educational collaboration was to design an APPE rotation for fourth year pharmacy students in a University dental clinic and assess its impact. Method: A total of 21 pharmacy students spent five weeks rotating through the Creighton University School of Dentistry dental clinic from August 2015 to July 2017. A descriptive checklist of interventions was kept on a daily basis by students for impact analysis. Results: The pharmacy students, with oversight from a pharmacy faculty member, assisted dental students with acquiring, correcting, and evaluating 2,708 medication histories. Pharmacy students identified 575 adverse reactions/drug interactions, 553 drug choice problems, 458 patient-related problems, and 228 dosing problems. A total of 74 recommendations were given on the prescribing of dental medications. Implications: The data demonstrates the impact pharmacy students can have in a dental clinic. The students were able to assist in identifying drug related problems that would have likely 


\section{American Journal of Pharmaceutical Education 2018; 82 (5) Article 7158.}

gone undetected. Allowing pharmacy and dental students to interact on a regular basis may result in an increased understanding of the role of each profession, increased interprofessional communication, and better outcomes for patients. Cultivating the dental and pharmacy student relationship may result in the professions utilizing each other to a greater degree for their expertise when they are licensed and practicing.

Development and Assessment of a Simulated Professional Meeting in the Experiential Curriculum. Lisa P. DeGennaro, University of Saint Joseph, Jennifer L. Luciano, University of Saint Joseph. Objectives: The objective of this project was to assess student preparation, performance, and engagement during a simulated professional meeting. Secondary objectives were to determine if this experience served as a valuable mechanism to develop professionalism, selfawareness, and communication skills among students. Method: A simulated professional meeting (Colloquium Day) was created in which APPE students were required to present a patient case, clinical topic or research poster. Faculty provided feedback via a rubric. A survey was designed to collect feedback for quality improvement and to evaluate student responses in the areas of professionalism, self-awareness, and communication. Some questions were specifically designed for selfreflection by students. This survey was distributed electronically to faculty and student participants after the experience. Results: A total of eight faculty and thirty-eight students completed the survey with a response rate of $36 \%$ and $48 \%$, respectively. Faculty and students agreed that the students performed at a level expected of APPE students and that students demonstrated a high level of professionalism throughout the day (faculty $4.25 / 5$ vs. students $4.21 / 5$; faculty $4.375 / 5$ vs. students $4.18 / 5)$. Survey responses from student presenters noted a high level of confidence in presentation skills (4.25/5) and the importance of seeking feedback from mentors prior to the presentation $(4.25 / 5)$. Overall, participants felt that the Colloquium Day was a valuable experience and should be continued (4.08/5). Implications: Results and feedback from this survey will shape the design of subsequent Colloquium Days and guide the development of future curricular and co-curricular activities.

Development of a Faculty-Led Health-System IPPE Pilot Program Including APPE Mentorship. Gretchen Jehle, MCPHS UniversityWorcester/Manchester, Karyn M. Sullivan, MCPHS UniversityWorcester/Manchester, Kara Bonaceto, MCPHS University-Worcester/ Manchester. Objectives: (1) Identify ways to expose Introductory Pharmacy Practice Experience (IPPE) students to components of the healthsystem by scheduling activities encompassing the pre-APPE core domains and Joint Commission of Pharmacy Practitioners: Pharmacists' Patient Care Process (PPCP). (2) Determine if mentoring a health-system IPPE student can result in benefits for Advanced Pharmacy Practice Experience (APPE) students relative to the Center for Advancement of Pharmacy Education (CAPE) Educational Outcomes subdomains 4.1 (Self-awareness), 4.2 (Leadership), and 4.4 (Professionalism). Method: A faculty-led health-system IPPE pilot was conducted as part of a fourweek rotation in which one student participated in activities designed to encompass the pre-APPE core domains and PPCP. Five APPE students had a role in mentoring the IPPE student. The IPPE and APPE students completed an optional post-survey consisting of closed and open-ended questions to obtain specific and reflective responses. Results: The IPPE student completed the survey and reported having exposure to ten of the eleven (91\%) pre-APPE core domains. The student also reported gaining an understanding of the PPCP in the health-system setting by shadowing in the Hepatitis C and travel clinics. All five APPE students (100\%) completed the survey and commented that they had improved their leadership (4.2) skills. Mentorship also allowed the students to self-reflect on their progression (4.1 Self-awareness) and to work with peers as a role model (4.4 Professionalism). Implications: The pilot IPPE encompassed nearly all pre-APPE core domains and led to benefits for APPE students in several CAPE areas. Next steps include a larger pilot with more students and adjunct faculty.

Development of an Evidence-Based, Longitudinal Interprofessional Education Program Integrated Into a PharmD Curriculum. Joseph A. Zorek, University of Wisconsin-Madison, Susanne G. Barnett, University of Wisconsin-Madison, Betty A. Chewning, University of Wisconsin-Madison, Casey Gallimore, University of Wisconsin-Madison, Lizeth Garcia-Jennings, University of WisconsinMadison, David Hager, University of Wisconsin-Madison, Robert Holland, University of Wisconsin-Madison, Beth K. Janetski, University of Wisconsin-Madison, Paula Jarzemsky, University of WisconsinMadison, Mara A. Kieser, University of Wisconsin-Madison, Karen Kopacek, University of Wisconsin-Madison, Amanda Margolis, University of Wisconsin-Madison, Beth A. Martin, University of WisconsinMadison, Michael E. Pitterle, University of Wisconsin-Madison, Jeanette C. Roberts, University of Wisconsin-Madison, Katherine Rotzenberg, University of Wisconsin-Madison, Katy Tomlinson, University of Wisconsin-Madison, Denise L. Walbrandt Pigarelli, University of Wisconsin-Madison, Alex Wontor, University of WisconsinMadison, Melgardt M. de Villiers, University of Wisconsin-Madison. Objectives: To develop an evidence-based, longitudinal, integrated interprofessional education (IPE) program. Method: An IPE committee with stakeholders throughout the PharmD curriculum and faculty partners from medicine, nursing, and an institution-wide IPE Center was charged to develop the program. A literature review was completed to identify theoretical frameworks upon which to build the program and validated measurement instruments to assess students' progress. The quantity and types of IPE instruction within the required curriculum was inventoried and categorized as part of a gap analysis. Consensus on program components was forged through an iterative process involving the IPE, Experiential, Curriculum, and Assessment committees. Results: An interprofessional socialization framework to facilitate dual identity formation was chosen as the foundation of the program, with a structure based on the Institute of Medicine's Interprofessional Learning Continuum Model. One hundred hours of pre-Advanced Pharmacy Practice Experience (APPE) learning activities intended to develop interprofessional competencies but completed uniprofessionally were identified and categorized as pre-IPE. Forty-two hours of intentionally-sequenced pre-APPE didactic, experiential, and co-curricular IPE activities targeting interprofessional competencies are being developed to augment pre-IPE instruction. An assessment plan linked to Kirkpatrick levels 1 (reactions), 2a (attitudes/perceptions), 2b (knowledge/skills), and 3 (behaviors) using validated measurement instruments appropriate for each Kirkpatrick level and vetted by the National Center for IPE will be administered at developmentally appropriate stages in the pre-APPE and APPE years. Implications: This programmatic approach is intended to ensure students' readiness for both teamwork prior to APPEs and entry-level interprofessional collaborative practice prior to graduation in accordance with accreditation requirements.

Development of an Innovative Critical Care Pharmacy Elective Course Utilizing Online Case-Based Patient Simulations. Paul Staffieri, Long Island University, Francesco Ciummo, Long Island University, Yoonsun Mo, Long Island University. Objectives: The primary objective of this study was to develop and implement an innovative critical care elective course that utilizes didactic teaching with case-based discussions using online patient case simulation technology. 


\section{American Journal of Pharmaceutical Education 2018; 82 (5) Article 7158.}

The secondary objective was to evaluate the impact of the course on student problem solving skills. Method: A 3-hour critical care elective course, consisting of didactic lectures and small-group case discussions and presentations, was offered during the 2017 fall semester to third professional year pharmacy students. Neehr Perfect Go!® technology was utilized to simulate patients and electronic health records. To assess for improvement in problem-solving skills, case-based test scores were compared before and after the course utilizing a modified version of the VALUEs problem solving assessment ranging from 1 to 4 . At the end of the course, an online post-course survey was administered to students to assess for perceived improvement in problem-solving skills. Results: The survey revealed that 11 out of 12 students found that Neehr Perfect ${ }^{\circledR}$ technology improved their problem solving skills, with all students agreeing that the problem solving skills developed in the course would better prepare them for their clinical rotations. Based on the modified VALUEs rubric, average scores improved in defining problem (2.2 to 2.6 ), proposing solutions (2.2 to 2.5 ), and evaluating potential solutions (2.1 to 2.6), but decreased in identifying strategies (2.4 to 2.3 ). Implications: This study suggests that the integration of online simulated patient cases into a critical care pharmacy elective course may improve student problem-solving skills.

DIcipher: Assessing Drug Information Skills Through Gaming. Cambrey Nguyen, The University of Kansas. Objectives: Using an "escape-room" themed challenge to assess drug information (DI) skills on an experiential rotation has not been reported in the literature. This innovative strategy incorporates concepts of serious gaming supported by AACP with the application of DI knowledge, critical thinking and teamwork. The objective of this study was to determine whether an "escape-room" themed challenge, DIcipher, assessed DI skills of P4 students on a DI rotation. Method: The 60-minute challenge featured DI questions pertaining to a rare infection that was not taught in the pharmacotherapy courses. The students worked as a team to solve puzzles and clues to obtain the DI questions staged in a room. The students were required to use DI resources to find the correct answer to each question in order to unlock a box with the antidote. A discussion session and a Likert-scale survey were used to document the students' experience. Results: There were a total of 15 students that participated in DIcipher from June to August 2017 and two groups reported 'winning' the challenge. The students struggled the most with finding the answer to the natural products and preventative vaccination questions. The majority of the students agreed the challenge promoted active learning while assessing knowledge of DI resources and identified specific resources that require additional practice. Implications: The students' responses indicated that DIcipher assessed their knowledge of DI resources in a fun and novel setting. Due to the positive feedback, DIcipher will be implemented in the drug information course in the spring.

Early Introductory Pharmacy Practice Experiences May Improve Empathy Among First-Year Student Pharmacists. Melissa M. Dinkins, Wingate University, Christopher Gillette, Wingate University, Jenna Brown, Wingate University, Michael L. Manolakis, Wingate University, Lisa S. Smith, Wingate University. Objectives: The purpose of this study was to examine: (a) student pharmacist empathy development over the first semester of pharmacy school, and (b) whether early introductory pharmacy practice experience (IPPE) impacts first-year student pharmacist empathy development. Method: A prospective randomized controlled trial was conducted to accomplish the study objectives. Students were randomized to first-semester IPPE enrollment (treatment group) or second-semester IPPE (control group). All students, regardless of group, completed the Jefferson Scale of
Empathy on the first day of class. During the first semester, IPPE students were exposed to formal empathy training while non-IPPE students did not receive empathy training. Empathy was then assessed at the end of the semester. Paired t-tests were used to examine change in empathy in both groups. A two-tailed independent samples t-test was conducted to examine whether early IPPE exposure and empathy training was associated with changes in empathy scores. Analyses were restricted to the main campus. Results: Data for 72 of 81 first-semester student pharmacists were analyzed ( $88.9 \%$ response rate). There were no significant differences in baseline demographic characteristics between the groups. In the IPPE group, there was a non-significant increase in empathy over the course of the semester $(t=0.63, p=0.53)$. The non- IPPE group had a statistically significant decrease in empathy $(\mathrm{t}=-2.86$, $\mathrm{p}=.01$ ). Comparing the educational groups, there was a significant difference in change of empathy scores $(1.13$ vs. $-4.55, \mathrm{t}=-2.37, \mathrm{p}=0.02)$. Implications: Early student pharmacist exposure to IPPE and empathy training may improve first-year student pharmacist empathy relative to no IPPE exposure.

Evaluating Interprofessional Education Outcomes of a Dental School Pharmacotherapy Consult Service. Kimberly A. Sanders, University of North Carolina at Chapel Hill, Christine L. Downey, University of North Carolina at Chapel Hill, Kathryn A. Fuller, University of North Carolina at Chapel Hill, Jennifer B. Harmon, University of North Carolina at Chapel Hill, Shannon H. Mitchell, University of North Carolina at Chapel Hill, Heidi N. Anksorus, University of North Carolina at Chapel Hill. Objectives: Promoting collaboration of future dental and pharmacy professionals has the potential to positively impact patient care. This study evaluated interprofessional education outcomes within implementation of a pharmacotherapy consult service involving doctor of dental surgery (DDS), dental hygiene $(\mathrm{DH})$, and doctor of pharmacy (PharmD) students in dental school-based clinics. Method: The pharmacotherapy consultation process was created for third year DDS and second year DH students to request medication-related consults for clinic patients throughout an academic year. Consult services were conducted by pharmacists and second year PharmD students which included reviewing patient medication histories and profiles, providing medication and disease state education, and answering medication-related inquiries. Pre-post experience surveys were conducted assessing DDS, DH, and PharmD students' knowledge of each discipline's scope of practice using confidence scale statements, interprofessional communication perceptions, and program evaluation. Assessment of student clinical experience was evaluated through self-reflection, focus groups, and consult tracking technology. Results: Of the 116 students $(\mathrm{DDS}=68, \mathrm{DH}=33$, PharmD $=15$ ) included, 75 completed pre-post experience surveys (DDS $=45, \mathrm{DH}=24$, PharmD $=6$ ). Significant increases in reported confidence from baseline were noted in $100 \%, 72.3 \%$, and $83.3 \%$ of statements for DDS, DH, and PharmD students, respectively $(p<0.05)$. Emerging qualitative themes included increased understanding of using medication lists to evaluate patients' medical problems and impact on oral health, reinforcement of didactic material in clinical settings, respect for differing roles, and appreciation of interprofessional communication. Implications: Dental school clinic settings provide a rich opportunity for interprofessional interaction and results indicate student learning benefits when pharmacy services are integrated within the dental clinic environment.

Evaluation of Unified Experiential Assessment Scale Across Three Pharmacy Programs in Indiana. Meghan Bodenberg, Butler University, Ahmed Abdelmageed, Manchester University, Brian M. Shepler, Purdue University. Objectives: To determine if the use of a unified 


\section{American Journal of Pharmaceutical Education 2018; 82 (5) Article 7158.}

experiential assessment scale clarifies preceptors' overall assessment of the student, improves understanding of the colleges' expectations for pass/fail, and increases preceptors' standardization of assessments across programs. Method: In 2016, all three pharmacy programs in Indiana (Purdue, Butler and Manchester) adopted a standard assessment scale for evaluating rotation students, which included details about what the schools defined as "exceeds", "meets" and "does not meet" expectations, as well as "automatic fail" categories. Preceptors who have taken students from all three colleges between the dates of May 2016 and May 2017 were invited to complete an online, anonymous survey. An email reminder was sent every two weeks during the six week recruitment period. Responses were analyzed using descriptive statistics. Results: Fifteen of 72 identified preceptors (20\% response rate) completed the survey. Ninety-three percent of preceptors strongly agreed or agreed with the following: additional definitions for the assessment scale are clear, unified assessment made it easier for the preceptor to evaluate students standardly across all three program, and the list of automatic failures has helped preceptors feel more comfortable justifying their decision to pass or fail a student. Additional suggestions from preceptors were to consider "needs improvement" rather than "does not meet expectations" and to define the number of failed evaluation sections that should result in failure of the rotation. Implications: Using a standardized assessment scale may help clarify expectations and make the evaluation process easier for preceptors taking students from multiple colleges of pharmacy.

Examining Tracked Patient Encounters During Advanced Pharmacy Practice Experiences and Skill Self-Assessment Using Entrustable Professional Activities. Jody L. Lounsbery, University of Minnesota, Bethany A. Von Hoff, University of Minnesota, Scott A. Chapman, University of Minnesota, Caitlin K. Frail, University of Minnesota, Jean Y. Moon, University of Minnesota, Ann M. Philbrick, University of Minnesota, Zachary Rivers, University of Minnesota, Chrystian R. Pereira, University of Minnesota. Objectives: To determine if the number of patient encounters during advanced pharmacy practice experiences (APPEs) relates to self-determination of patient care skills using entrustable professional activities (EPAs). Method: During 12-week acute care/institutional (AC/INST), 15-week combined community pharmacy and ambulatory care (CPAC), and three 5-week AC/INST or CPAC elective APPEs, fourth-year students completed patient tracking surveys. Students documented number of encounters, type of care provided, primary and secondary diagnoses, and special dosing/population considerations. Students completed selfassessment surveys based on 12 EPAs. Students rated their ability to perform each EPA using a four-point scale $(1=$ still developing this skill; 4 = can do this independently) at the start and after each APPE semester. Results: Students self-reported 79,896 encounters. During AC/INST and AC/INST elective APPEs, 280 students documented 67,557 encounters. During CPAC and CPAC elective APPEs, 289 students documented 12,339 encounters. The mean ratings for the 12 EPAs ranged from 3.2 to 3.7 at the start and 3.5 to 3.9 at the end of APPEs. Skills that students most frequently reported "can do this independently" at the end of APPEs included effectively communicate verbally with the patient and other healthcare providers (88\%; start: $52 \%)$ and provide medical information as written documentation (79\%; start 42\%). Implications: Increased exposure to patient encounters may improve student perceptions of their preparedness to perform various patient care skills. Tracking encounters highlights the need to ensure students see sufficient encounters to gain ability and confidence in their patient care skills.

Expanding International Rotations for Pharmacy Students. Keith DelMonte, St. John Fisher College, Christine R. Birnie, St. John
Fisher College, Lipika Chablani, St. John Fisher College, Kobi Nathan, St. John Fisher College, Andrea DiGiorgio, St. John Fisher College. Objectives: Pharmacists in any health setting will require the cross-cultural skills necessary to be effective in caring for their diverse populations. International Advanced Pharmacy Practice Experiences (APPE) provide students an opportunity to further develop those skills in the context of the practice setting. As a result, efforts were made to increase the number of international Advanced Pharmacy Practice Experiences (APPE) available to pharmacy students. Method: Over the last 10 years, new international APPE rotations were developed through various means. Service trips were redesigned to align with APPE rotation schedules, international students used their professional networks to identify potential rotation sites in their home countries, and faculty were encouraged to draw upon their international networks. Ongoing partnerships were developed with service organizations, international hospitals and universities. To increase student interest, awareness of the international rotations was highlighted through student presentations and media attention following the rotations. Results: The number of international rotations administered increased from three to nine annually over an eight year period, with participation increasing from six to 17 students. APPE rotations are now available in 14 different countries, with students participating in community, hospital and service-focused practice settings. Additional sites are currently under development. Implications: With targeted efforts in establishing new sites and raising awareness, student involvement in international APPE's has increased exponentially. These projects provide additional opportunities for students to build crosscultural skills that will aid them in any future practice setting.

Factors That Influence Advanced Pharmacy Practice Experience Selection. Kristina Powers, Wilkes University, Vicky Shah, Wilkes University. Objectives: To assess student perception of factors potentially influencing Advanced Pharmacy Practice Experience (APPE) selections. Method: Wilkes University Pharmacy students are required to complete 1440 hours of experiential education during their final year of pharmacy school. Students must choose from seven rotations which include ambulatory, community, inpatient general medicine, hospital/ health system and three electives. Third (P3) and fourth (P4) year professional students completed an anonymous survey to determine what factors impact their decision process when selecting required rotations. Factors include area of interest, size of institution, location, future employment, preceptor reputation, rotation hours, faculty rotation, nonfaculty rotation, peer recommendation, cost/housing, level of difficulty, size of institution, and if a residency is offered. The students ranked the top five factors which influenced their required APPE selection. Results: $100 \%$ of P3 (71) and P4 (72) students responded to the survey. Both classes selected location as the number one factor which influenced their decision for selecting required rotations. Cost/housing was second most important for P3's for all rotations except inpatient general medicine. P3's ranked faculty rotation as second most important for this rotation. Peer recommendation was second most important for P4's for all rotations except inpatient general medicine. P4's ranked preceptor reputation as second most important for this rotation. Implications: Location appears to be the driving factor for selecting APPE rotations. It would be beneficial to look into future rotation sites which potentially offer housing to help decrease costs. Further research into the factors that influence ranking on APPE electives is warranted.

Global Health Learning Outcomes and Competencies Among Pharmacy Students. David R. Steeb, University of North Carolina at Chapel Hill, Monica L. Miller, Purdue University, Ellen M. Schellhase, Purdue University, Jodie V. Malhotra, University of Colorado, Thomas 


\section{American Journal of Pharmaceutical Education 2018; 82 (5) Article 7158.}

A. Angelo, University of North Carolina at Chapel Hill. Objectives: The purpose of this study is to assess the learning outcomes and global health competencies of pharmacy students who participate in international Advanced Pharmacy Practice Experiences (APPE). Method: Fourth year pharmacy students $(n=46)$ participating in an international APPE at the UNC Eshelman School of Pharmacy, Purdue University College of Pharmacy, and University of Colorado Skaggs School of Pharmacy and Pharmaceutical Sciences were matched with fourth year students not completing an international APPE $(n=46)$ based on age and rotation schedule. A retrospective pre-post survey assessed each cohort's self-perceived ability to meet basic global health competencies as defined by the Consortium of Universities for Global Health on a 5-point Likert scale. Wilcoxon signed rank test was used for analysis within cohorts and the Mann-Whitney U test for analysis between cohorts. The international APPE cohort answered additional questions assessing knowledge, skills, and attitudes gained or enhanced from their international rotation. Results: The international APPE cohort had significant growth $(p<0.05)$ for all thirteen competencies compared to minimal growth for three competencies in the non-international APPE cohort. The largest pre-post differences in the international APPE cohort were for describing the role major entities play in global health $(+1.24, \mathrm{p}<0.001)$ and describing major public health efforts to reduce disparities in global health $(+1.15, \mathrm{p}<0.001)$. Limited resource utilization, interpersonal communication, and an appreciation for global and cultural differences were the most common growth areas across knowledge, skills, and attitudes respectively. Implications: International APPE rotations strengthen basic global health competencies and provide an opportunity for meaningful learning beyond pharmacy content areas.

Healthy Aging Fair: An Interprofessional Community Outreach Collaboration. Gina M. Baugh, West Virginia University, Gretchen M. Garofoli, West Virginia University. Objectives: (1) To describe an innovative interprofessional education IPPE community outreach initiative (2) To assess student attitudes toward interprofessional education through course evaluation. Method: Student pharmacists collaborated with Audiology, Music Therapy, Occupational Therapy, Physical Therapy, and Speech \& Language Pathology students to offer a Healthy Aging Fair to three rural communities. Interprofessional student teams were charged with developing and implementing twelve interactive stations involving health education, screenings, and immunizations. A 360-degree assessment of the project was completed with data from students, faculty and community participants. Results: Thirty-one student pharmacists completed the course evaluation survey that included questions from the IPEC Competency Survey, assessing their competency in two domains: roles and responsibilities (3 questions) and interprofessional communications (4 questions). Students responses were overwhelming positive, with $97-100 \%$ marking agree or strongly agree to the roles and responsibilities questions, and $100 \%$ marking agree or strongly agree in the communications domain. According to exit survey results, the health fair was rated excellent by $86.4 \%$ of the community participants. Verbal feedback to faculty facilitators from students and participants was positive and confirmed project success. Faculty debriefing sessions were held after each event leading to continuous quality improvements. Implications: Student teams working in collaboration are able to positively impact the community while learning valuable interprofessional skills including roles and responsibilities and communications. Community settings offer a dynamic learning environment and possibility for unique interprofessional partnerships that challenge students and faculty to be innovative in project design and implementation as well as flexible in meeting the various needs of the community.
Impact of a Flu Clinic Simulation on Student Readiness to Provide Immunization Services. Ashley N. Hannings, The University of Georgia, Kay L. Brooks, The University of Georgia, Michelle McElhannon, The University of Georgia, Russell Palmer, The University of Georgia, Katie Smith, The University of Georgia. Objectives: To assess the impact of a flu clinic simulation on student perceived readiness to provide immunization services and to assess immunization administration performance. Method: Second year student pharmacists participated in a flu clinic simulation following completion of the American Pharmacists Association's Pharmacy-based Immunization Delivery course. The simulation was completed prior to their immunization patient care experiences. The simulation consisted of five stations (patient, non-immunizer clinic role, vaccine administration, documentation, frequently asked questions). A 10-item survey was administered prior to and following the simulation. Students used a 3-point scale to indicate perceived readiness to perform each step of the immunization process independently. In addition, preceptors evaluated student technique and performance during the simulation at the administration station. Results: Eighty-nine percent of P2 students (125/141) consented to participate in the study. In the post-survey, students indicated an increased sense of readiness for all steps of the process, with the following showing the greatest improvement: organizing a mobile flu clinic, using the state registry, and documenting per state law ( $\mathrm{P}<.0001$ each). On the preceptor assessment, $54 \%$ of students scored above average at the administration station. As a result of preceptor feedback, $89.5 \%$ of students reported feeling more prepared for immunization practice. In addition, 92\% reported feeling more prepared because of the realistic qualities of the simulation. Implications: Simulation can be a useful tool to reinforce best practices for immunization services, especially for programs that may not have the resources to provide specific patient care experience in this area.

Implementation and Perceived Benefit of a Preceptor Fair to Showcase Experiential Education Opportunities. Jennifer Steinberg, Nova Southeastern University, Stacey M. Maravent, Nova Southeastern University. Objectives: Provide an opportunity for students to interact with preceptors while obtaining information on potential experiential sites and rotations. Method: Since 2014, the Experiential Education team at Nova Southeastern University (NSU) invites preceptors to showcase sites and experiences at Preceptor Fairs conducted at NSU campuses. Students are invited to attend fairs at any campus based on their professional and geographical interests and freely interact with preceptors to gather information at their own pace. Preceptors promote their experiences and are provided preceptor development continuing education following the fair. Upon exiting the 2017 fairs, students were surveyed on their perceived benefit of the event. Results: Eighty students attended two fairs held in November 2017 , with $85 \%$ responding to the survey. The majority were class of 2019 , indicating they were exploring advanced pharmacy practice experience options. Overall, $92.4 \%$ of respondents felt the preceptor fair was beneficial, and $72.7 \%$ felt more confident in their site selection plans after attending. Many respondents indicated their experiential plans were influenced, with $55.2 \%$ stating their plans for site selection changed and $61.2 \%$ stating they became interested in a new type of experience because of their interactions during the fair. Implications: The annual Preceptor Fair helps students better prepare for and plan experiences to help achieve their professional goals. Interactions with the experiential team provides opportunities for preceptor development and support. Networking, communication and engagement among students, preceptors, and experiential teams are important as the landscape of pharmacy continues to evolve. 


\section{American Journal of Pharmaceutical Education 2018; 82 (5) Article 7158.}

Implementation of a Communication Skills Module in PharmD New Student Orientation: Feasibility, Successes, and Implications. Sharon Rush, The University of Texas at Austin, Laura E. Brown, University of Texas at Austin, Erin Donovan, The University of Texas at Austin. Objectives: To determine the feasibility of a communication skills module during new student orientation as an academic leveling effort prior to pharmacy students' first year in a PharmD program. The module was part of a new interdisciplinary collaboration between the College of Pharmacy and the College of Communication at a Tier 1 research institution. Method: The two-hour module was composed of introductory comments, a large $(\mathrm{N}=125)$ group warm-up, and five interactive breakout sessions $(\mathrm{n}=25)$ facilitated by health communication educators. Breakout sessions included four interactive exercises and accompanying debriefs to discuss the communication skills students learned and practiced, and what the skills look like in an applied pharmacy setting. Students were surveyed post-module. Results: Students were taught communication skills such as rapport building, active listening, persuasion, and how to bridge health literacy issues by being audience-focused. Student survey results revealed that $89 \%$ agreed that the module improved their communication skills; $92 \%$ agreed that the activities were realistic; $93 \%$ indicated a preference to receive additional communication training. Implications: New student orientation that includes a communication skills academic leveling session is feasible. Interactive activities should include accompanying debrief discussions so that students gain the full benefit of the exercises. The module helped students connect with their classmates, consider how to adapt to others while communicating, and understand how their communication skills would be useful in professional settings, including during their PharmD program. Given the high percentage of students who want more communication training, additional programming is recommended.

Institutional Factors Associated With Global Health Education Across US Pharmacy Schools. David R. Steeb, University of North Carolina at Chapel Hill, Benjamin Y. Urick, University of North Carolina at Chapel Hill, Betsy L. Sleath, University of North Carolina at Chapel Hill, Pamela U. Joyner, University of North Carolina at Chapel Hill. Objectives: To determine whether institutional factors influence the offering and participation of international Advanced Pharmacy Practice Experience (APPE) rotations or global courses across US pharmacy schools. Method: Data for this study comes from a survey of US pharmacy schools conducted in 2014. The survey assessed international APPE rotation and global course offerings including student participation, interprofessional classification, and student demand. Responses were linked with institutional factors including tuition, class size, geographic location, academic medical center affiliation, year established, and ranking. Multivariate regression models were used to evaluate the relationship between individual institutional factors and international APPEs and global courses. Results: More institutional factors correlated with international APPE rotations than with global course offerings. In multivariate models, older and more established schools had a larger percent of the graduating class with an international APPE $(9.5 \%$ vs. $2.6 \%, \mathrm{p}<0.01)$ and a larger number of international APPE offerings (3.7 vs. 1.4, p $<0.01$ ). Schools with higher tuition had a larger percent of their graduating class with an international APPE ( 7.9 vs. 3.0, p $<0.05)$, a greater number of international APPEs (3.7 vs. $1.5 \mathrm{p}<0.05$ ), and more interprofessional international APPEs $(\mathrm{p}<0.001)$. Implications: Institutional factors of tuition and year of establishment have the strongest association as it relates to international APPE rotations, which could imply that these experiences are more dependent upon internal costs and the infrastructure of the institution. There are opportunities to overcome perceived cost and infrastructure barriers by focusing on global health opportunities in a local context and integrating global health principles into existing experiences and courses.

Integrating the Entrustable Professional Activities (EPAs) for Pharmacy Practice Into General Experiential Learning Outcomes. Erin L. Johanson, Roseman University of Health Sciences, Phillip Lawrence, Roseman University of Health Sciences, Catherine L. Oswald, Roseman University of Health Sciences, Darla Zarley, Roseman University of Health Sciences. Objectives: To integrate the ACPE Standards 2016, CAPE Outcomes 2013, PPCP Wheel, and AACP EPAs for New Pharmacy Graduates into college-level general experiential learning outcomes. Method: The Experiential Team spent a considerable amount of time review of what already existed, identified repetition/overlap in existing experiential learning outcomes, reviewed rubrics, assessment forms, evaluated preceptor and student feedback and completed past evaluation forms. The goal was to redesign for clarity/brevity for students, preceptors, and provide specific micro-level activities through EPA statements that would identify practice-based examples for preceptors to better assess student achievement of outcomes through specific behaviors and longitudinal progression through the experiential curriculum. Robust spreadsheets were created to mapped ACPE Standards 2016, CAPE Outcomes 2013, AACP EPAs for New Pharmacy Graduates, and experiential learning outcomes. Results: Nineteen general learning outcomes were created for all experiential rotations and implemented for 2017-2018 academic year rotations. For required IPPE and APPE Community and Institutional rotations, expectations for levels of progression ( $\mathrm{P} 1$ to $\mathrm{P} 2$ to P3) were identified and general learning outcomes further illustrated through specific mapping of EPAs to enable a robust picture of student progression through the experiential curriculum. Implications: EPAs provide specific examples to help students and preceptors determine what specific activities provide opportunity to meet a learning objective. EPAs are clear ways students can demonstrate progress in skills, abilities, and attitudes. General learning outcomes which incorporate EPAs allow opportunity to differentiate the level (introductory vs. advanced) of student learning on rotations.

Interprofessional Education and Collaboration Within the Experiential Curriculum. Erik Jorvig, Roseman University of Health Sciences, Erin L. Johanson, Roseman University of Health Sciences, Phillip Lawrence, Roseman University of Health Sciences, Catherine L. Oswald, Roseman University of Health Sciences, Darla Zarley, Roseman University of Health Sciences. Objectives: To quantify student exposure to interprofessional education and collaboration within the experiential curriculum. Method: Retrospective analysis of surveys submitted by students completing experiential rotations from August 10th, 2016 to September 1st, 2017. At the end of each rotation, students completed surveys describing site characteristics and patient population including the question "Please indicate which of the following allied health providers you had the opportunity to interact with at your site (select all that apply)". Responses were stratified by rotation type, program year, and healthcare provider type. Results: Evaluations $(2,525)$ were submitted by 672 students. Overall, 97\% of rotations involved interprofessional interaction and $100 \%$ of respondents indicated at least one interaction during the year. Of those responses, the predominant interaction was with physicians (78\%) with nurses close behind (74\%). Intriguingly, interactions between students and dentists in community settings was higher than expected at $43 \%$. Interactions with allied health students lagged behind other measures at only $32 \%$, despite being an amalgamated category. Interactions in 


\section{American Journal of Pharmaceutical Education 2018; 82 (5) Article 7158.}

hospital/clinic-based rotations were only slightly higher than those present in community settings with responses of $98 \%$ versus $96 \%$, respectively. Finally, interprofessional interactions showed relative consistency between academic years with a range of only $7 \%$ between program years. Implications: Data show substantial interprofessional activity by pharmacy students on experiential rotations throughout their academic tenure with physician interactions being most common in all settings. Community settings showed unexpected opportunity for interprofessional interaction with dental prescribers. More analysis is needed to further understand the relative lack of interprofessional interaction discovered with other allied health students.

Interprofessional Interaction With Social Workers Enhances APPE Student Understanding of Ambulatory Care Pharmacy. Rebecca M. Wise, Lake Erie College of Osteopathic Medicine. Objectives: The purpose of this research is to explore the impact of experiences with social workers on students attending ambulatory care APPE rotations and identify the different categories of care in which these experiences may occur. Method: The researcher will gather de-identified reflections written by students upon completion of ambulatory care APPE rotations from 2014 to 2017 where they had inter-professional interactions with social workers. All identifiers will be removed, including student names, dates, rotation block numbers, and site locations, before the researcher begins to analyze for data points which shall include (but are not limited to) categories of care noted, patient outcomes, and self-acknowledged student growth if present. Results: All student reflections noted areas in which ambulatory care pharmacists and social workers work together to improve patient outcomes. There were 42 different issues within 10 categories of care in which the two professions communicated, showing APPE students a level of inter-professionalism of which they were previously unaware. Implications: Interprofessional education is a current topic among pharmacy schools, but most of the interaction is between pharmacy students and physicians and nurses. This study can show the value of stretching Interprofessional experiences to social workers.

"I Want to Do Research! (Wait, What Is Research?)" - PharmD Candidate. Jaclyn Novatt, Long Island University, John M. Lonie, Long Island University, David E. Elkowitz, Hofstra University, Kathleen M. Frey, Long Island University. Objectives: We developed a medicinal chemistry research APPE where Pharm.D. students contribute to an ongoing research project. A portion of a project is chosen that can be completed within the five week APPE. Students learn the context of the project, laboratory techniques, and the theory behind the techniques. Students learn to understand and evaluate the primary literature and to connect the information therein with the hands-on experiments being done in the laboratory. Method: The course is separated into 2 concurrent parts: (I) Students are taught how to read and understand papers from the primary literature in order to learn the context of the project and the theory behind each laboratory procedure. (II) Students perform experiments in the laboratory that contribute to an ongoing research project and relate directly to the readings from part (I). Students submit weekly written summaries connecting the readings (I) to the laboratory experience (II). Results: This APPE is useful for both students and faculty. Students develop laboratory and metacognitive skills, while the collaborating laboratory is supplied with material required for further experiments. Because the APPE preceptor is not the professor in charge of the research effort, the preceptor is able to focus on the learning skills (both metacognition and hands-on) and the development portion of the APPE. Implications: This model using a non-PI preceptor may be applicable to schools of pharmacy that want to increase student exposure to research yet have limited lab space, limited resources, and limited mentors available to supervise student projects.

Keeping Healthy and Active in Your Senior Years: An Interprofessional Community Outreach Initiative. Gretchen M. Garofoli, West Virginia University, Kimeran W. Evans, West Virginia University, Gina M. Baugh, West Virginia University, Ashlee N. McMillan, West Virginia University, Morgan Petitte, West Virginia University. Objectives: To (1) assess student and faculty perceptions of the roles and responsibilities of other disciplines on a health care team and (2) describe the impact of an interprofessional experience on participants. Method: Faculty and students from pharmacy, medicine, dentistry, nursing, physical and occupational therapy collaborated to offer screenings and health education at a local senior center. An initial screening event was held with a follow-up session one month later. Student teams representing each discipline were assigned to a senior participant to rotate through stations including oral health, medication history, blood work, and fall risk. Students and faculty perceptions were assessed pre and post implementation using the Interdisciplinary Education Perception Scale (IEPS) and the Interdisciplinary Health Education Competencies (IHEC). Results: The IHEC survey showed statistically significant results when pre and post scores were compared for items related to the integration of professions and interprofessional collaboration. Forty- three students completed a post-project open-ended reflection, and these responses were analyzed and coded according to common themes. Students felt that the experience was overall very positive, relevant to their education, and impact on future work as a healthcare professional. Implications: This interprofessional activity allowed students to gain a better understanding of the roles and responsibilities of other disciplines and see the positive impact of collaboration on patient care. It was valuable for students, faculty, participants and the community partner. The future impact is that students who participated in this activity will better utilize the expertise of their colleagues to improve the quality of patient care.

Measurement of Faculty Consensus of EPA Importance and Level of Entrustability to Determine APPE Readiness. Lisa M. Meny, Ferris State University, Kali VanLangen, Ferris State University, David R. Bright, Ferris State University, Mandy R. Seiferlein, Ferris State University. Objectives: To identify the appropriate entrustable professional activities (EPAs) and corresponding level of entrustability necessary to demonstrate APPE readiness. Method: An electronic survey was distributed to college faculty to assess level of consensus regarding the role of specific EPAs in determining APPE readiness. The survey focused on identifying two measures of EPAs: 1) level of importance, and 2) level of entrustment prior to APPEs. Respondents rated the level of importance of each college-approved EPA so as to identify which specific EPAs are appropriate for measuring APPE readiness. Respondents also identified the level of EPA entrustability students should demonstrate in each EPA in order to begin APPEs. Data were analyzed and thresholds of consensus were determined to identify appropriate EPAs for inclusion and corresponding levels of entrustment for each EPA. Results: Of 44 faculty, 28 responded to the survey (63.6\%). Of the respondents, $57 \%$ reported the majority of their didactic teaching responsibilities were assigned in the third professional year. Of the 18 items assessed, $39 \%$ of items indicated strong consensus for level of importance while $61 \%$ indicated moderate consensus; therefore, 10 of 12 assessed EPAs were deemed important in defining APPE readiness. Conversely, regarding level of entrustment, no items demonstrated strong consensus. Implications: Substantial consensus was observed in determining level of importance of EPAs appropriate for determining APPE readiness. Due to the minimal 


\section{American Journal of Pharmaceutical Education 2018; 82 (5) Article 7158.}

consensus observed regarding EPA level of entrustment, a modified delphi approach will be utilized among faculty and non-faculty APPE preceptors to better elucidate an EPA-based APPE readiness assessment plan.

Organizational Structures to Promote SoTL in US 4-Year Colleges and Universities. Yumi So, The University of Texas at Tyler, Rahmat M. Talukder, The University of Texas at Tyler, Mohammed A. Islam, West Coast University. Objectives: The objective of this study is to explore the existing organizational structures in US higher education institutions that foster the practice of scholarship of teaching and learning (SoTL) and create campus cultures where SoTL is recognized as an important scholarly work. Method: A thorough Google search was conducted to identify institutions with teaching and learning centers using the keywords: "scholarship of teaching and learning", "SoTL", "teaching and learning" combined with "center". Each institution's website was visited to search pages with information on SoTL. All the identified pages on SoTL, faculty development, SoTL award, or SoTL grant were retrieved. Data extraction from website was performed utilizing a systematic content analysis method Results: Two-hundred thirty-seven US 4-year colleges and universities were identified with existing teaching and learning centers. Eighty-three percent $(n=196)$ of these institutions have structured programs that foster SoTL practices and faculty development. Common structures include SoTL forums, SoTL Community, SoTL Commons, faculty development center, educational research development unit, etc. They provide workshops, mini-conferences, forums, seminars, summer SoTL, and teaching and learning fairs. Fifty-one institutions $(22 \%)$ were identified that provide faculty awards and grants on SoTL accomplishments. Implications: Our results document the existing structures that promote SoTL at institutional level in US higher education institutions. Similarly, SoTL and faculty development programs can be structured within pharmacy academic units to enhance faculty pedagogical knowledge and skills and to provide opportunities for them to advance SoTL practice.

Pharmacy Student-Led Wellness Screenings for Underserved Populations: Barriers, Opportunities, Lessons Learned. Kristin A. Casper, The Ohio State University, Jennifer L. Rodis, The Ohio State University, Jessica Brumbaugh, The Ohio State University, Tyler Davis, The Ohio State University. Objectives: (1) Describe a model for student-led wellness screenings, (2) Identify characteristics of patients at free health screenings (3) Report type and frequency of student pharmacist recommendations, and (4) Determine impact characteristics of store have on screenings. Method: The Ohio State University and The Kroger Co., Columbus Division partnered to bridge underserved populations to the healthcare system through a student-led screening program. Third year pharmacy students conducted blood pressure and blood glucose screenings and counseled patients on healthy lifestyle and medication adherence. Fourth year pharmacy students provided peer-mentorship to these students and follow up phone calls for patients. Frequencies and percentages were calculated regarding out of range values, type of screening, and type of follow-up recommended. Follow up notes made by pharmacy students were analyzed for themes. Results: Investigators analyzed over 1900 individual screening encounters between July 2014 and May 2017. Nearly half of patients seen had at least one value out of range. Over $90 \%$ of patients received counseling on lifestyle in both the in-range group and out of range group. Students accurately directed patients to a healthcare provider or emergency department based on results. Barriers to followup identified were related to patients' contact number being unreachable and language barriers. Implications: This screening program provided experience for students to practice counseling techniques, documentation, and recognize the barriers to follow up. Underserved populations have difficulty accessing healthcare and student-led screening were able to bridge the gap and provide care and information to these patients.

Preceptor Acceptability and Fidelity of APPE Student Performance Evaluations Modeled on the Pharmacist's Patient Care Process. Amanda Margolis, University of Wisconsin-Madison, Robert M. Breslow, University of Wisconsin-Madison, Mara A. Kieser, University of Wisconsin-Madison, Denise L. Walbrandt Pigarelli, University of Wisconsin-Madison. Objectives: To determine preceptor acceptability and fidelity of a newly-implemented Student Performance Evaluation (SPE) for Advanced Pharmacy Practice Experiences (APPEs) modeled on the Pharmacist's Patient Care Process (PPCP). Method: A new SPE was implemented for preceptor evaluation of directly observed APPE student performance. The SPE contained six items based on the PPCP; students were rated using Miller's Learning Pyramid (5-point scale of does not know to does). After using the new evaluation, preceptors were surveyed with eight questions regarding their acceptance of the SPE. An audit was conducted to check preceptor fidelity. Mismatches were identified by comparing preceptor comments about student performance to their rating. All SPEs during a single rotation with scores lower than $93 \%$ and a random sample of SPEs with scores over 93\% were included in the audit. Results: Of the 48 preceptors who completed the survey, $81 \%$ were satisfied with the SPE; $85 \%$ responded that the new SPE accurately assessed student performance. Of the 57 SPEs that were audited, mismatches occurred between the preceptor rating and comments $12.3 \%$ of the time. Mismatch errors included potentially inappropriate low or high rating or when improvement was assessed rather than competency. For $27.5 \%$ of the audited items, the preceptor did not provide written feedback. There were 10 preceptor comments $(2.9 \%)$ suggesting confusion about the interpretation of specific PPCP items. Implications: Preceptor acceptability of the SPE was high with a low mismatch of ratings to comments. Further clarification of PPCP definitions and examples may further promote accurate preceptor evaluations.

Preceptor and Resident Perceptions of Entrustable Professional Activities (EPAs) for Postgraduate Pharmacy Training. Sarah K. Schweiss, University of Minnesota, Jean Y. Moon, University of Minnesota, Jody L. Lounsbery, University of Minnesota, Amy L. Pittenger, University of Minnesota. Objectives: To describe residency preceptors' and residents' perceptions of using an entrustable professional activities (EPAs) rating scale to assess resident performance. Method: An electronic survey was distributed to residency program preceptors and residents in a PGY-1 pharmacy residency program. Results: Both residents (15/25) and preceptors (26/61) responded and three preceptors had not yet used the new EPA rating scale and were excluded. Residents and preceptors found the EPA framework (66.7\% and $78.3 \%$ respectively) to be more helpful compared to the traditional model for rating levels of progression. Both groups also described that for certain learning objectives EPAs were less beneficial. Sixteen (66.7\%) preceptors felt the residency graduation standard for learning objectives or skill attainment should be an EPA entrustment level 4 or 5; seven $(29.2 \%)$ were unsure. Preceptor comments included finding the EPAs scale to be more descriptive, more objective, and easier to understand; however, it did not always apply to all skills and was more challenging to provide residents information regarding what they needed to improve upon. Residents commented that the EPAs scale was more descriptive, had better clarity, and gave them a better idea of where their progression was at on a continuum. Residents also commented that regardless of the rating scale, 


\section{American Journal of Pharmaceutical Education 2018; 82 (5) Article 7158.}

specific, day-to-day feedback is more helpful. Implications: Residents and preceptors perceive the EPA framework to be more helpful than the traditional rating scale when evaluating learning objectives. Both groups reported less frequently observable learning objectives to be less helpful.

Preceptor Fidelity to a Continuous Professional Development Program. Amanda Margolis, University of Wisconsin-Madison, Ruth H. Bruskiewitz, University of Wisconsin-Madison, Mara A. Kieser, University of Wisconsin-Madison, Anna Li, University of WisconsinMadison. Objectives: To determine preceptor fidelity to creating continuous professional development (CPD) plans. To determine topics of interest for preceptor improvement. Method: An online CPD program was launched teaching the process of CPD, encouraging preceptors to review student feedback, and requiring a precepting CPD plan. Preceptors used four guided questions to develop their plans. Fidelity to a CPD plan to improve precepting skills was assessed as: 1) having a plan for improving a precepting skill, 2) having a plan for improving precepting, but not skill based, 3) having a plan lacking a precepting focus. The audit was coded in duplicate and discrepancies were adjudicated. Topics of precepting improvement were identified. Results: Of the 331 preceptors completing usable CPD plans, 156 (47\%) described improvement in a precepting skill, 162 (49\%) described a nonskill based precepting improvement, and 13 (4\%) were not precepting focused. Of those who had skill-based plans, $62(40 \%)$ had vague resources listed. Primary topics of interest for preceptor development included: giving feedback 147 (44\%), improving rotation organization $76(23 \%)$, initiating scheduled discussions $66(20 \%)$, scheduling more time with students 65 (20\%), and setting expectations $57(17 \%)$. Implications: Most CPD plans described precepting and rotation improvements; however, more focus is needed on skills improvement and identification of specific learning resources. The topics of interest for preceptor improvement can be used to guide future preceptor development offerings.

Preceptor Orientation and Development: Highlights From a National Survey. Teresa A. O'Sullivan, University of Washington, Erin L. Johanson, Roseman University of Health Sciences, Melissa M. Dinkins, Wingate University, Andrea S. Joseph, Thomas Jefferson University, Patricia L. Darbishire, Purdue University, Susan S. Vos, The University of Iowa, Craig D. Cox, Texas Tech University Health Sciences Center. Objectives: This study was designed to identify current preceptor orientation and development programs at Colleges and Schools of Pharmacy. Method: An anonymous 28-item survey was administered in January 2017 to experiential education administrators (EEAs) of accredited US Schools and Colleges of Pharmacy. Data from the survey were tabulated and qualitative answers to openended questions were examined using thematic analysis. Results: Eighty-five EEAs participated in the survey (67\% response rate). Most preceptor orientation programs included requirements outlined in ACPE Standard 20.3; 53\% of programs didn't require preceptor orientation prior to student placement. Two-thirds of respondents offered annual, live preceptor development and $75 \%$ of programs used commercially-available online products. Approximately $39 \%$ of respondents collaborate with other schools or professional organizations to offer preceptor training. Only $29 \%$ of programs had specific requirements for pharmacists to maintain their active preceptor status. Seventy percent of respondents reported spending over $\$ 2500$ and 39\% over $\$ 5000$ annually on preceptor development. Programs with significant investment ( $>\$ 10,000 /$ year) generally featured multiple venues (live and online) for offering preceptor development. Programs with significant personnel commitment ( $>0.5$ FTE devoted to preceptor development) most frequently described dedicated site visitors who provided group or individualized preceptor development. Implications: Preceptor orientation programs are similar but development programs vary across the Academy. Successful programs invested significantly either in live and online programs or site visitors who provided individualized feedback or programs. Future studies should explore the cost-effectiveness of program options and their impact on preceptor learning and behaviors.

Preceptor Perception of EPA Competency Opportunities and Level of Entrustability. Connie L. Smith, The University of Louisiana at Monroe, Roxie L. Stewart, The University of Louisiana at Monroe, Gregory W. Smith, The University of Louisiana at Monroe, Glenn Anderson, The University of Louisiana at Monroe, Scott A. Baggarly, The University of Louisiana at Monroe. Objectives: Pharmacy Practice Experience (PPE) evaluations at our university were redesigned to incorporate Core Entrustable Professional Activities (EPAs). EPAs are essential activities new pharmacy graduates must perform without direct supervision upon graduation. The purpose of this study is to identify inconsistencies in perceived level of EPA opportunities by preceptor type. Method: Surveys were sent to all preceptors $(n=402)$. Preceptor types included community, institutional, clinical faculty and non-faculty, and others. In addition to demographic data, preceptors were asked questions regarding the opportunities for EPAs at their sites including their expected level of entrustment (Level 1 - Level 5) for each EPA. Chi square and logistic regression models were used to analyze preceptor responses $(n=52)$. Results: Overall, preceptor perceptions of student independent performance of EPAs during the APPE year was consistent by preceptor type. Inconsistencies of perception were notable in community pharmacists for: collect patient medication information $(\mathrm{OR}=18.2, \mathrm{p}=0.008)$, implement a care plan $(\mathrm{OR}=17.3, \mathrm{p}=0.025)$, monitor a care plan $(\mathrm{OR}=16.5, \mathrm{p}=0.04)$, identify at-risk patients $(\mathrm{OR}=44.0, \mathrm{p}=0.01)$, minimize adverse drug events $(\mathrm{OR}=126.0$, $\mathrm{p}=0.009)$, maximize appropriate medication use $(\mathrm{OR}=16.2, \mathrm{p}=0.008)$, ensure appropriate immunizations $(\mathrm{OR}=12.4, \mathrm{p}=0.018)$, participate in population-based health-care $(\mathrm{OR}=29.2, \mathrm{p}=0.009)$, use evidencebased information $(\mathrm{OR}=49.4, \mathrm{p}=0.007)$, represent patients' best interests $(\mathrm{OR}=61.7, \mathrm{p}=0.007)$, interprofessional team practice $(\mathrm{OR}=17.5$, $\mathrm{p}=0.01)$, recognize health disparities $(\mathrm{OR}=62.5, \mathrm{p}=0.001)$, effectively communicate $(\mathrm{OR}=262.3, \mathrm{p}=0.006)$. Two of these inconsistencies were also noted in clinical faculty: collect patient medication information $(\mathrm{OR}=20.8, \mathrm{p}=0.036)$ and ensure appropriate immunizations $(\mathrm{OR}=35.1, \mathrm{p}=0.021)$. Implications: Advantages in identifying gaps in preceptor perceived EPA opportunities and levels of entrustability can lead to focused preceptor development.

Preceptor Perceptions of Entrustable Professional Activities. Lena M. Maynor, West Virginia University, Hunter Highlander, West Virginia University, Ashleigh L. Barrickman, West Virginia University. Objectives: The primary objectives of this study were to determine preceptors' perceptions of the importance of the core entrustable professional activities (EPAs) for new pharmacy graduates and the expected level of supervision for students at the beginning of the Advanced Pharmacy Practice Experiences (APPEs). Method: This study was approved by the Institutional Review Board at WVU. All WVU APPE pharmacist preceptors were surveyed in December 2017. Respondents were asked to rank the importance of each EPA for entrylevel pharmacists from 1 (not at all important) to 5 (essential), and to rank the expected level of supervision for students beginning APPEs from 1 (observe only) to 5 (can supervise more junior students). Results: Surveys were mailed to 265 preceptors; 73(27.5\%) surveys were returned. Average scores for the importance of each EPA for entry-level pharmacists ranged from 4-4.76. Average scores for the expected level of supervision for each EPA for students beginning 


\section{American Journal of Pharmaceutical Education 2018; 82 (5) Article 7158.}

APPE ranged from 2.5-3.57. Differences were found between residency-trained preceptors compared to preceptors with no residency training and preceptors with PharmD degrees compared to BS degrees, with residency-trained preceptors and preceptors with PharmD degrees indicating need for more direct supervision on 9 of 13 items and 5 of 13 items, respectively. Implications: Our survey results indicate that our respondents view the EPAs as important for entry-level pharmacists and indicate an expectation for students to perform most tasks with reactive supervision at the beginning of APPEs. This has implications for the design of APPE activities and school-driven levels of expectation as the APPE year progresses.

Prescription for Patient Centered Care: Use of a Structured Narrative Life History Interview With Pharmacy Learners. Adam B. Woolley, Northeastern University, Susan Nathan, VA Boston Healthcare System, Jennifer Moye, Harvard University. Objectives: "My Life My Story" uses patient narrative and storytelling to tell a patient's life story in the electronic medical record. We have incorporated pharmacy trainees into this process and assessed the benefits of this innovative learning experience. Method: P-4 Pharmacy students, PGY-1 and PGY-2 residents at the VA Boston Healthcare system have participated in this program since July 2016. Learners are provided with an interviewer guide that provides suggested questions to structure the interview. Learners were tasked with identifying a patient to interview during the rotation and provided the autonomy to complete the interview and write up. The learners were asked to complete anonymous, voluntary, pre and post-surveys online to assess patient centered care competencies and overall perceptions of the experience. Results: Ten pharmacy students, four PGY-1, and two PGY-2 residents $(\mathrm{N}=16)$ documented 18 patient life stories. Five (31\%) learners completed the pre-survey and nine of $14(56 \%)$ completed the post survey. On a Likert scale (1 poor -5 excellent), learners reported improvements between average pre and post surveys ratings in their ability to let the patient tell their story (4.60 to 4.78 ), listen to the patient (4.4 to 4.67), view the patient as a whole person (4.60 to 4.89 ), and show care and compassion (4.60 to 4.78). Overall, participants reported that the learning experience was valuable and helped foster a positive relationship with patients. Implications: Integrating pharmacy learners into the innovative "My Life, My Story" program may assist in developing patient centered care skills in the clinical setting.

Qualitative Analysis of Service Learning Reflections. Jennifer D. Arnoldi, Southern Illinois University Edwardsville, Kate Newman, Southern Illinois University Edwardsville, Maithili Deshpande, Southern Illinois University Edwardsville, Amanda Schmidt, Southern Illinois University Edwardsville, Katie Davis, Southern Illinois University Edwardsville. Objectives: To evaluate the impact of a service learning experience through qualitative analysis of the content and extent of written reflections. Method: Using an inductive content analysis approach, two investigators independently reviewed reflection papers written by first year pharmacy students after a three-week service-learning experience. Predominant themes discussed were identified by the reviewers and quantified by the number of reflections in which the theme was discussed (mention) and the total number of references in all reflections (weight). Each reflection was also analyzed for depth using a four-point scale from non-reflection to critical reflection. Results: In a total of 81 reflections, six prevailing themes were identified: benefit to the pharmacy students' professional development $(\mathrm{n}=79)$, attitude towards an unpredictable experience $(n=57)$, importance of community involvement $(n=45)$, perceived benefit to the audience $(n=51)$, changes in students' attitudes or opinions $(n=39)$, and impact on students' career choice $(n=37)$. The depth of reflection achieved indicated that nearly all students exhibited understanding $(\mathrm{n}=48)$ or reflection $(\mathrm{n}=30)$ while several students did not achieve true reflection $(n=3)$. None of the essays were found to achieve critical reflection. Implications: The service learning experience led to students reflecting upon their professional development and pre-conceived notions of the opportunity. That students did not achieve critical reflection may not be surprising given the expectations of the assignment and the students' inexperience. Analyzing reflective writing will remain crucial given its pivotal role in self-awareness and continuing professional development.

Student Perceptions of Comfort of Immunization Administration Related to Experience. Ashlee N. McMillan, West Virginia University, Gretchen M. Garofoli, West Virginia University. Objectives: 1) Determine if practicing vaccine administration during Advanced Pharmacy Practice Experiences (APPEs) improves student comfort level. 2)Assess if students are providing immunizations during their APPEs. Method: Over the course of two years, students in this comparative study completed an immunization certificate program during the Spring semester prior to their APPEs. The first group of students wasn't able to administer immunizations during rotations while the second group was able to based on state legislative changes. Just prior to their respective graduations, students were given a survey to assess their readiness to perform immunizations before and after rotations. Results: There was no statistically significant difference between the comfort levels of providing immunizations between the two groups of students. However, the comfort level of the students who actually administered immunizations during their APPEs was statistically higher than those who did not administer immunizations during the same year. Of the group of students that were legally able to provide immunizations ( $\mathrm{n}=84)$, only $44(52.3 \%)$ actually provided immunization during their APPEs. Seventy percent of the total students completing the survey planned to practice in settings where immunizations are frequently provided. Implications: The results of this survey demonstrated practice increases student comfort level for administering immunizations even though their baseline didactic learning was the same. It is concerning that although students were legally permitted to provide immunizations, only approximately half administered them. These observations demonstrate a gap that should be addressed in APPE curriculum requirements to ensure students have the opportunity to practice this valuable skill.

Student Perceptions of Mindfulness Content as a Component of a Leadership APPE. Jane E. Wilson, The University of Oklahoma, Michael J. Smith, The University of Oklahoma, Christina F. Bulkley, Philip E. Looper, The University of Oklahoma, David L. George, The University of Oklahoma. Objectives: Practicing mindfulness has well-known benefits, including improved workplace performance. This study assessed fourth-year pharmacy student perceptions of a Mindfulness Retreat (MR) designed to translate their previously delivered didactic content on mindfulness into experiential learning and practice. Better understanding of student satisfaction of this content will help further APPE development. Method: Three leadership student cohorts ( $n=50$, classes 2015-2017) participated in the MR during their respective APPE year. Utilizing a modified Dillman method, each cohort received a 12-item questionnaire that included two free response questions. This abstract focuses on qualitative data from the free response items regarding student perceptions of the MR and recommendations for future MRs. Comments from the free response questions were analyzed using thematic analysis. Several factors were considered for nodes and themes: frequency of comments, specificity of detail, exhibited emotion, and extensiveness of ideas. Two qualitative analysts developed, compared, revised, and edited all nodes and 


\section{American Journal of Pharmaceutical Education 2018; 82 (5) Article 7158.}

themes until agreement was achieved. Results: Thirty-nine individuals completed the questionnaire (78\% response rate). Student comments fell under two broad domains: (1) general comments concerning the MR and (2) future recommendations for the MR. Three primary themes related to general comments concerning the MR emerged: (1) Setting, (2) Experience, and (3) Attitude. Two primary themes emerged related to future MR recommendations: (1) Opportunities and (2) Overall Perspective. Implications: The MR had a perceived positive influence on student leadership development. Coordinators should continue to refine and deliver mindfulness content and experiences as part of leadership curriculum.

Student Reported Institutional Practice (IP) Activities During a Blended Acute Care/Institutional (AC/INST) APPE. Scott A. Chapman, University of Minnesota, Zachary Rivers, University of Minnesota, Christene M. Jolowsky, University of Minnesota, Lauren E. Dubosh, Abbott Northwestern Hospital, Laura Vollmer, Spectrum Health. Objectives: To quantify student IP activities within our blended 12 week AC/INST APPE. Method: APPE students completed a 17 category IP survey weekly to report if they had or had not experienced each category and also the percent of total APPE time for each week involved in all IP activities. The number of IP categories addressed during a week, the weekly frequency and the average number of weeks the students experienced activities in a category, and the number of weeks the activity was reported were determined. Preceptor evaluations of IP learning goals were also evaluated. Results: 147 (88\%) students submitted1594 surveys (90.4\%, 10.6/student). Over $90 \%$ reported experiences in nine categories (medication distribution, medication preparation, transition of care, automated dispensing systems, medication safety, institutional policies of clinical services, inventory management, electronic medical records, drug information questions) and over $75 \%$ reported experiences in 13 (in addition to above: sterile preparation; P\&T committee; budget, acquisition, and procurement; regulatory organizations) during any week. Frequency of experiences were highest for medication preparation $(95.8 \%, 10.2$ weeks), electronic medical records (86.2\%, 9.1 weeks), and drug information questions $(87.8 \%, 9.3$ weeks). Frequency was less for sterile preparation $(24.7 \%, 2.5$ weeks), formulary monograph review $(9.7 \%$, 1.0 week), regulatory organizations $(23.2 \%, 2.5$ week), and pharmacy budget/medication acquisition (32.7\%, 3.5 weeks). Average percent APPE time participating in IP activities was $45 \%$ /week. Preceptor evaluations indicate students are meeting or exceeding IP learning goals. Implications: Blended experiences in AC/INST in an APPE curriculum can successfully achieve institutional practice and clinical practice educational goals.

Student Specific Remediation Plans for Introductory Pharmacy Practice Experiences. Courtney R. Caimano, Albany College of Pharmacy and Health Sciences, Megan Veselov, Albany College of Pharmacy and Health Sciences, Teresa H. Kane, Albany College of Pharmacy and Health Sciences, Sandra W. Rosa, Albany College of Pharmacy and Health Sciences. Objectives: To identify student deficiencies and create specific remediation plans to raise students to the level of competency needed for Advanced Pharmacy Practice Experience(APPE)-readiness. Method: At the completion of community and institutional Introductory Pharmacy Practice Experiences (IPPE), preceptors evaluated students on several competencies using a fourpoint scale: Exceeds Competency, Meets Competency, Needs Improvement (NI), or Significant Deficiency (SD). Students receiving at least one $\mathrm{NI}$ or $\mathrm{SD}$, while still receiving a passing grade for the IPPE, were identified as needing remediation. Students received individual remediation plans tailored to the competencies that were evaluated as a NI or SD. Community IPPE remediation activities included additional practice in the student operated pharmacies and review of the most frequently prescribed medications. Institutional IPPE remediation activities included journal article reviews, answering drug information questions, and reviewing medication related problems. Students in both community and institutional IPPEs that required remediation of communication skills completed simulations in the pharmacy skills lab. Results: Thirty three students were identified for remediation. Thirteen $(6 \%)$ P1 students $(n=212)$ remediated competencies from the Community IPPE while 20 (9\%) P2 students ( $n=220)$ remediated competencies from the Institutional IPPE. At the conclusion of the remediation, students were evaluated again and determined to meet competency. Implications: Students were offered additional instruction and practice opportunities and will be more likely to succeed in future rotations. Specific plans allow tracking of students' performance as they progress through the experiential curriculum to identify continued deficiencies or success. A formalized assessment for all activities is necessary.

Syllabus Development as a Component of PharmD Preceptor Development Program in Egypt. Toral Patel, University of Colorado, Jodie V. Malhotra, University of Colorado, Joseph J. Saseen, University of Colorado. Objectives: Develop rotation specific syllabi to meet requirements of the Children's Cancer Hospital Egypt (CCHE) PharmD program Method: CCHE is offering a PharmD program in collaboration with the University of Colorado Skaggs School of Pharmacy and Pharmaceutical Sciences (SSPPS). SSPPS faculty facilitated a live preceptor development program at CCHE in 2017 for select CCHE clinical pharmacists. These pharmacists were experienced in precepting baccalaureate students, but never precepted PharmD students. Syllabus development was a primary focus of the program, assignments, and final evaluation. Participants were instructed how to develop syllabi that aligned with the structure and objectives of Introductory and Advanced Pharmacy Practice Experiences (IPPE and APPE). Individual rotation syllabi were drafted as homework, reviewed by peers and SSPPS faculty for feedback, were revised and submitted as a component of the graded final evaluation. Performance on the final evaluation determined acceptance as PharmD preceptors. Syllabi were evaluated on rotation description, objectives, calendar, pre-requisites, and assignments. Results: Twenty pharmacists submitted a final syllabus. Developing rotation site specific learning activities was the most common area that needed improvement. Mean scores for individual components were: $88 \%$ (description), 93\% (objectives), $86 \%$ (calendar), $86 \%$ (prerequisites), and $83 \%$ (assignments). Seventeen pharmacists scored greater than $70 \%$ on the syllabi, with 16 also scoring $70 \%$ or greater on the cumulative final evaluation and identified as PharmD preceptors. Implications: Evaluating experiential syllabi enabled the selection of preceptors that understood requirements and structure of the PharmD program. This model was effective in ensuring preceptors incorporated experiential requirements into experiential syllabi.

Targeted Preceptor Feedback Developed Using an Annual Experiential Quality Assurance Process. Wesley R. Haltom, Wingate University, Melissa M. Dinkins, Wingate University, Eric F. Schneider, Wingate University. Objectives: To describe an annual process to review and provide targeted feedback to all volunteer preceptors for a school of pharmacy. Method: Student evaluations of preceptors and practice sites from 2016-2017 introductory pharmacy practice (IPPE) and advanced pharmacy practice experiences (APPE) were de-identified and randomly assigned to members of the Practice Experience Advisory Committee (PEAC) for review. PEAC members 


\section{American Journal of Pharmaceutical Education 2018; 82 (5) Article 7158.}

completed a form summarizing positive and negative aspects of rotation experiences and identifying preceptors requiring a second review and potential intervention by the experiential administrators. All preceptors received a summary report of student feedback, PEAC feedback, and comparator scores by rotation type and all preceptors. Preceptors were instructed to review the summary reports and set one to two goals for their precepting in upcoming rotations. Results: Student evaluations for 391 preceptors were reviewed for the 20162017 year. Nine preceptors required additional follow-up, including a phone call (1/9), email (4/9), face-to-face visit (2/9), or closely monitor (2/9). Reasons for additional follow-up included calendar/ organization of student activities, lack of preceptor involvement, challenges with non-preceptor staff, and rotation activities out of alignment with objectives. Implications: An annual quality assurance process provides individualized preceptor development opportunities, encourages improvements in practice experience offerings, and identifies preceptors in need of intervention from an experiential administrator. Similar processes to provide targeted feedback could be easily implemented in experiential offices around the country.

The Use of Two Interprofessional Survey Instruments at a Comprehensive Academic Health Center. Vincent C. Dennis, The University of Oklahoma, Stephen B. Neely, The University of Oklahoma, Melissa Craft, The University of Oklahoma, Dale Bratzler, The University of Oklahoma, Melody Yozzo, The University of Oklahoma. Objectives: To assess attitudes/perceptions of students at a comprehensive academic health center toward interprofessional practice. Method: Faculty from Colleges of Allied Health, Dentistry, Medicine, Nursing, Pharmacy, Public Health and the School of Social Work identified one class of respective students for assessment. Students were randomized within each discipline to receive one validated survey instrument; the 14 item Collaborative Healthcare Interdisciplinary Relationship Planning survey (CHIRP) on a 4 point scale or the 27 item Interprofessional Assessment Scale (IPAS) instrument on a 7 point scale. An Analysis of Means using Transformed Ranks procedure was used to determine if Pharmacy student responses differed from the overall campus. Results: Of 866 students randomized, 62.4\% (272 of 436) completed the CHIRP survey with an overall median response of 3 (interquartile range 2.6 to 3.4 ) and $58.6 \%$ (252 of 430) completed the IPAS instrument with an overall median of 6.1 (interquartile range 5.8 to 6.4 ). No statistically significant differences were seen between the campus-wide survey responses and those of Pharmacy students for CHIRP (identical median/range; 29 of 48 completed) or IPAS (lower median at 5.9 , range 5.6 to $6.1 ; 21$ of 47 completed). Pharmacy students submitted the lowest median response with greatest difference relative to the campus-wide response in the IPAS domain of patientcenteredness (6.4 vs. 6.8, respectively). Implications: A cross-section of students at a comprehensive academic health center display comparable responses for the CHIRP and IPAS instruments. Establishing benchmarks at a point in curricula allows for upstream/downstream assessment of attitudes/perceptions in relation to advancements in interprofessional education and practice.

Training the Trainers: A Preceptor Development Program Targeting Interprofessional Collaboration Competencies. Nicholas M. Fusco, University at Buffalo, The State University of New York, Peter M. Brody, University at Buffalo, The State University of New York, Patricia J. Ohtake, University at Buffalo, The State University of New York. Objectives: To evaluate the impact of a preceptor development program on pharmacy preceptors' self-reported skills in interprofessional (IP) collaboration. Method: Pharmacy preceptors were invited to participate in a two-hour preceptor development program followed by a brief survey. The objectives of the preceptor development program were to: define interprofessional education (IPE) and IP collaborative practice (IPCP); describe the benefits of IPCP for patients/clients, healthcare providers, and health systems; discuss the four IPEC Core Competencies; and, describe IPE student experiences aimed at increasing collaborative practice. The Interprofessional Collaborative Competencies Attainment Survey (ICCAS) was administered after the preceptor development program. Descriptive statistics were used to characterize the data and the Wilcoxon Signed-Rank test evaluated the pre-/postprogram scores. Results: Thirty (30\%) preceptors completed the survey. Median (range) age of preceptors was $40(31-47)$ years with $15(9-23)$ years as a licensed pharmacist. Preceptors had $8(5-14)$ years of experience. The majority of preceptors described their practice site as academia $(n=10,33.3 \%)$, followed by community pharmacy $(n=9,30 \%)$, acute care $(n=6,20 \%)$ and other $(n=5,16.7 \%)$. The majority of preceptors precept both advanced and introductory pharmacy practice experiences $(n=21,70 \%)$, followed by introductory only $(n=5,16.7 \%)$ and advanced only $(n=4,13.3 \%)$. Median (IQR) preceptor pre-program self-reported ability scores on the twenty ICCAS items (scored on a 7-point scale) were 5 (4-5) and increased by $\sim 1$ point following the program [6 (6-6); $\mathrm{p}<0.05]$. Implications: A preceptor development program targeting IPE and IPCP increased pharmacy student preceptors' opinions toward their IP collaboration ability.

Use of a Pre-Rotation Survey to Enhance Pharmacy Student Engagement in an Underserved Population. Jennifer L. Petrie, University of Colorado, Rhianna M. Tuchscherer, University of Colorado, Emily R. Kosirog, University of Colorado, Benjamin Chavez, University of Colorado. Objectives: To evaluate the effectiveness of using a pre-rotation survey to successfully identify students' perceived knowledge gaps, and subsequently tailor specific learning opportunities that best meet their needs over the course of a Federally Qualified Health Center (FQHC) Ambulatory Care rotation. Method: Prior to a 6-week experiential rotation, pharmacy students were provided an optional pre-rotation survey to gauge knowledge and confidence levels within 17 ambulatory care disease states, five patient care tasks, and nine underserved patient care tools and barriers. Responses were used to guide learning activities, projects, and presentations during the FQHC rotation. Following completion of the rotation, students were provided an optional post-rotation survey allowing for self-reflection of growth and development. Results: Forty students completed the pre-rotation survey; 33 completed the post-rotation survey. Within the pre-rotation survey, "not confident at all" was selected within 13/17 (76\%) ambulatory care topics, $5 / 5(100 \%)$ patient care tasks, and 7/9 (78\%) underserved patient care tools/ barriers. Within the post-rotation survey, "not confident at all" was selected within $6 / 17(35 \%)$ ambulatory care topics, $0 / 5(0 \%)$ patient care tasks, and $0 / 9(0 \%)$ underserved patient care tools/ barriers. Based on "not confident at all" selections, the greatest change of student perceived confidence included bipolar disorder (pre-38\%; post-12\%), preparing a professional handout (pre-8\%; post-0\%), and 340B Drug Pricing Programs (pre-18\%; post-0\%). Implications: A pre-rotation survey allows opportunity to identify students' perceived knowledge gaps and subsequently tailor learning activities. By the completion of their rotation, students' overall confidence improved in ambulatory care topics, patient care, and knowledge of barriers when treating the underserved.

Use of Technology and Analytics to Assess Inter-Rater Reliability Across Multiple Evaluators. Michael E. Pitterle, University of Wisconsin-Madison, Amanda Margolis, University of Wisconsin-Madison, Denise L. Walbrandt Pigarelli, University of Wisconsin-Madison, 


\section{American Journal of Pharmaceutical Education 2018; 82 (5) Article 7158.}

Katherine M. Chylla, University of Wisconsin-Madison. Objectives: To assess evaluator inter-rater reliability of student presentations on Advanced Pharmacy Practice Experiences (APPEs) using online rubric analytics. Method: An online rubric was developed to help improve inter-rater reliability among evaluators of APPE student project presentations. The online rubric was built using MySQL and PHP and integrated into the existing experiential education database. Two types of analytics were used to assess evaluator inter-rater reliability of project presentations. The first was to compare mean final presentation scores by evaluator and the second was the distribution of ratings by evaluator on specific presentation items. Distributions of specific item ratings was calculated as the proportion of full credit by evaluator. Students were randomized to evaluators. Results: Prior to the online rubric, evaluator presentation means ( $n=7$ evaluators) ranged from $79 \%$ to $100 \%$. Following the implementation of the online rubric presentation means ranged from $91 \%$ to $98 \%$. The proportion of full credit by evaluator for project methods ranged from $65 \%$ to $97 \%$ and for project outcomes ranged from $60 \%$ to $96 \%$. The proportion of full credit by evaluators for background and for presentation delivery both ranged from $80 \%$ to $100 \%$. Implications: Use of online rubric analytics can help identify how consistently evaluators are rating assignments and pinpoint exactly where in an assignment interventions are needed. In the case of this assignment, more training and clarification is needed for evaluators to improve presentation score consistency in several content items including presentation methods and outcomes.

Working SMARTer: Development of a Multispecialty StudentLed Curriculum for an Advanced Pharmacy Practice Experience. Patrick Bridgeman, Rutgers, The State University of New Jersey, Navaneeth Narayanan, Rutgers, The State University of New Jersey, Deepali Dixit, Rutgers, The State University of New Jersey, Mary M. Bridgeman, Rutgers, The State University of New Jersey, Evelyn R. Hermes-DeSantis, Rutgers, The State University of New Jersey. Objectives: To describe the impact of a Subject-based Multispecialty Acute-care Rotational Teaching (SMART) curriculum and student-led workshop designed for students completing an advanced pharmacy practice experience at a large academic medical center. Method: Participants in a multispecialty student-led curriculum on advanced pharmacy practice experience rotations were invited to partake in pre- and post-workshop participation assessments. This activity was designed to improve students' knowledge and exposure to a variety of sub-specialty topics over the course of their five-week rotation at the site to ultimately influence North American Pharmacy Licensure Examination (NAPLEX) competency and preparedness. Institutional review board approval was obtained prior to initiation of data collection. Results: A total of 23 students have currently completed rotations, participated in the SMART curricula, and completed both pre- and post-assessments. Based on this interim data, the average change in the scores on the 25 -item evaluation was 3.17 (12.7\%; $\mathrm{p}<0.001)$, with an improvement from a mean of 12.0 $(48 \%)$ on the pre-test to a mean of $15.17(60.7 \%)$ on the post-test. In an assessment of the individual questions, 18 questions improved, 4 questions did not improve, and 3 questions decreased in accuracy of response. There was no difference in improvement between the two rotation cycles evaluated (12.86\% vs. $12.4 \%)$. Implications: Participants of this multispecialty student-led curriculum demonstrated a significant change in performance on a short 25-question assessment. Educators seeking to incorporate a student-led curriculum on advanced pharmacy practice experience rotations could easily replicate this activity.

\section{Theoretical Models}

Framework for Collaborative Preceptor Development Programming From Northwest Pharmacy Experiential Consortium (NWPEC). Jennifer Chang, University of Washington, Anita J. Cleven, Pacific University Oregon, Tracy K. Pettinger, Idaho State University. Objectives: Pilot a new framework for schools to work together to develop and deliver preceptor development CE programs Method: Faculties from 3 different schools in NWPEC developed a new preceptor development CE program to meet the needs of the preceptors using the framework outlined below: I. Needs Assessment: Identified preceptor needs through preceptor self-assessment results II. Program Design and Development: Created a preceptor development CE program with topical modules III. Program Delivery: Delivered preceptor development CE program as a live presentation and online CE program through commercial vendor Results: I. The needs assessment identified the following topics: - Preceptor Professional Development and Training - Integrating students into practice II. A new preceptor development CE program with the following modules was created to help preceptors develop customized learning plans to integrate students into practice: - Introduction to Continuing Professional Development (CPD) - Preceptor Self-Assessment - Developing a CPD Plan III. The CE program was first presented live at Northwest Pharmacy Convention and then later adapted into an online program via CEI to make the presentation more accessible to NWPEC preceptors Implications: - Enhances collaborative preceptor development programming effort in NWPEC - Designs preceptor development program based on preceptor needs - Provides preceptors with a framework to encourage and support professional development - Supports individualized preceptor education in a group setting - Partners with local pharmacy organizations and commercial CE vendors to deliver preceptor development CE programs (benefits schools and preceptors with minimal cost).

The Loyalty of LEOs: Nurturing Student and Preceptor Relationships. Huy T. Hoang, Pacific University Oregon, Anita J. Cleven, Pacific University Oregon. Objectives: A novel approach to gather data from both students and preceptors via the use of Liaisons for Experiential Outreach (LEOs). Data gathered from LEOs are utilized to enhance experiential education at Pacific University School of Pharmacy and facilitate quality assurance of sites. Method: LEOs serve as liaisons and goodwill ambassadors for the School of Pharmacy Office of Experiential Education (OEE), and practicing pharmacists. They visit experiential sites as assigned and meet individually with preceptors and students to provide training, and assess quality of the educational experiences provided by the preceptor and site. LEOs provide continuous quality improvement, expansion of existing sites, and recruitment of new sites for introductory and advanced pharmacy practice experiences. They also support the OEE in areas of practitioner collaboration (e.g. Preceptor Advisory Board) and disseminate general information on School and Experiential programming to members of the pharmacy community. Results: Data gathered from LEOs are inputted in $\mathrm{E}^{*}$ Value, an online platform system to leverage aspects of experiential learning. Data gathered from site visits include but are not limited to: quality of pharmacy, preceptor and student information. These results yielded information that increased education in preceptors and students, increased more information in our office of experiential education, and more importantly, optimized student placement in their experiential sites. Implications: Achieving high quality experiential sites is critical for the future of pharmacy practice. LEOs help strengthen existing relationships and foster new ones between the School and Community. This, in turn, helps build long-lasting connections with students, preceptors, and the School. 


\section{American Journal of Pharmaceutical Education 2018; 82 (5) Article 7158.}

Utilizing Clinically-Focused Student Learning for Patient Medication Reconciliation and Improved Team Integration. Eiizabeth Trolli, The Ohio State University, Joshua W. Gaborcik, The Ohio State University, Claire Murphy, The Ohio State University, Tracy Wiczer, The Ohio State University. Objectives: In a time when preceptors are being asked to do more than ever before, it is essential to create a dynamic, patient-centered interprofessional clinical experience where doctor of pharmacy (PharmD) students become a vital team asset. The objective is to demonstrate the impact of utilizing train the trainer techniques for student integration into the clinical pharmacy team. Method: The Ohio State University College of Pharmacy (COP) partnered with The Ohio State University Wexner Medical Center (OSUWMC) to create quality learning experiences for students by integrating them into the patient care team as a pharmacist extender. By using the train the trainer technique and pharmacist oversight, students complete 20 hours of admission medication reconciliation. Results: The medication reconciliation learning experience pilot began in Spring 2016 with 23 students. Due to its success, all 138 second-year PharmD students were required to participate for a total of 2760 hours during the 2016/2017 academic year. For the 2017/ 2018 academic year all third-year pharmacy students were also included, allowing for 271 students to complete 5420 hours of admission medication reconciliation in 7 different clinical areas. Teams that were previously without pharmacy-based medication reconciliation services now have these services including oncology and hematology. Student and preceptor feedback from these experiences have been overwhelmingly positive and quarterly quality improvement updates continue to strengthen the experience. Implications: Colleges and schools of pharmacy can use this method to partner with local hospitals to obtain clinical experiences for students that can expand pharmacy services without the requirement for additional personnel resources.

\section{LIBRARY AND INFORMATION SCIENCE Completed Research}

Assessment of Attendance Encouragement on Attendance and Classroom Performance in a Drug Information Course. Christopher S. Wisniewski, Medical University of South Carolina. Objectives: To determine if encouraging attendance by using passwords for assignment access influenced attendance and performance. Method: In a distance-learning drug information course with recorded lectures, students are given passwords at the end of each class to access assignments. Passwords are not recorded nor made available to absent students. It is unknown if assignment passwords increase attendance or if students benefit scholastically from attending class. Password-protected assignments were used in 2016 and removed in 2017. Baseline knowledge, demographics, opinion on, and motivation for, attending class, and desired grade were assessed at the start of the semester for both years. Attendance, course grades, and motivating factors were compared via descriptive statistics. Results: Student performance at baseline was comparable $(2016=39.16 \%$ vs. $2017=40 \%)$, students were demographically similar, and demonstrated a similar proportion of desired grade distribution between years. In 2016, students were more motivated to attend when graded assignments were scheduled, while in 2017, students were more likely to be motivated by professionalism. For reasons to attend any course, instructor knowledge of attendance was rated higher in 2016 and difficulty of course content was rated higher in 2017. Attendance in 2016 was $89.8 \%$ (1679/1870) compared with $53.4 \%(977 / 1830)$ in 2017. Course average was $87.06 \%$ and $88.28 \%$ in 2016 and 2017, respectively. Implications: Password-protected assignments increased student attendance but did not impact course grade average. Students between years had slightly different reasons for attending class prior to starting the course.

Bibliometric Analysis of Pharmacology Publications: A State-byState Evaluation. Dennis F. Thompson, Southwestern Oklahoma State University, Krista G. Brooks, Southwestern Oklahoma State University. Objectives: Bibliometrics can be used to track the growth of scientific disciplines and reveal trends, resource utilization and productivity. The purpose of this study was to evaluate the pharmacology and pharmacy publications in the United States at a state-by-state level and determine correlations with economic and population parameters. Method: We utilized the Web of Science (WoS) database as a means of quantifying publications, citations, and bibliometric indices at the state level. The search was confined to the ten-year inclusive timespan of 2007-2016. The search was refined by limiting the results to the Pharmacology/Pharmacy subject category. Population data were obtained from the United States Census Bureau; Gross Domestic Product (GDP) was extracted from the United States Bureau of Economic Analysis; and total state expenditures were taken from Ballotpedia. Statistical analysis involved descriptive statistics and Pearson correlation analysis using ungrouped data. Results: Pharmacology publications represent a small percentage of total state publications (highest $5.4 \%$ ). Pharmacology publications are concentrated in a small number of states. Fifteen states (30\%) account for $71 \%$ of all pharmacology publications and $72 \%$ of all citations. A strong correlation was found between state pharmacology publications and GDP $(r=0.9)$. Most states have higher education institutions as their primary source of pharmacology publications, but 13 states have a biopharmaceutical company or government agency as one of their top three producers. Implications: Bibliometric data can provide insight on factors that correlate with scientific output and may help policy makers decide what strategies might attract economically stimulating industries to their states.

Development of a Mentorship Program Within the Library and Information Sciences (LIS) Section. Audrey Kostrzewa, Concordia University Wisconsin, Christopher S. Wisniewski, Medical University of South Carolina, Priya Shenoy, Drake University, Melissa L. Hunter, University of Wyoming. Objectives: To describe the development and characteristics of a videoconference mentorship program created to assist newer faculty and promote collegiality within the Library and Information Sciences (LIS) Section. Method: Under the direction of the LIS Membership Committee, a mentorship program was developed. An interest survey was sent to all section members; interested respondents were organized into groups of mentors and mentees. The mentors were tasked with organizing group meetings. Data on participation, number of groups, group makeup, meeting frequency, and topics discussed were collected. Informal feedback was gathered from participants. Results: Participation in the mentorship program was $12.6 \%(\mathrm{n}=15 / 119)$. Three groups of 5 members were created with 2 mentors ( 1 drug information pharmacist and 1 librarian) and 3 mentees each. Meeting frequency and topics were not stipulated by the Membership Committee. The groups each self-selected to meet approximately every other month via videoconference, with further correspondence by email as needed. Discussion topics included scholarship, job duties and expectations associated with an academic career, uncommon information resources, student evaluations and assignments, and stress management. Mentors report that the groups provided emotional support given the rigors associated with stressors of pharmacy academia. Mentees found value in networking with and 


\section{American Journal of Pharmaceutical Education 2018; 82 (5) Article 7158.}

learning from colleagues, particularly the mixture of pharmacists and librarians. Suggestions included formal agendas and documentation of discussions for future reference. Implications: The LIS Membership Committee supports the continued development and expansion of the mentorship program. Future opportunities include improved structure, formal evaluation, and further dissemination to other sections.

Effect of Drug Information Organization On Student's Knowledge and Preparedness. Jason W. Guy, University of Findlay, Andrew J. Hvizdos, University of Findlay. Objectives: To determine if participating in a drug information (DI) organization enhanced DI knowledge and preparedness. Method: A survey was sent to students participating in the Annual Review of Changes in Healthcare (ARxCH) drug information organization. The ARxCH was established to provide students with additional opportunities to learn about the publication process and literature evaluation to better prepare them for practice. The survey consisted of 28 questions using Likert scales to measure students' preparedness and confidence in their DI skills. Two groups of students were surveyed, ARxCH student participants and non-ARxCH student participants. Students needed to be enrolled in the professional years of the curriculum (i.e., non-undergraduate) to participate in the study. An alpha of 0.05 was used for statistical significance. Results: Students in $\mathrm{ARxCH}$ scored at least as high or higher in 27 of the 28 DI-related questions compared to non-ARxCH participants. $\mathrm{ARxCH}$ students felt more prepared to assess topics in pharmacy and perform literature reviews $(p=0.03)$. They also felt more prepared to conduct research in pharmacy $(p=0.025)$ and were more comfortable making recommendations using the biomedical literature $(\mathrm{p}=0.027)$. ARxCH students also felt the experience helped increase their therapeutic knowledge $(p=0.041)$. Implications: An organization devoted to literature evaluation and the publication process may be useful in Colleges/Schools of Pharmacy to help prepare students for DI-related activities on rotations and in practice. This enhanced preparedness could help students in their pursuit of post-graduation goals.

Evaluation of Student Pharmacists' Ability and Self-Confidence Using Drug Information Resources to Analyze Drug-Drug Interactions. Robert D. Beckett, Manchester University, Rozta Fawzy, Manchester University, Alissa Keillor, Manchester University, Rosary Ajaelu, Manchester University. Objectives: To evaluate student pharmacists' ability and self-confidence using a drug information database to investigate an unknown drug-drug interaction and recommend appropriate course of action. Method: A longitudinal cross-sectional study was conducted where didactic-level students were presented with three patient cases, each containing a previously unencountered drug-drug interaction. Students were asked to select a drug information resource and determine the mechanism, clinical effects, severity, level of documentation, and course of action for each interaction. Overall performance was assessed on a 15-point scale (1 point for each assessment item for each interaction), and students rated their confidence on a 5-point scale for each interaction, yielding a 15-point confidence scale. The Kruskal-Wallis test was used to compare performance and confidence scores based on demographic factors using a pre-set alpha of 0.05. A multiple logistic regression was performed to investigate factors associated with high performance (ie, age, GPA, sex, selected reference, confidence). Results: For 187 participants (90.8\% response rate) who completed each interaction assessment, median performance score was 14 (IQR 12-15); 58 (31.0\%) earned the maximum score. Median confidence score was 13 (IQR 12 to 14). There was no difference in overall performance $(p=0.12)$ or confidence $(\mathrm{p}=0.66)$. High performers were likely to have high confidence scores (ie, 14 or 15 points; OR 5.2, 95\% CI 2.0 to 13.8, p<0.001). No other factors were statistically significant. Implications: This crosssection of student pharmacists earned generally high performance scores; future efforts will focus on using confidence as a marker to drive students toward supplementary practice.

Longitudinal Assessment of Students' Skills Interpreting and Applying Results From Clinical Studies. Robert D. Beckett, Manchester University, Jennifer A. Henriksen, Manchester University, Sara N. Trovinger, Manchester University. Objectives: To describe students' skills in interpreting clinical studies following advanced pharmacy practice experiences (APPEs). Method: Following completion of required coursework in drug literature interpretation and evaluation in the P2 Spring semester, students in the class of 2017 at one pharmacy program completed a formal assessment (14 points, $70 \%$ passing threshold) where they interpreted a piece of primary drug literature and applied results to a patient case at 1) pre-P3 year, 2) post-P3 year in a high-stakes objective structured clinical examination (OSCE), and 3 ) during required post-APPE assessments. Students were required to complete two formal journal club presentations during the P3 year, and at least two during APPEs. Performance on each assessment was compared using appropriate tests for paired data and an alpha value of 0.05. Results: Fifty-five of 71 (77.5\%) graduating students completed all three assessments. Median scores (IQR) were 9 (7-11), 12 (11-14), and 11 (9-13), respectively ( $\mathrm{p}<0.001$ for each comparison). First time pass rates were $43.6 \%, 90.9 \%$, and $69.1 \%$, respectively $(\mathrm{p}<0.01$ for each comparison). More students earned full credit post-APPE compared to post-P3 for determining statistical significance of results ( $65.5 \%$ vs. $43.6 \%)$, but fewer for determining clinical relevance (50.9\% vs. $76.4 \%$ full credit) and selecting the best intervention for the patient (34.5\% vs. $69.1 \%$ full credit). Implications: Student performance on a drug literature interpretation assessment was highest during a high-stakes post-P3 OSCE compared to low-stakes post-APPE and pre-P3 assessments. Based on results, a remediation program is in development for APPE students who do not meet expectations for required journal clubs on APPEs.

PhD Graduate Students Self-Assessed Level of Information Searching Skills. Jennifer R. Martin, The University of Arizona, Patrick J. Campbell, The University of Arizona, Marion K. Slack, The University of Arizona. Objectives: To assess graduate student self-reported information searching skills critical for conducting PhD level research. Method: Pharmacy graduate students rated their level of ability for nine information searching items using a five-point unipolar response scale from no skill to a high level of skill. Students that participated in a library education series completed a retrospective pre-posttest version of the questionnaire. A Wilcoxon rank sum test was used to assess differences between graduate program tracks and a Wilcoxon signed rank test was used for the retrospective pretestposttest. Results: A total of 27 students (14 outcomes \& 13 lab-based graduate students) completed the assessment at baseline. Overall, most students (74\%) rated themselves with moderately high to high skill in formulating a research question, in comparing PubMed, Embase, and Google Scholar, ability to navigate PubMed (82\%) and Google Scholar (88\%), ability to identify 3 additional information sources, and using references $(82 \%)$. Students rated themselves lowest in navigating Embase (58\% with no or little skill), and low to moderate skill in conducting citation searches. Outcomes students had greater expertise in using Embase $(p<0.001)$ and lab-based students had greater expertise in citation searches $(\mathrm{p}=0.042)$. Overall, outcomes students (10) rated themselves significantly higher on the posttest measure than on the retrospective pretest $(\mathrm{p}<0.05)$ for 8 of 9 skills. Implications: Overall, most students had moderate knowledge of searching related to 


\section{American Journal of Pharmaceutical Education 2018; 82 (5) Article 7158.}

their research. Instruction in searching can increase skill levels to moderately high to high; a level of skill appropriate for a graduate in a PhD program.

Pilot Study of Student-Made Videos Describing Pharmacist Activities for Non-Pharmacist Faculty. Sherrill J. Brown, University of Montana, Lendelyn Ekholt, University of Montana, Liliya Vorobey, University of Montana, Jean T. Carter, University of Montana. Objectives: This initial study was performed to determine the best content and format of short informational videos about pharmacy practice in a community setting. If successful, the videos will be used to help orient nonpharmacist faculty to current pharmacy practice. Method: Two fourthyear student pharmacists prepared a 6-minute video about several aspects of community pharmacy practice for a pilot study. The video was produced at the recording studio provided by the campus library. Students and clinical faculty viewed the video and completed feedback forms during their weekly conference. Responses and suggestions will be used to guide preparation of several final informational videos about typical activities for a pharmacist in three settings: hospital, clinic, and community. Results: Fourteen faculty and students watched the video and provided feedback. While the majority (57\%) felt that the provided information was clear, comments focused on ways to improve the video, such as the addition of visual aids. The video length was considered just right by $64 \%$ of the viewers, although four people found the video was too short. While the conversational delivery style worked for $71 \%$ of the viewers, $64 \%$ felt the presentation was choppy and that the transitions could have been smoother. Comments indicated the videos were a good idea and perhaps should be shown in a faculty meeting followed by a Q\&A session. Implications: Based on the feedback, the fourth-year students will continue to produce short videos about typical pharmacist activities in community, clinic, and hospital settings for viewing by nonpharmacist faculty.

Student Skills Related to Critiquing Systematic Reviews: Experience From a Literature Evaluation Course Series at Utah. Kendra Lawrence, The University of Utah, Krystal Moorman, The University of Utah, Joanne LaFleur, The University of Utah. Objectives: A critical skill for future pharmacists is to accurately read, interpret, and critique the medical literature, arriving at sound interpretations of the evidence. In our professional program, a 6-credit, 2-semester course series is dedicated to teaching 2nd-year PharmD students to read and interpret clinical trials, non-inferiority studies, systematic reviews (SRs), cohort studies, case-control studies, and pharmacoeconomic studies. Given that SRs constitute the highest level in the evidence hierarchy, we asked students to conduct 2 comprehensive critical appraisals during the SR module. Method: We amended the validated AMSTAR critical appraisal tool and created a template for students to use on the Google Forms online platform. Most students (51/56) consented to share their data collected for this study. Results: Prior to this course, $76 \%$ of students reported receiving zero hours of training on SRs and 20\% reported never previously accessing SRs as part of their education or practice. In general, students were most adept at identifying clearly reported items (i.e., $>90 \%$ correct), but struggled to appraise the more nuanced appraisal items that required a critical approach. For example, $65 \%$ of students incorrectly stated that the quality of individual studies was adequately addressed in formulating both reviews' conclusions. Implications: A research gap exists concerning how to best facilitate students' SR critical appraisal skills. Qualitative data from this pilot study suggests that students believe SRs are essential to their understanding of medical evidence, but feel under-exposed to this type of research in their formal training, even after a module dedicated to training students about SRs.
The Evolution of a National Electronic Educational Resource for Informatics. Marie A. Rocchi, University of Toronto, Lisa Bishop, Memorial University of Newfoundland, Janet Cooper, Association of Faculties of Pharmacy of Canada, Harold Lopatka, LopAlta Ltd, Seema Nayani, Canada Health Infoway. Objectives: To improve the quality of the "Informatics for Pharmacy Students" e-Resource to allow relevant and valid teaching and learning about e-health in Canadian schools of pharmacy. Method: Against a backdrop of electronic health records, digital information resources, and expanded scopes of practice, the e-Resource was developed in 2012 by the Association of Faculties of Pharmacy of Canada (http://afpc-education. info/moodle/index.php). With the goal of updating and expanding the e-Resource, modifications and reorganization resulted in release of two subsequent versions. Multiple formal internal evaluations helped inform the directions taken to improve the e-Resource. Content modifications underwent a structured review process through an editorial advisory committee and peer review. Several other sources informed revisions: faculty case reports on integration and curriculum changes, learning management system analytics, and feedback from student users. Results: Version 3 was released in August 2017 with significant revisions and additional learning activity development. All chapters were enhanced and updated, with improved navigation and formatting for a streamlined learning experience. Four months after the release of Version 3, 746 new users had enrolled (with over 4800 enrolled prior to its launch). User testing revealed little or no technical difficulties and satisfaction with content and platform. Implications: The continuous improvement of the e-Resource has resulted in a robust, stable platform about information and communications technology for use in pharmacy education. Given the ever-changing world of informatics, it is important that it is relevant and up-to-date. The lessons learned through this design and content review process can be used by others.

Using Curriculum Mapping to Assess Individual Faculty Integration of a National Informatics Education Program. Lisa Bishop, Memorial University of Newfoundland, Marie A. Rocchi, University of Toronto, Janet Cooper, Association of Faculties of Pharmacy of Canada, Harold Lopatka, LopAlta Ltd, Seema Nayani, Canada Health Infoway. Objectives: The purpose of this project was to determine the extent to which informatics was being taught in pharmacy schools using a nation-wide curriculum mapping activity. Method: Six of the 10 Canadian schools of pharmacy from across the country participated in the curriculum mapping. Course learning objectives were used to identify teaching and learning activities with an informatics focus. The learning objectives were then aligned with national informatics competencies. The NFC (novice, functional, competent) framework was used to assign the expectation of the student performance for each informatics-related course objective. A description of the course, activity, area of curriculum, and year of study was collected. Lessons learned were also captured. Results: The competencies were categorized into the five key areas of the curriculum, with the most common area being pharmacy practice/therapeutics, then practice skills, followed by experiential learning. Informatics teaching was distributed over 4 years of most programs, with a greater emphasis in years 1-3. The performance levels were most often tagged at a novice or functional level, although it was often challenging to categorize retrospectively based upon the course learning objectives and the activities within the course. Implications: Given the focus on informatics under a number of the CAPE domains, the curriculum mapping process is a useful way to help determine the extent that the competencies are being addressed and where gaps exist. The results provided insight 


\section{American Journal of Pharmaceutical Education 2018; 82 (5) Article 7158.}

about situations where strategies might be used to improve uptake of informatics in the pharmacy curriculum.

Using Dual-Peer Review to Improve a Drug Information Response. Gregory W. Smith, The University of Louisiana at Monroe, Scott A. Baggarly, The University of Louisiana at Monroe, Glenn Anderson, The University of Louisiana at Monroe, Bryan Donald, The University of Louisiana at Monroe, Stephen R. Hill, The University of Louisiana at Monroe, Cassie Crew, The University of Louisiana at Monroe. Objectives: To determine the impact of incorporating a dual-peer review process using Examsoft ${ }^{\circledR}$ rubrics for improving medical-professional writing quality and content of a drug information (DI) response paper in a 1st professional year DI Retrieval course. Method: Pharm. D. candidates may lack experience in effectively formulating a written DI response, which is an essential component of patient-centered care as stated in Domain 2 of the Center for Advancement of Pharmacy Education (CAPE) outcomes. In a DI Retrieval course, each student authored a DI response and reviewed two peers' written DI responses using an Examsoft ${ }^{\circledR}$ rubric. Students used their peers' feedback to edit papers prior to final submission for faculty grading using an identical rubric. Peer reviewer grades were compared to the faculty grades using a t-test. The dual peer grades for each paper were analyzed for interrater reliability. Results: The interrater reliability of the rubric dimension ratings from the dual peer reviews was 0.525 . The average peer-reviewer grade before final edits was $84.25 \%$, and the average faculty grade after final edits was $92.25 \%(\mathrm{P}<0.001)$. Implications: A dual peer review process can benefit students as authors and as peer reviewers. Student authors receive benefit from feedback that can positively impact the content and writing quality of their DI responses. Peer reviewers benefit by experiencing the process of critically evaluating student authors' papers and providing feedback to improve medical-professional writing and evidence-based content. A dual peer review offers the advantage of having two unique perspectives and expectation levels in terms of professional writing and evidence-based content.

Utility Survey of the 2016 AACP Core Journal List. Neyda V. Gilman, Binghamton University, The State University of New York, Robert D. Beckett, Manchester University, John L. Redwanski, University of New England, Rebecca Hoover, Idaho State University, Scott Perkins, Campbell University. Objectives: Obtain feedback about the AACP Core Journal List that will then be used to update the list. Method: A nineteen question survey was drafted based on a 2015 survey. In order to reach as many users of the Core Journal List as possible, the final survey was shared with the AACP Library and Information Sciences Section, Medical Library Association, Special Library Association, and Consortium for the Advancement of Medication Information Policy and Research. Results: The survey had eighty-five respondents, sixty (70.6\%) of which answered all nineteen questions. $58.6 \%$ of the respondents work in academia and reported using the Core Journals List mostly for accreditation (35.6\%) and/or collection management (33.3\%) purposes. Fourteen of the twenty individuals $(70 \%)$ who stated they used the list for either accreditation or collections noted that the list influenced changes to their institution's collection within the last 18 months. The majority of respondents find the list to be accessible (ranked good or excellent by $83.3 \%$ ), relevant (good or excellent by $88.9 \%$ ), reliable (good or excellent by 94.5\%), and usable (good or excellent by $77.8 \%$ ). Implications: The results of the survey suggest the AACP Core Journal List is a valuable tool for maintaining quality resource access. Responses will be used to update the list and improve its visibility.

\section{Theoretical Models}

Implementation of Electronic Health Records in the First Professional Year. Scott Perkins, Campbell University, Kimberly E. Kelly, Campbell University. Objectives: To implement electronic health records (EHRs) into various courses in the first-year didactic course work of a new integrated curriculum at an established pharmacy program. Method: The use of EHRs was implemented in various courses in the first professional year of a new pharmacy curriculum in order to longitudinally impart the skills of identifying relevant patient information and making clinically-sound recommendations. Courses which used an EHR include Pharmacy Practice Skills courses, Introduction to Evidence-Based Medicine, and Integrated Pharmacotherapy. The implementation plan includes the development of 7 individual patient cases with escalating degrees of complexity. Results: Through the first semester, students accessed the EHR to solve 3 series of a progressive case scenarios involving 1 patient and 2 calculations-based cases involving 2 patients. Two courses used the EHR in the Fall and the average time to create a case was 1.2 hours. Course passage rates were $100 \%$. The plan for the second semester involves 4 patients as part of 13 individual case scenarios. Implications: The integration and use of EHRs in multiple pharmacy courses allows for early simulated clinical experiences. Due to the cost and time commitment related to using EHRs as part of didactic course work, an implementation strategy to maximize the effective and of these tools to provide early simulated clinical experiences is desirable.

\section{PHARMACEUTICS \\ Completed Research}

An In Vitro Release Test for Ketoprofen in Semisolids Using Immersion Cells With USP Apparatus 2. Jacob R. Dunbar, South College. Objectives: In vitro release tests (IVRT) quantify drug release from semisolid products and can discern differences in drug release due to formulation differences, process changes, processing errors, or product instability. The objective of this study was to develop an IVRT to compare drug release from $5 \%, 10 \%$, and $20 \%$ ketoprofen in a Lipoderm ${ }^{\circledR}$-based cream versus a pluronic lecithin organogel (PLO) using immersion cells with USP Apparatus 2. Method: Formulation samples were loaded into the sample compartment of immersion cells ( $n=3$ per formulation $x$ strength) covered with a $0.45 u$ Tuffryn ${ }^{\circledR}$ polysulfone membrane. The assembled immersion cells were placed in a flat-bottomed vessel containing $100 \mathrm{~mL}$ of phosphate buffered saline (PBS) at $32^{\circ} \mathrm{C}$, stirred at a paddle speed of $100 \mathrm{rpm}$. Ketoprofen concentrations were determined by HPLC in PBS samples taken at $0.5,1$, 2, 3, 4, 5, and 6 hours of testing. Results: Cumulative ketoprofen release from both formulations at all drug concentrations was linear versus square root of time ( $R 2 \geq 0.9962$ for all samples) and slopes of the regression lines were proportional to drug concentration. At each concentration, ketoprofen release was 3-4 $\mathrm{x}$ faster from PLO than from Lipoderm ${ }^{\circledR}$-based cream. Desired performance parameters for IVRT were achieved including acceptable sink conditions, NMT $30 \%$ of the sample's drug content released at the last testing time, drug release linear vs. $\sqrt{\text { time, }}$, slope variability $<15 \%$, and no significant lag time to linearity. Implications: The described IVRT offers a simple, reliable method for determining differences in ketoprofen release from different semisolid formulations.

Comparing the Compounding Process and the Product of a Novel Lidocaine Gel Formulation. Mamoon Rashid, Appalachian College of Pharmacy, Mehbuba Rahman, Appalachian College of Pharmacy, Faisal Hossain, Appalachian College of Pharmacy, Akinola Adeyemo, 


\section{American Journal of Pharmaceutical Education 2018; 82 (5) Article 7158.}

Appalachian College of Pharmacy. Objectives: 1. to evaluate the product quality of a newly developed formulation of lidocaine gel, a formulation with less alcohol than the conventional method. 2. To evaluate compounders' ease of compounding, satisfaction, and confidence of reproduction of this new formulation. Method: This cross-over study with P1 and P2 student-pharmacists compared a newly developed fivefold low alcohol formulation and the compounding procedure with a conventional carbomer-based $2 \%$ lidocaine dental gel that contains $75 \%$ alcohol. The product was analyzed using HPLC to assess the potency. A separate questionnaire was used to record the compounders' seven-point feedback including the compounding method, level of satisfaction and confidence of reproduction. Finally, the time spent in various sections of the two methods were compared. Results: Eleven lidocaine gel products were compounded from two groups of compounders which showed that the novel method has smaller standard error of mean in product potency. Moreover the compounders are able to finish the compounding in shorter time. Of the seven feedback questions, five did not receive any significant difference in compounding aspect, but in the remaining two the students found that the novel method is more favorable. Also, this new method is free from chance of formation of insoluble agglomerates which are difficult to handle by the compounders. Implications: This new method may be easily applied in compounding pharmacy as well as taught to student-pharmacists for the extemporaneous preparation.

Co-Precipitate Calcium Phosphate With Methotrexate in PLGA Microparticles for Better Sustained Release. Qian Zhang, MCPHS University, Mariam Khalife, MCPHS University, Guang Yan, MCPHS University-Worcester/Manchester. Objectives: Previous study showed that methotrexate (MTX) loaded poly(lactic-co-glycolic) (PLGA) microparticles can be administered intra-articularly for localized drug delivery to minimize systemic toxicity. Rapid burst release phase followed by a very slow release phase for the conventional MTX-PLGA microparticles was far from ideal to meet our clinical need. In this study, we intend to improve the MTX-PLGA microspheres with a method to coprecipitate MTX with calcium phosphate to lower burst release and improve the sustained release profile. Method: MTX, sodium phosphate, and calcium nitrate were mixed to create a precipitant, then the precipitant was added to PLGA in methylene chloride solution. The mixture was then added drop wise to a PVA solution under stirring condition to form the microparticles (MTX-CaP-PLGA). Separately, the MTX in PLGA microparticles (MTX-PLGA) were also prepared. The MTX encapsulation rate, the microparticles size and drug release from the microparticles were determined for both type of microparticles. Results: Both type of microparticles showed similar particle size. MTX -PLGA microparticles showed good encapsulation efficiency. MTX-CaP-PLGA microparticles had lower MTX encapsulation efficiency. MTX-PLGA microparticles showed high burst release. MTX-CaP-PLGA showed low burst release and better controlled release profile in the later stage. Implications: Precipitating MTX with calcium phosphate and then encapsulating in PLGA, the PLGA microparticles can improve the drug release profile with lower burst release and better sustained release. This improved drug release profile is advantageous in employing the MTXCaP-PLGA microparticles for local treatment of rheumatoid arthritis.

Design and Evaluation of Dihydroergotamine Nanoparticles Using Chitosan Polymer. Ahmed Alastal, Jordan University of Science and Technology, Ahmad Shennar, Jordan University of Science and Technology, Abeer M. Al-Ghananeem, Jordan University of Science and Technology. Objectives: The aim of this study was to develop and evaluate dihydroergotamine (DHE) loaded chitosan nanoparticles. Method: Chitosan nanoparticles were prepared based on the ionotropic gelation of chitosan with tripolyphosphate (TPP) anions. Different concentrations of DHE in chitosan solution were examined to enhance drug encapsulation efficiency. In vitro release profile was determined by measuring the cumulative concentration using dialysis bags. Detailed characterization of DHE-loaded chitosan nanoparticles was conducted using scan transmission microscopy (STM) and laser diffraction for particle size analysis. Zeta potential was further detected by Nanosizer. The encapsulation efficiency and in vitro release studies were conduct in phosphate buffer at pH 5.2 Results: DHE-loaded chitosan nanoparticles illustrated an average size of $213 \mathrm{~nm}$ and potential of $+15 \mathrm{mv}$ while mean poly-diversity index (PDI) was 0.254. Maximum Encapsulation Efficiency (EE) was $19 \%$. Particle size increased with increasing loading efficiency. However, the increase was not linearly proportional. Investigating the in vitro drug release showed almost $33 \%$ of the loaded DHE was released within 24 hours. Interestingly, the drug in vitro release studies revealed different rates and patterns with the change in \% drug loading and levels of chitosan cross-linking. Increasing chitosan cross-linking resulted in lowered the drug release profile under the experimental conditions. Implications: The results of the present work illustrated an attractive and reproducible formulation technology with chitosan nanoparticles. The formulation could be a good modality for further evaluation as a drug delivery system.

Determination of the $\mathbf{p H}$ Effect on Transdermal Penetration of Diclofenac. Thrir Lwin, MCPHS University, Faris Alhalwan, MCPHS University, Guang Yan, MCPHS University-Worcester/Manchester. Objectives: Diclofenac is a weak acid with a pKa around 4.15. Intuitively, the unionized form of diclofenac is more favorable for transdermal penetration. The objective of this study was to determine the transdermal penetration of diclofenac at different $\mathrm{pH}$ and thus to delinearize the contribution from the ionized and unionized forms. Method: In vitro flux study was conducted with Franz diffusion cells to determine the flux of diclofenac across the human cadaver skin, a CoTran 9712 membrane (polyethylene vinyl acetate), and a dialysis membrane (MWCO 3000) with 1\% diclofenac solution ( or suspension) at different $\mathrm{pH}(3.0,4.9,6.17,7.4$, and 10.2). The permeability of the ionized diclofenac and unionized diclofenac across the different membrane was then calculated. Results: Diclofenac showed maximum flux across skin at $\mathrm{pH} 7.4$, though the permeability of the unionized diclofenac is about 1000 fold of that of the ionized diclofenac. The permeability of the ionized diclofenac and unionized diclofenac across dialysis membrane are similar. For penetration across CoTran-9712 membrane, the permeability of the unionized diclofenac is about 2800 fold of that of the ionized diclofenac. Implications: Both unionized diclofenac and ionized diclofenac penetrate through the skin by partition into the skin. Unionized diclofenac has higher permeability across the skin. However, at higher $\mathrm{pH}$, ionized diclofenac can have large contribute the transdermal penetration because of its high solubility. The CoTran-9712 is a better synthetic membrane to mimic drug penetration across the skin.

Development of Plasmid Vectors Encoding Genes Betacellulin and Hepatocyte Growth Factor for Improving Islet Transplantation. Ravikiran Panakanti, Roosevelt University, Rimple Patel, Roosevelt University, Frederick K. Galarza, Roosevelt University, Yash Desai, Roosevelt University, Micky Patel, Roosevelt University. Objectives: Human islet transplantation has the potential to replace pancreatic endocrine function in patients with diabetes. The critical challenge here is to improve the survival and functionality of islets for a longer period of time. Betacellulin (BTC) is an epidermal growth factor promoting beta cell (insulin producing cells) proliferation, differentiation 


\section{American Journal of Pharmaceutical Education 2018; 82 (5) Article 7158.}

and growth. Hepatocyte growth factor (HGF) is a potent mitogen known to improve $\beta$-cell growth and function. It also shows anti-apoptotic activity. We aim to develop and assess functionality of the plasmid vectors encoding genes HGF and BTC in improving the outcome of islet transplantation. Method: Plasmid vectors were constructed by inserting genes BTC and HGF in the plasmid pcDNA3.1 separately by using restriction enzymes Bam HI-XhoI for BTC and Nhe I-Hind III for HGF. Rat Insulinoma cells (RIN-5mF) cells were transfected with plasmid vectors pcDNA3.1-BTC and pcDNA3.1-HGF respectively using lipofectamine 3000 reagent. MTS, Caspase -3 assay and ELISA were done to ascertain the function of plasmid vectors pcDNA3.1-BTC and pcDNA3.1-HGF respectively. Results: RIN-5mF cells transfected with plasmid vectors pcDNA3.1- BTC and pcDNA3.1-HGF with lipofectamine 3000 showed less toxicity. There was time dependent increase in expression of HGF and BTC from plasmid vectors. Plasmid vectors especially pcDNA3.1 -BTC showed antiapoptotic effect, when transfected with lipofectamine 3000 at $0.15 \mu 1$. Implications: Plasmid vectors pcDNA3.1 HGF and pcDNA3.1BTC showed good expression of genes BTC and HGF. They also showed anti-apoptotic effects by protecting the cells in the presence of cytokines. More tests need to be done to further ascertain the safety and efficacy of these vectors.

Effect of Batch Age on Potency and Dissolution of Levothyroxine Sodium Tablets. Mohamed I. Nounou, University of Saint Joseph, Bassant Abou-Taleb, Pharos University in Alexandria, Nawal Khalafallah, Alexandria University, Saleh Khalil, Alexandria University. Objectives: Less attention has been given to possible variation in quality and efficacy within the same brand depending on production site, storage condition, manufacturing condition, transportation and on product specification in different geographical locations. We have recently published a clinical study in patients comparing the efficacy of multisource $100 \mathrm{mcg}$ levothyroxine-sodium tablets. The results indicated that brand/source interchangeability could not be claimed. The aim of the present study was to investigate the relation between batch age and pharmaceutical quality including tablet potency and dissolution of multisource $100 \mathrm{mcg}$ levothyroxine sodium tablets from different origin locations, involving two brands and a total of twelve batches (1-4 batches/brand), purchased from local pharmacies in Egypt. Method: The study took into consideration both BP 2014 and USP 2014 conditions concerning levothyroxine tablets dissolution test. The pharmaceutical quality of multisource Brand I and Brand II levothyroxine sodium, $100 \mathrm{mcg}$ tablets, was assessed. Tablet potency, uniformity of content (BP 2014) and dissolution rate (BP 2014 and USP 2014) were determined for a total of twelve tablet batches. Correlation between generated data and batch age was investigated. Results: Tablets' potency, uniformity of content, and dissolution data showed inter-source, inter-batch and inter-brand differences. The contribution of batch age to the observed differences was assessed by linear and non-linear regression analysis. Implications: A trend was evident in the correlations examined indicating an adverse effect of batch age on potency and dissolution.

Evaluation of Celecoxib Release in an Acrylic Pressure Sensitive Adhesive Patch. Marissa E. Gooden, Xavier University of Louisiana, Natalie R. Faciane, Xavier University of Louisiana, Tommy C. Morris, Xavier University of Louisiana. Objectives: Celecoxib (CXB) is FDA approved for the treatment of arthritis and acute pain. However, its oral use is often restricted due to concerns of dose-related risks of negative GI and/or cardiac events. A transdermal patch would provide localized dosing that minimizes systemic exposure. We have conducted a study to evaluate impact of the pressure sensitive adhesive (PSA) choice on the in vitro release of CXB from a drug-in-adhesive transdermal patch.
Method: CXB patches were prepared with the following formula: $9 \%$ celecoxib, 19\% glyceryl monooleate and 72\% DURO-TAK ${ }^{\circledR}$ adhesive $(9301,2196$, or 2287). Each CXB patch was formed in a vertical diffusion cell dosage wafer. The in vitro release studies were performed using a vertical diffusion cell system and the following parameters: stirrer $(400 \mathrm{rpm})$, temperature bath $\left(32{ }^{\circ} \mathrm{C}\right)$, membrane filter $(0.45 \mu \mathrm{m})$, and dissolution media (PEG 400:water 20:80). Samples were run in triplicate and assayed spectrophotometrically at a wavelength of $252 \mathrm{~nm}$. Results: CXB samples that contained DURO-TAK ${ }^{\circledR} 2196$ showed the greatest rate of release from the patch. After 8 hours, the concentration of CXB in the receptor chamber was 3 times greater than the other two PSAs tested. Implications: The selection of the appropriate PSA is critical to the successful development of a transdermal patch. This study is important because it allows for the assessment of the impact of PSA properties such as crosslinkers and functional groups on release of CXB from the patch. The test method was specifically designed to allow for dissolution of CXB but also differentiate the PSAs.

Flipped Teaching in Pharmaceutical Calculations: Outcomes and Lessons Learned. Mohammad T. Nutan, Texas A\&M University, Elaine L. Demps, Texas A\&M University. Objectives: Flipped teaching has been implemented in pharmacy education in topics that range from pharmaceutics to drug literature evaluation. The objectives of this project were to measure student learning in a first-year pharmaceutical calculations course after flipping it and to understand the students' preference for the flipped course. Method: The Class of 2018 completed the traditional version of the course and served as the control group. Classes of 2019- 2021 experienced the flipped format and were the experimental groups who viewed lecture recordings prior to coming to class, completed a readiness assessment at the start of each class, solved multiple problems in class, etc. The following data were compared between the classes of students: course average grades and the pharmaceutical calculations section average scores from the annual benchmark exams. Classes of 2019-2021 students completed an anonymous survey designed to assess their preferences for flipped teaching. This survey was adapted from a validated instrument. The Texas A\&M University Internal Review Board approved this project. Results: The class average grade in the course for the experimental groups were either comparable to or higher than the control group. In addition, the experimental groups performed significantly better than the control group on the benchmark exams. The survey results indicate that students found the various aspects of flipped teaching effective. However, while some students strongly preferred the flipped format, many did not. Implications: Although flipped teaching can improve student learning, lessons learned include overcoming student perceptions that math courses are most effectively taught face-to-face.

Formulation and Stability of Extemporaneously Compounded Oral Suspension of Eslicarbazepine Acetate From Aptiom ${ }^{\circledR}$ Tablets. Vivek S. Dave, St. John Fisher College, Fang Zhao, St. John Fisher College, HongHao Li, St. John Fisher College, Mazin Mar, St. John Fisher College, Jonathan Perri, St. John Fisher College. Objectives: To develop oral suspension formulations using Aptiom ${ }^{\circledR}$ tablets. Method: Prototype formulations were prepared using commercially available vehicles such as ORA-Plus ${ }^{\circledR}$, ORA-SWEET ${ }^{\circ}$, ORA-SWEET ${ }^{\circledR}$ SF, Unispend Anhydrous, Unispend Anhydrous (sweetened), ORAL-MIX, ORAL-MIX SF, and SyrSpend ${ }^{\circledR}$ SF, either alone or in combination. The suspensions were prepared at $40 \mathrm{mg} / \mathrm{mL}$ concentration to provide the $200 \mathrm{mg} / 5 \mathrm{~mL}$ dose. The suspensions were evaluated for viscosity to assess a balance of suspending ability and 


\section{American Journal of Pharmaceutical Education 2018; 82 (5) Article 7158.}

flowability. The suspensions were also visually evaluated for settling behavior. The palatability of the suspensions was evaluated by a panel of two volunteers on a severity scale of 1-5 for bitter, sweet, sour, salty, oily, metallic, odor, texture, irritation, and bitter after-taste. HPLC was used to monitor the eslicarbazepine stability in the suspensions for two weeks. Results: The viscosity of the suspensions decreased with increasing shear-rate linearly. The suspensions were physically stable (no particle settling behavior) for at least 48 hours. From the palatability results, two suspensions [ORA-Plus ${ }^{\circledR}:$ ORA-SWEET $®(50: 50, \mathrm{v} / \mathrm{v})$ and ORA-Plus ${ }^{\circledR}$ : ORA-SWEET ${ }^{\circledR}$ SF $\left.(50: 50, \mathrm{v} / \mathrm{v})\right]$ were found to be acceptable. These suspensions were also found to be chemically stable for two weeks. The mean eslicarbazepine concentrations in these suspensions were found to be $101.8 \pm 1.4 \%, 102.1 \pm 0.4 \%$, and $100.9 \pm$ $0.1 \%$ for ORA-Plus ${ }^{\circledR}$ : ORA-SWEET $\AA$; and $100.4 \pm 0.3 \%, 100.8 \pm$ $1.3 \%$, and $101.5 \pm 2.1 \%$ for ORA-Plus ${ }^{\circledR}$ : ORA-SWEET ${ }^{\circledR}$ SF based suspensions at day 0, week 1 and week 2, respectively. Implications: The study showed that stable eslicarbazepine suspension formulations can be reliably prepared from the Aptiom ${ }^{\circledR}$ tablets by pharmacists.

Immersive Pharmaceutical Sciences Research Training for High School Students Through the Young Innovators Program. Jacqueline McLaughlin, University of North Carolina at Chapel Hill, Antonio A. Bush, University of North Carolina at Chapel Hill, Janice Anderson, Josh Corbat, Samuel K. Lai, University of North Carolina at Chapel Hill. Objectives: To examine outcomes of the 2017 Young Innovators Program (YIP), a 10-week summer program that immersed high school students in science, technology, engineering, and mathematics (STEM) research at the UNC Eshelman School of Pharmacy. Method: Twenty-four YIP participants were assigned a laboratory mentor and student mentor. Interns conducted research, attended workshops, and submitted an academic report and presentation. Each participant completed a pre-YIP survey, post-YIP survey, and 2 focus groups. Data were analyzed with descriptive statistics and thematic coding. Results: The 2017 cohort consisted of 54\% White/Caucasian, 34\% Black/African American, and a relatively balanced gender distribution ( $58 \%$ female $/ 42 \%$ male). In the post-YIP survey $(n=24$, response rate $100 \%$ ) most indicated they interacted with mentors at least daily $(n=16)$. Participants agreed YIP improved my knowledge in STEM $(n=24 ; 100 \%)$ and positively influenced my desire to pursue a STEM career $(n=21 ; 88 \%)$. All $(n=24,100 \%)$ agreed they would recommend a friend or family member to participate in YIP. Focus groups indicated that students saw YIP as one of diversity, warmth, and encouragement, with helpful support and opportunities provided. One student summarized YIP, saying "I learned how to be in a lab, and this is what I want to do with my life. . . No matter who you are, this is going to help you in some way decide if this is the path you want to go down or if it isn't." Implications: This study suggests that pharmacy schools can promote learning and encourage research pathways in high school students through immersive research programs.

Incorporation of the Pharmacists' Patient Care Process (PPCP) Into an Integrated Pharmaceutics Course Sequence. Bill J. Bowman, Midwestern University/Glendale. Objectives: To incorporate the Pharmacists' Patient Care Process (PPCP) into an integrated pharmaceutics course sequence. Method: Over the past two years, the pharmacy compounding lab component of an integrated pharmaceutics course sequence was modified to enable the incorporation of the PPCP. This incorporation was accomplished by revising and reformatting most of the previously created lab activities and corresponding application questions so that the students purposely worked through each component of the PPCP (i.e. collect, assess, plan, implement, and follow-up) as they completed a mock prescription. At the conclusion of each academic year, the students were also surveyed to determine the extent to which they agreed or disagreed with five different statements regarding the incorporation of the PPCP. Results: The survey response rate was $98 \%(n=298)$. Almost all respondents $(94-98 \%)$ either agreed or strongly agreed that incorporation of the PPCP appropriately modeled the PPCP, was consistent with the PPCP's inclusion in other courses, and improved their understanding of the PPCP. In addition, $94 \%$ of respondents either agreed or strongly agreed that incorporation of the PPCP enhanced the course material's applicability to practice and facilitated their understanding of the course material. Implications: The results of this study demonstrate the feasibility of incorporating the PPCP into pharmaceutics courses and/or pharmacy compounding labs using 'real world' examples that may facilitate both the teaching and the learning of the PPCP. The results also indicate that incorporation of the PPCP may enhance the applicability of these sciences to practice and may even facilitate learning of the course material itself.

In Silico Prediction of Calcitriol Pharmacokinetics: Potential Effects of Anticancer Agents. Subrata Deb, Larkin University, Hayden Hagemann. Objectives: Calcitriol $(1 \alpha, 25$-dihydroxyvitamin D3) is the biologically active form of vitamin D3 and its physiological levels are critical in exerting anticancer effects of vitamin D3. Although analytical assays for calcitriol are available, they are expensive and technologically demanding which precludes them from routine use in humans. The present study is aimed to predict select calcitriol pharmacokinetic parameters and understand their interaction with cytochrome P450 3A4 (CYP3A4) inhibitory anticancer agents. Method: The GastroPlus software 9.5 version (Simulations Plus, Inc., Lancaster, CA) with PKPlus and ADMET Predictor modules were used to simulate the calcitriol pharmacokinetics, whereas Drug-Drug Interaction (DDI) module was used to evaluate the potential of kinase inhibitor anticancer drugs (cabozantinib, dasatinib, imatinib, nilotinib, sorafenib, sunitinib) to influence calcitriol disposition. Results: Following simulation of an oral dose of 2 ug calcitriol for $24 \mathrm{~h}$, the Cmax was predicted as $9 \mathrm{ng} / \mathrm{ml}$ with the predicted Tmax at $5.2 \mathrm{~h}$. The fraction of calcitriol dose (Fa) absorbed was $8.6 \%$. Although the Cmax was relatively low, the AUC0-24h was $170(\mathrm{ng} * \mathrm{~h} / \mathrm{mL})$. The predicted hepatic level of calcitriol $(14 \mathrm{ng} / \mathrm{ml})$ was significantly higher than the plasma levels. The ADMET Predictor analyses showed that calcitriol is primarily metabolized by hepatic CYP3A4. Co-simulation of kinase inhibitors and calcitriol using DDITM module suggests weak to strong interaction between kinase inhibitors and calcitriol. Implications: The pharmacokinetic simulation of calcitriol indicates that calcitriol is poorly absorbed with the concentrations in the subtherapeutic level. The calcitriol plasma levels could be enhanced by co-administered CYP3A4 inhibitors such as kinase inhibitor anticancer drugs.

Longitudinal Appraisal of "Critical Thinking and Quantitative Reasoning Skills" in Professional Pharmacy Students. Ajoy Koomer, Marshall B. Ketchum University, Rajesh Vadlapatla, Marshall B. Ketchum University, Kevin Zhijun Wang, Marshall B. Ketchum University, Edward Fisher, Marshall B. Ketchum University. Objectives: To measure progression in attainment of "critical thinking and quantitative reasoning skills" in professional pharmacy students using HSRT-N Method: The COP has adopted a triangulation approach to appraising "critical thinking and quantitative reasoning skills" in pharmacy students. In addition to COP created assessments, "Health Sciences Reasoning Test- Numeracy (HSRT-N)", developed by Insight Assessment, San Jose, CA, was administered to the inaugural class of 2020 during their first professional year at the beginning of the fall quarter of 2016. This assessment was repeated a year later for 


\section{American Journal of Pharmaceutical Education 2018; 82 (5) Article 7158.}

the same cohort of students as a post-test vehicle. In addition to the average percentile score, the assessment also reports the overall scaled score and sub scores for eight focus areas. Results: Results indicated that the average percentile for COP's inaugural class was 45 in their second year as compared to the baseline score of 37 . Pre/Post comparison of mean scores indicated that the class of 2020 did score at least 1.5 points or higher in the overall and focus area scores, except for two sub categories. Paired t-test analysis revealed that the difference in mean scores between the same testing group in fall quarters of 2017 and 2016 was statistically significant for the "Overall", "Inference", "Explanation" and "Deduction" sections. Implications: This standardized assessment tool aid us in benchmarking the above-mentioned skills in COP students, in comparison to a national sample of professional pharmacy cohorts. In addition, it may act as a pointer for reorganizing curricular content to address the deficient focus areas.

Novel Microcomposite for Controlled Delivery of Antibiotics in the Treatment of Osteomyelitis Following Total Joint Replacement. Victoria K. Maskiewicz, Loma Linda University, Serkan Inceoglu, Loma Linda University, Gary Botimer, Loma Linda University. Objectives: Osteomyelitis remains a significant complication following total joint replacement surgery, requiring the delivery of a significant dose of antibiotic to the joint space to eradicate the infection. Current practice involves implanting an antibiotic-laden polymethylmethacrylate spacer for local delivery of drug, however these devices are of limited effectiveness due to incomplete/ subtherapeutic release of drug payload. We have developed a novel microcomposite spacer that can locally release sustained, high antibiotic concentrations to guarantee infection sanitation, while maintaining the requisite mechanical properties to preserve soft tissue limb length prior to subsequent definitive joint repair. Method: Vancomycin or gentamicin loaded microcomposite implants were fabricated by incorporating drug-loaded mesoporous silica microparticles into polymethylmethacrylate bone cement to a final drug loading of $10 \% \mathrm{w} / \mathrm{w}$. Release kinetics at $37^{\circ} \mathrm{C}$ were monitored by reverse phase high performance liquid chromatography, and compared to current therapy implants consisting of drug alone incorporated at $10 \% \mathrm{w} / \mathrm{w}$ into polymethylmethacrylate bone cement. Results: Novel microcomposite constructs eluted $65 \%$ to $95 \%$ of drug payload over 30 days for vancomycin and gentamycin respectively, vs. $11 \%$ to $13 \%$ elution from currently used implants. Bending modulus showed no statistical difference between microcomposite implants and current standard therapy implants prior to drug elution, and maintenance of acceptable strength of microcomposite implants post-drug elution. Implications: These results demonstrate that we have developed a novel microcomposite PMMA spacer that will release continuously high antibiotic concentrations over a prolonged period of time, offering the possibility to eliminate infection while maintaining the requisite mechanical properties for proper space maintenance and joint fixation.

Perceptions and Appropriate Consequences of Lapses in Academic Integrity in Health Sciences Education. Marina Galvez Peralta, West Virginia University, Tina Antil-Keener, West Virginia University, Melinda Smith, West Virginia University, Lauren Swager, West Virginia University, Sijin Wen, West Virginia University, Mariette Barbier, West Virginia University. Objectives: Academic dishonesty is a concern among higher education, including Heath Sciences (HSC). Research suggests that individuals who engage in academic dishonesty may continue to exhibit unethical behaviors in professional practice. Students and faculty perceive differently what constitutes academic dishonesty and its seriousness. The purpose of this study is to gain faculty and students' perceptions and the appropriate consequences of lapses in academic integrity. Method: Faculty and students from HSC were asked to complete the anonymous "Appropriate Consequences of Lapses in Academic Integrity Survey" in which 10 different academic and clinical scenarios were presented. For each scenario, students or faculty scored their concern and assigned the academic consequence that they considered appropriate (ranked from no consequence to dismissal). The survey was developed by an interdisciplinary health care team. Statistical analysis: A mixed-effects model was used to assess the difference of questionnaire scores between subgroups. (WVU IRB \#1607176862) Results: A total of 491 students and faculty completed the survey. Data analysis revealed a variety of factors affecting the concern or consequence: gender, being first generation students, and faculty or students status. Students witnessing cheating in the past did not significantly affect their answers, while there were significant differences on the answers between faculty who had or had not witness cheating. Nonclinical scenarios showed significantly higher differences between faculty and students than clinical scenarios. Implications: The findings from this study may serve as a resource to academicians and administrators in developing and upholding academic standards protocols.

Pharmaceutical and Clinical Assessment of Over-the-Counter (OTC) Weight Loss Ultraceuticals. Mohamed I. Nounou, University of Saint Joseph, Nada Ahmed, Alexandria University, Alaa AbouElfetouh, Alexandria University, Amal Amal El-Kamel, Alexandria University. Objectives: The global market of nutraceuticals was $\$ 198.7$ billion, as it was forecasted that it would be $\$ 285$ billion by 2021, indicating the wide-spread of the problem of the claimed to be natural herbal products globally. In this project, we designed a strategy of a full evolutional system including; pharmaceutical, microbiological and prevalence evaluations of some of herbal slimming products. Method: The pharmaceutical evaluation included analyzing the samples via High Performance Liquid Chromatography (HPLC) and Near Infra-Red (NIR) to determine any possible adulterants. Additionally, physical properties were assessed via SeDeM analysis and pharmacopeial tests. Finally, microbial evaluation and the retrospective prevalence cross-sectional observational study had been conducted to assess the safety and efficacy of the investigated products. Results: The products were adulterated with sibutramine, phenolphthalein, orlistat and Sildenafil and contaminated with fungi, Escherichia coli and Salmonella. According to the reported results, the analyzed weight loss products were found to be heterogeneous in their adulterant drug content and pharmaceutical quality, and with poor microbial quality. Last, the observational survey showed that a high percentage of adult participants suffered from common side effects such as depression, diarrhea and hypertension. Those products represent a major safety threat to the consumers' health. Implications: The herbal slimming ultraceuticals that are claimed to be $100 \%$ herbal represent a major threat to the health of the consumers. The majority of surveyed subjects used the illegal slimming products with a misconception of their safety profile. Common side effects of illegal slimming products such as depression, diarrhea and hypertension had been reported.

pH-Sensitive Polymeric Nanoparticles Fabricated by Dispersion Polymerization for Cancer Therapy. Emmanuel O. Akala, Howard University. Objectives: Studies on $\mathrm{pH}$-responsive nanoparticles capable of rapid degradation in the mildly acidic environments similar to those found in the endosomes and lysosomes of tumor tissues but are more stable in the physiological $\mathrm{pH}(\mathrm{pH}$ 7.4). Method: Three $\mathrm{pH}$-sensitive acetal crosslinkers were synthesized and characterized by 1H NMR, 13C NMR, FT-IR and high resolution mass spectroscopy (HR-MS). The nanoparticles were fabricated by free-radical 


\section{American Journal of Pharmaceutical Education 2018; 82 (5) Article 7158.}

dispersion polymerization technique. Hydrolysis studies were carried out on the crosslinkers and blank nanoparticles, and drug release studies were done on docetaxel-loaded nanoparticles in acetate buffer $(\mathrm{pH}$ 5.0) and phosphate buffer saline (pH 7.4). Statisitical experimental design used was randomized complete block design followed by analyses of variance with F-test of significance. Pairwise comparison test was used to locate specific differences among parameters of the crosslinkers and the nanopaticles. Results: Scanning electron micrographs showed the formation of spherical particles. Particle size analysis showed that the nanoparticles are within nanosize range with negative zeta potential. The rate of hydrolysis was faster at $\mathrm{pH} 5.0$ compared to $\mathrm{pH}$ 7.4, which confirms the pH-responsiveness of the crosslinkers. Hydrolysis and drug release studies were dependent on the structure of the acetals: $\operatorname{Di}(2-$-methacryloyloxyethoxy)-[2,4,6-trimethoxyphenyl] methane crosslinker showed the fastest rate of hydrolysis, followed by di(2-methacryloyloxyethoxy)-[2,4-dimethoxyphenyl] methane

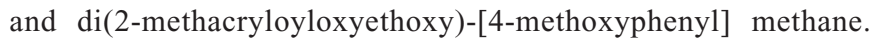
The docetaxel-loaded nanoparticles were internalized into cancer cells and were as effective as the free drug. Implications: The $\mathrm{pH}$-responsive nanoparticles are suitable for the delivery of bioactive agents and are expected to overcome the side effects related to toxicity of docetaxel and its commercial formulation.

Risk and Benefit Assessment of Expired Naloxone Products From First-Responders' Supplies. Justin J. Frey, Albany College of Pharmacy and Health Sciences, Schuyler Pruyn, Albany College of Pharmacy and Health Sciences, Benjamin Baker, Albany College of Pharmacy and Health Sciences, Michael R. Brodeur, Albany College of Pharmacy and Health Sciences, Carla Graichen, Albany Medical Center, Michael Dailey, Albany Medical Center, Hai-An Zheng, Albany College of Pharmacy and Health Sciences. Objectives: Naloxone is an opioid receptor antagonist that reverses opioid overdose, which can be life-threatening. Since 1970's, Naloxone products have been developed as injections, and more recently as nasal sprays. They have saved many lives at emergency settings, and have been routinely carried by first-responders including fire fighters, law enforcement officers and emergency medical services (EMS). While public safety supplies are monitored, community naloxone programs distribute naloxone to the public, who may have expired product on-hand in an emergency. Method: This study analyzed the quality and stability of expired Naloxone Solutions for injection, in order to assess their remaining efficacies and potential risks. The samples were collected from EMS or law enforcement supplies, with expiration dates from 1988 to 2018. Using UPLC-MS, the remaining Naloxone was quantified. The main degradation product Noroxymorphone and other possible species were systematically monitored and quantified. Results: All tested samples were found containing more than $90 \%$ of labeled Naloxone amount, including those stored over 20 years. The remaining Naloxone amounts is correlated with storage time. There is no significant degradation product was detected across all samples. Noroxymorphone was detected from some older samples, but all less than $1 \%$. Therefore, the risk caused by Noroxymorphone is low, although it is an opioid agonist. Implications: This exploratory risk and benefit assessment suggested that an expired Naloxone Solution for Injection may still be qualified by USP standards. Further pharmacology or clinical investigation may be conducted to confirm our assessment, especially for other naloxone dosage forms.

The Development and Assessment of a Point-of-Care Testing Laboratory Course in a Doctor of Pharmacy Program. Aladin A. Siddig, University of Charleston, Gannett Monk, University of Charleston, Ronaldo V. Ramirez, University of Charleston, Aymen
Shatnawi, University of Charleston, Tamer Fandy, University of Charleston, Dean Reardon, University of Charleston. Objectives: This innovative, required laboratory course introduces the concepts and processes associated with Point-of-Care Testing (POCT), and empowers students to use effective technology to aid their decision making at the "point-of-care" to improve patient care. Method: This required POCT laboratory course is taken by the first year PharmD students. The lab is structured into three major sections. An initial basic laboratory section introduces the student to key foundational concepts of physical assessment, and provides hands-on experience. The second section includes biologic sample collections, such as blood (Hepatitis C), oral swab (HIV), throat swab (Group A Strep) and nasal swab (influenza). A final section includes practical considerations such as risk management, development of POCT testing services, and testing limitations. Results: Assessment results and student evaluations from the Fall 2016 semester guided refinements to the course for the Fall of 2017. The mean course evaluation was 4.2 on a Likert scale anchored at Strongly Disagree (1) and Strongly Agree (5). In addition, the evaluation comments were positive, and will be used to improve the course for Fall 2018. Students demonstrated mastery of the material taught in both years, with $36 \%$ receiving an $\mathrm{A}$ in the course, $50 \%$ receiving a B, and $14 \%$ receiving a $C$. Implications: This course helps to prepare students for both the basic science and clinical portions of the curriculum, making this an invaluable tool to help the students see the connection between the two, and how each can help them make a positive impact on future patients.

Transdermal Delivery of Apomorphine Using Chitosan Nanoparticles and Microneedles. Ahmad Shennar, Jordan University of Science and Technology, Ahmed Alastal, Jordan University of Science and Technology, Abeer M. Al-Ghananeem, Jordan University of Science and Technology. Objectives: The aim of this study was to develop and evaluate Apomorphine-loaded chitosan nanoparticles. Furthermore, to investigate in vitro transdermal delivery of apomorphine through human skin using nanotechnology and microneedle technology. Method: Chitosan nanoparticles were prepared based on the ionotropic gelation of chitosan with tripolyphosphate (TPP) anions. Different concentrations of apomorphine in chitosan solution were examined to enhance drug encapsulation efficiency. In vitro release profile was determined by measuring the cumulative concentration using dialysis tubes. Franz cells were utilized to evaluate apomorphine formulations transdermal delivery in vitro using phosphate buffer, $\mathrm{pH}$ 6.7. Results: Apomorphine-loaded chitosan nanoparticles illustrated an average size of $297 \mathrm{~nm}$ with maximum encapsulation efficiency using $2 \%$ $\mathrm{w} / \mathrm{v}$ chitosan solution around $83 \%$. Accumulated release study revealed $60 \%$ of drug released within 24 hours. Transdermal delivery of apomorphine in vitro utilizing human skin illustrated enhancement in drug transdermal delivery when applying microneedle technology. Furthermore, nanotechnology enhanced apomorphine transdermal delivery but to a lesser extend when compared to microneedles, $\mathrm{P}<0.5$. Implications: The results in the present work suggest the feasibility of nanoparticles and microneedles technologies for the transdermal delivery of apomorphine. Microneedle rollers enhanced apomorphine delivery significantly compared to the nanotechnology.

\section{Theoretical Models}

Problem-Solving Skill Development for Solving Pharmaceutics Problems and for the Pharmacists' Patient Care Process. William C. Mobley, University of Florida, Robin M. Li, University of Florida. Objectives: The objectives of this innovation were to develop and implement a team-based, ill-structured problem-solving approach 


\section{American Journal of Pharmaceutical Education 2018; 82 (5) Article 7158.}

for achieving curricular goals of developing students' problemsolving skills, for achieving course-level objectives related to non-therapeutic and therapeutic problem-solving in a pharmaceutics course, and for developing skills used in the Pharmacists' Patient Care Process. Method: A problem-solving process was employed to train teams of 5-7 students to systematically solve ill-structured problems in four scaffolded problem-solving sessions in a pharmaceutics course. The process involved contributing factor identification, problem definition, SMART goal-setting, solution strategy selection and argumentation, and plan implementation and follow-up. The first session focused on a drug development problem and the next two sessions focused on patient case-based pharmaceutics problems. In the fourth session, the PPCP was applied to address patient case-based pharmaceutics problems. For all problem-solving exercises, an electronic form that provided just-in-time guidance, was completed and submitted for grading and feedback. Results: Teams successfully completed each step of the problem-solving process in each session. For the third, and least scaffolded session, the most common error made was inadequate contributing factor identification. For the subsequent PPCP-based session, contributing factor identification (in Collect) improved, and the most common deficit was with the Assess phase. Implications: Through the application of this problem-solving process, students developed team-based problem-solving skills that were successfully applied to solving ill-structured pharmaceutics problems and that are valuable for patient problem-solving using the Pharmacists' Patient Care Process.

\section{PHARMACY PRACTICE \\ Completed Research}

A Capstone Course Utilizing the Pharmacists' Patient Care Process (PPCP) to Develop Patient Care Skills. Beth Phillips, The University of Georgia, Christopher Bland, The University of Georgia, Andrea S. Newsome, The University of Georgia, Russell Palmer, The University of Georgia, Katie Smith, The University of Georgia, Stephanie Phan, The University of Georgia. Objectives: The 2016 Accreditation Standards place emphasis on producing practice ready graduates. This project aimed to design case-based instruction using the PPCP, identify how it can support third year pharmacy students in developing advanced patient work-up skills for future practice, and inform the revision of instruction for future re-implementation. Method: Cases were assigned weekly in a simulated electronic health record. A student template was developed for patient work-up following the PPCP. A focus group interview was conducted with students enrolled in the first course offering. The interview was conducted using a semi-structured interview guide, and a two phase analysis process using open and focused coding was employed to identify relevant themes in the data. Results: Students $(n=9)$ spent 3 to 4 hours weekly working up the cases. Based on student perceptions, three themes were essential in supporting students' development of advanced patient work-up skills: Skills were advanced by engaging in cases that were structured similarly to realworld practice and included authentic resources. A relatively small class size made discussion less intimidating than in the large student cohort, promoting engaging and productive class-sessions. Individual rather than group case preparation led to increased preparedness and more productive in-class discussion. Implications: Students perceived that newly designed case-based instruction using the PPCP enhanced their patient care skills. Future re-implementation of the course with a larger cohort of students will emphasize individual case preparation, student activity that aligns with real-world practice, and a class format that promotes engaging case discussions with large groups of students.
Accessibility and Coverage of Pharmacogenomic Tests by Private Health Insurance Companies. Sharon K. Park, Notre Dame of Maryland University, Jonathan Thigpen, Notre Dame of Maryland University, Insong J. Lee, Notre Dame of Maryland University. Objectives: Pharmacogenomic testing is implicated in the practice of personalized medicine but the extent of its accessibility and payment by insurance companies is largely unknown. The objective of this study was to assess the accessibility and coverage of clinically relevant pharmacogenomic tests by the top 41 private insurance companies in the United States. Method: Websites of insurance companies were searched for medical policies addressing 34 common and clinically relevant pharmacogenomic tests referenced by the Clinical Pharmacogenetics Implementation Consortium (CPIC), PharmGKB, and/or FDA product labeling. Accessibility was analyzed by the number of mouse clicks to locate medical polices. Policies were subsequently reviewed to determine coverage of pharmacogenomic tests by genedrug pair and insurance company. Results: A total of 223 medical policies mentioning pharmacogenomic tests were retrieved, representing 34 unique policy sets from 41 companies. Only 33 companies had their policies accessible on their website, with a moderate amount of effort required to retrieve them (average of 5 mouse clicks). Approximately $50 \%$ of gene-drug pairs were unanimously mentioned in all policies, but were only covered $<20 \%$ of the time. When mentioned in a policy, 7 gene-drug pairs were uniformly covered and 11 gene-drug pairs were uniformly not covered. Overall, insurance companies covered approximately $40 \%$ of gene-drug pairs mentioned in their polices. Implications: Medical policies addressing common pharmacogenomic tests were not readily accessible on websites of the top, private health insurance companies. Coverage determinations for common, clinically relevant pharmacogenomic tests varied by insurance company and gene-drug pair and remains suboptimal for patient care.

A Conversation Analysis of Web-Based and Face-to-Face Interprofessional Team Communication During a Standardized Patient Encounter. Kelly Lempicki, Midwestern University/Downers Grove, Christine Holland, Rosalind Franklin University of Medicine and Science, Marilyn Hanson, Rosalind Franklin University of Medicine and Science. Objectives: In a previous study comparing webbased (WB) and face-to-face (FTF) interprofessional team encounters with a standardized patient (SP), student teams' communication assessment scores were similar; however, differences in the teams' communication were noted by investigators. This conversation analysis was therefore conducted to further describe interprofessional student teams' communication during a WB and a FTF SP encounter. Method: Recordings of a WB and a FTF interprofessional team SP encounter were transcribed by a study investigator. These transcriptions were then reviewed for accuracy by the other two investigators. Recordings were subsequently analyzed using conversation analysis, focusing on observations related to turn-taking, alliances formed, and power relations developed. Results: The FTF team used gaze and head-turning to indicate turn-taking, while the WB team used pauses and smiles. In the WB encounter, there were 32 pauses, averaging 5.25 seconds in length. In the FTF encounter, there were 22 pauses, averaging 2.59 seconds in length. Alliances of the IP team with the SP formed in both the FTF and WB encounters, with the medical student gaining authority as the leader in the FTF encounter. In the WB encounter, all members seemed of equal authority. Minimal, if any, power struggles occurred between team members on either platform. Implications: Differences may exist in how interprofessional student teams communicate during a WB and a FTF SP encounter. Additional 


\section{American Journal of Pharmaceutical Education 2018; 82 (5) Article 7158.}

studies analyzing a larger number of encounters are warranted to further understand these implications prior to implementation of curriculum using web-based encounters, such as for telehealth.

Active Learning Video Assignment to Impact Pharmacy Students' Knowledge of Natural Products. Kelly M. Shields, Ohio Northern University, Amber Etzel, Ohio Northern University, Hannah Lamb, Ohio Northern University, Kayla Rieman, Ohio Northern University, Emma Talley, Ohio Northern University, Corey Thrush, Ohio Northern University, Yong Wang, Ohio Northern University. Objectives: To design and assess a small-group active-learning video project and its impact on student knowledge of specific natural products. Method: Students in a required course were provided with general information about some common natural products. Students were then divided into small groups and asked to create a 2 to 3 -minute video on selected specific products that were not covered in course material. Each group received a different topic (natural product/usage combination). Students were also expected to review two other groups' videos to gain exposure to additional topics. Results: Students completed a pre and post intervention assessment $(n=108)$ with knowledge based questions on topics that were not covered in class but were elements of student-created videos. The raw percentage of students obtaining the correct answer increased from baseline in each of the questions $(27.8 \%$ to $57.4 \%, 46.3 \%$ to $63.9 \%, 8.3 \%$ to $50 \%, 45.37 \%$ to $50 \%$ ). The change in correct responses was recoded to determine if students who created the video content did better on the question related to that content area. It appears that the improvement from baseline is not associated with the types of exposure (video creation, video viewing only or no video). The uneven distribution of students per treatment group likely impacted results. Implications: Small group, self-directed learning of a specific natural product did not appear to significantly impact the ability to answer a specific fact based question. It did however appear to overall improve general performance in questions about natural products.

Adapting Exam Wrappers to a Prescription Rubric in a OneSemester Skills Lab Course. Lauren M. Caldas, Virginia Commonwealth University, Abigale T. Matulewicz, Virginia Commonwealth University, Teresa M. Salgado, Virginia Commonwealth University, Jacob F. Ellerbrock, Virginia Commonwealth University, Laura M. Morgan, Virginia Commonwealth University. Objectives: Exam wrappers are a self-reflection tool used to increase student learning. Our aim was to apply the exam wrapper concept to prescription assessments in the first Foundations course and to assess the association between preparation time, preparation activities, student confidence, and the final grade. Method: After filling each prescription, students were graded as unsatisfactory (U), student pharmacist (SP) or pharmacist $(\mathrm{P})$ and then completed an exam wrapper. Equal variance two-sample t-tests were conducted to assess differences in the mean number of preparation time and proportion of time spent in each preparation activity for groups $\mathrm{U}$ and $\mathrm{SP} / \mathrm{P}$. A homogeneity test was performed to assess preparedness ('not at all/somewhat', 'adequately', 'very') across the two groups. Results: Of the 120 students, 81 (67.5\%) obtained a U, $26(21.7 \%)$ a SP, and $13(10.8 \%)$ a P. The mean (SD) number of preparation hours in group $\mathrm{U}$ was 1.94 (1.0) versus 2.08 (1.1) in group $\mathrm{SP} / \mathrm{P}$. This difference was not statistically significant $(p=0.4826)$ nor was the proportion of total study time allocated to each activity. Significant differences were found across levels of preparedness $(p=0.0002)$, with $96.7 \%$ students who reported being 'not at all/ somewhat' prepared scoring unsatisfactory, while $39.7 \%$ and $48.3 \%$ of those who reported being 'adequately' or 'very' prepared, respectively, scoring SP or P. Implications: Adapting exam wrappers to prescriptions encourages students' self-reflection on their preparation and performance. Performance does not seem associated with preparation time/activities, but rather with students' self-reflection of preparedness. Future studies should examine other factors associated with prescription errors.

Addition of Nutrition and Healthy Eating Skills-Based Learning in a Pharmacy Curriculum. Nicholas J. Messinger, University of Cincinnati, Bradley E. Hein, University of Cincinnati, Shauna M. Buring, University of Florida, Ana L. Hincapie, University of Cincinnati. Objectives: To assess the impact of adding a nutrition and healthy eating module in the pharmacotherapy and skills lab curriculum on pharmacy student knowledge, confidence, and perception of the pharmacist's role in nutrition counseling. Method: A 1-hour lecture on nutrition therapy was incorporated into the third-year pharmacotherapy course, followed by hands-on learning activities in the longitudinal skills lab course. Activities centered on patient cases and included assessment of energy balance, meal planning, carbohydrate counting, and insulin dosing. Patient teaching tools, such as food replicas and nutrition facts labels, were utilized. A 23-item knowledge and attitudinal survey was administered before and after completion of the module. Wilcoxon and McNemar tests were used to assess pre- and post-survey differences. Results: Ninety-three students completed the pre- and post-survey. Statistically significant differences were found in student confidence in ability to counsel healthy patients $(\mathrm{p}<0.001)$, patients with diabetes $(\mathrm{p}<0.001)$ and patients with obesity $(\mathrm{p}<0.001)$ on nutrition. At baseline, most students $(99 \%)$ agreed or strongly agreed that pharmacists should be competent in nutrition counseling and pharmacists can help patients manage chronic illnesses through nutrition counseling. These attitudes did not change after completion of the module. With exception of 4 items associated with dietary fat, more than $70 \%$ of students correctly answered the knowledge-based items on the post-survey. Ninety-two students (99\%) recommended the nutrition and healthy-eating module remain in the curriculum. Implications: Nutrition education can be incorporated into core pharmacy curricula to prepare students for current pharmacy practice, and the expectation to provide nutrition counseling.

Administration of an Integrated Examination and Its Utility in Outcomes Assessment and Curricular Quality Improvement. Siu-Fun Wong, Chapman University, Khaled A. Elsaid, Chapman University, Jason M. Yamaki, Chapman University, Rennolds Ostrom, Chapman University. Objectives: Integrated exam (IE) is a block examination that includes materials from multiple courses that is administered on a fixed schedule throughout each trimester. We herein describe the development and administration of IEs and highlight the optimal utilization of the outcome measure reports to: 1) provide student feedback; 2) deliver faculty feedback for course quality improvement; and 3) conduct continual curriculum improvements. Method: IEs are administered every 3 weeks, total of five exams per trimester using ExamSoft ${ }^{\mathrm{TM}}$. IEs consist of items from multiple courses in the trimester, simulating the random question sequence of the NAPLEX exam. Each IE item undergoes peer-review to ensure clarity and conformation to exam standards. IE items are coded to a maximum of 10 categories, which allow for performance analyses (cross-sectional and longitudinal) and identify gaps. Results: Quality parameters in a total of 55 IEs administered showed excellent reliability as demonstrated by mean Kuder-Richardson formula 20 (KR-20) more than 0.8 , mean biserial index of 0.22 , and mean post-examination item adjustment rate of $1.45 \%$. Students receive immediate feedback and strength reports, both cross-sectional and longitudinal. Faculty receive reports summarizing alignment of curriculum maps to 


\section{American Journal of Pharmaceutical Education 2018; 82 (5) Article 7158.}

assessments in ACPE Appendix I, institutional student learning outcomes, cognitive levels, and student performances. Correlative analysis with standardized exams and student GPA allows benchmark validation of IE as strong predictors to provide means for early intervention to improve student academic performances. Implications: IE is an effective assessment approach to provide robust feedback for individual and program improvement, and prepare students to take standardized exams.

A Hybrid Learning Approach for Tobacco Cessation Counseling Training. Karen S. Hudmon, Purdue University, Nicole M. Lodise, Albany College of Pharmacy and Health Sciences, Ingrid Allard, Albany Medical College, Gregory Vis, Hudson Simulation Services, Henry Pohl, Albany Medical College, Heather Frenz, Albany Medical College, Francis M. Vitale, Robin L. Corelli, University of California, San Francisco. Objectives: To implement and evaluate the impact of a hybrid learning approach to tobacco cessation training. This pilot study of pharmacy students is part of an NIH-funded curriculum development project among six sites nationally ( 2 pharmacy, 2 medicine, 2 nursing) prior to nationwide dissemination of the shared educational teaching and assessment tools. Method: To accompany the existing $\mathrm{Rx}$ for Change tobacco cessation training program (http://rxforchange. ucsf.edu), an interprofessional faculty team developed a series of six virtual patient simulations and six standardized patients/OSCE assessments. At Albany College of Pharmacy and Health Sciences, these were implemented in an elective course in combination with 15 hours of live training (including role playing with case scenarios). Students $(\mathrm{n}=17)$ completed a survey at baseline, after the live training, and after OSCE assessments with three standardized patients. Results: Overall, students achieved a $72.1 \%$ on the three virtual patients and addressed $75.7 \%$ of items in the OSCE checklists (averaging across all three scenarios). Self-efficacy scores increased significantly from baseline to post-OSCE ( 4.9 vs $8.1, \mathrm{p}<0.0001)$. All students agreed that (a) virtual patient exercises effectively prepared them for the tobacco OSCEs, (b) pharmacy students at other institutions would benefit from this OSCE experience, and (c) the OSCEs were a valuable tool to develop their skills for providing tobacco cessation counseling. Implications: A hybrid learning approach for tobacco cessation is associated with enhanced student confidence for cessation counseling. Sharing educational teaching and assessment tools across healthcare disciplines has the potential to increase interprofessional collaboration in the provision of tobacco cessation counseling.

An Activity to Improve Pharmacy Students' Ability to Care for Non-English-Speaking Patients Using a Translator. Jennifer G. Smith, The University of Louisiana at Monroe, Savannah K. Posey, The University of Louisiana at Monroe. Objectives: To evaluate an activity developed to provide students with hands-on practical experience working with a translator in a simulated patient interaction. Participants' perceptions of the activity will be collected to provide baseline information of feasibility and efficacy for implementation into the core curriculum to improve students' cultural competency. Method: Students completed 2 surveys: one prior to the activity and one immediately after activity completion. Graduate student volunteers who served as the "translator" and non-English speaking "patient" completed a single survey following the activity. Descriptive statistics were used to summarize the results. This study was approved by the institution's IRB. Results: Eight students and 4 volunteers completed the activity. Prior to the activity, students' average rating of confidence in providing care to a non-English speaking patient using a translator was $2.375(1=$ not at all confident and $5=$ very confident $)$. After the activity, the students' average rating increased to 3.625 .
Students felt that the activity improved their confidence and ability to work through a translator (average 4.625 , scale: $1=$ strongly disagree to $5=$ strongly agree). Student and volunteer feedback was very positive and highlighted the utility of this activity in preparation for clinical practice. Implications: Although the activity was developed and implemented in a small elective course focused on medical outreach in developing countries, the experience and knowledge gained will be beneficial throughout a pharmacist's career. The positive feedback and results support further development of a similar activity for use in the core curriculum.

Analysis of Self-Reported Scholarly Publications Among US Pharmacy Practice Faculty. Kacey K. Hight, University of Arkansas for Medical Sciences, Nalin Payakachat, University of Arkansas for Medical Sciences, Melanie A. Reinhardt, University of Arkansas for Medical Sciences, Amy M. Franks, University of Arkansas for Medical Sciences. Objectives: Data from 2006-2010 describe an average of 0.51 peer-reviewed publications annually among pharmacy practice (PHPR) faculty. However, these data may not reflect the productivity of current PHPR faculty. We sought to characterize self-reported scholarly publishing among U.S. PHPR faculty and identify characteristics associated with number of publications. Method: A 62-item online questionnaire was distributed to U.S. PHPR faculty via department chair emails. Peer-reviewed publication history (total and firstauthor publications) and participant demographic information were collected. Descriptive statistics were used to characterize participants, and t-tests and ANOVA were used for comparisons. Results: 238 participants were included with an average (SD) age of $41.7(10.5)$ years. Participants had 11.0(9.1) years faculty experience, with 4.8(4.6) years in current rank. The majority were female $(67.0 \%)$ and white $(81.4 \%)$. Academic ranks of assistant, associate, and full professor were well-represented $(37.0 \%, 37.0 \%$, and $21.3 \%)$. Participants reported averages of 2.0(2.1) total publications/year and 1.0(1.3) first-author publications/year. Total publications were significantly greater for tenured faculty compared to tenure-track (not yet tenured) and non-tenure-track (31.3(17.5) v. 18.0(16.2) v. 11.9(13.1), $\mathrm{p}<0.001)$ and those with $\mathrm{PhD}$ degree versus not $(28.9(18.1)$ v. 15.4 (15.7), $\mathrm{p}<0.001)$. Faculty from public universities had more total publications than those from private institutions (19.2(17.2) v. 14.1 $(15.5), p=0.036)$. Faculty with administrative appointments had more total publications than non-administrators $(24.8(18.5)$ v. 13.3(14.3), $\mathrm{p}<0.001)$. Publication rates for total and first-author publications did not differ among academic ranks. Implications: While data are selfreported from a faculty sample, they may indicate an increase in the average number of publications per year among PHPR faculty than previous estimates.

Analysis of Student Pharmacists' Errors When Simplifying Complex Medication Regimens. Clark Kebodeaux, University of Kentucky, Jamie Woodyard, Purdue University, Marlowe D. Kachlic, University of Illinois at Chicago, Sheila M. Allen, University of Illinois at Chicago, Aric Schadler, University of Kentucky, Scott M. Vouri, St. Louis College of Pharmacy. Objectives: Complex medication regimens with distinct dosing quantities and frequencies can lead to poor patient outcomes. We analyzed documented errors during a standardized laboratory exercise measuring student pharmacists' ability to organize a complex prescription regimen. Method: First year (P1) and third year (P3) student pharmacists at 3 US schools of pharmacy completed a required activity to organize a complex medication regimen. Students organized 7 fictitious medications with varying frequencies, dosing quantities, and instructions over a 24-hour period. Errors were categorized as a medication quantity or frequency error. 


\section{American Journal of Pharmaceutical Education 2018; 82 (5) Article 7158.}

Chi-square analyses compared performances between medications, students, and schools. Results: A total of 372 P1 and 459 P3 students $(99.9 \% \mathrm{RR} ; \mathrm{n}=831)$ consented across 3 schools. $25.2 \%$ of student pharmacists had an error when completing the activity (Mean 0.59 Errors; SD 1.27; Range 0 - 10). Medication instructions with 2 tablets compared to 1 tablet were significantly more likely to have quantity errors $(7.05 \%$ vs. $0.74 \%, p<0.001)$, and $>1 \mathrm{x} /$ day regimens were significantly more likely to have frequency errors than $1 \mathrm{x} /$ day regimens ( $1.01 \%$ vs. $0.24 \%, p=0.028)$. P1 students were more likely than P3 students to have frequency errors $(1.29 \%$ vs. $0.69 \%, \mathrm{p}<0.001)$ but not quantity errors $(2.65 \%$ vs. $2.76 \%, p=0.699)$. There were significant differences between schools for quantity errors (3.63\% vs. $2.01 \% \mathrm{vs}$. $2.41 \%, \mathrm{p}<0.001)$ and frequency errors $(0.91 \%$ vs. $0.49 \%$ vs. $1.50 \%$, $\mathrm{p}<0.001)$. Implications: New educational opportunities may be needed to help student pharmacists further improve organizing complex medication regimens. Similar simulations can potentially be implemented into practice to determine if patient outcomes can be improved.

An Analysis of P1 Student Reflections on the Impact of White Coat Ceremony Participation. Laurie L. Briceland, Albany College of Pharmacy and Health Sciences, Jeffrey M. Brewer, Albany College of Pharmacy and Health Sciences, Angela C. Dominelli, Albany College of Pharmacy and Health Sciences. Objectives: A formal White Coat Ceremony (WCC) is one means of welcoming students into the pharmacy profession. The impact of the WCC on students' professionalization has been sparsely documented. The objective of this study was to document the impact of WCC participation on student professionalization. Method: P1 students participated in the college's WCC at the conclusion of $\mathrm{P} 1$ Orientation program. Students were instructed on reflective practice, and assigned to reflect, in $<300$ words, on the impact of participation as the first graded assignment in their Foundations of Pharmacy (FoP) course. A grading rubric to discern critical reflection (cites future behavioral change); reflection; understanding; or non-reflection was developed for FoP to assess the reflections in a standardized fashion. Based on a word cloud generated from key reflection terms, the six most common reflection themes were extracted: Professionalism; Welcome to Pharmacy; Personal Achievement; Life-Long Learning; Patient Care; Code of Ethics. Authors then classified $<3$ themes per reflection to discern prevalence; "negative connotation" and "eye-opening experience" were also denoted. Results: 218 of 225 students in Class of 2020 submitted valid reflection assignments; of these, $92 \%$ met critical reflection criteria, $8 \%$ reflection criteria. Four percent offered negative reflection on WCC, while $75 \%$ expressed an eye-opening experience/realization. Of 483 thematic classifications, the most prevalent were Personal Achievement (26\%), Professionalism (21\%), and Welcome to Pharmacy (18\%). Implications: For the majority, the WCC is an eye-opening experience with positive impacts on several aspects of student professionalization, as evidenced by Critical reflection.

An Analysis of Self- Assessed Confidence Pre- and Post-APPE Rotation in the Ambulatory Care Setting. Tayla Rose, Northeastern University, Kaitlyn Gallant, Northeastern University, Tiffany Moy, Northeastern University, Danielle M. Miller, Northeastern University. Objectives: Ambulatory care pharmacists are essential members of the health care team who help improve patient outcomes by optimizing medication regimens, managing chronic disease states, and promoting health and wellness. As a required advanced pharmacy practice experience (APPE), an assessment of student's knowledge and skills related to the ambulatory care practice setting is crucial to prepare practice-ready pharmacists. We aim to determine pharmacy students' self-reported confidence in the ambulatory care setting before and after completion of their ambulatory care APPE. Method: Students assigned to two ambulatory care faculty members completed paper based pre- and post- APPE surveys self-assessing their confidence in the ambulatory care setting. Students were asked to complete the presurvey as part of routine practice to evaluate baseline knowledge of ambulatory care pharmacy and confidence in performing essential ambulatory skills. A post-survey was collected to evaluate progression. Results: Of 43 paper surveys administered, 25 pre-surveys $(58 \%)$ and 18 post- surveys (42\%) were completed. Pre-survey findings indicated that the majority of students $(>50 \%)$ strongly agreed that ambulatory care pharmacists conduct face-to-face visits and complete post-graduate training. Post-survey findings indicated an increase in confidence in: obtaining vital signs, performing point of care testing, discussing the role of an ambulatory care pharmacist, and explaining/ defining related terminology. Implications: The required ambulatory care APPE is essential in transitioning didactic knowledge and applying clinical skills in the practice setting. While some aspects of ambulatory care can be introduced in the classroom setting, application of knowledge and skills are best learned in practice.

An Elective Course in LGBT Health and Practice. Michael W. Jann, University of North Texas Health Science Center, Scott R. Penzak, University of North Texas Health Science Center, Annesha White, University of North Texas Health Science Center, Amulya Tatachar, University of North Texas Health Science Center. Objectives: To design, implement, and assess a Lesbian, Gay, Bisexual, and Transgender (LGBT) health and practice elective course for secondand third-year doctor of pharmacy (Pharm.D.) students. Method: A LGBT health and practice course was developed, focusing on health promotion, recognizing barriers to health care, disease prevention, and treatment throughout an LGBT person's lifespan. Course format included didactic topic discussions, reading assignments, in-class activities, peer teaching, small group presentations, an OSCE assessment with a transgender person, and participation from the LGBT community Results: Students were evaluated by the Course Learning Outcomes, class session participation, active-learning methods, and the Capstone Topic Presentation. Students exhibited significant improvement in the Course Learning Outcomes $(p<0.05)$. Student portfolios contained general themes of discrimination, health care access problems, advocacy, inclusive pharmacy environments, and desire to be a better practitioner. Students actively participated in each class session with the guest speakers from the LGBT community. Five quizzes were employed during the course that focused on course content and sexual terminology. The OSCE with a transgender person was well-received by the students. The Capstone Topic Presentations had a wide variety of topics including Pharmacists' Roles in Transgender Persons and LGBT in Islam. Implications: Students demonstrated knowledge of the different unique health care issues among the LGBT community. The availability of health care resources, barriers to treatment, and development of strategies for advocacy were among the most improved areas for the course learning outcomes. Student evaluations showed that the students viewed the elective course as a meaningful learning experience.

An Interprofessional Continuing Education Initiative: The Chicagoland Critical Care Conference (C4)*. Rosalyn P. Vellurattil, University of Illinois at Chicago, Jill R. Wilson, University of Illinois at Chicago, Scott T. Benken, University of Illinois at Chicago. Objectives: National meetings exist having few dedicated lectures intended for critical care pharmacists and nurses. C4 is a blend of critical care expertise in pharmacy and nursing delivered under the 


\section{American Journal of Pharmaceutical Education 2018; 82 (5) Article 7158.}

framework of systematic educational design by the Office of Continuing Education and Meeting Services. The objective is to describe the approach to interprofessional planning for a continuing education initiative, and share results and outcomes for quality improvement. Method: An interprofessional planning committee developed the conference agenda by analyzing needs and identifying speakers. The program chair and planning committee identified educational gaps and learning objectives to target. Content mapping was linked to clinical standards and competencies for pharmacists and nursing, and interprofessional competencies. Outcomes reporting included Moore's outcome levels (participation, satisfaction, learning and competence). Results: A total of 160 participants attended C4 $(n=92$ pharmacists, $\mathrm{n}=40$ nurses, $\mathrm{n}=2$ physicians). Ninety-three percent indicated overall satisfaction was good or very good. Ninety-four percent indicated their understanding of topics after the activity was good or very good. Pharmacists and nurses $(n=32)$ reported interest in optimizing and evaluating their quality improvement projects post-session. After attending, $84 \%$ of participants reported C4 will help improve patient outcomes, and $74 \%$ were very committed to enacting changes in their practice. Implications: C4 was effective in achieving its overall goals as evidenced by higher level outcome data. Opportunities for the future include integrating physicians into the target audience to enhance team learning. *This initiative was the winner of the Award for Excellence in Educational Design from ACEhp in January 2018.

A Novel Naloxone Training Compared to Current Recommended Training on Overdose Simulation Readiness and Results. Thomas S. Franko, Wilkes University, Danielle Distefano, Wilkes University, Lauren Lewis, Wilkes University. Objectives: The aim of this study is to determine if a novel training program with a focus on situational stress management yields better results than the currently recommended training on a simulated overdose response. Method: Students were randomized to receive either the state sanctioned training or a novel training developed by the Nesbitt School of Pharmacy. Each student individually completed a live simulated overdose response with an added stressor of a panicked bystander. A checklist was used to evaluate students during the simulation; results of which were compared. Results: The novel training group completed the simulation on average 23 seconds faster than the state group. The average grade for the novel training students was $92 \%$ compared to $69 \%$ for the state training students. Implications: Students who underwent the novel training completed the simulation in a more timely and appropriate manner than those that received the state training despite similar perceptions of readiness. Further research is needed to determine efficacy of this type of training on long term retention.

A Peer-Assisted Learning (PAL) Teaching Approach in Immunization Delivery Training. Eva Wong, Marshall B. Ketchum University, Monica Trivedi, Marshall B. Ketchum University. Objectives: To assess the impact of peer-assisted learning (PAL) teaching model on alleviating student pharmacists' apprehension of receiving and administering needle injections, and improving student pharmacists' confidence and skills with immunization delivery. Method: First-year (P1) student pharmacists $(n=53)$ and second-year (P2) student pharmacists $(n=40)$ participated in the immunization technique training activity. P1 and P2 students were assigned in 1:1 and 1:2 groups. P2 students who successfully completed certification the previous year shared their experiences and created a comfortable learning environment to practice immunization technique. Faculty trainers were available to answer questions. Assessment measures include a peer evaluation rubric, technique assessment rubrics, and student reflections. Results: 53 of the $53 \mathrm{P} 1$ students demonstrated competency in intramuscular and subcutaneous injections verified by $\mathrm{P} 2$ mentors and faculty trainers. Both P1 and P2 student cohorts gave the respective mentee/mentor full scores, which validated the P1-P2 teams shared a connected mentorship experience and developed collegiality in a shared effort to learn/teach immunization technique. Student reflections from both the P1 and P2 cohort support the effectiveness of peer-assisted learning, and the positive experience of peer mentorship. Implications: There is a paucity of current literature focused on the concept of peer-assisted learning in pharmacy and health professions education. This teaching approach explored the benefits of gaining knowledge and skills from peers. Receiving and administering needle injections can be a daunting experience, and peer-assisted learning demonstrated effectiveness in relieving student pharmacists' fears and improving confidence in immunization delivery.

A Pilot Activity of Providing an Academic APPE Elective Via Videoconferencing. Lea S. Eiland, Auburn University, April Staton, Auburn University, Lynn Stevenson, Auburn University. Objectives: To describe an academic advanced pharmacy practice experience (APPE) provided via videoconference between off-campus faculty and students and to assess students' experiences and learning outcomes. Method: A 5-week APPE was developed using backwards design and delivered via videoconferencing with the preceptor and students physically located in different experiential regions. Students participated in teaching, scholarship/research and service activities. After the APPE, students completed a survey comparing their perspectives and learning outcomes pre- and post-APPE and provided qualitative feedback in addition to the standard APPE feedback evaluation. The preceptor's and experiential administration's perspectives were documented. Results: Six students completed this APPE over three academic years. Students spent approximately five hours per day videoconferencing with the preceptor in various activities. All students stated there was 'no hindrance' with this rotation being conducted via videoconference or with the geographical distance between preceptor and students. Student ratings of nine learning outcomes increased after completing the APPE, demonstrating improvement in students' perception of knowledge in all topic areas $(p<0.05)$. After the APPE, five students $(83 \%)$ stated their interest in a full-time academic career increased; one student's interest remained the same. Students, the preceptor, and experiential administration deemed this videoconferenced academic APPE effective and successful. Implications: This experience affords students, without regard for physical location, the opportunity to pursue personal interests in academia, adds to the variety of APPEs and allows students to learn about academic pharmacy careers, which is an ongoing area of need in our profession. Potential challenges include occasional technology connectivity issues and limited in-person interactions.

A Pilot Modular Experiential Education Program for First-Year Health Professions Students to Develop Interprofessional Competencies. Therese M. O'Neil-Pirozzi, Northeastern University, Jamie L. Musler, Northeastern University, Mary Carney, Northeastern University, Leslie Day, Northeastern University, Pauline C. Hamel, Northeastern University, Jennifer Kirwin, Northeastern University. Objectives: A continuum of interprofessional education (IPE) is important to develop skills needed for collaborative practice. This project developed, implemented, and evaluated an IPE module introducing Interprofessional (IP) Communication and Teams and Teamwork IPE competencies to first-year undergraduate health professions students. Method: The module included an online introduction to IPE, structured in-class IP activities and discussion, CPR training emphasizing IP competencies, and an IP simulation with reflective debriefing. One group $(\mathrm{N}=52)$ participated in the module, while the other $(\mathrm{N}=75)$ did 


\section{American Journal of Pharmaceutical Education 2018; 82 (5) Article 7158.}

not, serving as a control for outcome comparisons. Impact of the IPE module on students' self-efficacy and confidence was evaluated before and after using the Self-Efficacy for Interprofessional Experiential Learning (SEIEL) scale and the Interprofessional Collaborative Competencies Attainment Survey (ICCAS), respectively. Results: IPE self-efficacy and confidence were both relatively high at baseline, with most respondents indicating moderate-to-strong agreement with all SEIEL statements and moderate-to-high levels of confidence with their ability to carry out IP duties on ICCAS. Compared to the presurvey, students who completed the module showed greater levels of agreement with self-efficacy statements on all questions and increased levels of confidence on the assessment. In the non-module group, students' responses on the ICCAS were similar or decreased compared to the pre-survey. Implications: An experiential IPE module may positively impact first-year health professions students' development of skills and knowledge needed for interprofessional practice. Early IP experiential learning activities may produce positive results on the development of undergraduate IPE competencies.

Are Pharmacy Schools Adequately Preparing Graduates for Roles in Industry? Tina Kanmaz, St. John's University, Hilary D. Mandler, University of the Sciences, Sam Rasty, Suzanne Soliman, Touro College of Pharmacy-New York. Objectives: (1) Determine the background characteristics of pharmacists entering the pharmaceutical industry [specifically medical affairs (MA)]; (2) Identify gaps in pharmacy education for pharmacists entering industry; (3) Outline factors contributing to pharmacists pursuing a career in pharmaceutical industry/MA. Method: A 10-question survey was distributed via SurveyMonkey to 400 pharmacists working in pharmaceutical industry. Names/email addresses were randomly selected from LinkedIn`c. Demographic questions and Likert-scale questions were utilized. The study received IRB approval. Results: A total of 66 (16.5\%) pharmacists practicing in pharmaceutical industry responded. Nearly $76 \%$ graduated from pharmacy school between 2010-2017. Approximately $73 \%$ disagreed/strongly disagreed that their exposure to pharmaceutical industry careers was adequate during their pharmacy education. More than half of respondents $(54.5 \%)$ completed residency $(15 \%)$ or fellowship (39.5\%) programs. Seventy nine percent of fellowshiptrained versus $33 \%$ of residency-trained respondents found their training valuable for a career in pharma. If available, $79 \%$ would have enrolled in a course on pharmaceutical industry as a student and $64 \%$ would have entered a board certification program as a student to help pursue a career in pharma. Implications: The majority of pharmacists surveyed indicated that pharmacy school did not offer adequate or sufficient training and education for a career in industry. The majority agree that fellowships are valuable for a career in pharma but less than half completed one. The majority of respondents would have enrolled in a board certification program in medical affairs or educational program on the pharmaceutical industry.

Are Students Practicing Self-Awareness? An Inventory of Critical Reflection Throughout the Curriculum/Co-Curriculum. Aimee F. Strang, Albany College of Pharmacy and Health Sciences, Laurie L. Briceland, Albany College of Pharmacy and Health Sciences, Gina Garrison, Albany College of Pharmacy and Health Sciences, Teresa H. Kane, Albany College of Pharmacy and Health Sciences, Jeffrey M. Brewer, Albany College of Pharmacy and Health Sciences, See-Won Seo, Albany College of Pharmacy and Health Sciences. Objectives: To assess Self-Awareness in the required curriculum and examine how existing assignments contribute to a meaningful, intentional thread. Method: An inventory of existing assignments was taken for the required didactic, experiential, and co-curriculum. Faculty described assignments where students reflected on an activity, analyzed participation, and summarized applicability toward future behaviors. Assignment instructions, type (required/bonus/remedial), frequency, grading approach, and feedback mechanisms were collected. A gap analysis was performed using a curricular design framework developed to assess the existing curricular thread. Results: Eleven assignments were identified in the required curriculum ( 7 required, 2 bonus, 2 remedial) with most occurring multiple times. A typical ACPHS student will complete at least 40 reflections upon graduation $(\mathrm{P} 1=12, \mathrm{P} 2=10, \mathrm{P} 3=11, \mathrm{P} 4=7)$, with bonus assignments, elective course activities, and course remediation allowing for more. Reflection topics include P1 orientation, white coat ceremony, habits of mind, exam or assignment performance, experiential learning, and co-curricular domains; remediation reflections involve professionalism and IPPE/APPE performance. Grading rubrics were used in 6 of 11 assignments $(54.5 \%)$. While most reflection assignments are evaluated for completion, faculty feedback on student reflections is atypical. The gap analysis revealed need for further development to: (1) more clearly define outcomes for self-awareness, (2) document curricular organization and structure for critical reflection (3) collect and analyze student achievement data on a more consistent, prospective basis to inform quality improvement. Implications: SelfAwareness is well developed in the ACPHS required curriculum and students meet the outcome through reflection assignments. The gap analysis revealed areas for quality improvement of the Self-Awareness curriculum.

Assessing Pharmacy Student Knowledge of Kratom: The FDA's Newest Opioid. Jessica Gardea, University of North Texas Health Science Center, Annesha White, University of North Texas Health Science Center, Ayyappa Chaturvedula, University of North Texas Health Science Center, Russell Coyle, Scott R. Penzak, University of North Texas Health Science Center. Objectives: To assess pharmacy student knowledge of kratom, a legal botanical substance recently designated as an opioid by the FDA. Kratom has gained popularity in the United States for the self-treatment of opioid addiction and as a substance of abuse. Method: An online survey was used to assess P1-P4 pharmacy student knowledge of kratom including medical uses, knowledge source, and clinical effects using Likertscale and yes/no questions. Data were analyzed via Chi Square analysis. $\mathrm{P}<0.05$ was accepted as statistically significant. Demographic information was also collected. Results: The survey was completed by 143 pharmacy students; $85 \%$ of whom rated themselves as "not at all knowledgeable or not very knowledgeable" of kratom. In contrast, $80 \%$ of P3 students reported having heard of kratom, which was significantly greater than P1 (5\%), P2 (5\%) and P4 (6\%) students. However, only $30 \%$ of $\mathrm{P} 3$ students rated themselves as "knowledgeable or very knowledgeable" about kratom. P3 students were significantly more likely to have heard of kratom via pharmacy school curriculum versus P1, P2 and P4 students whose knowledge came from other sources such as lay publications. Although kratom is indigenous to Southeast Asia, of the six students who grew up in that region, five had not heard of kratom. Implications: Despite lay press popularity, and a failed DEA ban, most pharmacy students had never heard of kratom. Kratom education represents an important teaching opportunity in pharmacy school curriculums. Future studies will assess kratom knowledge in health care students and professionals beyond our university.

Assessing Student Learning of Leadership and Teamwork Skills Via a Community-Sponsored, Team-Based Management Project. Kyle Turner, The University of Utah, Craig P. Henchey, The University of Utah. Objectives: Assess the impact of a leadership and management course with a real-world management project on PharmD 


\section{American Journal of Pharmaceutical Education 2018; 82 (5) Article 7158.}

students' leadership and teamwork skills. Method: In a leadership and management course, third year pharmacy students were divided into groups of five to six to complete a project proposed by local pharmacists as a "lab" for students to practice leadership and teamwork skills. A survey of the students assessed the perceived importance of the course's principles for effective teams and of knowing their Gallup StrengthsFinder results. Teamwork effectiveness was assessed using a previously validated Team Performance Survey (TPS). Project sponsors were surveyed to assess the quality of the students' work, likelihood of implementation, benefit of participation, and willingness to sponsor future projects. Results: All 58 students and all 10 sponsors completed the assessments. Greater than $75 \%$ of students rated eight out of the nine course's teamwork principles as "extremely" or "very important" for team effectiveness. The majority of students commented that knowing their strengths aided dividing up team roles. More than $85 \%$ of students stated that all 18 activities of effective teams in the TPS were performed either "every time" or "almost every time". All project sponsors were willing to participate again, and the majority listed interacting with students and increasing their connection to the College as benefits. Eight of the ten sponsors said they were "extremely" or "somewhat likely" to implement the student project. Implications: This course with a management project can serve as a model for developing students' leadership and teamwork skills within pharmacy curricula.

Assessing Student Pharmacist Performance of Rapid Strep, HIV, and Influenza Point-of-Care Testing. Kimberly C. McKeirnan, Washington State University, Jennifer C. Czapinski, Washington State University, Taylor G. Bertsch, Washington State University, Christina R. Buchman, Washington State University, Julie Akers, Washington State University. Objectives: The goals of this study were to implement point-of-care testing training for student pharmacists and to evaluate student performance on performing a point-of-care streptococcus pharyngitis and influenza swab. Method: Student pharmacists were trained to perform point-of care testing for streptococcus pharyngitis and influenza. A posterior pharynx and tonsil swab was utilized for streptococcus pharyngitis testing and a nasal swab was utilized for influenza testing. Students were then assessed on technique performing these two swabs on a partner while observed by a faculty evaluator. If students did not successfully complete the swab on the first attempt they were provided coaching and then repeated the swab. The Office of Research Assurances reviewed this protocol and found the project to be exempt from the need for IRB review. Results: One-hundred sixty student pharmacists across two campuses completed the point-of-care training. The majority $(86 \%)$ of student pharmacists performed the posterior pharynx swab correctly on the first attempt while some students required a second attempt (11\%) or a third attempt (3\%). Results were similar for the nasal swab with $88 \%$ of students demonstrating correct technique on the first attempt. A second attempt was required to correctly perform the test for some students (12\%). Implications: As point-of-care testing for influenza and streptococcus pharyngitis becomes more wide-spread in pharmacy practice, utilizing student pharmacists to reduce workflow burden may soon be a way to increase the number of patients treated for these illnesses. Student pharmacists can be successfully trained to perform point-of-care test swabs.

Assessing the Impact of an Interactive Learning Activity on the Teaching of Aliquots. Daniel J. Hansen, South Dakota State University, Surachat Ngorsuraches, South Dakota State University. Objectives: Evaluate the impact of an interactive learning activity on the teaching of aliquots Method: In Fall 2017, a faculty member incorporated an interactive learning activity (making of snack mix) centered around aliquots in to a first semester pharmacy practice course. Prior to coming to lecture, students were asked to read a chapter in the required textbook. The lecture period started with a short introduction of aliquots, which included an overview of the information in the textbook. Then, supplies were distributed and students worked to compound a snack mix that contained the medication prescribed. Finally, the lecture ended with a review of the aliquot process, an evaluation of accuracy, and some practice problems. A self-administered questionnaire survey with a 5-point Likert scale was developed to examine student perceptions before and after the interactive learning activity. In addition, the pre-assessment asked students to identify whether or not they completed the assigned reading prior to coming to class. Paired-samples t-tests were used to analyze the perception data. Results: Eighty P1 students took part in this project. These students showed significantly higher positive perceptions (p-values $<$ 0.05 ) in being able to define what an aliquot is (3.04 to 4.46), how comfortable they felt in completing the aliquot process (2.38 to 4.13) and the clinical application of aliquots (3.48 to 4.68). In addition, on average, students said that they completed parts of the assigned reading prior to coming to class. Implications: The interactive learning activity positively impacted the students' understanding of aliquots, the aliquot process, and the clinical application of aliquots.

Assessment of a Competitive Study Website to Enhance Student Pharmacists' Study Time. Patricia L. Darbishire, Purdue University, Ming Yang, Purdue University, Lindsey N. Eble, Purdue University. Objectives: To develop, pilot and assess perceptions of a competitive study website designed to help first professional year pharmacy students learn and retain medication information. Method: This IRB-approved project called "Study in a Flash" was a collaboration between pharmacy, engineering, and computer science students. The website was piloted in a first year, experiential pharmacy course at Purdue University. The voluntary participants provided information on their current study habits in the course, created an account on the website, played at least one round of quizzes, and gave feedback on their experience through a series of multiple choice, free response, and 5-point Likert-like questions. Results: Seventy-four students who were enrolled in the experiential course participated in the pilot and completed the survey. In regard to current study habits, $66 \%$ of students revealed they studied 2 hours or less per week learning the medication-related material prior to using the website and $41 \%$ expected to retain $60 \%$ or less of the material. In regard to feedback on the study tool, $70 \%$ found the website fun and engaging and $69 \%$ were interested in using it as a study tool. Aspects of the website users liked most included ease of use, the question format, and the concept/ competitive aspect of the tool. Implications: This pilot program demonstrated that further investigation of the use a competitive study tool for this course may be warranted. Further research will demonstrate if this study tool actually increases study time, and results in increased knowledge and retention of commonly used medications.

Assessment of a Joint Pharmacy and Theater Led Standardized Patient Training Program. Sarah Lynch, Binghamton University, The State University of New York, William Sticht, KarenBeth $\mathrm{H}$. Bohan, Binghamton University, The State University of New York. Objectives: Standardized patients (SPs) are used in healthcare education to deliver consistent scenarios. Often multiple SPs are required to meet needs within a skills lab session but there is little published literature regarding assessment of the SPs' performance to assure uniformity. This study sought to analyze accuracy and consistency in SPs' verbal and nonverbal portrayal of patients in a first-year pharmacy skills lab. Method: Undergraduate non-pharmacy students were 


\section{American Journal of Pharmaceutical Education 2018; 82 (5) Article 7158.}

trained as SPs by instructors from the pharmacy and theater departments and employed in a first-year pharmacy lab. SPs were surveyed about their perspectives after the training. Pharmacy instructors reviewed videos of the SPs' performances. A technical skills checklist of verbal and nonverbal acting was used to determine adherence to the scripted patient case and instructions. Variance in intra- and inter-SP performance was analyzed. Results: According to the survey, most SPs strongly agreed or agreed the training improved their verbal (72.8\%) and nonverbal (72.8\%) communication skills. SPs gave the correct scripted answers $98.5 \%$ of the time. Their individual performances were consistent $86 \%$ of time across their assigned students, but there were major variances in nonverbal affect between SPs. Implications: Student achievement in skills training may depend on the consistency of the SP's delivery. Our assessment of SPs indicated the joint theater-pharmacy training program developed accurate and consistent verbal performances. However, there was high inter-SP variance in nonverbal affect, indicating the need for further training in this area. Individual coaching and additional training is planned. Other healthcare programs can use these methods to assess their SPs' performances.

Assessment of Student Compounding Knowledge in a One Semester Skills Lab Course. Abigale T. Matulewicz, Virginia Commonwealth University, Lauren M. Caldas, Virginia Commonwealth University, Fawaz Alotaibi, Virginia Commonwealth University, Giulia Barlow, Carolinas Medical Center, Laura M. Morgan, Virginia Commonwealth University. Objectives: With pharmacists' expanding clinical role taking a priority in many curricula, the methods of educating our future pharmacists in the skill of compounding have been less defined. The goal of this study was to demonstrate the effect of a stand-alone non-sterile compounding course on student knowledge and assess development of student confidence in compounding knowledge and skills. Method: First year Doctor of Pharmacy students were given a pre- and post- assessment as part of a required, stand-alone 15-week compounding course. The assessment included questions in three areas: legal requirements, calculations, and application with knowledge-based questions and a confidence question for each area. Descriptive statistics and McNemar's test were used for data analysis. Results: A total of 122/122 (100\% response rate) students completed the pre- and post-assessments. All three areas showed an improvement in student performance, with an $18 \%$ increase in correct responses for legal and application based questions and 52\% increase for calculation based questions. There was also a significant improvement in reported confidence, with a $61 \%$ increase in application-based confidence and a $75 \%$ increase in legal knowledge confidence. Implications: A standalone, single semester, compounding focused laboratory course was successful in increasing first-year students' knowledge in legal, calculation, and application-based concepts, as well as perceived selfconfidence in independent compounding knowledge and skills.

Assessment of Two Delivery Methods of a Teaching and Learning Curriculum (TLC) as a Global Partnership. Darin C. Ramsey, Butler University, Tracy Sprunger, Butler University, Amy H. Sheehan, Purdue University, Alex N. Isaacs, Purdue University, Robert D. Beckett, Manchester University, Norman Fenn, Purdue University, Jasmine D. Gonzalvo, Purdue University. Objectives: The Indiana Pharmacy Teaching Certificate (IPTeC) program offered a live, twoday conference to pharmacy residents, fellows, and preceptors of Indiana, while simultaneously live-streaming to participants in Doha, Qatar. The objective is to compare conference evaluation results from the live conference to those that participated internationally via live-stream. Method: The program was simultaneously live-streamed to the Hamad Medical Corporation in Doha, Qatar. International participants were able to view the live conference in real time and able to utilize the chat feature to ask questions of the presenters. Participants were asked to complete an evaluation to assess participant satisfaction and to identify differences between the Qatar and Indianapolis attendees using a nine item questionnaire with a 5-point Likert scale. Results: Twelve participants from the Qatar cohort and 67 participants from the Indianapolis cohort completed program evaluation (overall response rate $=80 \%$ ). All responders agreed the program was scientifically sound and free from bias or commercial interest. The most common responses (Qatar vs. Indianapolis) were "improve current practices" (83.3\% vs. $89.6 \%, \mathrm{p}=0.62)$, "provided new ideas or information I expect to use" $(50.0 \%$ vs. $65.7 \%, \mathrm{p}=0.30)$, "enhanced my current knowledge base" (33.3\% vs. $52.2 \%, \mathrm{p}=0.35)$, and "reinforced my current practice" $(25.0 \%$ vs. $38.8 \%, p=0.52)$. Implications: The findings of this study demonstrate a successful collaboration for a live teaching and learning curriculum administered to pharmacists in two countries. With the global expansion of pharmacy residency programs, distance education expands the opportunities for pharmacist involvement in a teaching and learning curriculum.

Association Between Pharmacy Students' Implicit Bias Scores, Explicit Bias Scores, and Responses to Clinical Cases. Jennifer A. Santee, University of Missouri-Kansas City, An-Lin Cheng, University of Missouri-Kansas City, Kylie N. Barnes, University of Missouri-Kansas City, Mark T. Sawkin, University of MissouriKansas City. Objectives: To summarize implicit and explicit bias of pharmacy students in a professional communication course and to identify if there is an association between student biases and responses to clinical scenarios. Method: Students were presented with two cases, including a picture of a White or Black patient for each case, and asked to rate the patient's pain in one, and the reliability of the patient's family in the other. Students then completed the Implicit Association Test (IAT). Students were also asked three questions to assess explicit bias which included their preference for Whites or Blacks, and a ranking of how warm they felt towards Blacks and Whites respectively. Spearman's rho was used to determine the association between implicit bias and response to cases and between explicit bias and response to cases. Results: Data was generated from spring 2017. Of 81 students completing the study, $85 \%$ demonstrated implicit bias scores favoring Whites. The majority of students did not express explicit bias. Correlations were not seen between the explicit bias score and responses to cases ( $p=0.579$ for pain, $p=0.074$ for reliability) or IAT scores and responses to cases ( $p=0.986$ for pain, $p=0.507$ for reliability). Implications: Whether pharmacy student IAT scores correlate with their clinical decisions remains to be seen. Further analysis is warranted to investigate the association between IAT scores and clinical decision making. Schools and colleges of pharmacy should still address bias, as implicit bias may exist amongst some pharmacy students.

A Survey of Post-Graduate Trainees and APPE Students on Dosing Knowledge Requirements in PharmD Programs. Deepali Dixit, Rutgers, The State University of New Jersey. Objectives: To examine perspectives of recent post-graduates in training and advanced pharmacy practice experience (APPE) students regarding their experiences with the extent of exposure and responsibility for required dosing knowledge for commonly used medications. Method: A survey was administered to recent pharmacy graduates currently completing a post-doctoral training program (residency or fellowship) and APPE students. The survey population included participants from the "Teaching and Learning" certificate program, the Pharmaceutical Industry Fellowship professional development program and APPE 


\section{American Journal of Pharmaceutical Education 2018; 82 (5) Article 7158.}

students. The participants were asked questions related to dosing regimens taught in therapeutic classes, tested, and held responsible for dosing knowledge during APPEs. A qualitative analysis was performed. Results: 217 surveys were returned, 76 post-graduate trainees and 141 APPE students, representing 31 pharmacy schools. Majority of participants reported although dosing regimens were taught in therapeutic classes, yet, were not tested and were held responsible during APPEs. $84 \%$ agreed that learning common dosing regimens would have been beneficial for rotations and practice. $88 \%$ agreed that as future preceptor, students should be held responsible for knowing dosages of common medications during their education and $78 \%$ reported moderate to extensive dosing knowledge was necessary for pursuing any career path in pharmacy. Despite being taught in class, less than 50\% reported being held responsible for dosing in cardiology/ pulmonology, oncology or endocrine therapeutic areas on examination. Implications: This survey demonstrated that requirements for dosing knowledge in general are not standardized in the PharmD curriculum. Re-evaluation of dosing knowledge requirements in therapeutic areas is warranted.

A Web-Based Review Game as a Measure of Overall Course Knowledge in Pharmacotherapeutics. Kamila A. Dell, University of South Florida, Melissa Chudow, University of South Florida. Objectives: To determine if the scores on a comprehensive web-based review game are representative of overall Pharmacotherapeutics course and course series grades. Method: Two cohorts of students in Pharmacotherapeutics IV, the last course in a four course series, were administered a web-based quiz review game (Kahoot!) at the end of the course. Student performance on the game was compared to grades throughout the Pharmacotherapeutics course sequence to determine if the games are reflective of the students' performance in the Pharmacotherapeutic sequence. All students enrolled in Pharmacotherapeutics IV in 2016 and 2017 were included in the analysis. Pearson correlation was performed on the scores from the review game, conglomerate and segregated by the course the content was covered in, were compared to the grades in Pharmacotherapeutics series courses. The comparisons were preformed using a 2-tailed test with a significance level set at an alpha of 0.05 . Results: A total of 197 students, 111 in the 2016 cohort and 86 in the 2017 cohort, were included in the analysis. The correlation coefficient (r) for the review scores and Pharmacotherapeutics IV course grade was 0.399 and 0.461 for the 2 cohorts $(\mathrm{p}<0.001)$. All component comparisons between the review scores and the other Pharmacotherapeutics course grades were also significantly correlated $(\mathrm{p}<0.01)$. The R squared values were between 0.1 and 0.2 for the comparisons. Implications: Student performance on the review game significantly correlates with Pharmacotherapeutics course grades. Review games are fun tools for determining student understanding, progression, and knowledge and may be used as a formative assessment technique.

Collaborative Use of a Mock-Trial as a Learning and Assessment Activity in Two Pharmacy Programs. Ettie Rosenberg, West Coast University, Hoai-An Truong, University of Maryland Eastern Shore, Shih-Ying (Audrey) Hsu, West Coast University, Reza Taheri, West Coast University. Objectives: To compare results of a mock-trial as a learning and assessment activity in a first-professional year (P1) required course at two pharmacy programs. Method: After two iterations in a P1 course at one institution, a semester-long mock-trial project was expanded to another institution. Faculty from both institutions collaborated on topic selection, standardization of procedures, and assessment tools. Each cohort of students was divided into teams that researched, prepared, and debated "for" and "against" two distinct controversial topics in courtroom format. Descriptive analyses were conducted for faculty-judge scores, student-juror scores, student-peer evaluations, and overall student evaluations of the mock-trial experience. An Institutional Review Board approval was obtained. Results: Sixty P1 students from one institution and 57 P1 students from another debated two controversial topics in their respective cohorts. Evaluations indicated students performed well with overall mean scores by judges and jurors above $90 \%$ of the total score. Scores from both institutions were comparable; mean judge and juror scores and peer evaluations all showed a point difference less than 0.5 . Student feedback surveys administered at one institution revealed positive results where $89.3 \%$ of students agreed the mock-trial provided opportunity to demonstrate responsibility and accountability toward achieving team goals; and $89.5 \%$ agreed the mock-trial assessed ability to demonstrate professional attitudes and behaviors. Implications: A previously reported mock-trial shown to serve as an effective mechanism for selfdirected learning at one institution was successfully implemented at another. Results demonstrate the mock-trial as learning and assessment strategy could be generalizable to other programs.

College of Psychiatric and Neurologic Pharmacists Students Educate Peers in a First-Year Pharmacy Wellness Course. Lindsey Peters, Ohio Northern University, Amy M. Fanous, Ohio Northern University. Objectives: During the first year at Ohio Northern University, a direct-entry pharmacy program, pharmacy students are required to take Lifetime Wellness for Pharmacy Professionals. A component of this wellness course is a discussion of common mental health conditions. An innovative approach was utilized incorporating various teaching methodologies including: layered learning, TED-like talks, and personal stories or experiences with mental illness. Method: Nine CPNP student pharmacists volunteered and were selected to participate in educating first year students. Each group self-selected a mental health condition in which they had personal experience. Groups researched, created, and presented a TED-style talk using a combination of PowerPoint, pictures, videos, and storytelling. After the lecture, a survey was provided to the first year students. Results: A total of 113 surveys $(64 \%)$ were completed. Using a likert scale $(1=$ strongly disagree, $5=$ strongly agree), students indicated the content of the lecture was delivered effectively (4.7) and the delivery was engaging (4.5). First year students would like future content delivered by older pharmacy students (4.6) and through first-hand accounts (4.6). Students identified they felt more knowledgeable about mental health conditions (4.6), are more likely to seek help for potential mental health conditions (4.6), and know where to seek help (4.8) after the lecture. First year students found this teaching approach worthwhile (4.8). Implications: A multi-faceted, innovative teaching approach was used during a first year pharmacy wellness course. Results support future use of this teaching style.

Comparing Student Interventions During Medicine Advanced Pharmacy Practice Experiences (APPE) in High-Income Versus Low-Income Settings. Rakhi Karwa, Purdue University, Monica L. Miller, Purdue University, Ellen M. Schellhase, Purdue University, Imran Manji, Shelby Wilkinson, Purdue University. Objectives: Compare and contrast the number and type of interventions made by student pharmacists who participated in an adult internal medicine APPE in the US to an internal medicine APPE in Kenya. Method: A US-based faculty-led, four-week internal medicine APPE with interprofessional team rounding that provides care for underserved patients in an urban setting and hosts 16 students annually was compared to a faculty-led, eight-week internal medicine APPE in Kenya that hosts on average 24 students annually. Starting May 2017 - January 


\section{American Journal of Pharmaceutical Education 2018; 82 (5) Article 7158.}

2018 a standard clinical intervention collection form was used by all students on these two APPEs starting the first full week of the rotation and continued until the last day. Descriptive statistics were used to compare the intervention data. This study is Institutional Review Board approved. Results: Data were collected from 13 students for an average of 15 days for the US-based APPE and 23 days for the Kenya-based APPE. US-based students documented an average of 2.2 interventions per patient per day, while Kenya-based students documented 2.8 interventions per patient per day. The most common interventions were patient chart reviews and treatment sheet rewrites for the US-based and Kenya-based students, respectively. Two notable interventions that were documented on the Kenya-based APPE, but not the US-based APPE, were intravenous administration instructions and obtaining patients' blood pressures and fasting blood sugars. Implications: Regardless of setting, student pharmacists are able to have a profound impact on patient care and further demonstrates that the Kenya-based internal medicine rotation is a comparable learning experience to its US-based counterpart.

Comparison of Baseline Professionalism Among Student Pharmacists to Inform a Co-Curricular Professional Engagement Program. Maria M. Thurston, Mercer University, Jill M. Augustine, Mercer University, C. Lea Bonner, Mercer University. Objectives: The purpose of this study was to assess and compare student pharmacists' professionalism at one college of pharmacy prior to the implementation of a co-curricular professional engagement program in accordance with Standards 2016. Method: At the start of the school year, two validated instruments (Behavioral Professionalism Assessment Instrument [BPAI] and Pharmacy Professionalism Instrument [PPI]) were administered to assess professional attitudes and behaviors prior to program implementation. Kruskall-Wallis tests compared responses among the three pharmacy classes. Analysis was conducted using SPSS. This research was approved by the University's Institutional Review Board. Results: A total of 362 students completed the survey (75\% response rate), of which 131 were first-year, 139 were second-year, and 92 were third-year students. Significant differences in agreement were found among these classes of students regarding whether they felt a program focused on professional engagement was important and helpful $(\mathrm{p}<0.001)$. The level of agreement among the professional years was significantly different for seven BPAI statements and five PPI statements. These behaviors include: completing assignments independently and without supervision ( $\mathrm{p}=0.004)$; commitment to helping others $(p=0.004)$; reporting a medication error $(p=0.005)$; using time efficiently $(p=0.02)$; and being respectful $(p=0.04)$. Implications: There were significant differences in the level of agreement for 12 statements among the three pharmacy classes at baseline. Differences may be explained by perceived professionalism (in first-year students) versus actual professional behaviors (second- and third-year students) as well as breadth and depth of experience. Student awareness of professionalism and professional behaviors could be enhanced by a tailored co-curricular professional engagement program.

Comparison of Grit Scores to Strengths Amongst Two Cohorts of the Academic Leadership Fellows Program. Kurt A. Wargo, Wingate University, Michael Thomas, Samford University. Objectives: To determine the relationship between grit and strengths from StrengthsFinder in Academic Leadership Fellow (ALFP) cohorts Method: An electronic survey was used to elicit responses to an 8-item grit scale, ranking of the 34 strengths from the StrengthsFinder assessment, and demographics. The survey was sent to all members of ALFP cohorts 13 (2016-2017) and 14 (2017-2018). Grit scores were calculated according to standard practice and could range from 1-5 where 5 is extremely gritty. The top five strengths of each subject were compiled and average grit scores were calculated for those subjects. Results: Forty respondents completed all questions ( $67 \%$ response rate) with $58 \%$ being female, $65 \%$ having the PharmD as the terminal degree, and $90 \%$ at the rank of associate or full professor. The average grit score of the population was $4.1+/-0.48$. The passion domain of the grit score was $3.17 \pm$ 0.92 compared to $4.65 \pm 0.36$ for the perseverance domain, $\mathrm{p}<0.0001$. There were four strengths that occurred with the highest frequency, learner, achiever, relator, and responsibility. On the other hand, there were four strengths that occurred most frequently in the subjects bottom five, woo, adaptability, context, and empathy. The average grit score with learner in their top five was $4.13 \pm 0.45$, achiever $4.33 \pm 0.37$, relator $4.11 \pm 0.62$, and responsibility $4.41 \pm$ 0.31 . Implications: In this group of academic leaders, the top four strengths were associated with high grit scores. Overall, academic leaders may exhibit more perseverance, but less passion.

Comparison of Three Non-Cognitive Measures to Predict Scholarly Writing Among US Pharmacy Practice Faculty. Nalin Payakachat, University of Arkansas for Medical Sciences, Kacey K. Hight, University of Arkansas for Medical Sciences, Melanie A. Reinhardt, University of Arkansas for Medical Sciences, Adam Pate, The University of Mississippi, Amy M. Franks, University of Arkansas for Medical Sciences. Objectives: Few existing validated non-cognitive measures exist in the literature. We compared the ability of two existing measures and a newly developed measure to predict scholarly writing among pharmacy practice (PHPR) faculty. Method: Two existing measures identified from the literature, Grit-S and Global Self-Efficacy (GSC) scales, were compared with the newly developed Scholarly Writing Motivation Scale (SWMS). A cross-sectional online survey study was conducted in October 2017 among U.S. PHPR faculty. Performance of the three measures was evaluated by how well each Tobit regression model predicted the outcome of scholarly writing (i.e., total number of self-reported publications). Tobit regression determined associations between the outcome and scores from each measure, controlling for demographic characteristics of participants (age, gender, race, education, tenure status). Variance (R2) was reported for each model, with higher $\mathrm{R} 2$ representing a better prediction. Results: 210 participants were included in the final analysis with an average age of $41.7(\mathrm{SD}=10.5)$ years. The majority was female $(68 \%)$, white $(85 \%)$, from public institutions $(66 \%)$, and in non-tenure track positions $(62.9 \%)$. Average total publications and years in academia were $18.3(\mathrm{SD}=1.3)$ and $11.0(\mathrm{SD}=9.1)$, respectively. Most $(88.6 \%)$ had a PharmD degree, and a few (14.8\%) had a $\mathrm{PhD}$ degree. Only the SWMS scores were significantly associated with total publication number $(\mathrm{p}<.001)$. R2 for Grit-S, GSC, and SWMS were 0.48, 0.46, and 0.54 , respectively. Implications: The SWMS performed the best among the three measures and may be used as a tool to explore noncognitive factors for scholarly writing in this sample.

Correlation Between Pharmacy Students' Resilience Scale for Adults (RSA) Scores and Experience, Leadership and Academic Performance. Lana Dvorkin-Camiel, MCPHS University-Boston, Maria D. Kostka-Rokosz, MCPHS University-Boston, Gary Tataronis, MCPHS University-Boston, Yulia A. Murray, MCPHS UniversityBoston, Catherine Taglieri, MCPHS University-Boston, Lisa Padgett, MCPHS University-Boston, Steven J. Crosby, MCPHS UniversityBoston, Michele L. Matthews, MCPHS University-Boston, Joseph Ferullo, MCPHS University-Boston, Jennifer Goldman, MCPHS University-Boston. Objectives: To examine if there is a correlation between students' RSA scores and their demographics, work 


\section{American Journal of Pharmaceutical Education 2018; 82 (5) Article 7158.}

experience, leadership style, Myers Briggs personality types and academic performance. Method: Prior to the start of the semester, students completed the RSA and answered demographics questions. At the end of the semester, students' academic performance data (via GPAs, exam/quiz scores) were evaluated to find any correlations with the resilience scores and demographics. Results: Of 309 students enrolled, 301 consented to participation. Based on the five categories of the RSA, students scored $81.4 \%$ on personal competence, $71.5 \%$ on social competence, $83.4 \%$ on family coherence, $88.7 \%$ on social support, and $78.3 \%$ on personal structure. There was a difference in RSA scores between leadership styles (leader vs. balanced vs. follower $-85.2 \%$ vs. $80.9 \%$ vs. $77.5 \%$ ) and work experience (a lot and some vs. little/none $-81.9 \%$ and $81.4 \%$ vs. $77.9 \%$ ) There was no difference in RSA scores between genders and most elements of the Myers Briggs personality types except for extrovert/introvert $(85.1 \%$ vs $76.8 \%$ ). GPAs, exam/quiz averages, participation and presentation scores were also evaluated. Implications: Among the five categories of the RSA, the strongest scores were for social support, family coherence and personal competence, with lower scores reported for social competence and social structure. RSA resilience scores in pharmacy students seem to positively correlate with leadership style, work experience and extrovert/introvert characteristics. We believe that resilience skills require development, and further research should focus on whether encouraging students to gain more work experience and develop leadership skills will enhance their overall resilience.

Correlation of Pharmacy Curriculum Outcomes Assessment (PCOA) Scores With School of Pharmacy GPA. Amber Verdell, West Coast University, Jennica Zaro, West Coast University, Richard d'Assalenaux, West Coast University, Kyle Sousa, West Coast University, Fred Farris, West Coast University, Ruth Martinez, West Coast University. Objectives: Many Schools of Pharmacy are investigating applications of PCOA scores. One possible application is to provide students with formative feedback. We sought to determine if there was a correlation between PCOA scores and GPA. Method: At our School, the PCOA exam is administered to each cohort in the Spring of their first, second, and third years. We performed a correlation with students' GPA and PCOA scores using the Pearson Correlation Coefficient ( $r$ ) over three years of data. We compared GPA with overall PCOA score and score among the four PCOA domains: Basic Biomedical Sciences, Pharmaceutical Sciences, Social/Behavioral/ Administrative Sciences, and Clinical Sciences. We interpreted the Pearson Correlation Coefficient based on the following values: $\mathrm{r}=0.40-0.59$ moderate and 0.60-0.79 strong correlation. Results: Student GPA was moderately to strongly correlated with overall PCOA score $(r=0.54$ to $r=0.68)$ for each year. First year correlation between GPA and PCOA score were $r=0.63$ and $r=0.61$, second year $r=0.54$ and $\mathrm{r}=0.68$, and third year $\mathrm{r}=0.64$. PCOA domains showed less correlation with GPA ( $r=0.34$ to $r=0.72$ ). Implications: The moderate to strong correlation between PCOA overall score and GPA supports the use of PCOA score to provide formative feedback to students. We plan to perform future correlations to investigate other applications for PCOA scores at our School.

Current Status of Infectious Diseases Education at United States Colleges of Pharmacy. Jonathan C. Cho, The University of Texas at Tyler, Conan MacDougall, University of California, San Francisco, Lauren R. Biehle, University of Wyoming, Navaneeth Narayanan, Rutgers, The State University of New Jersey, Meghan N. Jeffres, University of Colorado. Objectives: To describe content and pedagogical strategies employed for didactic infectious diseases (ID) education at United States pharmacy schools. Method: A 23-question cross-sectional electronic survey was distributed via e-mail to ID course coordinators and faculty at 137 pharmacy schools. Up to two follow-up emails and one telephone call were made to non-responders during the data collection period. Results: A total of $106(77 \%)$ pharmacy schools responded to the survey. ID curriculum was allotted 60 (IQR 40-80) hours of classroom time and primarily taught in the third year of the curriculum (41\%). ID fundamentals was allocated 33\% (IQR 25-40) of curricular time compared to $67 \%$ (IQR 60-75) allocated to disease states. The majority of classroom hours were dedicated to traditional lecture (75\% [IQR 57.5-80]) with 25\% (IQR 20-42.5) spent using active learning. Most common active learning strategies either consistently or frequently used were patient case application (98\%) and audience response systems (77\%). Tier one ID topics from the ACCP Pharmacotherapy Didactic Curriculum Toolkit were taught in $94 \%$ of pharmacy schools. Schools with block schedules dedicated more curriculum hours to ID content compared to semester and quarter systems (78 vs. 48 vs. 44 hours, respectively). Implications: Although pharmacy schools taught most tier one ID topics, increased use of active learning modalities may enhance ID education. Additionally, an overallocation of curricular time dedicated to fundamentals compared to disease states may exist.

Curricular Mapping to ACPE Standards 2016 Appendix 1 and NAPLEX Blueprint. Jonathan Hernandez-Agosto, University of Puerto Rico, Edna N. Almodovar, University of Puerto Rico. Objectives: To evaluate the alignment of the curriculum content with ACPE Standards 2016- Appendix 1. To evaluate if the curriculum content addresses the revised NAPLEX blueprint competency statements. To determine breadth and depth of the curriculum content when compared to Appendix 1 and NAPLEX blueprint. Method: A curricular mapping process was conducted during the second semester of 20152016 for the required courses of the curriculum, the Appendix 1 Key Elements, and the NAPLEX Blueprint. Data was collected through an electronic platform, during a workshop offered to the faculty. Data was extracted and summarized from the electronic database. A tabular display of the map/cross-walk was developed and reviewed by the Curriculum Committee (CC). A descriptive analysis of the data was performed and results were presented to the faculty. Results: Faculty response rate was $85 \%$. All Appendix 1 content areas are represented within the curriculum. Appropriate breadth and depth is suggested by the number of courses that address each key element at different years in the curriculum. All NAPLEX competency statements are represented within the curriculum, however, percentage of distribution for Area 2 is significantly lower (16\%) than the $33 \%$ of weight distribution for this area in the NAPLEX. Results for Area 2 show that competency statements related to the compounding of sterile and non-sterile products $(2.2 .2,2.2 .3$, and 2.2.5) may need to be reinforced. Implications: This analysis provides the basis for the correlation between students' performance on PCOA, NAPLEX and curricular alignment.

Delivery of a Co-Curricular Licensure Preparedness Program in the Final Professional Year: Incorporating Pharmacy Student Feedback. Jill M. Augustine, Mercer University, C. Lea Bonner, Mercer University, Candace W. Barnett, Mercer University. Objectives: To describe changes made to a required licensure preparedness program (LPP) based on student feedback. Method: In 2017, 50 final-year student pharmacists volunteered to participate in a co-curricular LPP. At LPP completion, students answered a 28 -item questionnaire, which assessed programmatic features for Class of 2018 delivery. Respondents evaluated the pace of the LPP; stressfulness and management of program; resources used; and amount of time spent on each module. Frequencies were calculated and open-ended questions 


\section{American Journal of Pharmaceutical Education 2018; 82 (5) Article 7158.}

were analyzed for common themes. The project was approved by the University's institutional review board. Results: Overall, a majority of students had positive attitudes about the LPP and provided positive comments about the program's implementation. Students reported the LPP was not "stressful" (52\%), yet most students indicated that the program was worth their time (88\%). Students reported spending 1-2 hours on modules $(70 \%)$ and used online tutorials and the textbook to review topics (87\%). Almost all students suggested that the LPP be a requirement for the subsequent cohort (88\%). Program administrators made changes based on feedback for the Class of 2018, including: changing the LPP to a longitudinal program (from one semester to two); increasing the number of required modules; and adding required clinical disease-state modules. Implications: Student volunteers provided helpful feedback on the use and implementation of a LPP. Student feedback allowed program administrators to make changes for full implementation, including making the LPP a required co-curricular activity. Administrators will continue to monitor students' performance and attitudes for ongoing programmatic evaluation.

Demonstrating Value: Evaluating Outcomes of Attendance at the Nonprescription Medicines Academy Conferences. Mary M. Bridgeman, Rutgers, The State University of New Jersey, Jenny A. Van Amburgh, Northeastern University, Seena L. Haines, The University of Mississippi, Katherine K. Orr, The University of Rhode Island. Objectives: To examine the impact of attendance at the Nonprescription Medicines Academy (NMA) conferences provides pharmacy faculty members. Method: The NMA provides relevant and impactful information on nonprescription medicines and self-care conditions, and is designed to promote networking and informationsharing among participants. To address the absence of outcomes data and with IRB approval, investigators developed an anonymous electronic survey for individuals previously attending a NMA meeting. Results: Of 178 attendees invited to participate, 77 (43.3\%) consented to survey participation and $69(38.8 \%)$ completed the assessment. Survey participants were predominantly females $(55,79.7 \%)$, between the ages of 31-40 years $(39,56.5 \%)$, had attended three or more conferences $(35,50.7 \%)$, and, at the time of first attendance, were at the rank of assistant/clinical assistant professor (54,78.3\%). A majority of survey respondents agreed or strongly agreed attendance at a NMA conference impacted their teaching $(64,92.8 \%)$, relationships (56, $81.2 \%$ ), perspectives, self-awareness, and research and scholarship (60,87\% for all three domains). Areas of faculty development most frequently identified by participants as having been strongly influenced by attending a NMA conference included competency in teaching self-care $(43,20.7 \%)$; incorporation of active learning-based teaching methods $(43,20.7 \%)$; opportunities for collaboration in scholarship (26, 12.5\%); and opportunities for collaboration in teaching $(22,10.6 \%)$. Implications: NMA meeting participation is a valuable experience that participants describe as influencing their careers and professional advancement. Results of this instrument will be useful in informing continued meeting planning and in promoting NMA meeting attendance for individuals interested in professional and career advancement.

Description and Evaluation of a Co-Curricular Diversity Program Focused on Patient Care. Faria Munir, The Ohio State University, Emmanuel Osei, The Ohio State University, Kristina Naseman, The Ohio State University, Chelsea R. Pekny, The Ohio State University, Alexa Valentino, The Ohio State University, Anna C. Haas-Gehres, The Ohio State University, Jennifer L. Rodis, The Ohio State University. Objectives: The Ohio State University College of Pharmacy (OSU COP) hosted Diversity Week in September 2017 focused on understanding diversity to improve patient care. Authors aim to describe Diversity Week and share perspectives on programming by faculty, staff, and students. Method: The week long event featured practitioners, guest speakers, and faculty discussing diversity topics to enhance skills in patient care and foster self-improvement and selfreflection. An anonymous, written post-survey was provided to participants at each session. A comprehensive, online survey was sent to all faculty, staff, and students following the week. Surveys examined influence on attendees' perceptions and behaviors. Descriptive statistics were used to report frequencies, percentages, and means. Thematic analysis was conducted for open-ended responses. Results: A total of 389 individual written and 227 individual online surveys were collected. Most participants indicated that Diversity Week positively impacted their perception of diversity at OSU COP (74\%) as well as patient care $(76 \%)$. Major themes identified in open-ended responses were increased knowledge and awareness, self-reflection and improvement, and a willingness to change future actions when providing care to patients from diverse backgrounds. Implications: The Diversity Week co-curricular programming model highlights the importance of incorporating diversity topics into health-professional institutions; the survey results show promising data about the influence it has on participants. Further innovative approaches to diversity programming should be considered as a means of achieving Domains 3 \& 4 CAPE Outcomes 2013. Additional assessments may determine the lasting impact diversity programming has on participants.

Description of a Book Club Faculty Development Program and Faculty Opinions Regarding the Process. Michelle O. Zagar, The University of Louisiana at Monroe, Laurel A. Sampognaro, The University of Louisiana at Monroe, Courtney A. Robertson, Gina C. Craft, The University of Louisiana at Monroe. Objectives: To implement a faculty development book club and evaluate faculty opinions of the process Method: A year-long pharmacy faculty development book club was conducted. Monthly meetings examined a text addressing many areas of pharmacy education. Like traditional book clubs, discussions centered on reflective questions submitted by chapter facilitators. Faculty members completed pre- and post-surveys regarding attitudes and opinions of faculty development and utilization of a book club format. The Kruskal-Wallis, Dunn's multiple comparisons, Mann Whitney, and Wilcoxon tests were used to analyze results. Results: Of $26(54.2 \%)$ and $19(38.8 \%)$ respondents to the pre- and post-surveys respectively, $73 \%$ agreed they would like a faculty development book club on the pre-survey, while $86.3 \%$ of participants were satisfied or highly satisfied with the book club on the post-survey. Females and clinical faculty felt they would be more engaged in the book club than they had been in more traditional development programs $(\mathrm{p}=0.0491$ and 0.0314 respectively) on the pre-survey; the same groups indicated more engagement on the post-survey $(p=0.0536$ and $p=0.0320$ respectively). Males indicated greater improvement in teaching abilities than females as a result of the book club $(p=0.0406)$. Paired pre- and post-surveys revealed that 12 respondents were more able to describe characteristics of a student-centered approach to teaching after the book club ( $p=0.0391)$. Implications: Faculty found a book club format to be an enjoyable, effective, and engaging way of developing skills and knowledge as a pharmacy academician. This alternative delivery method for faculty development programming can be replicated by other institutions.

Design and Implementation of a Remediation Program to Improve Competence in a Self-Care Course. Katelyn M. Alexander, East Tennessee State University, Cortney M. Mospan, Wingate University, Kathryn A. Mueller, East Tennessee State University. Objectives: To 


\section{American Journal of Pharmaceutical Education 2018; 82 (5) Article 7158.}

develop an innovative remediation program for students who received a failing grade in a self-care course to improve knowledge and skills. Method: As a result of students being referred for remediation, faculty created and implemented a remediation program to ensure mastery of content. A standardized plan was designed that focused on principal areas for development, all mapped to key course objectives. Areas of deficiency were identified for students using exam score data and metrics and incorporated into an individualized plan. The program consisted of a self-directed review of material and on-site session. Students reviewed patient cases for each content area identified, triaged and recommended appropriate treatment for the patient, and provided counseling points to faculty reviewers. Faculty developed cases and rubrics for assessment of proficiency. Results: A total of 8 students have progressed through the remediation program successfully. One student required a second remediation, while the other 7 students passed upon first attempt. Of those students requiring remediation, seven students $(88 \%)$ have been retained in the program since completing subsequent coursework with usual progression. Faculty time investment has varied year to year, but is generally kept to a few hours per student. Implications: While academic progression policies vary amongst institutions, the option to address student knowledge deficits through remediation are becoming more popular as a means to ensure competence while also retaining the student in the program. The remediation strategy implemented here has shown to be successful at overcoming deficits and also developing student for ongoing success.

Designing and Implementing Student Focus Groups for Longitudinal Evaluation of Renewed-Curriculum Implementation Over P1-P2 Years. Minakshi Lahiri, Wayne State University, Justine S. Gortney, Wayne State University, Candice L. Garwood, Wayne State University, Lynette R. Moser, Wayne State University. Objectives: To investigate student learning experiences longitudinally, for evaluation of the renewed P1 - P2 curriculum implementation, in order to improve curriculum, encourage teaching excellence and promote student-success. Method: Focus group methodology has wide acceptance for curriculum evaluation in health education settings. Non-directive moderator-style exploratory focus groups (Stalmeijer, McNaughton \& Van Mook, 2014) was used to assess student perspectives on the learning experiences with the renewed-curriculum after every semester of P1 and P2 years. Purposive sampling was done to recruit participants $(\mathrm{n} 1=24 ; \mathrm{n} 2=22 ; \mathrm{n} 3 \& 4=24)$. Pre-designed question prompts were used to match each semester specific curricular aspects. Focus groups were facilitated by non-faculty academic personnel to eliminate bias. Sessions were audio recorded; additional feedback from participants and facilitators notes were collected. All qualitative data were coded, analyzed and triangulated using Miles \& Huberman's data analysis model (1994). Results: Participants expressed satisfaction with achieving the learning objectives of the renewed-curriculum, physical resources and the faculty support. Areas for improvement were identified with pharmacy calculations, group assignments, access and communication with some faculty, faculty advising, problem based learning courses and tracking of co-curricular activities. The findings were communicated to the Curriculum Committee for inclusion with recommendations in the course review. Implications: Using the rich, qualitative student focus group feedback data as a component for evaluating longitudinal curricular effectiveness offers a model for a program improvement that can be easily implemented with minimal resources and could benefit many pharmacy programs.

Developing a Consistent Grading Scale Across a Doctor of Pharmacy Program. Cameron C. Lindsey, University of Missouri-Kansas City, Brandon Reynolds, University of Missouri-Kansas City. Objectives: The objectives of this review were to define the different grading scales currently in use at the school and to evaluate the impact on student grade point averages (GPA) a change to a consistent scale would impart. Method: Grading scales in syllabi for required, didactic coursework were analyzed. Grading scales were defined as a 10-point (wholeletter), a 7-point (plus/minus), or a non-traditional scale that was set by the course coordinator. Comparisons of grade point averages using a 10point, a 7-point, and the current grading scales within each individual course were applied to a proxy student pharmacist who earned a $92 \%$, $88 \%, 82 \%$ and $78 \%$ through the entire program. Results: Thirty-five courses were reviewed. Ten courses each documented having a wholeletter or a plus-minus grading scale. The remaining courses $(n=15)$ had either plus only scales $(n=8)$ or variations of all three $(n=7)$. Students who earned a $92 \%, 88 \%, 82 \%$ and $78 \%$ through the entire program would earn GPAs of 3.93, 3.25, 2.94, and 2.18, respectively, based on the current grading scales that vary. Students who perform at the high range (e.g., $88 \%$ or $78 \%$ ) would benefit from a plus/minus scale over the current varying structure and a whole-letter scale. Those that perform at a lower range (e.g., 92\%, 82\%) would have a slightly lower GPA versus the current structure or a whole-letter scale. Implications: One grading scale allows for consistency across the program to reduce student confusion, potentially decrease grade inflation, and increase student differentiation.

Development of an Instrument to Measure Academic Resilience Among Student Pharmacists. Christina A. Spivey, The University of Tennessee, Marie A. Chisholm-Burns, The University of Tennessee, Erin H. Sherwin, The University of Tennessee, Jennifer S. Williams, The University of Tennessee, Stephanie J. Phelps, The University of Tennessee. Objectives: Resilience is suggested as an important criterion in academic success. However, there is no valid and reliable scale to measure academic resilience in student pharmacists. Therefore, the objective was to develop a valid and reliable academic pharmacy resilience scale adapted for use in the didactic portion of the pharmacy curriculum (prior to Advanced Pharmacy Practice Experiences). Method: A cross-sectional survey was conducted among first-, second-, and third-year student pharmacists to assess psychometric properties of a 30-item adapted academic resilience scale; data were also collected using the Short Grit Scale (Grit-S). Demographic characteristics were collected from student records. Exploratory factor analysis was applied to determine number of underlying factors responsible for data covariation. Principal components analysis was used as the extraction method. Varimax rotation method was used and Cronbach's alpha was estimated. Validity testing was conducted by calculating Pearson's r correlations between the adapted academic resilience scale and Grit-S. Results: The response rate was $84 \%$. The final version of the scale, the Academic Pharmacy Resilience Scale (APRS-16), had 4 subscales and 16 items (14 items failed to load on any factors and were deleted). Cronbach's alpha was .84, indicating strong internal consistency. APRS-16 and its subscales were significantly correlated to the Grit-S and its subscales, providing evidence of effective convergent validity. Implications: Evidence supports the reliability and validity of the APRS-16 as a measure of academic resilience in student pharmacists. Future studies should use the APRS-16 to investigate the relationship between academic resilience and performance outcomes among student pharmacists.

Development of a Required Interprofessional Education Course Sequence. James W. McAuley, The Ohio State University, Katherine A. Kelley, The Ohio State University. Objectives: Describe the planning, implementation and assessments of a required four-course Interprofessional Education (IPE) sequence that was developed to address a need to enhance didactic IPE Method: After purposeful planning with willing campus partners, we developed a required 


\section{American Journal of Pharmaceutical Education 2018; 82 (5) Article 7158.}

course sequence that was designed to teach P2 and P3 students how to advance collaboration and quality of patient care by providing interprofessional opportunities for students to learn about, from, and with other members of the healthcare team. Each semester, the half-credit course has three interactive collaborations with at least one other healthcare discipline. Results: We have completed two of the four required courses to date. Our P2s have had six IPE sessions; they have worked in teams with a. medical students focusing on scope of practice using team-based learning, b. medical students on a medication adherence exercise and prescription writing, c. $700+$ health professional students focused on the opioid crisis, $d$. nurse practitioner students focused on implicit bias, e. social work students with a focus on motivational interviewing and team-based care, and f. medical dietetics and physician assistant students focused on roles and responsibilities in this triad. For the remaining 2 courses, P3s will work in IPE teams on simulations and electronic medical record exercises. Assessments have demonstrated significant increases in student knowledge and perceptions. Implications: Through efficient efforts, didactic coursework can be incorporated into curriculum to meet accreditation standards while also addressing IPEC competencies. These IPE sessions also advance students' knowledge and skills around contemporary healthcare topics.

Development of a Rubric to Assess Metacognition in Fourth Year Student Pharmacists. Rebecca Maxson, Auburn University, Kristen L. Helms, Auburn University. Objectives: According to Flavell's learning theory on metacognition, students must develop awareness and control of their own thinking (American Psychologist 1979). The goal of this project was to develop a rubric to assess metacognitive characteristics of a reflective essay assigned as part of an APPE rotation. The reflective essay is based on assigned readings that challenge the student's perceptions of health and ethics. Students reflect on how these readings impact personal perceptions and their future practice. Method: Two P4 students on an academic APPE rotation were provided with a description of the assignment and goals of the rubric. One of the students had completed the assignment in a prior rotation. Results of a literature search were used by students to construct the initial drafts of the rubric. After refinement by APPE preceptors, the rubric was peer reviewed for content validity. Three peer reviewers were chosen based on expertise in pharmacy and/or assessment. The rubric was then validated and piloted using reflections from previous students. Results: The final rubric contains domains reflecting adequacy of Introduction, Reflective Analysis (including themes and changes to perspectives), Conclusions, Language/Construct and Professionalism. All domains except Professionalism are assigned to one of four categories reflecting level of competency. Professionalism is a pass/fail component of the rubric. Implications: Development of a rubric that focuses on the metacognition in pharmacy students will allow for evidence-based, criteria-referenced, and student-specific feedback as well as insight into students' metacognitive skills.

Development of a Situated Learning Activity Using Chatroom and Videos to Simulate SOAP Note Documentation. Kathryn Zaborowski, University of Wisconsin-Madison, Edward Portillo, University of Wisconsin-Madison, Andrea L. Porter, University of Wisconsin-Madison, Amanda Margolis, University of WisconsinMadison. Objectives: To provide students with a situated learning opportunity utilizing technology to facilitate student learning and to assess student self-efficacy in documenting a patient interaction via the SOAP note method. Method: A three-week longitudinal blended learning SOAP Note Workshop was developed and implemented for 150 P2 students to simulate a virtual ambulatory care clinic visit from patient interaction to documentation in Spring 2018. The first session consisted of a didactic overview of SOAP note writing. In between sessions, students attended a virtual clinic visit by watching an online video simulating an ambulatory care clinic encounter. Students then submitted written SOAP Note components reflecting the encounter prior to workshop sessions two and three. During sessions two and three, students completed a think-pair-share activity with a partner to review their SOAP notes and posted final versions to a live SOAP Note Chatroom to promote interactive group discussion. Student self-efficacy was analyzed at two time points during the semester using 10 multiple choice questions with a five point scale $(1=$ not confident at all to $5=$ extremely confident) before workshop session two and after session three. Results: A total of 130 students completed both surveys with a response rate of $86.67 \%$. Survey questions were analyzed using the Wilcoxon Signed-Rank Test. It was determined that the mean change in self-efficacy was statistically significant in all question subject areas (Prob $>|z|=0.0000 ; P<0.001)$. Implications: Use of situated and blended learning activities increase student self-efficacy in documenting patient interactions via the SOAP note method.

Didactic Performance Before and After Implementation of an Integrated Curriculum. John Bossaer, East Tennessee State University, Samuel C. Karpen, East Tennessee State University, Adam C. Welch, East Tennessee State University, Nicholas E. Hagemeier, East Tennessee State University, Jeffrey A. Gray, East Tennessee State University. Objectives: To assess the effect of a revised curriculum on didactic performance, as measured by PCOA scaled scores. Method: Under the revised curriculum, medicinal chemistry, pharmacology, and pharmacotherapy were integrated into a series of courses spanning the P2 \& P3 years. The PCOA was administered in a low-stakes manner during spring of the P3 year to 3 cohorts of students in the old curriculum and 2 cohorts of students in the revised curriculum. Doubly-robust estimation was used to determine the effect of curriculum version on PCOA while controlling for PCAT scores and pre-pharmacy GPA (ppGPA). Additionally, researchers conducted ordinary least squares regression to determine whether the revised curriculum affected PCOA scores differently in students with higher PCAT scores and higher ppGPA. IRB approval was granted and informed consent was obtained for all participants. Results: There was no effect of the revised curriculum on PCOA performance $(p=0.136)$ after controlling for PCAT and ppGPA. Students with high ppGPAs scored lower on the PCOA under the new curriculum than under the old curriculum while students with low ppGPAs performed equivalently under both curricula $(p=0.009)$. Students with high PCAT scores performed similarly on PCOA compared to students with low PCAT scores. Implications: The revised curriculum did not improve student performance on PCOA. Future analyses to further assess the effect of the revised curriculum will focus on experiential performance, licensure exam performance, and residency match rates compared to the old curriculum.

Diversifying Student Groups Based on Learning Style: Results of Student Perceptions on Group Dynamics. Nicole R. Winston, Marshall University, Brian Train, Marshall University, Christopher J. Booth, Marshall University. Objectives: Our objective of this study was to compare group dynamics of pharmacy students when team formation was based on diversified learning style groups as compared to randomization or grade point average (GPA). Method: An online survey was administered to first year (P1), second year (P2) and third year (P3) pharmacy students to evaluate individual learning styles based on Felder and Solomon's Index of Learning Styles Questionnaire. Students were then stratified based on learning style results and heterogeneously grouped, controlling for GPA. Students evaluated 


\section{American Journal of Pharmaceutical Education 2018; 82 (5) Article 7158.}

group dynamics based on the Global Team Process Questionnaire (GTPQ) through another online survey before and after the learning style grouping method. Results were compared to previous grouping methods of randomization (P1s) and GPA (P2s and P3s) using paired sample t-tests. Themes from open-ended questions were developed using the Glaser's constant comparative method. Results: P1 $(n=60)$ and P3 $(n=39)$ students indicated significant increases in trust within their groups $(p=0.047$ and $p=0.011$, respectively), while P2 $(n=60)$ student results demonstrated significant increases in equal distribution of work $(p=0.043)$. In addition, P3 students also indicated significant improvements in team-based skill acquisition. Open-ended question analyses supported Likert question results; however, the theme of improved teamwork was mentioned almost twice as often with the learning style grouping method $(n=15$ versus $n=8)$ even though this did not reach significance in our quantitative analysis for P1 and P2 students. Implications: Our results suggest that trust among group members, team-based skills and work distribution significantly improve when learning style is used to create heterogeneous groups, controlling for GPA.

Do Worksheets Work? Evaluation of the Correlation Between Optional Worksheet Completion and Calculation Performance. Holly D. Robison, Manchester University, Sara N. Trovinger, Manchester University. Objectives: Primary objectives included the measurement of effects of integrated calculation worksheets on first year pharmacy student performance within a required calculation course and on a high-stakes summative examination (OSCE calculation station). A secondary objective was to assess for any direct correlations between the student scores on the OSCE calculation station and within the calculation course. Method: Students concurrently enrolled in a pharmacy practice laboratory (PPL) course and a calculation course were given five optional opportunities to complete calculation worksheets within the PPL course. Student completion of worksheets determined three student groups as follows: group A completed no worksheets; group B completed 1-2 worksheets; group C completed $\geq 3$ worksheets. Student performance in the calculation course and on the OSCE calculation station were compared among the three groups. Results: Study enrollment consisted of 74 students and analysis found that group $\mathrm{C}$ students had calculation course scores which were statistically significantly higher than students in groups A and B. There were no significant differences found between the groups when comparing performance on the OSCE. There was no correlation found between the OSCE calculation station and calculation course grades. Implications: When worksheets are optional, there is potential for higher performing students to pursue extra opportunity more than lower performing students. Future assessment may include making worksheets mandatory for one semester and comparing findings to current data. The lack of correlation findings between the OSCE and the calculation course has prompted reevaluation of OSCE calculation content.

\#drugfree: Using Social Media Advertisements to Spread Health on a College Campus. Amy M. Fanous, Ohio Northern University, Ben D. Aronson, Ohio Northern University, Jessica L. Hinson, Ohio Northern University, BreAnna Meinzer, Ohio Northern University, Lindsey Peters, Ohio Northern University, Zachary Woods, Ohio Northern University, Michael Rush, Ohio Northern University. Objectives: To implement a novel social media campaign aimed at reducing prescription stimulant misuse and tobacco use in college students. Method: As part of an interdisciplinary student wellness program, pharmacist faculty disseminated social media health messages to increase awareness of the campus smoke-free policy, impact of tobacco use, and effects of stimulant misuse. Images and social media hashtags were developed prior to campaign implementation utilizing principles of behavior change, communication, and graphic design. Advertising space was purchased on social media platforms (Facebook, Twitter, and Snapchat), and advertisements were distributed to students based on geographic and demographic filters. Analytics were collected from these platforms in order to assess the message reach. A post-campaign survey was administered to a convenience sample of students in high-traffic areas of campus to evaluate message sentiment and attitudes. Results: The month-long campaign resulted in 31,945 impressions on Facebook, 18,290 impressions on Twitter, and 147 Snapchat views. The survey was completed by 114 students. Tobacco advertisements received generally positive sentiment, with greater than $80 \%$ of respondents reporting a positive reactions to the graphics. However, only $40 \%$ of respondents indicated a positive reaction to the stimulant advertisements. Implications: Social media advertisements reach a considerable audience which makes it a unique way to directly distribute public health messages. Increasing the frequency and availability of educational content on social media platforms created health messages that students could easily access. More research is needed to understand the long-term effects of these interventions on student behavior.

Early Career Exploration in the Pharmacy Curriculum. Kristy L. Brittain, Medical University of South Carolina, Stacey Cohen, Medical University of South Carolina, Lyndsey Milburn, Medical University of South Carolina, Miranda Miles, Medical University of South Carolina. Objectives: Assess student interest and knowledge of career paths for pharmacists following brief in-class presentations by classmates Method: First year-first semester students in the Introduction to Pharmacy course completed a Pecha Kucha on career opportunities in pharmacy. Students were paired with practicing pharmacists, including alumni and other community partners to explore career opportunities. Practice areas included academia, ambulatory care, community management, community staff, clinical specialist (cardiology, neurology, infectious disease, critical care, psychiatry), compounding, government/federal, health coaching, medication safety, health-system staff, independent, medical science liaison, and institutional administration. Students were asked to focus on how the pharmacist interfaces with the JCPP Pharmacists' Patient Care Process and could collect information through interviewing and/or shadowing. The presentation was assessed with a rubric on content, quality and creativity. A survey assessing interest and knowledge of each practice area was administered prior to the presentations and directly after. Results: The highest areas of interest were ambulatory care (91\%), health-system staff (85.7\%), infectious disease clinical specialist (71.4\%), cardiology clinical specialist (67.8\%), compounding (67\%) psychiatry clinical specialist (66.1\%), and independent pharmacy (66\%). Following the presentations, students indicated low levels of knowledge in the areas of: institutional administration (58.9\%), health coaching $(50 \%)$, government/federal $(35.7 \%)$, medication safety $(30.3 \%)$ and medical science liaison (25\%). Implications: This in-class activity can incorporate many required curricular elements, all while increasing exposure of students to different areas of practice. The results of this research will highlight potential areas of focused exposure for our students especially in emerging areas of practice.

Effect of a Study Skills Course on Student Self-Assessment. Leah C. Sera, University of Maryland, Shannon R. Tucker, University of Maryland, Mary Lynn McPherson, University of Maryland. Objectives: To determine whether a study skills course taken by first professional year pharmacy students improved their self-assessment of 


\section{American Journal of Pharmaceutical Education 2018; 82 (5) Article 7158.}

study skills and strategies. Method: This study analyzed student responses to the Learning and Study Strategies Inventory (LASSI), an online assessment with questions in 10 subject areas: anxiety (ANX), motivation (MOT), concentration CON), test strategies (TST), study aids (STA), selecting main ideas (SMI), attitude (ATT), self-testing (SFT), information processing (INP), and time management (TMT). Scores for each subject are reported as percentiles. Students who took an elective study skills course in 2012-2017 completed the self-assessment twice; prior to the start of the course and at the end of the course. Wilcoxon singed-rank test was performed to compare pre- and postcourse scores. Results: Over a 5-year period, 312 students who were enrolled in the study skills course completed both the pre- and postcourse LASSI assessment. Median percentile scores increased significantly in 8 of 10 areas. Specifically, paired pre-post differences in median scores were 14 in ANX $(p<0.0001)$; 0 in ATT $(p=0.57), 5$ in CON ( $p=0.018), 20$ in INP $(p<0.0001), 20$ in MOT $(p<0.0001), 5$ in SFT $(\mathrm{P}=0.11), 5$ in SMI $(\mathrm{p}<0.0001), 5$ in STA $(\mathrm{p}=0.033), 10$ in TMT $(p=0.0009)$, and 10 in TST $(p<0.0001)$. Implications: This evaluation shows that a study skills course improves students' self-assessment of skills and attitudes associated with success in postsecondary education. Future studies will look at the effect of such a course on academic outcomes.

Effect of Team-Building Activities on PharmD Students' Perception of Teamwork on a Team-Based Learning Course. Marina Galvez Peralta, West Virginia University, Werner J. Geldenhuys, West Virginia University, Mary K. Stamatakis, West Virginia University. Objectives: To implement and evaluate team-building exercises at the start of an integrated pharmaceutical sciences/pharmacotherapy course series that utilizes team-based learning and group activities as instructional methods. Team-building exercises in the session exposed students to situations requiring teamwork. Objectives were to: 1] improve group communication skills; 2] instill confidence in speakingup; 3] handle differences of opinions; and 4] develop group expectations. Method: Sixty-seven second-year pharmacy students starting the course series and seated with their semester-long team completed five team-building exercises in the first week of class (6-7/team). Session evaluation included 3 parts: a satisfaction survey using a 5-point Likert scale ( 1 =poor, $5=$ excellent $)$; a retrospective prior-after evaluation on their learning; and qualitative measurements regarding team participation. (WVU IRB protocol \# 1802001503) Results: Students positively valued the activity, perceived acquisition of new knowledge, organization, and format (100\% survey participation). Mean values from retrospective prior-after evaluation showed an improvement in: level of confidence (3.1 to 4.4), sharing information (3.6 to 4.3), handling conflicts (3.3 to 4.2), and providing feedback (3.5 to 4.3). Forty-four percent of students said that they would change their team dynamics after the experience, and eighty-eight percent agreed that the activity increased their confidence in working on teams. Issues identified by students: equity of effort/work, communication (either need to speak up more or listen more), and fear of conflict. Implications: Students' perceptions of team-building activities geared towards communication and working together were improved.

Effects of Social Desirability on Grit Survey and Grit Grid Scores in Pharmacy School Applicants. Elizabeth M. Lafitte, The University of Louisiana at Monroe, Corey M. Guidry, The University of Louisiana at Monroe, Sara C. Pearson, Courtney A. Robertson, David J. Caldwell, University of Arkansas for Medical Sciences. Objectives: To determine whether social desirability influenced grit survey scores in pharmacy school applicants. Method: An 8-item grit survey was administered to applicants in the 2016-2017 admission cycle during on-site interviews.
Overall grit scores and subscale scores, consistency of interest and persistence of effort, were recorded. A social desirability scale (SDS) containing four yes/no statements was co-administered to gauge the likelihood of applicants providing socially desirable answers to the grit survey. To calculate the grit grid score, students were also asked to list three activities most important to them and their highest level of achievement in each on the supplemental admissions application. Results: Grit survey, grit grid and SDS data were collected for 45 students, of which $30(67 \%)$ had an SDS score of four, and $15(33 \%)$ had a score of $<3$. Applicants with an SDS score of four had higher mean grit survey scores than applicants with SDS scores of three or less (34.1 vs 31.6, $\mathrm{p}=0.007)$. Mean subscale scores for consistency of interest $(16.2$ vs $14.6, \mathrm{p}=0.01)$ and persistence of effort (17.9 vs $17.0, \mathrm{p}=0.04)$ were also different among the SDS groups. Grit grid scores were not significantly different $(p=0.25)$ between SDS groups. Implications: Grit grid scores appear to be less affected by social desirability than do grit survey scores. The results of this study confirm that grit survey should be used cautiously in admissions decisions, but that grit grid may have utility.

Embedded Assessments and Stress in the Curriculum: Too Much, Too Little, or Just Right? Rosalyn P. Vellurattil, University of Illinois at Chicago, Marieke D. Schoen, University of Illinois at Chicago, Nicole K. Ozturk, University of Illinois at Chicago. Objectives: The UIC College of Pharmacy implemented a new PharmD curriculum in Fall 2016. With this implementation, there has been a push for enhanced active learning and student engagement in the classroom. The objective is to assess the frequency of embedded assessments in the new and old curriculum and evaluate whether this corresponds to excessive student workload, and affected student performance and perceived stress. Method: Final grades and assessment scores were downloaded from Blackboard for all core courses (Fall 2012 to Spring 2017). Descriptive statistics were employed to evaluate the frequency of assessments longitudinally, and Chi-square and Fisher exact tests to determine significance. The Perceived Stress Survey (PSS-10) was administered to all students in Spring 2016. Results: Assessments increased from 26 in 2016 to 91 in $2017(\mathrm{P}<0.01)$ in the P1 Spring new curriculum term, while maintaining the number of $\mathrm{D}$ and $\mathrm{F}$ grades earned from $2016(n=26)$, to $2017(n=21)(P=0.08)$. During the P2 Spring 2016 old curriculum term, an increased number of assessments $(\mathrm{n}=125)$ were administered, and $\mathrm{P} 2$ students earned the most grades of D (26\%), relative to P1's (20\%) and P3's (6\%), during the 2015-16 academic year $(\mathrm{n}=116)$. The PSS-10 demonstrated P2's experienced the highest stress levels. In contrast, P1's in the new curriculum earned only $11 \%$ and $13 \%$ of D's, in fall and spring, respectively $(n=135)$. Implications: Student workload, academic performance and stress will continue to be assessed as the new curriculum rolls out. The data will inform quality improvement measures and educational policies moving forward.

Emergency Preparedness and Response to a Natural Disaster by a School of Pharmacy: Lessons Learned. Wanda T. Maldonado, University of Puerto Rico, Edna N. Almodovar, University of Puerto Rico, Myriam Gonzalez, University of Puerto Rico, Sacha RiveraSarate, University of Puerto Rico, Frances M. Rodriguez, University of Puerto Rico. Objectives: Describe preparedness efforts in anticipation of an imminent atmospheric phenomenon, and the contingency plans implemented to respond to a major hurricane, in order to preserve the integrity of the academic programs. Method: Describe how a school of pharmacy prepared for and responded to the landfall of a Category 4 Hurricane. This atmospheric phenomenon resulted in a significant effect on communications, basic utilities, access to 


\section{American Journal of Pharmaceutical Education 2018; 82 (5) Article 7158.}

transportation and other basic needs. Results: Preparedness efforts included securing the workspaces, allowing students faculty and staff ample time to prepare in their homes, and establishment of a communication strategy at the campus level with the upper administration. Preserving the institution's research laboratories infrastructure was also addressed. Contingency plans developed after the phenomenon included but were not limited to; (1) immediate needs assessment for P4 students regarding their advanced pharmacy practice experiences status and adjustment of their plan for the academic year if needed, (2) reaching out to pharmacy academia for assistance in the placement of P4 students, (3) assessment of student body needs, (4) revision of the first semester academic calendar for P1-P3 students, (5) development of a secondary contingency plan in the event of sporadic power and communications interruptions, (6) development of alternate methods to communicate with faculty, students and personnel when routine methods were not available, and (7) support efforts implemented for students, faculty and staff. Implications: Proactively preparing for natural disasters and developing an immediate response plan is a matter of the utmost importance in order to provide continuity to the academic programs.

Enhancing a Pharmacotherapy Course With Educational Methods Based on Cognitive Science to Make Learning "Stick." Sarah Shrader, The University of Kansas, Robyn Kelton, The University of Kansas, Crystal Burkhardt, The University of Kansas. Objectives: Determine the impact of teaching methods based on cognitive science of learning on student performance and experience in a pharmacotherapy course. Method: Evidence-based teaching methods grounded in cognitive science are emerging as best-practice to make learning "stick". Three of these educational methods (1) flipped classroom (2) practice quizzes and (3) exam-wrappers were implemented in the women's health block (active group) of a pharmacotherapy course for all 132 third-year pharmacy students. The remaining blocks in the course used traditional lecture-based methods (control group). Differences on exam question mean performance were compared between the active group ( $\mathrm{n}=22$ questions) and control group ( $\mathrm{n}=88$ questions) using independent t-tests. Student experience was determined by a voluntary survey administered during the final week of the course. Voluntary focus groups were conducted that included a purposeful sample of above average/average/below average students based on course scores and a conventional content analysis was conducted. Results: Students performed better on active group exam questions (mean $94 \%$ correct, $\mathrm{SD}=0.06$ ) compared to the control group (mean $83 \%$ correct, $\mathrm{SD}=0.14)$; this was statistically significant $(\mathrm{p}=0.001)$. One-hundred twenty-eight students completed the survey $(97 \%$ response rate). The percent of students that agreed the flipped classroom, practice quizzes, and exam-wrappers helped them learn were $88 \%$, $79 \%$, and $46 \%$, respectively. Thirty students participated in the focus groups and themes emerged that confirmed the survey findings. Implications: Implementing evidence-based educational methods positively impacted student performance and experience in a pharmacotherapy course. As a result, the entire course will use these methods in Fall 2018.

Enhancing Empathy: Using Simulation to Introduce Students and Healthcare Professionals to Geriatric Loss. Mary M. Bridgeman, Rutgers, The State University of New Jersey, Donna Prete, Robert Wood Johnson University Hospital, Marcus G. Sturgill, Rutgers, The State University of New Jersey. Objectives: To measure empathy amongst pharmacists, nurses, and students of either profession prior to and after participation in a narrative simulation exercise focused on geriatric loss. Method: Participants in the geriatric loss simulation, an exercise designed to introduce physiological, financial, social, and psychosocial losses faced by the older adult, were invited to partake in pre- and post-exercise assessments. The validated Jefferson Scale of Empathy (Health Professional and Student Versions) was used to measure levels of empathy amongst participants. Demographic information and self-reported aggregate empathy scores (calculated using a Likert scale, $1=$ strongly disagree, $7=$ strongly agree) on this 20-item survey instrument were evaluated; on the post-exercise assessment, additional qualitative questions were included. Results: A total of 153 students and 108 healthcare professionals completed both preand post-exercise assessments. For students, a significant increase in median (interquartile range) aggregate empathy scores was observed [112 (106, 119.5) to $118(109,125.5), \mathrm{P}<0.001]$, with female students having a significantly higher aggregate empathy score both prior to and after exercise participation. Similarly for healthcare professionals, a statistically significant difference in pre- and post-activity scores was also observed [118 $(111.25,127)$ vs. $126(116,132), \mathrm{P}<0.001]$. Implications: Participants of this narrative simulation demonstrated a significant change in self-reported levels of empathy, regardless of professional designation or whether student or practitioner. Educators seeking to increase student awareness of losses facing the older adult, or interested in incorporating simulation as part of a geriatrics-based training program for students or healthcare professionals, could easily replicate this activity.

Enhancing Interprofessional Education at a Rural University: An Elective Course With a Service Learning Component. Michelle R. Musser, Ohio Northern University, Emily Eddy, Ohio Northern University, Megan Lieb, Ohio Northern University, Lisa Walden, Ohio Northern University. Objectives: Interprofessional education (IPE) is essential for health professional students to develop the skills needed for interprofessional (IP) practice. Incorporation of opportunities for patient care in the learning environment enhances IPE, but these activities may be difficult to implement in rural universities. An elective IPE course was developed at small, private, rural university to provide an enriched experience. Method: Objectives for the course was developed in alignment with the Interprofessional Education Collaborative (IPEC) domains and profession specific educational outcomes. The course was offered to senior health professional students and fifth year pharmacy students (of a 0-6 program). A variety of activities are offered in the course including discussion and simulation. Students worked in groups to develop presentations on IP approaches to healthcare issues and implement a service learning event in the local community. The course is evaluated using a standard course evaluation and IPEC Competency Survey Instrument as a pre-and post-test. Additional post-test questions further assessed students' feedback. Results: Student feedback regarding the course was positive overall. All students in the course $(n=15)$ completed the competency survey. Students showed significant improvement in 32 of 41 competency areas, including improvement in all communication related competency statements. Implications: Incorporation of an elective course at a rural university allows an enhanced IPE experiences for students. Exposure to patient care experiences was vital to practical learning and development of IP competencies.

Enhancing Students' Critical Thinking Skills With a Self-Care Activity on Over-the-Counter (OTC) Migraine Treatments. Tiffany R. Shin, The University of Kansas, Brianna Long, Brandon Blehm, The University of Kansas, Brittany L. Melton, The University of Kansas. Objectives: To evaluate the impact of directed learning questions on students' rationales and choice of migraine treatment. Method: The study is a retrospective evaluation of a yearly activity 


\section{American Journal of Pharmaceutical Education 2018; 82 (5) Article 7158.}

where first-year pharmacy students visited a community pharmacy to find and recommend self-care products for a migraine case. In 2015, students had no directed learning questions. In 2016 and 2017, directed learning questions (DLQ) were added to the activity. The DLQ guided students to compare and contrast products, including cost, onset of action, active ingredients, directions for use, and maximum daily doses. It also asked students to form their own conclusions about OTC products labeled for "migraine". Students' course grades and activity grades were compared to product recommendations using Mann-Whitney U and course grade was predicted by DLQ status and product choice using linear regression. Results: A total of 453 students participated, 155 without DLQ and 298 had DLQ. There was a significant difference between groups for overall course grade $(p=0.031)$, but not for the migraine case assignment $(p=0.989)$. The DLQ group also had a greater proportion who chose generic nonmigraine $(\mathrm{p}<0.001)$. When both DLQ status and product choice were included in the regression model, only product choice was retained as a predictor of course grade however $(\mathrm{p}<0.001)$. Implications: Overall the addition of DLQ was successful. Future improvements to the assignment include rewording questions, setting clear expectations, and enhancing the post-activity debrief. This assignment could be used in other self-care courses to help students think critically about the product recommendations for patients.

Enhancing Success in the Pharmacy Residency Match Through Implementation of a Residency Preparatory Course. Monica L. Miller, Purdue University, Ashley S. Crumby, The University of Mississippi, Alex N. Isaacs, Purdue University. Objectives: Evaluate the impact of a residency preparatory course on pharmacy residency match rates for enrolled students compared to students not in the course. Method: Retrospective review of the American Society of Health-System Pharmacy (ASHP) residency match results. Match rates from 2007 through 2017 were evaluated. National pharmacy match data was compiled from the ASHP National Matching website and institution-specific data was collected from annual reports provided by ASHP. Data was separated into students graduating prior to (2007-2014) and after the course (2015-2017). The primary endpoint was the evaluation of residency match rates for students in the course compared to other students at the institution not enrolled in the course. Secondary endpoints included match rates within the course and institution compared to the national match rates. Data was analyzed using a Chi-square test in SPPS version 24. Results: For 2015-2017, the match rate was significantly higher at the institution with students who completed the course compared to those not enrolled ( $88.7 \%$ vs. $70.7 \%, \mathrm{p}<0.01)$. For the students enrolled in the course $(2015-2017)$, the match rate was higher than the national average ( $88.7 \%$ vs. $66.4 \%$, $\mathrm{p}<0.01)$. The institution's match rate was similar to the national match rate prior to the course $(71.2 \%$ vs. $65.3 \%, p=0.78)$, but was significantly higher after implementation $(78 \%$ vs. $66.4 \%, \mathrm{p}<0.01)$. Implications: The residency preparatory course increased the rate of successful attainment of a pharmacy residency for students in the course compared to students not in the course and increased the match rate compared to the national average.

Evaluating an Interprofessional Education (IPE) Curriculum Using a Simulated Job Interview. Jessica E. Burchette, East Tennessee State University, Adam C. Welch, East Tennessee State University, Rick Hess, East Tennessee State University, L. Brian Cross, East Tennessee State University, Ivy Click, East Tennessee State University, Jodi Polaha, East Tennessee State University. Objectives: To evaluate whether practicing clinicians were able to differentiate medical and pharmacy students who participated in an advanced interprofessional education curriculum from those who received the standard curriculum based on a simulated job interview. Method: Twelve medical and 12 pharmacy students completed a 4-day advanced IPE curriculum over two years in addition to the standard curriculum including a semester long communications course involving all medical and pharmacy students. Controls were students who received the standard curriculum from the same graduating class. A 15-minute simulated job interview was completed for student volunteers. Students were interviewed by one actor and all interviews were recorded and transcribed. Twelve clinicians with team care experience were recruited (4 medical prescribers, 4 pharmacists, and 4 psychologists) to review transcripts for knowledge, skills and values around team-based practice and education. Reviewers were also asked to predict which students had received advanced IPE training. Results: Eight students from each discipline were recruited (4 advanced IPE and 4 controls per discipline). Overall mean clinician ratings for advanced IPE students were statistically higher than controls (student's t-test, $\mathrm{p}=$ 0.006 ). Clinicians were able to accurately predict $83.3 \%$ of students receiving advanced IPE training versus $45.8 \%$ of control students. (x2, $\mathrm{p}=0.007)$. Implications: Clinicians were able to distinguish students with advanced IPE training compared to standard curriculum controls. These data show that the knowledge and values learned by students in an IPE program are evaluated more positively by practicing clinicians who work in team care.

Evaluating Mentoring Needs of Applicants and Recipients of AACP's Pharmacy Practice Section New Investigator Awards. Christina A. Spivey, The University of Tennessee, Sweta M. Patel, Mercer University, Lakesha M. Butler, Southern Illinois University Edwardsville. Objectives: To evaluate mentoring needs of previous Pharmacy Practice Section New Investigator Award (NIA) applicants. Method: A 28-item survey was developed and approved by members of the AACP Pharmacy Practice Scholarship and Research Committee. The survey included two sections: (a) mentoring during the NIA application process; and (b) mentoring during the NIA research project. The survey was distributed to 84 past NIA applicants and recipients via SurveyMonkey ${ }^{\circledR}$. Item frequency distributions were calculated. Results: The response rate was $32 \%$. The majority (63\%) reported having a mentor during the NIA application process. Of those who had a mentor, $70.6 \%$ described this relationship as informal, with only $41 \%$ meeting with their mentors on a regular basis. Eighty-eight percent believed they benefited from having a mentor during the application process. Seven of the 8 NIA recipients indicated they benefited from having a mentor during their project. Respondents generally believed having a mentor during both the application process and research project would be helpful, and that mentors should have a track record of competitive grant funding and peer-reviewed publications, expertise in the mentee's field, and provide guidance on various aspects of the research plan. Respondents tended to disagree or were neutral as to whether mentors should be past NIA recipients. Implications: NIA applicants and recipients were strongly in favor of mentoring as part of the NIA process. Based on these results, a formal list of criteria for NIA faculty mentors should be developed, and a process for assigning mentors to NIA applicants/recipients should be considered.

Evaluating Ohio Pharmacy Faculty Perceptions of Knowledge, Skills, and Qualities Necessary for a Career in Academia. Lindsey Peters, Ohio Northern University, Emily Eddy, Ohio Northern University, Brittany L. Long, Ohio Northern University. Objectives: Due to demands for qualified faculty, more pharmacy residency programs have adopted teaching certificate programs. There is a lack of 


\section{American Journal of Pharmaceutical Education 2018; 82 (5) Article 7158.}

standardization and published research on what should be included in the program. The primary objective of this study is to survey current Ohio faculty about their perceived gaps in knowledge when starting their academic pharmacy careers and gather concrete evidence to direct residency teaching certificate program design. Method: An IRB approved Qualtrics survey was developed and emailed to faculty at Ohio Schools of Pharmacy. Faculty email addresses were obtained from each college's website or department chair. The survey was open for two weeks starting January 2018 with two reminder emails. Informed consent was obtained. Participants were entered to win one gift card as incentive. Results: A total of 64 surveys (21.3\%) were completed with representation from all seven colleges. When asked to select from a provided list, the top six selected gaps in knowledge at the beginning of their career were: institutional finance $(78.3 \%)$, admissions/marketing (78.3\%), programmatic assessment (78.3\%), accreditation (76.7\%), academic legal issues (76.7\%), and experiential site recruitment/retention (76.7\%). However, when asked to list five gaps as a free response, the most common themes were tenure, teaching, research, curriculum, and work life balance/time management. Additional questions assessed skills, qualities, resources, and faculty development techniques. Implications: This research is the first step in a program review and inquiry into the most effective and needed topics for a residency teaching certificate program.

Evaluating Pamphlets Emphasizing Cardiovascular Prevention to Assess a Health Literacy Module in a Pharmacy Elective. Elizabeth Harris, University of the Sciences, Grace L. Earl, University of the Sciences, Mohak Dave, University of the Sciences, Jessica S. Estriplet-Jiang, Essex County College. Objectives: The objective of this research is to evaluate the impact of a health literacy (HL) module on written patient education material developed by student pharmacists. Method: A 4-hour HL module was added to a cardiovascular (CV) prevention elective course to introduce HL concepts, develop patient communication skills, and apply these skills at the university campus community health fair. Faculty analyzed 2 cohorts of pamphlets before and after implementation of the HL module using readability tests, and $15 \mathrm{HL}$ criteria identified from the literature. Two faculty evaluators resolved discrepancies by consensus. Data were analyzed using parametric tests in this IRB approved project. Results: Pamphlets from 2013 pre- $(\mathrm{n}=15)$ and 2016 post- $(\mathrm{n}=23)$ implementation of the HL module were evaluated. The mean (SD) Flesch Grade level decreased favorably $(8.08+1.20$ vs. $7.26+1.37, \mathrm{p}<0.031)$ but Reading Ease Score $(51.4+8.62$ vs. $54.9+8.59, \mathrm{P}=0.11)$ was similar between pre-/post-module pamphlets. The mean HL criteria met was 9.2 pre- and 10.2 post-module $(\mathrm{p}<0.029)$. Pamphlets from the post-module cohort contained significantly more statistical information on $\mathrm{CV}$ risk factors: "Avoiding Large Amounts of Statistical Information" (86.6\% vs. $47.8 \%, \mathrm{p}<0.015)$. There were no differences for criteria "Selection of Simple Illustrations Appropriate for Audience" (60\% vs. 78.2\%). Implications: The students' ability to develop written patient education material using universal precautions to address HL barriers improved. This reinforces developing new methods to enhance skills in using "Plain Language," and selecting meaningful and literate-appropriate illustrations to support text content.

Evaluating the Impact of an Interprofessional Education Poverty Simulation. Brittney A. Meyer, South Dakota State University, Surachat Ngorsuraches, South Dakota State University, Teresa M. Seefeldt, South Dakota State University, Linda Kang, South Dakota State University, Leann Horsley, South Dakota State University, Bernadette Olson, South Dakota State University, Jessica Meendering, South Dakota State University, Mary Beth Zwart, South Dakota State
University, Kristine Ramsay-Seaner, South Dakota State University, Becky Jensen, South Dakota State University, Chris Comstock, South Dakota State University. Objectives: To evaluate changes in pharmacy students' attitudes towards poverty, understanding of poverty as a social determinant of health, and interprofessional competencies following participation in an interprofessional poverty simulation. Method: Second-year pharmacy students participated in the 3-hour Missouri Association for Community Action (MACA) Poverty Simulation with students from athletic training, counseling and human development, exercise science, nursing, and nutrition and dietetics disciplines. Students completed self-administered web-based pre- and post-surveys. Results: A total of 213 students attended the simulations with 108 completing both surveys. Participants were mostly white (92\%) females $(72 \%)$ with a median age of 22 . Close to half reported a family household income of $\$ 75,000+(42 \%)$. Of the matched results, 45 pharmacy students showed significant improvements in 6 of 16 items on the MACA survey examining attitudes towards poverty (overall change in mean score 2.39 to $2.65 ; \mathrm{p}=<0.001$ ) and 3 of 8 questions on Beliefs about the Relationship between Poverty and Health, specifically the behavioral influences related to motivation and structural influences related to stress and lack of health care. No significant differences existed for any of the 19 IPEC Competencies in the Values/Ethics Domain and Roles/Responsibilities Domain. Implications: This one experience may not have a significant impact on helping achieve the Center for the Advancement of Pharmacy Education (CAPE) outcome subdomain of Interprofessional Collaboration; however, the interprofessional poverty simulation can positively impact students' perceptions of poverty and understanding of poverty as a social determinant of health to enhance achievement of CAPE outcome subdomains of Cultural Sensitivity and Patient Advocacy.

Evaluating the Impact of a Peer Writing Accountability Group on Scholarly Productivity Among Pharmacy Faculty. Wei C. Yuet, University of North Texas Health Science Center, Annesha White, University of North Texas Health Science Center. Objectives: To determine the impact of a peer writing accountability group (WAG) on scholarly productivity among pharmacy faculty. Method: This study was approved by the University of North Texas Health Science Center IRB in June 2017. The WAG consisted of a small number of pharmacy faculty who met weekly from June 2017-August 2017 to work on scholarly activity. Pre- and post-surveys were administered to all participants. Descriptive analyses were performed to assess writing session duration and quantity of scholarly activity. A paired t test was used to determine differences before and after WAG participation. For all statistical tests, alpha level of significance was set at 0.05 . Qualitative data based on responses to open ended questions was content analyzed to identify themes. Results: Ten (100\%) faculty involved in the WAG completed pre- and post-surveys. Average writing session duration was 2.1 to 2.9 hours, while number of pages written, abstracts submitted, and presentations provided was 17-24, 1-2, and 1, respectively. Mean scores for the pre- and post-test were: average number of publications accepted $(2,1)$; writing frequency where $0=$ Daily and $3=$ Rarely $(2.4,1.6)$; current time management is sufficient $(3,1.7)$, current organization skills are sufficient $(2.3,1.5)$ where $0=$ Strongly Agree and $4=$ Strongly Disagree $(\mathrm{p}<0.05$ for all). Qualitative analysis of open ended responses revealed the themes "enjoyed participation," "protected time," and "increased productivity." Implications: WAG participation increased productivity, time management, and organization skills with regards to scholarship. WAG could be an effective tool to improve scholarly productivity at a pharmacy program. 


\section{American Journal of Pharmaceutical Education 2018; 82 (5) Article 7158.}

Evaluation of an Interprofessional Poverty Simulation Experience. Cheryl L. Clarke, Drake University, Shelby Derrick, Drake University. Objectives: To evaluate the results of poverty simulation exercises when conducted as an interprofessional education (IPE) activity. Objectives were to determine if participating students demonstrated changes in 1) Attitude toward poverty and 2) Perceptions of interprofessional teamwork. Method: Two Missouri Association for Community Action Poverty Simulation exercises were conducted for an interprofessional student mix including Doctor of Pharmacy, Doctorate of Occupational Therapy, and Bachelor of Science in Nursing students. Students were randomly assigned to simulated family units with various life circumstances, while ensuring a mix of healthcare professional students per family. The families simulated typical daily living activities during 4 periods designated as weeks. Following the simulations, students participated in debriefing sessions to discuss their experience, including questions specific to interprofessionalism. Prior to and following the simulations, students completed two surveys: Attitude Toward Poverty Short Form (ATP-SF) and Refinement of the Interprofessional Socialization and Valuing Scale (ISVS-9). Paired-samples t-tests were used to determine the results. Results: Statistically significant improvements in attitude toward poverty were noted in 14 items on the 21-item ATP-SF. The domains of stigma and structural perspective showed statistically significant improvement while the personal deficiency domain did not. Statistically significant improvements in student perceptions related to interprofessional teamwork were not found using the ISVS-9 scale. Implications: An improved attitude toward poverty was measured for the domains of stigma and structural perspective following participation in a poverty simulation. An improvement in interprofessional teamwork was not found. The ISVS-9 may not have been an appropriate tool for this purpose.

Evaluation of a Peer- and Self-Grading Process for Clinical Writing Assignments. Tara Storjohann, Midwestern University/ Glendale, Erin C. Raney, Midwestern University/Glendale, Elizabeth K. Pogge, Midwestern University/Glendale, Alyssa M. Peckham, Midwestern University/Glendale, Jeff Barletta, Midwestern University/ Glendale. Objectives: As pharmacy class sizes increase, faculty must develop new assessment strategies for essay writing assignments. This study evaluated accuracy and student perceptions of an innovative grading process that utilizes peer- and self-assessment. Method: Four SOAP note sessions were evaluated. Each session included a writing workshop, assessment workshop, and reflection session. For each assessment workshop students scored their note and a blinded peer's note using a grading form, facilitated by a faculty-led discussion. In a subsequent reflection session, students reviewed their peer- and selfassigned grades and could petition for faculty review if desired. The average self-, peer-, and final-grades were compared for each of the 4 SOAP note sessions using ANOVA. After the fourth session, students completed an anonymous 10-question Likert-scale survey regarding their perceptions of the process and 3 open-response questions. Survey results were analyzed with descriptive statistics. Results: Approximately 140 students participated. No difference was found between the average self-, peer-, and final-grades for all 4 sessions $(\mathrm{p}>0.05)$. The survey response rate was $65 \%(91 / 140)$. Survey questions were grouped into 3 themes. The majority of students either strongly agreed or agreed that sessions were well organized and effective $(\geq 84 \%)$, assessment workshops enhanced learning ( $\geq 68 \%$ ), and the scoring method was fair $(\geq 72 \%)$. The lowest score (mean 2.53 on a 4-point scale) reflected satisfaction with peer-provided feedback. When asked what they liked most, respondents most commonly cited that faculty-led review and discussion enhanced clinical knowledge.
Implications: The peer- and self-assessment process was accurate, well-received, and is transferable to other clinical writing assignments.

Evaluation of a Structured Interprofessional Education Program at a Non-Academic Community Hospital. Angela O. Shogbon, Mercer University, Bobby C. Jacob, Mercer University, Jonathan Perkins, DeKalb Medical Center, Matthew Hogan, DeKalb Medical Center. Objectives: To evaluate students' perceptions of physician to pharmacist interprofessional clinical education and its effectiveness towards their learning. Method: A 3-tier structured approach to interprofessional education (IPE) was designed consisting of daily pre-rounds, daily teaching rounds, and once to twice weekly lunchand-learn sessions between pharmacy and medical students on their Medicine clinical rotation at a non-academic community hospital. Preand post-surveys were administered assessing students' perceptions of physician and pharmacist collaboration in IPE using the Student Perceptions of Physician-Pharmacist Interprofessional Clinical Education, Version 2 (SPICE-2) instrument, and perceptions were rated on a 5-point Likert scale (strongly disagree $=1$ to strongly agree $=5$ ). Students' perceptions of the educational intervention were also assessed. Data were analyzed using descriptive statistics and pairedstudent's t-test for comparative data. Results: A total of 21 students, consisting of 12 fourth-year student pharmacists and 9 third-year medical students, provided informed consent and were enrolled over 6 months. The majority of students (81\%) were $25-29$ years, and $62 \%$ were females. On the SPICE-2 survey, even though the majority of students had positive perception scores, students showed significant improvement in their average perception scores on survey items related to the Interprofessional Education Collaborative competency on roles/responsibilities for collaborative practice $(\mathrm{P}<0.05)$. In addition, the majority of students found all three components of this structured IPE program effective to very effective (91-95\%). Implications: Expansion of this program to all pharmacy and medical students and incorporation of other healthcare professions within the practice site may aid with further enhancement of IPE and collaboration among the health professions.

Evaluation of Clinical Activities and Interventions Completed by Pharmacy Students During a Physician-Precepted APPE Rotation. Cynthia Moreau, Nova Southeastern University, Alejandro Nieves, Nova Southeastern University, Stacey M. Maravent, Nova Southeastern University, Tina Joseph, Nova Southeastern University, Genevieve Hale, Nova Southeastern University, Jennifer Steinberg, Nova Southeastern University. Objectives: To evaluate activities and interventions completed by pharmacy students during a physician-precepted advanced pharmacy practice experience (APPE) within an accountable care organization (ACO). Method: Students were part of an ACO-based APPE rotation block. Students first completed a four-week rotation in Ambulatory Care or Outpatient Cardiology precepted by pharmacy faculty followed by a four-week Interprofessional Quality Outcomes (IPQO) elective precepted by a primary care physician. Students documented daily tasks and interventions recommended and accepted by the physician. Patients were surveyed to assess perception and satisfaction with the pharmacy student visit. Additionally, each student was surveyed at the end of the their rotation experience. This study was approved by the NSU IRB. Results: Five students participated in the rotation with two physicians between August 2017 and January 2018. Students participated in a number of clinical activities, including medication reconciliations $(58.9 \%)$, disease-state focused visits $(26.3 \%)$, and medication/device counseling (14.7\%). Students completed 100 combined interventions, of which $98 \%$ were accepted by physicians. Overall, patients agreed 


\section{American Journal of Pharmaceutical Education 2018; 82 (5) Article 7158.}

that they felt comfortable speaking with pharmacy students and that the interaction was beneficial. Students perceived the rotation experience as positive and felt that their activities contributed to improved patient outcomes. Implications: Data suggests that physicians utilize pharmacy students for a variety of clinical activities. Interventions performed by pharmacy students in this setting have a high physician acceptance rate. Embedding pharmacy students within an ACO with a physician preceptor can foster interprofessional collaboration and promote team- and practice-readiness among pharmacy graduates.

Evaluation of Disease State Topics Taught in a Doctor of Pharmacy Curriculum Using the ACCP Toolkit. Krista L. Donohoe, Virginia Commonwealth University, Benjamin W. Van Tassell, Virginia Commonwealth University, Leigh A. Hylton-Gravatt, Virginia Commonwealth University, Donald F. Brophy, Virginia Commonwealth University, Gretchen M. Brophy, Virginia Commonwealth University, John D. Bucheit, Virginia Commonwealth University, Lauren M. Caldas, Virginia Commonwealth University, Ericka L. Crouse, Virginia Commonwealth University, Dave L. Dixon, Virginia Commonwealth University, Rachel W. Flurie, Virginia Commonwealth University, Sharon S. Gatewood, Virginia Commonwealth University, Jean-Venable R. Goode, Virginia Commonwealth University, Lauren Grecheck, Virginia Commonwealth University, Cynthia K. Kirkwood, Virginia Commonwealth University, Abigale T. Matulewicz, Virginia Commonwealth University, MaryPeace McRae, Virginia Commonwealth University, Laura M. Morgan, Virginia Commonwealth University, Nicole Omecene, Virginia Commonwealth University, Amy L. Pakyz, Virginia Commonwealth University, Kacie Powers, Virginia Commonwealth University, Emily P. Peron, Virginia Commonwealth University, Evan Sisson, Virginia Commonwealth University, Patricia W. Slattum, Virginia Commonwealth University, Casey S. Washington, Virginia Commonwealth University, Nancy S. Yunker, Virginia Commonwealth University, Kristin Zimmerman, Virginia Commonwealth University. Objectives: To assess the disease state topics covered in the Virginia Commonwealth University (VCU) Doctor of Pharmacy didactic curriculum. Method: The disease state topics proposed in the 2016 American College of Clinical Pharmacy (ACCP) Pharmacotherapy Didactic Curriculum Toolkit, known as the ACCP Toolkit, were compared to lectures taught in the Clinical Therapeutics Modules and Self-Care course during the 2016-17 academic year. Course coordinators were asked to use a Google Sheet to identify all Tier 1 (should be included in education and training), Tier 2 (additional skills may be required after graduation), and Tier 3 (may not be covered in didactic curriculum) disease state topics in required courses. An in-depth review using course syllabi was conducted to verify content in the courses. Results: Of the 13 Clinical Therapeutics Modules, Self-Care course, and Skills Lab, $99 \%(n=85)$ of the Tier 1 disease state topics from the ACCP Toolkit were covered in the didactic curriculum. Seventy-seven percent $(n=103)$ of the Tier 2 topics were covered. Of the Tier 3 topics, $16 \%(n=9)$ were covered in the didactic curriculum. Implications: Completing a comprehensive evaluation of the ACCP Toolkit disease state topics in our curriculum identified topics that are inadequately covered and conversely those included in the curriculum, but may be better left for students to learn on APPEs or during residency training. Comparing VCU's results with other colleges and universities may help evaluate the implementation of the ACCP Toolkit and improve the balance of disease state topics within the didactic curriculum.

Evaluation of Long Term Retention and Utilization of the SBAR Communication Technique. Thomas S. Franko, Wilkes University, Vicky Shah, Wilkes University, Ajay K. Bommareddy, Wilkes
University, Ka L. Hong, Wilkes University. Objectives: The aim of this study is to determine if students will utilize the SBAR communication technique during an interprofessional skills assessment one year following initial instruction. Method: Second-year pharmacy students were trained in the situation, background, assessment and recommendation (SBAR) communication technique. One year later, these students participated in an objective structured clinical examination (OSCE) focused on communicating a medication error to a prescriber. Along with the technical aspects of the case, students were evaluated on their utilization of each component of the SBAR technique to communicate with the provider. Descriptive statistics were used to analyze the data. Results: Sixty-nine students participated in the study. The mean score for the SBAR skill set utilized by the students was 3.65 with $85.5 \%$ of the students completing the interaction utilizing all four components of the technique. Implications: Students were able to demonstrate retention of the SBAR communication technique one year after initial instruction. Consideration should be given to train students in this technique early in the curriculum with regular intervals of assessment.

Evaluation of Scholarship Output Resulting From a Research Capstone Requirement. Margarita V. DiVall, Northeastern University, Elizabeth Yimam, Northeastern University, Jennifer Kirwin, Northeastern University. Objectives: A 2016 curricular revision allowed P3 students their choice of project to fulfill the required $4 \mathrm{SH}$ research capstone course. Capstone projects are guided by a common syllabus and completed under the mentorship of a faculty instructor. This study aimed to evaluate scholarly productivity derived from capstone projects. Method: A survey was designed to evaluate scholarly output from capstone projects conducted in AY2016-17, measured by number of poster/platform presentations and manuscript preparation/submissions. The December 2017 survey also asked about project characteristics and faculty perceptions regarding students' achievement of capstone course outcomes. Faculty instructors for all sections of the capstone course were invited to participate. Descriptive statistics were used to evaluate survey results. Results: Twenty-eight faculty (93\%) responded to the survey, representing 50 capstone projects. Sixty-eight percent of projects resulted in some form of scholarly output. Most commonly, posters were presented at the university's scholarship expo (66\%). Additionally, 17 (34\%) of the projects were presented at a national/regional conferences. Nineteen projects $(38 \%)$ were reported to be at various stages of manuscript preparation, one has been published and another has been accepted for press. Most faculty agreed that students were well prepared to conduct a research project, met capstone course outcomes, and $94 \%$ agreed that the outcomes justified the efforts of mentoring the project. Implications: The capstone course led to significant scholarly output despite the short time frame post project completion. The curricular change that required more faculty workload to advise capstone projects was successful in increasing scholarship opportunities for students and faculty.

Evaluation of Student Accuracy and Changes in Confidence From a Simulated Drug Utilization Review Process. Cortney M. Mospan, Wingate University, Katelyn M. Alexander, East Tennessee State University, Christopher Gillette, Wingate University. Objectives: To (1) assess the impact of a pharmacy skills lab on third-year student pharmacists' confidence in completion of a drug utilization review (DUR) during the dispensing process and (2) assess student accuracy in identifying the therapeutic issue in each case. Method: An electronic preand post- survey was administered to assess change in confidence and to collect case answers. The exercise included a lecture explaining the DUR process. Students then completed 12 simulated DURs with 


\section{American Journal of Pharmaceutical Education 2018; 82 (5) Article 7158.}

a faculty debrief. All responses were anonymous and matched via selfgenerated code. Identification of therapeutic issues are decision to dispense are summarized using descriptive statistics and Wilcoxon signed rank tests were used to analyze matched pre- and post- data. Kruskal-Wallis tests were used to analyze implications of duration of pharmacy experience on initial confidence. Results: A total of 75 surveys were usable for analysis ( $96 \%$ response rate). All six confidence components showed statistically significant improvement from pre- to post- intervention ( $\mathrm{p}=<0.0001-0.0333$ ). Previous pharmacy experience was not associated with intial student DUR confidence assessed via six skill components $(\mathrm{p}=0.0944-0.5404)$. Student ability to correctly identify the therapeutic issue in each DUR case ranged from $26.32 \%$ to $92.11 \%$ (average $66.34 \%$ ). Student intention to dispense the medication ranged from $14.67 \%$ to $82.89 \%$ (average $51.00 \%$ ), showing confounding issues affecting decision to dispense and evaluation of clinical significance of the interactions. Implications: A simulated DUR exercise was associated with increased student confidence regarding ability to perform a DUR. Student variance in dispensing decisions shows additional opportunity to refine DUR skills despite increased confidence.

Evaluation of Student Pharmacist Participation in Advanced Practice Experience (APPE) With a Dual Licensed PharmacistPhysician Assistant. Gary Manley, Roseman University of Health Sciences, Evan Williams, Roseman University of Health Sciences, H. Stephen Lee, Roseman University of Health Sciences, Elizabeth J. Unni, Roseman University of Health Sciences. Objectives: Many state boards of pharmacy prohibit direct preceptorship of student pharmacists by non-pharmacists, despite benefits reported in the literature regarding precepting by a non-pharmacist. Roseman University College of Pharmacy provides experiential rotation with a clinical faculty member who is a dual-licensed pharmacist-physician assistant (PhPAs). The purpose of this study was to understand the benefits of this advanced pharmacy practice experience (APPE) as perceived by the students. Method: Students who completed the rotation with the PhPA faculty member were invited to a focus group interview shortly before the completion of the PharmD program. A qualitative thematic analysis was conducted to determine various themes and trends from the interviews by three investigators. Results: The description of the experience by students from one focus group $(n=13)$ resulted in four major categories, including: (1) improved ability to interact and communicate with prescribers and patients, (2) improved ability to apply pharmacotherapeutic knowledge, especially for patients with more complex medical needs, (3) better understanding and appreciation of practice pressures faced by providers, and (4) increased desire to become more proactive in the medical care of patients and more supportive of the prescriber-patient relationship. Self-reported changes in perceptions and attitudes of the students towards prescribers, patients, and themselves were also identified. Implications: The student pharmacists who participated in this ambulatory rotation led by a duallicensed pharmacist-physician assistant, reported a favorable and unique learning experience. The study also brought out the need for increased self-awareness and advocacy for the profession.

Evaluation of Student Pharmacists' Clinical Interventions in Advanced Pharmacy Practice Experiences at a Nonteaching Community Hospital. Nicole L. Ryba, Fairleigh Dickinson University, Jesse B. Sullivan, Fairleigh Dickinson University. Objectives: To determine the number, type, and acceptance rate of student pharmacists' clinical interventions in advanced pharmacy practice experiences at a large, non-teaching community hospital. The secondary objective was to measure the value of interventions. Method: A retrospective review of clinical interventions of fourth-year (P4) student pharmacists $(n=64)$ assigned to faculty-driven acute care rotations over a 27-month period was performed. All interventions were analyzed for intervention type and acceptance rate. A secondary analysis was performed to assess hard dollar cost savings and assigned relative value unit (RVU). Results: A total of 64 student pharmacists attempted a total of 1411 interventions (22 interventions /student) with an acceptance rate of $81.0 \%$. Majority of interventions were classified as therapeutic $(n=676$, $48.0 \%$ ) followed by drug information and education $(n=314,22.2 \%)$. Total hard dollars saved was $\$ 5,462$ with majority of the interventions having an RVU of five $(n=709,50.0 \%)$ followed by three $(n=405$, 29.0\%). Implications: Student interventions were considered valuable and were well-received by physicians. These interventions also involved an overall cost savings to the institution.

Evaluation of Students' Personal and Professional Development in an Enhanced Student Advising Process. Justine S. Gortney, Wayne State University, Minakshi Lahiri, Wayne State University. Objectives: To evaluate performance of a novel Faculty Advisor Assessment of the Advisee (FAAA) tool within a didactic curriculum. Method: We developed a simple-to-use evaluation rubric, the FAAA, for longitudinal assessment of students on professionalism and personal development. The FAAA is a 5-item tool with questions about values, engagement, self-awareness, professionalism, and leadership. The anchors to all questions describe characteristics exhibited in the student that match categories of beginning, emerging or engaged; 2 have addition of not engaged. Prior to meetings with their advisor, advisees complete a semester-specific self-inventory that ties to advisee's learning and professional development and is discussed during advising. The FAAA is then filed by the advisor on EValue after the winter-semester meeting and provides feedback to students. De-identified aggregate cohort reports were run within EValue. The hypothesis was that for each year progress in didactic curriculum, a larger percentage of students would categorize as "engaged." IRB designated as exempt. Results: Data was analyzed for 261 students (P1 n=97, P2 n=80, P3 n=84) Greater than $90 \%$ of advisors completed FAAAs for before semester deadlines. Stepwise increases aligning to "engaged" were observed comparing class years in all 5 question characteristics measured (for example, values, P1-12, P2-38, P3-51\% were engaged respectively). Similar trends were observed for questions that had "not engaged" as an extreme. Implications: A simple to use 5-item tool showed cohort growth in personal and professional development. This tool could be used effectively to monitor student engagement and development of professional skills to promote and enhance student-success.

Evaluation of the Impact of a Novel, Hands-On Basic Science Exercise on Students' Learning. Yousif B. Rojeab, Ohio Northern University, Tarek M. Mahfouz, Ohio Northern University, Kelly M. Shields, Ohio Northern University, David H. Kinder, Ohio Northern University, Hannah Lamb, Ohio Northern University, Deirdre L. Myers, Ohio Northern University, Emily Loudermilk, Ohio Northern University. Objectives: Our students spend a significant amount of time learning pharmaceutics principles and the impact of $\mathrm{pH}$ on drug ionization, solubility and consequently oral absorption. While students demonstrate competency based on exam scores, they do not seem to retain this information very well over time. The primary objective of this study was to evaluate whether incorporating a novel, hands-on exercise would increase students' understanding of the impact of $\mathrm{pH}$ on drug solubility. Method: The Institutional Review Board granted this study an exempt status. Prior to beginning the exercise, students were given a pre-survey containing an informed consent and a 15question survey. The first five questions were subjective and asked 


\section{American Journal of Pharmaceutical Education 2018; 82 (5) Article 7158.}

each student to use a Likert scale to assess their comfort level with solubility concepts. Questions 6-15 were knowledge-based, pertinent to drug solubility. Afterwards, students were instructed on the procedure for the hands-on laboratory exercise. Finally, they were given a post-survey containing the same 15 questions and all responses were recorded. Results: The three main question groups (subjective questions, objective questions, and total survey) all showed statistically significant increases in score from the pre-survey to the post-survey ( $\mathrm{p}<0.001$, paired t-test, Minitab ${ }^{\circledR}$ version 18.1, Minitab, Inc.). However and when studied individually, those differences were attributed to seven of the 15 survey questions. Implications: Involving students in a hands-on, active learning exercise seem to significantly enhance their comfort level and knowledge base with regard to drug solubility and we intend to make this exercise a permanent component in our PharmD curriculum.

ExamSoft ${ }^{\circledR}$ Use as a Curricular Assessment and Learning Analytics Tool. Ashok E. Philip, Union University, Margarita V. DiVall, Northeastern University, Roopali Sharma, Touro College of Pharmacy-New York, Kimberly K. Daugherty, Sullivan University, Jaime L. Maerten-Rivera, University at Buffalo, The State University of New York, Bethany Kalich, University of the Incarnate Word, James G. Henkel, University of Saint Joseph, Sukhvir J. Kaur, California Northstate University, Surajit Dey, Roseman University of Health Sciences. Objectives: To describe trends in ExamSoft ${ }^{\circledR}$ data use for curricular assessment and personalized student feedback. Method: A Qualtrics ${ }^{\circledR}$ survey was distributed to US pharmacy programs with questions related to the use of ExamSoft ${ }^{\circledR}$ as an assessment tool. Aggregate data were analyzed using descriptive statistics. Results: Sixty-two programs (45\%) responded with 47 (78\%) using ExamSoft ${ }^{\circledR}$. Majority of programs require use of ExamSoft $\AA$ in all courses $(47 \%)$ or most courses (32\%). Programs report using ExamSoft ${ }^{\circledR}$ for secure online testing (93\%), to collect learning outcomes data $(93 \%)$, accreditation (80\%), and to enhance student learning (79\%). Most $(70 \%)$ mandate exam item tagging, while the rest recommend it. Instructors are most commonly responsible for tagging (80\%) and $87 \%$ of programs provide faculty development to support item tagging. Tagging category systems are complex with majority tagging to Bloom's taxonomy, program outcomes, CAPE 2013 outcomes, and ACPE Appendix 1 content areas. ExamSoft ${ }^{\circledR}$ data are being used to document student learning (62\%), justify curricular revision (49\%), and to modify instructional methods and course assessments (34\%). Student learning analytics reports are released by $81 \%$ of programs, although only $28 \%$ train students on interpretation and $6 \%$ require students to view these. Five programs (11\%) report having item tagging validation process in place and being confident in validity of tagging. Implications: Data suggest that many are using potentially erroneous ExamSoft data to revise curricula and instructional methodologies, document achievement of learning outcomes, and provide feedback to students. Further exploration of best practices for item tagging, quality assurance of tagging, and data utilization is warranted.

Exploring Curricular Integration in Pharmacy Schools: A Survey of Integration. Bradley Wright, Auburn University, Julaine Fowlin, Auburn University, Brittany Horton, Auburn University, Rachel Horne, Auburn University, Salisa C. Westrick, Auburn University. Objectives: Accreditation Council for Pharmacy Education (ACPE) recommends an integrated curriculum. This study aims to investigate curricular integration in US pharmacy schools, including identifying the organizational thread used to integrate knowledge across the curriculum, determining what pedagogies are used, determining how pharmacy schools evaluate the success of integration, and determining the challenges and solutions. Method: This institutional review board approved, cross-sectional study employs a mixed methods approach in two phases. Phase 1 consists of an online survey and will be reported here. Phase 2 consists of interviews with a sample of participants from phase 1 . The top 50 pharmacy schools in the US according to the US News and World Report were targeted, with a goal sample population of 30 schools. Schools including the terms "integrative, integration, or integrated" in curriculum information on the school's website were included in the sample population. Results: The total response rate to Phase 1 was $46 \%(n=14)$. The findings indicate that most schools had varying levels of integration and diverse pedagogies. The majority indicated using organ systems as the primary way to achieve curricular integration, with pharmacologic drug class being the least utilized. The most common methods for determining success were similar across programs, with all schools using residency match rates and few schools utilizing GPA (23\%). Numerous challenges, such as workload were reported with frequent and open communication with all stakeholders being a dominant solution. Implications: Findings from Phase 1 of this study can be used as an implementation guide for pharmacy schools to achieve curricular integration.

Face-Validity of AACP's Entrustable Professional Activities for New Pharmacy Graduates: Survey of Experienced Pharmacy Preceptors. Stuart T. Haines, The University of Mississippi, Brenda L. Gleason, St. Louis College of Pharmacy, Amy L. Pittenger, University of Minnesota, Melissa S. Medina, The University of Oklahoma, Stephen B. Neely, The University of Oklahoma. Objectives: Determine the face validity of the core entrustable professional activities (EPAs) for new pharmacy graduates published by the American Association of Colleges of Pharmacy in 2017. Method: A 28-item questionnaire was sent to experienced pharmacy practitioners affiliated with four schools of pharmacy. In addition to demographic information regarding education, training, credentials, and practice setting, participants were asked whether each EPA statement was pertinent to pharmacy practice and an expected activity that all pharmacists should be able to perform. Questions regarding the secondary attributes of the EPA statements examined whether the activity was observable, measureable, transferable to multiple practice settings, and an integration of multiple competencies. Results: The questionnaire was distributed to 137 eligible participants and 71 usable survey responses were received. Participants consistently agreed ( $\geq 75 \%$ agreement) that the fifteen EPA statements for new pharmacy graduates describe activities that are pertinent to pharmacy practice and that pharmacists are expected to perform. A consistent level of agreement was observed regardless of preceptor's employment with a college or school, board certification status, or completion of postgraduate training and no statistical differences in level of agreement were found based on these attributes. There was consistent agreement $(\geq 60 \%)$ across the four schools. No statistical differences in agreement were found between preceptors who practice in acute versus ambulatory care settings. Implications: The fifteen core EPA statements have strong face validity. Experienced pharmacy preceptors believe the EPAs are pertinent to pharmacy practice and pharmacists should be expected to perform these activities, regardless of practice setting.

Facilitation of a Multisite Topic Discussion Series Using WebBased Conferencing Technology. Lydia C. Newsom, Mercer University, Sweta M. Patel, Mercer University, John D. Bucheit, Virginia Commonwealth University. Objectives: Incorporation of technology into didactic curriculum is required by the Accreditation Council for Pharmacy Education. However, the use of technology during Advanced Pharmacy Practice Experiences (APPE) is less well defined. 


\section{American Journal of Pharmaceutical Education 2018; 82 (5) Article 7158.}

The primary objective of this study was to assess students' perceptions of learning through a multisite topic discussion (TD) series using webbased conferencing during ambulatory care APPEs. Method: A five session faculty-led TD series was conducted during each five week Ambulatory Care APPE at three distinct clinical sites. Students were assigned readings and case-based activities to complete prior to each TD. The TD series utilized $\mathrm{WebEx}{ }^{\circledR}$ that provided audio and visual feeds for all attendees. At the end of the TD series, each student completed an online survey to assess their perceptions of learning. Results: A total of 20 students completed the survey. The majority of students felt that web-based TD series enhanced their learning ( $N=19,95 \%$ strongly agreed or agreed). All students stated the TD series enhanced their confidence to provide patient care and think critically. While $70 \%$ of students $(\mathrm{N}=14)$ stated they would prefer to learn as a web-based community, $60 \%$ of students $(\mathrm{N}=12)$ felt that this format introduced unnecessary technological complications. Implications: The faculty-led web-based TD series during ambulatory care APPEs enhanced student perceptions of learning and confidence in providing patient care. To ensure this technology is a viable format, technological issues must be minimized. Future studies assessing the impact of web-based TDs on student learning are needed.

Faculty and Student Perspectives on Collaborative Design and Teaching in an Integrated Curriculum. Bradley Wright, Auburn University, Julaine Fowlin, Auburn University, Lea S. Eiland, Auburn University. Objectives: Interdisciplinary collaboration between faculty in course design and teaching is necessary in an integrated curriculum, but can also be challenging. The process used to develop and engage interdisciplinary teams of faculty who designed and taught in a new curriculum is described as well as faculty and student perceptions of this integration. Method: A new integrated curriculum, referred to as the Practice Ready Curriculum, was designed and developed by interdisciplinary faculty teams. The teams were further sub-divided into interdisciplinary teaching teams for the design and teaching of individual disease state units within each course. The new curriculum was implemented in Fall 2017. Faculty perceptions of the collaborative design and teaching process were identified through surveys. In addition, student feedback through the use of course evaluations, unit instructor evaluations, and surveys (asking them to self-report their understandings and what helped them to learn) was obtained and analyzed. Results: Thirty faculty members participated with representation from all three academic departments. Faculty reported that they enjoyed collaborative teaching and felt it created a richer experience for students. Students also reported that they enjoyed seeing the connections between all disciplines in one experience. Time constraints was the biggest challenge and resulted in some inconsistencies with teaching units. However, through a systematic course review process, improvements will be made to future semesters. Implications: The systematic process used as well as lessons learned from faculty and student feedback can assist other schools in implementing collaborative teaching needed to achieve true curriculum integration.

Gamification of Patient Cases to Simulate Longitudinal Chronic Disease Management. Rebecca Schoen, Texas Tech University Health Sciences Center, Wajiha Khan, Texas Tech University Health Sciences Center, Lisa Chastain, Texas Tech University Health Sciences Center. Objectives: At Texas Tech University Health Sciences Center, a third-year pharmacy ambulatory care elective focuses on chronic disease state management. Innovative teaching methodology strived to integrate gamification with longitudinal patient care management to facilitate application of material with decision making and critical thinking. This pilot project assessed the impact of gamification on student self-reported confidence and abilities in anticoagulation and diabetes management. Method: Students completed game-based activities on two separate days for antidiabetic and anticoagulation management. Both activities required longitudinal management of a fictitious patient allowing different therapy selections and associated outcomes. Incorrect or dangerous selections presented reflection opportunities before choosing an alternate pathway. A post-game survey collected student reported confidence and improvement in abilities of disease state objectives. Demographics, satisfaction, and engagement were also addressed. Results: Of the 57 enrolled students, 50 students completed the diabetes survey and 42 students completed the anticoagulation survey. Approximately half of the students had prior ambulatory care rotations. Overall, seventy percent and seventy-seven percent of students felt more confident managing non-insulin therapies and insulin therapies, respectively. Seventy-eight percent and sixtynine percent of students felt more confident managing warfarin and peri-procedural anticoagulation. For the diabetes case, eighty-eight percent enjoyed participating and ninety-two percent felt the game made lecture material more relevant. For the anticoagulation survey, eighty-three percent and eighty-eight percent agreed with these measures. Implications: Students indicated improved confidence and skills, enjoyment, and engagement. Future directions could include comparison to other active learning methodologies and objective measurement of skills.

Health Care Professionals' Decision-Making to Recognize Potentially Inappropriate Prescribing in Renal Insufficiency. Nervana Elkhadragy, Purdue University, Amanda P. Ifeachor, Richard L. Roudebush VA Medical Center, Julie B. DiIulio, Applied Decision Science, Karen J. Arthur, Richard L. Roudebush VA Medical Center, Laura Militello, Applied Decision Science, Michael Weiner, Richard L. Roudebush VA Medical Center, Peter A. Glassman, VA Greater Los Angeles Healthcare System and University of California, Los Angeles, Alan J. Zillich, Purdue University, Rachel Dismore, Richard L. Roudebush VA Medical Center, Alissa Russ, Purdue University. Objectives: To examine the cognitive strategies that health care professionals (HCPs) use to recognize medication-related problems in patients with renal insufficiency. Method: Cognitive task analysis (CTA) interviews were conducted with HCPs (physicians, nurses, and pharmacists) who had recently addressed a potential medication-related problem in patients with renal insufficiency. We used inductive, qualitative analysis of CTA interviews to identify cues that HCPs used to recognize potential problems related to nephrotoxic or renally eliminated medications. Results: We completed CTA interviews with 21 HCPs. Most HCPs used multiple cues to recognize potential medication-related problems. In spite of the prevalence of computerized alerts, they were not the most common recognition method (only 2 HCPs). Rather than alerts, the most common method was 'active inspection' (13 HCPs), where participants initiated a search for patients' "renal drug" issues. Another common cue (8 HCPs) was noticing abnormal laboratory values for renal function. Barriers to recognizing problems included the amount of time needed to inspect patients' information in electronic health records (EHRs), such as renal function history, and documented notes from other HCPs regarding medications to avoid and patient counseling about medications. Participants indicated a need for improved collaboration between HCPs and advanced methods for quickly finding key information in EHR documentation. Implications: This study provided important insight into HCPs' decision-making processes and identified considerable challenges to recognizing potential renal drug problems in a timely fashion. Results emphasize the importance of easy 


\section{American Journal of Pharmaceutical Education 2018; 82 (5) Article 7158.}

access to key patient information and call for stronger efforts to improve alerts and enhance collaboration between HCPs.

Identifying Patients With Uncontrolled Asthma Via Telephone Interview. Mimi Mukherjee, MCPHS University-Worcester/ Manchester, Deval Shah-Canning, Edward M. Kennedy Community Health Center. Objectives: Objectives: This study was conducted to identify and characterize the adult patients with uncontrolled asthma at the Edward M. Kennedy Community Health Center- Framingham (EMKCHC-Framingham) and to assess whether a telephone interview consisting of 6 questions based on the Asthma Therapy Assessment Questionnaire and national asthma guidelines was an effective way of identifying them. Data on asthma control is limited in indigent patients. EMKCHC-Framingham serves a multicultural and multilingual indigent population. All patients seen there have a primary care provider and access to medications at low cost. Method: Method: Fifteen pharmacy students on their advanced pharmacy practice experiences conducted the telephone interviews, often via telephonic interpreter since $69.2 \%$ of the patients did not speak English. The answers to the questions were tied to points and patients with a score of greater than 1 were determined as having uncontrolled asthma. Each patient with uncontrolled asthma was called by an EMKCHC-Framingham team member who linked them to appropriate care. Results: Results: Of the 266 patients with asthma that were eligible to be interviewed, 21 patients declined, 127 patients were interviewed, and 119 patients were deemed unreachable via phone during daytime business hours. Of the patients interviewed, 53.5\% had uncontrolled asthma. Implications: Implications: In conclusion, the telephone interview effectively identified patients with uncontrolled asthma and improved their care if patients could be reached by phone. However, over half were not interviewed, which means other interventions are needed to ensure that the majority of patients are assessed.

Impact of a Health Information Technology Skills Lab on Second Year Student Pharmacists. Linda D. Logan, The University of Georgia, Andrew Darley, The University of Georgia, Gregory B. Seagraves, The University of Georgia. Objectives: To describe the development and results of a health system informatics skills lab designed to (1) expose 2nd year PharmD students to technology used for medication distribution, use, and management processes and (2) enhance student readiness for operational components of health system practice experiences. Method: College of pharmacy faculty partnered with industry representatives to develop a skills lab designed to provide students hands-on exposure to current and emerging health system informatics at no cost to the institution. The lab was structured to include a discussion on the importance of health technology in optimizing medication use processes, small group hands-on product demonstrations on campus, and debriefing with college faculty. Students completed a pre- and post-survey describing the impact of this activity on their understanding of health system information technology. Paired t-tests and descriptive statistics were used to analyze study results. Results: Of the 137 students enrolled in the course, 133 completed pre-and post-surveys. Student responses showed statistically significant improvement $(\mathrm{p}<0.05)$ in items rating understanding of different aspects of health information technology (use, safety, efficiency, cost impact, working knowledge of technology, and benefits to the pharmacy profession). Students agreed or strongly agreed the design of this lab improved their understanding $(97 \%, 129 / 133)$ and confidence in using $(89.5 \%, 119 / 133)$ health technology. Implications: Partnering with industry representatives to provide hand-on experience successfully enhanced student knowledge and confidence in the use of health system technology. Based on student feedback, this lab will continue to be offered to future classes.

Impact of an Academic Pharmacy Elective on Student Interest in a Career in Academia. Gina Bellottie, Thomas Jefferson University, Lindsay M. Fitzpatrick, Thomas Jefferson University, Elena M. Umland, Thomas Jefferson University. Objectives: To determine the impact of participation in an academic pharmacy elective on student interest in pursuing academic careers. Method: Student interest in careers in academia was measured using a 7-item survey administered on the first and last class days in an academic pharmacy elective delivered in Spring 2014, 2015, and 2016. Students rated how likely they were to consider a career in academia on a scale from 1 to $10(1=\mathrm{I}$ have not given it any thought; $10=\mathrm{I}$ have given it lots of thought and it is high on my list of career options) and identified which factors would influence them to pursue a career in academia and what they perceived as the main responsibilities and top attributes of an individual with an academic pharmacy career. Pre- and post-course results were compared and a thematic analysis was completed. Results: Fifty-five students completed both surveys: $n=16$ (2014), $n=19$ (2015), and $n=20$ (2016). After course completion, $76.3 \%$ of students maintained or increased their interest in pursuing a career in academia (21.8\% maintained and $54.5 \%$ increased). The most commonly identified reasons to pursue a career in academia included love for teaching $(n=23$, precourse; $n=24$, post-course) and altruism ( $n=19$, pre-course; $n=27$, post-course). Implications: In order to cultivate the next generation of pharmacy educators, it is important to understand the motivations and perceived barriers towards pursuing a career in academia, as well as provide education related to this career option.

Impact of an Interprofessional Education Session on Bridging Gaps in Asthma Care: A Pilot Study. Christina Gauthreaux Angel, Washington University in St. Louis, Justinne Guyton, St. Louis College of Pharmacy, Sara K. Richter, St. Louis College of Pharmacy, Patricia Kao, Washington University in St. Louis, Megan Wren, Washington University in St. Louis. Objectives: To evaluate the change in asthma and inhaler-related knowledge in addition to student perceptions of physician-pharmacist interprofessional collaboration, following an interprofessional education (IPE) activity. Method: This cohort pilot study enrolled second-year medical and pharmacy students after completion of pulmonology-related coursework. Students worked in groups of four (two medical students paired with two pharmacy students) to complete a patient case focused on asthma management. Groups were provided a patient mannequin, stethoscopes, and inhaler demonstrators during the activity. Each profession was instructed to provide education within their group; medical students provided instruction about the physical exam and lung sounds, while pharmacy students provided instruction about inhaler devices and administration technique. Asthma and inhaler-related knowledge was assessed preand post-activity with a pulmonary quiz developed by the study investigators. Student perceptions were assessed pre- and post-activity with the Student Perceptions of Interprofessional Clinical Education (SPICE) questionnaire. Differences in knowledge were analyzed with paired and unpaired t-tests, as appropriate. Results: Six students from each profession participated in the activity. Medical and pharmacy students had significantly higher mean scores on the post-activity pulmonary quiz compared to pre-activity (16.5 vs. 13 and 16.7 vs. 14.2, respectively). Overall impressions on the post-activity SPICE questionnaire improved, and every student "strongly agreed" that participating in IPE activities enhanced their future ability to work on interdisciplinary teams and that IPE activities should be used in 


\section{American Journal of Pharmaceutical Education 2018; 82 (5) Article 7158.}

education. Implications: Incorporation of IPE activities may be beneficial in enhancing student knowledge and perceptions of IPE experiences.

Impact of a Pharmacist-Led Intervention Program on the Readmission of African-American Men With Heart Failure. Pamela M. Moye, Mercer University, DeAngelo McKinley, Mercer University, Ayman Akil, Mercer University. Objectives: This study was aimed to evaluate whether the incorporation of a pharmacist component to the standard of care, would reduce readmission rates in African American males with heart failure. Method: A prospective study using a historical control was conducted at an urban community hospital. Patients were enrolled from May 2012 to December 2015. The intervention group received standard of care, with the addition of a pharmacist. The pharmacist's contribution included: providing medication reconciliation, discharge medication counseling, and post-discharge follow-up on days 14 and 30. The dataset was modeled using the machine learning tools available for Python 3.6. All-cause readmission within 30-days was the outcome variable. Several classifiers were tested during model building including random forest, lasso, k-nearest neighbor $(\mathrm{KNN})$, and support vector machine. Results: The KNN classifier performed best, and able to correctly classify readmission status in $82.5 \%$ of patients with a C-statistic of 0.82 . The intervention was found to be predictive of reduced 30-day readmission. Other attributes found to be predictive were ejection fraction $<40 \%$, obesity, dyslipidemia, pacemaker, nitrate and other cardiovascular medication use, and smoking. Implications: The modest predictive ability of the KNN model suggested that the listed attributes were associated with increased 30-day readmission, and the pharmacist-led intervention program was predictive of reduced 30-day readmission in African American men with heart failure. Future studies can focus using this type of pharmacist-led intervention program to reduce readmission rate of other disease states that are prevalent in African-American men.

Impact of a Relaxed Absence Policy on Class Attendance. Kim G. Adcock, The University of Mississippi, Liz A. Carlson, The University of Mississippi. Objectives: The third-year pharmacotherapy course is taught in a problem-based learning format through small group sessions. Since participation in this format is collaborative and interdependent, absences are detrimental to team learning. Students absent from small group sessions are required to submit an absence form indicating reason for absence. In the 2017-2018 academic year, the absence policy was relaxed to be more inclusive of personal life experiences. The impact of a relaxed absence policy on student attendance in a small group, problem-based learning course was determined. Method: A retrospective review of absences submitted by students from 2014 through Fall semester 2017 was conducted. Absences were categorized based on reason provided including medical illness, funerals, weddings, and professional conferences. The 2017-2018 academic year was compared to each of the previous 3 academic years. Results: A total of 250 absence forms were submitted over 3.5 academic years $(46,2014-2015$; 59, 2015-2016; 69, 20162017; 76, Fall 2017-2018). Of these 250 absences, $30 \%$ were related to medical illness, $12 \%$ to funerals, $4 \%$ to weddings, and $32 \%$ to professional conferences. Professional conferences accounted for the greatest increase in student absences in 2017-2018 (49\%) compared to the other academic years $(15 \%, 37 \%, 22 \%$ respectively). Implications: Even with an absence policy and a pedagogy dependent upon student interactions, absenteeism continues to rise with each subsequent class. A relaxed absence policy for personal life experiences (ie, weddings) had little impact on this increase. Instead, the school's promotion of leadership and professional development appear to have a greater impact on student absenteeism.

Impact of SOAP Note Teaching Styles on Student Perception and Knowledge. Dawn M. Battise, Wingate University, Heather A. Kehr, Wingate University, Sharmeen Ali, Wingate University, Christian R. Dolder, VA Northern California Healthcare System. Objectives: The objective of this study was to assess if incorporating more interactive teaching skills and peer-evaluation to teach SOAP notes improved student perceptions of preparedness to write SOAP notes and/or student knowledge. Method: In 2016, SOAP notes were taught through written case examples reviewed in class. Feedback was offered on a graded project. In 2017, SOAP notes were taught using written and video case examples. External feedback was offered from guided peer-evaluation and on a graded project. A questionnaire gathered student demographics, perceptions of ability to write SOAP notes and objective measures of knowledge. A final exam assessed knowledge. Student demographics, perceptions and knowledge were compared between years. Results: No significant difference was found in student perceptions of overall ability to write a SOAP note with either teaching method $(\mathrm{p}=0.488)$. A significant difference was found in student perceptions regarding skills to write the subjective $(\mathrm{P}=0.001)$ and objective $(\mathrm{p}=0.004)$ portions of the SOAP note. However, post-hoc analysis found no significant differences. No significant difference was found in knowledge using survey questionnaire $(p=0.423)$ or final exam scores $(p=0.810)$. Implications: ACPE Standards highlight the role of communication; however, little data exists evaluating ideal documentation teaching methods. Some data indicate more interactive cases and peer-feedback may increase student interest and confidence. This study demonstrates two methods utilizing varying interaction and feedback that are equally effective in teaching SOAP notes in regards to student perceptions and knowledge.

Implementation of a Multi-Station Diabetes Self-Care Workshop to Assess First-Year Student Pharmacists' Competency Using Standardized Checklists. Christina Mnatzaganian, University of California, San Diego, Panteha Kelly, University of California, San Diego, Eduardo S. Fricovsky, University of California, San Diego, Nathan A. Painter, University of California, San Diego, Candis M. Morello, University of California, San Diego, Jennifer Namba, University of California, San Diego, Renu F. Singh, University of California, San Diego. Objectives: To implement and evaluate the effectiveness of a multi-station diabetes workshop incorporating standardized checklists within a self-care course for student pharmacists. Method: In the 2016-2017 academic year, a multi-station diabetes workshop was implemented as part of a comprehensive Diabetes Self-Care Education Program in a Pharmacy Practice (PP) course. PP faculty identified 8 key diabetes-related skills and developed checklists to assess student competency. Fifty-seven first-year students completed the 2-hour workshop facilitated by 8 faculty and volunteer preceptors. Workshop topics included insulin pharmacokinetics and administration, self-monitoring of blood glucose, interpreting a logbook, hypoglycemia treatment, and nutrition. Two weeks prior to the workshop, students attended lectures on diabetes topics and completed home assignments that included home glucose monitoring and watching a pre-recorded lecture and faculty-developed demonstration videos. Students were divided into 8 groups and rotated through stations in 15-minute increments. Facilitators at each station reviewed skills and demonstrated techniques. Students practiced skills using peer-to-peer teach back and then facilitators signed off on competencies. Results: Exam grades assessing diabetes self-care topics improved (92\% in 2017 versus 84\% in 2016). Students demonstrated 


\section{American Journal of Pharmaceutical Education 2018; 82 (5) Article 7158.}

knowledge retention on a diabetes OSCE in the following quarter with a mean score of $89.5 \%$ in their ability to identify, assess, and resolve hypoglycemia. Implications: Implementing a multi-station diabetes workshop with standardized checklists maximized exposure to diabetes self-care management within a limited time frame and standardized evaluation of student competencies. The workshop improved diabetes exam performance and diabetes knowledge retention was observed.

Implementation of a Strengths-Based Leadership Program for Third-Year Pharmacy Students: A Pilot Study. Amy Pick, Creighton University, Shawn M. Cook, Creighton University, Kimberley J. Begley, Creighton University, Kevin T. Fuji, Creighton University. Objectives: To develop and implement a strength-based leadership program for third-year pharmacy students that enhances their professional and personal development and addresses ACPE 2016 Standard Section 4. Method: Twenty students were randomly selected and participated in three, one-hour workshops on strength-based leadership. Students completed the Clifton Strengths for Students assessment prior to participation, receiving access to their top five strengths. The sessions were facilitated by two investigators who had completed strengthbased coaching, and focused on understanding, applying and incorporating strengths in leadership. Facilitation of topics occurred through group interaction, hands-on-application, individual reflection, and dialogue. After each session students submitted a leadership portfolio that included self-awareness and leadership-based questions. A preand post-leadership perception survey was administered to all students participating in the leadership program. Quantitative data of the survey was analyzed descriptively. Results: Twenty-students completed the strength-based leadership program. The student feedback for this program was overwhelmingly positive. Pre-leadership survey results showed students had diverse perceptions of leadership and limited knowledge of strengths. Throughout this program, student views on leadership evolved with $100 \%$ of students viewing themselves as a leader in the post-assessment survey. The students could articulate and apply their strengths to their professional and personal life. Implications: This pilot study suggests a strength-based leadership program can increase the students' awareness of their own and others' strengths and may enhance their leadership skills. This program may be incorporated into the school's professional development course to address the ACPE Standard Section 4.

Implementing and Evaluating Hand Hygiene Practices in a Pharmacy Skills Lab. Nicholas Zupec, Concordia University Wisconsin, Lynne A. Fehrenbacher, Concordia University Wisconsin. Objectives: To determine if educating students on the importance of proper hand hygiene and evaluating them via a rubric translates into improved compliance with appropriate hand hygiene practices in a pharmacy skills lab. Method: Hand sanitizer dispensers were installed inside the entrance of each patient care simulation room in a school of pharmacy. Signage was placed on the door and above each dispenser reminding students to perform hand hygiene both before and after simulated patient encounters. The P1 and P2 students were formally educated on proper hand hygiene and graded via a rubric. The P3 students did not receive education beyond the posted signs and were not aware that they were being evaluated via the rubric. Results: The compliance rate with proper hand hygiene criteria varied by cohort. Students who received formal education and were aware of rubric grading performed better than those who did not. First and second year students met criteria for proper hand hygiene $97.1 \%$ and $92.5 \%$ of the time respectively. The $\mathrm{P} 3$ students sanitized their hands appropriately only $10.1 \%$ of the time. Implications: Formally educating students on the importance of proper hand hygiene and evaluating them via a rubric resulted in a significant increase in compliance with appropriate hand hygiene practices in a pharmacy skills lab.

Implementing Mental Health First Aid Training Into the Doctor of Pharmacy Curriculum. Amber D. Frick, University of North Carolina at Chapel Hill, Suzanne C. Harris, University of North Carolina at Chapel Hill, Heidi N. Anksorus, University of North Carolina at Chapel Hill, Charlene Williams, University of North Carolina at Chapel Hill, Leah Osae, University of North Carolina at Chapel Hill, Philip T. Rodgers, University of North Carolina at Chapel Hill. Objectives: Engaging strategies to improve student pharmacist views, including Mental Health First Aid (MHFA), are necessary to revolutionize perceptions, confidence, and knowledge when interacting with individuals with mental illness and to subsequently enhance patient care. Method: We surveyed third-year student pharmacists enrolled in a required patient care experience course who also participated in MHFA training. The survey instrument examined attitudes, social distancing, health literacy, and empathy towards individuals with mental disorders. Student pharmacists were also surveyed on their self-perceived capability in performing tasks related to the ALGEE acronym, a mnemonic for the five-step action plan central to MHFA. Paired pre- and post-intervention responses were analyzed with Wilcoxon signed-rank tests. Results: Twenty-one students were included in the first cohort of MHFA training. Of student pharmacists completing the survey (response rate 95\%), 95\% agreed that MHFA training will be useful in their personal life and during their clinical immersion experiences, and 100\% agreed that MHFA training will be useful in their professional lives and the doctor of pharmacy curriculum. Significant increases from pre- to post-survey responses occurred in student pharmacists' confidence in helping someone who is developing a mental health problem or experiencing a mental health crisis $(p=0.0008)$, assessing an individual for risk of suicide or harm $(p=0.001)$, giving reassurance and information $(p=0.0007)$, encouraging appropriate professional help $(p=0.002)$, and encouraging self-help or additional mental health support strategies $(p=0.04)$. Implications: Implementation of MHFA in the curriculum is a feasible solution in helping prepare student pharmacists to support peers and members of the community with mental illness.

Improved Learning Experience With Modified Case Studies Courses in a Professional Pharmacy Program. Jiehyun Lee, Philadelphia College of Osteopathic Medicine, Sonia A. Thomas, Philadelphia College of Osteopathic Medicine, Drew W. Cates, Philadelphia College of Osteopathic Medicine, Candis McGraw-Senat, Philadelphia College of Osteopathic Medicine, Louise Jones, Mercer University. Objectives: Six Case Studies (CS) courses at PCOM School of Pharmacy are offered to pharmacy students during their second and third years (one per trimester). These courses have been constantly changing in attempts to improve overall design of the courses. However, the frequent changes from one CS course to another have led to inconsistency and poor learning experience for the students. The purpose of this study is to assess student learning experience between the newly modified CS courses during the 2017-18 academic year (CSAY17-18) and the previous CS courses during the 2016-17 academic year (CSAY16-17). Method: CS courses 2 through 5 were redesigned to have same course structure, schedule, and assessment methods (quiz, SOAP note, and short-answer questions addressing medicationrelated problems), as well as improved faculty-student interactions. Survey questions were developed to evaluate and compare student learning experiences between CSAY16-17 and CSAY17-18. The survey was administered to pharmacy students, who had completed both designs of CS courses. Results: 72 students have completed the survey $(80 \%$ response 


\section{American Journal of Pharmaceutical Education 2018; 82 (5) Article 7158.}

rate). The survey shows the students prefer the following components of CSAY17-18 over CSAY16-17: classroom small group discussion (94.4\%), faculty-facilitated case review (93.1\%), and detailed objective SOAP note rubric (97.2\%). The survey also shows CSAY17-18 encouraged more collaboration within a group, provided better learning experiences, and resulted in more student participation than CSAY16-17. Implications: The students overall prefer the format of the newly modified CSAY17-18 over the previous CSAY16-17. The newly modified CS courses also provide better learning experiences for the students.

Improving Empathy in Pharmacy Learners Through the Use of a Polypharmacy Simulation Exercise. Amie Blaszczyk, Texas Tech University Health Sciences Center, Rebecca Mahan, Texas Tech University Health Sciences Center, Jamie McCarrell, Texas Tech University Health Sciences Center, Rebecca Sleeper, Texas Tech University Health Sciences Center. Objectives: Empathy is key to effective and meaningful patient interactions and outcomes. The discordance in age and health-status between the majority of pharmacy learners and the older adult patient may impact empathy when a patient struggles with adherence/persistence. The Jellybean Polypharmacy Simulation Exercise (JPSE) was designed to simulate the experience of managing a complex medication regimen, with the hope empathy would be inculcated in pharmacy learners. Method: The JPSE is an 8-"prescription" regimen students manage for 7 consecutive days. All 3rd-professional year pharmacy students $(2015-2017)$ completed this exercise within a required course. The Kiersma-Chen Empathy Scale (KCES) and assessment of perceived/actual difficulty of the regimen were collected before and after the exercise. Open-ended questions sought input on lessons learned after the exercise. Results: Participants $(n=415)$ significantly improved $(p<0.001)$ in KCES scores from pre- to postintervention. Changes in the perceived/actual difficulty question were highly inversely correlated to their values at enrollment $(\mathrm{rho}=-0.70$, $\mathrm{p}<0.001$ ); participants who rated less difficulty at pre-intervention showed larger differences at post-intervention. Open-ended responses reflected themes of improved empathy and quality of life impact. Implications: This simulation activity had a positive impact on overall empathy and open-ended responses reflected personal growth in many participants. Interestingly, individuals who showed the largest change in their reflection on the difficulty of the regimen also showed the largest positive change in KCES scores. The JPSE is relatively inexpensive, easy to set up and can increase empathy for those managing complex medication regimens, especially those who may overestimate their ability to manage it.

Improving Student Perception of a Top (200) Drugs Course by Better Curricular Alignment. Sarah Billings, University of MissouriKansas City, Steven C. Stoner, University of Missouri-Kansas City, Tatum N. Mead, University of Missouri-Kansas City, Maureen E. Knell, University of Missouri-Kansas City. Objectives: Recently, our Top Drugs course has evolved several times with different teaching methods, assessment styles, and variations in curricular placement. Yet, according to student perceptions, these revisions did not enhance the Top Drug learning experience due to a lack of alignment with other didactic coursework. Anecdotally, the perceptions lowered student engagement in the course. To address these concerns and improve students' perception regarding the value of this learning experience, the course underwent revision to improve alignment with other didactic coursework. Method: Top Drugs coordinators matched drug coverage with simultaneous coursework (i.e. pharmacology, pathophysiology) to create groupings of 10-15 top drugs weekly that aligned with course content of these classes. Strategically, this required placement of the Top Drugs class in the second professional year to enhance alignment with selected courses. Weekly lectures reinforce concepts from selected courses by demonstrating relationships between drug characteristics (pharmacology, pharmacokinetics, dosages, contraindications/precautions, adverse effects, etc.). Assessments of students' new and cumulative content knowledge is tested weekly using peer-reviewed multiple-choice questions. Results: Student evaluation of overall course performance data from 2017 (redesign)/2016/2015/2014 based on a 5 point Likert Scale were: 1 . I believe the course objectives were met (4.4/4.1/NA/4.1) 2. The coordinator was available and effectively responded to student concerns $(4.5 / 4.1 / \mathrm{NA} / 3.5) 3$. The course was well organized by the coordinator (4.4/3.9/NA/3.4) 4. The examinations were well managed (4.3/4.4/ NA/4.2) Implications: The revised course structure and curricular placement improved students' course perceptions. Opportunities to further improve student engagement through innovative assessment strategies and course management have also been identified.

Incorporating Physical Manipulatives for Tactile Learning Into an Integrated Pharmacotherapy Curriculum. Jaime A. Foushee, Presbyterian College, Erika Tillery, Presbyterian College, Amber Giles, Presbyterian College, Mandy McCaslan Couch, University of North Carolina Charlotte. Objectives: To describe differences in academic performance in an infectious diseases (ID) integrated course following implementation of kinesthetic blocks as a learning tool. Method: Students enrolled in the Fall 2017 ID module were provided the opportunity to utilize a set of kinesthetic building blocks for inclass activities. A key was included with each set of blocks, colorcoding each block to represent a different bacterial pathogen. Blocks were used in class to model and compare differences in antimicrobial agent spectrum of activity (SOA), as well as model common bacterial pathogens requiring empiric coverage for particular infections, allowing students to produce "bug-drug" matches. Course data was utilized to compare pre-implementation aggregate performance from Fall 2016 with post-implementation data from Fall 2017 for students who utilized the blocks. Overall performance and performance on SOA related questions were evaluated, both initially and cumulatively, using unpaired t-tests. Results: Fifty-nine students accepted blocks for utilization during Fall of 2017 (80.8\%). Overall performance on initial examination of material was statistically higher post-implementation (mean $80.42 \%$ ) compared to pre-implementation ( $\mathrm{n}=74$; mean $75.5 \%$; $\mathrm{p}=0.031$ ). When comparing initial assessment of SOA questions, average performance was statistically higher post-implementation on questions tested both years (mean 74.2\%) compared to pre-implementation (mean $57.2 \% ; \mathrm{p}<0.001$ ). However, no difference was observed for performance on cumulative SOA questions post-implementation (mean 71.5\%) compared with pre-implementation (mean 70.3\%). Implications: Kinesthetic blocks may be a useful tool when used inside class time as an active learning technique to improve short-term performance on SOA related questions.

Incorporation of Electronic Health Record Patient Cases to Address the Pharmacists' Patient Care Process. Justin W. Cole, Cedarville University, Stephanie M. Cailor, Cedarville University, Zachary N. Jenkins, Cedarville University, Andrew Straw, Cedarville University. Objectives: Per the ACPE 2016 Standards, schools must prepare students to effectively provide patient-centered care as part of a healthcare team using the Pharmacists' Patient Care Process (PPCP). Thus, the objective was to examine the effects of written versus educational electronic health record (EHR) cases on student performance and confidence related to the PPCP through the formulation of patient care plans. Method: After completing patient care plan assignments from written and EHR cases during a capstone course, third-year professional pharmacy students completed the Student Perceptions of 


\section{American Journal of Pharmaceutical Education 2018; 82 (5) Article 7158.}

Utilizing an Educational EHR Survey (27 items, 7-point Likert-type scale). Patient care plans were graded using a standard rubric mapped to the PPCP for assessment. Data were analyzed in SPSS using Wilcoxon and Kruskal-Wallis tests as appropriate. Results: Of the 26 student respondents (79\% response rate), most (69.2\%) agreed that the EHR prepared them more effectively for practice. Students agreed or strongly agreed that the EHR cases were effective for all PPCP components and felt that EHR cases were more effective than written cases for practicing the "collect" component $(\mathrm{p}=0.034)$. Overall student grades on plans were higher for cases utilizing the EHR $(p<0.001)$. Student performance in all components of the PPCP was also significantly higher for EHR cases $(\mathrm{p}<0.05)$. Implications: Utilizing an EHR to create patient care plans may better prepare students for practice, especially by enhancing competence in the ability to collect information as part of the PPCP. EHR-based patient care plans may also be a useful method for assessing student performance in various components of the PPCP.

Incorporation of Interprofessional Quality Improvement Education Experiences for Third-Year Pharmacy Students at a Distance Location. Erica J. Ottis, University of Missouri-Kansas City, Kelly A. Cochran, University of Missouri-Kansas City, Linda A Headrick, James P. Koller, Carla A Dyer, Gretchen Gregory, University of Missouri. Objectives: Interprofessional QI experiences have been incorporated into the $\mathrm{P} 3$ year. This study aims to describe previous QI experience and the impact of interprofessional QI education on attitudes and confidence with QI skills. Method: In the P3 didactic and experiential curriculum, interprofessional QI education was embedded. Didactically, interprofessional teams from 5 disciplines participated in a four week quality and safety curriculum. Pre and post assessment was completed using a 5 point Likert scale. Experientially, a subgroup of students were incorporated into a longitudinal QI project coupled with dedicated work sessions facilitated by IP faculty. A baseline survey evaluated past experiences in QI,current level of interest and engagement in QI work. Results: The matched pre and post data $(n=17)$ from the didactic IP curriculum revealed increased confidence with performing a root cause analysis $(p=0.019)$. Students also expressed increased confidence in identifying contributing factors to an adverse event (pre mean 3.88; post mean 4). Attitudes regarding belief that health care professionals should spend time working to improve care (pre 4.53; post 4.76), and that an interprofessional approach to QI training enhances understanding of different professional perspectives (pre 4.53; post 4.65), also increased. On the baseline experiential survey $(n=6), 83 \%$ of students had no prior QI experience, but were interested or enthusiastic about QI in clinical work. 67\% reported likely participation in QI and $83 \%$ highly prioritized QI in clinical work. 33\% reported never incorporating QI in clinical work. Implications: Offering interprofessional QI education may improve attitudes, build confidence in QI skills, and mimic the clinical setting.

Innovative Technique in Traditional and Distance-Site Classroom Settings Improves Student Pharmacists' Self-Perception of Communication Skills. Lisa Goldstone, University of Southern California, Caitlin K. Cameron, The University of Arizona. Objectives: The objective of this study was to assess the effectiveness of an innovative communication skills and human behavior course in improving student pharmacists' self-perception of the ability to communicate in four skill areas, as well as compare student improvement between on-site only (OS) and on-site plus distance campus (OSDC) cohorts. The four skill areas self-assessed by the students were listening skills, emotional intelligence, verbal communication, and communication in groups. Method: A two-credit hour communication skills and human behavior course for first-year student pharmacists was developed. The course consisted of a weekly mini-lecture on a designated communication topic immediately followed by active learning exercises which included improvisation techniques. Students from two separate cohorts, OS and OSDC, completed a pre- and post-questionnaire in which they rated their perception of their ability to communicate and received a score (0-100) for each of the four skill areas. An independent t-test was used to compare pre- and post-scores in the four skill areas and to assess for any differences between the two cohorts. Results: Both cohorts reported a significant improvement in the perception of their ability to communicate in all four skills areas $(\mathrm{p}<0.03)$. There were no significant differences between the OS and OSDC cohorts in pre- or post-assessment scores for any of the skill areas. Implications: An innovative course in communication skills and human behavior can positively impact the self-perception of student pharmacists regarding their communication abilities. This educational approach is effective for both on-site and distance learning environments.

Integrating Electives Through Simulation to Increase Student Confidence and Enhance Intraprofessional Communication. Gwendolyn Wantuch, University of South Florida, Kamila A. Dell, University of South Florida, Gwendolyn Wantuch, University of South Florida, Kamila A. Dell, University of South Florida. Objectives: To integrate two electives through four simulations to increase intraprofessional communication, and confidence and preparation for inpatient rotations. Method: Four scaffolded simulations were developed to integrate critical care elective and clinical nutrition elective students. Students from each elective were paired to provide a patient consult for their respective specialty. Care plans were assessed to identify if intraprofessional collaboration occurred, via frequency of discrepancies. To evaluate knowledge impact, grades in the last 2 courses of the Pharmacotherapeutics series were evaluated between those in the electives and those who were not enrolled. Perception of confidence was assessed on tasks related to working up critically ill patients. Descriptive statistics were utilized for survey data and independent $t$ tests were used to evaluate the differences in grades. Significance was set at an alpha of 0.05. Results: An increase in student awareness and ability to demonstrate intraprofessional communication was shown. The frequency of intraprofessional communication increased by $26.6 \%$ over the course of two simulations. Student confidence increased after every simulation. There was a significant difference in grades for students enrolled in the electives versus those who were not enrolled in Pharmacotherapeutics IV (88.04, 85.95\% respectively, $\mathrm{p}<0.05$ ), a course held the same semester as the electives. No difference was seen between groups in the grades for Pharmacotherapeutics III (82.04\%, 80.72\% respectively, $\mathrm{p}=0.113)$. Implications: Electives combined through simulation serve as an example for teaching intraprofessional communication and function to increase student knowledge and confidence. The outline of these simulations may serve as a template for other colleges.

Integration of Interprofessional Education in the Doctor of Pharmacy Curriculum. Edna N. Almodovar, University of Puerto Rico, Yasmin Pedrogo, University of Puerto Rico, María Padilla, University of Puerto Rico, Arlene Sanchez, University of Puerto Rico, Wilda Guzman, University of Puerto Rico. Objectives: To describe how interprofessional education is incorporated and evaluated throughout the Doctor of Pharmacy program. Method: Based on the Interprofessional Education Collaborative (IPEC) competency framework, activities address the core domains of ethics and values; roles and responsibilities; communication; and teamwork. Activities are incorporated longitudinally throughout the curriculum, in didactic and 


\section{American Journal of Pharmaceutical Education 2018; 82 (5) Article 7158.}

practice experiences. P1 students are introduced to IPEC competencies and patient safety concepts, through videos and small group discussions with medicine students. P2 students further reinforce these concepts by discussing an HIV case in small groups and during a panel with medicine students. P2 and P3 students practice these concepts through the IPPE: Longitudinal Care, in which pharmacy students are paired with dental medicine students to follow-up a patient during one semester. During P3 medicine and pharmacy students practice an error disclosure with standardized patients. In addition to participating as health care team members in APPE's, P4 students participate in a simulation as members of a rapid response team. Most activities include a debriefing session. Students complete reflections and evaluate the activities. Results: APPE's rubrics show excellent performance of students in items related to interprofessional collaboration. Students' reflections and debriefing sessions indicate that students develop a better understanding of the roles and responsibilities of team members and the principles of team dynamics. In addition, students learn to value the importance of communication and mutual respect in order to improve patient safety and achieve better health outcomes. Implications: These activities prepare students to participate as competent interprofessional healthcare team members.

Interprofessional Debate Activity as a Method of Literature Evaluation: An Interim, Year-Long Analysis. Taylor D. Steuber, Auburn University, Haley M. Phillippe, Auburn University, Bradley Wright, Auburn University, Miranda R. Andrus, Auburn University. Objectives: To assess the impact of interprofessional debates as an alternative activity to journal club on perceived pharmacy-physician relationships and literature evaluation skills. Method: Third year family medicine residents and fourth year pharmacy students participated together in an interprofessional debate activity, debating a controversial topic in the medical literature. A pre-survey using the 10-item SPICE-R2 instrument (Likert scale; 1 =strongly disagree through $5=$ strongly agree) was administered to the participants via Qualtrics to gauge interprofessional teamwork perceptions. Postsurveys were administered directly after the debate and matched to assess changes in perceived pharmacist-physician relationships, and included questions to gauge perceptions of literature analysis and other skills via this method. Matched survey results were analyzed using descriptive statistics. Changes in perceptions of the SPICE-R2 instrument were analyzed using Wilcoxon Signed Rank test. Results: Twenty-eight participants (10 medical residents and 18 pharmacy students) have participated in 5 unique interprofessional debates. Twenty-six participants completed the post-survey (93\%) and of those, $68 \%(n=19)$ completed both the pre- and post-surveys. Level of agreement increased on the post-survey for each item on the SPICE-R2 instrument, and was statistically significant for 4 out of 10 questions $(\mathrm{p}<0.05)$. Overall, participants agreed or strongly agreed that the debate activity improved their literature evaluation, problem-solving, critical thinking, teamwork and communication skills. Implications: The debate was well received as a method of literature evaluation by both family medicine residents and pharmacy students. Participants reported positive and improved perceptions of working with an interprofessional team, as well as improved literature evaluation skills and other skills.

Interprofessional Diabetes Education and Awareness (IDEA) Initiative: Improving Students' Abilities and Comfort Within Interprofessional Teams. Andrea Levin, Nova Southeastern University, Sandra Dunbar, Nova Southeastern University, Terry Morrow-Nelson, Nova Southeastern University, T. Lucas Hollar, Nova Southeastern University, David Quinn, Nova Southeastern
University. Objectives: In an effort to combat the diabetes epidemic and expose students to interprofessional education (IPE), Nova Southeastern University and the American Diabetes Association of Broward County joined forces with community organizations to establish the Interprofessional Diabetes Education and Awareness (IDEA) Initiative. Students from thirteen health-related graduate programs, forming eleven interprofessional teams, were surveyed at the beginning and end of the IDEA Initiative academic year to assess their perceptions of interprofessional team functioning. Method: Student participants completed the Bristol Entry Level Interprofessional Questionnaire at the beginning and end of their participation in IDEA (academic year 2015-2016). Wilcoxon signed-rank tests were conducted to determine statistically significant changes in survey questionnaire scores from baseline to follow-up. Tests were two-tailed, and p-value of less than 0.05 was deemed statistically significant. Results: Thirty-one student participants (approximately 41\%) completed both baseline and follow-up questionnaires. Upon completion of the IDEA initiative's academic year, students experienced significant improvements in their reported abilities to adapt their communication styles to particular situations/audiences ( $\mathrm{p}$-value $=0.022$ ) and to become quickly involved in new teams/groups ( $\mathrm{p}$-value $=0.005$ ). Students also reported significant improvements in feeling more comfortable working in groups ( $\mathrm{p}$-value $=0.042$ ); expressing opinions in a group, even when they know others may not agree $(p$-value $=0.006)$; and justifying recommendations/advice face-to-face with more senior people ( $p$-value $=0.012$ ). Implications: By engaging students in interprofessional groups to plan and execute IDEA, this IPE co-curricular initiative improved aspects of students' abilities to perform in interprofessional teams and settings, while improving their comfort in doing so as well.

Interprofessional Education: An Integration of Pharmacy and Medicine Courses to Create Team-Ready Students. Susan S. Vos, The University of Iowa, Chase D. Kooyman, The University of Iowa, Michael E. Ernst, The University of Iowa, Michael W. Kelly, The University of Iowa, George R. Bergus, The University of Iowa, Elizabeth M. Bald, University of Iowa Hospitals and Clinics, Allison M. Bernard, The University of Iowa, Mary C. Schroeder, The University of Iowa. Objectives: To describe and evaluate a two-week, interprofessional course designed to promote team-ready pharmacy and medical students, delivered immediately prior to commencing advanced experiential education. Method: An elective, case-based course in the college of medicine was integrated with a required pharmacy course. Teams with a 1:1-2 medical-to-pharmacy student ratio were created. Each day teams developed comprehensive care plans utilizing collaborative decision-making, evidence-based practice, and foundational sciences. In a grand-rounds format, student teams presented their care plans in a 90-minute session. Following each session, new pharmacy and medical student teams were formed, a new case given, and teams created their care plans for presentation the subsequent day. Pharmacy and medicine faculty facilitated discussions using Socratic questioning. Students completed a pre-and post-selfassessment survey using a Likert scale $(1=$ strongly disagree to $4=$ strongly agree) to address their comfort in providing collaborative care to patients. Data were compared using paired t-tests. Results: Eight cases were completed over eight days. Each pharmacy student $(n=103)$ was involved in two cases (one as observer; one as participant) and the medical students $(n=9)$ were involved with all eight cases. Both pharmacy and medical students' agreement with teambased readiness questions improved ( $p r e=3.3$ vs. post $=3.7, p<0.01$; pre $=3.4$ vs. post $=3.8, p=0.02$, respectively). The largest increase for 


\section{American Journal of Pharmaceutical Education 2018; 82 (5) Article 7158.}

students was in their reported ability to develop an effective care plan with other professionals (change $=0.44 ; \mathrm{SD}=0.61$ ). Implications: As one component of an interprofessional curriculum, this 2-week, integrated medicine and pharmacy case-based course was effective at enhancing pharmacy and medical students' team-readiness prior to advanced experiential education.

Interprofessional Education Simulation Day and Its Impact on Residents' Perceptions of Pharmacist-Physician Collaboration. Taylor D. Steuber, Auburn University, Bradley Wright, Auburn University, Haley M. Phillippe, Auburn University, Miranda R. Andrus, Auburn University. Objectives: To assess the impact of an interprofessional simulation day on medical interns' perceptions of pharmacist-physician collaboration. Method: Family and internal medicine interns, and nurse practitioner students were divided into interprofessional teams to solve simulated patient cases. Cases ranged from emergency situations to day-to-day cases commonly seen in hospitals and were approximately 20 minutes each. In each of the cases a pharmacist or $\mathrm{P} 4$ pharmacy student was present in the room to act as a resource and answer any drug information questions. Immediately following the case, a debriefing session occurred with medicine, nursing, and pharmacy faculty. Following the simulation day, the family and internal medicine interns were asked to anonymously complete the "Scale of Attitudes Toward Pharmacist-Physician Collaboration". This survey was a Likert scale ranging from 1-strongly disagree to 4-strongly agree. Baseline characteristics were collected and results were analyzed using descriptive statistics. Results: Sixteen medical interns, 16 nurse practitioner students, 2 pharmacy students, and faculty from each discipline participated in the activity. Five internal medicine and 10 family medicine interns completed the survey ( $75 \%$ response rate) and the perceptions of pharmacistphysician collaboration were positive. The median response was "strongly agree" for 12 of 16 questions. For 3 additional questions, the median response was "agree." Respondents disagreed when asked: "The primary function of the pharmacist is to fill the physician's prescription without question." Implications: Medical interns had positive perceptions of pharmacist-physician collaboration after an interprofessional simulation.

Interprofessional Team-Based Learning Activities in the Ambulatory Setting. Talitha L. Pulvino, Temple University, David Koren, Temple University Hospital, Elizabeth L. Lee, Temple University, Alia Chisty, Temple University Hospital. Objectives: Advanced Pharmacy Practice Experience (APPE) students and medical residents work alongside each other to deliver care; however, they have limited opportunities to learn together. We sought to introduce a modified teambased learning (TBL) module including teams of medical residents and pharmacy students, role model interprofessional collaboration with joint development of the teaching module, determine if team-based learning promotes interprofessional trust and engenders positive relationships, and ascertain whether learning with other professionals makes the learner a more effective member of the healthcare team. Method: Teams worked together on the topic of female contraception during dedicated ambulatory sessions over three consecutive days. Students drew on prior knowledge from didactic lectures and provided pharmacy-specific input. Participants were encouraged to complete a survey at the conclusion. Results: 48 learners participated in the modified TBL exercise. 24 medical residents $(77.4 \%)$ and 7 pharmacy students $(22.6 \%)$ responded to the survey. $93.6 \%$ of participants agreed that team members actively participated. $100 \%$ of participants found the information to be helpful, and $93.6 \%$ of participants would like more interprofessional involvement in future sessions. $86.7 \%$ agreed that shared learning helped them to think positively about other healthcare professionals. $90.3 \%$ of participants believed that learning with other students/professionals will make them more effective members of the healthcare team and will increase their ability to understand clinical problems. Finally, $100 \%$ of participants believed that team-working skills are vital for healthcare professionals to learn. Implications: This TBL activity highlights the advantages of creating opportunities for pharmacy students to engage in interprofessional education in the clinical setting.

Interprofessional Telehealth Case Studies: An Innovative Way to Assess Clinical Knowledge and Interprofessional Collaboration. Kimberley J. Begley, Creighton University, Amy Pick, Creighton University, Kathleen A. Packard, Creighton University, Ann M. Ryan Haddad, Creighton University. Objectives: To develop and implement telehealth case activities using interprofessional teams comprised of pharmacy students, physician assistant students, and standardized patients. Method: Faculty met to develop interprofessional education (IPE) cases which were incorporated into Pharmacotherapeutics II case study sessions. Fourteen virtual rooms were created and students signed up for a scheduled time to meet for their case. Each virtual room contained a standardized patient so healthcare students could ask further questions. On the scheduled date, paired participant teams logged onto WebEx and entered their virtual room to discuss their patient case. Pharmacy and physician assistant faculty were utilized to moderate the sessions and provide feedback. Results: Students were assessed in two ways. The teams were evaluated on their clinical knowledge using faculty-developed rubrics. Feedback was given on the appropriateness of the team's recommendations. Faculty also evaluated team behaviors using the Creighton Interprofessional Collaborative Evaluation (C-ICEC) instrument to assess team behaviors which was set at an $80 \%$ to pass. The average team score was $93 \%$ and only one team did not meet the $80 \%$ minimum. Qualitative data was gathered to examine pharmacy student perceptions of the interprofessional case study sessions and standardized patient feedback regarding the experience. Implications: With new accreditation requirements, schools of pharmacy may find it challenging to develop efficient and effective ways to incorporate meaningful IPE experiences into their curricula. This telehealth IPE case activity was easily integrated into an existing course and uses an innovative platform that provides students with interprofessional opportunities to practice patient-centered, team-based care.

Keeping the Patient at the Center of the Pharmacists' Patient Care Process. Lisa Charneski, University of the Sciences, Tyan F. Thomas, University of the Sciences, Jean M. Scholtz, University of the Sciences, Amy McLaughlin, University of the Sciences. Objectives: To describe the use of combined video and electronic health record (EHR) simulation with debrief as a method to develop student skills related to the Pharmacists' Patient Care Process (PPCP) Method: Inclusion of PPCP into 2016 Accreditation Council for Pharmacy Education (ACPE) resulted in curricular revisions at the Philadelphia College of Pharmacy. Within the case studies series, traditional paper cases were replaced with simulated patient cases containing a mock EHR and either a corresponding video simulation of a pharmacist-patient interaction (created by faculty/staff) or a standardized patient encounter ( 2 of 14 weeks). Each case week, students completed all steps defined in PPCP with targeted formative assessments. As supported by simulation literature, most class periods concluded with faculty lead debrief discussions. Summative assessment occurred at the end of each semester. Results: Student satisfaction and engagement was assessed using a survey (response rate $71.8 \%$ ). Overall, students responded positively 


\section{American Journal of Pharmaceutical Education 2018; 82 (5) Article 7158.}

to the course design with $80.4 \%$ of students agreeing that simulations "seemed realistic" and $85 \%$ agreeing that simulations "provided insight into patient care in different settings". Assessments were designed to measure skill development as defined in PPCP. Metrics related to "collect", "assess", and "plan" showed student growth between the fall and spring semesters. Mean overall course grades were $>90 \%$ for both semesters. The mean scores for the final summative assessments were $88 \%$ for fall and $89.8 \%$ for spring. Implications: In a didactic setting, simulation can keep the patient at the center of PPCP. Video simulation of pharmacist-patient interactions was effective, well received, and had minimal financial implications.

Measuring ACPE Standard 11: Programmatic Evaluation of Interprofessional Activities in Advanced Pharmacy Practice Experiences. Veronica S. Young, The University of Texas at Austin, Jennifer L. Ridings-Myhra, The University of Texas at Austin, Emma Gugala, The University of Texas at Austin, Rochelle M. Roberts, The University of Texas at Austin. Objectives: ACPE Standard 11 mandates all pharmacy students participate in IPE activities in experiential learning involving prescribers/student prescribers, including physicians/medical students. Ensuring experiential IPE activities is challenging because it is dependent on existing collaborations at practice sites. An assessment tool was successfully piloted to determine the extent of IPE on rotations. This tool has since been adopted for programmatic evaluation. The objective is to determine the utility of this tool in assessing the extent and types of IPE and existing gaps by rotation types. Method: APPE students complete the IPE assessment as part of a required end-of-rotation questionnaire. Descriptive analyses were conducted to analyze responses by rotation types and region to determine gaps and growth opportunities. Results: Overall, students most frequently interacted with prescribers/student prescribers: physicians (89\%), medical residents (41\%), medical students (30\%), PA/ students $(33 \%)$, and nurse practitioners/students $(38 \%)$. Students placed in interprofessional teams were more likely to interact with physicians (93\%). Students on acute care and selective rotations were more likely to be placed in teams. Most frequently reported IPE activities include bedside/sit-down rounds, responding to drug inquiries and case discussions. Not all IPE activities conducted were expected of students by their preceptors. Implications: Findings demonstrated the utility of a student self-reporting tool to determine the extent of IPE experiences on rotations. While future evaluations may target preceptors' responses, this analysis supports the importance of querying students since they are involved in IPE not expected by their preceptors. Findings will be used to guide placement of required IPE activities.

Measuring APPE Readiness: A Survey of US Colleges of Pharmacy. Kali VanLangen, Ferris State University, Lisa M. Meny, Ferris State University, David R. Bright, Ferris State University, Mandy R. Seiferlein, Ferris State University, Ashok E. Philip, Union University, Roopali Sharma, Touro College of Pharmacy-New York, Yae-Ji J. Kim, Margarita V. DiVall, Northeastern University. Objectives: To describe current trends in how Colleges of Pharmacy (COPs) assess student APPE readiness. Method: A web-based survey was distributed to assessment leads at all US COPs regardless of accreditation status. Respondents were asked questions related to current approach to assessing student APPE readiness, including existence of intentional assessment plans, competencies used, assessment methods, benchmarks, and remediation. Aggregate data were analyzed using descriptive statistics. Results: Forty-nine COPs (34\%) responded to the survey. The majority (89.8\%) were fully accredited schools. Most schools have an intentional plan to assess APPE readiness (69.4\%) although the duration since plan implementation varied. There was no consensus among schools on what competencies informed APPE readiness with $67 \%$ listing CAPE outcomes, 61\%-Appendix A Guidance for Standards 2016, 53\%-Pre-APPE domains (Standards 2007), and 31\%-Entrustable Professional Activities. Course level embedded assessments (68.9\%) and skills-based embedded assessments (71\%) are most frequently used for measuring student APPE readiness. Twenty-eight COPs (57\%) have individual level evidence of student APPE readiness and provide individualized student feedback. Fewer schools reported using assessment data on APPE readiness to modify curriculum $(41 \%)$ or pre-APPE assessments $(27 \%)$. Most common methods of validation of student APPE readiness are preceptor $(51 \%)$ and student surveys (47\%), while only $39 \%$ of school use APPE preceptor evaluations. Implications: Low response rate to the survey may over-estimate the proportion of COPs with APPE readiness assessment plans; however, survey data provides commonly used competencies, methods, and plans, and challenges in assessing student APPE readiness and will be of interest to the academy.

Measuring Professional Pharmacy Student Understanding of Leadership Principles and Self-Awareness of Their Leadership Abilities. Meghan Bodenberg, Butler University, Trish Devine, Butler University. Objectives: To determine if online leadership modules incorporated into the first professional year (P1) pharmacy curriculum improve students' understanding of basic leadership principles and self-awareness of their leadership abilities. Method: In spring 2017, P1 pharmacy students at Butler University College of Pharmacy and Health Sciences were invited to participate in an anonymous, online survey assessing perceptions of their leadership abilities (based on a 4-point Likert scale) and understanding of basic leadership principles ("choose all that apply" or multiple-choice questions). Students completed three different online leadership modules during the semester: StrengthsFinder assessment, introduction to leadership and practices of exemplary leaders, and professional organization opportunities. Students were also required to complete reflection questions on material from each module. A post-survey was then administered using the same questions regarding knowledge and perception, and two additional questions about which module was the most and least helpful. Scores were analyzed using a Wilcoxon t-test for the perception questions and a student t-test for the knowledge questions. Results: Ninetyone of 114 invited students ( $80 \%$ response rate) completed both the pre-and post-survey response. Student perception of their own leadership abilities increased significantly between the pre- and post-surveys ( $p=0.0039$ ). While students' overall knowledge of leadership improved, it was not statistically significant. The majority of students (54\%) felt the StrengthsFinder assessment module was the most helpful. Implications: Introducing leadership modules during the first professional pharmacy year helps to increase student's self-awareness of leadership abilities.

Medication Therapy Management Services in PGY-1 Community Pharmacy Residency Programs in the US. Brooke D. Fidler, Long Island University, Fraidy N. Maltz, Long Island University, Nadine Dandan, Kings Pharmacy, Keren Darzi, Long Island University. Objectives: Medication Therapy Management is an integral part of PGY-1 Community Pharmacy Residency Programs (CPRP). The purpose of this study was to gather information from ASHP-APHA accredited CPRPs regarding MTM platforms utilized to provide MTM , types of therapeutic interventions, and other services offered. Method: A survey was administered through Campus Labs via email to all CPRP directors in the US identified utilizing the ASHP Online Residency Directory. Results: A total of 140 CPRP directors were e-mailed, and 39 completed the survey. Approximately 54\% of 


\section{American Journal of Pharmaceutical Education 2018; 82 (5) Article 7158.}

respondents' residency programs were established within the last 1-5 years. In almost $70 \%$ of CPRPs a PGY-1 community resident was in charge of MTM services, which predominately took place over the phone (64\%). While there were a variety of other MTM platforms noted in the survey, $45 \%$ and $40 \%$ used OutcomesMTM and MirixaPro, respectively. CPRPs who performed greater than 10 MTM activities per month reported doing more monthly targeted interventions as compared to comprehensive medication reviews (53\% vs. 33\%). Adherence issues (51\%), inappropriate standard of care treatment (49\%) and drug interactions $(36 \%)$ were the most common targeted interventions. Implications: While CPRPs provide various services to meet program competencies, MTM has been a primary focus. Providing MTM allows CPRP residents and pharmacists to work with healthcare providers to ensure that patients are receiving appropriate and affordable medications. With only 140 CPRPs in the US, it is important to know how fellow programs provide MTM services to improve the experience of the CPRP residents and to promote patient care.

Mentor Objectivity When Grading Mentees. Samuel C. Karpen, East Tennessee State University, Kathryn A. Mueller, East Tennessee State University. Objectives: To determine whether faculty assign higher capstone presentation grades to their mentees than to students who they do not mentor, and if so, why. Method: Researchers recorded: 1) capstone presentation grades. 2) whether or not the grade was assigned by a mentor. 3) graders' responses to the following questions about the presenter/presentation. The capstone presentation topic is one of my professional interests. I may conduct research on this topic in the future. I feel personally invested in the success of this presentation. My experiences with the presenter have been positive. We hypothesized graders would answer these questions in the affirmative to a greater extent when evaluating mentees than when evaluating non-mentees. Results: Graders assigned higher grades to mentees $(M=90.08, S D=7.40)$ than to non-mentees $(M=88.89$, $\mathrm{SD}=7.43), \mathrm{p}=.015,95 \% \mathrm{CI}[0.24-2.16], \mathrm{d}=0.21$. This effect could be explained by the fact that mentors had more positive experiences with mentees $(\mathrm{M}=4.22, \mathrm{SD}=0.83)$ than with non-mentees $(\mathrm{M}=3.66$, $\mathrm{SD}=0.49), \mathrm{p}=.0001,95 \% \mathrm{CI}[0.43-0.69], \mathrm{d}=0.85$. Specifically, mentees' graders were 1.33 points higher than non-mentees' grades as a result of the grader having more positive experiences with them. The bootstrapped $95 \%$ CI for this effect was $0.54-2.24$. The remaining three presenter/presentation questions were also answered in the affirmative to a significantly greater when mentees were evaluated than when non-mentees were evaluated, but they did not have a significant mediating effect on grades. Implications: The current research calls into question whether mentors can objectively evaluate their mentees, not only during presentations, but in any setting.

Metacognitive Activities on a Primary Care APPE Rotation. Rebecca Maxson, Auburn University. Objectives: Two of the eight abilities-based outcomes at Auburn University describe goals for students' professionalism and independent pharmacy practice. Specifically, students must "develop practice and leadership" and "advance the profession." Two metacognition activities were developed for students participating in a Primary Care APPE rotation to achieve these goals. The purpose of this project is to evaluate student perceptions of the usefulness of these activities. Method: Two assignments were created as part of rotation requirements: (1) read four short articles and provide two reflective discussions based on these articles and (2) read a nonfiction healthcare-based book, compose a reflective paper on themes in the book that will influence future practice, and participate in active discussions of these themes with the preceptor. A post-rotation survey was developed to assess impact of these activities on students' perceptions. Anonymous survey results from students in the 20162017 and 2017-2018 academic years were analyzed. Results: The cohort includes 24 students with $88 \%$ (21/24) completing the survey. $95 \%(20 / 21)$ strongly or somewhat agreed that the short articles activity was useful. $86 \%(18 / 21)$ strongly or somewhat agreed that the book activity was useful. $95 \%(20 / 21)$ strongly or somewhat agreed that these activities encouraged reflection on their thoughts and beliefs about being a pharmacist. The students' top benefit from these activities were in considering others' point of view (81\%) and reflecting on their experience as a pharmacist (81\%). Implications: The survey results demonstrate that the APPE students perceived that these activities helped them reflect on their future profession.

Motivating Influences for Truancy in the Age of Technology. Steven C. Stoner, University of Missouri-Kansas City, Maqual R. Graham, University of Missouri-Kansas City. Objectives: Most contemporary student learners are digital natives who enter programs with diverse learning backgrounds and experiences. The expansion of pharmacy academia has necessitated the integration of distance education technology and the use of lecture capture programs at many institutions. Such programs allow for learning to occur within the timeframe of learners' personal activities, potentially decreasing their desire to attend the live delivery of education. Learning in this manner may impede relationship building between students and faculty. The purpose of this study is to explore whether lecture capture promotes truancy and determinants of classroom attendance. Method: Students from consecutive classes of third professional year students were asked to voluntarily complete an attendance survey and provide feedback regarding attendance. Results: Of the 239 respondents, $62.3 \%$ reported attending class regularly, while $24.6 \%$ attended only for assessments. Respondents (64.4\%) perceived benefits of attending class. When not attending class $81.5 \%$ of students used lecture capture technology, and $59.4 \%$ reported lecture capture was not a motivating factor in attending. The primary reasons for truancy were upcoming exams (24.7\%), lecture capture (16\%), no topic interest (14.7\%), and work (10.8\%). Respondents (56.5\%) believed attendance was not a professionalism issue; $59 \%$ felt classroom attendance was not area of concern and $49.4 \%$ thought it should not be part of assessment $(49.4 \%)$. Implications: Truancy occurs for other reasons unrelated to technology. Performing in class assessments mitigate truancy and other innovative or engaging instructional methods are needed to motivate attendance.

Multicenter Assessment of Cultural Competency Active Learning Strategies for Pharmacy Students. Lakesha M. Butler, Southern Illinois University Edwardsville, Aleda M. Chen, Cedarville University, Anastasia L. Armbruster, St. Louis College of Pharmacy, Bethanne Brown, University of Cincinnati, Nancy Borja-Hart, The University of Tennessee, Sally Arif, Midwestern University/Downers Grove, Natasha J. Petry, North Dakota State University. Objectives: Given the increasingly diverse population and a global focus in healthcare, it is important to prepare students to engage effectively with different cultures. Thus, the objective of this project was to assess effectiveness of two different learning activities in enhancing cultural competency of pharmacy students at multiple institutions. Method: Pharmacy students (P1-P3) at seven different pharmacy schools participated in two learning activities, Global Beads and Trading Places, in their respective, elective or required courses during Spring 2017. The revised Clinical Cultural Competency Questionnaire (CCCQ) was distributed as an online, anonymous pre-survey prior to the learning activities. After the activities, students were requested to complete the post-survey CCCQ to provide qualitative and quantitative assessments 


\section{American Journal of Pharmaceutical Education 2018; 82 (5) Article 7158.}

including demographic information and perceptions of the activities. IRB approval was obtained. Data were analyzed using SPSS. Since pre- and post-data were not paired and not normally distributed, differences in the pre- and post- assessments were analyzed using a Mann-Whitney U test. Results: Students' ( $N=669$ pre-test, $N=624$ post-test) cultural competence significantly improved from pre- to post-test ( 12 out of 12 questions, $\mathrm{p}<0.001$ ). There was no significant change in the importance of receiving training in cultural diversity $(p=0.835)$ or motivation to receive training $(p=0.592)$. Implications: Incorporation of learning activities across institutions and at different stages of pharmacy education resulted in improved cultural competence. These activities require minimal time and resources to implement and could assist programs in addressing both ACPE standards related to cultural competency and preparing students for future practice.

Multi-Centered Study Evaluating Pharmacy Students' Perception of Palliative Care and Clinical Reasoning Using Script Concordance Testing. Rabia S. Atayee, University of California, San Diego, Dawn K. Lockman, The University of Iowa, Cara M. Brock, Roosevelt University, Daniel T. Abazia, Rutgers, The State University of New Jersey, Tracy L. Brooks, Manchester University, Jayne Pawasauskas, The University of Rhode Island, Kyle P. Edmonds, University of California, San Diego, Christopher M. Herndon, Southern Illinois University Edwardsville. Objectives: This study evaluates studentperceived importance and confidence in palliative care and changes in students' clinical reasoning before and after completion of a palliative care elective and survey. Method: P2 and P3 students from six accredited schools of pharmacy participated in surveys before and after completion of a palliative care didactic elective at each school taken between August 2016 to August 2017. Script concordance tests (SCT), which is a novel written assessment of clinical reasoning under conditions of uncertainty, were used to assess clinical decision-making skills on patient cases, created by the lead investigator, related to palliative care topics. Student scores on the SCT were compared to those of a reference panel of experts, excluding the study investigators. Results: A total of 89 students completed the pre-/post-surveys and were included in data analysis. There was no statistically significant difference in student perceived importance of palliative care skills before and after the elective $\left(\chi^{2}=1.031, p=0.597\right)$. Students from all six institutions showed significant increase in confidence in their palliative care skills at the end of the course $(p<0.001)$. There was significant improvement across all institutions in clinical reasoning skills upon completion of the elective in most of the script concordance test questions used to assess these skills. Implications: Delivery of a palliative care elective in the pharmacy curriculum significantly increases student confidence in their palliative care skills and clinical reasoning skills in these areas. SCT may be a useful clinical assessment tool in pharmacy school curricula.

Naloxone Study - A Gap Between Students' Perception and Ability in Opioid Overdose Education and Naloxone Use. Mikiko Y. Takeda, The University of New Mexico, Amy Bachyrycz, The University of New Mexico, James J. Nawarskas, The University of New Mexico, Joe R. Anderson, The University of New Mexico, Mary Vilay, The University of New Mexico, Melanie A. Dodd, The University of New Mexico, Kristina M. Wittstrom, The University of New Mexico, Barry Bleske, The University of New Mexico. Objectives: Opioid overdose (OD) is a critical public health issue. Pharmacists in New Mexico (NM) can help prevent OD by dispensing naloxone via a standing order. Students at the University of NM College of Pharmacy are taught this through a naloxone training program launched in the third-year pharmacy skills lab in 2017. This project assessed the impact of that program on student knowledge, skills, and confidence regarding OD. Method: Student training included a review of the NM Board of Pharmacy protocol for pharmacist prescribing naloxone; discussion of a case series to identify risks of OD; and demonstration and practice of naloxone kits. Before and after the training, students completed a questionnaire to assess their confidence level to discuss OD and naloxone. At the end of the semester, students' ability to identify risk of OD and make a naloxone recommendation for a geriatric patient on chronic opioid treatment was assessed. Results: After training, there was a significant increase in students' $(\mathrm{n}=78)$ confidence level to discuss OD, explain proper use of naloxone, and identify high-risk patients of OD who need naloxone ( $\mathrm{p}<0.001$ for all). However, at the end of the semester, only $13 \%$ of students identified the risk of $\mathrm{OD}$ and made a proper naloxone recommendation for the patient case. Implications: A naloxone training produces short-term learning gains, but students were largely unable to apply this knowledge in a new situation after a period of time. These results indicate that more constructive training is necessary to improve transfer of learning.

National Survey of Pharmacy Services Offered in Student-Run Free Clinics (SRFC). Amy H. Schwartz, University of South Florida, Vanessa Ulloa, University of South Florida, Danielle Dantuma, University of South Florida, Kristina Larizadeh-Poole, University of South Florida, Samantha Tobitt, University of South Florida. Objectives: Primary: to identify the number of pharmacy programs involved in SRFC, and types of services provided. Secondary: to describe pharmacy and other discipline involvement, and quantify administrative processes. Method: Cross-sectional analysis utilizing a survey developed from the literature. Peer review of questions (face-validity); format included dichotomous response, multiple-choice, and open response. Qualtrics survey distributed via email to Pharmacy Practice Chairs at AACP affiliate institutions. Descriptive analyses; duplicate responses combined when possible. Project was deemed exempt by IRB. Results: Forty-three programs responded (44\% private, $56 \%$ public). Pharmacy services: patient education (100\%); disease management $(78 \%)$; formulary management, OTC medications or PAP (61\%); immunizations and lab monitoring (56\%); or prescription medications (44\%). Discipline participation: medicine $89 \%$, nursing $83 \%$, physician assistants and dentistry $50 \%$. Sixty-five percent reported pharmacy students participated in patient encounters with other disciplines. Student participation per academic year varied; however, noted need for at least one preceptor. The majority operated one day per week, serving approximately 300 patients. Use of a schedule varied, as did average wait time and encounter duration. Seventy-five percent had a budget, $46 \%$ divided based on pre-determined parameters; $36 \%$ equal distribution. Challenges included: funding and preceptor participation (63\%); patient adherence and student participation (50\%); and student transitions (44\%). Those not involved with SRFC noted the reason being funding/resources (59\%) or without facility (50\%). Most had considered, however remained without timeline. Implications: Limited information pertaining to pharmacy inclusion in SRFC has been published. The information may help identify additional opportunities for enhancements and/or student engagement.

Need for Case Studies Aimed at Improving Students' Readiness in Delivering Culturally Sensitive Pharmacotherapy. Anna Dushenkov, Fairleigh Dickinson University, Lillian Rozaklis, Fairleigh Dickinson University. Objectives: Cultural awareness coupled with cultural knowledge are foundational constructs for delivery of culturally sensitive healthcare (Campinha-Bacote, 2002). We hypothesized that P3 students are culturally aware-defined as confidence in one's ability 


\section{American Journal of Pharmaceutical Education 2018; 82 (5) Article 7158.}

“to safely and appropriately incorporate patients' cultural beliefs and practices into health and wellness care plans" (CAPE Outcome 3.5. Cultural sensitivity (Includer), Learning Objective 3.5.4)—but lack factual knowledge of socio-cultural factors impacting patient's decisions regarding one's pharmacotherapy. Method: Before discussing a Worlds Apart case on cross-cultural aspects of oncologic pharmacotherapy, 77 P3 students rated their ability to achieve Learning Objective 3.5.4 on 5-point confidence scale. A survey was administered with embedded logic, where only "confident" rankings prompted respondents to provide illustrative examples. "Correct" illustration included patient population, disease state and medications involved. Coauthors evaluated examples independently and blindly. Results: All respondents demonstrated confidence: $9.1 \%$ "completely confident" (CC), $36.4 \%$ "very confident" (VC), 42.9\% "moderately confident" (MC), $11.7 \%$ "somewhat confident" (SC). An example was provided by $36.4 \%$ of respondents; mostly from MC and VC groups. No SC students submitted an example. One CC student submitted an example, but it was "incorrect." Of VC and MC examples, $67.9 \%$ were "correct." Implications: Results revealed a sizable gap between a high level of students' confidence in performing the Learning Objective 3.5.4 and low observable knowledge of cultural issues in healthcare. Inclusion of case studies that address socio-cultural determinants of medication therapy management is desirable. The intervention would provide additional educational opportunities aimed at improving students' preparedness in carrying out culturally sensitive pharmacotherapy.

Needs and Resources for Successful Deprescribing Identified by Health Care Trainees in an Interprofessional Course. Kristin Zimmerman, Virginia Commonwealth University, Teresa M. Salgado, Virginia Commonwealth University, Courtney A. Bell, Virginia Commonwealth University, Krista L. Donohoe, Virginia Commonwealth University. Objectives: To explore healthcare trainees' perspectives on needs and resources necessary to successfully deprescribe in practice and to assess their performance on a clinical deprescribing case. Method: A survey assessing interprofessional roles, attitudes, preparedness, and confidence to deprescribe was disseminated to 482 final-year trainees (medicine, pharmacy, and nursing) enrolled in a capstone course at Virginia Commonwealth University. The survey also included a clinical case (graded 0-20) followed by two openended questions about needs (What would better prepare you for the deprescribing process and how to initiate it?) and resources for successful deprescribing (What resources do you foresee needing in practice to support deprescribing?). Kruskal-Wallis and Mann-Whitney tests were used to assess differences in case scores across trainee groups and between those who felt confident/not-confident to deprescribe, respectively. Responses to questions were thematically analyzed by two investigators. Results: Eighty-two of 108 responses received were usable (35\% pharmacy, 27\% medicine, 13\% nursing). Median case scores did not differ across trainee groups nor between those who felt confident/not-confident to deprescribe. To enhance deprescribing, trainee needs included: additional experiential (clinical training) and curricular education/training (case activities, discussions, lectures, interprofessional training); further knowledge about medications and designing a deprescribing plan; and enhanced communication. Necessary resources included: training, knowledge/skills (implementation, risks/benefit assessment), supportive interprofessional dynamics, authority to deprescribe, access to evidence-based drug information, technology (integrated medical record, ability to contact prescriber) and infrastructure (administration support, time, practice setting). Implications: Additional interprofessional deprescribing education and training should be implemented to better prepare trainees to deprescribe in practice.

Needs Assessment of National Sabbatical Program for Pharmacy Practice Faculty. Lakesha M. Butler, Southern Illinois University Edwardsville, Brittany L. Riley, Marshall University, Christina A. Spivey, The University of Tennessee, Gary Milavetz, The University of Iowa. Objectives: To examine the utilization of sabbaticals among pharmacy faculty To determine the need for a national sabbatical program for pharmacy faculty Method: A 22-item electronic survey was created by the AACP Pharmacy Practice Section Scholarship and Research Committee and distributed to all Section members to determine utilization and awareness of sabbatical programs at their institutions. Questions inquired about eligibility for sabbatical, length of time for sabbatical, funding for sabbaticals, and interest in participation in a sabbatical program. Frequency counts and descriptive statistics were conducted. Results: 177 survey participants responded to required questions 1-2, however, participants decreased to 128 for optional questions 3-22. Respondents were evenly divided among tenure-track and clinical track faculty and $93 \%$ in the pharmacy practice field. $92 \%$ of respondents had not taken a sabbatical and only $2 \%$ had applied for a sabbatical. Average length of sabbatical was 6 months. The majority of institutions (79\%) provide funding during sabbaticals, with twothirds providing partial or full salary. $83 \%$ of respondents expressed interested in participating in a sabbatical with the ideal length of 4 to 6 months. Implications: Significant interest in sabbatical programs exist among pharmacy practice faculty, however further research on the perceived barriers to participation is needed. Sabbatical programs may not include all pharmacy practice faculty as eligible participants and may provide justification to create a national policy for AACP member institutions on sabbatical leaves for these faculty. Further investigation into why faculty are interested in sabbaticals and the impact of funding on the utilization of sabbaticals is also needed.

Opioid Overdose Attitude Changes in Third-year Pharmacy Students. Krista L. Donohoe, Virginia Commonwealth University, Archana Raghavan, Virginia Commonwealth University, Thuy T. Tran, Virginia Commonwealth University, Fawaz Alotaibi, Virginia Commonwealth University, Kacie Powers, Virginia Commonwealth University, Laura M. Morgan, Virginia Commonwealth University. Objectives: To determine changes in attitudes of third-year pharmacy students on opioid overdoses following a training session and laboratory activity. Method: All third-year pharmacy students $(\mathrm{n}=130)$ participated in an opioid overdose and naloxone education training lecture followed by a laboratory activity that included: mock naloxone counseling, a case-based Prescription Drug Monitoring Program (PDMP) discussion and equianalgesic opioid dose conversion scenarios. A preand post-assessment, which included a survey on attitudes about opioid overdose management and naloxone, was administered. The Opioid Overdose Attitudes Scale, a validated survey instrument, has 28 questions. Students were asked to rate their level of agreement with each statement. For data analysis, completely disagree and disagree as well as completely agree and agree were combined. McNemar test was performed to test the statistical differences in two proportions at 0.05 level of significance. Results: A total of 129 students (response rate: $99 \%$ ) completed the attitudes survey before and after the lecture and laboratory session. A majority of questions $(n=21)$ on the Opioid Overdose Attitudes Scale statistically improved from baseline. The biggest change was found in being able to inject naloxone $(+84 \%)$, effectively dealing with an overdose $(+69 \%)$, having enough information to manage an overdose $(+69 \%)$, and knowing what to do in an overdose $(+67 \%)$. There was no significant change in attitudes for six 


\section{American Journal of Pharmaceutical Education 2018; 82 (5) Article 7158.}

questions, while there was a statistical decline in not wanting to help because of the responsibility if the patient died. Implications: Incorporating a naloxone training session and laboratory on opioid overdose management improved students' attitudes.

Outcomes of a Required Longitudinal Interprofessional Education Program in Teamwork (LIPET). Demi L. Rissmiller, Wilkes University, Edward F. Foote, Wilkes University. Objectives: To evaluate outcomes of the LIPET experience among pharmacy students. Method: The LIPET begins spring P1, MD1 and sophomore nursing years. In session one, "Got Ethics" (https://www.mededportal.org/ publication/9331/), students are placed into teams of 6-8 learners, these teams are kept together for subsequent sessions. Session two (fall P2, MD2 and junior nursing) introduces TeamSTEPPS and SBAR (situation, background, assessment, recommendation). Session three (springs P2, MD2 and junior nursing) introduces CUS (Concerned, Uncomfortable, Safety issue). For session four, fall P3 and senior nursing students participate in an SBAR/CUS simulation. Pharmacy students finish LIPET in the P3 spring semester with a four week IPE skills lab series. LIPET was assessed using student opinion surveys and a final OSCE. Results: After "Got Ethics", over $90 \%$ of students had a better understanding of the importance of IPE. After the second session, $98.5 \%$ of nursing, $96 \%$ of pharmacy, and $82.5 \%$ of medicine students agreed that working in interprofessional teams enhanced learning. Greater than $80 \%$ of students felt an increased sense of team in their interprofessional groups after the third session. $94.91 \%$ of pharmacy students and $100 \%$ of nursing students stated the simulation increased their appreciation of the value of interprofessional collaboration. In the OSCE, $100 \%$ of pharmacy students successfully demonstrated the use of SBAR while while only 30\% appropriately performed CUS. Implications: The LIPET program is an innovative approach to teaching team skills. Students place a high value on the program and we were able to demonstrate increased interprofessional communication skills.

Passport to Health: Evaluating a Comprehensive Service-Based Health Fair Targeting Medicare Beneficiaries. Carly A. Ranson, University of the Pacific, Cynthia S. Valle-Oseguera, University of the Pacific, Bradley F. Ramos, University of the Pacific, Kristina M. Hoh, University of the Pacific, David G. Carranza, University of the Pacific, Donna J. Cruz, University of the Pacific, Sheel M. Patel, University of the Pacific, Olivia Page, University of the Pacific, Amandeep S. Raikhy, University of the Pacific, Edward L. Rogan, University of the Pacific, Rajul A. Patel, University of the Pacific. Objectives: Provide a model for creating a comprehensive service-based health fair that enhances student learning, optimizes patient care, and assesses patient outcomes. Method: In the Fall of 2017, 14 health fairs targeting Medicare beneficiaries were held in 10 cities throughout Northern/ Central California. Upon entry, patients received a card ("Healthcare Passport") containing a color-coded list of 13 available student-driven health screenings/services including Medication Therapy Management (MTM), Blood Pressure, Cholesterol, Diabetes, Immunizations, Bone Density, and several others. Each "Passport" was assigned a unique identification number to record screening/service uptake and facilitate patient tracking throughout the event. Results: In total, 910 patients utilized MTM services, with $822(90 \%)$ reporting a chronic condition for which a corresponding screening/service was offered. The most prevalent chronic conditions observed included hypertension 624(69\%), hyperlipidemia 491(54\%), and diabetes 197(22\%). On average, MTM patients took advantage of 2 screenings/services. The uptake of hypertension, hyperlipidemia and diabetes screenings by those with the disease was 488(78\%), 146(30\%), 52(26\%), respectively. Moreover, of those taking advantage of the service with the disease state 309(63\%) blood pressure, 133(91\%) cholesterol, and $43(83 \%)$ blood glucose readings were controlled according to current practice guidelines. Implications: Providing a student-driven, comprehensive service-based health fair using a "Passport" identification tool has multiple advantages, including: allowing tracking of screening/service uptake, providing insight into disease management, exposing students to a broader scope of patient care, developing practice-ready student pharmacists, and fulfilling Introductory Pharmacy Practice Experience requirements.

Pediatric Degree Option Program Graduates in a Doctor of Pharmacy Curriculum: Confidence and Employment Characteristics. Peter N. Johnson, The University of Oklahoma, Brooke L. Gildon, Southwestern Oklahoma State University, Michelle E. Condren, The University of Oklahoma, Jamie L. Miller, The University of Oklahoma, Tracy M. Hagemann, The University of Tennessee, Teresa V. Lewis, The University of Oklahoma, Barnabas John, Children's Hospital at Saint Francis, Kevin C. Farmer, The University of Oklahoma. Objectives: To survey curricular track graduates (Pediatric Degree Option program) to 1) identify confidence with pediatric pharmacotherapy knowledge statements, 2) compare confidence among residency trained versus non-residency trained graduates, and 3) categorize initial employment position. Method: A survey was conducted in the 30 program graduates from 2011-2016. A 29-item questionnaire was developed to assess confidence pertaining to pediatric pharmacotherapy knowledge statements and post-graduation employment characteristics. An analysis of variance (ANOVA) was utilized to compare responses between residency and non-residency trained graduates. Results: Nineteen $(63.3 \%)$ graduates responded; all expressed "very high" or "high" confidence with calculations, selection of first-line treatment for otitis media, and counseling caregivers. Whereas, $<75 \%$ of graduates expressed "very high" or "high" confidence with identification of pharmacokinetic differences and with counseling children. Ten $(52.6 \%)$ graduates completed a postgraduate year-one (PGY1) residency with five $(50 \%)$ of these completing this residency in a children's hospital. Seven (36.8\%) graduates completed a second year of postgraduate training with three (42.1\%) of these completing a pediatric PGY2 or fellowship. When comparing responses between graduates with and without post-graduate training, the only statement with a difference was identification of pediatric drug information sources with those completing post-graduate training reporting higher confidence $(p=0.08)$. Implications: Graduates noted high confidence in pediatric pharmacotherapy knowledge statements. Many graduates completed post-graduate training. However, there were limited differences in knowledge confidence statements between graduates who did and did not complete residency training. The Pediatric Degree Option program was well received and provides an opportunity for additional knowledge and skill development.

Perceptions and Attitudes of Graduating/Recent Graduate Pharmacists on the Pharmacist's Role in Combating Antimicrobial Resistance. Yuman Lee, St. John's University, Jordan Denas, St. John's University, Ming San Ho-Zhen, St. John's University, Emily M. Ambizas, St. John's University. Objectives: The objective of this research is to evaluate the perceptions and attitudes of graduating and recent graduate pharmacists on antimicrobial resistance (AR), antimicrobial stewardship (AS), and the pharmacist's role in these specified areas. Method: A voluntary, anonymous electronic survey was distributed to P4 students and 2016/ 2017 PharmD graduates from St. John's University. Results: Of 86 respondents, majority agreed that $\mathrm{AR}$ is a global public health crisis and antibiotics are overused in the 


\section{American Journal of Pharmaceutical Education 2018; 82 (5) Article 7158.}

general population, $95 \%(82 / 86)$ and $91 \%(78 / 86)$, respectively. $86 \%$ (74/86) agreed that AS reduces AR and $88 \%(75 / 86)$ agreed that AS improves patient care. Majority disagreed that physicians are the only discipline responsible for AS and agreed that pharmacists have a major role in AS and that their individual efforts impact AR, 93\% (80/86), $92 \%$ (79/86), (62\%) 53/86. 83\% (71/86) would like additional education on AS. In regards to current practice, 56\% (48/86) always/often question the appropriateness of antibiotics, but only $24 \%(21 / 86)$ always/often communicate with prescribers regarding antimicrobial related issues. Less than half report educating patients and/or healthcare professionals on the optimal use of antimicrobials and making an effort to prevent/reduce inappropriate antimicrobial use, 44\% (38/86) and $45 \%(39 / 86)$, respectively. 64\% (55/86) reported not being involved in AS activities. Implications: Respondents are aware of $\mathrm{AR}, \mathrm{AS}$, and the pharmacist's role, however, majority reported not being actively involved. A stronger emphasis on AR/AS in the pharmacy curriculum incorporating active learning is crucial in preparing pharmacists in our combat against AR. Further promotion of AS certification programs may also be beneficial.

Pharmacist Intervention for Blood Pressure Control in Patients With Diabetes and/or Chronic Kidney Disease. Tyler H. Gums, The University of Texas at Austin, Maxwell Anderegg, The University of Iowa, Eric J. MacLaughlin, Texas Tech University Health Sciences Center, Oralia V. Bazaldua, The University of Texas Health Science Center at San Antonio, Timothy J. Ives, University of North Carolina at Chapel Hill, Barry L. Carter, The University of Iowa. Objectives: The objectives of this study were to 1) determine if hypertensive patients with comorbid diabetes mellitus (DM) and/or chronic kidney disease $(\mathrm{CKD})$ receiving a pharmacist intervention had a greater reduction in mean blood pressure (BP) and improved BP control at 9 months compared to those receiving usual care and 2) compare Seventh Report of the Joint National Committee on Prevention, Detection, Evaluation, and Treatment of High Blood Pressure (JNC-7) guideline and 2014 guideline (JNC-8) BP control rates in patients with DM and/ or CKD. Method: This cluster, randomized trial included 32 medical offices in 15 states. Clinical pharmacists made treatment recommendations to physicians at intervention sites. This post-hoc analysis evaluated mean BP and BP control rates in the intervention and control groups. Results: The study included 335 patients (227 intervention, 108 control). When JNC-8 inclusion and control criteria were applied, 241 patients (165 intervention, 76 control) remained and were included in the analysis. The pharmacist-intervention group had significantly greater mean SBP reduction compared to usual care at 9 months (8.64 $\mathrm{mm} \mathrm{Hg}$ [95\% Confidence interval $(\mathrm{CI})=-12.8$, $-4.49, \mathrm{p}<0.001])$. The pharmacist-intervention group had significantly higher BP control at 9 months than usual care by either the JNC-7 or JNC-8 inclusion and control groups (adjusted odds ratio (OR) 1.97 $[95 \% \mathrm{CI}=1.01,3.86] ; \mathrm{p}=0.0470$ and $2.16[95 \% \mathrm{CI}=1.21,3.85]$; $\mathrm{p}=0.0102$, respectively). Implications: This study demonstrated that a pharmacist-lead intervention was effective in reducing mean systolic $\mathrm{BP}$ and improving BP control in patients with uncontrolled hypertension with $\mathrm{DM}$ and/or $\mathrm{CKD}$, regardless of which $\mathrm{BP}$ guidelines were used.

Pharmacist-Led Education of New Medical Residents. Leslie A. Hamilton, The University of Tennessee, Michael L. Behal, The University of Tennessee, Weston $\mathrm{P}$. Keen, The University of Tennessee. Objectives: New medical residents enter programs with vast amounts of knowledge; however, with the small amount of pharmacy-based education included in medical school curricula, it is important residents have a foundation of pharmacy-based knowledge prior to beginning their program. The purpose of this study was to assess the effectiveness of a pharmacist-led education session in improving medical resident knowledge of medication-related concepts. Method: New medical residents were presented a one-hour session on pharmacy-based topics in 2016 and 2017. Prior to the session, an eightquestion pre-test was administered. Following the session, the same eight questions were administered as a post-test. One group of residents was only administered a pre-test and did not receive the intervention, serving as the control. Descriptive statistics were used to assess the efficacy of the education session. Results: Overall, pre-test and post-test averages were $69.5 \%$ and $88 \%$ for 2016 and $68 \%$ and $88.9 \%$ for 2017 . Similar pre-test scores were seen in the control group with $70.1 \%$ in 2016 and $71 \%$ in 2017 . Improvements of $18.5 \%$ and $20.9 \%$ were seen in the 2016 and 2017 intervention groups respectively. Individual questions with the most improvement focused on topics such as anticoagulation and insulin management, while questions with the least improvement dealt with antibiotic therapy. Implications: The pharmacist-led education session significantly improved medical resident knowledge. More frequent or in-depth sessions could further improve results. Residency programs should consider implementing similar sessions to provide new medical residents with a better foundation of medication-related knowledge.

Pharmacy and Dental Student Interprofessional Collaboration: The Impact on Patients. Kathleen Hitchcock, Lake Erie College of Osteopathic Medicine, Kelly L. Scolaro, Lake Erie College of Osteopathic Medicine, Madge Potts-Williams, Lake Erie College of Osteopathic Medicine, Crystal Toloza, Lake Erie College of Osteopathic Medicine, Ian Toloza, Lake Erie College of Osteopathic Medicine, Stephanie Peshek, Lake Erie College of Osteopathic Medicine. Objectives: To evaluate the patients' experience, as well as the quantity and type of counseling interventions, when pharmacy students and dental students collaborate for new patient dental screening appointments. Method: Pairs of third year pharmacy students and third year dental students screened new patients in an academic dental clinic setting. Each screening was comprised of a comprehensive medical history, a medication review with patient counseling, collection of vital signs, and an oral exam. At the end of each visit, the pharmacy student recorded the quantity and type of counseling interventions made during the visit on the bottom of the patient survey. The survey was then provided to each patient to gauge their perception of the experience. Results: Patient surveys were returned by 105 of the 112 patients screened. The patients' overall satisfaction with the experience was $96.4 \%$. Of the patients that were screened by the interprofessional pharmacy and dental student teams, $27.6 \%$ were provided with smoking cessation counseling, $44.8 \%$ were provided recommendations for healthier eating, and $45.7 \%$ were given education about exercise. In regards to medication-related counseling, $39.3 \%$ of the patients were counseled about OTC or herbal products, $32.4 \%$ were given tips to increase adherence to their medications, and $14.8 \%$ were counseled regarding adverse drug reactions. Implications: Combining pharmacy and dental students to screen new patients resulted in a high level of patient satisfaction, as well as meaningful health and medication-related counseling interventions.

Pharmacy Student-Patient Encounters Involving Spirituality During Advanced Practice Rotations. Brian Cryder, Midwestern University/Downers Grove, Michelle Lee, Midwestern University/ Downers Grove, Gloria Workman, Deployment Health Clinical Center, Nathaniel Krumdick, Midwestern University/Downers Grove. Objectives: To identify if spirituality is a current component of pharmacy students interaction with patients during advanced practice 


\section{American Journal of Pharmaceutical Education 2018; 82 (5) Article 7158.}

rotations. Method: Fourth year pharmacy students were asked to complete a web-based questionnaire defining the frequency and setting of patient care activities that incorporated spiritual beliefs during advanced pharmacy practice experience (APPE) rotations. Student demographics, prior spirituality and health coursework, and measures of religiosity and spirituality were collected. Data analysis utilized descriptive statistics with nonparametric exploratory analysis. Results: Sixty-three students completed the survey (31.7\% response rate). While $11 \%$ of students asked patients about spiritual needs, $25.4 \%$ of students reported inclusion of spiritual factors in therapeutic planning creation. The general medicine rotation was the most common setting in which spirituality was incorporated into therapeutic plans. Overall, the inclusion of spirituality in patient care activities was limited in frequency for most students, with only $6 \%$ of students identifying $>5$ incidents addressing spirituality. Student frequency of religious service attendance had the greatest association with student comfort and perceived importance of asking patients about spiritual needs. Implications: Due to the positive health benefits cited in medical literature, students are considering patients' spiritual needs in their patient care activities. While this does not occur frequently with many patients, it was experienced by several students at least once. Student personal spiritual practices seemed to have greater influence on comfort asking patients about spiritual beliefs than past curricular training. Results highlight the need for pharmacy instructors to consider how students' religious characteristics and patient care setting influence addressing spiritual concerns in patient encounters.

Pharmacy Students' Perception and Attitudes Towards Opioid Overdose and Naloxone Rescue Therapy. Jeeseon Kim, Touro College of Pharmacy-New York, Ronnie Moore, Touro College of Pharmacy-New York, Maria A. Sorbera, Long Island University, Roopali Sharma, Touro College of Pharmacy-New York. Objectives: To investigate pharmacy students' perception and attitudes towards opioid overdose and dispensing naloxone rescue therapy without a prescription Method: Prior to a mandatory online training on naloxone rescue therapy, a pre-training survey was distributed to assess students' perception and attitudes towards opioid overdose and naloxone standing order. After the training, a post-training survey was distributed to evaluate whether their perception and attitudes towards the topic changed compared to pre-training. Data were analyzed using descriptive statistics, paired t-test, and chi-squared tests. Results: A total of 105 and 90 students completed pre- and post-training surveys, respectively. Overall, students perceived opioid abuse as a disease that requires treatment (pre 93\% vs. post $91 \% ; \mathrm{p}=0.363$ ). Most students stated that the availability of naloxone rescue therapy as a standing order would not be a means to promote opioid overdose (pre $65 \%$ vs. post $79 \% ; p=0.458)$. Students exhibited high confidence in providing education and counseling to the public and healthcare professionals in pre- and post-training $(\mathrm{p}>0.05)$. However, significantly more students responded that they feel confident/very confident in identifying high risk patients after the training $(\mathrm{p}<0.05)$. Implications: Students perceived opioid abuse as a disease that requires treatment, and naloxone standing order would not be a means to promote opioid overdose. Additional training improved students' confidence in identifying high risk patients, suggesting it may be helpful in improving their overall confidence in this topic, which can impact their ability to contribute in combating the opioid crisis as student pharmacists and future healthcare professionals.

Preparing Advanced Practice Pharmacists: Developing Physical Assessment Competence Through Simulation, Team-Based Learning and Interprofessional Education. Nicole M. Nielsen, California
Health Sciences University, Will Ofstad, California Health Sciences University. Objectives: To develop and assess physical assessment training delivered through simulation and team-based learning (TBL) to prepare for the role of advanced practice pharmacist and work within an interprofessional team. Method: A 15-week two-unit course was created to prepare pharmacy students for advanced practice. Students were trained through a blended simulation and TBL pedagogy. After completion, students were asked to complete a survey consisting of 10 questions adapted from the Kirkpatrick Outcomes Topology focusing on 1) preferences for the learning activities, 2) change in knowledge, skills and attitudes, 3) the utility of the course for future practice, 4) understanding where the training could improve patient care. Results: All students passed the course based on individual and team simulated performance assessments, showing competency across a series of physical assessment and patient interview skills. Survey results demonstrated learner satisfaction, perceived improvement in skills development and excitement to further use this training during rotations. The curriculum developed will be shared as a potential model for other programs. Implications: The growing need for pharmacists to practice at the top of their license calls for more training in physical assessment. Methods must be geared to prepare students for success as an interprofessional patient-centered caregiver and readiness for the advanced practice pharmacist certification. This expanded pharmacist role supports interprofessional practice, particularly in underserved areas where provider shortages may exist. Physical assessment training delivered through blended simulation and TBL methods may serve as a model for preparing advanced practice pharmacists.

Process for Assisting Schools of Pharmacy for Strategic Planning. Therese I. Poirier, Southern Illinois University Edwardsville, Radhika Devraj, Southern Illinois University Edwardsville. Objectives: Conducting a gap analysis (GA) survey is an essential and important first step in Strategic Planning (SP). Generating items for the GA survey is cumbersome and no established process currently exists. We performed a retrospective review of gap analysis items generated during our SP process by comparing it to ACPE standards and Mattingly's Quality criteria Method: Each of the 49 GA items generated were mapped to the appropriate ACPE standard and to Mattingly's Quality criteria via consensus by two reviewers at two different time points. Results: About 80\% ( $39 / 49)$ items aligned with the ACPE standards, and $67 \%$ ( $33 / 49$ ) of them aligned with quality criteria, while $16 \%$ $(8 / 49)$ items did not map to either ACPE standards or Quality criteria. The most frequent quality measures addressed by the gap analysis items were stakeholder feedback, curriculum and operational measures. Two ACPE standards did not map to GA items or Quality criteria. These included Standard 5: eligibility and reporting requirements and Standard 17: progression. Implications: Our retrospective approach successfully mapped gap analysis items to most standards and quality criteria. To make the GA survey item generation process less burdensome, and to increase alignment with ACPE standards and quality criteria, we suggest schools engaging in Strategic Planning use a prospective approach to generate items for GA.

Promoting Change in Interprofessional Socialization and Valuing Through Roundtable Discussions on Opioid Misuse and Abuse. Marta J. Brooks, Regis University, Suzanne Holm, Regis University, Amy Rich, Regis University, Christine Feltman, Regis University, Shelene Thomas, Regis University. Objectives: To utilize an interprofessional panel presentation and roundtable discussion to quantitatively and qualitatively assess student beliefs, attitudes, and behaviors regarding interprofessional collaboration to address opioid misuse and 


\section{American Journal of Pharmaceutical Education 2018; 82 (5) Article 7158.}

abuse. Method: This IRB approved study used a mixed method, cohort design to explore the collaborative environment simulated by this interprofessional experience (IE) of a panel and small focus group discussions. Graduate students (OT, PT, Pharmacy) were assessed before and after the IE. The validated tool, Interprofessional Socialization and Valuing Scale (ISVS) 9A/B was used to measure participant self-perceptions pre/post-IE. The ISVS identifies three constructs as related to student perception of interprofessional relationships: beliefs, attitudes and behaviors. Qualitative measures (individual responsibility, the severity of the opioid epidemic, modeling of interprofessional communication) were obtained through data analysis of audiotaped focus group discussions using NVivo. Multiple Wilcoxon signed-rank tests were conducted to compare ISVS relationship behaviors before and after the IE. Results: A statistically significant difference was found pre/post event for the entire audience of students, form, ISVS9A compared to ISVS-9B $(\mathrm{P}<0.001 ; \mathrm{z}=-4739)$. Regarding individual questions, each profession demonstrated improvement in interprofessional valuing based on median scoring comparing pre and post educational event experience for 7 of the nine questions. Qualitatively, Students reported needing to work on open and active communication, making time to develop relationships, advocating for clients, and deepening their understanding of other professional's roles. Implications: At our university, this IE is an effective way to foster students to learn about, from, and with each other regarding the opioid crisis.

Promoting Empathy With Glucometer Use in a Diabetes Laboratory. Ashlee N. McMillan, West Virginia University. Objectives: To assess students' learning and perceptions of a glucometer exercise and laboratory. Method: One week prior to a diabetes education laboratory, first year professional students were given a glucometer, glucose $\log$, and 14 test strips and lancets. Students were not given any instructions on how to use the glucometers and were only instructed to test their blood glucose twice daily for one week and bring their glucose log and glucometer the following week for the diabetes education laboratory. Students were taught how to correctly use their glucometers and interpret blood glucose values during the diabetes education laboratory. At the end of the session, students completed a survey that assessed their glucometer usage and their thoughts regarding this educational experience. Results: A total of 68 students (95.8\% of class) completed the survey. Prior to this experience 44 students $(64.7 \%)$ had never used a glucometer, and all but four students learned how to properly use the glucometer prior to being taught in class. Sixty-six students (97.1\%) either agreed or strongly agreed that this was a valuable learning experience. Sixty-seven students (98.5\%) either agreed or strongly agreed that this experience made them feel more compassionate towards individuals that need to test their blood glucose daily. Implications: This laboratory activity was a valuable learning experience that also helped students have more empathy for patients who have to test their blood glucose multiple times daily. This experience will also hopefully motivate students to effectively counsel their patients on glucometer use in practice.

Providing Interdisciplinary Academic Detailing to Improve Pneumococcal Immunization Rates. Kimberly C. McKeirnan, Washington State University, Karen Colorafi, Washington State University, Shannon G. Panther, Washington State University. Objectives: To develop academic detailing material and to provide academic detailing to physicians, nurses, clinic staff, pharmacists, and pharmacy staff in rural areas. To improve pneumococcal immunization rates in two rural Washington state counties. Method: An interprofessional team of faculty and students lead by clinical pharmacists was trained in academic detailing. The team performed needs assessments and clinic workflow assessments; developed educational materials including a patient-centered exam room poster and a provider-targeted guideline based handout; delivered training including best practices for incorporating pneumococcal immunizations into workflow using an electronic health record; and collected Immunization rates for the two intervention clinics during a four-year period. Results: Academic detailing presentations and materials were well received. The exam room posters in particular were well liked by providers and patients and are being displayed in clinics and pharmacies in rural Washington. Immunization trends steadily increased yearover-year during the reference period. Implications: Immunizations, such as pneumococcal vaccine, are a low-cost, high-impact intervention, according to the Task Force on Community Preventative Services. While academic detailing has been utilized in the areas of diabetes and mental health, the impact on immunization practices is limited. The use of academic detailing as a technique to boost immunizations therefore represents a significant contribution to the literature, demonstrates an approach to utilizing academic skills in community settings, and provides an opportunity to involve health science students in interprofessional research.

Publication Records and Bibliometric Indices of Canadian and US Pharmacy Deans. Dennis F. Thompson, Southwestern Oklahoma State University. Objectives: As leaders and role-models in Schools and Colleges of Pharmacy, CEO Deans must have a sufficient background and experience in research and scholarship. The primary purpose of this research was to characterize and compare the publication records and bibliometric indices of the current CEO Deans at the Schools and Colleges of Pharmacy (SCOP) in Canada and the United States. Method: This was a cross-sectional study of pharmacy dean publication records and bibliometric indices using the Web of Science (WoS) database. Deans were identified using the AACP Roster and institutional web sites. The Phase 2 methods of Thompson and Nahata (AJPE 2012;76(9): Article 173) were used in conducting the WoS searches. The software programs developed by Soler (Scientometrics 2007;72(2):281-290) were used to separate homologues and calculate bibliometric indices. Bibliometric indices generated included: lifetime publications, publications/year, h-index, m-quotient, lifetime citations, citations/year, and average citations/paper. The Kruskal-Wallis analysis of variance for nonparametric data was used to assess differences between groups. Results: Median bibliometric indices for Canadian pharmacy deans $(\mathrm{N}=10)$ vs. United States pharmacy deans $(\mathrm{N}=124)$ were as follows: No. of publications $=57.5$ vs. 20.5, Publications $/$ year $=3.5$ vs. 0.5 , h-index $=14.5$ vs. 8 , Total citations $=$ 628.5 vs. 223.5 , Citations/year $=38.2$ vs. 11.2. None of the differences were significant at $\mathrm{p}<0.05$. Implications: Median bibliometric indices from Canadian pharmacy deans were higher but not significantly different from U.S. pharmacy deans.

Raising the Bar for Student Performance in a 6-Semester Pharmacy Skills Sequence. Teresa H. Kane, Albany College of Pharmacy and Health Sciences, Jane K. Boyd, Albany College of Pharmacy and Health Sciences, Lee Anna S. Obos, Albany College of Pharmacy and Health Sciences. Objectives: To develop a comprehensive support program for the Pharmacy Skills courses to assist students in meeting the new course passing standard of $70 \%$. Method: Practice sessions were held prior to grading new compounding techniques. Student attendance was voluntary. Frequent assessments were administered to identify areas where students needed additional instruction. Students scoring $<70 \%$ were required to complete in-course remediation for specific content areas. Remediation consisted of group or individual sessions with Teaching Assistants, content specific worksheets, 


\section{American Journal of Pharmaceutical Education 2018; 82 (5) Article 7158.}

review of recorded instructional material or additional skill-based practice activities. In addition, students with a midpoint average $<70 \%$ met with faculty to develop a personal remediation plan. Results: In 2016, prior to the new passing standard and restructured support system, the number of students with a final course grade $<70 \%$ was: 20 P1 students (10.8\%), 5 P2 students (2.6\%) and 1 P3 student (0.44\%). After implementation in 2017, the number of students with a final course grade $<70 \%$ was: 9 P1 students (5.2\%), 0 P2 students and 0 P3 students. Implications: Raising the passing requirement to $70 \%$ motivated students to attend optional practice sessions. This combined with in-course remediation resulted in improved student overall performance.

Recognizing Excellence in Teaching in Pharmacy Education. Jaime A. Foushee, Presbyterian College, Jennifer N. Clements, Presbyterian College. Objectives: To describe various practices for recognizing excellence in didactic and experiential teaching at pharmacy schools nationwide. Method: A voluntary survey was distributed electronically in January 2018 to pharmacy school administrators. The 13 questions survey included demographic information and questions regarding procedures for recognizing teachers of the year. Results: Thirty-seven (26\%) of 142 institutions participated in the survey. All participating institutions recognize teachers of the year annually, with the majority $(n=35 ; 94.6 \%)$ separately recognizing experiential teachers. The number of teachers of the year awarded annually differed from institution to institution, with $15(40.5 \%)$ recognizing one individual, $11(29.7 \%)$ recognizing one teacher per pharmacy school class, $5(13.5 \%)$ recognizing two individuals, and $6(16.2 \%)$ describing an alternative number. Numerous processes are utilized for identifying teachers of the year, with student popular vote being employed most frequently at 23 institutions (62.2\%). The number of faculty sent to the annual American Association of Colleges of Pharmacy (AACP) teacher of the year luncheon was also variable. Rewards for teaching award recipients included awards ceremonies, individual plaques, perpetual plaques at institution, printed recognition, AACP Annual Meeting expenses, and/or monetary awards. The majority of institutions $(\mathrm{n}=21 ; 56.8 \%)$ do not limit awards, allowing for administrators to receive awards and recipients to receive awards in consecutive years. Implications: A variety of strategies are utilized in pharmacy education to select and recognize quality pharmacy educators. Guidance from the academy on optimal strategies may standardize processes.

Re-Designing Classroom Peer-Evaluation Tools to Strengthen the Peer-Review Process. Lea S. Eiland, Auburn University, Allison Helmer, Auburn University, Kristi W. Kelley, Auburn University, E. Kelly Hester, Auburn University. Objectives: To assess faculty's utility and perceptions of re-designed classroom peer-evaluation tools that enhance the formative peer-review process. Method: Our institution developed a classroom peer-evaluation form in 2011. In 2017, due to a changing curriculum, four new peer-evaluation tools were developed to encourage a stepwise, intentional approach for the peer-evaluation process in any didactic teaching role. These tools were created following a faculty survey and review of published literature, websites of peer-evaluation tools, the 2016 ACPE curriculum standards, and AACP's entrustable professional activities. Pre-assessment and postteaching self-reflection questions were created for the faculty instructor to complete. The peer-evaluator guidance document encompassed five domains with example questions of teaching elements to evaluate. A draft letter provided framework for the peer-evaluation response. All tools encouraged faculty planning, assessing, reflection and discussion. Faculty were educated on the new tools prior to implementation. A follow-up faculty survey was distributed in 2018 to assess the utility and perceptions of the new tools. Results: Twenty-four faculty completed the follow-up survey. Since November 2017, four faculty conducted eight peer-evaluations using the new tools, with each tool used at least four times. All domains within the guidance document were used at least five times and $93 \%$ of faculty stated the example questions prompted assessment of additional teaching elements than they typically provided. Faculty were highly satisfied (63\%) and found benefit (92\%) in the re-designed peer-evaluation tools. One faculty member requested additional explanations. Implications: Faculty used all four tools developed for the peer-review process. Education is essential for faculty's understanding of the forms.

Reducing Costs by Using Simulated Medications Instead of Commercial Products in a Pharmaceutical Care Lab. Anthony L. Walker, The University of Louisiana at Monroe, Raghda Elsayed, The University of Louisiana at Monroe, Stephen R. Hill, The University of Louisiana at Monroe. Objectives: To determine if compounding simulated medications by pharmacy school faculty and/or staff, for use by student pharmacists in pharmacy school practice labs, reduces costs compared to paying the cost for original medications from commercial sources. Method: Students had the opportunity to utilize inhouse prepared simulated antibiotics to dispense their final products during three laboratory lessons. The simulated antibiotics prepared for the labs included ampicillin, ceftazidime, and amoxicillin powders for reconstitution/suspension. Previously, antibiotics were purchased from wholesale suppliers at contracted prices. For the most recent year, changes were made to utilize in-house simulated medications, which were prepared from basic ingredients and supplies already available. Subsequently, the costs of the two methods were compared and results were reported using descriptive statistics. Results: In-house prepared simulated ampicillin, ceftazidime, and amoxicillin powders for reconstitution/suspension were $73 \%, 34.9 \%$, and $4.32 \%$ less costly in comparison to commercially available generic products. Preparation time for 100 vials/bottles of each simulated medication was two hours for amoxicillin and one hour for each of the other two products. Preparing in-house simulated medications for use in pharmacy school practice labs is cheaper than purchasing commercially available products. Implications: Despite the added prep time of 1 to 2 hours to get simulated medications ready for use in lab, per lab costs decreased. In addition to cost benefits, the lab is no longer contributing to the national drug shortage.

Revising an Experiential Peer-Evaluation Form to Evaluate Preceptor, Practice Site, and Student Activities. Allison Helmer, Auburn University, E. Kelly Hester, Auburn University, Kristi W. Kelley, Auburn University, Lea S. Eiland, Auburn University. Objectives: To assess the utility and faculty perceptions of a revised experiential peerevaluation form created to ensure quality student education through formative preceptor evaluation Method: Our institution developed an experiential peer-evaluation form in 2011 and completed major revisions in 2017 following a faculty survey, review of published literature, websites of peer-evaluation tools, the 2016 ACPE curriculum standards, and AACP's entrustable professional activities. Revisions were made to evaluate preceptor qualities (eg, engaging, professional), practice site qualities (eg, patient load, interprofessional interactions), patient care learning (eg, direct patient care, critical thinking), student activities (eg, projects, presentations), preceptor's feedback, and quality assurance in experiential structure. Peer-review was provided by selected pharmacy practice and professional program assessment faculty prior to implementation. Faculty were educated on the revised form prior to implementation. A follow-up survey was distributed in 2018 to assess the utility and faculty perceptions of the revised form. 


\section{American Journal of Pharmaceutical Education 2018; 82 (5) Article 7158.}

Results: Twenty-four faculty completed the follow-up survey. Since May, four $(16.67 \%)$ faculty conducted a peer-evaluation using the revised form. All sections of the form were used at least once, with all faculty evaluating experiential structure. Most (61-99\%) faculty were extremely satisfied with the six domains of the form and found it extremely easy to use. All faculty plan to modify experiential teaching based on peer feedback. Most faculty reported peer evaluation positively impacts planning for teaching, provides new ideas, and improves ability to get promoted. Implications: Faculty found the feedback from the revised form easy to use and beneficial for modifying teaching. Additional evaluator training may increase faculty satisfaction.

Sign of the Times: Preparing Students for Emerging Roles With a Contraceptive Prescribing Activity. Sarah Lynch, Binghamton University, The State University of New York, Kathleen Vest, Midwestern University/Downers Grove, Brooke Griffin, Midwestern University/ Downers Grove. Objectives: Several existing state laws increase the pharmacist's role in hormonal contraceptive access. There is little published evidence of effective ways to prepare students for this role. This study sought to determine the utility of a simulated contraceptive prescribing activity for pharmacy students. Method: Students enrolled in a third-year women's health elective course in 2017 and 2018 participated in a required simulated prescribing experience. Using an existing state protocol, students interviewed and assessed standardized patients. An introductory lecture reviewed contraceptive therapeutics and discussed prescribing as part of the patient care process. Pre- and post-surveys were administered to collect students' perspectives and self-assessed confidence. Grades were analyzed to assess academic performance. Results: Students performed well on the activity with average grades of $95 \%$ in 2017 and $86.5 \%$ in 2018 . The response rate was $65 \%$ on the pre-survey and $49 \%$ on the post-survey. Most respondents strongly agreed or agreed that the activity was challenging $(72.7 \%)$ and realistic $(90.9 \%)$. The majority of students agreed that they felt more confident in their ability to prescribe hormonal contraceptives $(90.9 \%)$. After completing the simulation activity, more students agreed that they felt comfortable evaluating and recommending a specific hormonal contraceptive per protocol (Pre-survey 63.7\%; Post-survey 94\%). Implications: Students found this activity to be realistic and reported feeling more confident and comfortable prescribing hormonal contraceptives per protocol after completing the simulation. Other colleges of pharmacy may consider a similar model for simulation activities to prepare students for this change in pharmacy practice.

Simulation Improves Student Pharmacists' Knowledge and SelfConfidence in Managing a Community-Based Opioid Overdose. James W. Torr, Lipscomb University, Emily K. Harris, Lipscomb University, Meagan Singletary, Lipscomb University. Objectives: A 2016 survey found only $20.4 \%$ of community pharmacists were comfortable selling naloxone without a prescription, indicating an educational gap in this area. The nationwide opioid epidemic, accompanied by changes to naloxone accessibility such as dispensing under a collaborativepractice agreement, imply community pharmacists should be prepared to recognize and manage an opioid overdose. Our objective was to develop and evaluate the impact of a simulation-enhanced exercise on student pharmacist knowledge, skills, and self-confidence when managing a community-based opioid overdose. Method: Third-year student pharmacists $(\mathrm{n}=14)$ participated in a 2-week Community-Based Opioid Overdose Management exercise enhanced with high-fidelity simulation. Participants completed pre-/post-knowledge assessments, pre-/post-self-confidence instruments, and a post-curriculum satisfaction survey. Participants completed an online Opioid Antagonist Training Program prior to participating in an opioid overdose simulation. Participants were randomized into teams of 2 to 3 for the simulation and assessed with an Objective Structured Clinical Examination (OSCE). The OSCE was mapped to the Pharmacists' Patient Care Process. Statistical analysis was performed to measure changes in pre-/postsimulation scores on the knowledge and self-confidence instruments. Results: Knowledge-based assessment scores increased significantly from pre-test to post-test $(69.4 \%$ and $90.3 \%, \mathrm{p}=0.0002)$. Students' scores increased significantly on 4 of 5 items from pre-to-post on the self-confidence survey. The mean score for the OSCE skills assessment was 91\%. Implications: This exercise demonstrates the positive impact of simulation-enhanced learning on student pharmacists' knowledge and self-confidence for recognizing and managing a community-based opioid overdose. Additionally, simulation may be an effective method to evaluate student pharmacists' competency.

Skills Lab Curriculum Impact on Student Confidence to Navigate Health Records and Present Patients. Caroline Schweitzer Quinn, University of Wisconsin-Madison, Susanne G. Barnett, University of Wisconsin-Madison, Edward Portillo, University of Wisconsin-Madison. Objectives: To evaluate the impact of a patient presentation skills lab curriculum on student confidence to perform skills necessary to navigate a patient's electronic health record (EHR) and present pertinent, relevant, and complete information, including the identification and resolution of drug-related problems. Method: Four patient case presentation lab sessions were implemented in a third-year Doctor of Pharmacy (P3) pharmacotherapy laboratory course. Students $(n=146)$ were invited to complete baseline, mid-semester, and end-of-semester electronic surveys to measure self-perceived confidence in ability to identify and present relevant components within an EHR to a pharmacy preceptor, including the identification and resolution of drug-related problems (34-item, 7-point Likert scale). The baseline survey included questions relating to relevance and importance of this skill using a 7point Likert agreement scale. Changes in confidence were measured using the Wilcoxon Signed-Rank Test, with statistical significance determined by alpha $\leq 0.016$. Results: Baseline, mid-semester, and end-of-semester surveys were completed by $96 \%, 86 \%$, and $99 \%$ of students, respectively. A significant increase in all confidence items was seen between baseline and end-of-the-semester. Comparing baseline to mid-semester confidence, 32 of 34 items had significantly increased. At baseline, $76 \%, 81 \%$, and $82 \%$ of students reportedly agreed or strongly agreed that patient case presentations were an important skill for outpatient, ambulatory, and inpatient practice settings, respectively. Implications: Incorporating patient case presentation lab sessions into a pharmacotherapy lab course resulted in increased student confidence to perform this skill over time. Students agree the ability to present a patient case to a preceptor is important to multiple pharmacy practice settings.

Student Anxiety Towards Performance-Based Assessment With Repeated Exposure. Daniel S. Longyhore, Wilkes University. Objectives: State-anxiety is not linked to outcomes in performancebased assessment (PBA). However, students still experience differing levels of anxiety when new PBAs are introduced into a curriculum. The objective is to identify the difference in student anxiety between those first exposed to a moderate-risk summative PBA versus those with repeated exposure. Method: Student anxiety was assessed using the Spielberger State-Trait Anxiety Index (STAI). Students completed this survey in their third-professional year, with Group A completing it in 2015 and Group B in 2017. Both groups completed the survey two weeks prior to their end-of-semester OSCEs, in the semester prior to 


\section{American Journal of Pharmaceutical Education 2018; 82 (5) Article 7158.}

entering APPEs. Group A was new to OSCEs and had not undergone simulation as a progression-related exam. Group B had experienced OSCEs as a progression-related exam since starting pharmacy school. Anxiety scores for trait (STAI-T) and state (STAI-S) were compared between groups. Results: STAI results were available for 64 and 67 students in Groups A and B, respectively. STAI-T scores did not significantly differ between student groups (41.34 vs. 44.10, $\mathrm{p}=0.214$ ), serving as evidence that baseline anxiety characteristics were not different between groups. However, Group B's survey did identify a significantly lower STAI-S score (47.83 vs. $39.69, \mathrm{p}=0.001)$, signaling that repeated exposure may have lessened student anxiety leading up to OSCEs. Implications: From a student-concern perspective, anxiety secondary to PBA is less with repeat exposure. Faculty and curriculum committee members concerned about student anxiety when introducing new PBAs should expect this anxiety to lessen over time.

Student Perceptions and Engagement Level in Flipped Learning vs. Lecture-Based Instruction. Cheyenne C. Newsome, Washington State University, Brenda S. Bray, Washington State University, Connie M. Remsberg, Washington State University. Objectives: To examine how student perceptions on class preparation, engagement, and learning changed from instruction in a lecture-based classroom to a flippedlearning classroom. Method: This study was certified as exempt by the Washington State University IRB. A voluntary, anonymous survey was administered to students in a third-year pharmacotherapy course at the conclusion of the semester via Qualtrics ${ }^{\circledR}$. This survey was administered to two cohorts of students. One cohort received lecturebased instruction style and the other received flipped-classroom style. Survey items asked students to score how they prepared for class, how preparing for class affected their learning, and how in-class activities affected learning. A Mann-Whitney U test was used to identify significant differences between the two cohorts. Results: Response rates were $64 \%(81 / 131)$ and $73 \%(112 / 154)$ for the lecture and flipped cohorts, respectively. Sixty-eight percent of students in the flipped classroom cohort frequently completed assigned materials prior to coming to class in comparison to $32 \%$ in the lecture-based $(\mathrm{p}<0.01)$. More students in the flipped cohort than the lecture cohort felt preparing for class improved their learning during class time $(90 \%$ vs $63 \%, \mathrm{p}<0.01$ ) and improved their performance on exams ( $77 \%$ vs. $61 \%, p=0.01)$. The different teaching approaches did not appear to impact student confidence on their ability answer to assessment items at different Bloom's taxonomy levels. Implications: Students within this pharmacotherapy course find value in preparing for class and believe the flipped learning model enhances their learning. This information should encourage faculty considering implementation of a flipped classroom model.

Student Perceptions and Outcomes Associated With a Simulated Electronic Medical Record Pharmacotherapy Case Studies Elective. Kimberly E. Kelly, Campbell University, Scott Perkins, Campbell University, Manali Patel, Campbell University, Paul DiMondi, Campbell University. Objectives: To compare the Clinical Foundation Scores (CFS) between students who completed a simulated pharmacotherapy case studies (SPCS) elective course compared to the standard curriculum. Secondary objectives included perceptions of the elective course and preparedness for APPE experiences, and to compare the individual components of the CFS. Method: This was a prospective, open-label, cohort study of third-year student pharmacists. All students enrolled in the core-curriculum therapeutics course were eligible for inclusion. Students voluntarily enrolled in a 7-week SPCS elective which used and electronic medical record (EMR) to deliver content and guide discussion. The primary endpoint was the CFS, which was designed to assess knowledge, decision making, and critical thinking. The CFS was the composite of a student's final numeric therapeutics course grade, a script concordance test, and the health science and reasoning test. Results: Students average age and cumulative GPA were similar between SPCS group versus control (26.2 vs. 29.6 years; $p=0.157$ and 3.31 vs 3.36; $\mathrm{p}=0.764)$. More females enrolled in the SPCS course ( $93 \%$ vs. $44 \%, p=0.0067)$. A trend favoring the SPCS group was seen in the overall CFS ( 70.7 vs. $67 ; p=0.082$ ). No difference was seen in the three individual components of the CFS. Students' confidence in solving cases, using EMRs, and identifying the primary problem improved. Implications: A trend towards greater clinical aptitude was seen in the intervention group with the use of the EMRs and simulated cases. Extending these activities throughout the curriculum may further improve readiness to practice.

Student Perceptions of Active Learning Strategies Used in a First Year Pharmacy Course. Pramodini B. Kale-Pradhan, Wayne State University, Minakshi Lahiri, Wayne State University. Objectives: To investigate the student perceptions of active learning strategies used in a first year Doctor of Pharmacy course in order to improve student learning and promote student success. Method: Participants of the study were recruited from 100 first year students enrolled in the first Pharmacotherapy module. The students were provided a list of nine questions as instructor provided items with the university conducted anonymous Student Evaluation of Teaching (SET) form. Data was collected from student responses to instructor provided items using the university's scale for SET $5=$ Strongly Agree, $4=$ Agree, $3=$ neutral/undecided, 2 = disagree, $1=$ strongly disagree. Participation in the study was voluntary and had no impact on their course grade. Results: There were five different active learning strategies utilized in the course including team based learning (TBL), case study (CS), group activities with discovery question prompts (GA), prework with in class presentations (PCP) and interactive technology (IT). 90\% of the students responded to each of the individual questions. $81 \%$ indicated that prework was helpful toward learning. The participants strongly agreed/agreed that the active learning strategies were effective for their learning with $64 \%$ agreement for TBL, $80 \%$ for CS, $63 \%$ for GA, 52\% each for PCP and IT. Implications: Results indicate that the active learning strategies were beneficial for student learning. More longitudinal data will be collected. Student perspectives from this study indicate that active learning strategies will be helpful for student success in other professional programs.

Student Perceptions of a Mobile Spaced Education and Testing Platform in a Pharmacotherapy Course. Jennifer Steinberg, Nova Southeastern University, Cynthia Moreau, Nova Southeastern University. Objectives: To evaluate the perceived utility of mobile spaced education and testing as a tool to enhance students' studying in a Pharmacotherapy course Method: Using a commercially-available mobile platform, spaced learning and testing effects were utilized to reinforce concepts in a Pharmacotherapy course for third-year pharmacy students. Via smartphone app, email, or online portal, students received 4 multiple-choice questions approximately every 2 days to align with select topics covered during the semester, which repeated in a spaced manner until mastery. Students were surveyed prior to the intervention to obtain baseline demographics and at the end of the semester on the perceived utility and benefit. Results: A total of 146 students completed the intervention in Fall 2017 and 84 (57.5\%) completed the postsurvey. Overall, $89.3 \%$ of respondents perceived the intervention as a helpful or very helpful study strategy and $64.8 \%$ felt the platform improved their overall performance in the course. When comparing spaced versus bulk presentation of questions, $67 \%$ reported increased 


\section{American Journal of Pharmaceutical Education 2018; 82 (5) Article 7158.}

benefit from spaced learning. In addition to pharmacotherapy, students most frequently selected NAPLEX (89.7\%) and top 200 drug review $(53.4 \%)$ as useful alternative applications. Implications: Mobile spaced education and testing was well received among students in a Pharmacotherapy course. Preliminary data suggests students perceived the study tool had a positive impact on their course performance. Future evaluation should examine correlation between student engagement with the intervention and course grades. Additionally, the intervention may provide benefit for other pharmacyrelated applications.

Student Perceptions of an Application-Based Exercise on Use of an Infectious Disease Clinical Reference Guide. Sarah Eudaley, The University of Tennessee, Leslie A. Hamilton, The University of Tennessee. Objectives: Antimicrobial resistance has propelled stewardship to the forefront of healthcare. An application-based exercise was developed to introduce students to an infectious disease clinical reference guide. We aimed to evaluate confidence and perceptions of second-year pharmacy students in their ability to use the reference. Method: A digital Sanford guide app was provided. Prior to an in-class application-based activity in a required skills-based course, students were required to view a recorded tutorial. During class, students answered 16 case-based questions using the app, after which answers were discussed. An online survey was emailed to students following course completion. Data was analyzed using descriptive statistics. Results: Overall, 120 and 127 students completed the confidence portion and activity evaluation of the survey, respectively. Most (89\%) felt moderately/very confident in using the reference to select an empiric $(89 \%)$ or alternative regimen $(90 \%)$ based on diagnosis. In addition, $77.5 \%$ felt moderately/very confident in de-escalating therapy. Most (94\%) agreed/strongly agreed that the application-based activity, compared to the tutorial, better prepared them for use in patient scenarios. Most (67.7\%) reported using the app in other courses, and the majority agreed/strongly agreed (94\%) that it enhanced their learning. Notably, $54 \%$ of students who work do so in the community and $49 \%$ $(n=34)$ reported referencing the app while working. Overall, $>90 \%$ agreed/strongly agreed they feel prepared to use the reference for patient care $(\mathrm{n}=117)$ and using the reference will facilitate antimicrobial stewardship $(n=121)$. Implications: Application-based skills exercises can impact antimicrobial use and are important given the expanding role of pharmacists in optimization of antimicrobial use.

Student Perceptions of the PCOA-A Multi-institutional Sample. Kimberly K. Daugherty, Sullivan University, Adam C. Welch, East Tennessee State University, Justine S. Gortney, Wayne State University, Ashley Castleberry, University of Arkansas for Medical Sciences, Elizabeth A. Coyle, University of Houston, Jennifer M. Trujillo, University of Colorado, Melissa S. Medina, The University of Oklahoma, Julie M. Sease, Presbyterian College, Uyen M. Le, California Northstate University, Michael J. Rudolph, Marshall University, Angela Brownfield, University of Missouri-Kansas City. Objectives: Questions regarding use of the PCOA to identify at risk students and serve as a high stakes exam have been debated in the literature, but no data exists regarding students' perceptions of the exam. The purpose of our study was to assess students' perceptions of the PCOA exam. Method: A 21-question, electronic survey was developed and beta-tested by faculty representing geographically diverse S/COPs. APPE students from $5 \mathrm{~S} / \mathrm{COPs}$ completed this survey regarding their PCOA exam study habits, feedback, and results use. Results: 131 students complete the study with the majority being female. A majority of students indicated that they did not study for the PCOA (65\%) or use results of PCOA to prepare for APPE $(93 \%)$ or NAPLEX (81\%) nor did they review results with an advisor
(78\%). Most students were neutral or disagreed with the statement that doing well on this test was important to them $(65 \%)$ but most agreed they gave good effort on the test (61\%). Students agreed that the following incentives would influence their motivation on the PCOA: if it affected progression $(70 \%)$, APPE placement $(65 \%)$, or required remediation (69\%). Implications: This study revealed that a majority of students do not study for the PCOA exam and do not discuss their results with faculty advisors in order to improve their area(s) of weakness for APPEs. It is unclear if student perceptions are the result of a lack of understanding of the PCOA's goals, student time, exam result interpretation and mentoring, or tangible incentives for strong performance.

Student Perceptions of the Reinforcement of Immunization Technique Throughout a Pharmacy Curriculum. Gretchen M. Garofoli, West Virginia University, Ashleigh L. Barrickman, West Virginia University, Ashlee N. McMillan, West Virginia University. Objectives: To 1) determine student perceptions about how immunizations and immunization technique are taught throughout the curriculum, 2) determine immunization experience outside of the classroom, and 3) evaluate student confidence regarding immunization administration as a result of the didactic curriculum. Method: The American Pharmacists Association Pharmacy-Based Immunization Certificate program is delivered during the first professional year of the curriculum. Practicum sessions were developed for the second and third years of the curriculum to improve students' knowledge about immunizations and to reinforce administration technique. A questionnaire was given to students after completing the immunization reinforcement classes to assess the value of these sessions. Descriptive statistics were utilized to evaluate the data. Results: Forty-five students completed the survey, and the majority of students found that the reinforcement of immunization technique and concepts was valuable (37\%) or very valuable (61\%). Only $36.96 \%$ of students were able to vaccinate during their Introductory Pharmacy Practice Experiences (IPPE), and 39.13\% of students do not have internships that allow them to provide vaccinations. Most students agreed (41\%) or strongly agreed (48\%) that their confidence level in administering vaccines improved as a result of the immunization sessions throughout the curriculum. Implications: Introducing students to immunization concepts and technique in the first year and reinforcing these concepts in the second and third years of the curriculum has been effective in increasing student confidence and is perceived as a valuable learning experience. Reinforcement is necessary in the curriculum based on the limited experience that students obtain outside of the classroom.

Student Perceptions of the Use of CATME: An Online Peer Evaluation Program. Kimberly A. Ference, Wilkes University, Edward F. Foote, Wilkes University, Blake Mackesy, Wilkes University, Paul Reinert, Wilkes University. Objectives: To assess student perceptions of giving and receiving feedback using CATME and the significance of written peer-to-peer comments. Method: Teams of P1 students participate in a year-long project-based learning activity. Students are taught about feedback, oriented to CATME (www.catme.org) and complete self and peer evaluations three times during the project. The system collects feedback consisting of numerical ratings in various dimensions of teamwork as well as written self and peer feedback. After the evaluation data was reviewed by course faculty, all students received the numerical ratings but only one section of the class received the (anonymous) peer comments electronically through the system. An online, end-of-project survey (likert based statements and open ended questions) was used to determine student opinions about the process. Results: Student response rate to the online survey was $85 \%$. The group that received written peer feedback agreed or 


\section{American Journal of Pharmaceutical Education 2018; 82 (5) Article 7158.}

strongly agreed that the feedback: 1) helped them understand their own performance ( $83 \%$ vs. $47 \%, p<0.05), 2$ ) improved group/team performance ( $79 \%$ vs. $44 \%, \mathrm{p}<0.05$ ), and 3 ) prepared to them to provide feedback in the future ( $86 \%$ vs. $59 \%, \mathrm{p}<0.05)$. Answers to openended survey questions supported the quantitative data where $86 \%$ of students who received written peer feedback felt the process had a positive impact on their performance compared to $55 \%$ in the nonwritten feedback group. Implications: CATME is a useful tool for providing feedback. Written comments to peers had the most meaningful impact on student perceptions of giving and receiving feedback.

Student Pharmacists' Perceptions of the Pharmacists' Patient Care Process (PPCP) for Hypertension-Focused Curricular Activities. Anita Siu, Rutgers, The State University of New Jersey, Lucio Volino, Rutgers, The State University of New Jersey, Lucy Lin, Rutgers, The State University of New Jersey, Rupal Mansukhani, Rutgers, The State University of New Jersey. Objectives: Compare faculty expectations to students' perceptions of the PPCP in a blood pressure objective structured clinical examination (OSCE). Method: Third professional year student pharmacists completed three Institutional Review Board-approved surveys related to the PPCP for blood pressure screening: (1) pre-lecture quiz assessing baseline PPCP knowledge and demographics, (2) post-lecture quiz assessing students' PPCP knowledge, and (3) post-OSCE PPCP utilization survey. The primary endpoint was the difference in students' perceptions of the PPCP elements completed compared to faculty's intended expectations of the OSCE. The secondary endpoint was change in students' baseline knowledge of the PPCP. Results: Response rates for the preand post quizzes, and post-OSCE survey were $87.9 \%(182 / 207)$, $82.1 \%(170 / 207)$, and $89.3 \%$ (185/207), respectively. The majority of participants were $20-24$ years old $(84.7 \%)$, female $(73.5 \%)$, and Asian $(68.8 \%)$. The average pre-quiz was not significantly different from that of the post-quiz score ( $80.6 \%$ vs. $81.4 \%$, respectively). The post-OSCE PPCP utilization survey revealed $79.5 \%$ (147/185) of students agreed that all five components of the PPCP were addressed in the OSCE. Collect was the most common (98.4\%) PPCP element identified, while Implement was the least common (81.6\%). This may be due to the lack of collaboration with other health care professionals. Implications: Although the faculty expected students to fulfill all five principles of the PPCP, 20.5\% (38/185) of students disagreed that the OSCE identified all areas. This study shows that students' and faculty members' understanding of the PPCP differ. Obtaining student feedback and reinforcing application could improve future activities utilizing the PPCP.

Student Preparation for and Perceived Value of the Pharmacy Curriculum Outcomes Assessment (PCOA). Michael G. Kendrach, Samford University, Elizabeth A. Sheaffer, Samford University. Objectives: Assess students' PCOA preparation, their anticipated results, and their perceived value of the PCOA (currently a low-stakes assessment) at one school. Method: Second- and third-year students received an email containing a link to an internet-based survey and were instructed to complete the survey immediately after finishing the PCOA (taken Jan 19, 2018). The students were informed of the survey purpose and that results would remain anonymous. University IRB approval was obtained. Results: A total of 213 of 216 students (98.61\%) completed the survey. The majority of the students devoted zero hours to prepare for the PCOA prior to, during, and after Christmas break $(95.31 \%, 92.02 \%$, and $67.14 \%$, respectively). Although, $26.76 \%$ of the students committed 1-2 hours of PCOA preparation after the break. The primary study resource was class notes $(\mathrm{n}=$ 55). Many (73.24\%) did complete the NABP practice PCOA. The mean self-assessment scores (5-point scale) were: level of preparation, 1.86; and attempt to answer all questions correctly, 3.66. The two most common responses for using the PCOA results were: personal awareness of my strengths and weaknesses $(n=116)$; and prepare for next year's PCOA or NAPLEX $(n=81)$. The mean score for students' perceived value of the PCOA was 5.33 (10-point scale). Implications: Our students did not devote significant time to prepare for the 2018 PCOA. However, they did attempt to answer the questions correctly to provide personal feedback. The mean perceived value of the PCOA was mid-range.

Student Professional Development Program's Effects on Student Self-Efficacy in Goal Setting. Bethany Kalich, University of the Incarnate Word, Russell T. Attridge, University of the Incarnate Word. Objectives: Evaluate a professional development program's effect on student self-efficacy in goal setting. Method: A structured goal setting session was provided to first-year student pharmacists as a part of a professional development program in fall 2017. A validated, 8-item, 5-point likert scale pre- and post-semester survey was completed by 73 student pharmacists to assess self-efficacy in goal setting. Sixty-six (90\%) consented to study inclusion. Matched responses were assessed using paired t-tests, and a simple repeated measures analysis was used to control for the effect of meeting with a faculty mentor to discuss goals. Results: Post-semester (vs. pre-) surveys demonstrated increases in self-efficacy for setting specific goals (3.92 vs $4.39 \mathrm{p}<$ 0.01 ), setting moderately-difficult goals ( 3.67 vs $3.91, \mathrm{p}=0.03)$, setting goals that challenged learning performance ( 3.53 vs $4.02, \mathrm{p}<0.01$ ), competence in achieving study goals $(4.21$ vs $4.65, \mathrm{p}<0.01)$, and receiving regular feedback on performance toward goals ( 2.86 vs 3.48 , $\mathrm{p}<0.01)$. The structured goal-setting activity had no effect on selfefficacy with commitment to accomplishing goals, pride in receiving feedback on goals, or setting deadlines. Faculty-student mentor meetings did not have an effect on student self-efficacy. Implications: Pharmacy programs are expected to facilitate student personal and professional growth necessary to demonstrate self-awareness, leadership, innovation, entrepreneurship and professionalism. This professional development activity led to improvements in self-efficacy related to goal-setting.

Student's Perceived Ability to Implement Pharmacist's Patient Care Process in a Redesigned Case Studies Series. Amy Henneman, Palm Beach Atlantic University, Samantha Axtell, Palm Beach Atlantic University, Jamie L. Fairclough, Palm Beach Atlantic University. Objectives: To determine if a redesigned problem based learning case studies series impacted student's perceived ability to implement the pharmacist's patient care process (PPCP). Method: This study was approved by the Palm Beach Atlantic University Institutional Review Board. A redesigned problem based learning, four semester case studies series was implemented to foster critical thinking and formally integrate the PPCP into the curriculum. Students completing the original case studies series and students completing the redesigned course were given a 12 item survey to assess perceived ability to integrate the PPCP. Student's perceived ability to implement the PPCP after completing the second course of the series were compared to perceptions of students completing the case studies course prior to being restructured. Statistical analyses were conducted using IBM SPSS v.25. Student perceptions were analyzed using the nonparametric Mann-Whitney U test. A summated scale score was computed for each student to capture perceived abilities overall. Descriptive statistics were utilized for demographic data. Results: One hundred seven students completed the survey, including 61 students (57\%) from the original case studies series and 46 students (43\%) from the redesigned course. Results of the 


\section{American Journal of Pharmaceutical Education 2018; 82 (5) Article 7158.}

nonparametric Mann-Whitney U test suggested students completing the redesigned case studies series had significantly higher perceptions of their ability to integrate the PPCP compared to students completing the original series $(\mathrm{p}<0.001)$. Implications: Our data suggests student's perceived an improved ability to implement the PPCP after completing the restructured course. Further study is warranted to determine if this translates into improved patient care during advanced practice experiences.

Students' Perceptions of an Active Learning Session in Pharmaceutical Calculations. Catherine C. Van, West Coast University, Rahul Nohria, West Coast University, Diem Thai, West Coast University. Objectives: To describe the development and implementation of an active learning session, and students' perceptions on their engagement, teamwork, and confidence in pharmaceutical calculations. Method: Third-year pharmacy students were placed in groups of four. Each student completed a unique calculation problem set comprised of seven questions and combined his or her answers with teammates' answers to generate a lock combination and obtain prizes. The problem sets covered percentage strengths, ratio strengths, dilution, flow rates, milliequivalent and osmolarity. Students completed an anonymous, voluntary 12 -item post activity survey distributed in class. The survey utilized the Likert scale ranging from strongly disagree to strongly agree. Results: Forty-four students participated in the activity. Thirty-four students completed the survey (77\% response rate). Students felt more comfortable with performing calculations ( $82 \%$ to $88 \%$ agree/strongly agree on six survey items). Students felt their teammates were engaged (94\% agree/strongly agree). Students felt this activity improved their ability to work in a team setting (91\% agree/ strongly agree). Students would like to do this activity in other courses (88\% agree/strongly agree). Implications: Students demonstrated positive learning experience during this active learning session. This activity promoted individual accountability while providing an opportunity for peer collaboration and teaching on pharmacy calculations. The activity can be expanded to other classes to reinforce calculations and teamwork.

Survey of Recent Pharmacy Graduates on NAPLEX Preparation Strategies. Paula Zeszotarski, University of Hawaii at Hilo, Donna Ohora, University of Hawaii at Hilo, Lara Gomez, University of Hawaii at Hilo, Linda Connelly, University of Hawaii at Hilo. Objectives: A survey of recent PharmD graduates was conducted to determine the most effective strategies and resources for preparing for NAPLEX. Graduates of the Class of 2016 at The Daniel K. Inouye College of Pharmacy had been required to complete a randomized series of 600 questions from PharmacyLibrary and to take the PreNAPLEX on a voucher provided by the College prior to their graduation. Method: In August and September, 2016, all members of the Class of 2016 were invited to complete a survey about their preparation for the NAPLEX. Subjects who had not yet taken the exam were excluded. Results: Respondents reported using a variety of free (PharmacyLibrary question bank available through College subscription) and commercial (e.g. Kaplan, RxPrep) resources as well as PreNAPLEX. 95.74\% selected a commercial test preparation service as the best resource. Respondents reported that they could have better prepared by starting to study earlier (more than 2 months) and structure daily study time. Notably, the seven students who did not pass NAPLEX on the first attempt did not reflect (in their survey responses) on what they could have done better. Implications: The implications of these findings are two. The PreNAPLEX requirement has been dropped and the funds diverted to supplement students' contribution to enrollment fees in a commercial test preparation vendor. Starting in May 2017, the College began offering a one-unit NAPLEX Review APPE course that requires a minimum passing score on biweekly quizzes on topical modules from the commercial test preparation vendor.

Test-Enhanced Learning in a Therapeutics Course. Jaekyu Shin, University of California, San Francisco, Katherine Gruenberg, University of California, San Francisco. Objectives: To determine whether increasing the number of graded written assessments results in a significant change in median exam score over a course and to determine students' perceptions and preferences with a greater number of written assessments. Method: This was a prospective study of second year pharmacy students enrolled in a therapeutics course with four written graded exams in 2017. A historical control group, who completed the course a year prior with 2 written graded exams, was used to compare the change in median score from the first to the last assessment in this course. Students in the 2017 cohort completed a questionnaire about their perceptions and preferences with an increased number of graded written assessments. Results: The first assessment score was comparable between the 2016 and 2017 cohorts. The median score of the last assessment in the 2016 cohort was not significantly different from that of the first assessment, however the median assessment score in the 2017 cohort increased significantly from the first assessment (84.0\% vs. $76.9 \%$ ). Students overwhelmingly preferred having more than two assessments in a therapeutics course and reported improved time management skills and reduced levels of stress and test anxiety with more assessments. Implications: Our data suggest that increasing the number of graded written assessments in a therapeutics course may enhance student academic performance, improve time management skills, and reduce stress and test anxiety. To maximize test-enhanced learning, educators should consider the timing of assessment feedback, types of assessment questions, and overall academic workload.

The Validation of an OSCE Assessment to Measure Student Pharmacist Competencies of Pre-APPE Domains. Rick Hess, East Tennessee State University, John Bossaer, East Tennessee State University, Adam C. Welch, East Tennessee State University, Sam Harirforoosh, East Tennessee State University, Samuel C. Karpen, East Tennessee State University. Objectives: To determine the validity of newly created objective structured clinical examination (OSCE) assessments designed to measure student pharmacist pre-advanced pharmacy practice experiences (APPE) competency domains. Method: Eleven OSCEs were developed over 18 months through an accepted process of case writing, validation, and standard setting. Twentyseven faculty received formal OSCE training during this interval with 4 faculty serving on an OSCE Committee. Four OSCEs were then piloted with eight APPE students selected by faculty. OSCEs were recorded and evaluated by a single faculty member live and with up to two faculty members by recording. A different faculty member engaged the OSCE at each stage: case writing, validation, standard setting, and piloting. Validity in the piloted OSCE was defined as $\geq 75 \%$ agreement between faculty pass/fail ratings and $\geq 75 \%$ of students passing. Cumulative faculty time was estimated by the summation of hours scheduled multiplied by number of faculty involved. Results: Of the four OSCEs tested, 50\% were determined to be valid. The percent agreement between faculty ranged from $62.5-87.5 \%$ with one OSCE failing to reach the $\geq 75 \%$ threshold. Students passing ranged from $0-100 \%$ with two OSCEs failing to reach $\geq 75 \%$ threshold. A total of 518 cumulative hours has been invested during this 18-month interval. Implications: The development of a high stakes level OSCE assessment requires significant faculty time and resources. In our pilot study, 50\% of OSCEs were 


\section{American Journal of Pharmaceutical Education 2018; 82 (5) Article 7158.}

not considered valid assessment tools according to how they were originally created.

Three-Year Study on the Impact of the Progression of Prescription Verification Activities in Skills-Based Laboratory Course. Leah C. Sera, University of Maryland, T. Joseph Mattingly, University of Maryland, Deanna Tran, University of Maryland. Objectives: To determine how a progression in the number and complexity of prescription verification activities (PVA) in a skills-based laboratory course affects students' confidence during introductory community pharmacy rotations. Method: Motivated by the 2016 ACPE Standards, a skillsbased laboratory course further incorporated PVA. Over the course of 3 years, students who recently completed their IPPE community pharmacy rotation were asked to fill out an anonymous survey. In Year 1, students had 1 semester of PVA prior to their introductory community pharmacy rotation, in Year 2 students had 2 semesters, and in Year 3 students had 3 semesters. We evaluated the effects of class year and professional pharmacy experience on student responses using the twoway ANOVA. Results: Over a 3-year period, 395 students completed the survey. The majority (89.4\%) had professional pharmacy experience. Our study found that students with 2 or 3 semesters of PVA were significantly more confident than those with 1 semester in the following areas: verifying prescriptions on rotation $(p=0.02)$; determining whether a prescription is legal or valid $(p=0.01)$; fixing a label that does not match the hardcopy prescription $(p=0.04)$; and correcting discard/expiration dates $(p=0.026)$. Students without professional pharmacy experience agreed more strongly that the lab activities prepared them for rotations compared to those with experience $(p=0.04)$. Implications: This study showed that PVA had a positive impact on student confidence in several areas pertaining to prescription verification. Students without pharmacy experience found greater value in these activities. Future research will focus on whether performance indicators agree with student self-assessment of confidence.

Through the Eyes of Faculty Delegates: Enhancing Faculty Engagement With AACP. Jane E. Krause, Purdue University, Emily K. Frederick, Sullivan University, Yolanda M. Hardy, Chicago State University, Jared L. Ostroff, Western New England University, Katie J. Suda, University of Illinois at Chicago. Objectives: To identify how schools/colleges of pharmacy (S/COP) engage faculty with AACP and potential opportunities for increasing involvement. In addition, to describe current roles/responsibilities and operationalization of AACP faculty delegates at S/COPs. Method: An anonymous 12-item electronic questionnaire was emailed to the 141 AACP faculty delegates. The questionnaire consisted of multiple-choice and open-ended questions. Faculty delegates were identified through the AACP website. Results: A total of 94 responses were received (66.7\% response rate). The majority of the delegates were Associate Professors (44.7\%) with a mean of 11 years (range of 2-40 years) in academia. The majority of $\mathrm{S} / \mathrm{COPs}$ nominate/elect their AACP faculty delegate (86.2\%). Beyond attending the AACP annual meeting of the House of Delegate meeting, other roles of faculty delegates include discussion of policy proposals with faculty prior to the annual meeting $(32.2 \%)$ and providing a report following the annual meeting $(33.3 \%)$. S/COPs encourage faculty engagement with AACP by promoting research dissemination at AACP meetings $(23.4 \%)$ and forwarding AACP emails/communications to all faculty $(20.9 \%)$. Example recommendations to enhance faculty engagement include: intensifying faculty delegate responsibilities and training; creating additional opportunities for new faculty and science-based faculty; and partnering with the S/COP's administrative delegate to promote engagement. The cost to attend AACP meetings was indicated as a potential barrier to faculty engagement.
Implications: AACP faculty delegates provided insights for enhancing faculty engagement and involvement with AACP. To continue to facilitate growth, these opportunities should be explored within the AACP governance.

Training Student Pharmacists to Recommend and Dispense Naloxone: Is a Written Continuing Educational Model Enough? Elizabeth T. Skoy, North Dakota State University, Heidi Eukel, North Dakota State University, Jeanne E. Frenzel, North Dakota State University, Amy B. Werremeyer, North Dakota State University. Objectives: To determine if pharmacy students' knowledge of recommending and dispensing naloxone is retained after completion of a one-hour written continuing education module and to determine if knowledge and confidence increases after participation in an additional lecture and laboratory activity. Method: Third-year professional pharmacy students completed a one-hour written continuing education model on naloxone and opioid overdose. This is the standard for some states to authorize registered pharmacists to prescribe naloxone. Three months following the completion of this module, students took a pre-survey to measure knowledge retained and their confidence of recommending and dispensing naloxone. Students then participated in a 1-hour lecture and 2-hour laboratory activity focused on all naloxone dosage forms and how to identify, recommend and counsel patients on naloxone. Following the activity, students took the same survey measuring their knowledge and perceived confidence. Results: Seventy students completed the pre/post survey. The paired results were analyzed using a Paired Sample T-Test. Of the 6 questions assessing knowledge, 4 showed statistically significant gain $(p<0.05)$. All of the 11 survey questions assessing students' perceived confidence increased significantly after participating in the activity $(\mathrm{p}<0.05)$. Implications: Pharmacy students' knowledge and confidence of recommending and dispensing naloxone gained significantly after participating in a 1-hour lecture and 2-hour laboratory activity. Students had completed a 1-hour written continuing education model before taking the survey. This could warrant further investigation that additional training should be available for those prescribing naloxone.

Translating From Employee Wellness to Student Wellness: A First-Year Pharmacy Course. Amy M. Fanous, Ohio Northern University, Michael Rush, Ohio Northern University, Ben D. Aronson, Ohio Northern University, Karen L. Kier, Ohio Northern University. Objectives: To describe a novel wellness course for student pharmacists designed based on principles of an employee wellness program. Method: A 1-credit required course was developed for first year student pharmacists at a 6-year direct entry program. Elements of an existing employee wellness program were tailored to fit student needs. The course focused on eight dimensions of wellness, acclimating students to campus resources and teaching skills for achieving a lifetime of health and wellness. Grades were awarded for attendance, and from wellness activities documented on a student version of the employee wellness portal. Through the portal students earned points by tracking participation in a variety of wellness activities including tutoring, writing center visits, mental health screenings, religious services, and preventive health visits. After the course, students completed a survey assessing their participation in wellness activities and healthy behaviors. Results: In 2016, there were 164 students enrolled in the course. The average overall grade was $96.98 \%$. The average number of wellness points obtained was 30 . Students reported high levels of participation in wellness-related activities pertaining to preventive health (100\%), academic health (83\%), mental health (76\%), and religious/spiritual health $(70 \%)$. The majority of students reported healthy eating practices $(74 \%)$, and $58 \%$ reported exercising 3 or more 


\section{American Journal of Pharmaceutical Education 2018; 82 (5) Article 7158.}

times per week. Implications: Prior evidence shows that worksite wellness programs ensure employees are receiving appropriate screenings and preventive care. Utilizing this model as a course for entering student pharmacists encourages them to participate in activities and behaviors to improve their wellbeing while introducing campus resources.

Trends in Glyburide Prescribing: A Five-Year Retrospective Claims Data Analysis in a Large Regional Healthcare Organization. Mitchell Barnett, Touro University California, Kristen Herzik, Touro University California, Danielle Thanh, Touro University California, Shadi Doroudgar, Touro University California, Eric Ip, Touro University California. Objectives: In 2012, the American Geriatrics Society's Beers Criteria recommended avoiding glyburide in patients over 65 years. Objectives of this study are to: 1) compare rates of glyburide-use in patients with type 2 diabetes mellitus (T2DM) prior to, during, and after the 2012 recommendation; and 2) examine differences in rate of glyburide-use over time among regional sites. Method: A multi-center retrospective study conducted on T2DM patients over 65 years of age from four Sharp HealthCare regional areas in and around San Diego, California. Patients taking glyburide, glipizide, or glimepiride $\geq 30$ days in 2011, 2013, and/or 2015, who had a prescription claim in the following year, were included. Differences in glyburide-use stratified by the four regions over the three study years were analyzed. A series of multivariate models were created to control for patient differences, including age, gender, ethnicity, and comorbidity factors (Elixhauser Index). Results: A total of 3,166 unique patients $(n=1,026, n=1,039$ and $n=940$ for 2011, 2013 and 2015, respectively) were included. Overall glyburide-use fell from $35.8 \%$ to $4.2 \%$ between 2011 and 2015 . Unadjusted glyburide-use varied by as much as $63.3 \%$ by regional site per study year. Significant differences in glyburide-use by regional site remained after adjusting for patient differences. Implications: Glyburide-use decreased after the 2012 recommendation. However, different regions appear to have implemented the recommendation at varying rates. Further research is needed to examine reasons for these differences, and may include participation of clinical pharmacists, additional prescriber training, and/or more readily-accessible encounters for patients.

Use of a Mock Pharmacy and Therapeutics Committee Activity as an Entrustable Professional Activity. Nancy Borja-Hart, The University of Tennessee, Justin Gatwood, The University of Tennessee, Anthony S. Rowe, The University of Tennessee, James Wheeler, The University of Tennessee. Objectives: To describe an entrustable professional activity (EPA) conducted on a multisite campus aimed at improving utilization of evidence based medicine (EBM) skills. Method: Teams, comprised of 6-7 students $(n=28$ teams over 3 campuses), were asked to deliberate a formulary decision. To accomplish this task, pairs of teams were assigned a drug and instructed to complete the following activities: (1) background literature search; (2) drug monograph; 3$) 5$ minute presentation to simulate a live pharmacy and therapeutics (P\&T) committee meeting; and 4) vote on a formulary drug per drug class. A scoring rubric assessed monograph and presentation elements $(\max$ points $=30)$. Students completed a pre/post 5 point likert scale self-efficacy survey describing their confidence in their practice of EBM. Descriptive statistics were used for assignment grades. Wilcoxon signed-rank tests analyzed pre and post self-efficacy surveys. Results: The median monograph and presentation rubric score were 20 points (range 13-24) and 5 points (range 5-6), respectively. One hundred and six (55\%) students completed the pre-post self-efficacy survey. Students perceived statistically significant improvement $(\mathrm{p}<0.001$ for all items) in their 1) medical writing skills,
2) ability to cite sources, 3) ability to develop a concise response, 4) ability to evaluate primary literature, 5) ability to use libraries resources, 6) resource availability, 7) application of study results, 8) Medline searching skills, and 9) ability to prepare a drug monograph. Implications: Research concerning the development, conduction, perceptions and efficacy of EPAs is sparse. A mock P\&T activity represents an innovative way to validate an EPA within the curriculum.

Use of a Post-Exam Survey to Encourage Pharmacy Student Metacognition in Therapeutics Courses. Laura M. Morgan, Virginia Commonwealth University, Teresa M. Salgado, Virginia Commonwealth University, Nancy S. Yunker, Virginia Commonwealth University, Rachel W. Flurie, Virginia Commonwealth University. Objectives: Evaluation of a post-exam survey to determine associations between performance and study habits and reasons for missed questions on therapeutics exams. Method: Second year pharmacy students completed a 2-part exam wrapper following the first cardiology exam and the last nephrology exam. Part 1 asked about study methods and preparedness. Part 2 asked students to identify reasons for missed questions. Differences in study time and methods, and reasons for missed questions were compared by performance groups $(\mathrm{A}=90-100 \%, \mathrm{~B}=80-89 \%, \mathrm{C}=70-79 \%, \mathrm{~F}=<70 \%)$. Results: Exam scores improved between the cardiology and nephrology exams across all letter grades ( $63.4 \%$ vs $85.3 \%$ for $\mathrm{A} / \mathrm{B}$ and $36.6 \%$ vs $14.7 \%$ for $\mathrm{C} / \mathrm{F}$ ). No significant difference in study methods was observed in cardiology but in nephrology, those earning a $\mathrm{C} / \mathrm{F}$ were more likely to re-read the textbook $(p=0.0032)$, review class application exercises $(p=0.0096)$ and review the study guide $(\mathrm{p}=0.0463)$. Reasons for missing questions differed with those receiving a $\mathrm{C} / \mathrm{F}$ in cardiology reporting difficulty with understanding the question $(\mathrm{p}=0.0438)$ and changing answers $(p=0.0379)$ more frequently than $A / B$ students. In both exams, test anxiety and difficulty choosing between two answers were reported significantly more in $\mathrm{C} / \mathrm{F}$ students. Perceived level of preparedness differed only in the cardiology module with $37.8 \%$ of $\mathrm{C} / \mathrm{F}$ students reporting feeling 'not at all/somewhat' prepared compared to $90.6 \%$ of A/B students feeling 'adequately/very' prepared $(\mathrm{p}=0.0028)$. Implications: Exam performance improved between therapeutics exams. Significantly, lower performing students reported test anxiety and difficulty in choosing between answers. Faculty may consider additional training/resources be made available to help these students.

Use of Mannequin-Based Simulation to Improve Skills and Retention of Knowledge in a Toxicology Elective. Abbie Lyden, Rosalind Franklin University of Medicine and Science, Kevin O. Rynn, University of Illinois at Chicago. Objectives: There exists a growing body of evidence documenting the successful use of mannequin-based simulation learning in pharmacy schools including improved understanding of topics, increased retention of knowledge, elevated confidence in clinical skills, and enhanced interprofessional communication. In this project, we describe the use of mannequinbased simulation in a Toxicology elective. The purpose of our project is to assess the impact of high-fidelity mannequin simulation on student knowledge and confidence, demonstrated by pre-test, immediate post-test, three month ('retention of knowledge') post-test, and student surveys. Method: A mannequin-based simulation was implemented in the Toxicology course to provide students the opportunity to practice and apply knowledge in a high-stress, real-world environment. Toxicology is a 1-quarter, 2 credit hour elective offered to third year Doctor of Pharmacy students. The simulation cases are all toxicologic in nature and in past years have included both drug and chemical agent overdoses. The study was single-center and included a total of 113 students enrolled in the toxicology course from October 2014 to 


\section{American Journal of Pharmaceutical Education 2018; 82 (5) Article 7158.}

January 2017. Results: The primary outcome demonstrated quiz grade improvement between: pre- and immediate post-simulation $(65.24 \%$ vs $91.90 \%, \mathrm{p}<0.05)$ and pre- and 3-month post-simulation $(65.24 \%$ vs $81.08 \%, \mathrm{p}<0.05)$. Secondary outcomes included an evaluation of survey results, abbreviated findings: $100 \%$ pharmacy students felt the simulation increased an understanding of the clinical decision-making process, $90.7 \%$ of students felt it improved their retention of knowledge and $68.8 \%$ students felt that it enhanced APPE performance. Implications: This study provides evidence that mannequin-based simulation activities may positively impact student learning and retention of knowledge.

Using Blended Learning to Deliver Interprofessional Education (IPE). Jane Shtaynberg, Long Island University, Bojana Beric, Long Island University, Scott Henkle, Long Island University, Peggy Lynam, Long Island University, Amerigo Rossi, Long Island University. Objectives: To evaluate the effectiveness of a blended learning module on the delivery of interprofessional education. Method: Firstyear students from Pharmacy and Physical Therapy programs participated in interprofessional activities focusing on IPEC competencies of Roles and Responsibilities and Interprofessional Communication. The first portion was completed electronically via a learning management system. It included watching educational presentations, researching professions, and participating in discussion boards. The second portion required face-to-face group navigation of an interactive patient scenario. Students were divided into groups of 10 ( 5 from each profession) and given access to a patient scenario game. Game progression relied on the group's consensus for a decision about each stage of the patient's care and recommendations for health professionals' involvement. Student beliefs, behaviors, and attitudes were assessed before and after using the Interprofessional Socialization and Valuing Scale (ISVS). Basic knowledge of IPE was assessed by quiz after viewing provided presentations. Qualitative feedback was also collected from the students. Results: Eighty-two students participated in the IPE activities. Majority of the students (85\%) scored more than $70 \%$ on the knowledge quiz with $52 \%$ of the students receiving a perfect score. Results from the ISVS assessment were analyzed using Wilcoxon Signed rank test. Statistically significant improvements were seen with all 21 items (Mean score increase from 5.6 to 6.1. Effect size $(r=0.34))$. Students also expressed positive feedback about their participation. Implications: Blended learning can be an effective way to deliver interprofessional education and can partially alleviate logistical challenges related to fully synchronized sessions.

Utilizing Academic Electronic Health Records for Assessment in the Didactic Curriculum. Kali VanLangen, Ferris State University, Kimberly G. Elder, Sullivan University, Mark Young, Ferris State University. Objectives: To examine the current utility and factors impacting selection of academic electronic health records (EHRs) in the didactic curriculum of doctor of pharmacy programs in the United States. To describe how EHRs are used to assess curricular components. Method: An electronic survey was distributed to all US schools/ colleges of pharmacy. Content areas in the 35-question survey included current EHR utilization in the didactic curriculum, EHR use in curricular assessment including the Pharmacists' Patient Care Process (PPCP), and factors related to EHR implementation. Aggregate data were analyzed using descriptive statistics. Results: A total of 56 of 144 schools completed the survey (38.9\%) with $62.5 \%$ of respondents indicating current EHR use. Of current non-users (37.5\%), 57\% plan to integrate EHRs into didactic coursework within three years. Users frequently reported assessing students' ability to collect subjective and objective data $(83 \%)$ and document within the chart
$(80 \%)$. EHRs are used for summative skills-based assessments by $40 \%$ of users. Evaluation of the PPCP steps "Collect" $(85.7 \%)$ and "Assess" (82.9\%) were most common although all steps were reported as being assessed by an EHR. "Implementation" was the least frequently assessed step (65.7\%). Available EHR functions and cost were the most important factors to consider when selecting a product. Implications: Selecting an EHR product to meet course needs, including assessment, is critical. EHRs are being utilized to assess steps of the PPCP and for summative skills-based assessments. Future research should focus on evaluating levels of satisfaction and best practices for utilizing EHRs for skills-based assessments.

Validation of the ShahTech NAPLEX Risk Prediction Model. Kalin Clifford, Texas Tech University Health Sciences Center, Iverlyn Peng, Texas Tech University Health Sciences Center, Sachin Shah, Texas Tech University Health Sciences Center. Objectives: To validate a NAPLEX outcome predictive model that is measureable, reliable, and reproducible. Method: This study consisted of validating pre- and post-implementation of model in groups of students who graduated from the TTUHSC-SOP who had taken their first NAPLEX attempt during 2012-2016 and 2017 Trimester II. The eligible students had taken NAPLEX and their first-attempt examination score were available to the SOP. Primary outcome was to validate the student specific factors within the NAPLEX Risk predictive model to affirm the risk groups are distinct. Descriptive and comparative statistics were utilized to confirm validity. Results: 433 students were in the 2012-2016 and 124 students in 2017 groups. The 2012-2016 group, had clear and significant differences among ShahTech model risk groups (Mean scaled score: Low risk: 106; Intermediate-1: 97; Intermediate-2: 87; High risk: 75). ANOVA analysis showed $\mathrm{p}$ value of 0.000 and post-hoc Bonferroni analysis between groups identified a maximum statistically significant difference of $p=0.0002$. The actual results of 2017 Trimester II were compared with the class of 2017 predicted NAPLEX outcomes by the model risk groups, and results were identified to be comparable. Overall, $14.57 \%$ of students were predicted to performer poorly compared to actually $15.32 \%$ who performed poorly. Implications: NAPLEX outcome predictive model is easy to use and validated by significant differences of high risk group compared to other risk groups. Further preliminary 2017 data supports predictability of the model.

Which Preadmission Variables Predict Pharmaceutical Calculations Success? Kamila A. Dell, University of South Florida, Gwendolyn Wantuch, University of South Florida. Objectives: To determine which preadmission student variables best predict success in pharmaceutical calculations. Method: A retrospective analysis of student preadmission data and performance in a stand-alone pharmaceutical calculations course was conducted. Successful performance was defined as a passing grade of $\geq 80 \%$. Students included in the analysis consisted of those enrolled from 2011-2016 and those who failed were only included the first time they were enrolled in the course. Fifteen independent preadmission variables were evaluated including demographics, grade point average, PCAT scores, prior degrees, and number of pre-professional credit hours completed. All data were de-identified prior to analysis and the Institutional Review Board determined the study to be exempt. Statistical analysis was performed using SPSS and the R Project for Statistical Computing. Descriptive statistics were used for baseline characteristics and relative importance analysis was used to examine association between the grade in the course and the preadmission variables. Statistical significance was set at an alpha of 0.05 . Results: Including 506 students in the analysis resulted in 7 of the 15 preadmission variables being significantly 


\section{American Journal of Pharmaceutical Education 2018; 82 (5) Article 7158.}

correlated with the pharmaceutical calculations final grade. The strongest weighted factors affecting the final grade were science and cumulative GPA, and the PCAT quantitative and composite scores. The R squared value for the relative importance analysis model was 0.236 . Implications: Preadmission grades and PCAT scores are the best predictors of pharmaceutical calculations success. Unfortunately, only $23.6 \%$ of the success can be predicted from these variables. Other predictors need to be investigated to more precisely predict success.

\section{Theoretical Models}

An EPIC Alternative: Using an ePortfolio System to Create Mock Electronic Medical Records. Adam B. Woolley, Northeastern University, Alexa A. Carlson, Northeastern University, Stephanie L. Sibicky, Northeastern University, Jason W. Lancaster, Northeastern University, Mark Douglass, Northeastern University, Michael J. Gonyeau, Northeastern University. Objectives: To develop mock electronic medical records (EMRs) within an existing ePortfolio system to facilitate learning in a principles of general medicine elective. Method: Formal and informal feedback from students and course faculty identified the need to develop real world patient assessment skills through simulation of examining a patient case using EMRs. Potential options for incorporating EMRs available within our institution were assessed. We concluded that utilization of our readily available ePortfolio system (Digication (C) was the most feasible solution. A template EMR was developed to ensure that each mock patient medical record retained a consistent format and appearance. Results: The ePortfolio system allowed for multiple faculty members and APPE students to create, access, and edit simulated patient EMRs utilized in the elective course during interactive class sessions and a mockrounding experience. EMRs included progress notes, labs, medication administration records and other pertinent patient information. EMRs were shared via web hyperlinks and designed to release new information during class for just-in-time learning. Based on informal feedback, the EMRs functioned effectively and were well received by both faculty and students. Implications: The novel implementation of an EMR using a readily-available platform may be easily replicated at other institutions. Our dynamic model leveraged available resources while providing convenience and added flexibility to meet the unique needs of our general medicine elective, and could be implemented easily throughout the curriculum. Future studies will assess the impact of this system on learning outcomes.

An International Collaboration on Sustainable Workforce Development and Implementation of a Doctor of Pharmacy Program. Gina M. Prescott, University at Buffalo, The State University of New York, Tyler R. Mullen, University at Buffalo, The State University of New York, Cameil F. Wilson-Clarke, University of the West Indies, John Lindo, University of the West Indies, Brian T. Tsuji, University at Buffalo, The State University of New York, Jeffrey R. Lombardo, University at Buffalo, The State University of New York, Eugene D. Morse, University at Buffalo, The State University of New York, Maxine D. Gossell-Williams, University of the West Indies. Objectives: The University of the West Indies (UWI), Mona Campus, and University at Buffalo (UB) School of Pharmacy and Pharmaceutical Sciences have identified educational and implementation research priorities to expand education and research approaches for pharmacists to participate with public health prevention initiatives. Method: In 2016-2017, a needs assessment was conducted between UB SPPS and the UWI Research Task Force. Needs were identified in the following areas: UWI Post-BS PharmD students and PharmD student teaching and experiences, UWI-UB Fellow training, Jamaican Pharmacists Advanced
Training, and Jamaican STEM student training. Common areas of scholarship and curricular exchange included: Antibiotic resistance, cannabinoid sciences, cancer, liver disease, noncommunicable diseases, and health information technology (HIT). Results: Five programs have been developed between the partnership. A pilot program utilizing HIT to computerize patients $(n=100)$ with Type 1 DM has been developed and one with for HIT and rural clinics/ short term global health experiences is under development. Post BS Training $(n=x x)$ for UWI in the areas of hepatitis, infectious disease, and oncology is ongoing. Pharmacist immunization training has been developed to train pharmacists in practice. Competency based experiential learning opportunities for student exchange ( $n=4$ in $2017 ; n=8$ in 2018) between the UWI and UB has been developed. Implications: International collaborations can assist universities with building a PharmD program, and promoting research to build capacity. Financial constraints and can be addressed through internal, global health, and educational agencies grants.

Applying the Principles of Engagement to a Partnership of Caring and Education. Kristin A. Casper, The Ohio State University, Jennifer L. Seifert, Charitable Pharmacy of Central Ohio, Jennifer L. Rodis, The Ohio State University. Objectives: 1) Describe a charitable pharmacy practice model, 2) Recognize the benefits of a partnership between a College of Pharmacy and a charitable pharmacy on educational and research initiatives, and 3) Identify opportunities to apply the engagement principles of awareness, integration, stewardship, accountability, and advocacy to the College of Pharmacy and charitable pharmacy partnership. Method: The Charitable Pharmacy of Central Ohio (CPCO) functions to provide medications and pharmacy services to underserved individuals. Students from the Ohio State University College of Pharmacy enhance their communication skills, better understand the needs of vulnerable patients, and apply the Pharmacists' Patient Care Process through participation in direct patient care at CPCO. Pharmacy residents, College of Pharmacy faculty, and CPCO leadership apply the principles of engagement while participating in shared strategic planning, educational enhancement, and practicebased research. Results: Pharmacists and pharmacy students have partnered for over seven years at CPCO, and they currently serve over 700 unique patients per month. Approximately 45 advanced pharmacy practice students and two PGY1 residents per year contribute to the care of patients. Application of the engagement principles have resulted in national recognition, funding for practice-based research, and numerous opportunities for dissemination of research findings. Implications: This unique partnership effectively applies the principles of engagement to improve patient care, enhance student learning, and contribute to community engaged research. This model of engagement can be utilized by other colleges to create mutually beneficial community partnerships that foster student learning and advance the practice of pharmacy.

Coordination and Development of a Collaborative Effort to Assess Global Health Curricula. Sharon E. Connor, University of Pittsburgh, Regine Beliard, Notre Dame of Maryland University, Rebecca L. Brynjulson, North Dakota State University, Jordan R. Covvey, Duquesne University, Negar Golchin, University of Washington, Lauren J. Jonkman, University of Pittsburgh, Abby A. Kahaleh, Roosevelt University, Yardlee S. Kauffman, University of the Sciences, Michele Klein-Fedyshin, University of Pittsburgh, Sharon K. Park, Notre Dame of Maryland University, Melody H. Ryan, University of Kentucky. Objectives: Most pharmacy programs provide global health educational opportunities for students; however, clear strategies for assessment are not readily available. The objectives of this project 


\section{American Journal of Pharmaceutical Education 2018; 82 (5) Article 7158.}

were to (1) execute a collaborative effort to retrieve and evaluate assessment methods used in didactic global health education in the health professions, and (2) leverage a software tool to support the collaboration among a large workgroup. Method: The project was initiated by the Global Health committee within the AACP Public Health SIG involving a group of ten pharmacy academics and one health science librarian across eight different institutions. The group committed to a longitudinal initiative to complete a systematic review utilizing an online software product, DistillerSR (Evidence Partners; Ottawa, Ontario). A protocol was registered with the International Prospective Register of Systematic Reviews (PROSPERO; CRD42017065379). Search construction was formulated by the medical librarian using collaborative input. Resulting search strategies were translated into multiple database syntaxes and delivered via EndNote. Regular team conference calls were scheduled to assess progression, discuss strategies arising with execution of the review, and ongoing deadlines and goals. Results: The search constructed by the medical librarian resulted in 9805 abstracts for review by the team. Experience with the software was positive and provided effective opportunities for transparency, oversight and documentation of progress by the project leader. Implications: The use of a diverse project workgroup served as a useful strategy to review educational literature while collaborating across multiple settings. The results will be used to recommend robust assessment tools in global health education.

Development and Implementation of an International Introductory Pharmacy Practice Experience to Model Student Exchange. Gina M. Prescott, University at Buffalo, The State University of New York, Tyler R. Mullen, University at Buffalo, The State University of New York, Cameil F. Wilson-Clarke, University of the West Indies, Eugene D. Morse, University at Buffalo, The State University of New York, Maxine D. Gossell-Williams, University of the West Indies. Objectives: An international, introductory pharmacy practice experience (IPPE) pilot was designed to educate students on global health implementation research, social determinants of health, and Jamaican disease burden and serve as a template for student exchange. Method: A global health implementation fellow was the onsite preceptor, with University at Buffalo (UB)-University of the West Indies (UWI) faculty oversight for a one-week experience. Components include: a pre- and post-travel Likert-scale survey, a Skype IPPE-1 Jamaican/global health introduction, a pre-travel training video, activities and assessments with outcomes derived from the Consortium of Universities for Global Health (CUGH) and International Pharmaceutical Federation (FIP) competencies. Learning activities included: clinical pharmacy and research experiences, rural health, global health drug information questions, journaling, and a presentation. Modifications have been made to this template to develop an individual training plan for exchange of the UWI PharmD students to UB. Results: Four students completed the rotation in June, 2017. Two students will come from the UWI program in 2018. All students were well versed on the following: sustainable developmental goals (SDGs), social determinants of health, universal health care, and access to health care. Students were most aware of: pharmacists' role in Jamaica and SDGs (mean $=4.5,4.75)$, nutrition and Type $2 \mathrm{DM}$ (mean=3.75), and less informed on public health policy programs $($ mean $=3)$. All students would recommend this rotation. Implications: International collaborative partnerships can assist universities with building a PharmD program, and promote educational exchange. Financial considerations are the largest constraint and can be addressed through internal, global health, and educational grants

Development of an Interprofessional Personalized Education and Collaboration Experience (iPEACE) Complementing a CompetencyDriven PharmD Curriculum. Nicole M. Schroeder, University of the
Sciences, Jennifer N. Smith, University of the Sciences, Brooklyn Cobb, University of the Sciences, Diane Hadley, University of the Sciences, Cathy Y. Poon, University of the Sciences. Objectives: To describe the framework and process for implementing a "parallel" interprofessional education (IPE) program alongside a Doctor of Pharmacy (PharmD) curriculum. Method: A taskforce was created to develop a "parallel" IPE program to complement a competency driven curriculum and co-curriculum at University of the Sciences Philadelphia College of Pharmacy (PCP). A comprehensive literature search was conducted to review IPE standards for implementation, assessment tools, and existing models within professional curricula. The task force created a tool to align the 2016 Interprofessional Education Collaborative (IPEC) competencies and sub-competencies with the 2016 Accreditation Council of Pharmacy Education (ACPE) Standards and PCP Measurable Abilities. The IPEC sub-competencies were linked to one of three learning benchmarks (introductory, reinforcement, and emphasis) to guide successful completion of the "parallel" IPE Program. Results: A framework was created to showcase the proposed method for implementation of a "parallel" IPE Program. It utilized learning benchmarks to guide sequential placement of activities throughout the program. Validated assessment tools were selected to assess competencies at each of the three learning benchmarks and four IPEC domains. A request form was created to enable faculty to accurately develop and map proposed IPE experiences within the "parallel" IPE Program. Implications: This proposed experience offers insight to developing an IPE framework within professional curricula. It provides data from a structured co-curricular IPE experience to assess the depth of learning needed for IPE considering the 2016 ACPE standards. This framework serves as an example for proposed development, required resources, and implementation adjacent to a competency-driven PharmD curriculum and co-curriculum.

Exploration of Lightboard Recordings for Student Engagement Outside the Classroom. Jeremy R. Fox, Shenandoah University, Jennifer E. Bryant, Shenandoah University. Objectives: In pharmacy education, several methods have been implemented to increase student engagement such as flipped classroom and team-based learning. The objective of this theoretical model is to explore alternative ways to deliver content outside of the classroom. Method: The lightboard provides a new variation to the type of media used to prepare students for the classroom. A lightboard is an illuminated glass sheet, similar to a whiteboard, where the presenter can provide annotated lists and drawings while facing a camera to record a presentation. The camera reverses the image so the text/drawings are readable to the viewer. The advantage of a lightboard is that the presenter faces the audience while providing commentary and dynamically creating material. This method may promote increased student engagement and greater understanding of concepts presented. Recorded lightboard presentations were delivered to students as pre-class work, supplemental material, or as an alternative way for learning difficult material. Results: The initial development of content using lightboard technology required dedicated planning time, several recording sessions, and time for editing prior to student viewing. Preliminary feedback from students collected informally and statistics from our learning management system and YouTube was very positive and student interest in more lightboard presentations was very high. Future studies will include a survey of student perspectives regarding the lighboard and a plan to study the effect on student learning. Implications: If the impact of this new technology on student learning is beyond just novelty, positive results could prompt replacement of traditional methods of delivery with lightboard presentations. 


\section{American Journal of Pharmaceutical Education 2018; 82 (5) Article 7158.}

Implementation of a Post-Graduate Fellowship Focused on Teaching and the Scholarship of Teaching and Learning. Connie M. Remsberg, Washington State University, Taylor G. Bertsch, Washington State University, Yili Zhong, Washington State University, Micah E. Miller, Washington State University, Nicole M. Perea, Washington State University, Angela Stewart, Washington State University, Kimberly C. McKeirnan, Washington State University, Christina R. Buchman, Washington State University. Objectives: To establish a post-graduate fellowship program that prepares fellows for a future career in pharmacy academia. The goal of the two-year fellowship program is to provide fellows with a mentored experience in teaching, professional development, and the scholarship of teaching and learning (SOTL). Method: The Teaching Fellows Program was established in 2016. At the start of the program, each fellow reflects on their goals, selects a mentoring team, and works with mentors to create an individualized plan for their fellowship experience. Fellows are given numerous opportunities to teach within the PharmD curriculum including as content developers, facilitators, and/or preceptors. Fellows participate in scheduled professional development including 1) new instructor trainings, 2) a biweekly curriculum focused on teaching and scholarship skills, and 3) journal clubs. All fellows work with their mentors to develop SOTL projects. Progress of the fellows is evaluated by the mentoring team and the director of the program. Teaching abilities are evaluated using the college's peer review of teaching rubric and student evaluation feedback. Teaching portfolios maintained by each fellow are frequently reviewed. Progress on scholarship projects are evaluated throughout the year. Results: Four fellows total have been accepted into the program: two in 2016 and two in 2017. All fellows have gained extensive teaching experience within the PharmD program and have tracked teaching accomplishments within teaching portfolios. All fellows have active and ongoing SOTL projects. Implications: The Teaching Fellows Program is a unique fellowship that creates a pipeline for developing future pharmacy educators.

Innovative Approach to Implement Interprofessional Education Through Co-Curricular Certification Programs. Angela Bingham, University of the Sciences, Cathy Y. Poon, University of the Sciences. Objectives: To implement interprofessional education (IPE) through two co-curricular certification programs with health professional students within and external to the University of the Sciences that expand on traditional incorporation within curricula and align with ACPE Standards, CAPE Educational Outcomes, and Core Competencies for Interprofessional Collaborative Practice. Method: The CPR Leadership Team, an interprofessional student-led Basic Life Support (BLS) certification program, was established at USciences in Fall 2014. Students are recruited annually from all health professional programs on campus and trained as American Heart Association (AHA) BLS instructors who certify other health professional students (e.g., occupational therapy, pharmacy, physician assistant), faculty/staff, and community members. Interprofessional Advanced Cardiac Life Support (iACLS) was implemented with the University of Pennsylvania's Schools of Medicine and Nursing to deliver the AHA's ACLS certification course with an interprofessional focus in March 2016. iACLS augments the traditional course with interprofessional activities (e.g., teamwork exercise, TeamSTEPPS overview, discussion of education/ training and future roles for each profession, vignettes such as a difficult team member incorporated into megacodes to facilitate assessment and debriefing of interprofessional performance). Results: The interprofessional student-led BLS program has certified over 1,800 participants with research demonstrating a positive impact on skills performance and retention, knowledge acquisition, and attitudes regarding interprofessional collaboration. iACLS has certified over 200 participants who have demonstrated development of interprofessional behaviors and TeamSTEPPS skills domains. Implications: Based on our demonstrated outcomes, other institutions may consider expanding current IPE opportunities through co-curricular certification programs. Future research should assess generalizability of our findings to other co-curricular certification programs.

Transitioning From a Stand-Alone Self-Care Course to an Integrated Self-Care and Pharmacotherapeutics Course. Kaelen C. Dunican, MCPHS University-Worcester/Manchester, Aimee Dietle, MCPHS University-Worcester/Manchester, Cheryl Abel, MCPHS University-Worcester/Manchester. Objectives: Discuss rationale for transitioning from a stand-alone self-care therapeutics course to an integrated pharmacotherapeutics course. Describe revised course design including methods of delivery and assessment. Compare course grades before and after revised curriculum. Method: Self-Care Therapeutics was a 3-credit course required during the third semester of an accelerated Doctor of Pharmacy program immediately prior to the Introductory Pharmacy Practice Experiences. The curriculum revision task force suggested that an integrated, modular-based curriculum would enhance student learning outcomes. Course coordinators were tasked with integrating the pharmacotherapeutics sequence with self-care therapeutics. Course coordinators from self-care therapeutics, pharmacotherapeutics, physiology, and pharmacology/medicinal chemistry worked to align the lecture schedule. During the transition, coordinators and lecturers collaborated on a template used for all course sessions. The template utilized the original self-care course methods of delivery and assessment while integrating pharmacotherapeutic topics. The method for delivery included a flipped classroom approach requiring students to complete reading assignments, online lectures, and online assessments prior to class. The live class session included a brief lecture clarifying important concepts followed by cased-based discussions. Course assessments included video cases where students provide recommendations via short answer and traditional multiple-choice exams. Results: Scores on exams declined on 2 out of 3 exams. Remediation opportunities were offered to reinforce key concepts. Overall course grades went down one percent. Students expressed concerns about being overwhelmed with the volume of material. Implications: Transitioning from a stand-alone self-care therapeutics course to an integrated pharmacotherapeutics course is challenging. Faculty must be aware of student workload.

Use of Lightboard Technology to Introduce Vaccine Topics. Carolyn Jung, Butler University, Kristen Nichols, Butler University. Objectives: To describe the use of lightboard technology as a method for reviewing immunology concepts and introducing basic vaccine topics Method: Adult and pediatric vaccine topics are taught in the Self-Care and Health Promotion I course at Butler University. A separate elective course is offered for students to become immunization certified, so this lecture is meant as exposure to the topic for all students, including those who do not take the elective. Desired student outcomes include gaining knowledge about each of the vaccines and vaccine-preventable illnesses and using the information to make patient-specific recommendations. Previously, limited time was dedicated to these topics. Time spent reviewing immunology concepts limited the lecture material to just the core information needed to understand and use the vaccination schedules, with little opportunity for application. In fall 2017, lightboard video technology was utilized to create and record a series of short key concept videos for students to view prior to class. The videos contained immunology review and introductory concepts. Students were required to view the videos prior 


\section{American Journal of Pharmaceutical Education 2018; 82 (5) Article 7158.}

to lecture and submit a one-page document of notes with at least three key points from each video. During lecture and recitation, the material built on the key concepts in the videos with more application scenarios. Results: Implementation of the lightboard technology videos allowed a change from a knowledge-based approach of teaching vaccine topics to an application-based format. Implications: Lightboard technology can be an effective method for teaching introductory concepts to students.

We Come Together: Integration of Adult Internal Medicine Inpatient APPEs. Stephanie L. Sibicky, Northeastern University, Alexa A. Carlson, Northeastern University. Objectives: To describe an innovative practice model where advanced pharmacy practice experiences (APPE) from different hospitals integrated face-to-face and webbased rotation-related activities. Method: Two, adult internal medicine rotations integrated student projects, journal clubs, topic discussions, and formal presentations to increase student and preceptor feedback, enhance learning experiences for rotation students across two hospital campuses, help foster collaboration between students, and reduce faculty workload. Initially, Blackboard was used to integrate rotation specific information and training. Face-to-face encounters included all students and faculty in one location for topic discussions and presentations. The use of Web 2.0 technology, including Facebook, ClassDojo, Google Sheets, and WordPress incorporated social media to increase interactivity between students and faculty, share drug information, and collaborate on projects. Students were paired across rotations to increase exposure to different hospital policies and procedures. Results: Based on informal and formal feedback from evaluations, student and faculty perceptions of integrating have been overwhelmingly positive. Students felt like their learning was enhanced by integrating with students and faculty from another hospital. Interactivity using Web 2.0 technologies enhanced learning by requiring students to post, comment, blog, and vlog about drug information and their experiences. Feedback was increased for all students as faculty shared the responsibility of providing feedback to all students. Implications: The integration of similar advanced pharmacy practice experiences offered students and faculty the opportunity to increase information exchanges across hospital campuses, feedback on presentations, interaction using Web 2.0 technologies, and responsibility sharing for faculty.

You are Invited to Train in Our Dojo: ClassDojo Applied to Didactic and Experiential Education. Alexa A. Carlson, Northeastern University, Stephanie L. Sibicky, Northeastern University. Objectives: To describe the use of ClassDojo, a communication application, in a team-taught, didactic elective course and an advanced pharmacy practice experience (APPE) for recording attendance, providing feedback, and communicating with students. Method: ClassDojo allows for increased communication between faculty and students. When creating a team-taught, general medicine elective, an analysis of participation tracking was undertaken, and ClassDojo was selected for organizing quantitative and qualitative weekly feedback on student participation. Based on this positive experience in the classroom, two faculty have adopted ClassDojo for collating paper rubrics through the photograph function, communicating with students, and providing real-time feedback to APPE students in various rotation activities. Results: Since 2015, 40 students have taken the general medicine elective and since 2017, 12 students and 1 pharmacy practice resident in an integrated APPE have received feedback using ClassDojo. In the general medicine elective, 16 positive and 9 negative feedback options were created. For APPEs, faculty created 15 positive (streamlined from 24) and 12 negative feedback options. Formal and informal feedback on the use of ClassDojo from faculty and students has been positive. Experiential faculty have appreciated the ability to store copies of completed rubrics and view the feedback "story" for students which facilitates final grading. Implications: This intervention could be utilized in small didactic classrooms or experiential settings and offers an alternative for recording attendance, giving feedback, and communicating with students.

"You Decide": An Elective Course Designed Using StudentSelected Topics. Charles Mosler, University of Findlay, Ryan A. Schneider, University of Findlay, Richard W. Dudley, University of Findlay. Objectives: In many pharmacy curricula, student choice in regards to what is covered in courses is often not considered when preparing topic outlines. We sought to design and implement an elective course in which the students determined the course content. Method: A one credit hour elective course was designed and offered to second and third professional year pharmacy students. The intent was for topics to not be review of material covered in required courses, but rather to take a more in depth look at topics or to cover topics not included in the required curriculum. The course was team-taught by three faculty members with diverse backgrounds. Outside speakers were invited to cover topics within their areas of expertise. Prior to the semester, students were asked to submit a list of three topics that they felt should be covered in the course. A list of the most frequently voted topics was then created to form the basis of the topic outline. Results: Students identified several interesting topics in what was the highest enrolled elective class that semester. The most frequently rated topics identified by students included: continual professional development after graduation, Zika, hospice and the dying process, and medical marijuana. Implications: Pharmacists have a growing role in healthcare and often times become "siloed" within their specialty. This elective helped to express the need for continual professional development and application of foundational knowledge so students are prepared to address new issues as they come up such as with Zika and medical marijuana.

\section{SOCIAL AND ADMINISTRATIVE SCIENCES Completed Research}

A Longitudinal Analysis of Pharmacy Student Wellbeing: The First Professional Year. Nicholas E. Hagemeier, East Tennessee State University, Chelsea L. Beavers, East Tennessee State University, Tucker S. Carlson, East Tennessee State University. Objectives: 1) To longitudinally describe the domain-specific and overall wellbeing of pharmacy students across the first semester of the first-professional (P1) year; 2) To determine the extent to which domain-specific wellbeing perceptions predict overall wellbeing perceptions. Method: At the beginning of a required, weekly course that spans the fall and spring semesters of the P1 year, pharmacy students $(\mathrm{N}=74)$ selfreported their career, community, financial, physical, social, and overall wellbeing using a 7-point response scale $(1=$ extremely poor; 7 =extremely good). Wellbeing domains were adapted from Gallup's Wellbeing: The Five Essential Elements. Multiple imputation approaches were employed to handle missing data. Wellbeing scores were mapped chronologically to curricular activities (e.g., exams). Repeated measures statistical tests and generalized estimating equations were used for longitudinal analyses. Results: At the beginning of the fall semester, career $(p=0.016)$ and physical wellbeing $(p<0.001)$ were statistically significant predictors of overall wellbeing whereas physical $(p=0.002)$ and social wellbeing $(p=0.001)$ were significant predictors of overall wellbeing at semester end. While variations in domain specific wellbeing were noted, average domain-specific and overall wellbeing trended downward across the fall semester. Statistically significant decreases were noted for all wellbeing scores except 


\section{American Journal of Pharmaceutical Education 2018; 82 (5) Article 7158.}

for social wellbeing ( $p$-values $<0.03$ ). Spring semester analyses are ongoing. Implications: To our knowledge, this is the first study to evaluate pharmacy students' wellbeing longitudinally. Physical wellbeing, in particular, was a routine predictor of weekly overall wellbeing. Assessment of student wellbeing across the curriculum could inform development and integration of interventions to sustain and/or maximize wellbeing as students progress in the professional program.

An Examination of the Implementation of Co-Curriculum Programs in US Schools and Colleges of Pharmacy. Jaime L. Maerten-Rivera, University at Buffalo, The State University of New York, Aleda M. Chen, Cedarville University, Jill M. Augustine, Mercer University, Antonio A. Bush, University of North Carolina at Chapel Hill, Richard d'Assalenaux, West Coast University, Justine S. Gortney, Wayne State University, Kelly C. Lee, University of California, San Diego, Cameron C. Lindsey, University of Missouri-Kansas City, Laurie S. Mauro, The University of Toledo, Daniel R. Malcom, Sullivan University, Nina Pavuluri, Lake Erie College of Osteopathic Medicine, Michael J. Rudolph, Marshall University, Siu-Fun Wong, Chapman University, Jacqueline M. Zeeman, University of North Carolina at Chapel Hill, Paula Zeszotarski, University of Hawaii at Hilo. Objectives: The ACPE 2016 Standards require programs to track and assess co-curricular activities, but limited information is available regarding how schools are implementing this requirement. This study, conducted by a committee within the Assessment SIG, examined how co-curriculum is implemented and evaluated. Method: A Qualtrics survey was administered to assessment representatives at all US schools/colleges of pharmacy examining the following aspects of co-curriculum: a) structure and personnel, b) types of activities, c) assessment, d) level of confidence that the ACPE co-curriculum requirement is satisfied, and e) challenges. Descriptive statistics were examined. Results: The response rate to date is $21 \%$. Many schools reported having a standing $(38 \%)$ or ad-hoc $(38 \%)$ co-curriculum committee/subcommittee/task force. The most common office/department reported as primarily responsible were academic/curricular affairs (28\%) and student affairs/services (28\%). Most programs described the model used as a hybrid of pre-specified activities and a list of activities that fit a category (48\%). The types of outcome measures used included reflections (30\%), self-assessment surveys $(20 \%)$, checklists $(12 \%)$, and IPPE/APPE evaluations $(10 \%)$. On a scale of $1-10(1=$ unconfident, $10=$ confident $)$, schools had a mean of $7.93(\mathrm{SD}=1.44)$ on confidence in their ability to satisfy the ACPE co-curriculum requirement. Schools reported that closing the loop was an area of concern (25\%), followed by documentation $(25 \%)$ and assessment (19\%). Implications: The structure, activities, and assessment of the co-curriculum varies across the academy, but most programs report confidence in their ability to satisfy the new ACPE requirements. The information from this study will contribute to establishing best practices for co-curriculum.

Applying Generalizability Theory to Provide Validity Evidence for a Pharmaceutics Course Grade: Combining Exam Reliability Coefficients. Michael J. Peeters, The University of Toledo, M. Kenneth Cor, University of Alberta, Sai Hanuman Sagar Boddu, The University of Toledo, Jerry Nesamony, The University of Toledo. Objectives: The vast majority of pharmacy education uses Classical Test Theory (CTT), where reliability is limited to each exam's scores; in CTT, individual exam reliabilities cannot be combined over multiple exams/occasions. While rarely reported in pharmacy education, Generalizability Theory (Gtheory) can combine results from multiple exams - reliability can be estimated for more complex measures. This study illustrated Gtheory use to combine reliability of three separate exams, into reliability for one overall pharmaceutics course-grade. Reliability trade-offs were also investigated. Method: First-year PharmD students took a pharmaceutics course, with 12-weeks of lectures and three exams. Kuder-Richardson Formula-20 (KR20) estimated the CTT-based reliability of each exam. For Gtheory, we used G-String-IV. Results: 101 students took two midterms and one finalexam; exam $1=50 \mathrm{MCQ}$ with $\mathrm{KR} 20=0.69$; exam2=43MCQ with $\mathrm{KR} 20=0.65$; exam $3=67 \mathrm{MCQ}$ with $\mathrm{KR} 20=0.67$. Using Gtheory to model items nested within exams/occasions (p x i:o), the G-coefficient/ reliability was 0.71 for course-grade. Data analysis further demonstrated how reliability changed as a function of number of exams/occasions and items per exam. For a g-coefficient of 0.80 , options include four exams of 80MCQ, five exams of 60MCQ, or six exams of 40MCQ. As the number of exams increased, fewer questions were needed per exam, and fewer questions over all exams combined. Implications: Gtheory provides reliability evidence for test AND course validation-vital generalization evidence. Final course-grades appear reasonably reliable mainly from multiple additive examinations,; with more exams, each can have fewer questions and fewer questions over all the exams combined. Notably, reliability of grade-point-average for a program is likely improved further by combining multiple courses.

A Prescription for Improved Leadership Skills: An Assessment of the PILs Program. Youness R. Karodeh, Howard University, LaMarcus T. Wingate, Howard University, Muhammad J. Habib, Howard University, Imbi Drame, Howard University, Bryan K. Sackey, Pharmacy Initiative Leaders (PILs), Inc. Objectives: To evaluate and determine the impact of a peer-mediated mentorship program on student professional development and leadership readiness at a predominantly minority pharmacy school. Method: A cross-sectional study design was utilized in evaluating student attitudes towards the Pharmacy Initiative Leaders (PILs) program and to evaluate the impact of PILs on participation in student organizations. First and second year students were asked to complete a survey eliciting information on involvement in student organizations and attitudes towards PILs. Results: Over 115 students completed the survey for a response rate of over $80 \%$. Students mentored through the PILs program participated in 2.2 student organizations during pharmacy school, which was significantly more than the 1.3 student organizations participated in among students not participating in PILs $(p=0.005)$. Approximately $54 \%$ of PILs participants agreed or strongly agreed that PILs helps to establish a culture of leaders, and over $75 \%$ of those mentored through PILs said they would recommend that future students participate in the program. Implications: Based upon the results of this study, students who participated in a tailored professional development and leadership program for pharmacists called PILs, were significantly more likely to participate in student organizations than those not participating. The vast majority were also likely to recommend that future students participate in the PILS program, demonstrating high favorability of PILs amongst first and second-year pharmacy students. Further study may be warranted to determine impact of PILs on matriculation rates.

Assessing Critical Thinking Skills of Pharmacy Students and Its Association With Students' Academic Performance. Estela Lajthia, Howard University, Miranda G. Law, Howard University, LaMarcus T. Wingate, Howard University, Mary Maneno, Howard University, Muhammad J. Habib, Howard University, Youness R. Karodeh, Howard University. Objectives: To evaluate the association between pharmacy students critical thinking skills, remediation rates and Pharmacy Curriculum Outcomes (PCOA) test scores. Method: The Health Sciences reasoning test with Numeracy (HSRT-N) provided through Insight Assessment (California Academic Press) and Schuhfried 


\section{American Journal of Pharmaceutical Education 2018; 82 (5) Article 7158.}

Publishing, Inc, was utilized to assess critical thinking skills. All pharmacy students in their second, third, and fourth year were asked to complete the HSRT-N survey. Descriptive statistics, Pearson's correlations, and multivariate logistics regression analyses were used to evaluate the effect of critical thinking skills on remediation, and PCOA scores. Results: A total of 145 out of $176(82.4 \%)$ students completed the HSRT-N. Students with a critical thinking score $<86 / 100$ on the HSRT-N had 5.97 times the odds of remediating a course compared to those with a HSRT-N score of at least 86/100. Critical thinking skills appear to be effective at predicting pharmacy school GPA and PCOA performance. After adjusting for other factors, each additional point on the critical thinking score test is associated with 1.6 more points on the PCOA exam. Implications: Critical thinking skills appear to be a possible predictor of pharmacy school performance. Currently, critical thinking skills are not routinely evaluated among pharmacy students. Efforts geared towards improving critical thinking skills in student pharmacists may decrease remediation rates, increase PCOA scores and thereby, increase NAPLEX passing rates. Future research further exploring the role of critical thinking on student performance will provide additional clarity on the impact of critical thinking skills in pharmacy students performance.

Behavioral Intent Among Health Professionals in Assessing Patients for and Addressing Medication Nonadherence. Connie F. Rust, South College, Joseph H. Ford, South College, Brian D. Esters, South College, Tyler S. Dougherty, South College, Rebecca Yarnell, South College, Louisa Hope Bruce, South College, Robert Mindrup, The University of Tennessee, Knoxville, Ragan Schriver, The University of Tennessee, Knoxville. Objectives: The objective of this project is to determine the behavioral intent of health professionals in assessing patients for and addressing medication nonadherence. Method: The Theory of Planned Behavior can be used to assess the impact of three domains - behavioral beliefs, normative beliefs, and control beliefs - on behavioral intent and thus, by inference, actual behavior. For this project, a pre-post cross-sectional analysis of nurses, pharmacists, social workers, and students was conducted before and after an interprofessional seminar to gauge participants' intent to assess patients for and address medication nonadherence. A three-page, 31-item questionnaire based on the Theory of Planned Behavior was developed to gauge behavioral intent as assessed on a seven-point Likert scale. Results: Compared with pre-seminar responses, post-seminar data were notable for positive changes in behavioral intent among participants. Changes in post-seminar scores for the behavioral beliefs, normative beliefs, and control beliefs domains were also observed. Implications: Behavioral intent, and by extension, actual behavior, represents an important outcome for interventions designed to positively impact the behaviors of health professionals. As in this study, interprofessional educational interventions can be used to address significant public health concerns such as medication nonadherence.

Cheating and Academic Dishonesty Among California Pharmacy School Students and Faculty. Paul Gavaza, Loma Linda University, Sophia Dinh, Loma Linda University, You Been Kim, Loma Linda University, Simon Situ, Loma Linda University, Lawrence Chui, Loma Linda University, Justin Thurber, Loma Linda University, Maria Santiago, Loma Linda University, Jennifer Nguyen, Loma Linda University, Farnoosh Zough, Loma Linda University, Nancy E. Kawahara, Loma Linda University, Quang Vu, Loma Linda University. Objectives: To determine pharmacy student's definition of cheating, their motives, views, and perceived long-term consequences of cheating. Method: Two separate electronic surveys were distributed to each of the 14 California pharmacy schools; one targeted students, while the second targeted pharmacy school faculty and professionals. The surveys measured the respondents' views and motivations on cheating, but also included items unique to each target group. Demographics data were also collected. Results: A total of 251 students and 90 professionals completed the survey. Most pharmacy students (68.82\%) and professionals (58.8\%) agreed that cheating would affect their judgment as pharmacists. Some students (16.27\%) and professionals (25.8\%) admitted to dishonest behavior in pharmacy school. A significant number of respondents (66.01\% students, $66.3 \%$ professionals) knew of individuals who acted unethically in pharmacy school, while $49.4 \%$ of professionals knew of licensed pharmacists who cheated in school. Implications: Students and professionals have varying definitions of academic dishonesty and agree that that dishonest behavior by a pharmacy school student may be persist after licensure and into the professional setting. More should be done to guard the integrity of the pharmacy profession.

Comparison of Select All That Apply to True/False Items Administered to PharmD Students. Scott A. Baggarly, The University of Louisiana at Monroe, Jeffery D. Evans, The University of Louisiana at Monroe. Objectives: To evaluate the difficulty and discrimination ability of Select All that Apply (SAA) items compared to True/False (TF) items in a first professional year pharmacy law class. We hypothesized that SAA questions are more difficult but offer greater discrimination between higher and lower performing students. Method: Two instruments were developed, each containing 4 SAA law items and 1 SAA control item from an unrelated topic. Three parallel TF items were derived from each SAA item. Form A contained TF items derived from SAA items of Form B while Form B contained TF items derived from SAA items of Form A. The instruments were administered to volunteers enrolled in the course. Item statistics were tabulated and analyzed statistically with t-tests. Results: Sixty-eight of eighty-seven students $(78 \%)$ participated. Difficulty index scores in pooled item analyses indicated TF items were less difficult (higher scores) than SAA ( $70 \%$ vs $41.5 \% ; \mathrm{p}=0.0153)$, and course-related items were less difficult than control $(66.1 \%$ vs. $27.5 \%$; $=0.0052)$. Point biserial correlation coefficients (RPB) were similar between TF and SAA items ( 0.279 vs. $0.230 ; p=0.5292)$ and between course-related and control items ( 0.267 vs. $0.179 ; \mathrm{p}=0.2178)$. The discrimination index (point biserial) was similar between TF and SAA items (0.176 vs. $0.239 ; \mathrm{p}=0.4396)$ and between course-related items and control items ( 0.192 vs. $0.129 ; p=0.3926)$. Implications: These data demonstrate that SAA items were more difficult than corresponding TF items without a significant difference in the ability to distinguish between higher and lower performing students. Further studies are needed to evaluate the discrimination ability of SAA items.

Concomitant Use of Central Nervous System Stimulants and Depressants. Aram Babcock, The University of Rhode Island, Xuerong Wen, The University of Rhode Island, Nickolas Belviso, The University of Rhode Island, Amala Murthy, The University of Rhode Island, Stephen Kogut, The University of Rhode Island. Objectives: This study describes the concurrent use of CNS stimulants and depressants, and to determine predictors of the combined use of these medications. Method: We conducted a cross-sectional study using deidentified data from a state PDMP for the year 2015. We included all patients who filled at least 1 prescription for a stimulant or depressant medication. The outcome of interest was the concomitant use of these medications, defined as any stimulant(s) and any depressant(s) with at least 60 days of combined continuous use (less than 15 days' gap in filling), with overlapping fills. Results: The study cohort consisted of 131,476 patients who filled both stimulants and 


\section{American Journal of Pharmaceutical Education 2018; 82 (5) Article 7158.}

depressants. Most $(\mathrm{N}=127,087,96.7 \%)$ received these medications separately, while $3.3 \%(\mathrm{~N}=4,389)$ filled stimulants and depressants concomitantly. Compared to patients without overlapping use, the patients filling both stimulants and depressants concurrently tended to be younger (mean age \pm SD: $43 \pm 13$ years vs $49 \pm 21$ years, $\mathrm{P}<.0001)$, more often female gender (68\% vs $58 \%, \mathrm{P}<.0001)$, and used private pay (cash) more frequently ( $33 \%$ vs $24 \%, \mathrm{p}<.0001)$. Results from multivariate regression analyses revealed that the average daily dose of stimulant greater than $25 \mathrm{mg}$ was significantly associated with concomitant use of both stimulants and depressants as compared to those who filled only (OR $1.7895 \%$ CI 1.93 - 1.64), after adjusting for age, female gender, and insurance type. Implications: As compared with patients receiving dispensings for either prescription CNS stimulants or CNS depressants, those using these medications concurrently received a higher doses of stimulants.

Contagious Education: Characterization of an Infectious Diseases Pharmacist Educator Network. Katherine Gruenberg, University of California, San Francisco, Conan MacDougall, University of California, San Francisco, Wesley D. Kufel, Binghamton University, The State University of New York, Meghan N. Jeffres, University of Colorado. Objectives: To characterize membership, participation through listserv activity, and shared repository access of the Infectious Diseases Educator Network (ID-EN). Method: In September 2017, ID faculty at 137 United States colleges or schools of pharmacy were invited to participate in the ID-EN via an online survey. Survey respondents were subsequently invited to participate in the ID-EN. This network provides listserv communications and an educational resource repository, including active dissemination of great educational materials (GEMs) for members to adapt and utilize. Results: Between November 27, 2017 to February 20, 2018, a total of 157 respondents confirmed participation in the ID-EN, representing 105 colleges or schools of pharmacy. Survey respondents were primarily full-time faculty specializing in ID (81\%); $32 \%$ completed a post-graduate year two ID residency and 14\% completed an ID fellowship. To date, 68 listserv messages have been sent, and 39 documents have been uploaded and curated by the ID-EN in the shared repository. Five GEMs have been disseminated with 138 views and 32 downloads. Originating through listserv communication, a collaborative educational research project about pharmacokinetics education is actively underway. Implications: The ID-EN is comprised of $>150$ ID pharmacist educators nationwide. This network provides a forum to share educational materials and to develop collaborative educational research initiatives. The ID-EN is poised to open membership to educators in ID pharmacotherapy among all professions, and may serve as a template for other specialty educators.

Crosswalk Between the ACPE Standards and the FIP Nanjing Outcomes. Abby A. Kahaleh, Roosevelt University, Miranda G. Law, Howard University, See-Won Seo, Albany College of Pharmacy and Health Sciences, Toyin S. Tofade, Howard University. Objectives: The purpose of this study is to compare and contrast the 2016 Accreditation Council for Pharmacy Education (ACPE) Standards with the 2017 Nanjing Outcomes. Specifically, to streamline the pharmacy education standards globally by mapping the International Pharmaceutical Federation (FIP) Nanjing Outcomes to the ACPE Standards. Method: Three members of the GPE SIG conducted a thorough review of the ACPE Standards and Nanjing Outcomes to identify which Nanjing Outcomes mapped to the ACPE Standards. The reviewers highlighted recurring themes of pharmacy education and areas of uncertainty that revealed residual outcomes that were unable to be mapped. A fourth reviewer, an expert on pharmacy education, evaluated the results of the mapping to ensure face validity of the findings. Results: A total of 25 ACPE Standards and 8 Nanjing Outcomes clusters were reviewed to create a crosswalk of overlapping standards and outcomes. Select outcomes from 20 different ACPE statement categories remained unmapped to the Nanjing Outcomes, whereas 8 statements within five of the 8 clusters remained unmapped. Implications: These preliminary findings may help pharmacy educators to better understand how global competencies correspond to the national standards. These results identified competencies that may require further evaluation. Notably, the Nanjing Outcome (1.1) recommends larger strategic workforce planning on local and national levels, to better meet the local health needs. In summary, both ACPE and FIP created these competencies to continuously improve the quality of education. Ultimately, all accrediting organizations can collaborate on identifying strengths and areas for improvement in global pharmacy education.

Cultural Competence Instruction in Didactic Settings: Exploring the Perceptions of URM PharmD Students. Antonio A. Bush, University of North Carolina at Chapel Hill, Danielle J. Allen, University of North Carolina at Chapel Hill. Objectives: Schools/Colleges of pharmacy $(\mathrm{S} / \mathrm{CoP})$ have often acknowledged the rapid diversification of the US population and the rise of health disparities. Furthermore, accreditation bodies are mandating the inclusion of cultural competence (CC) content in curricula. Funded by AACP's New Investigator Award, this study explored how Pharm.D. studentes identifying as underrepresented racial minorities (URM) perceived CC instruction at a S/CoP. Method: Twenty students from a S/CoP within a researchintensive institution participated in 60-90 minute interviews. Following audio transcription, the data were analyzed using the Sort and Sift, Think and Shift $\subset$ method. Multiple coders used several rounds of open coding to develop a codebook, which guided the final rounds of coding and theme identification. Trustworthiness procedures included a dependability audit, peer debriefing, and analytic memos to promote reflexivity. Results: The students interviewed $(n=20)$ represented $40 \%$ of the total population of URM students within the S/CoP $(\mathrm{n}=50)$. Students felt the curriculum lacked a CC emphasis (e.g., lack of cultural diversity in patient cases), and that $\mathrm{CC}$ was optional. In addition, students sensed that the responsibility to gain $\mathrm{CC}$ awareness had been placed upon them. Students often supplemented didactic CC instruction with co-curricular activities and intentionally sought immersion experiences in culturally diverse communities. Implications: Previous research shows that curriculum committee chairs and student leaders believe that $\mathrm{CC}$ should be required, however incremental changes have been implemented in curricula. This work provides insight into how URM pharmacy students perceived CC instruction, provides strategies to bolster CC engagement, and demonstrates how co-curricular experiences may be employed to advance $\mathrm{CC}$ delivery.

Defining and Exploring the Role of Social Incentives for Health Behavior Change Including Medication Adherence: FocusGroups. Justin K. Owensby, Auburn University, Kimberly B. Garza, Auburn University, Richard A. Hansen, Auburn University, Brent Fox, Auburn University, Ana Franco-Watkins, Auburn University, Joshua C. Hollingsworth, Edward Via College of Osteopathic Medicine. Objectives: To explore perceptions of social incentives for healthful behaviors in adults, assess technology utilization, and characterize the types of interventions perceived as most relevant. Method: Focus groups were conducted with adults receiving prescription medications. Baseline survey assessed demographics, number of medications, perspectives of differing incentives, and smartphone use. A facilitated discussion explored participants' conceptualization of social incentives, associated 


\section{American Journal of Pharmaceutical Education 2018; 82 (5) Article 7158.}

facilitators/barriers, and how these motivated medication adherence. Sessions were audio recorded and transcribed verbatim. Data were analyzed using ATLAS.ti. Thematic analysis identified relevant themes; Krippendorf's Alpha assessed inter-coder reliability. Results: 17 participants were assigned to one of two sessions by age $(<50, \geq 50)$. Session 1 included seven participants $(6 \mathrm{~F}, 1 \mathrm{M})$ with a mean age of 31 ; session 2 included 10 participants $(8 \mathrm{~F}, 2 \mathrm{M})$ with a mean age of 58 . Thematic analysis identified four themes: Accountability, Motivation, Barriers/ Solutions, and Technology. Inter-coder reliability was favorable overall (Krippendorff's $\alpha=0.787$ ) and by theme (range:0.755-0.853). Participants reported using apps/smartphones or wearables to stay accountable with health goals. Very few track medication adherence using an app, and most disapproved of having social incentive features associated with a medication-taking app, stating medication-taking behaviors are "private" and sharing is "too personal". Others felt medication-taking was not necessarily a goal or social features attached to medication-taking are not trendy or appealing. Implications: Despite endorsing social incentives, with/without technology, to motivate diet or exercise-related behaviors, participants were not receptive to incorporating social features for medication adherence or into a medication-taking app. Further study is needed to determine features of social incentives for medication adherence that might improve acceptability.

Development of an Escape the Room Learning Activity in a Therapeutics Course Series. Michael J. Gonyeau, Northeastern University, Jim Huang, Northeastern University, Jason Jenwen Kuang, Northeastern University, Vanessa Le, Northeastern University, Taylor Wright, Northeastern University. Objectives: An escape room is a time-limited ludic game, team played, whose objective is to 'escape' from a challenge-filled room, encouraging students to think creatively and critically. Our objectives were to design an immersive experience to stimulate and deepen knowledge, skills and attitudes while promoting collaboration in a live simulated setting. Method: A literature search revealed a paucity of data regarding escape room designs in health education. Data obtained revealed mutiple design strategies, foremost that puzzles must collaborate while providing diverse challenges connected to a theme. Inherently team activities, puzzle design must ensure balanced and meaningful contributions. An iterative process developed in consultation with instructional designers incorporated physical and technological puzzles to simulate varied learning experiences. Results: A 1 hour escape room was developed with specific rules and five puzzles integrating hyperlipidemia pharmacology, pathophysiology, and evidence based therapeutics. To win, students must construct a prescription based on ASCVD risk-assessment of a video introduced patient. Puzzles included: medication reconciliation via pharmacy call, finding/completing a non-statin therapy table, electronic drug maze on password locked ipad, statin dose and \%LDL lowering jenga blocks, and a crossword puzzle. An open-path design was employed allowing puzzles completion in any order, but the final puzzle could not be ascertained until all other puzzles were solved. Implications: As constructed, escape rooms help develop skills in team working, creative problem solving and critical thinking. The inherent multi-modal and team-based mechanics increase likelihood of student engagement. The activity enhances student attitudes regarding collaboration/teamwork and applied knowledge confidence.

Differences Among Hypertensive Patients in Two Urban Community Health Centers in Their Beliefs About Medicines. Judith T. Barr, Northeastern University, Sharon Callender, Mattapan Community Health Center, Michael Conley, Northeastern University, Borna Fatehi, Northeastern University, Tayla Poretta, Northeastern University. Objectives: To determine in two urban community health centers
(CHC) whether patients with hypertension (HTN) had differences concerning HTN medication use as revealed by Beliefs about Medicine Questionnaire responses (BMQ, Horne, 1999) Method: Following Northeastern University's IRB approval, a convenience sample of patients was asked to complete the BMQ prior to routine HTN followup appointments at two Boston CHCs. Researchers screened for eligibility (English speaking, taking anti-hypertensive medication), described the study, and requested informed consent. Patients completed background demographic/sociologic questions and a hypertension-modified BMQ (18 items; 5 levels: $5=$ strongly agree, $1=$ strongly disagree; 4 factors). T-tests and ANOVA followed by Fisher's LSD were used in bivariate analyses. Results: 186 patients averaged 56.78 yo $\pm 11.63,58.1 \%$ female. Overall BMQ factor scores were Specific Necessity (SN), 3.39 \pm 0.80 ; Specific Concerns (SC), 2.70 \pm 0.77 ; General Overuse (GO), 2.9 \pm 0.81 ; and General Harm (GH), 2.41 \pm 0.70 . Initial bivariate analyses indicate no difference in impact of demographic/ sociologic variables on SN. However, those "Living with Someone" had more HTN drug-related concerns (SC: Yes $=2.82 \pm 0.75, \mathrm{No}=$ $2.45 \pm 0.76 ; \mathrm{p}=0.004)$. Blacks believed that there was more General Overuse of drugs (GO: Blacks $=3.03 \pm 0.80$, Whites $=2.70 \pm 0.81$; $\mathrm{p}=0.011)$. Patients were more likely to believe that drugs in general caused harm $(\mathrm{GH})$ when they had less education $(<$ high school= $2.71 \pm 0.76,>$ high school $=2.22 \pm 0.63 ; \mathrm{p}=0.005)$, parents hadn't had stroke (No stroke $=2.49 \pm 0.65$, had stroke $=2.28 \pm 0.75 ; \mathrm{p}=0.048$ ), were black (black $=2.53 \pm 0.74$, white $=2.15 \pm 0.55 ; \mathrm{p}=0.001$ ), and were nonadherent (nonadherent $=2.77 \pm 0.79$, adherent $=2.32 \pm 0.66 ; \mathrm{p}=0.001$ ). Implications: HTN patients have different beliefs concerning General Overuse and Harm of drugs. BMQ results may help clinicians tailor patient-specific medication counseling programs and to reduce patient-specific medication harm/overuse concerns.

Effects of Video and Text Educational Material on Knowledge, Perceptions, and Intention of Vaccination. Heba A.T. Aref, Auburn University, Kimberly B. Garza, Auburn University, Salisa C. Westrick, Auburn University, Chiahung Chou, Auburn University, Fred Kam, Auburn University, Debra Worthington, Auburn University. Objectives: This study aims to compare the effect of multi-modal (audiovisual with text) with single mode (text-only) online health messages on college students' knowledge, perceptions, and intention to obtain the Meningitis B (Men B) vaccine. The Health Belief Model guided the development of this study. Method: We recruited 132 first-year college students using student email listserv for a twogroup randomized controlled trial. Participants received either online audiovisual with text or online text only-based educational material that described the Men B disease and the benefits of Men B vaccination. In both groups, participants' knowledge, perceptions, and intention of Men $B$ vaccination were assessed at baseline and after the intervention using an online survey. Paired t-tests were used to examine the pre-post intervention difference within each group, and difference-in-differences were used for between two groups. Results: Participants in both groups had similar levels of baseline knowledge, perceptions, and intention $(\mathrm{p}>0.05)$. Both audiovisual-with-text and text-only groups showed significant post-test mean score improvement in perceived susceptibility, severity, benefits, barriers, self-efficacy, and intention to obtain Men B vaccine $(\mathrm{p}<0.05)$. The audiovisual-with-text group also showed a significant post-test mean score improvement in knowledge and perceived cues to action $(\mathrm{p}<0.05)$. No significant difference was found in mean score improvement of knowledge, perceptions, and intention for multi-modal compared to single mode educational material $(\mathrm{p}>0.05)$. Implications: Both audiovisual-with-text vehicle and text-only vehicle are effective educational tools for promoting vaccination among college students. 


\section{American Journal of Pharmaceutical Education 2018; 82 (5) Article 7158.}

Using these educational techniques has the potential to positively impact disease prevention.

Entrepreneurship Versus Intrapreneurship: Comparisons of Business Planning Experiences for Student Pharmacists. Justin Gatwood, The University of Tennessee, Kenneth C. Hohmeier, The University of Tennessee, Sohul Shuvo, The University of Tennessee, Glen E. Farr, The University of Tennessee. Objectives: To compare student impressions of business planning projects within a required pharmacy practice management course and describe change in perceptions of a novel, real-world business planning experience over its first two years of implementation. Method: Teams of student pharmacists were given two options for completing a semester-long business planning project as part of the pharmacy practice management course: 1) traditional projects allowed groups to develop a pharmacy concept or product of their choosing; 2) applied projects paired groups with a Tennessee-based pharmacy-related firm to provide consulting and propose an internally-relevant concept to firm stakeholders. All students were surveyed by Likert-type questions at the end of the course to gauge impressions of their project. Descriptive statistics, MantelHaenszel tests, and chi-square tests compared impressions within and between years. Results: The applied concept was well-received by students, and positive impressions were more pronounced in the second year. Compared to traditional project groups, a higher proportion of applied group members agreed their project provided a more valuable (2016: $76 \%$ vs. $50 \%$; 2017 : $82 \%$ vs. $48 \%$ ) and real-world learning experience (2016: $92 \%$ vs. 44\%; 2017: 86\% vs. 50\%), helped them better understand pharmacy management principles (2016: $68 \%$ vs. $50 \% ; 2017: 67 \%$ vs. $47 \%$ ), made them more marketable to employers (2016: $52 \%$ vs. $29 \%$; $2017: 63 \%$ vs. $15 \%$ ), and assisted them in seeing how they could apply the skills gained in their future, desired position (all listed comparisons $\mathrm{p}<0.05$ for trend). Implications: Connecting student pharmacists with pharmacy-related firms can effectively add needed context to the topics covered within management coursework.

Ethical Issues in Consenting Cognitively Intact and Independent Older Adults: Academic Researchers and Community Perspectives. Shoroq M. Altawalbeh, Jordan University of Science \& Technology, Fadi M. Alkhateeb, The University of Texas at Tyler. Objectives: to evaluate ethical issues and strategies in consenting older adults in Jordan as perceived by both academic researchers as well as older adults Method: Academic researchers in the Jordanian medical and pharmacy colleges were interviewed and asked to complete the study survey if had ever conducted clinical research with older adult population. The study survey included items eliciting demographics and professional characteristics, and perceptions regarding: the consenting process in older adults, consent related skills in elderly, and strategies to improve the consenting process in older adults. The survey was then modified to assess the consent related ethical issues and challenges as viewed by a sample older adults after explaining the concept of the consenting process to them (in a hypothetical scenario). Results: A total of 250 academic researchers and 233 older adults have participated in the study. Both researchers and older adults reported that signing the written forms and the impact of age related physical impairments were the most challenging in consenting older adults. Lack of consistency and repeating questions were the most frequently encountered by researchers in consenting older adults. Ensuring privacy, providing extra time and efforts patiently, treating older adults as autonomous individuals and respecting their cultural believes were the most helpful strategies recommended by both academic researchers and older adults Implications: This study was the first in obtaining researchers' attitudes and views regarding the process of informed consents in Jordan. The findings of this study highlight the importance of considering age related physical and emotional characteristics in the consenting process

Evaluation of an Online Short Course to Prepare Pharmacy Students to Communicate Effectively With Youth. Delesha M. Carpenter, University of North Carolina at Chapel Hill, Julia Gilmartin-Thomas, Monash University, Stacy C. Bailey, University of North Carolina at Chapel Hill, Angel Chater, University of Bedfordshire, Catherine MacAllister, University of North Carolina at Chapel Hill, Oksana Pyzik, University College London, Brandi Wayman, University of North Carolina at Chapel Hill, Izabela Annis, University of North Carolina at Chapel Hill, Felicity Smith, University College London, Betsy L. Sleath, University of North Carolina at Chapel Hill. Objectives: We describe the development and evaluation of an online short course focused on training pharmacy students to communicate effectively with youth. The course was designed to be readily incorporated into university pharmacy curriculums and contribute toward the international agenda of achieving youth-friendly pharmacy services. Method: The course, entitled "Communicating with Youth in Pharmacy Settings" (https://pharmalliance.cips.unc. edu), was developed by pharmacy faculty and students from the USA, Australia, and England. The course includes two modules: "An overview of counseling young people in pharmacies" and "Strategies for effectively counseling young people." Pharmacy students from the USA and Australia $(\mathrm{N}=31)$ completed surveys with knowledge and self-efficacy questions before and after completing the course. Student changes in knowledge and self-efficacy were assessed using a paired samples t-test. Open-ended questions assessed satisfaction. Results: Students were mostly female $(67.7 \%)$ with an average age of 24.9 years and were primarily in the third (51.6\%) year of their pharmacy program. Over $80 \%$ of students somewhat or strongly agreed that the course increased their comfort speaking with young people in pharmacy settings. Both knowledge $(\mathrm{p}<0.01)$ and selfefficacy in communicating with young people $(p<0.01)$ significantly improved after taking the course. On open-ended questions, students expressed that the course was useful and provided new information on communicating with youth. Implications: The course was positively received by pharmacy students and improved students' knowledge and self-efficacy in communicating with young people. Pharmacy schools should consider using the course to complement existing coursework to help prepare students to communicate effectively with youth.

Examining Math Credits as a Predictor of PY1 Student Performance. Kevin B. Sneed, University of South Florida, Natasha Baloch, University of South Florida. Objectives: This analysis examines the relationship between first year student performance and pre admission factors, specifically prior math credit hours affecting student's performance in a Doctor of Pharmacy Program. The research specifically examines students having an interruption in PY1 while analyzing two groups of students; students with academic interruption vs students with no academic interruption. Method: The data used in this analysis comes from our most recent classes of 2020 and 2021. A total of 192 students were included in the analysis. Descriptive statistics and independent t-tests were conducted to explore relationships between prior math credit hours, other pre admission variables and academic progression. Preliminary logistic regressions were also conducted to confirm the findings. Results: Students with academic interruptions had on average significantly $(\mathrm{p}<$ 0.01 ) higher number of math credit hours suggesting a higher number of repeated math classes. Students held back also had lower performance in other pre admission variables including PCAT scores, prior university 


\section{American Journal of Pharmaceutical Education 2018; 82 (5) Article 7158.}

GPA and overall PY1 GPA. Findings are also supported by regression analysis. Implications: Results support findings from a prior study that examined the relationship between prior math credits and student interruption in PY1 to PY4. Pharmacy programs can provide pre matriculation enrichment programs to at risk students in order to provide a foundational review of math and sciences to prevent interruptions.

Exploring How Underrepresented Racial Minority PharmD Candidates Were Exposed to and Gained Interest in Pharmacy. Mauriell H. Amechi, University of North Carolina at Chapel Hill, Danielle J. Allen, University of North Carolina at Chapel Hill, Antonio A. Bush, University of North Carolina at Chapel Hill. Objectives: As evidenced by the 2013-14 Argus Commission report, the American Association of Colleges of Pharmacy has shifted its focus to a broad examination of the issue of diversity, including the applicant pipeline. The purpose of this exploratory study was to understand the pre-pharmacy experiences of underrepresented racial minorities (URMs). One research question guided this work: How are URM students exposed to and gain interest in pharmacy education and health professions? Method: Semi-structured, interviews were conducted with 20 URM pharmacy students at a large, public, southern research institution. Audio-recorded interviews, which ranged between 45-90 minutes, were professionally transcribed and a constant comparative method was used to code data. Multiple validation strategies, as recommended by Creswell, were employed by the research team to ensure the accurate representation of students' experiences and beliefs including peer debriefing, multiple coders, and a dependability audit. Results: The overall findings suggest that URM students gained exposure to and interest in pharmacy as a result of formative experiences and co-curricular activities: (a) participation in a URM-specific health professions pipeline programs; (b) working or volunteering in a healthcare environment; and (c) being motivated by parents, relatives, and friends and coached by primarily URM persons in health professions. Implications: Implications Given recent calls to examine diversity and inclusion in pharmacy, this work provides insights on co-curricular activities and formative experiences that compelled URM students to pursue career pathways in pharmacy. Insights from this study might inform practice and policy efforts nationally that focus on enhancing access to quality education for all students, especially URMs.

Facilitating Student Pharmacists' Personal and Professional Development in a 3-Year Course Series. Julie Truong, Keck Graduate Institute. Objectives: To describe the development and assessment of the Professional Development Series (PDS). Method: A 3-year longitudinal course was developed at Keck Graduate Institute School of Pharmacy to address ACPE Standards 4.1-4.4 - Personal and Professional Development. The series aims to provide students with the knowledge, resources, and experiences to guide their personal and professional development. The PDS focuses on self-awareness during the first year, leadership during the second year, innovation during the third year, and reinforces professionalism throughout the series. Additionally, students learn about various areas of pharmacy practice, participate in interdisciplinary clinical experiences, provide community service, and partake in continuous professional development activities. During the last semester of the series, students completed an anonymous Google questionnaire to provide feedback on the series. Data was collected and analyzed by the course coordinator and reported as percentages. Results: Sixty-one (91\%) students completed the questionnaire. Seventy-nine percent of respondents stated that PDS was enjoyable and engaging, $85 \%$ stated that PDS provided information that they expect to use, and $87 \%$ stated that they were committed to using information from PDS beyond graduation. Topics that students found most useful included CV writing (100\%), leadership (98\%), writing professional thank you letters (96\%), and 7 Habits of Highly Effective People (93\%). Some biggest takeaways from PDS included professionalism, teamwork, professional and personal development, and the 7 Habits of Highly Effective People. Implications: The Professional Development series positively affected students' personal and professional development and provided them with useful information that they could use beyond graduation.

Factors Associated With Burnout in Health Professional Students: Implication for Pharmacy Students. Shih-Ying (Audrey) Hsu, West Coast University, Monica Hwang, St. John's University. Objectives: To investigate factors associated with burnout in health professional students. Method: A systematic literature review was conducted using an electronic database, PubMed. Search terms "burnout", "students", and "factors" were used in combination to identify peer-reviewed articles in English language from 2008 to 2018. Out of the 164 publications extracted for abstract review, 32 publications were deemed relevant for the study objective and were selected for analysis. Publications were included if the study examined health professional students. Publications focusing on non-healthcare students, clinicians and/or residents were excluded. Results: Health professional students being studied were from medical $(n=22)$, dental $(n=4)$, nursing $(n=$ $3)$, speech therapy $(n=1)$, and veterinary $(n=2)$ programs. Eleven major aspects were identified in the literature regarding factors associated with burnout: demographics; learning/educational factors; years of study; support; coping methods; perceived stress and stressors; lifestyle; career motivation; sense of control; self-efficacy; and sleep condition. Implications: Pharmacy students are at risk for burnout due to high work demand from school and job; however, this systematic literature review did not find any peer-reviewed publication related to burnout in pharmacy students. Given the negative consequences of burnout, such as decrease in productivity, loss of empathy, and thoughts of dropping out, understanding potential factors associated with burnout could help develop Interventions that promote well-being among pharmacy students. A study is needed in the near future to examine the level of burnout and the associated factors among pharmacy students.

First Professional Year Pharmacy Students' Perceptions and Knowledge of Older Adults. Siddhi A. Korgaonkar, The University of Mississippi, Lori Ward, The University of Mississippi, Lauren Pohl, The University of Mississippi, Alisha S. Nicks, The University of Mississippi. Objectives: Prior studies examining health profession student perceptions of older adults have reported a general negative attitude towards older adults. Educational interventions can potentially improve pharmacy students to perceptions, interactions, and knowledge of older adults. Literature focusing on pharmacy students' perceptions of older adults is limited. The objectives of this study were to assess first professional year pharmacy students' perceptions and knowledge of older adults. Method: First professional year pharmacy students conducted interviews with older adults (65 years or older). The pre-interview questionnaires and post-interview questionnaires, including the Geriatric Attitudes Scale (GAS) and the Facts on Aging Quiz (FAQ), were administered to assess students' perceptions and knowledge of older adults. Paired t-tests were used to evaluate differences in students' perceptions and knowledge before and after the interview. Results: Eighty students completed both the pre-interview and post-interview questionnaires. The majority of the sample was female (65\%) and aged 21-23 years (88.75\%). A little over onehalf $(51.25 \%)$ had prior pharmacy work experience. No significant 


\section{American Journal of Pharmaceutical Education 2018; 82 (5) Article 7158.}

difference was found in students' pre-interview and post-interview attitudes. However, students' knowledge of older adults significantly increased after the interview (FAQ pre-interview score versus FAQ post-interview score $(64.25$ vs 67.00$), p=0.005)$. Increase in geriatric knowledge was significant among females (64.50 vs $67.38, \mathrm{p}=0.006)$ and among students with no prior pharmacy work experience ( $64.20 \mathrm{vs}$ $67.38, \mathrm{p}=0.011)$. Implications: Geriatric education interventions and exposure to older adults outside of regular coursework can improve pharmacy students' understanding of older adults and enable them to serve the geriatric population better.

First-Year Pharmacy Student's Public Health Knowledge Before and After a Required Public Health Course. Stephanie Lukas, St. Louis College of Pharmacy, Peter D. Hurd, St. Louis College of Pharmacy. Objectives: This project was designed to: 1) determine what students already knew about public health; 2) demonstrate an increase in knowledge over the semester; and 3) determine areas where students struggled with content. Method: Participants were first-year pharmacy students taking a required public health course. (Enrollment: Fall $=129$; Spring = 127) In the fall, students completed a 20-question pretest on the first day of class. The same questions were incorporated into the final exam for credit so students were motivated to perform. Pretest and final exam scores were compared. Students also took the pretest in the spring and a comparison was done to the fall. Results: Pretest scores were similar both semesters $($ Fall $=50.6 \%$; Spring $=$ $53.6 \% ; \mathrm{p}=0.19$ ) with minimum scores Fall $=25 \%$; Spring $=0 \%$ and maximum scores Fall $=80 \%$; Spring $=80 \%$. Final scores showed improvement in knowledge (pretest $=50.6 \%$; final $=84.4 \%$; $\mathrm{p}<0.01$ ). Pretest scores were partially determined by knowledge retained on topics previously covered in the Introduction to Healthcare course ( 7 questions; 5 of which had pretest scores $\geq 77 \%$ ), and improvement was noted even on questions with high "correct" answers on the pretest. Areas that students found challenging included research methods and analytic epidemiology. Implications: These results show the need for public health education in the curriculum. Research methods material was included in this P1 course to address a gap in the student knowledge of research, and while scores improved in this area, it is still a challenge for students.

Got My Mind Set on You: Measuring Mindset in Student Pharmacists. Ben D. Aronson, Ohio Northern University, Robert Bechtol, University of Minnesota, Bethany A. Von Hoff, University of Minnesota, Gardner A. Lepp, University of Minnesota, Kristin K. Janke, University of Minnesota. Objectives: Mindset, beliefs that basic qualities like intelligence are either fixed or can grow, is related to resilience and motivation in learners. This potent concept may affect effort students expend in learning, yet exploration in pharmacy education is limited. Thus, the purpose of this study was to establish validity evidence for a measure of mindset in a sample of student pharmacists. Method: First year students in a professional development course completed the 8-item implicit theories of intelligence scale in spring 2016 and 2017. Evidence for structural validity was established through confirmatory factor analysis. A two-factor model was tested where entity beliefs items (i.e. fixed mindset) loaded on one latent factor, and incremental beliefs (i.e. growth mindset) loaded on another. Cronbach's alpha was calculated as evidence of internal consistency. Results: Approximately 35\% of students (115/326) could be categorized as holding fixed beliefs about intelligence. The confirmatory model had marginally acceptable fit. Modification indices were explored where justified. One modification, correlating two similarly worded items, was accepted. The final model had an RMSEA of 0.060 (90\% CI 0.034-0.086), CFI of 0.990, and SRMR of 0.021.
Cronbach's alpha was 0.924 and 0.905 for the entity and incremental beliefs subscales, respectively. Implications: This study establishes evidence supporting use of the implicit theories of intelligence scale in student pharmacists. Programs may use this scale to determine the need for interventions to shift students from fixed to growth mindset, thus creating more resilient and motivated learners.

Graduating Student Perceptions of Professional Competencies and Outcomes in an Innovative $2+2$ PharmD Curriculum. Tara L. Jenkins, Touro University California, Rae R. Matsumoto, Touro University California. Objectives: To identify the perceived value of an innovative $2+2$ curriculum on professional competencies and outcomes by graduating students in the Class of 2017 Method: AACP Graduating Student Survey data from an innovative $2+2$ PharmD curriculum (two years of didactic, two years of pharmacy practice rotations) were compared to national responses for educational outcomes. Chi-square tests were used to compare the proportion of "strongly agree" responses in the four CAPE domains. Results: Students graduating from the $2+2$ curriculum expressed a statistically significantly higher proportion of strongly agree responses to $84 \%$ of the competencies/outcomes assessed in the AACP graduating student survey compared to national data from 127 other pharmacy schools. With regard to Foundational Knowledge (Domain 1), 2+2 graduates indicated a significantly higher proportion of strongly agrees in $2 / 3$ competencies $(\mathrm{P}<0.0005)$. In Essentials for Practice and Care (Domain 2), 2+2 graduates had a significant higher proportion of strongly agrees for $5 / 5$ competencies $(\mathrm{P}<0.05-0.0002)$. In Approach to Practice and Care (Domain 3), $2+2$ graduates rated significantly more strongly agrees in 5/7 competencies compared to national responses $(\mathrm{P}<0.05)$. For Personal and Professional Development (Domain 4), 2+2 graduates had a significantly higher proportion of strongly agree responses in $4 / 4$ competencies $(\mathrm{P}<0.05-0.005)$. Implications: With a higher proportion of students in a $2+2$ program expressing strong agreement that the curriculum prepared them to achieve professional competencies, and a consistency of this design having NAPLEX pass rates above the national average, both student perceptions and outcomes appear stronger with this curricular structure.

Health Professions Students' Attitudes and Perceptions of Interprofessional Biases. Christopher Gillette, Wingate University, Melissa M. Dinkins, Wingate University, Rebecca Bliss, Wingate University, Michele Pfaff, Wingate University, Elizabeth Maupin, Wingate University, Aseel Badran, Wingate University, Michael L. Manolakis, Wingate University, Lisa S. Smith, Wingate University, Melissa Sweetman, Wingate University. Objectives: The purpose of our study was to examine: (a) first-year health sciences student attitudes and perceptions about inter-professional teamwork and biases, and (b) examine differences about attitudes/perceptions of teamwork and biases among the disciplines. Method: We administered an online, anonymous survey to all first-year health sciences students (graduate and undergraduate) on the first day of class, regardless of discipline at a private university. Students completed the Interprofessional Attitudes Scale (IPAS), which was based on competencies from the Interprofessional Education Collaborative (IPEC). We present descriptive statistics of attitudes/perceptions of the interprofessional teamwork and bias subscales and then present differences in attitudes/perceptions among the disciplines using Kruskal-Wallis tests. Higher scores denote more of the measured concept. Results: Two hundred eleven students $(\mathrm{n}=211)$ across all health professions completed the survey and had usable data (92.1\% response rate). Without any previous interprofessional education exposure, the mean 


\section{American Journal of Pharmaceutical Education 2018; 82 (5) Article 7158.}

teamwork score was $55.19(\mathrm{SD}=5.29)$ while the bias subscale mean was $11.74(\mathrm{SD}=3.27)$. Between groups, student Physician Assistants scored the lowest on teamwork, compared to student pharmacists, physical therapists, and nurses (Chi-square $=13.11, \mathrm{df}=3, \mathrm{p}=0.004)$. Further, student nurses and physical therapists reported the highest inter-professional biases (Chi-square $=10.90, \mathrm{df}=3, \mathrm{p}=0.012$ ). Implications: There are significant differences between first year health professions students on the importance and effectiveness of being part of a healthcare team as well as perceptions of bias amongst team members. Future work should investigate methods to reduce bias and increase importance of teamwork in healthcare delivery.

Human Papillomavirus (HPV) and HPV Vaccine Knowledge, Attitudes, and Uptake in Pre-Professional Pharmacy Students. Marie Barnard, The University of Mississippi, Lori Ward, The University of Mississippi, Phillis L. George, The University of Mississippi, Mandy L. Perryman, The University of Mississippi. Objectives: Human papillomavirus (HPV) is a highly prevalent infection associated with increased cancer risks. Uptake and completion of the HPV vaccination has been suboptimal. Community pharmacies provide an opportunity to improve vaccine coverage, however little is known about pharmacy students' perceptions related to HPV and the HPV vaccine. The purpose of this study was to examine HPV and HPV vaccine knowledge, attitudes, and uptake in pre-professional pharmacy students to inform educational efforts to prepare students to be effective partners in initiatives to increase vaccine uptake and completion. Method: Pre-professional pharmacy students $(n=52)$ completed a survey assessing knowledge, attitudes, and perceptions associated with HPV/HPV vaccine, as well as vaccination status and intentions. Descriptive statistics were utilized to examine response patterns. Results: Most students were aware of HPV (91.5\%) and the vaccine $(74.5 \%)$, but only $48.9 \%$ had been vaccinated. Few students felt they were at risk for getting HPV (17.0\%) and most unvaccinated students had never seriously thought about getting vaccinated (71.4\%). Students reported some agreement that the vaccine has significant side effects $(19.2 \%)$ and is likely to cause health problems $(14.9 \%)$, but most agreed it was safe $(61.7 \%)$ and is an effective way to prevent HPV infection (70.2\%). Implications: Pharmacists can serve an important role in improving HPV vaccination coverage. To fulfill this role, the results of this study indicate pharmacy students must be educated about the safety and efficacy of the vaccine, as well as HPV risk factors. Efforts to increase vaccination coverage in preprofessional students are also warranted.

"I'm Surviving. I Don't Know What Else to Say": URM Students' Perceptions of School Climate. Antonio A. Bush, University of North Carolina at Chapel Hill, Danielle J. Allen, University of North Carolina at Chapel Hill. Objectives: The purpose of this research was to understand URM Pharm.D. students' perceptions of the professional school climate, their interactions with pharmacy school faculty and administrators, and the actions they took to navigate the environment at a school of pharmacy (SoP) within a public, research-intensive institution. This work was framed by agency theory, graduate and professional student socialization framework, and anti-deficit perspectives. Method: In 2017, 20 students from the study site participated in 60-90 minute recorded interviews. Audio recordings were transcribed and analyzed using the Sort and Sift, Think and Shift $\subset$ method. Open coding techniques and constant comparative analysis were used to identify emergent themes. To ensure the trustworthiness of the findings, researchers used multiple coders, maintained a dependability audit, employed peer debriefing, and utilized analytic memos were written to promote reflexivity. Results: The participants represented
$40 \%$ ( $n=20$ out of 50 ) of the URM students within the SoP. While students felt supported academically, participants described a "chilly climate" and low sense of belonging due to a lack of URM affinity groups, lack of diversity, and perceived limited social awareness. Further, their academic issues were compounded due to the pressure of being a "representative" of their race. Students navigated this environment by receiving socio-emotional support from URM faculty and code-switching. Implications: This work provides critical insight into the experiences of URM pharmacy students and the actions they take to navigate their environment. The results may inform policies and programmatic activities that may assist SoPs in fostering environments conducive for URM students.

Incorporation of a Health Literacy Exercise Into First Year Pharmacy Students' Introductory Pharmacy Practice Experiences. Aleda M. Chen, Cedarville University, Stephanie M. Cailor, Cedarville University, Thad Franz, Cedarville University, Nicole G. Harper, Cedarville University. Objectives: Given the importance of patient-centered communication, the objective was to assess the impact of using the AHRQ Health Literacy (HL) Assessment Tool during an Introductory Pharmacy Practice Experience (IPPE) on students' confidence in applying HL skills and on their understanding, perceptions, and application of HL compared to students who did not utilize the tool. Method: After a semester of introducing HL concepts, first professional year student pharmacists evaluated their IPPE site for its effectiveness with limited HL patients using the AHRQ tool and shared the results with their preceptor. Students' perceptions, understanding, and application of HL concepts and confidence in the application of these concepts were assessed with a HL questionnaire (29 items, 7-point Likert-type). The questionnaire was administered at the start and end of the fall semester and end of the spring semester. Data were analyzed using a Friedman's test. Results: Students from two cohorts (no exercise, $\mathrm{N}=40$; health literacy exercise, $\mathrm{N}=49$ ) had significant improvements on 22 items $(\mathrm{p}<0.05)$ across all 3 assessments, with improvements seen at the end of fall sustained in the spring semester. When comparing these cohorts, there were no significant differences any items after completing the HL exercise. Implications: Incorporating a HL exercise into IPPEs sustained students' perceptions, understanding, or application of HL concepts, as well as their confidence in the application of these skills but did not result in further improvements. Further research should be conducted to continue to improve students' confidence in applying these skills.

Not So Flawless: The Impact of Item Writing Flaws in Multiple Choice Examinations. Michael D. Wolcott, University of North Carolina at Chapel Hill, Jessica M. Greene, University of North Carolina at Chapel Hill. Objectives: To describe the influence of item writing flaws (IWFs) on item psychometric properties using classical test theory (CTT) and item response theory (IRT). Method: Guidelines for multiple-choice item writing were used to create a checklist to evaluate items for the presence of IWFs. Sixty-nine multiple-choice questions were reviewed by a panel of assessment experts to determine the number and type of IWFs present. Psychometric properties (difficulty and discrimination) were estimated using CTT principles and compared to estimates from a 2-parameter IRT model. Psychometric properties were compared based on the number of IWFs present per item. IRT was also used to estimate the item information generated by each question and estimates of examinee ability. The university institutional review board approved this study. Results: Examinee response data $(\mathrm{N}=144)$ was analyzed using the CTT and IRT approach for all 69 items. Individual item parameters were similar based on the CTT and IRT approaches and examinee ability as predicted by CTT and IRT 


\section{American Journal of Pharmaceutical Education 2018; 82 (5) Article 7158.}

were highly correlated $(r=0.92)$. According to the IRT analysis, test information was maximized at a theta of -2. Estimates of item parameters did not follow a consistent pattern based on increasing or decreasing number of IWFs. Implications: The number of IWFs in test questions can impact the psychometric properties of items; this effect is difficult to characterize and appears to be dependent on the type of IWF instead of overall number. IRT can be a valuable and feasible strategy in evaluating item quality by offering additional information compared to the CTT approach.

P1 Students' Interventions in a Disadvantaged Community in Puerto Rico After Hurricane Maria. Myriam L. Gonzalez-Cordero, University of Puerto Rico. Objectives: To expose P1 students to the community health needs in Puerto Rico after Hurricane Maria. Contribute to the enhancement of community health through the implementation of primary care interventions. Method: After Hurricane Maria, and as part of Health Promotion and Disease Prevention course, visits by $\mathrm{P} 1$ students to a disadvantaged community were made with the coordination of a faculty member and two established community based organizations who serve the elderly and children. Their needs were assessed and prioritized. Among the most pressing needs identified were water purification, hygiene (hand washing), optimal use of food ingredients, safe drug handling and storage, prevention against infectious diseases like dengue, zika, chikungunya, and leptospirosis, and health promotion for diabetes and stress management. Small talks and demonstrations were offered by P1 students. Written materials were also distributed. Results: Between the two community organizations, 55 elders and 45 children participated in the students' interventions. Evaluations showed that the participants as well as the administrators of the organizations were very satisfied with the students' interventions. Implications: Hurricane Maria devastated the Island of Puerto Rico. P1 students implemented a primary care intervention addressing the immediate needs of a disadvantaged community. Through this intervention, they learned about the community needs and the possibilities they have as future pharmacists to contribute to the quality of life of the people they serve when devastating climactic events occur. They also learned how to prepare to proactively respond to future events. This content will be formally incorporated to the doctor of pharmacy curriculum.

"People Just Gravitate Towards Themselves. ..": Understanding the Social Peer Experiences of URM PharmD Students. Danielle J. Allen, University of North Carolina at Chapel Hill, Antonio A. Bush, University of North Carolina at Chapel Hill. Objectives: AACP's 2014 Argus Commission report examined diversity and inclusion from five perspectives, including that of current students, and found little empirical research on the experiences of underrepresented minority (URM) students in doctor of pharmacy programs. This study seeks to fill a void in the research on the social peer experiences of URM Pharm.D. students, and the strategic actions they take to navigate the complex social environment. Method: This qualitative study used 60-90 minute semi-structured interviews with 20 Pharm.D. students at a large, highly-ranked institution in the south. Interview recordings were transcribed and analyzed using constant comparative analysis and thematic coding. To maximize trustworthiness of the study, the research team used multiple coders, peer debriefing, and a dependability audit. Results: Three major findings emerged from the data: 1) The perception of self-segregation among students of various races/ethnicities was prevalent; 2) URM students often saw formal and informal student groups with other URM peers as critical to their survival in the doctor of pharmacy program; 3) and there were unique patterns of URM students building friendships and breaking barriers with their
non-URM peers. Implications: This study adds to the body of knowledge as it relates to URM student experiences in doctor of pharmacy programs. The findings contained here can be used to help faculty and administrators implement the necessary social structures and supports to ensure students are being welcomed into a diverse and inclusive environment.

Perceived Appropriateness of Medication Adherence Incentives. Tessa J. Hastings, Auburn University, Natalie Hohmann, Auburn University, Ruth N. Jeminiwa, Auburn University, Richard A. Hansen, Auburn University, Jingjing Qian, Auburn University, Kimberly B. Garza, Auburn University. Objectives: $50 \%$ of prescriptions dispensed in the US are not taken as prescribed leading to approximately 125,000 deaths and $10 \%$ of hospitalizations/year. Incentives are effective in improving medication adherence; however, information about patient perceptions regarding incentives is lacking. The objectives of this study were to 1)explore patients' perceived appropriateness of incentives and 2)examine associations between perceived appropriateness and patient characteristics. Method: A cross-sectional online survey was administered via Qualtrics Panels to US adults taking at least one prescription medication for a chronic condition. The survey included three domains: 1)perceived appropriateness of adherence incentives(5-point Likert scale); 2)self-reported adherence(Medometer); and 3)demographics. Analyses included descriptive statistics with chi-square and independent $t$-tests comparing characteristics between participants who perceived incentives as being appropriate vs. inappropriate, and logistic regression to determine predictors of perceived appropriateness. Results: 1,009 individuals completed the survey. The majority were female(53\%), white(74\%), and non-Hispanic (42\%). 740 participants ( $73 \%$ ) perceived medication adherence incentives as being appropriate, or acceptable as a reward given for taking medications at the right time each day, while 95 (9\%) perceived incentives as being inappropriate. Remaining participants were neutral. Logistic regression revealed Hispanic ethnicity $(\mathrm{OR}=1.66 ; 95 \% \mathrm{CI}$, $1.03-2.66)$, annual income $<\$ 50,000(\mathrm{OR}=2.08 ; 95 \% \mathrm{CI}, 1.07-4.04)$, no college degree $(\mathrm{OR}=1.93 ; 95 \% \mathrm{CI}, 1.21-3.09)$, and lower level of adherence $(\mathrm{OR}=0.99 ; 95 \% \mathrm{CI}, 0.98-0.99)$, as significant predictors of perceiving incentives as appropriate vs. inappropriate. Implications: Perceived appropriateness for medication adherence incentives may be most effective among certain groups of patients, such as those with lower annual income. These characteristics should be taken into account when structuring incentives.

Pharmacy Students' Perceived Stress and Its Impact on Academic Performance in a Flipped Classroom Environment. Omar F. Attarabeen, Marshall University, Isha Patel, Marshall University, Michael J. Rudolph, Marshall University, Glenn Anderson, The University of Louisiana at Monroe, Kimberly A. Broedel-Zaugg, Marshall University, Cynthia B. Jones, Marshall University, Hasan Koc, Marshall University, Caroline A. Gaither, University of Minnesota, M. Omar F. Khan, Marshall University. Objectives: Investigating the association between pharmacy students' perceived stress level and their grade point average (GPA) is critical to ensure adequate learning outcomes. Thus, this study aimed to investigate this association in a flipped classroom environment. Method: PharmD students currently enrolled in a public university in the US were invited to participate in a cross-sectional survey study in February 2018 after IRB approval. GPA, which ranges from 0 to 4 , was the outcome variable. Self-efficacy, Pharmacy College Admission Test (PCAT) scores, undergraduate degree, undergraduate major, year in the PharmD program (P1-P4), age, gender, race/ethnicity, and marital status were the independent variables. Univariate statistics and multivariable linear regression 


\section{American Journal of Pharmaceutical Education 2018; 82 (5) Article 7158.}

analyses were conducted. Data analysis was conducted using SPSS version 24. A p-value of less than 0.05 was considered significant. Results: One hundred ninety-two students completed the survey, reflecting a response rate of $63.2 \%$. On a 6-point Likert scale $(1=$ never or not applicable $-6=$ multiple times a day), P1, P2, P3, and P4 students exhibited stress levels of 2.8, 2.8, 3.2, and 2.7, respectively, with a mean value of 2.9. GPA was inversely associated with students' perceived stress using univariate linear regression. However, this relation lost statistical significance after controlling for self-efficacy. In multivariable analyses, GPA was significantly associated with two independent variables: PCAT scores and year in the PharmD program. Implications: Providing stress-coping seminars may reduce the negative impact of students' perceived stress on their academic performance. Effect of stress-coping activities on students GPA will be studied in the future.

PharmD/MBA Dual Degree Student APPE Project to Build a Pharmacist Value-Impact Calculator. Marie A. Smith, University of Connecticut, Daniel Brush. Objectives: 1. Build/prototype a pharmacist value-impact calculator for ambulatory care services that synthesizes clinical practice, critical-thinking, and problem-solving skills. 2. Test the value calculator with executive-level leaders at 4 health care delivery organizations. Method: We developed a pharmacist valueimpact calculator for a new population heath and practice-level service. Elements of the calculator included: revenue and cost-saving opportunities, pharmacist expenses, quality metrics, and return-oninvestment. The calculator default data was from publicly-available sources (e.g., CMS Medicare payments, 2016 ACO performance data, meta-analysis of publications, state health registries, publications). The calculator prototype was validated with executives in four caredelivery provider organizations (i.e., ACOs, physician multispecialty groups). Results: The validation process included 8 executives (CEO, COO, CMO, VP-Strategy, CMIO, VP - Population Health, and Director - Clinical Pharmacy Services) who verified that the assumptions and default data used for the prototype were rational. Some provider executives had site-specific data to validate the calculator. Executives stated the calculator could be used to forecast new/expanded clinical pharmacist services or track impact of existing services. Pharmacist services had an average ROI of 2-8:1. The student's feedback was "beside the clinical impact of pharmacist services that is emphasized in pharmacy school, I realize that we need to advocate the corresponding financial impact". Implications: - Use real-world problems from care providers/organizations as APPE research projects. - Valueimpact calculator is a useful tool to engage provider executives in discussions on new clinical pharmacist services. - The calculator elements can be adapted to justify other clinical pharmacist service opportunities.

Pilot Study to Assess Academic Rigor of Cultural Sensitivity Training of Fourth Year Pharmacy Students. Nicole R. Winston, Marshall University. Objectives: Our objective of this study was to evaluate cultural sensitivity using the validated Clinical Cultural Competency Questionnaire (CCCQ) of fourth year pharmacy (P4) students to serve as a benchmark for didactic and experiential curriculum for quality improvement at a new school of pharmacy. Method: An online version of the CCCQ was administered to P4 students graduating in the spring of 2017. The 45-question survey assessed knowledge, skills, encounters/situations and attitudes pertaining to cultural diversity training using a 5-point Likert scale ( $1=$ not at all; $5=$ very). Descriptive statistics and bivariate associations were used to analyze results. Results: We received 35 completed surveys (54\% response rate). The majority of responses were from students 25 years or younger $(43 \%)$, female $(66 \%)$ and Caucasian $(83 \%)$, which were not significantly different from the P4 class demographics. On average, students rated attitudes toward improving various health disparities the highest $(4.01 \pm 0.5)$, followed by encounters $(3.04 \pm 0.83)$, skills (3.02 \pm $0.86)$ and knowledge $(2.96 \pm 0.61)$. Students indicated the least familiarity with the standards for Culturally and Linguistically Appropriate Services (CLAS) for healthcare $(1.91 \pm 0.85)$. Visiting or living outside the United States or bilingualism did not correlate with responses in all categories. Implications: Our results demonstrated similarities with previously published survey data using the CCCQ. However, deficiencies in the area of knowledge related to cultural diversity have been addressed by adding laboratory activities in our Integrated Laboratory series.

Predictors of High Performance on the NAPLEX at a Historically Black College of Pharmacy. LaMarcus T. Wingate, Howard University, Mary Maneno, Howard University, Salome B. Weaver, Howard University, Muhammad J. Habib, Howard University, Youness R. Karodeh, Howard University, Earl Ettienne, Howard University, Michael A. Marcus, Howard University. Objectives: To determine which preadmission and pharmacy school factors predict success on the NAPLEX at a historically black college of pharmacy. Method: A retrospective analysis was conducted using data from four graduating classes that took the NAPLEX from 2011 to 2015. Descriptive statistics were conducted in addition to correlational and chi-square analysis to determine which variables had a significant effect on NAPLEX scores and first time passage rate. Results: There were 195 students with available data on scores. The mean NAPLEX scores was 99.9 and 93.8\% of students achieved passing scores on the first attempt. Approximately two thirds $(65.6 \%)$ of students were black, and approximately half $(51.3 \%)$ were male. Cumulative PCAT score, probation during the 1 st year of pharmacy school, and cumulative GPA during graduation were significantly correlated with the NAPLEX score. Students put on probation during their first year were significantly more likely to fail when compared to students not put on probation during this time $(p=0.002)$. Students graduating with a GPA in the lower quartile (less than 2.94) were more likely to fail when compared to graduating students with a GPA in the upper two quartiles $(p=0.001)$. Implications: Students who were not placed on probation and had a graduating GPA in the higher quartile scored higher on the NAPLEX at an HBCU.

Preparing Practice-Ready Graduates: An Integrated Approach to Health Systems Sciences. Kimberly B. Garza, Auburn University, Julaine Fowlin, Auburn University, Channing R. Ford, Auburn University, Bradley Wright, Auburn University, Nicole Slater, Auburn University. Objectives: To describe outcomes of an integrated learning experience (ILE) for first-year student pharmacists focused on the US healthcare system, developed using backward design and the theory of situated learning. Method: Performance-based competencies were created from a shared understanding of what the "practice-ready graduate" should know, understand, and be able to do. Using the theory of situated learning, classroom activities including student reflection, group case analyses, and role-play, were used to "situate" the learning into a pharmacy practice context, with rubrics developed to guide assessment. Both quantitative and qualitative assessment measures included objective structured clinical examination (OSCE) scores, final exam scores, faculty reports via online survey of evidence of integration of material across disciplines as well as reports comparing previous non-integrated approach with the integrated approach, and student self-reported understandings as an open-ended exam item. Results: Aggregated data from authentic assessment indicated that 


\section{American Journal of Pharmaceutical Education 2018; 82 (5) Article 7158.}

students $(n=152)$ performed well on exams $($ mean $=85.9 \%$; $\mathrm{SD}=6.5 \%)$ and OSCEs $($ mean $=82.9 \% ; \mathrm{SD}=7.2 \%)$. Faculty $(\mathrm{n}=6)$ reported that student presentations and discussions reflected a deeper understanding of the US Healthcare system and implications for the profession of pharmacy than in previous years at the P1 level, and that, even when not asked to do so, students included relevant knowledge from their clinical and basic science courses. Student self-reported understandings were also in alignment with faculty-created overarching understandings used in development of the course. Implications: We propose a systematic process to create more meaningful integrated experiences to prepare the practice ready graduate to apply knowledge of the social/behavioral/health systems sciences.

Serious Attempts to Quit Smoking in a Sample of Adult Muslim Smokers in the US. Omar F. Attarabeen, Marshall University, Fadi M. Alkhateeb, The University of Texas at Tyler. Objectives: Guided by the Social Cognitive Theory, we investigated the associations between the number of serious cigarette smoking cessation attempts and cognitive as well as environmental factors in adult Muslim smokers in the United States (US). Method: This cross-sectional study was based on a convenience sample of adult ( $\geq 18$ years) US Muslim smokers. Data were collected using an on-line survey from November 2016 to March 2017. We conducted unadjusted Poisson regression followed by adjusted multivariable Poisson regression analyses. Results: One hundred thirty-two eligible smokers completed the questionnaire. Sixty-two smokers $(47.0 \%)$ seriously attempted to quit cigarette smoking at least once over the past 12 months, half of which reported attempting to quit cigarette smoking without any assistance. Smokers reported more serious cigarette smoking cessation attempts if they 1) had more knowledge about the consequences of cigarette smoking cessation, 2) had more positive attitude regarding quitting, and 3) reported greater religiosity. Additionally, smokers reported fewer serious cigarette smoking cessation attempts if they 1) were employed, 2) affiliated with Sunnah sect, 3) reported better self-assessed health, 4) reported higher perceived value for quitting, and 5) indicated that using tobacco was not allowed inside the home. Implications: Suboptimal utilization of behavioral support and prescription medication while attempting to quit cigarette smoking may indicate inadequate utilization of provider professional assistance in US Muslim smokers. Knowledge of the consequences, more positive attitudes, and greater religiosity can be influential constructs in future interventions that aim to encourage serious cigarette smoking cessation attempts in US Muslim smokers.

Social Marketing in Human Immunodeficiency Virus PreExposure Prophylaxis Literature. Heba A.T. Aref, Auburn University, Kimberly B. Garza, Auburn University, Chiahung Chou, Auburn University, Brent Fox, Auburn University, E. Kelly Hester, Auburn University. Objectives: We evaluated the utilization of "social marketing" as a means for increasing awareness and knowledge of human immunodeficiency virus pre-exposure prophylaxis (PrEP) and the essential features of PrEP social marketing messages in the literature. Method: PubMed, EBSCO and Google Scholar were searched, from 1961 to January 2018, for studies with variations of following keywords: "social marketing" and "pre-exposure prophylaxis." Studies were excluded if not focusing on PrEP, not in English language, or not a scientific research article. A thematic analysis was conducted for the social marketing content. Results: Of the 23 studies identified, $65.2 \%$ were nonsystematic reviews $(n=15), 26 \%$ were qualitative studies $(n=6), 4.3 \%$ were quantitative studies $(n=1)$, and $4.3 \%$ were systematic reviews $(n=1)$. The targeted populations for the social marketing campaigns were: HIV high risk individuals $(65.2 \%)$, policy makers $(17.3 \%)$, youth $(13.2 \%)$, and healthcare providers
(4.3\%). Essential features identified for social marketing PrEP messages included being brief, straightforward, comprehensible, referring to PrEP as a pill, describing efficacy results, more focused on the individuallevel, and omitting sexually transmitted diseases or epidemiological terms. Seventeen percent of the studies identified stigma as an essential factor to address in social marketing campaigns or messages. Implications: Although social marketing has potential to influence the awareness and motivation for increasing PrEP use, robust assessments of the effectiveness of PrEP-social marketing campaigns are lacking in the literature. Studies are needed to evaluate the impact, provocation, and barriers of PrEP social marketing messages.

Student Attitudes Towards a Licensure Preparedness Program: Voluntary vs. Required. C. Lea Bonner, Mercer University, Jill M. Augustine, Mercer University, Candace W. Barnett, Mercer University, Susan W. Miller, Mercer University. Objectives: To assess students' attitudes about a licensure preparedness program (LPP) after required co-curricular participation. Method: A 28-item questionnaire surveyed 2 groups of student pharmacists: Class of 2017 volunteer pilot group; and Class of 2018 required group. The LPP consisted of a schedule of assignments and activities using a commercial product containing videos, a text book, and assessments. Students were surveyed regarding their attitudes about the LPP using a 5-point Likert scale (Strongly agree to Strongly disagree) at the program's midpoint. Comparisons between groups were analyzed for each attitudinal question using Wilcoxon Rank Sum test. The project was approved by the University's institutional review board. Results: Forty-nine pilot group students and 134 required group students completed the questionnaire (response rate was $99 \%$ and 94\%, respectively). Overall, students had a high opinion of the LPP. Students felt the program was not "too fast pace" or "stressful". More pilot group students strongly agreed the LPP was "worth their time" (48.9\%) compared to the required group $(19.6 \%, \mathrm{p}=0.003)$. More pilot group students agreed the LPP helped "contribute to my ability to manage my time wisely" $(70.2 \%)$ compared to the required group $(58.0 \%, \mathrm{p}=0.022)$. A similar percentage of students in both groups agreed the LPP was difficult to manage alongside final-year rotations (65.9\% and $52.4 \%$, respectively, $p=0.05$ ). Implications: Overall, both groups of students had positive attitudes about the LPP regardless of enrollment method. Program administrators will continue monitoring student attitudes as well as licensure examination pass rates.

Student Pharmacists' Experiences of Teamwork in a Professional Pharmacy Program. David Rhys Axon, The University of Arizona, Patrick J. Campbell, The University of Arizona, Terri L. Warholak, The University of Arizona. Objectives: To assess second-year (P2) and fourth-year (P4) student pharmacists' teamwork experiences in a quality improvement (QI) or research project. Method: A retrospective pre-post paper-based survey was conducted at a college of pharmacy with P2 and P4 students. Students reported their level of agreement with 17 statements to assess teamwork perceptions after project planning and after project completion. They also responded to demographic questions (age, gender, race, and previous teamwork experiences). A Kuder-Richardson 20 (KR20) was calculated. Differences between demographic variables for $\mathrm{P} 2$ and $\mathrm{P} 4 \mathrm{~s}$ were assessed using a t-test, chi-square test, or Fisher's test while item differences between time points were assessed using McNemar's test. An alpha level of 0.05 was used and a Bonferroni correction was applied when appropriate. Institutional Review Board approval was granted. Results: Sixty P2 and 29 P4 students (mean age 25.8 ( \pm 3.5 ) years; $64 \%$ female) responded. The majority indicated agreement with good teamwork qualities and disagreement with poor teamwork qualities for most items. There were no significant differences 


\section{American Journal of Pharmaceutical Education 2018; 82 (5) Article 7158.}

between responses after project planning and after project completion. Sixty-six percent of respondents indicated they would like to do collaborative work again while $24 \%$ indicated imbalances in member contributions caused team conflict. The survey demonstrated satisfactory internal consistency (KR20 $=0.79$ pre and 0.85 post). Implications: Most respondents reported positive experiences working in student pharmacist teams, but further work is needed to explore and prevent member contribution imbalances and to encourage students to work on future collaborative projects.

Systematic Review of Studies Using Social Cognitive Theories to Explain and Predict Pharmacists' Intentions and Behaviors. Paul Gavaza, Loma Linda University, Mayur D. Mayur, Loma Linda University. Objectives: Little is known about the extent of utilization of social cognitive theories and their effectiveness in explaining and predicting pharmacists' intentions and behaviors. This study was conducted to systematically review the literature of utilization of social cognitive theories in predicting pharmacists' intentions and behaviors. Method: A literature search was performed from August to December 2016 to discover pharmacy-related articles published in English in PUBMED, EMBASE, CIHNAL, PsycINFO, MEDLINE, and Cochrane Library databases. A data collection form developed for the study was utilized to extract the information from the studies. For all the articles, two reviewers were able to agree through discussion on a coded response for each item. Results: Fifty-four articles met our inclusion criteria. Most studies investigated clinical practice behaviors $(n=30,55.6 \%)$ and included mostly retail pharmacists $(n=40,74.1 \%)$. Most studies utilized the Theory of Reasoned Action $(\mathrm{N}=5)$ and its extension the Theory of Planned Behavior $(\mathrm{N}=25)$ in explaining and predicting pharmacists' intentions and behaviors. Thirty-two $(59.3 \%)$ studies reported that the use of model or theory was effective in predicting pharmacists' intentions and behaviors. Counseling $(n=30)$ and acceptance of technology $(n=11)$ were the most investigated behaviors. Implications: This study was the first known systematic review of studies explaining and predicting only pharmacists' intentions and behaviors. Attitude was the most common predictor of intention for pharmacists, and the theory of planned behavior as the most utilized theory. More research is needed to explain and predict pharmacist's intentions and behaviors.

The Assessment of Interprofessional Education Activities Across a 3-Year Concentrated Curriculum. Lana Sherr, University of Maryland Eastern Shore, Frederick R. Tejada, University of Maryland Eastern Shore, Hoai-An Truong, University of Maryland Eastern Shore. Objectives: Describe the assessment of various Interprofessional Education (IPE) activities that were developed and implemented in the pharmacy curriculum in collaboration with seven health care programs. Method: To ensure all students receive IPE experience, the University of Maryland Eastern Shore School of Pharmacy and Health Professions facilitated a variety of interprofessional educational activities through different didactic courses. These activities include: IPE Scope of Practice Seminar and Point of Dispensing Drill in the first professional year, Geriatric Assessment Interdisciplinary Team Workshop and Annual Health and Wellness Festival in second year, and Interprofessional Collaborative Competencies Attainment Seminar in third year. Currently, each IPE activity is evaluated separately using an activity-specific survey instrument. Most recently, the Interprofessional Curriculum Committee developed its IPE goals and mapped them to the four IPEC Core Domains. Selected surveys were then updated to reflect the ICC goals and IPEC Core Domains. Results: To consolidate the results, the ICC identified the "International Perception, Knowledge, and Attitude Scale (IPKAS)" as a comprehensive survey and mapped student responses from the different IPE surveys to IPKAS. Based on that, only $70 \%$ of current surveys captured "Roles and Responsibilities" and "Interprofessional Communication", 40\% included "Team and Teamwork", and $0 \%$ reflected "Values and Ethics". The next step is to develop a global assessment approach that could potentially be used to assess all the IPE activities. Implications: The ICC will adapt IPKAS as a global assessment and quality improvement approach for all IPE activities.

The Determinants for Community Pharmacists' Positive Image and the Needs for Community Pharmacists' Services in Taiwan. Kuang-hsun Tseng, Ever Health Pharmacy, Yu Ko, Taipei Medical University, Elizabeth H. Chang, Taipei Medical University. Objectives: To examine the specific factors that the general public believes have an effect on the image of community pharmacists and also to identify community pharmacists' services that truly fulfill people's needs. Method: This study was a cross-sectional survey that used a newlydesigned questionnaire. A total of five community pharmacies in Taipei participated in this study, and a convenient sample of 401 pharmacy customers completed the questionnaire. Results: Among the survey respondents, $98.3 \%$ reported correct dispensing as the most important factor that helped establish a positive image for a community pharmacist, followed by cordial attitude $(95.5 \%)$, wearing clothes showing pharmacist's license with a photo $(94.6 \%)$, wearing a clean uniform (93.3\%), and providing enough communication time $(91.8 \%)$. Among the services provided by community pharmacies, most people think that the most needed service is a consultation explaining drugdrug interactions $(93.8 \%)$, followed by a consultation explaining a doctor's prescription ( $92.3 \%$ ), the dispensing of a physician's prescription $(91.5 \%)$, and a check for drug-food interactions (91.2\%). Furthermore, $312(77.8 \%)$ respondents indicated that there is a need for comprehensive pharmaceutical services, and 280 of them $(89.7 \%)$ wanted to receive such service at a community pharmacy near home. Implications: Nearly $80 \%$ of the respondents reported that they needed comprehensive pharmaceutical services and were willing to pay for it. The results of this study could aid future planning of relevant pharmaceutical care programs by the government in Taiwan.

Tobacco Use Among a Sample of Adult Muslims in the United States. Omar F. Attarabeen, Marshall University, Fadi M. Alkhateeb, The University of Texas at Tyler. Objectives: To investigate the Social Cognitive Theory (SCT) factors that are associated with tobacco use among a sample of adult Muslims in the US since Muslims in the US exhibit elevated rates of tobacco use as compared with the general US population. Method: Data were collected (November 2016 - March 2017) using a cross-sectional, on-line survey from a convenience sample of adult ( $\geq 18$ years) US Muslims. Participants with a lung cancer history were excluded. Associations between SCT factors and tobacco use were investigated with bivariate analyses and multinomial logistic regression models Results: Eligible participants $(n=271)$ from 30 states completed the survey; $52.8 \%$ reported current tobacco use. A higher rate of current tobacco use was reported by men $(62.8 \%)$ as compared to women $(41.3 \%), x 2(1, N=271)=12.49, \mathrm{p}<0.001$. In terms of cognitive factors, individuals who 1) expect more personal consequences for tobacco use on health, and 2) have more confidence regarding ability to abstain from tobacco use, were less likely to report current tobacco use. In terms of environmental factors, individuals whose family members do not use tobacco were less likely to report current tobacco use. An interaction between sex and attitudes indicated that women with negative views about tobacco were less likely to report current use compared to men with negative views about tobacco Implications: Several cognitive and environmental factors 


\section{American Journal of Pharmaceutical Education 2018; 82 (5) Article 7158.}

can influence tobacco use. The study findings suggest that familyoriented interventions to prevent tobacco use can potentially be effective in reducing tobacco use rates in the adult US Muslim population.

Use of Vitalograph Aerosol Inhalation Monitor (AIM) in a Pharmacy Practice Laboratory. Monica Hwang, St. John's University, Carmela Avena-Woods, St. John's University, William Maidhof, St. John's University. Objectives: To explore the use of Vitalograph Aerosol Inhalation Monitor (AIM) in training pharmacy students on metered-dose inhaler(MDI) usage. Method: Pharmacy students enrolled in a pharmacy practice lab were exposed to the AIM device during the patient assessment component of the laboratory. AIM is a device that assists individuals to learn proper inhaler techniques. It consists of a digital monitor with tubing that is attached to a mouthpiece fitted with a canister that contains a non-medicated propellant. Utilizing multi-colored indicator lights, the monitor identifies a specific step in the inhalation process where further inhaler training may be necessary. At the end of the laboratory, students were asked to complete a survey that assessed perception of AIM as an effective tool for learning (5-items), self-efficacy in counseling on the use of MDI (7-items), and demographic characteristics (11-items). Results: Total of 242 surveys were usable for data analysis. Approximately $67 \%$ of the students were Asian and majority of the students were not currently using a MDI (93\%) nor have used it in the past (80\%). Approximately $65 \%$ of the students strongly agreed and $30 \%$ agreed that AIM is an effective tool for learning and majority of the students $(90 \%)$ indicated high self-efficacy in counseling patients on a MDI at the end of the laboratory. Implications: AIM seems to be a great device to use in teaching students about proper inhaler usage techniques. Further study is needed to examine how use of AIM in a pharmacy laboratory course can truly impact students' learning.

Validity Evidence Using Generalizability Theory for an Objective Structured Clinical Examination (OSCE): A Multiple Occasions Rationale. Michael J. Peeters, The University of Toledo, M. Kenneth Cor, University of Alberta, Bryan M. Bishop, The University of Toledo, Sarah E. Petite, The University of Toledo, Michelle N. Schroeder, The University of Toledo. Objectives: Pharmacy colleges/schools should be generating validation evidence for assessments used to make important decisions; this includes OSCEs. Using Generalizability Theory (Gtheory), pharmacy educators can produce reliability evidence as a part of the validation process - though it has been rarely reported in pharmacy education. This investigation illustrates Gtheory to evaluate how reliability changes depending on number of stations over multiple weeks/occasions. Method: To evaluate practice-readiness in the semester before final-year rotations, third-year PharmD students took an OSCE. This OSCE included 14 stations over three weeks. Each week had four or five stations; one or two stations scored by faculty-raters while three stations required students' written responses. For Gtheory, we used mGENOVA. Results: Ninety-seven students completed this OSCE; stations were scored independently. Student's scores were modeled as stations nested within three weekly occasions $\left(\mathrm{p}^{\bullet} \mathrm{x} \mathrm{w}^{\circ}\right)$. The estimated g-coefficient (reliability) for the total score based on 3 weeks and approximately 5 stations per week was estimated at 0.74 . Further analysis revealed how reliability changed depending on combinations of stations per week. For g-coefficient $>0.80$, seven stations per week were needed. Reliability was higher for stations in week\#3 than week\#1. Week\#2 showed lowest reliability; improving stations especially week\#2 should also help improve reliability (and use fewer stations). Implications: In test validation, evidence of reliability is vital for generalization; Gtheory can provide this. Our results illustrate that increasing the number of stations each week and/or the number of weeks (occasions) can both improve reliability. Regardless, students accounted for only some OSCE score variation.

What Influences College Students to Seek Prescription Drug Information From the Internet? Ankit Shah, St. John's University, Monica Hwang, St. John's University. Objectives: To examine the impact of attitude, subjective norm, and perceived behavioral control on college students' intention to use the Internet in obtaining prescription drug information. Method: Cross-sectional survey design was used to collect data at a private university. Survey items were created based on the theory of planned behavior (TPB); items assessed participants' attitude (2items), subjective norm (3-items), perceived behavioral control (4-items), intention (3-items) and past behavior (2-items) of obtaining prescription drug information from the Internet. All of the items were measured using a 7-point summated scale. Multiple linear regression was used to examine the relationship between the TPB constructs and intention of obtaining prescription drug information from the Internet. Results: Total of 196 surveys were considered usable for data analysis. Attitude, subjective norms, and perceived behavioral control were statistically significant $(\mathrm{p}<0.05)$ and had positive effect on intention. Attitude was found to be the strongest predictor of intention. Intention had a strong positive correlation with past-behavior of obtaining prescription drug information from the Internet $(r=0.758, p=0.000)$. Implications: Attitude, subjective norms and perceived behavioral control are important factors in predicting college students' intention to use the Internet in obtaining prescription drug information. Companies should utilize the Internet to present drug information in a user-friendly way to younger generations.

"You're Always Trying to Prove Yourself": Exploring URM PharmD Students' Group Work Experiences. Danielle J. Allen, University of North Carolina at Chapel Hill, Antonio A. Bush, University of North Carolina at Chapel Hill. Objectives: Group work is becoming increasingly common as pharmacy schools move to team-based learning. This qualitative study explored how URM Pharm.D. students make sense of their academic experiences, and how they exercise various forms of agency in order to persevere through challenging dynamics. The study draws from an anti-deficit approach, a conceptualization of the graduate and professional student socialization process, and notions of agency. Method: Purposeful sampling was used to recruit 20 URM Pharm.D. students from a highly-ranked school of pharmacy. Data collection included a demographic survey and a semi-structured interview whereby participants described their experiences as pharmacy students. Following interview transcription, constant comparative analysis was used to identify themes emerging from the data. In order to promote trustworthiness of the data, the research team used multiple coders, peer debriefing, and a dependability audit. Results: Challenging group work experiences emerged as a prevalent theme across a number of participants, with students commonly feeling dismissed during group work and under pressure to prove their intellectual competence. In order to overcome these dynamics, students assumed several agentic perspectives and behaviors such as "working twice as hard and having to be twice as good" and navigating the perceived consequences speaking up or remaining silent about challenging group work experiences. Implications: The findings of this study fill a critical void in the literature on the academic experiences of URM pharmacy students. Faculty and administrators can use these findings to anticipate challenges URM students may face and prepare all students to work more cohesively in groups.

\section{Theoretical Models}

Using the Theory of Planned Behavior to Predict Pharmacy Students' Intentions to Utilize Health Outcomes. Minghui Sam Li, The University of Tennessee, Z. Kevin Lu, University of South Carolina, 


\section{American Journal of Pharmaceutical Education 2018; 82 (5) Article 7158.}

Richard M. Schulz, University of South Carolina, Junling Wang, The University of Tennessee. Objectives: Health outcomes is the general name given to a body of knowledge and skill set that examine the clinical, economic and humanistic consequences of healthcare decisionmaking. The objectives of this study were to examine pharmacy students' opinions on health outcomes and to use the theory of planned behavior (TPB) to predict pharmacy students' intentions to utilize health outcomes in future jobs. Method: This study surveyed a total of 376 second-year pharmacy students in 2015 and 2016. Four constructs (attitude towards the behavior, subjective norm, perceived behavioral control, and behavioral intention) were measured in the TPB. Questions in the TPB were measured using seven-point Likert scales and standardized on a 0 to 1 scale. A linear regression model was used to predict pharmacy students' intentions to utilize health outcomes in future jobs. Results: A total 229 pharmacy students responded to the survey (response rate: $60.90 \%$ ). They had positive attitude towards the behavior (mean: 0.77 , SD: 0.16), high level of subjective norm (mean: 0.75, SD: 0.18 ), high level of perceived behavioral control (mean: 0.74 , SD: 0.15 ), and high level of behavioral intention (mean: 0.74, SD: 0.21). Significant predictors of utilizing health outcomes in future jobs were attitude towards the behavior $(0.21 ; 95 \% \mathrm{CI}: 0.03,0.40)$, subjective norm $(0.38 ; 95 \% \mathrm{CI}: 0.23,0.54)$, and perceived behavioral control $(0.45 ; 95 \%$ CI: $0.27,0.63)$. Implications: All three constructs in the theory of planned behavior (attitude towards the behavior, subjective norm, and perceived behavioral control) were significantly associated with the intention to utilize health outcomes in future jobs.

\section{6-2017 NEW INVESTIGATOR AWARD WINNERS}

Biological Sciences

Histamine Release in Traumatic Brain Injury: Neurotoxic or Neuroprotective. Matt Baier, The University of Oklahoma, Megan R. Lerner, The University of Oklahoma, Hibah O. Awwad, The University of Oklahoma \& Oklahoma Center for Neuroscience. Objectives: Histamine (HA); a neurotransmitter and inflammatory mediator, modulates memory, pain and sleep by activating its histamine $\mathrm{H}_{3}$ receptors $\left(\mathrm{H}_{3} \mathrm{R}\right)$ in the brain. Increases in $\mathrm{HA}$ and $\mathrm{H}_{3} \mathrm{R}$ levels following a traumatic brain injury (TBI) have been reported for both their neurotoxic and neuroprotective properties. HA's role in TBI pathology and neurobehavioral deficits is still not clear. Our aim was to determine the relationship between $\mathrm{HA}, \mathrm{H}_{3} \mathrm{R}$ and acute TBI-induced deficits in a controlled cortical impact model of mild TBI in Sprague-Dawley male rats. Method: Deficits were measured on day 1 post-injury in sham and TBI rats treated with vehicle (normal saline), HA (40 $\mu \mathrm{g})$ or Thioperamide (10 $\mu \mathrm{g}$; a $\mathrm{H}_{3} \mathrm{R}$ inverse agonist) i.c.v. 5 min post-TBI/ sham surgery. The modified neurological severity score (mNSS), righting reflex (RR) time (an indicator of unconsciousness), post-TBI seizure parameters and the anxiety index via elevated plus maze were assessed. Results: HA and Thioperamide treatment post-TBI had no effect on overall TBI severity. HA treatment increased RR time for both sham and TBI groups compared to vehicle treated groups, whereas Thioperamide treatment improved RR time. Furthermore, thioperamide treatment reduced the anxiety index and time of the last seizure experienced in TBI rats compared to vehicle treated rats, whereas HA treatment increased both parameters. Implications: These initial results on the acute effects of TBI suggest that elevated HA levels in the CSF may aggravate TBI -induced anxiety, unconsciousness and seizures following a mild TBI. Alternatively, thioperamide treatment may improve these acute consequences post-TBI.
Neuronal Injury and Parkinson's Disease: Role of Purinergic Signaling. Allison Cook, Ferris State University, Jessica Goldsworthy, Ferris State University, Trevor Karbowski, Ferris State University, Megan Rodewald, Ferris State University, Colton Zwart, Ferris State University, Jennifer Lamberts, Ferris State University. Objectives: The neuropathologic hallmark of Parkinson's disease (PD) is intra-neuronal accumulation of $\alpha$-synuclein ( $\alpha$-syn), a small, soluble protein that is mainly expressed in the brain. A recent study showed that $\alpha$-syn accumulation is increased in cells exposed to ATP, a condition known to arise during traumatic brain injury. These results suggest a potential link between neuronal injury, purinergic signaling, and the pathogenesis of PD, yet there remains limited information about this phenomenon. The primary goal of this project was to determine which purinergic receptors are involved in ATP-induced accumulation of $\alpha$-syn in mammalian cells. Method: N2a and SH-SY5Y mouse neuroblastoma cells were treated with purinergic agonists and $\alpha$-syn expression was evaluated using cellbased ELISA. Secretion of $\alpha$-syn was measured in conditioned media using sandwich ELISA. Results: Treatment with ATP, ADP, AMP or adenosine for $48 \mathrm{~h}$ increased $\alpha$-syn expression in differentiated N2a cells. Only ADP increased $\alpha$-syn expression in undifferentiated N2a and SH-SY5Y cells. Purinergic agonists had no effect on $\alpha$-syn expression following $24 \mathrm{~h}$ treatment. In preliminary experiments, $48 \mathrm{~h}$ treatment with ATP, but not ATP $\gamma$ S, stimulated $\alpha$-syn secretion from differentiated N2a cells. Implications: These studies suggest that purinergic signaling is altered following neuronal differentiation. Moreover, the data reveal that long-term exposure to purinergic agonists increases $\alpha$-syn expression. This work provides new insight into the role of purinergic signaling in PD pathogenesis.

Pharmacologic Modulation of Ocular Mucins for the Treatment of Dry Eye. Ajay Sharma, Chapman University, Kiumars Shamloo, Chapman University, Ashley Barbarino, Chapman University. Objectives: Mucins are high molecular weight glycosylated proteins that are crucial for ocular surface hydration. Severe dry eye is a frequent manifestation of Graft versus host disease (GVHD), a serious complication of bone marrow transplantation. The aim of this study was to test the hypothesis that GVHD dry eye is caused by proinflammatory cytokine-mediated decrease in ocular surface mucins and rebamipide, a mucin secretagogue, can be a potential treatment to alleviate GVHD dry eye. Method: A mouse model of allogeneic bone marrow transplantation was used to induce GVHD dry eye. The mice were treated with topical rebamipide eye drops. Tear secretion and fluorescein staining of corneal/conjunctival epithelial defects was used for quantification of dry eye. Corneas were harvested for mucins mRNA and protein quantification. Human corneal and conjunctival cells were exposed to inflammatory cytokines IL6 and IFN $\gamma$ to test their effect on mucin gene expression. Results: Our data shows that allogeneic bone marrow transplantation caused a severe GVHD dry eye in mice (50-80\% reduction in tear secretion and an increase in ocular surface epithelial defects). This was accompanied by a substantial decrease in mucins gene and protein expression. Rebamipide eye drops significantly alleviated the symptoms of dry eye. Exposure of human corneal and conjunctival cells to IL6 caused a dose-dependent decrease in mucin $16 \& 19$ but an increase in mucins was observed with IFN $\gamma$. Implications: A decrease in ocular surface mucins contributes to the pathogenesis of GVHD-associated dry eye and can be a mechanismbased therapeutic approach for its treatment.

Prospective Evaluation of Preclinical Anti-HIV Agents for Mutational Resistance. Syeda Maryam Azeem, Long Island University, Alecia N. Muwonge, Long Island University, Michael Ivatorov, Long Island University, Matatiya Yunayev, Long Island University, 


\section{American Journal of Pharmaceutical Education 2018; 82 (5) Article 7158.}

Kathleen M. Frey, Long Island University. Objectives: Non-nucleoside reverse transcriptase inhibitors (NNRTIs) are used in combination therapies for the treatment of HIV. We have established a computational, structure-based method to predict resistance mutations to NNRTIs nevirapine (NVP), efavirenz (EFV), and investigational drug doravirine (DOR). The objective of this study is to predict resistance for 2 types of NNRTIs: rigid (NVP, EFV) and flexible inhibitors (DOR). Method: We selected NVP and EFV as rigid inhibitors and DOR as the flexible inhibitor. In our study, we employed computational residue scanning to predict resistance to all 3 NNRTIs. These predicted results were further validated through structural analysis, molecular dynamics (MD), and an enzymatic reverse transcription assay. Results: Results from residue scanning predict that the K101P mutation confers high level resistance to rigid inhibitors, but flexible DOR remains susceptible. Our predictions were further validated by $\mathrm{MD}$ and an experimental reverse transcription assay. Both results indicate that rigid inhibitors NVP and EFV are resistant to RT (K101P), while flexible DOR remains susceptible. These results are in agreement with recently published antiviral and clinical data. Implications: Our results show that this method can accurately predict mutational resistance. As a proof of concept, we believe that our computational and structure-based approach may be used to predict resistance to diverse inhibitors in development.

\section{Chemistry}

Dual Opioid-FAAH Inhibitor Analgesic Ligands. Matthew D. Metcalf, MCPHS University-Worcester/Manchester, Kenneth Lowe, MCPHS University—Worcester/Manchester, Joseph F. Biga, MCPHS UniversityWorcester/Manchester, Joseph D. Pirani, MCPHS UniversityWorcester/Manchester. Objectives: Synergistic analgesia produced by the combination of opioid and cannabinoid (CB) agonists has been well established over the past several decades. Fatty acid amide hydrolase (FAAH) inhibitors, which prevent the degradation of endocannabinoids (eCB), lack the limiting side effects associated with direct acting $\mathrm{CB}$ agonists but provide less than ideal analgesia for acute pain. While FAAH inhibitor synergistic combinations with opioids have proven difficult, recently, the author reported FAAH inhibition (by itself non-efficacious) combined with sub-efficacious doses of morphine produces robust, synergistic analgesia in an acute pain model. The objective was to produce a series of single molecule combination opioid-FAAH inhibitors which initially function as a FAAH inhibitor which as part of the FAAH inhibition mechanism, release the unmasked opioid agonist and test those compounds in a FAAH inhibition assay. Method: A series of opioid-carbamates was synthesized using isocyanates varying the size and lipophilicity of the $N$-portion of the carbamate while keeping the opioid portion constant. The novel compounds were tested in an in vitro FAAH inhibition assay (Cayman Chemical, FAAH inhibitor screening assay kit) for activity. Results: The novel compounds were synthesized (moderate to good yields), isolated and characterized. Compounds were screened in the FAAH inhibitor assay and inhibited FAAH at $[20 \mu \mathrm{M}]$. Implications: The initial synthesis produced a series of opioid pharmacophore based compounds which demonstrate FAAH inhibitory activity. They will assayed in mice and serve as the lead compounds for clinically useful, analgesic drugs for the treatment of human pain which lack side effects and abuse liability.

Targeting Sirtuin 7 in BRCA-Deficient Breast and Ovarian Cancers. Raphael Franzini, The University of Utah, Yuen Lik Hang, The University of Utah, Dana Srikanta, The University of Utah, Schuler Herwig, Karolinska Institutet. Objectives: The efficient discovery of hit compounds for diverse target proteins is a key challenge in the development of chemical probes and therapeutic agents. DNAencoded chemical libraries are emerging as a powerful hit discovery technology. It is widely believed that platforms of several ultra-large DNA-encoded libraries are needed to achieve consistent screening productivity, which are inaccessible to most medicinal chemists. We instead pursue the development of DNA-encoded chemical libraries designed for specific target classes. Method: Based on this concept, we designed and synthesized a DNA-encoded chemical library to specifically target NAD+-binding pockets. Results: Despite its simple design and small overall size, this library enabled the discovery of potent and structurally novel inhibitors of several sirtuin and ADPribosyl transferase enzymes. Implications: The presented data provides evidence that even small and inexpensive DNA-encoded chemical libraries can provide robust screening success when designed carefully.

\section{Experiential Education}

Best Practices for Assessment of Interprofessional Team-Ready Behaviors on APPEs. Ashley N. Crowl, The University of Kansas, Crystal Burkhardt, The University of Kansas, Sarah Shrader, The University of Kansas. Objectives: To determine best practices for assessment of team-ready behaviors of pharmacy students participating in interprofessional advanced pharmacy practice experiences (APPEs). Method: Preceptors received training on two interprofessional behavioral assessment tools, modified interprofessional collaborator assessment rubric (mICAR) and individual teamwork observation and feedback tool (iTOFT). Preceptors evaluated students at midpoint and end of rotation using each tool during two different months. Preceptors completed a utility survey at the end of the month on each tool to determine a preference. Focus groups were conducted with a subset of preceptors to elicit further perspectives. IPE experts and accrediting board members were interviewed to determine perceptions of best practices. Results: Thirteen preceptors completed the study. No significant difference was found comparing utility of mICAR to iTOFT; though mICAR trended towards being easier to use/understand $(p=0.29)$. Focus group participants $(n=8)$ felt using a tool led to enhanced feedback and interprofessional experiences and all preferred mICAR. From midpoint to end of rotation, ITOFT showed a difference in teamwork $(p=0.016)$. IPE experts and accrediting board members perceived best practices to include: using a tool that aligns with IPEC competencies, is imbedded into an existing APPE evaluation, and can be applicable to all health professional learners/evaluators. Implications: Preceptors preferred mICAR to iTOFT, though no significant difference was observed likely due to small sample size. Additional research is needed to confirm these results and for best practices to emerge. Future steps include evaluating interprofessional teamwork using these tools in a 360-evaluation of the individual learner by preceptors/clinicians outside of their own profession.

\section{Library and Information Science}

Drug Information Education for Non-Pharmacy, Non-Prescribing Health Professions. Rebecca Hoover, Idaho State University, Kalon Ludvigson, Idaho State University. Objectives: Assess the ability and perception of students in non-pharmacy, non-prescribing health professions in drug information resource identification, utilization, and quality before and after formal didactic training by a drug information specialist. Method: Students studying nursing (bachelor level), physical therapy, or dental hygiene completed a questionnaire assessing their perceptions of drug information, importance of quality of drug information resources, and potential utility of resources. Following the 


\section{American Journal of Pharmaceutical Education 2018; 82 (5) Article 7158.}

perception portion of the questionnaire, the students were asked to name the three most common resources they use for information about medications and to apply their knowledge to a drug information case. After the pretest, each group received a formal drug information lecture consisting of a 60-90 minute presentation. Upon conclusion, students were given the same questionnaire as a posttest. Results: A total of 146 participants underwent training. $73 \%$ of students reported using an Internet search engine (such as Google, Bing, or Yahoo!) to answer drug information questions on at least a monthly basis. $34 \%$ of students reported observing a clinical instructor answer a drug information question using a search-engine strategy. The top 3 resources identified and/or used for drug information case on the pretest were "Google" (43\%), "Internet" (18\%), and "[text/hand]book" without further identifier $(13 \%)$. For the posttest, the top resources were: MedlinePlus $(19 \%)$, Google (17\%), Epocrates (17\%), and Facts and Comparisons (17\%). Implications: Drug information education in non-prescribing health professions is needed as students report routinely utilizing drug information but may be unable to identify reliable resources without training.

\section{Pharmaceutics}

DNA Nanoparticles for Ischemic Stroke Therapy. Wanzhu Zhao, Duquesne University, Younsoo Bae, University of Kentucky, Devika S. Manickam, Duquesne University. Objectives: The objective of our research is to develop a safe neurorestorative therapy to effectively treat ischemic stroke. Brain-derived neurotrophic factor (BDNF) has the capability to sustain neuronal cell survival, facilitate synaptic function, and improve overall neuroplasticity after brain injury. We hypothesize that DNA nanoparticle (NP) transfection of brain microvessel endothelial cells will result in secretion of BDNF protein, which will increase neuronal cell survival in an in vitro oxygen glucose deprivation stroke model. Our DNA NPs are not designed to cross the BBB per se and deliver cargo to the brain. Our strategy is to transfect the brain endothelial cells with BDNF DNA NPs and make them as a depot secreting neuroprotective factors-mimicking the BBB's natural function. Methods: DNA NPs were prepared using poly(ethyleneglycol) $)_{\mathrm{x}}$-block-poly(aspartate diethyltriamine) $\left(\mathrm{PEG}_{5 \mathrm{~K}}-\mathrm{DET}_{48}\right.$, where $\mathrm{x}$ and $\mathrm{y}$ denote molecular weight and the number of repeating units) as the polycation and Pluronic P84 (P84) as an excipient. hCMEC/D3 monolayers were used as an in vitro model of the human blood-brain barrier (BBB). We used Lucifer Yellow permeability assay to confirm tight junction (TJ) integrity in transfected cells and used an ATP assay to determine cell viability upon NP exposure. Results: We demonstrate that the observed increase in P84-induced transfection occurs with minimal (insignificant) perturbation of endothelial TJs (when comparing NP-transfected cells to untreated cells) and that the NPs are cyto-compatible. Implications: DNA NP transfection is a safe and effective strategy for delivery of therapeutics to an in vitro model of the human BBB.

Maturation and Spatial Expression of Intestinal MCT1 in Obesity. Melanie Felmlee, University of the Pacific, Michael $\mathrm{Ng}$, University of the Pacific, Jieyun Cao, University of the Pacific, Hao Wei, University of the Pacific. Objectives: Monocarboxylate transporters (MCTs) are involved in intestinal drug absorption, and have altered regulation in adult obesity, yet maturation and spatial expression data is not available in obese children. Our objective was to quantify the maturation and spatial expression of intestinal MCT1 in obese and non-obese rats from birth through sexual maturity. Method: We evaluated MCT1 expression in a diet-induced model of pediatric obesity with standard chow (NC) or high-fat diet (HFD) administered to pregnant dams from gestational day 7. Samples were collected from male pups at PND1 through 42, and mRNA, whole cell protein expression were evaluated by qPCR and western blotting. Results: mRNA expression in the jejunum and ileum was significantly increased in both NC and HFD fed animals pre-weaning and the declined to adult values. Duodenal mRNA expression demonstrated nonlinear maturation with the highest expression observed at PND14. Significant regional differences were observed with mRNA expression highest in the ileum. Total cellular MCT1 expression varied significantly with intestinal region and age. Maturation of protein expression was not consistent with maturation of mRNA expression with nonlinear maturation patterns observed in the jejunum and ileum. Differences between NC and HFD rats were observed in the jejunum and ileum; however, the directionality differed. HFD rats had greater expression in jejunum as compared to age-matched NC rats. In contrast, NC rats had higher expression in the ileum. Implications: Results demonstrated that maturation and spatial expression of MCT1 is altered in obesity potentially leading to variations in intestinal drug absorption.

\section{Pharmacy Practice}

Creation and Assessment of an Interprofessional Activity Using ThinkShare. Janet Cooley, The University of Arizona, Alice Min, The University of Arizona, Herman Gordon, The University of Arizona. Objectives: Interprofessional education (IPE) is required in health sciences education, and programs are exploring how to incorporate IPE into curricula. This project describes a hybrid online/in-person case-based interprofessional (IP) activity for use in ThinkShare, a commercial social learning program. Method: A team of IP faculty developed an IP case, which, along with the evaluative survey, was validated using a think-aloud protocol. Volunteer IP students first worked through the case asynchronously (online in ThinkShare) and then in-person in small groups. A retrospective pre-post Likert scaled and open-ended question survey determined student satisfaction, sense of teamwork, and preference for online or in person activities. Results: Fourteen students participated in the activity and 13 completed the survey $(92 \%)$. Survey respondents found the activity to be moderately or extremely useful in helping them learn across professions. Respondents found the in-person discussion to be more useful than specific online features. Four of the eight items showed a statistically significant difference toward a more positive impression about IP collaboration and teamwork after the activity (with the other four items showing a similar trend). Six of the seven items that compared student opinions about working online compared to the in-person groups showed statistically significant differences with a preference toward the in-person discussion groups. Implications: The pilot project was successful in teaching students IPE competencies and students had a preference for in person learning activities. Future iterations will include a more substantial group introduction (such as an online icebreaker, focus on individual student roles, and more thorough instructions).

Exploring Patient Perceptions of Pain Contracts and the Role of the Pharmacist. Adriane Irwin, Oregon State University, Natalea Suchy, Oregon State University, Daniel Hartung, Oregon State University. Objectives: Risk management strategies are an expected component of opioid prescribing. The objective of this study was to describe patient attitudes towards controlled substance agreements (a.k.a., pain contracts) and characterize perceptions of their impact on patient care, behavior, communication with prescribers, and engagement with the healthcare system. Method: A qualitative study was conducted on patients seen by a clinical pharmacist to review and sign a controlled substance agreement at a multi-site federally 


\section{American Journal of Pharmaceutical Education 2018; 82 (5) Article 7158.}

qualified health center. Data was collected through semi-structured, one-on-interviews of 20 patients. Themes were identified through a crystallization approach. Results: Patients were generally supportive of controlled substance agreements and felt these agreements helped educate patients on the risks of opioid therapy, set expectations, and manage prescriber liability. Respondents with positive perceptions frequently mentioned a trusting relationship with their primary care provider (PCP) and/or health center and did not feel these agreements affected these relationships. Respondents with more neutral perception generally provided less information on these relationships and viewed these agreements as a paperwork requirement for opioid prescribing. Patients had mixed feelings as to whether these agreements translated to behavior changes for themselves or others. Patients were generally supportive of completing these agreements with a pharmacist rather than their PCP. Implications: Patients consistently identified positive outcomes from controlled substance agreements and did not articulate any unintended negative outcomes. The degree of support for these agreements seemed to align with the patient's relationship with the PCP and/or health center and may be an opportunity for future research.

\section{Social and Administrative Sciences}

Duration of First-Line Chemotherapy for Advanced Non-Small Cell Lung Cancer (NSCLC) and Survival Among the Elderly. Pramit A. Nadpara, Virginia Commonwealth University. Objectives: Lung cancer is a significant contributor to cancer mortality in the US. Approximately $81 \%$ of those living with lung cancer are 60 years of age or older. Among elderly diagnosed with Non-Small Cell Lung Cancer (NSCLC), chemotherapy is the primary treatment modality. The objective of this study was to determine the association between duration of first-line chemotherapy and survival among elderly in the United States. Methods: We used the NCI's Surveillance, Epidemiology, and End Results (SEER) cancer registry linked Medicare (SEER-Medicare) claims data files for this study. Using the HCPCS and CPT codes, we identified patients who had received first-line chemotherapy. Survival time was calculated as the number of months from the cancer diagnosis date to the Medicare date of death or the five year follow-up. Results: We identified 168,212 elderly patients diagnosed with lung cancer during the study years. The patterns of first-line chemotherapy duration in the cohort varied significantly $(\mathrm{p}<0.05)$. Survival outcomes also differed significantly among patients by the duration of their first-line chemotherapy $(p<0.05)$. Implications: Although lung cancer in the elderly is associated with a poor prognosis, several treatment strategies can cure, or at least prolong survival. Therefore, we need to increase our understanding of comparative effectiveness of lung cancer treatments in the elderly. Duration of first-line chemotherapy should be optimized to benefit the patient.

Psychotropic Polypharmacy and its Impact on Healthcare Costs Among Cancer Patients in the US. Ami M. Vyas, The University of Rhode Island, Hilary Aroke, The University of Rhode Island, Stephen J. Kogut, The University of Rhode Island. Objectives: To estimate average costs in the year following cancer diagnosis among cancer patients with psychotropic polypharmacy (PP) as compared with those without PP. Method: A retrospective cohort study was conducted of patients age $\geq 18$ diagnosed with the most commonly occurring cancers (breast, prostate, lung, colorectal) during 2011-2012 using the Optum Clinformatics ${ }^{\circledR}$ Data Mart (OptumInsight, Eden Prairie, MN) deidentified commercial claims database. PP was defined as concurrent use of two or more psychotropic medications for at least 90 days.
Healthcare utilization costs were estimated as total medical payments derived from claims files. Generalized linear regressions with log link and gamma distribution were performed to model average costs, controlling for patient demographic and clinical covariates. BlinderOaxaca decomposition was conducted to examine the extent to which independent variables contributed to the differences in the average costs. Results: Average costs for cancer patients with PP $(\$ 53,615)$ were higher compared to those without PP $(\$ 38,453)$, an unadjusted difference of $\$ 15,162$. In the adjusted regression, the difference in the costs shrunk to $\$ 5,888$, and remained significant. Only $3 \%$ of the difference in the costs between those with and without PP were explained. The presence of physical and mental comorbidities, type of cancer, age, and use of chemotherapy accounted for the majority of the explained portion of the differences in costs. Implications: Overall medical care costs were higher among cancer patients with PP compared to those without PP. The difference in costs was largely driven by clinical factors, while a majority of the difference remained unexplained.

Same Destination. Different Journey: An Exploration of URM Students' Experiences to Become Pharmacists. Antonio A. Bush, University of North Carolina at Chapel Hill. Objectives: In recent years, there have been numerous calls to examine diversity in pharmacy education. However, little research has been conducted in this area. This study explored the experiences of students identifying as underrepresented racial minorities (URMs) in a S/CoP and the strategic actions they take to navigate towards degree completion. Method: Twenty students from a S/CoP within a research-intensive institution participated in 60-90-minute interviews. Multiple coders used several rounds of open coding to develop a codebook, which guided the final rounds of coding and theme identification. Trustworthiness procedures included a dependability audit, peer debriefing, and analytic memos to promote reflexivity. Results: The following themes emerged. Prepharmacy school experiences: pipeline programs, work experiences, family, and URM health professionals impacted participants' interest in and encouragement to attend pharmacy school. Pharmacy school experiences: lack of diversity, feeling unwelcomed, and concerns about cultural competency and group work challenges were expressed. Agentic perspectives: motivated by URM faculty, belief in themselves, and a sense of purpose. Inhibited by mental impact of social events, pressures of representing race, and feeling inferior. Actions taken to be successful: Code-switching, finding solace and support with URMs, seeking cultural competence experiences, and strategically remaining silent or speaking up during group work. Implications: This exploratory study provides the foundation for a theoretical framework to better understand URM students' journey to pharmacy school and the experiences thereof. Findings from this study can be used to develop and implement practical solutions to increase the diversity of S/CoPs and create environments conducive to the success of URM students.

\section{INNOVATIONS IN TEACHING COMPETITION-WINNERS}

A Second Year Capstone Assessing the Pharmacists' Patient Care Process. Kathryn J. Smith, University of Florida. Objectives: To provide an overview of a second year capstone designed to assess each step of the Pharmacists' Patient Care Process (PPCP). Methods: Students collect information from a standardized patient (SP) and electronic health record (EHR), assess the patient's medical conditions and plan for treatment in an inpatient setting. The students follow up with the patient in the outpatient setting, collect additional information from the patient and collaborate with dental students to assess the 


\section{American Journal of Pharmaceutical Education 2018; 82 (5) Article 7158.}

patient's problems and develop a plan for a toothache complicated by other medical concerns. Students document their care plan via a SOAP note. Multiple assessment methods are used throughout including use of both an SP and EHR for the collection step of the PPCP, use of eMCQs as a format to efficiently assess clinical reasoning skills in the assessment and planning steps of the PPCP, and use of a rubric for grading the Medication History interview and SOAP note. Results: Results from two years of the capstone will be reported. Students performed well on the assessments overall, especially collecting information from the patient and documenting their care in a SOAP Note. There was no significant change in students' attitude towards interprofessional collaboration but students stated value was added to their educational through this experience. Conclusions: Incorporating each step of the PPCP into a summative assessment can provide feedback for improvement to students and faculty.

Use of an Escape Room as a Disruptive Instructional Strategy to Enhance Pharmacy Students' Knowledge About Diabetes. Heidi Eukel, North Dakota State University, Jeanne Frenzel, North Dakota State University, Dan Cernusca, North Dakota State University. Objective: To fully engage students in learning topics focused on diabetes disease management by immersing them in hand-on applications of diabetes-related skills. The escape room live gaming experience was chosen as a contemporary means to increase professional pharmacy students' knowledge of diabetes disease management. Methods: Faculty created an innovative serious game, the diabetes escape room. An authentic escape room gaming environment was established through the use of a locked room, an escape time limit, and game rules within which student teams completed complex puzzles focused on diabetes disease management. Results: Data from two iterations of the diabetes escape room $(n=169)$ focused on the gains from pre to post- treatment diabetes knowledge, overall attitude toward the serious game as well as students' reflective views of the impact of the diabetes escape room. Analysis of the 2016 and 2017 quantitative data indicated a statistically significant gain in diabetes knowledge from pre to posttest as well as a statistically significant perceived value when compared to an indifference level related to this activity. Qualitative analysis of team-based interviews revealed four major themes: strong teamwork, effective team communication, distributed leadership, and hands-on knowledge gains. Conclusion: The diabetes escape room proved to be a valuable serious game which engaged students in learning and which has adaptability and transferability potential to teaching and learning topics, other health professions education, and other universities.

\section{INNOVATIONS IN TEACHING COMPETITION-HONORABLE MENTIONS}

\footnotetext{
A Novel Process for Guiding Student Critical Reflection on Learning Habits Through Exam Performance Analysis. Gina Garrison, Albany College of Pharmacy and Health Sciences, Aimee Strang, Albany College of Pharmacy and Health Sciences. Objective: Develop and assess a process to foster student metacognition, study skill development, and learning through critical reflection on exam performance. Methods: A process was created to engage pharmacy students in critical reflection of individual exam performance. The process included creating instructions for students to access, interpret, and reflect on exam Strength and Opportunity (S\&O) reports available with electronic examination delivery systems. Investigators developed a written guide and video tutorial to promote student understanding and success with the process. A bonus assignment was developed to motivate students to complete the reflection and document self-directed plans
}

to improve learning habits. Exam grades were used to assess impact on subsequent course exam performance. Results: More than $85 \%$ of students completed the reflection assignment after each exam. In two out of three exams, mean grades were $5 \%$ higher for students who completed the reflection. Paired analysis of subsequent exam scores revealed a trend for decreased performance for students who did not complete the reflection from exam 1 to exam $2(p=0.052)$ and from exam 2 to exam $3(p=0.012)$. Neither group demonstrated any difference in performance from exam 3 to exam 4 . Sixty-four percent of survey respondents considered the reflection assignment helpful with improving study approaches and $88 \%$ agreed it should be available for exams in all courses at the College. Conclusions: Critical reflection of exam performance using $\mathrm{S} \& \mathrm{O}$ reports fosters student metacognition and study skill development and appears to improve student exam performance.

Objective, Structured Management Exams (OSMEs): An Innovative Way to Learn Business and Management Skills. Jill M. Augustine, Mercer University, Terri Warholak, The University of Arizona, Marion Slack, The University of Arizona, Erin Holmes, The University of Mississippi, Sandipan Bhattacharjee, The University of Arizona. Objective: To describe how an innovative OSME: 1) was developed using results from preceptor focus groups; 2) measured student performance using a scoring rubric; and 3 ) was evaluated using pre- and post-student survey. Methods: Using the results from preceptor focus groups, an OSME was developed. Third year Doctor of Pharmacy students learned about business and management skills, participated in an OSME, and were graded using a 3-level scoring rubric. Upon OSME completion, students completed a retrospective pre-post ability and confidence questionnaire. The scoring documents and questionnaire underwent psychometric analysis to assess scale and item reliability and validity. A linear regression was used to determine if student characteristics were associated with better OSME performance score. Results: Ninety-six students completed the OSME (out of 96) and 95 completed the questionnaire (99\%). All students passed the role-playing OSME. A significant change was seen in the self reported ability and confidence of 44 students (46\%) from pre- to post-OSME. Three student characteristics predicted higher performance OSME score: 1) previous management experience; 2) previous leadership experience; and 3) educational lecture quiz grades. Conclusions: To our knowledge, this project was the first to elevate role-playing cases into an Objective, Structured Management Exam. The findings provide a model for the use of OSMEs at other schools. Exposure to management scenarios was associated with higher self-perceived performance; thus, educators should consider including these skills in the education of student pharmacists and students should seek opportunities to practice management skills to prepare for future managerial responsibilities.

Student Pharmacist Entrepreneurship: Planning and Piloting Pharmacy Services. Betty A. Chewning, University of WisconsinMadison, Nisaratana Sangasubana, University of Wisconsin-Madison. Objectives: This innovative course "Pharmacist Communication: Educational and Behavioral Interventions" seeks to strengthen 3rd-year PharmD students' entrepreneurial and communication skills, and ability to work successfully with a collaborating pharmacy by planning pharmacy services for patients. Method: Using basic problem-solving principles (i.e., assess, adapt and evaluate) and their own creativity, student pharmacists plan a Pharmacy Capstone Project. Across the semester and working in groups, students systematically complete a set of structured templates to select a partner community pharmacy and plan a service to increase patient expectations of pharmacists by identifying and assessing site needs and resources. Next, they design 


\section{American Journal of Pharmaceutical Education 2018; 82 (5) Article 7158.}

a service according to site needs and make plans for quality improvement. Finally, a key innovative element of the course is its focus on skill development by having students actually pilot test and implement their proposed pharmacy service for patients and evaluate its feasibility. Results: Student skills are evaluated through several means. First, student groups' executive summary and oral presentation of their projects are graded to evaluate project content as well as process of delivery. Second, collaborating pharmacy managers have evaluated positively the quality of student pharmacists' skills. Telephone interviews with pharmacy managers found more than $60 \%$ had continued to implement the pharmacy services during the year following the course. Hence, a number of student projects had impacted their partners' service delivery. Implications/Conclusions: Offered to 3rd-year students immediately preceding their APPE rotations, the course seeks to increase their ability and confidence to create systems and services that support their expanded roles more fully in the future.

\section{AWARD FOR EXCELLENCE IN ASSESSMENT-WINNERS}

Multimodal Assessment of a Co-Curricular Professional Engagement Program. Maria Miller Thurston, Mercer University, Jill M. Augustine, Mercer University, C. Lea Bonner, Mercer University. Objectives: To assess a co-curricular Professional Development Network-Professional Engagement Program (PDN-PEP). Methods: Internal and external stakeholders were tasked with the development of a co-curricular professional engagement program for student pharmacists. All faculty and pharmacy students are required to participate, with the assistance of interested alumni members. Assessment of the impact of the program has occurred using students' year-specific tracking form, a validated professionalism survey, and evaluation surveys of students, faculty, and alumni. Descriptive statistics and frequencies were used to compare completion rates of different activities and survey responses. Results: Over 1,000 pharmacy students participated in PDNPEP. Since its inception, there has been a $100 \%$ completion rate for all students through program participation and/or remediation. Results from the student, faculty, and alumni surveys have provided many opportunities to improve the program in order to target necessary professionalism attitudes and behaviors. Conclusions: A multi-modal approach to the development and assessment of PDN-PEP has involved multiple stakeholders, including students, faculty, and alumni. This allows for continuous quality 2 improvement of the program, in which to match the needs of the school and the educational outcomes set forth by accreditation standards.

Unique Assessments for Unique Experiences: Content Validation of Three Assessment Tools for Advanced Pharmacy Practice Experience Rotations. Eric H. Gilliam, University of Colorado, Jason M. Brunner, University of Colorado, Wesley Nuffer, University of Colorado, Toral C. Patel, University of Colorado, Megan E. Thompson, University of Colorado. Objective: Competency-based education necessitates valid instruments for assessing student performance in the clinical setting. Three unique assessment tools, specific to three required advanced pharmacy practice experience (APPE) settings, were developed to produce meaningful and valid student assessment. This content validation study gathered evidence of tool validity using three distinct evaluation strategies. Methods: Each assessment tool was developed by subject-matter experts. Proposed content underwent a job-task analysis by external APPE preceptors, evaluating each outcome in terms of importance and frequency of opportunity for student demonstration. After implementation, tool performance was evaluated using a measure of item applicability and student achievement.
Finally, a two-step grade verification process was developed and the rate of grade acceptance by preceptor was determined. A priori benchmarks for acceptable tool performance were established for each strategy. Results: The job-task analysis was completed by 131 preceptors ( $52 \%$ response) with items achieving a composite score between 1.08 -11.83 (possible range: 1 (most valuable) - 25 (least valuable)). Item applicability ranged from $91.3 \%$ to $94.1 \%$ ( $n=849$ student assessments) during the first year. Preceptors accepted the tool-generated grade in 798 (94\%) assessments, indicating good to very good agreement between tool-generated and preceptor-intended grade: Ambulatory, $\kappa=0.702$ (95\%CI, $0.592-0.812)$; Community, $\kappa=0.825(95 \%$ CI, 0.657 - 0.993); Health-System / Inpatient Care, $\kappa=0.772$ (95\%CI, 0.690 - 0.853). Implications: Data from these evaluation strategies provide evidence of valid design and use of three unique APPE settingspecific student assessment tools. Pharmacy programs can adopt these strategies when improving assessment tools.

\section{AWARD FOR EXCELLENCE IN ASSESSMENT-HONORABLE MENTIONS}

Gatekeeper or Facilitator: Empowering Students to Optimize Experiential Learning through Structured Summative and Formative Assessments. Tanya L. Ostrogorsky, Oregon State University, Gary E. DeLander, Oregon State University. Objective: Curricular reform designed to enhance management of complex clinical cases and provide opportunities to develop personal strengths prior to graduation, created urgency to assure student readiness for expanded advanced experiential education opportunities. Methods: A four-week Pre-APPE (PAR) block was designed to provide students greater selfawareness of specific abilities required for excellence in practice. Foundational concepts in calculations, pharmacy law, and use of common drugs were assessed in summative assessments; as were more advanced abilities such as literature evaluation and clinical problems solving. Small group formative assessments challenged students to engage collaboratively in solving complex patient cases and resolution of ethical dilemmas. Throughout the PAR block, students progressively created a Personal and Professional Development Plan designed to assist them in guiding experiential learning with preceptors. Results: The process of creating the PAR block engaged faculty in a collaborative, focused effort to assure student readiness for advanced practice experiences. Analysis of aggregate data highlighted areas for immediate curricular attention, and also identified assessments requiring adjustments to assure validity. Individual performance data, good and bad, provided students confidence through self-awareness of strengths and areas for improvement; and tools to lead discussions of learning strategies with preceptors. Conclusion: Assessment of practice readiness can be viewed from a perspective of the gatekeeper to opportunity, or facilitator to optimize learning. Student perspectives and performance suggest the PAR block facilitated a newfound confidence, allowing students to take ownership of their learning and creating a framework for life-long learning.

Leveraging a Required E-Portfolio Course to Meet Multiple Needs: Student Assessment, Curricular Improvement, and Accreditation. Mary Elizabeth Ray, The University of Iowa, Lisa DuBrava, The University of Iowa, Mary Starry, The University of Iowa. Objective: Learning portfolios enable pharmacy students to connect programmatic outcomes and related learning experiences to future practice as healthcare providers. Assessment programs need data to make decisions regarding curriculum quality and to maintain accreditation. Our goal was to create a portfolio that would meet the needs for 


\section{American Journal of Pharmaceutical Education 2018; 82 (5) Article 7158.}

learning improvement, curricular assessment, and accreditation. Methods: We designed a required, longitudinal, learning e-portfolio course. Students upload artifacts and reflect on how the corresponding learning experiences link to program outcomes and their own continuous professional development. Baseline and annual self-assessment ratings and guided reflections document progress toward outcome attainment. Individualized formative feedback from a consistent pharmacist reviewer provides reinforcement of metacognitive skills. De-identified quantitative and qualitative data is shared with the Assessment Committee and others with the purpose of improvement. Results: $13 \%$ of required artifact reflections in the first two years of the course required revision with the highest need occurring in the fall semesters of each year (approximately $19 \%$ ). The outcome requiring the largest percentage of revisions was Population and Public Health; the Scholarship \& Research outcome required no revisions and Self-awareness required one. Self-assessment ratings toward attainment of PharmD Learning Outcomes showed annual increases from baseline and year to year. Faculty made improvements in designing learning experience/artifacts based on quantitative and qualitative information, with additional benefits possible for the collective curriculum. Conclusion: Though no one can dispute the value that learning eportfolios bring to the student experience, consideration should be taken to maximize information available for overarching assessment and accreditation needs during the planning process.

\section{STUDENT POSTER COMPETITION}

A Novel Approach to Pharmacy Practice Law Instruction. Matthew Carl Deneff, University of Connecticut, Lisa Holle, University of Connecticut, Jill M. Fitzgerald, University of Connecticut, Kathryn E. Wheeler, University of Connecticut. Objectives: UConn School of Pharmacy students receive pharmacy law education in the second professional year, followed by a review course after graduation. UConn graduates' Multistate Pharmacy Jurisprudence Examination scores have trended downward from 2009 to 2016, indicating need for additional and different preparation methods. This study was designed to determine the baseline gap in functional understanding of pharmacy practice laws through exercises with the MyDispense Community Pharmacy Simulator. Method: MyDispense was integrated into a P3 case study course. Students were assigned eight individual exercises to complete before and five group exercises to complete in assigned sections. Exercises covered common practice topics, including prescription fraud, emergency fills, and federal counseling requirements. Assignments were graded as correct, partially correct, or incorrect based on specific parameters. Additionally, trends in errors were recorded. Results: Initial data analysis show students completed more exercises correctly working in groups compared to individually. Numbers of students correctly completing individual exercises in one section ranged from $27 \%-90 \%(n=22)$ and $55 \%-100 \%$ for all group-completed exercises $(n=18)$. Common errors included providing incomplete or incorrect information on patient counseling and DEA record-keeping requirements and misinterpreting controlled substance prescribing and dispensing regulations. Final data to be presented. Implications: A need for additional law education exists as shown by students' functional knowledge following completion of formal didactic law education during a previous school year. These data will help inform restructuring of UConn's School of Pharmacy law curriculum, such that continual emphasis will be placed on "muddiest" points and applicability to real practice.

A Pilot Program Featuring Formative Peer Review of Faculty at a College of Pharmacy. Pooja Sonali Kumar, University of Michigan, Jolene R. Bostwick, University of Michigan, Kristin C. Klein, University of Michigan. Objectives: To help students and faculty attain the highest level of success and involvement in the classroom, a pilot program of faculty peer review of teaching was conducted. Method: This initiative targeted individuals at the ranks of Clinical Assistant or Assistant Professor who taught during the 2016-2017 academic year. As part of the process, the peer reviewer(s) attended a class taught by the faculty member under review. During the observation, the peer reviewer(s) completed rubrics and noted strengths and areas for improvement. Participating faculty members were asked to complete a post-evaluation survey promptly following completion of the process to evaluate the pilot program and offer suggestions for enhancement. Results: Based on a $64.3 \%$ response rate $(9 / 14)$ from reviewers and $92.9 \%(13 / 14)$ from faculty members under review, 100\% (9/9) of reviewers and $92.3 \%(12 / 13)$ of faculty members under review would recommend the peer review program to their colleagues. However, $77.8 \%(7 / 9)$ of the reviewers and only $46.2 \%(6 / 13)$ of faculty members under review supported the use of the peer review method as part of the annual faculty evaluation and development process. Implications: Ultimately, this process of peer review will be implemented across the College, benefiting faculty evaluation and development, as part of the promotion and tenure process. With our positive feedback and suggestions for improvement, the authors hope this will serve as a guide for institutions to develop peer review programs that will positively supplement student ratings and provide an additional, meaningful form of evaluation for self- improvement and promotion/tenure.

A Survey of Pharmacy Student Perceptions, Involvement and Interest in Teaching Activities. Andrea Haugtvedt, The Ohio State University, Colleen A. Dula, The Ohio State University, Nicole Kwiek, The Ohio State University. Objectives: To assess student involvement and interest in activities related to teaching and to gauge perceptions of the value of potential teaching experiences offered during the PharmD curriculum. Method: A survey was developed and distributed via email to all professional pharmacy students at The Ohio State University. Students were asked about their knowledge of careers in academia, perceptions of the value of teaching, past teaching experiences, intention to teach after graduation, and interest in specific teaching activities. Results: 194 students across all professional years responded. The majority (95.8\%) agreed that teaching was an important skill for pharmacists. After graduation, 38\% of respondents reported interest in serving as a faculty member with even more students interested in precepting $(72.7 \%)$. Of those that responded "Maybe" or "No" $(26.3 \%)$ to precepting, most $(72.5 \%)$ identified a lack of prior teaching experience as a barrier. The most common prior teaching experiences were serving as a tutor (35\%) or mentor (34\%), while $28 \%$ of respondents identified no teaching experience. Students were most interested in a "teaching track" throughout the PharmD curriculum (59.3\% very or extremely interested), while the next most popular teaching opportunity was serving as a teaching assistant in a course (49\%). Implications: While not all students will pursue a career in academia, they acknowledge the value of teaching opportunities during their PharmD training. Colleges of Pharmacy should continue to expand teaching opportunities in their curricula to better prepare students for precepting, residency and careers in academia.

A Systematic Review of Pre-Clinical Cultural Competence Educational Interventions Involving Pharmacy Students. Grace Wang, Northeastern University, Sadik Owolewa, Northeastern University, Mark Douglass, Northeastern University. Objectives: To determine the effectiveness of pre-clinical cultural competence educational interventions on pharmacy students' knowledge, skills, and attitudes. 


\section{American Journal of Pharmaceutical Education 2018; 82 (5) Article 7158.}

Method: A systematic review of analytic studies evaluating preclinical cultural competence educational interventions on pharmacy students was performed by searching CINAHL and PubMed databases and the American Journal of Pharmaceutical Education and Currents in Pharmacy Teaching and Learning. No date restrictions were applied and only studies published in English were included. Results: Thirty-three of 791 total studies met inclusion criteria based on the results of the literature search. Of the 33 studies evaluated, 27 assessed student perceptions and attitudes, 16 studies evaluated student knowledge, and 10 assessed student skills. Interventions targeting mental health cultural competencies were most commonly assessed, and student reflections and group discussions were the most widely utilized interventions. Thirty-two of 33 studies demonstrated a statistically significant improvement in student knowledge, skills, attitude, or a combination of these outcomes. Implications: A review of key pre-clinical pharmacy student cultural competence studies revealed positive outcomes associated with interventions targeting students' knowledge, skills, and attitudes. The lack of a standardized outcome measure was a barrier to comparing results across studies. Further research in designing and standardizing a validated survey tool is needed. Additionally, the lack of diversity in cultural competency educational interventions calls for future interventions targeting topics of religion and $\mathrm{LGBT}+$.

An Inter-Professional Elective: Screening, Brief Intervention and Referral to Treatment. Lisa Ashley Daniel, University of Cincinnati, Karissa Y. Kim, University of Cincinnati. Objectives: An interprofessional Screening, Brief Intervention and Referral to Treatment (SBIRT) elective has been offered at the University of Cincinnati Academic Health Center since 2016. The objective of this study is to describe this elective and evaluate pharmacy students' knowledge and competence after course completion. Method: Students completed an 18-question pre- and post-course knowledge assessment related to substance abuse and SBIRT. Students also completed a survey related to effectiveness of training and perceived competence with SBIRT at course completion and 30 days after. Results: Of 30 pharmacy students enrolled over 3 semesters, 24 (80\%) consented to participate. In the knowledge assessment, the mean number of questions answered correctly before and after the course was $12.8 \pm 1.4$ and $15.7 \pm 1.4$, respectively. The mean score on the SBIRT Proficiency Checklist was 8.21 \pm 1.9 (Max Score 11). Interestingly, students' perceived level of competence in asking about and screening for substance use declined from pre- and post-course $(p=0.15)$, but students felt more competent in using pharmacologic methods for ongoing management of alcohol or other substance dependence post-course. At course completion, all students were either "very satisfied/satisfied" with the training and felt SBIRT was relevant to pharmacy. All students "strongly agreed/agreed" that this training enhanced their skillset; $95 \%$ expected to use the information. At 30 days, $60 \%$ of students had applied what they had learned in their pharmacy practice setting. Implications: This elective was well received by students and increased knowledge on substance abuse and SBIRT. Students felt that SBIRT was relevant to pharmacy practice.

Analysis of Student Performance Outcomes Using Virtual Dispensing Exercises. Sophia Brown, University of Kentucky, Clark Kebodeaux, University of Kentucky, Keenan Beaumont, Monash University, Aric Schadler, University of Kentucky, Keith Sewell, Monash University. Objectives: MyDispense is a community pharmacy simulation program recently implemented into the new Patient-centered Care Experience (PaCE) course at the University of Kentucky College of Pharmacy. This study aimed to compare outcomes between PY1 and
PY2 students on an identical exercise required during their final objective structured clinical exam (OSCE). Method: 285 students (148 PY1; 137 PY2) completed the OSCE. Anonymized data was assessed using a t-test to identify differences in PY1 and PY2 performance including variables measuring the medication use process. The study was designed to identify whether PY2 students would have superior outcomes to PY1 students due to increased exposure. Analysis was conducted with SPSS (IBM Corporation, Armonk, NY, Version 23.) Results: PY2 students reached the correct dispensing outcome significantly more than PY1 students (71\% vs. $25 \%$; p < 0.001$)$. PY2 students were more likely to collect all essential patient information through patient fact finding than PY1 students $(90 \%$ vs. $71 \%$; $\mathrm{p}<0.001)$ and were more likely to successfully complete a comprehensive prescriber interaction through required prescriber fact finding (39\% vs. $21 \% ; \mathrm{p}<0.001)$. Significantly more PY2 students selected the correct outcome rationale than PY1 students (63\% vs. $18 \%$; $\mathrm{p}<0.001$ ), while PY2 students spent more time on average per exercise than PY1 students ( 411 vs. 329 seconds; $p<0.001$ ). Implications: Variables essential to the medication use process such as patient fact finding, prescriber fact finding, and accurate outcome and rationale were significantly higher in the PY2 cohort. Greater experience was associated with higher performance outcomes in this OSCE MyDispense Exercise.

Applying the Kirkpatrick Model to a Pharmacist Seminar on Opioid Misuse Screening in Community Pharmacies. Siri E. Burck, North Dakota State University, Mark A. Strand, North Dakota State University, Heidi Eukel, North Dakota State University. Objectives: Pharmacy best practices for caring for patients receiving an opioid prescription are a topic of concern. The hypothesis for the present research was that an education seminar followed by implementation of a guided pilot project could empower community pharmacists to provide evidence-based opioid misuse prevention care to patients receiving an opioid prescription. Method: The Opioid Misuse Risk Prevention Toolkit 3-hour continuing education seminar was designed and delivered to community pharmacists to prepare them to screen patients receiving opioid prescriptions for risk of opioid use disorder, and provide a series of follow-up services according to their risk level. The hypothesis was tested through implementation of a 6-week pilot project using the toolkit. Results: 54 pharmacists and 16 pharmacy students completed the training. Using the levels of the Kirkpatrick Model, participants were satisfied with the seminar (Level 1 - Reaction), had an $8.3 \%$ increase in those who agreed that screening for opioid misuse can be an important tool for the pharmacist (Level 2 Learning), 107 patients were screened, with $15.6 \%$ being counseled on support services based on their opioid risk profile (Level 3 - Behavior), and 5 were prescribed naloxone, with pharmacists reporting that the screening tools provided an objective and unbiased way to help their patients (Level 4 - Results). Implications: The Opioid Misuse Risk Prevention Toolkit demonstrates potential for a process that community pharmacists can utilize in practice. This process can play a role in the upstream prevention of the opioid epidemic.

Assessing Associations Between First Year Job Placement in Pharmacy and Clifton's StrengthsFinder 2.0. Kathryn Petersen, Northeastern University, Tatiana Bujnoch, Northeastern University, Jenny A. Van Amburgh, Northeastern University, David P. Zgarrick, Northeastern University. Objectives: The purpose of this study was to investigate the association between specific strengths based on Clifton's StrengthsFinder 2.0 (CliftonStrengths) and first year job placement within the pharmacy profession. Previous literature has evaluated prevalence of specific strengths of pharmacy students, but none have 


\section{American Journal of Pharmaceutical Education 2018; 82 (5) Article 7158.}

investigated associations between strengths and future employment. Method: This retrospective cohort study was conducted using CliftonStrengths data consisting of PharmD students' strengths collected during the pharmacy care management course for students that graduated between 2013 and 2016. Each students' top five CliftonStrengths were compared to their respective first year employment collected after graduation. Chi-square analysis was performed to determine if associations exist between CliftonStrengths and first year employment. Results: A total of 513 students were analyzed from four cohorts. Statistically significant positive associations included: being employed in any pharmacy-related profession with achiever, input, intellection, and learner; employment in community pharmacy with activator; employment in hospital pharmacies with consistency; employed as a pharmacy resident with achiever, focus, and learner; and employed in a post-graduate fellowship program with achiever, futuristic, and self-assurance. Statistically significant negative associations included: employment in community pharmacy with learner; employed as a pharmacy resident with adaptability and restorative. Implications: While certain CliftonStrengths are associated with first year placement within the pharmacy profession, differing strengths do not necessarily suggest pursuit of one pharmacy setting and position over another. Further studies are necessary to determine if CliftonStrengths can be used as guidance in choosing an area of practice within the pharmacy profession.

Assessing Hepatitis B Vaccination Status and Barriers to Vaccination Among Asian Americans in San Diego. Kaishan Ding, University of California, San Diego. Objectives: Hepatitis B (HBV) is a viral infection that disproportionately affects Asian and Pacific Islanders in the United States. This study aims to assess the HBV vaccination status of Asian populations in San Diego and to determine whether the patient's perceived knowledge, awareness, socio-economic status, and access to healthcare affect their vaccination status. Method: A cross-sectional study was conducted. Consenting participants were asked 2 survey questions - (1) whether they were vaccinated and (2) if HBV can be prevented by vaccination. Blood samples were collected to corroborate findings from participants' self-reports with HBV immunoassays. Results: Among the 224 participants, more than half (51\%) did not know their vaccination history. A majority of participants $(61.3 \%)$ incorrectly reported being vaccinated. Of the 32 individuals who answered the knowledge assessment question whether HBV was preventable by vaccine, $66 \%$ did not know the answer. Access to care has a significant effect on vaccination status $(\mathrm{P}=0.02)$. However, there was no significant association between participants' vaccination status and socio-economic status $(\mathrm{P}=0.26)$. Implications: The patient knowledge gap about vaccination status is a potential barrier to preventing HBV infection. The relatively large fraction of unvaccinated individuals with access to healthcare points to the need for expanding awareness of the availability and importance of HBV vaccination in San Diego.

Assessing Introduction of Team-Based Learning in Drug Development Course. Taylor G. Bertsch, Washington State University, Travis Denton, Washington State University, Nicole M. Perea, Washington State University, Ayesha Ahmed, Washington State University, Kimberly C. McKeirnan, Washington State University. Objectives: To identify students' perception of implementing Team-Based Learning (TBL) in a Drug Development course and to compare current class aggregate test results to historical non-TBL test results held within the course. Interpreting student opinions and test aggregates post TBL implementation in Drug Development will serve to assess its value. Method: TBL was incorporated into a one-semester Drug Development course in Spring 2018. Seven weeks of the course was taught using TBL principles, assessing students individually and in groups. The remainder of the Spring 2018 course will be trained traditionally, in a flipped classroom environment, using no weekly assessments and only bi-weekly exams. At the end of the semester, feedback will be collected via student surveys utilizing a five-point Likert scale. Student pharmacists will be asked to assess whether they strongly agreed, agreed, were neutral, disagreed or strongly disagreed with the implementation of TBL practices within the course. In addition, aggregate exam data will be collected from the 2017 and 2018 academic year, it will then be compared to measure quantitative impact. Results: Student feedback surveys will be received at the end of the Spring 2018 semester. This study will interpret and represent both qualitative and quantitative findings within the results. Implications: Faculty from other colleges of pharmacy who teach Drug Development or similar pharmaceutical science courses could potentially utilize these findings to tailor a similarly designed course, and provide an improved student learning experience.

Assessment of an Academic Dishonesty Seminar for First Professional Year Pharmacy Students. Norman Fenn, Purdue University, Natalie Gadbois, Purdue University, Kimberly S. Plake, Purdue University. Objectives: The primary objective of this study was to evaluate the impact of an academic dishonesty seminar on first-year pharmacy students' knowledge and perceptions of academic dishonesty. Method: First professional year students attended an academic dishonesty seminar during their professional program orientation. Students were engaged in active discussion through eight case-based scenarios depicting different scenarios involving aspects of academic dishonesty. A 45-item survey using a 7-point Likert scale was administered to students prior to and after the seminar to assess behavioral attitudes and intentions of pharmacy students towards academic dishonesty. Descriptive statistics were used to assess demographic information, while paired t-tests were conducted to assess variation between student responses before and after the seminar. Results: One hundred forty-nine students participated in the seminar and completed the surveys. The majority of the class were female (63\%), Caucasian $(66 \%)$, and 20 years of age $(60 \%)$. There were a number of statistically different changes in pre- and post-survey responses. Students stated they were more prepared to recognize and understand what academic dishonesty looks like, as well as hold themselves, their colleagues and faculty to a high ethical standard. Implications: The use of a seminar to prevent academic dishonesty improved student knowledge regarding this issue. This approach has the potential to influence the incidence of academic dishonesty as students progress through the curriculum.

Assessment of a Self-Paced Web-Based Pharmaceutical Calculation Practice Tool. Mamoon Rashid, Appalachian College of Pharmacy, Sally Riyadh Konja, Appalachian College of Pharmacy, Mehbuba Rahman, Appalachian College of Pharmacy, Craig R. Mullins, Appalachian College of Pharmacy. Objectives: Design and development of a self-paced augmented learning platform of pharmaceutical calculation. Method: This prototype learning platform was created using server-side computer language PHP and scripting languages HTML5, CSS and Javascript. The website is available online rxcal. org/practice and allows anyone to practice representative calculation problems without any login barrier. The platform provides the same type of problem with a randomly generated new set of numbers for practicing. The student is required to first solve the problem and then verify the answer which is visible only upon clicking of a button. Another button may be pressed to reveal the step-by-step calculation 


\section{American Journal of Pharmaceutical Education 2018; 82 (5) Article 7158.}

of the problem. The first year students of the ACP were given access to this learning platform and various aspects of the website were assessed through their feedback. Results: 74 students used this website during their course of Pharmaceutical Principles and participated in an anonymous survey to evaluate various attributes. The level of satisfaction was quantified in the scale of 0-100. The graphical interface, Readability of the texts and the Level of explanations were quantified and the responses were received from four subgroups of students based on (a) their frequency of using online resources and (b) an approximate number of times they used this platform. The mean ranged from $95.78 \%$ to $100 \%$ with a few outliers above 100 . Implications: Learning of Pharmaceutical Calculation may be simplified using this online self-paced platform.

Assessment of Cultural Competency \& Cultural Intelligence in Health Professional Students Studying Abroad. Nicole K. Stute, Cedarville University, Austin Lail, Cedarville University, Melody Hartzler, Cedarville University. Objectives: It is important that healthcare students are appropriately prepared to interact with an increasingly diverse patient population. Thus, the objective was to determine if short-term study abroad experiences improve cultural competency and cultural intelligence in health professional students. Method: Six students (5 pharmacy, 1 nursing) completed a monthlong study abroad experience in Valencia, Spain. During this time, students completed elective coursework on diabetes and health behaviors and beliefs, which included specific intercultural development sessions, and were hosted by a Spanish-speaking family. They completed a pre- and post-survey adapted from Clinical Cultural Competency Questionnaire (CCCQ) and the Cultural Intelligence Scale (CQS, 19 items, 4 subscales: strategy, knowledge, motivation, behavior). These surveys tested the confidence and comfort of the students performing care in a cross cultural setting and predicted cultural judgement, decision-making, as well as task performance. Differences between pre-post data was analyzed using Wilcoxon-signed rank tests. Results: Students showed significant increases in knowledge, skills, and encounters/situations ( $p<0.05$ ), as assessed by the CCCQ. Significant improvement also was shown in two strategy, one motivation, and two behavior items on the CQS $(p<0.05)$. Implications: Health professional students participating in a study abroad experience increased their cultural competency, especially their knowledge, skills and comfort with another culture in health care. More research is needed to look at other types of study abroad experiences, groups of larger size and data compared to taking the same courses in a domestic setting.

Assessment of Student Pharmacists' Perception of High Volume, Conceptual Polling Questions. Andrea Whitaker, Manchester University, Andrea L. Wilhite, Manchester University, Robert D. Beckett, Manchester University. Objectives: To describe students' perceptions of high-volume, conceptual in-class polling questions (PQ) and determine the correlation between in-class and examination performance. Method: Hypertension was taught to second-year pharmacy students in a required integrated pharmacotherapy module; active participation was promoted using in-class PQ. In-class and exam questions were mapped to Bloom's taxonomy to classify as factual or conceptual (i.e., "Apply" level or higher). The lecture incorporated at minimum 8 PQ per lecture hour and $\geq 60 \%$ of the questions were conceptual. Student perceptions were assessed using 5-point Likerttype questions (i.e., completely true or highly confident to not at all true or confident) in a survey distributed following the related examination. Performance correlation was assessed using Spearman's rank-order. Results: Of the 72 students in the course 70 (97\%) completed the survey. Students strongly agreed in-class PQ required them to use critical thinking, had a focus on conceptual concepts, volume of questions were higher than typical, and helped them retain the information (median 5/5, IQR 4-5). Students agreed the questions helped them feel confident or highly confident in their ability to compare/contrast pharmacologic treatments to determine the best therapy and use critical thinking in future (median 4/5, IQR 4-5). Students' performance on conceptual questions in-class moderately correlated with overall exam performance and conceptual items $(r=0.490$ and $r=0.531$, respectively; $\mathrm{p}<0.001)$. The correlation was weaker with factual in-class PQ $(r=0.0 .357$ and $r=0.370$, respectively; $\mathrm{p}<0.002)$. Implications: High-volume, conceptual PQ were positively perceived by students and helped improve self-confidence. In-class performance on these activities moderately correlated with examination performance.

Assessment of the Perceived Benefits of a Professionalism Development Program. Lauren Nicole Latten, Howard University, Rut Beyene, Christine Tran, Michael A. Marcus, Howard University, Oluwaranti R. Akiyode, Howard University. Objectives: The objective of this study is to assess how a professionalism development program that spans from P1-P4 year of pharmacy education at Howard University College of Pharmacy influences student professionalism. Method: Pharmacy students participated in professional development program throughout their P1-P4 year. The program development program initiative included white coat ceremony, seminars, professional development essay, professionalism chairs, annual professionalism/ leadership workshop, newsletter, and recognition award. A cross sectional study design was implemented using a 20-item survey in April 2017. The survey was distributed to the fourth year pharmacy students during their last semester of pharmacy school. The assessment was limited only to the fourth-year students because of their 4 years of exposure to the program when it was initiated in Fall 2013. Statistical analysis was completed with SPSS and Tableu statistical software. Results: Overall, the response rate was $59.7 \%$, composed of 37 participants. Approximately $80 \%$ of the participants self-identified as Black/African-American/African. Additionally, $97.2 \%$ of participants agreed that the professionalism development initiatives provided insight on conflict resolution management, $91.9 \%$ agreed that the professional development initiatives contributed to their professional development, and $100 \%$ agreed that professional development initiatives provided them with knowledge directly applicable to their growth as a student pharmacist. Implications: The outcome of the study supports a professional development program in its ability to positively impact pharmacy students. The future plan includes assessing other cohorts of fourth year pharmacy students on the program's benefit on professionalism development.

Assessment of the Use of a Patient Simulation on Pharmacy Student HIV Treatment Knowledge. Juanita A. Draime, Cedarville University, Anna Staudt, Cedarville University, Zachary N. Jenkins, Cedarville University, Nicole K. Stute, Cedarville University, MeiLing Norfolk, Cedarville University. Objectives: With 1.1 million people living with HIV, pharmacists can play a crucial role in monitoring, counseling, and adherence checks across pharmacy settings. Thus, the objective of this study was to assess if a HIV patient simulation increases pharmacy student knowledge of HIV in preparation for future practice. Method: Using an observational design, second-year pharmacy students $(\mathrm{N}=47,98 \%$ response rate) were given the validated HIV Treatment Knowledge Scale to establish baseline knowledge before participating in a four-hour HIV team-based learning session. Post-session, students were administered the scale again. Three days later, students participated in an HIV patient counseling simulation 


\section{American Journal of Pharmaceutical Education 2018; 82 (5) Article 7158.}

where they proposed a new antiretroviral plan while also addressing any monitoring, barriers, and maximizing adherence for the patient. Post-simulation, students were again administered the scale. Total scale scores were summed, and data were analyzed using descriptive statistics, Wilcoxon and paired t-tests, as appropriate. Results: HIV knowledge increased significantly post-lecture $(\mathrm{p}<0.001)$. Postsimulation, scores improved but not significantly $(p=0.291)$. Knowledge on 15 of the 21 items on the HIV Treatment Knowledge Scale significantly improved from pre-lecture to post-simulation ( $\mathrm{p} \leq 0.025)$. Implications: The results of this study suggest that the TBL session produced an increase in HIV knowledge, which was sustained after completing a simulation experience. Further research should examine the impact on skills and abilities through this experience, and similar activities at other institutions may assist in preparing students for future practice.

Best Practices for Creating and Utilizing Pharmacokinetics Course Materials in an Active- and Collaborative-Learning Classroom. Yili Zhong, Washington State University, Jeannie Padowski, Washington State University, Gary M. Pollack, Washington State University, Connie M. Remsberg, Washington State University. Objectives: To determine student perception and utilization of course materials/ resources, and how these relate to educational background and performance, in an active- and collaborative-learning pharmacokinetics course taught across two campuses. Method: All students enrolled in a first-year pharmacokinetics course were invited to participate. Of 174 students ( 144 on one campus, 30 on the other), 80 consented to participate (54 and 26). One background survey and six post-test surveys were administered online through Qualtrics ${ }^{\circledR}$ throughout the semester. Responses were analyzed in aggregate and by campus for each post-test survey. Results: Participants did not differ from non-participants in terms of final course grade ( $p>0.05$; two-sample t-test). Sixty percent respondents spent 1-2 hours on pre-class preparation; $84 \%$ indicated that the amount of material was appropriate. Practice exams, homework questions, and in-class activities were consistently ranked as the top three most-useful resources for facilitating understanding of course content throughout the semester. The most commonly-cited reasons were "allows for practice and testing my knowledge," "relevant to the exam," and "simple/concise." Recommended textbooks were consistently ranked least-useful. When content questions arose, $62 \%$ of responders most commonly consulted with classmates. Implications: Students' preferences of materials and study habits were consistent across the two campuses, despite substantial differences in class size. As expected, in-class activities were valued as part of the active-learning experience; the degree to which students consulted with each other was consistent with the collaborative-learning philosophy. Pre-class materials that were designed specifically to prepare students for in-class activities were more highly-valued than generic textbooks.

Boosting Student Confidence in Core Curricular Competencies Through Student-Led Journal Clubs. Clare T. Dyczkowski, Lake Erie College of Osteopathic Medicine, Emma Wysocki, Lake Erie College of Osteopathic Medicine, Kristen Gawronski, Lake Erie College of Osteopathic Medicine. Objectives: Officers of the Student College of Clinical Pharmacy introduced quarterly, student-led journal club sessions during the 2017-2018 academic year. Student leaders aligned articles with concurrent topics being taught in the Pharmacotherapeutics course in an attempt to maximize impact and increase student interest. We hypothesized that student-led journal club attendance would positively impact students' confidence in assessing primary literature. Method: Journal Club sessions were advertised via social media and student e-mail. An anonymous and voluntary tenquestion, 5-point Likert-scale survey was administered following the student-led session. The quantitative data was dichotomized and analyzed using Chi-squared test to determine statistical significance. Institutional Review Board exemption was obtained. Results: Thirteen out of fifteen students in attendance completed the survey. Participants perceived student-led journal clubs to be a valuable use of time $(\mathrm{p}<$ $.001)$. Those sampled reported that attending the sessions improved their confidence in assessing primary literature $(\mathrm{p}<0.01)$ and increased their knowledge of biostatistics and research methods $(\mathrm{p}<$ $.001)$. Furthermore, participants reported that the coordination of article topics with Pharmacotherapeutics coursework improved their understanding of concurrent Pharmacotherapeutics subjects $(\mathrm{p}<.001)$. Implications: Student-led journal clubs show promise as a valuable addition to student programming. Colleges of pharmacy currently hosting student-led journal clubs, or planning to add such programming, should consider coordinating article topics with Pharmacotherapeutics coursework to enhance the overall learning experience of students in attendance. Our sample size was small for this pilot study; sessions are ongoing and final results will be presented at the meeting.

Characterization of a Multi-Kinase Targeting N4-phenylsubstituted7H-pyrrolo[2,3-d]pyrimidin-4-amine Anticancer Derivative in Various Models of Upper Gastrointestinal Cancers. Amruta S. Samant, Long Island University, Tanvi Visal, Dana-Farber Cancer Institute, Priya Pancholi, Dana Farber Cancer Institute, Samhita Bapat, Memorial Sloan Kettering Cancer Center, Dhvanir Nikunj Kansara, Long Island University, Tanmay Dichwalkar, Koch Institute for Integrative Cancer Research, Robert Senones, Roosevelt University, Sonali Kurup, Roosevelt University, Vikas Sehdev, Presbyterian College. Objectives: Upper Gastrointestinal Cancers (UGCs) exhibit resistance to conventional chemotherapy due to constitutive overactivity of multiple oncogenic kinases (EGFR, ERBB2/HER-2, Aurora kinases, and JAK2). In this study, we investigated a series of N4phenylsubstituted-7H-pyrrolo[2,3-d]pyrimidin-4-amines and selected a promising novel investigational derivative (RS-41) that targets multiple kinases and exhibits significant anticancer activity in both P53 mutant (FLO-1) and wild type (AGS) models of UGC. Method: Target kinase inhibition and selectivity screening (T-KISS) and MTT-cell viability assays were performed to identify RS-41 as the most promising agent with significant multi-kinase inhibition and anticancer efficacy. Next, clonogenic cell survival assay, cell cycle analyses, and western blot analyses were done to evaluate the effect of RS-41 treatment on cell survival, cell cycle progression, and expression of apoptotic markers in FLO-1 and AGS UGC cells, respectively. Results: Evaluation with T-KISS showed that RS-41 derivative selectively inhibits AURKA (IC50-0.96 $\pm 0.03 \mu \mathrm{M})$, JAK2 (IC50-1.21 $\pm 0.17 \mu \mathrm{M}$ ), and EGFR (IC50-5.92 $\pm 0.75 \mu \mathrm{M}$ ) kinases, respectively. The cell viability and clonogenic cell survival data indicated that treatment with RS-41 mediates significant $(\mathrm{P} \leq 0.05)$ inhibition of FLO-1 and AGS UGC cell viability and survival. The cell cycle data and western blotting data also exhibited a marked increase $(\mathrm{P} \leq 0.05)$ in the percentage of sub-G1-phase (cell death) and expression of various apoptotic markers in FLO-1 and AGS cells. Implications: Our T-KISS data indicate that multi-targeted inhibition of EGFR, AURKA and JAK2 can be achieved with the pyrrolo[2,3-d]pyrimidine scaffold. In addition, we demonstrate that inhibition of various oncogenic kinases with RS-41 could an effective therapeutic strategy for treatment of UGC.

Characterization of Near-Peer-Assisted Learning in US Pharmacy Schools. Carrolyn K. Cowey, University of the Incarnate Word, Gwendolyn L. Wilkening, University of the Incarnate Word, Sarah 


\section{American Journal of Pharmaceutical Education 2018; 82 (5) Article 7158.}

Rinehart, Hansita B. Patel, University of the Incarnate Word. Objectives: Describe use of near-peer-assisted learning (NPAL) in doctor of pharmacy curricula in the United States. Method: A survey was developed utilizing published data about NPAL use in allied health profession education (i.e., nursing, medicine). The survey was adapted for discipline-specific outcomes, and modified based on feedback and question validation with administrative colleagues. A list of current pharmacy programs was collected from the American College of Pharmacy Education. The online survey was then distributed via email to a curricular representative at each school on 01-22-2018, and participants had 4 weeks to respond. Data were evaluated using descriptive statistics. Results: Responses were recorded from 37 pharmacy programs $(n=37 / 142$, $26.1 \%$ response rate). Among respondents, most programs $(n=23$, $62.6 \%$ ) utilized some form of NPAL. Reported settings for NPAL included clinical skills labs $(\mathrm{n}=11)$, patient care and communication labs $(n=10)$, academic electives $(n=10)$, therapeutic lectures $(n=9)$, and experiential rotations $(n=8)$. In the majority of cases, near-peer teachers taught material previously presented by faculty $(n=15)$, as opposed to novel material $(n=5)$. Respondents reported using P2 $(n=14)$, P3 $(n=15)$, and $\mathrm{P} 4(\mathrm{n}=11)$ students as near-peer teachers. Ten programs selected near-peer teachers based on academic performance or faculty preference, while three programs reported a formal application process for near-peer teachers. Implications: The majority of pharmacy programs utilize NPAL, but curricular placement and near-peer teacher selection criteria varies between institutions. More data are needed to assess NPAL effectiveness and to describe methods for optimal curricular integration.

Comparison of Student and Professor Perceptions in Healthcare Simulations: A Mixed Methods Design Study. Kelly Vassallo, Northeastern University, Emily Bitton, Northeastern University, Samantha Hubley, Northeastern University, Shakira Kareem, Northeastern University, Shannon Moloughney, Northeastern University, James Murphy, Northeastern University, Kristin Greenwood, Northeastern University, Jennifer Kirwin, Northeastern University. Objectives: To explore how student and professor perceptions regarding simulation experiences compare. Method: Five College of Health Sciences professors were recruited to participate via convenience sampling. Student perceptions were captured via a reliable outcome assessment and data was collected and analyzed using Qualtrics survey software. Professor perceptions were investigated through semistructured interviews conducted after their simulation. Responses were qualitatively evaluated using an open coding, two-step content thematic analysis to draw common themes for each simulation and across the five simulations. Data from the outcome assessment was compared to the qualitative analysis to examine how student and professor perceptions aligned. Results: Professors discussed the importance of a realistic simulation. Among the students who completed the outcome assessment $(\mathrm{N}=339), 76 \%$ agreed that the simulation felt like a real-life situation. More so, professors emphasized how the simulations were rather ambiguous, and only $60 \%$ of students felt prepared for the simulations. Professors also described how the simulation increased emotions, and $80 \%$ of students reported feeling nervous during the simulation. Students and professors were in modest agreement that the simulation facilitated self-reflection, challenged the students, addressed the students' communication skills, and would assist the students' future performance. Implications: In our population, there was modest alignment between student and professor perceptions of a simulation experience. Comparison of teaching and learning perceptions is essential to ensure best practice and refinement of teaching and learning assessments. By providing the professors with results of this study, they will have the opportunity to enrich their use of simulation.

Correlation Between Medication Regimen Complexity and Quality of Life in Patients With Heart Failure. Pablo Federico Saenz, University of the Incarnate Word, Lourdes M. Vega, University of the Incarnate Word, Gwendolyn L. Wilkening, University of the Incarnate Word, Sonja M. Brune, The University of Texas Health Science Center at San Antonio, Bethany Kalich, University of the Incarnate Word. Objectives: To assess the correlation between medication regimen complexity index (MRCI) and quality of life/functional status in patients with heart failure, as evaluated by the Minnesota Living with Heart Failure questionnaire (MLHFQ). Method: A review of electronic medical records was conducted for patients who received care at the heart failure clinic between 2012 and 2017. Patients who had completed the MLHFQ survey were included in the study. Demographic data (age, race, ethnicity and heart failure ejection fraction classification) and MLHFQ scores were collected. An MRCI was calculated based on the patient's medication regimen documented during the same visit the MLHFQ was conducted. Inferential statistics will be used to assess correlation between MLHFQ and MRCI (overall and category A, B and C), with subgroup analysis based on heart failure classification. A secondary analysis of correlation between change in MRCI and change in MLHFQ will be conducted for patients with follow-up MLHFQ scores available. Results: Data collection is currently in progress. Data analysis to be completed by May 1, 2018. Implications: Pharmacists play an integral role in optimizing medication therapy which includes a focus on minimizing complexity. A correlation between patients' MRCI and MLHFQ would justify future studies evaluating the effect of reducing medication regimen complexity on quality of life.

Correlation Between Pharmacy Learner Interventions and Time Spent at an Academic Medical Center. Johanna C. Dresser, Wingate University, Meredith J. Welty, Lisa F. Brennan, Wingate University, Amy Loken, Wake Forest Baptist Medical Center, Sarah A. Nisly, Wingate University. Objectives: The objective of this study was to describe the incidence and types of pharmacy learner interventions per academic year and correlate the frequency and cost-analysis associated with interventions over time. Method: Patient care interventions are logged by students in the electronic medical record (EMR) as part of routine practice. Intervention data was collected through a monthly EMR report. All student rotations completed within the medical center system during the study period were eligible for inclusion. The primary endpoint was change over time in the frequency of interventions made per student, per rotation block. The main secondary endpoint was change over time of the cost analysis of interventions per student, per rotation block. Results: During the study period, 33 students were eligible for inclusion. The average number of interventions documented per eligible student decreased by an average of 15.3 interventions per student per block from the first through fourth blocks $(p=0.49)$. The average cost-savings of documented interventions documented per student per block decreased by an average of $\$ 2,849$ per block from the first through fourth blocks $(p=0.42)$. A total of 736 interventions were documented during the study period, for a total cost-savings of $\$ 113,117$. Implications: Pharmacy learner interventions result in cost savings to the institution at which they practice. Despite the decrease in the number of interventions documented over time, the impact and cost-savings that learners provide to an institution are still evident. Additional longitudinal data throughout the entirety of an academic year is forthcoming. 


\section{American Journal of Pharmaceutical Education 2018; 82 (5) Article 7158.}

Cost-Effectiveness Analysis of Umeclidinium/Vilanterol for the Management of Patients With Chronic Obstructive Pulmonary Disease (COPD). Yiran Rong, The University of Mississippi, David D. Allen, The University of Mississippi, Yi Yang, The University of Mississippi. Objectives: To compare the cost and effectiveness of umeclidinium/vilanterol (UMEC) $62.5 / 25 \mathrm{mcg}$ with fluticasone propionate/salmeterol (FP/SAL) $250 / 50 \mathrm{mcg}$ for the management of patients with COPD. Method: A Markov disease transition model was developed to compare costs and effectiveness of UMEC and FP/SAL for patients with COPD using TreeAge Pro. Model inputs for drug efficacy (change in utility as measured using EQ-5D, initial EQ-5D scores, and rates of exacerbations) were obtained from two published clinical trials. Drug cost data was obtained from Red Book Online. Data on quality of life changes associated with exacerbations was obtained from literature. Model outputs over four 12-week cycles include drug costs and change in EQ-5D scores. All costs are presented in 2017 U.S. dollars. A sensitivity analysis was conducted on the utility of each treatment, using three willingness to pay (WTP) values. Results: UMEC was associated with lower medication costs $(\$ 3,064)$ over four cycles when compared against FP/SAL $(\$ 5,944)$. UMEC was associated with higher cumulative EQ-5D scores (3.64) compared with FP/ SAL (3.56). UMEC dominated FP/SAL in the model due to its higher utility and lower cost. UMEC had lower rates of exacerbation in the clinical trials which was reflected in our model. Results from sensitivity analysis has shown that after adjusting utility score improvements for both medications, UMEC was more cost-effective at three WTP values. Implications: The results suggest that UMEC is the dominant treatment regimen when compared with FP/SAL. The sensitivity analysis showed that UMEC was a more cost-effective option even when the change in utility is decreased below FP/SAL's.

Decitabine Suppresses Viability and Causes Reexpression of Novel Hypermethylated Genes in Human Prostate Cancer Cells. Meghan N. Armstrong, Western New England University, Jessica LeClair, Victoryn S. Williams, Jeffrey Rovatti, Western New England University, Maya Leiva, Western New England University, Shannon Kinney, Western New England University. Objectives: Gene specific DNA hypermethylation and transcriptional repression in prostate cancer $(\mathrm{CaP})$ may be linked to important tumor suppressor activities, thus serving as potential therapeutic targets and indicators of individual's disease aggressiveness. We have previously identified a list of hypermethylated putative tumor suppressor genes (TSG) in a mouse model of CaP (TRAMP). The goal of the current study was to examine the role of DNA methylation, as well as these genes in human $\mathrm{CaP}$ cell lines. Method: We grew $\mathrm{LnCaP}$ and $\mathrm{PC} 3 \mathrm{CaP}$ cell lines as recommended and treated daily with $0.05 \mu \mathrm{M}, 0.5 \mu \mathrm{M}, 2.5 \mu \mathrm{M}$, and $/$ or $5 \mu \mathrm{M}$ Decitabine (DAC) for 72 or 144 hours. Cell viability was quantified using the MTS tetrazolium assay. Flow cytometry was used to determine the more specific effects of DAC on apoptosis and cell cycle. We also measured DNA methylation of the hypermethylated genes, CLPTM11 and SENP2, using restriction enzyme digestion of DNA followed by quantitative Polymerase Chain Reaction (qPCR) and measured their expression by quantitative reverse transcription (RT) PCR. Results: We found that even the lower concentrations of DAC used inhibited cell viability and induced apoptosis, and higher concentrations tended to cause cell cycle arrest. In addition, DAC treatment resulted in hypomethylation and reexpression of the putative TSGs in the treated cells, as compared to untreated cells. Implications: Our results suggest that reexpression of TSGs may lead to altered human $\mathrm{CaP}$ cell viability. Additionally, these genes may be useful prognostic biomarkers to distinguish aggressive and indolent forms of $\mathrm{CaP}$, which can improve therapeutic decisions.

Defining Innovation: Using Qualitative Interview to Inform Innovation Assessment in Admissions. Mary Nannette Walton, The University of Louisiana at Monroe, Stephen R. Hill, The University of Louisiana at Monroe, Elizabeth M. Lafitte, The University of Louisiana at Monroe. Objectives: To assess the understanding of innovation and its importance among key stakeholders of the University of Louisiana Monroe School of Pharmacy. Method: Students, pharmacists and school of pharmacy faculty were interviewed and asked to define innovation, suggest how it should be measured, and provide their thoughts on the role of innovation in the field of pharmacy. Interview was chosen over survey, so that participants could provide open-ended responses. Qualitative analysis was conducted on the interview transcripts for trends in opinions among stakeholders. Results: Both students and pharmacists expressed that innovation involved generating new ideas or improving current processes. When asked how innovation should be measured, students suggested that the success or impact of an idea should be measured after implementation. Pharmacists agreed that an objective should be established to measure innovation against. The results of the interviews will be used to create a station for multiple mini interview in which candidates will be given a scenario and assessed on their ability to solve a problem in an innovative way. Implications: In order to measure a program's achievement of ACPE Standard 4, schools need a baseline assessment of students' ability to innovate. Multiple mini interview provides an opportunity to assess a number of soft skills, including innovation. Future research should focus on an validated, affordable and generalizable measures of innovation in student pharmacists.

Design and Evaluation of a Council Structure and Meeting Format for Pharmacy Student Government. Sabrina Dunham, University of North Carolina at Chapel Hill, Jacqueline M. Zeeman, University of North Carolina at Chapel Hill, Bradford L. Wingo, University of North Carolina at Chapel Hill, Wendy C. Cox, University of North Carolina at Chapel Hill. Objectives: To evaluate student perceptions of a transformed Senate Council (i.e., pharmacy student government) structure and meeting format. Method: In August 2016, the Student Senate (pharmacy student government) structure and general assembly meeting format at the UNC Eshelman School of Pharmacy underwent a transformation in an effort to address perceived limitations, such as unidirectional information exchange, minimal discussion, and unengaged student leaders. To address these limitations, the Student Senate transformed into a formal council where one leader from each student organization served as a Council member at meetings held in a round-table format. To evaluate student perceptions of the new Senate Council, members who attended at least one Council meeting during the 2016-2017 academic year $(n=29)$ completed a 12-item Qualtrics survey evaluating inter-organization relations, communication, collaboration, and efficiencies. Descriptive statistics were used to summarize agreement frequencies among survey items. Results: Ninety-seven percent of Senate Council members $(n=28)$ completed the survey. The majority of students agreed or strongly agreed that the Senate Council facilitated inter-organizational relations $(n=26)$, communication $(n=26)$, and collaboration $(n=24)$. Ninety-three percent of respondents agreed or strongly agreed the Senate Council improved efficiencies $(n=26)$, while $96 \%$ preferred (agreed or strongly agreed) the new Senate Council meeting format over the previous Senate meeting format $(n=27)$. Implications: The results of this study suggest a council structure and meeting format may effectively support inter-organization relations, communication, collaboration, and efficiencies 


\section{American Journal of Pharmaceutical Education 2018; 82 (5) Article 7158.}

within student body government. These findings address a gap in the literature and provide insight into opportunities for enhancing student government in a professional pharmacy program.

Designing a "Room Escape" Event to Enhance Teamwork in Interprofessional Education. Micah E. Miller, Washington State University. Objectives: To utilize the teamwork building challenges and benefits inherent within the "Room Escape" activity paradigm to create an innovative approach to learning and applying skills required to meet the IPEC Core Competencies of Teams/Teamwork and Roles/ Responsibilities between healthcare program students. Method: A group of 3-to-5 third-year Pharmacy and second-year Doctor of Osteopathy program students will attempt to work as team to solve a series of nested, medically-related puzzles. The experience will take place within a simulation room and utilize audiovisual technology and a simulant mannequin to enhance immersion. Groups will have a 30-minute time limit to complete the activity. The initial puzzles focus on discovering the identity of an unconscious, ventilated patient within the room and unlocking the tools required to resuscitate him. The following puzzles revolve around diagnosing a previously unknown condition in the patient which, when properly identified and combined with information that can only be obtained by interviewing the patient, solves the final puzzle and successfully ends the scenario. Results: All required materials are built, and the scenario is ready to begin pilot testing. Implications: If well received and successful in its objectives, this pilot scenario could be utilized as a stand-alone module to be used nationwide. The core experience was designed to remain intact even in absence of simulation room technology, and can function in a low-fi setting with minimal required expenditures. Additionally, the general design would be a useful template for any faculty wishing to design a similar experience suited to their environment's specific needs and capabilities.

Development and Content Analysis of Film Dosage Form of Adrenaline. Amanda Allon, Larkin University, Jean J. Vincent, Larkin University, Samir A. Kouzi, Wingate University, Kalyan C. Nagulapalli Venkata, Larkin University, Mohammad N. Uddin, Larkin University. Objectives: Adrenaline, which is commonly used as Epinephrine, is available in an intramuscular injection form. The disadvantages of this route are low patient compliance, high cost, and side effects. The goal of this study is to evaluate a quickly dissolvable oral form of epinephrine for buccal administration. An oral film has several advantages that includes systemic administration, consistent drug concentration, immediate onset of action and avoidance of first pass effect. The objective of this study is to develop and conduct the content analysis of the film dosage form of Adrenaline. Method: The films were prepared using a polymer and applying evaporation method. The content of the drug was determined by the UV spectrophotometers. The absorption of drug in buccal area was imitated in a Franz cell using a synthetic membrane Polysulfone, (PSU), with the thickness of $0.025 \mathrm{~mm}$ (Sigma Aldrich). The absorbed drug was quantified using UV spectrophotometer. Results: The resulting films had smooth textures, thicknesses of 5-200um, and a desired tensile strength. The films were stable at room temperature, refrigerated, and freezer conditions. The disintegration study showed films were fully disintegrated in a saliva solution within 30 seconds. The absorption study shows most of the drug was absorbed through the synthetic polymer membrane. Implications: The results shows the film dosage form is quickly soluble and drug is able to pass through the polymeric membrane. This indicates a more efficient quickly soluble oral film can be developed which will increase patient compliance. The next step of the study will involve in vivo study.
Development and Evaluation of a Top 200 Drugs Learning Activity. Nicollette M. McMann, Concordia University Wisconsin, Kassandra M. Bartelme, Concordia University Wisconsin, Nicia Lemoine, Concordia University Wisconsin. Objectives: The primary objective is to determine the impact of two interventions to improve knowledge of top drug information: study materials provided for students to use individually and an in-class review activity. The secondary objective is to determine perceived usefulness of the activities. Method: Secondyear students enrolled in Applied Patient Care (APC) III at Concordia University School of Pharmacy were administered a 12-point electronic pre-intervention quiz to assess their baseline knowledge of renal and cardiovascular top drug information. They were then given access to an online flashcard deck to review relevant brand/generic names, adverse drug reactions, dosing information, black box warnings, and indications. Subsequently, students played a Jeopardy game in class that reflected flashcard content. Students were then administered an identical 12-point electronic post-intervention quiz. The pre- and post-quizzes were analyzed for improvement in scores. Students' responses to a post-activity online survey were analyzed to determine perceived usefulness of activities. Results: Of 70 students enrolled in APC III, 58 (82.9\%) completed both preand post-intervention quizzes. Overall, quiz scores increased an average of 3.63 points with an effect size of 1.99. On the survey, students were asked to select the activities (quizzes, flashcards, and/or Jeopardy game) that were most useful to their learning. Students selected the online flashcards $34.7 \%$ of the time, followed by selecting the Jeopardy game $30.7 \%$ of the time. Implications: Student performance and feedback on this activity will help identify useful methods to provide students tools for reviewing top drug information during didactic coursework.

Development and Implementation of Enhanced Evaluation Rubrics in the Multiple Mini-Interview Process. Ahmed M. Salem, University of Arkansas for Medical Sciences, Schwanda K. Flowers, University of Arkansas for Medical Sciences, Seth D. Heldenbrand, University of Arkansas for Medical Sciences, Angie Choi, University of Arkansas for Medical Sciences, Mrinmayee Lakkad, University of Arkansas for Medical Sciences. Objectives: Increasing the validity of the Multiple Mini-Interview (MMI) process by developing and implementing enhanced evaluation rubrics. Method: Retrospective MMI scenario video scoring was collected during a pilot phase to assess the effectiveness of enhanced evaluation rubrics to this type of interviewing technique. Inter-rater reliability was assessed during the pilot phase. A t-test was conducted after the full implementation phase to assess the variance and the effectiveness of the evaluation rubrics via comparison of the 2016 evaluation rubric cohort and the 2017 enhanced evaluation rubric cohort. Results: During the pilot phase, a significant degree of inter-rater reliability was found for all of the themes evaluated. Results after the implementation phase were (mean $+/-\mathrm{SD}$, p-value): Ethical Decision Making 2016 (5.9 +/- 1.13) vs 2017 (5.6 +/- 0.84), $\mathrm{p}<0.0088$, Critical Thinking $2016(4.9+/-1.25)$ vs 2017 (5.7+/- 0.97), $\mathrm{p}<0.0001$, Conflict Resolution $2016(5.3+/-1.41)$ vs $2017(5.2+/-1.44), \mathrm{p}=0.64$, Empathy $2016(4.9+/-1.45)$ vs 2017 $(4.8+/-1.49), p=0.57$. Implications: The implementation of enhanced evaluation rubrics into the MMI process will improve the admission process by providing a more non-bias approach to scoring. The rubrics allow graders to uniformly quantify scores while decreasing bias and other external factors that have the potential to influence scoring. The effectiveness of using evaluation rubrics can also be studied for use in other academic healthcare selection processes, where non-cognitive skills are tested.

Development and Validation of a New Measure of Scholarly Writing Motivation: The Scholarly Writing Motivation Scale. Kacey K. 


\section{American Journal of Pharmaceutical Education 2018; 82 (5) Article 7158.}

Hight, University of Arkansas for Medical Sciences, Nalin Payakachat, University of Arkansas for Medical Sciences, Amy M. Franks, University of Arkansas for Medical Sciences, Melanie A. Reinhardt, University of Arkansas for Medical Sciences. Objectives: No validated measure of motivation for scholarly writing exists in the literature. We developed and validated a new measure, the Scholarly Writing Motivation Scale (SWMS), to explore scholarly writing motivation among pharmacy practice (PHPR) faculty. Method: The 20-item Research Motivation Scale (RMS) was modified with the author's permission to focus on scholarly writing. The online SWMS was pre-tested for clarity, readability, and content, then sent to PHPR faculty via department chair emails. Internal consistency reliability, dimensionality, and construct validity were assessed using Cronbach's alpha, exploratory factor analysis, and confirmatory factor analysis. Discriminative validity was evaluated using participants' self-reported total number of publications. We hypothesized that participants with a higher publication number would have higher SWMS scores. Results: Construct validity was confirmed with good model fit (root mean square error of approximation $=0.07$ [95\% CI: 0.05-0.08], Bentler comparative fit index=0.96). The final SWMS has 16 items categorized into two domains: Joy (10 items) and Challenge (6 items), with factor loading $>0.35$ for all items. The two domains are moderately correlated $(\mathrm{r}=0.5)$. SWMS total scores range from 16 to 80 , with higher scores representing higher motivation for scholarly writing. Average SWMS score of participants in the high publication group was significantly higher than the low publication group (59.3 vs. 50.1, p<0.001). Implications: The SWMS is a valid and appropriate measure to explore motivation for scholarly writing among PHPR faculty. Understanding faculty motivation may assist department chairs in developing a plan and activities to promote scholarly writing for PHPR faculty.

Development of a Multi-Variable Admissions Factor to Identify Students At Risk for Academic Difficulty. Ahmed M. Salem, University of Arkansas for Medical Sciences, Brent D. Curry, University of Arkansas for Medical Sciences, Schwanda K. Flowers, University of Arkansas for Medical Sciences, Angie Choi, University of Arkansas for Medical Sciences. Objectives: To determine the correlation between pre-admission criteria and academic difficulty within the PharmD curriculum and to compare the association of academic difficulty to established markers (GPA and PCAT) to GPA-Factor (GPA-F), an intra-institutional value of pre-pharmacy scholastic achievements. Method: Academic difficulty is defined as having a D or F as a final grade in any course for this study. GPA-F was determined for each student enrolling in UAMS College of Pharmacy 2013-2016 by analyzing the difference between the students' cumulative undergraduate GPA and their GPA in the core classes (Chemistry, Biology, Organic Chemistry, and Math) required for admission into the Doctor of Pharmacy program. GPA-F was also compared to traditional markers for academic difficulty (GPA, PCAT, and MMI) using a chi-square test and a logistic regression analysis. Results: GPA-F for the study group was normalized, and the highest value was listed at 1.5. For each class except for the class of 2016, the number of students in academic difficulty increased in each GPA-F subset, as GPA-F increased. The probability of academic difficulty for each student became higher as GPA, PCAT and MMI decreased and GPA-F increased. The highest increase in probability of academic difficulty was seen with the increasing GPA-F. Implications: GPA-F allows for an intra-institutional comparison of pre-pharmacy scholastic achievements for each student. GPA-F along with GPA, PCAT, and MMI can help identify students that did not excel in their pre-requisite classes, indicating that they may not be prepared for the PharmD curriculum and could potentially struggle through the program.

Development of an Animal Replacement Assay: Phytotoxicological Effects of NSAIDs on Pisum sativum Seedling Growth. Tyler M. Holmes, MCPHS University-Worcester/Manchester, Abimbola Olaqunju, MCPHS University-Worcester/Manchester, Matthew D. Metcalf, MCPHS University-Worcester/Manchester. Objectives: IACUC vertebrate animal testing protocols call for the minimization of animals used and use/development of alternate procedures and tests if possible. Here, we present the development of an animal replacement drug assay for testing NSAIDs on Pisum sativum (sugar snap peas). Method: Pisum sativum seeds were germinated, allowed to sprout for two days, and then exposed to varying concentrations of drug in a liquid growth solution. Growth characteristics such as root length and shoot length were then measured. Results: NSAIDs produced a phytotoxicological concentration dependent effect on root growth in Pisum sativum sprouts similar to the effects of the plant hormone indole-3-butyric acid (I3BA) but not gibberelic acid (GA3). In the root growth assay naproxen LD50 $=23.1 \mu \mathrm{M}(95 \% \mathrm{CI}$ 16.6-32.2, $\mathrm{p}<0.0001)$, diclofenac LD50 $=36.5 \mu \mathrm{M}(24.7-53.8$, $\mathrm{p}<0.0001)$, I3BA LD50 $=1.7 \mu \mathrm{M}(1.1-2.8, \mathrm{p}<0.0001)$ inhibited growth where GA3 had no significant effect on root growth. In the shoot growth assay naproxen, diclofenac, and I3BA inhibited growth $(\mathrm{p}<0.0001)$, while GA3 enhanced shoot growth $(\mathrm{p}=0.0002)$. Implications: NSAIDS displayed a dose response phytotoxicological relationship similar to an auxin, and opposite of gibberellin. The mechanism of action of NSAID phytotoxicity appears to be specific and selective, and is posited to occur through the IAA pathway (auxin based mechanism). The results indicate a plant replacement drug testing assay has significant potential for eliminating animal and animal product ethical concerns while simultaneously being dramatically cheaper than alternative cell based drug testing. These results suggest that Pisum sativum is a suitable choice for further development as a plant-based replacement assay.

Development of a Tool to Systematically Assess Student Perception of Near-Peer-Assisted Learning. Sarah Rinehart, Gwendolyn L. Wilkening, University of the Incarnate Word, Carrolyn K. Cowey, University of the Incarnate Word, Hansita B. Patel, University of the Incarnate Word. Objectives: Develop a tool to evaluate student perceptions of near-peer-assisted learning (NPAL), including: effectiveness, relationships with near-peers, and general value. Method: An on-line survey was developed utilizing published data on NPAL use within allied health profession education (i.e., nursing, medicine). Content validation by NPAL experts, cognitive interviews with students, and psychometric review were performed. The survey was piloted on first-year pharmacy students with NPAL exposure and responses were tracked using Google ${ }^{\circledR}$ Surveys. The final instrument consisted of demographic questions and twelve statements assessing student perception of NPAL effectiveness, relationships with nearpeers, and general value. Survey statements were evaluated using a five-point Likert scale. Additional student feedback was solicited using an open-comment box. Survey reliability was assessed using Cronbach's alpha coefficient $(\alpha)$. Results: Pilot sample responses ( $\mathrm{n}=81 / 88,92 \%$ response rate) demonstrated internal consistency and reliability overall $(\alpha=0.86)$, and for each domain [effectiveness $(\alpha=0.84)$; relationships with near-peers $(\alpha=0.61)$; general value $(\alpha=0.82)]$. Median scores for each of these domains were 4,5 , and 4 , and mode scores were 5, 5, and 4, respectively. Twenty-one of twenty-two open-comments were positive, and described near-peer teachers as relatable, approachable, less intimidating, and easier to understand due to ability to communicate at the same level. 


\section{American Journal of Pharmaceutical Education 2018; 82 (5) Article 7158.}

Implications: Although NPAL is utilized by many allied health programs, there is no published tool to assess learner perception of NPAL. Our survey demonstrated consistency and reliability in assessing this metric, and may be useful to other allied health programs that utilize NPAL within their curriculum.

Diabetes Themed Escape Room: Perceived Knowledge Gains and Improvement Suggestions for Future Implementation. Brooke D. Schotters, North Dakota State University, Carolyn G. Marg, North Dakota State University, Dan Cernusca, North Dakota State University. Objectives: In 2017, several NDSU faculty members implemented a diabetes themed escape room gaming activity into their pharmacy curriculum. This poster presents the results of the qualitative analysis of student team interviews to assess knowledge gained from immersing in this activity and students' feedback for game improvements. Method: After completion of the escape room gaming activity, interviews of student teams were conducted, recorded and transcribed. Students were asked a series of questions to gauge their perception of learning during the game. Transcribed interviews were uploaded into NVivo(R) software for qualitative analysis. Through coding common themes across all student teams. Some of these themes emerged as suggestions for helpful future improvements to support strong student learning. Results: The results of qualitative analysis showed both aspects of perceived knowledge and suggested improvements for the gaming activity. Approximately 42 codes recorded the following improved student learning: prior knowledge being applied (18), new hands-on skills built (15), and learning while working as a team (4). Students also indicated specific improvements to improve the gaming activity: better technology to communicate with the faculty (22), adding a post-game debriefing (7), and explanation of the escape room itself prior to game (6). Implications: By qualitatively analyzing the student feedback we determined how to improve the impact of the gaming activity on students' perceived learning as well as the most beneficial improvements that will enhance learning. Implementing these findings will allow increased success of this educational activity. It will also allow for easier implementation at other pharmacy colleges.

Effective Isolation of Bioactive Diterpenes From Andrographis paniculata by High Performance Flash Chromatography. Mensah Boakye Agyemeng, South College, Zhihong Xu, South College. Objectives: Andrographis paniculata (Burm. f.) Nees has been well known for its multiple therapeutic applications in Asian countries for many years. In clinical treatment, this medicinal plant has been mainly used in anti-inflammation therapies. It also exhibited a number of other bioactivities, such as anti-cancer, anti-diabetic, anti-hypertensive, anti-venom, anti-thrombotic, anti-retroviral, anti-microbial, antipyretic, anti-malarial, and immunomodulatory activities. Recent reports showed that it could be used as an anti-oxidant agent, and had hepato/cardio-protective effects. The main antibacterial compounds from this plant include two diterpenes, namely andrographolide and 14-deoxy-11,12-didehydroandrographolide. The purpose of our research is to develop an efficient method to isolate these two diterpenes from the plant. Method: Here we report the development of the isolation of these two diterpenes by high performance flash chromatography (HPFC) from A. paniculata stems used in Chinese clinics. The eluting solvent system and time for the title compound isolation by HPFC were based on TLC calculation, and the purity of each isolated diterpene was investigated with HPLC on a RP C-18 analytical column. Results: An improved isolation method by HPFC has been developed to obtain bioactive diterpenes from the plant A. paniculata methanol extract. Our data indicated that an ideal purity of the title compounds may be achieved after HPFC separation based on HPLC analysis. Implications: HPFC can be used for the isolation of target compounds in a larger quantity than HPLC. Our isolation method may be applied for the preparation of other natural diterpenes from A. paniculata in large quantities with satisfactory purities.

Effect of Waterproof Footwear on Prevalence of Foot Related Complications in South Georgian Migrant Workers. Arielle Alanna Charles, The University of Georgia, Trina J. von Waldner, The University of Georgia. Objectives: The objective of this study is to evaluate the impact of waterproof boot footwear donations on the prevalence of foot related complications in migrant workers during Farm Worker Family Health Program from 2015 to 2017. Method: For each year, the prevalence of foot related ICD-9 codes, chief complaints, and dispensed medications were compared. Foot related ICD-9 codes included those pertaining to sprains and pain of the ankle/foot, indicating dermatophytosis of the feet, wounds on the foot, and dermatitis related to chemical or pesticide exposure. Foot related chief complaints included pain in the feet or ankles, itching and/or burning of the feet, sores and blisters, fungal infections (tinea pedis), chemical burns to the feet, and rashes on the feet. Foot related medications were those with foot related indications and/or those with specific foot related patient instructions. Data was analyzed for statistical significance using a chi-square test ( $\mathrm{p} \leq 0.05)$. Results: Foot related ICD-9 codes decreased from $7.14 \%(n=504)$ in 2015 to $6.67 \%(n=345)$ in 2017 $(p=0.098)$. ICD-9 codes specifically indicating foot/toe fungal infections or dermatophytosis decreased from $5.36 \%(n=504)$ in 2015 to $4.93 \%(n=345)$ in $2017(p=0.091)$. Foot related chief complaints decreased from $13.3 \%(n=465)$ in 2015 to $5.39 \%(n=334)$ in 2017 $(\mathrm{p}<0.001)$. Medications dispensed for foot related conditions decreased from $14.4 \%(n=450)$ in 2015 to $1.9 \%(n=419)$ in 2017 $(\mathrm{p}<0.001)$. Implications: Statistically significant decreases were seen with foot related chief complaints and medications. Statistically significant decreases were not seen with ICD-9 codes since the introduction of rubber boot donations in 2015 .

Effects of Brominated Flame Retardants on Cytokine Production by Mast Cells. Jonathan Mark Alan Mahoney, Western New England University, Dylan Krajewski, Western New England University, Warren Smith, Western New England University, Clinton Mathias, Western New England University, Diptiman Bose, Western New England University. Objectives: Polybrominated diphenyl ethers (PBDEs), extensively used as flame-retardants, have been shown to cause neurotoxicity, immunotoxicity and endocrine disruption; however, the exact mechanisms are largely unknown. Store-operated calcium entry (SOCE) channels are essential in the development and regulation of immune responses. In this study, we propose to examine the link between dysfunctional SOCE channels and PBDE-mediated immunotoxicity in MC/9 mast cells. Method: We examined the effects of two of the most commonly occurring brominated diphenyl ether (BDE) congeners, BDE-47, -99, and 6-OH-BDE-47. MC/9 cells treated with BDE-47, 6-OH-BDE-47 and -99 $(1,20 \mu \mathrm{M}, 24 \mathrm{~h})$ were stimulated with IgE/antigen $(1 \mu \mathrm{g} / \mathrm{ml}, 12 \mathrm{~h})$ and levels of IL3 and TNF $\alpha$ were measured. The changes in intracellular calcium was measured using calcium sensitive dye fluo-4 in freshly isolated bone marrow-derived mast cells (BMMCs) exposed to BDE-47. Results: Our results indicate PBDEs modulate cytokine levels in MC/9 cells in a concentra-

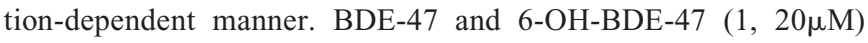
significantly increased IL3 concentrations whereas BDE-99 decreased levels. Interestingly, BDE-47 decreased TNF levels while -99 and 6-OH-BDE-47 exposure increased concentrations. BMMCs treated with BDE-47 $(1 \mu \mathrm{M})$ showed an increase in SOCE-mediated calcium influx. These results indicate BDE mediated modulation of 


\section{American Journal of Pharmaceutical Education 2018; 82 (5) Article 7158.}

calcium homeostasis could be responsible for BDE immunotoxicity since SOCE mediated calcium influx is essential for the development of mast cell immune response. Implications: In mast cells, calcium influx initiates multiple signaling cascades resulting in cytokine production and cellular degranulation. The overarching goal addresses the need to understand the molecular and cellular mechanisms by which exposures to PBDEs can impair and trigger immune responses.

Effects of "Superfoods" on Protein Disulfide Isomerase Activity and Colorectal Cancer Cell Viability. Kathleen Silveira, Western New England University, Christine N. Galinski, Western New England University, Daniel R. Kennedy, Western New England University, Shannon Kinney, Western New England University. Objectives: Protein disulfide isomerases (PDI) are located in the endoplasmic reticulum and catalyze the formation and breakage of disulfide bonds, which play a role in oxidative protein folding and cell viability. Studies suggest that PDI inhibitors may slow the progression of cancer and that PDI activity is necessary for cancer growth and proliferation. In the current study, our goal was to test various "superfoods" for their effectiveness in inhibiting PDI and reducing viability of human colorectal cancer cells. Method: PDI inhibition was verified using the insulin turbidity assay with freeze-dried "superfoods" at $0.3 \mathrm{mg} / \mathrm{mL}$. HCT116 human colorectal cancer cells were grown as recommended, treated with the same "superfoods" at a final concentration of $0.1 \mathrm{mg} /$ $\mathrm{ml}$ for 48 hours, and cell viability was measured with the PrestoBlue ${ }^{\mathrm{TM}}$ assay. Results: Most (8/10) of the "superfoods" inhibited PDI activity by at least $50 \%$. In addition to a known PDI inhibitor (positive control), a number $(5 / 10)$ of the "superfoods" reduced HCT116 viability by at least 20\%. Several (3/10) "superfoods" had both PDI inhibitory activity and efficacy against HCT116 cancer cells, such as strawberry and acai. Implications: Our results suggest that various "superfoods" inhibit PDI and may prevent colorectal cancer cell growth through this mechanism. Since intestinal cancer cells are likely to have the highest exposure to dietary components, our findings support further examination of these "superfoods" for preventing, or compounds derived from them for treating, this disease. Furthermore, our data provide a potential contributing mechanism for epidemiological studies indicating that dietary components reduce colorectal cancer incidence.

Eight-Year Trends in Research Presented at the American Association of Colleges of Pharmacy Annual Meetings. Angela A. Antoniello, Erin Dudolevitch, Stephanie L. Sibicky, Northeastern University, Alexa A. Carlson, Northeastern University, Michael J. Gonyeau, Northeastern University. Objectives: Professional conferences offer opportunities to network, educate, and support professional advancement. Innovative research in pharmacy education is critical to the evolution of the profession. Our objectives were to identify trends in posters presented at AACP annual meetings from 2010-2017 and identify publication trends. Method: A retrospective analysis of poster abstracts from 2010 to 2017 were categorized into 27 specific topic areas including assessment, curriculum, course development, teaching techniques, and others. Abstracts were allocated into categories as appropriate and aggregate and individual data analyzed for trends. Additional bibliometric analysis was conducted to identify publication rates. Data collected included study category, publication database(s), time to publication, publication type, and journal. Descriptive statistics were used. Results: Based on 3,781 abstracts, areas of highest presentation rate included assessment (13.1\%), instructional design $(12.5 \%)$, and curriculum/programmatic endeavors (8.6\%). Sub-areas of sustained presentation included professionalism, active learning and PBL/TBL, while areas of low presentation included academic dishonesty and academia as a career. Approximately $9 \%$ of research presented did not include an educational focus. The publication rate was $18.3 \%(n=681)$, with section variation. The majority $(46.3 \%)$ were published in AJPE, followed by CPTL (23.9\%). Implications: Many research posters are presented at the AACP Annual Meeting each year, yet some areas of research may benefit from increased attention. Only one in five posters resulted in publication and most within pharmacy academic journals. Innovative research and publication in additional education journals should be encouraged as part of the association's future research agenda.

Employing a Near-Peer Pharmacy College Admission Test Preparatory Workshop Series to Support Recruitment and CoCurricular Goals. Erin L. Hickey, University of Kentucky, Sharon D. Gonzales, University of Kentucky, Jason Mitchell, University of Kentucky, Kelly M. Smith, University of Kentucky. Objectives: Challenges of pharmacy education include increasing the qualified applicant pool and improving current student competency using cocurricular activities. University of Kentucky College of Pharmacy (UK COP) created a novel Pharmacy College Admission Test (PCAT) preparatory workshop series delivered by PharmD students to engage pre-pharmacy students through relational recruiting and provide professional development for PharmD students via near-peer instruction. Method: The Office of Enrollment Management and a pharmacy resident with PCAT instructional experience mobilized to form a steering committee. Relying upon student focus groups, evaluation of resources and best practices, and prior instructional experience, the committee identified the major design elements of strategically establishing a delivery platform and instructors, workshop content, workshop coordination, and outcome evaluation. Participants were regional pre-pharmacy students, and first and second-year PharmD students were engaged as workshop leaders and facilitators. Results: Fiftythree pre-pharmacy students participated in the series, which was structured as three 90-minute sessions and one practice examination. Instructors included four PharmD student "leaders" and six PharmD student "facilitators." Content incorporated strategy demonstrations followed by interactive group-work. Workshops and facilitator training were coordinated by the pre-pharmacy advisor and pharmacy resident. Formative assessment was used at the series midpoint to improve pre-pharmacy and PharmD student experience and preparedness. Implications: High pre-pharmacy student participation rates supported relational recruiting. Participant feedback and improvement in near-peer teaching and facilitation skills indicated that this method provides an authentic co-curricular experience with reflective-based student development elements. Participant admission rates and student instructor skill and career development will serve as future program outcomes.

End Stage Retention Disease (ESRD) When Re-Watching Lectures Does Not Improve Long-Term Retention. Shannon Palmer, University of North Carolina at Chapel Hill, Youn Chu, University of North Carolina at Chapel Hill, Adam M. Persky, University of North Carolina at Chapel Hill. Objectives: This study compared post-class study strategies, re-studying from a recorded lecture versus recalling information from the lecture. We hypothesize, recalling information will lead to better long-term retention than reviewing lecture. Method: One-hundred and three $(n=103)$ students participated in the study. Students attended two 80-minute pharmacokinetic lectures on two different topics; both lectures were recorded. Approximately one week later, students re-studied from one of the recorded lectures and recalled information from the stated learning objectives for the other lecture. All students completed both conditions in a randomized, block design. Half the students were tested immediately after restudy and the other 


\section{American Journal of Pharmaceutical Education 2018; 82 (5) Article 7158.}

half were tested one-week later. A paired t-test was used to compare treatments. The primary outcome was performance on the measure of long-term retention. The secondary outcome was a cost-effect analysis. Results: On average, students spent 34 minutes reviewing the recorded lecture and 11 minutes recalling information $(d=3.2$, $\mathrm{p}<.001$ ). On a measure of long-term retention, there was no difference between restudying the recorded lecture and those that recalled information $(\mathrm{d}=.10, \mathrm{p}=1)$. However, on the immediate test the restudied topic outperformed $(\mathrm{M}=72, \mathrm{SD}=20)$ the recalled topic $(\mathrm{M}=51$, $\mathrm{SD}=14)(\mathrm{d}=1.2, \mathrm{p}<.001)$. Students perceived the restudy was more effective for learning (Median 3 vs 4, p <.001). Implications: Students perceive restudying from video is more effective in learning than retrieval practice. For students that attend class, restudying improved short-term but not long-term performance. However, students restudying is not a cost-effective practice for long-term retention with 3 times more time spent for no learning gain.

Enhancing Pharmacy Personnel Immunization-Related Confidence, Perceived Barriers, and Perceived Influence: The We Immunize Program. Tessa J. Hastings, Auburn University, Lindsey Hohmann, Auburn University, Kimberly B. Garza, Auburn University, Sally A. Huston, Keck Graduate Institute, David R. Ha, Keck Graduate Institute, Salisa C. Westrick, Auburn University. Objectives: Pharmacy personnel are in need of practical strategies to incorporate nonseasonal vaccination services into pharmacy workflow. The purpose of the We Immunize Program was to improve participants' confidence, perceived barriers/facilitators, and perceived influence on immunization services. Method: 62 pharmacist-technician pairs from community pharmacies in Alabama and California participated in the We Immunize Randomized Control Trial. All participants were offered a one-hour live immunization update. Intervention participants were also offered a two-hour enhanced training delivered via online modules and tailored monthly feedback. To evaluate the impact of the program, a survey was administered at baseline and six-months. Likert-type scales were used to rank level of agreement related to confidence, perceived barriers/facilitators, and perceived influence on immunization services. Differences were analyzed using pairedsample t-tests and repeated measures ANOVA. Results: 67 participants completed both baseline and six-month surveys (37 intervention; 30 control). Within the intervention group, participants' confidence in determining pneumococcal vaccine appropriateness $(p=0.027)$, confidence in pneumococcal vaccine-related patient interactions $(p=0.041)$, perceived external support $(p=0.016)$, and perceived influence on immunization services $(p<0.001)$ significantly improved. No change was observed within the control group. When compared to control participants, a greater degree of change in perceived external support $(\mathrm{p}=0.023)$ and influence on immunization services $(p=0.005)$ were observed from baseline to six-months among intervention participants. Implications: Immunization training including educational interventions and tailored feedback can be used to positively impact pharmacy personnel's confidence in providing pneumococcal vaccinations and perceptions related to environmental support and influence on immunization services. This, in turn, may increase immunization activities within community settings.

Evaluating Echinacea purpurea as an Adjuvant for Microparticle Formulations. Theresa J. El-Murr, Samford University, Bernadette D'Souza, Samford University. Objectives: In this study, we measured the efficacy of an herbal supplement for targeting dendritic cells (DCs) of the innate immune system. Our research is based on optimizing immunotherapy formulations that stimulate DCs to release IL-12 (cytokine) for a robust cytotoxic T-cell (CTL) adaptive immune response. We screened multiple aqueous and ethanolic herbal extracts and determined their efficacy in stimulating IL-12 release from DCs. This would give us a non-toxic and cheaper substitute for the costprohibitive synthetic adjuvants available. Method: Thirty-four herbals were screened for DC stimulation and IL-12 release. DC cells were treated with herbals for 24 hours, supernatants were collected and immunosorbent assays were performed for IL-12 secretion. The herbals secreting highest IL-12 levels were tested at different concentrations for possible cell toxicities. Further, IL-6, TNF-a, and IL-1B levels were measured to confirm DC stimulation. Cell surface analysis was performed using BD Accuri C6 Plus analyzer to measure surface expression of the stimulated DCs. Results: DCs treated with Echinacea purpurea (EP) showed the highest IL-12 secretion in a concentration-dependent pattern. The cytotoxicity study showed the $100 \mathrm{ug} /$ treatment yielded the best release of IL-12 with least toxicity. Cell surface expression analysis showed rightward shifts signifying increased marker expression in DCs. Implications: The release of IL-12 by DCs of the innate immune system causes activation of the adaptive immunity. Higher proliferation levels of CD8 + CTLs have been detected in previous literature. We wish to incorporate EP into our microparticle formulation to produce optimized amounts of IL-12 secretion with reduced amounts of expensive adjuvants.

Evaluating Factors Associated With Interest in Healthcare or STEM Professions Among Rising 9th and 10th Graders. Jessica Pinchinat, Howard University, Mary Maneno, Howard University, LaMarcus T. Wingate, Howard University, Earl Ettienne, Howard University, Beverly C. Mims, Howard University, Tawanna Terry, Howard University, Lawander McFarland, Howard University, Jasmine Lopes, Howard University, Toyin S. Tofade, Howard University. Objectives: To evaluate factors associated with interest in Health care or Science Technology Engineering and Math (STEM) professions among rising 9th and 10th grade students. Method: A cross-sectional evaluation of data collected from the 2016 and 2017 offerings of the Health Occupation Program (HOP), the STEM Career Intervention Program (SCIP), and the Junior URM Mentoring Program (JUMP) within the Howard University National Workforce Diversity Pipeline program (NWDP). The two outcomes evaluated were interest in STEM professions and interest in Health Care professions. Descriptive statistics of all study participants were conducted as well as chi square tests to determine bivariable associations between the study outcomes and independent variables. Analysis was conducted using SPSS at an alpha of 0.05 . Results: A total of 76 rising 9 th and 10th graders were included in this evaluation. The mean age was 14.3 years and $69.7 \%$ were female. Majority of the students rated influence of family members as important/extremely important. Additionally a high proportion of participants reported high income, job security, helping people, and spending time with family as factors they considered important in career selection. Bivariable analysis showed a statistically significant association between gender and interest in STEM professions $(p<0.05)$ with male participants having a higher odds of high/extreme interest compared to females. No significant gender differences were found with regards to interest in healthcare professions. Implications: This study found family members as key influencers in career selection within the early high school age group. Gender specific differences in interest particularly in STEM professions was also observed.

Evaluating First-Year Pharmacy Students' Philosophy of Practice. Deborah Pestka, University of Minnesota, Keri D. Hager, University of Minnesota. Objectives: A philosophy of practice serves as the foundation for any patient care practice. It is a set of professional 


\section{American Journal of Pharmaceutical Education 2018; 82 (5) Article 7158.}

values and beliefs that guides a practitioner's actions and decisions in practice. The objective of this work was to evaluate how first-year pharmacy students conceptualize their philosophy of practice as future pharmacists. Method: In 2017, students at the University of Minnesota taking a required first-year course were asked to complete a reflection exercise and write their philosophy of practice that best described them as a future practitioner. The investigators independently and inductively coded a sample of 20 student responses to develop an initial coding scheme. These codes were applied and refined to a random sample of 32 student responses (20\%) where inter-rater reliability(IRR) testing between the two coders was carried out at three stages of the coding process to ensure acceptable levels of IRR had been achieved (Kappa $\geq 0.80$ ). The final codebook was applied to all responses. Results: The exercise was completed by 157 (93\%) students. Thirteen unique codes emerged from student responses describing their philosophy of practice. The most frequently occurring codes were "Working as a collaborative member of an interprofessional team," "Patient-centered care," and "Providing quality care." Students also included expanded roles like "Promoting public health" and "Community engagement." Implications: Students' philosophies of practice reveal their perceived roles as future pharmacists. Pharmacy curricula as well as official oaths, such as the Oath of a Pharmacist, may require adaption to support the aspirations of future practitioners.

Evaluating the Impact of PharmD Industry Fellows Integration Within a School of Pharmacy. Sarah Yong Scott, Northeastern University, Madeline Merkel, Alnylam Pharmaceuticals, Ruthvik Malladi, Alnylam Pharmaceuticals, Amy Monpara, Alnylam Pharmaceuticals, Mark Douglass, Northeastern University, Angela M. Partisano, Alnylam Pharmaceuticals. Objectives: To assess the perceptions of the Northeastern University pharmacy faculty on the integration achieved by industry fellows and to identify potential opportunities to expand collaboration. Method: A voluntary, anonymized, online survey was distributed to all faculty members at the Northeastern University School of Pharmacy regardless of their previous interactions with the Fellowship. The survey contained multiple choice and open response questions involving the extent of fellows' current level of participation (small group teaching/facilitation, large-group facilitation, precepting) and faculty perception of identified future teaching, scholarship, and service needs. Results: Fifty five percent (30/55) of faculty responded to the survey and $40 \%(11 / 30)$ indicated that they have previously collaborated with a fellow. Small-group teaching/facilitation (39\%), research projects (28\%) and large-group class facilitation $(17 \%)$ were identified as the most common faculty/fellow interactions. Opportunities identified by faculty for greater collaboration included research projects, small-group teaching/facilitation, and service at the School and/or University level. The Value and Evidence Strategy fellow was identified by faculty as being most suited to assist with the Economic Evaluation of Pharmaceuticals and Pharmacy Practice courses, and the Medical Information fellow best matched to contribute to the Evidence Based Medicine course. Implications: Industry fellows are currently being utilized in ways that best fulfill the identified teaching needs. Although most faculty have not yet worked with fellows, efforts are underway to better align faculty research interests and teaching opportunities with the developing expertise of the fellows.

Evaluation of a Clinical Track Program in the Experiential Learning Curriculum. John Jeon Lee, University of Maryland, Patricia A. Ross, The Johns Hopkins Hospital, Hyunuk Seung, University of Maryland, Agnes A. Feemster, University of Maryland. Objectives: The objective of this study was to evaluate participating site coordi- nator and student perceptions of a clinical track program Method: Online surveys were developed and distributed to clinical track participants for the graduating classes of 2017 and 2018. The site coordinator survey instrument consisted of 24 questions aimed to assess satisfaction from the coordinator's perspective while the student survey of 27 questions assessed satisfaction from the student perspective. All coordinators were sampled; a collection of students was sampled. The survey was analyzed using descriptive statistics. The ordinal responses were assigned score ranging from 1 ('most negative attitude') to 5 ('most positive attitude'). Results: Seven (64\%) coordinators participated, and $80 \%$ of invited students responded. Coordinators were confident of the average clinical track students' preparation for a PGY-1 program (4.57 of 5.0) and students reported benefit from participation (4.5 of 5.0). Additionally, $100 \%$ of student participants were motivated to participate in the program by the opportunity to expand their clinical knowledge, and $100 \%$ of coordinators responded that students were better prepared to pursue a health- system pharmacist position immediately post- graduation. $87.5 \%$ of students participated in a scholarly project at their site, and $100 \%$ of students received a letter of recommendation from a clinical track preceptor or participated in education/professional development at the site. Implications: Participation in the clinical track program was perceived as beneficial by both site coordinators and students for professional development. Students exceeded the goals and objectives of the site coordinators, and students were satisfied with their clinical track experiences.

Evaluation of Commonly-Visited Patient-Focused Medical Information Websites. Bryce Ryan Adams, Rutgers, The State University of New Jersey, Sandhya Balachandar, Steve Caproni, Samantha Pomponi, Pavit Singh, Keith Wittstock. Objectives: To evaluate accuracy, completeness, and readability of consumer drug information websites using the FDA approved package inserts (PI) of the most commonly prescribed drugs by volume. Method: Consumer druginformation websites were evaluated in comparison to the FDA approved PI for the most commonly prescribed drugs by volume. A standardized rubric was developed based on the PI, the Suitability Assessment of Materials (SAM) tool, and clinical judgement of a team of 6 pharmacists. Results: In our preliminary analysis we assessed the top 3 drugs' PIs against the top 6 websites as described in the methods section. Preliminary analysis shows gaps in accuracy, completeness, and readability of consumer drug-information websites, particularly in safety. Additionally, preliminary data shows Healthline.com is the most reliable source for consumer drug-information. Healthline.com also had the best score for readability, falling within the FDA's suggested range of 6-8th grade reading level for consumer drug information. The poorest score was received by MedicineNet.com, due largely to a lack of safety data. Implications: These results show that not all websites provide equally accurate and complete information. Our results show that the leading consumer website for the most reliable source of consumer drug-information is Healthline.com with a score of $86.76 \%$, and the least reliable was MedicineNet.com at $44.61 \%$. The biggest downfall of all websites was in the area of safety. The results from this study could influence consumers to search for their drug-information on websites with more accurate and complete information.

Evaluation of Screening, Brief Intervention and Referral to Treatment Training Amongst Student Pharmacists. Lisa Ashley Daniel, University of Cincinnati, Stacy Colleen Sullivan, Karissa Y. Kim, University of Cincinnati. Objectives: Students enrolled in a Substance Abuse Elective received 2 hours of training on Screening, Brief Intervention and Referral to Treatment (SBIRT). The objective of this 


\section{American Journal of Pharmaceutical Education 2018; 82 (5) Article 7158.}

study was to evaluate students' knowledge of SBIRT and perceived competence in SBIRT. Method: To assess the impact of this training, students completed an 18-item pre- and post-training SBIRT knowledge assessment and an 11-item survey assessing perceived competence in SBIRT. The post-training survey also assessed perceived ability to implement SBIRT. Results: Of 57 students enrolled, 47 and 22 students took the pre- and post-training survey, respectively. Twenty-eight students $(60.9 \%)$ had no prior substance abuse education while $13(28.3 \%)$ had 1-4 hours of prior substance abuse education. The average knowledge assessment score was $71.8 \%$ (SD 10.0\%) before and $88.4 \%$ (SD 6.7\%) after training. For perceived competence in asking patients about substance abuse, 11 students (23.4\%) reported being moderately or very competent before training while 12 students $(54.5 \%)$ reported being moderately or very competent after training. More students reported competence in screening clients for substance use problems using a formal screening instrument after $(n=14,63.6 \%)$ than prior to training $(n=7,14.9 \%)$. A majority of students $(95.5 \%)$ saw the potential benefits of screening patients using SBIRT in a pharmacy setting and many $(66.7 \%)$ felt that they can incorporate SBIRT in their pharmacy jobs. Implications: This 2-hour SBIRT training appears to improve students' knowledge of SBIRT and improved perceived competence in SBIRT. Students felt that SBIRT has potential for use in a pharmacy setting.

Evaluation of Student Professionalism in a Pharmacotherapy Skills Course. Catherine Kuecker, William S. Middleton Memorial Veterans Hospital, Casey Gallimore, University of Wisconsin-Madison, Andrea L. Porter, University of Wisconsin-Madison, Amanda Margolis, University of Wisconsin-Madison. Objectives: Evaluate impact of professionalism grading within a skills-based course on student self-evaluation of professionalism, and ability to self-identify strengths and areas for improvement. Method: A standardized rubric was used to grade and provide feedback on student professionalism. All enrolled P2 students were invited to complete pre- and post-semester surveys to self-evaluate professionalism using a modified Miller's Taxonomy (knows, knows how, shows, shows how, teaches how). Students self-rated 32 items within four domains of the Professionalism Assessment Tool (relationships with others; integrity and respect; reliability, responsibility, and accountability; lifelong learning and adaptability). Statistical analysis on pre- and post- data was completed using Wilcoxon signed rank test. A thematic analysis was conducted to identify student-perceived strengths and areas for improvement. Results: A total of 144 students $(99.3 \%)$ completed the pre-semester and $129(89 \%)$ completed the post-semester survey. There was a statistically significant increase in students' perceived performance level for each professionalism item $(\mathrm{p}<0.05)$ following course participation. Perceived pre-semester strengths occurred within domains of integrity and respect; and reliability, responsibility, and accountability including professional appearance and accountability. Perceived strengths post-semester occurred in domains of relationships with others and reliability, responsibility, and accountability including communication, preparedness, and accountability. Identified areas for improvement pre- and postsemester occurred within domains of relationships with others and lifelong learning and adaptability, including providing feedback, communication, and self-reflection. Implications: Students selfreported professionalism improved following professionalism grading and feedback. Developing activities that target student-perceived areas of weakness, including interpersonal relationships and continuing professional development, is a mechanism to enhance students' professionalism development.
Examining California Pharmacy Students' Attitudes Towards Academic Dishonesty In Pharmacy Education. Lawrence Chui, Loma Linda University, Sophia Dinh, Loma Linda University, Paul Gavaza, Loma Linda University, Nancy E. Kawahara, Loma Linda University, You Been Kim, Loma Linda University, Jennifer Nguyen, Loma Linda University, Maria Santiago, Loma Linda University, Simon Situ, Loma Linda University, Justin Thurber, Loma Linda University, Quang Vu, Loma Linda University, Farnoosh Zough, Loma Linda University. Objectives: To explore the California pharmacy students' opinions of academic dishonesty in pharmacy education and its implications beyond graduation. Method: An electronic survey was distributed to all currently enrolled students at each of the 14 California pharmacy schools. The survey evaluated students' definition of cheating, their motives, views, and perceived long-term consequences of academic dishonesty. Demographics data (e.g. age, gender) were also collected. Results: 251 usable responses were collected from 14 California pharmacy schools. Students held varying definitions of academic dishonesty, and there was disagreement even on more straightforward items. Many students indicated that they had heard of or witnessed cheating in school (66.01\%); students also admitted to being tempted to cheat in certain classes $(28.63 \%)$ and also indicated that they would cheat if it meant passing a class $(12.78 \%)$. A majority agreed that cheating in school would cloud their judgment as pharmacists in the future $(66.82 \%)$. Implications: Academic dishonesty is highly prevalent in the pharmacy academic setting. This is highly concerning, as dishonest behavior may translate into the workplace once these students graduate. More needs to be done to prevent cheating and encourage students to report dishonest behavior.

Filling the Gaps: Development of a Simulation to Better Prepare Students for Clinical Rotations. Caitlin Marie Caulfield, Northeastern University, Stephanie L. Sibicky, Northeastern University. Objectives: The purpose of this project was to identify potential gaps in the Northeastern University School of Pharmacy curriculum where the implementation of a simulation during a large, cohort therapeutics course could fill these gaps. Method: In order to determine a simulation topic to fill curricular gaps, a survey was distributed to students in their final year on clinical rotations. The survey asked students to describe specific aspects of their required rotations (ambulatory care, community, internal medicine, health system), where they felt unprepared for and how the curriculum could be supplemented with a simulation experience. Results: Six percent $(n=8)$ of students completed the survey. For the ambulatory care rotation, students felt more practice with inhalation and injectable devices, anticoagulation and motivational interviewing would be beneficial. For the community rotation, all students said practice with insurance would be helpful. For the internal medicine and health system rotations, multiple students said more preparation for rounding would be useful. When asked to rank possible simulation topics, a majority of students listed adjudicating an insurance claim and medication reconciliation as the top choices. Implications: Based on the results of the survey, a simulation is being developed to improve student's knowledge and familiarity with injectable devices, rounding and insurance. The simulation will mimic rounds and be completed in two parts, with the first part focused on insurance and the second part focused on counseling. The assessment of this simulation will be determined after the effective delivery of this new simulation experience, with an anticipated delivery in 2018 or 2019.

First Evidence for a Pharmacist-Led Anticoagulant Clinic in a Transitional Care Environment. Vivien Navin Patel, East Tennessee State University, Jeffrey A. Gray, East Tennessee State University, 


\section{American Journal of Pharmaceutical Education 2018; 82 (5) Article 7158.}

David W. Stewart, East Tennessee State University, Cindy Pohland. Objectives: Evaluate time in therapeutic range (TTR) for a pharmacist-led anticoagulant clinic in a short-term transitional care environment (TCE). Method: A retrospective review of all warfarin patient admissions from a large long-term care pharmacy's anticoagulant clinic was conducted for residents between July 2016 and December 2017. All INR results (admission to discharge), test dates, INR goal range, age, indication for warfarin, interacting medications, number of medical errors, adverse bleeding events, and vitamin $\mathrm{K}$ doses administered were collected. TTR was calculated by the Rosendaal method. Additional variables included the percentage of INR measurements within range, INR values below 1.5 on admission, number of subtherapeutic and supratherapeutic INR values, and number of antibiotic courses during stay. Results: For all patients $(n=104)$, the overall TTR was $46.7 \%$ (43\% in range). Results based on indication were: Atrial fibrillation $(n=64) 45.2 \%(42.3 \%$ in range); deep vein thrombosis or pulmonary embolism $(n=16) 53.1 \%(48.2 \%$ in range $)$; and mechanical valve $(n=7) 48.7 \%$ (44.4\% in range). Patients with INR $<1.5$ and $\square 1.5$ at time of admission had a TTR of $32.1 \%$ and $52.6 \%$, respectively. Overall INR range was $0.89-6.26$ (mean =2.2) with 5-6 drug interactions per patient. Out of range INR values included 382 subtherapeutic and 106 supratherapeutic values. Average age was 78.7 years with a mean stay of 19.5 days. Vitamin K was not administered. Implications: Managing anticoagulant therapy in advanced age patients in partnership with a short- term TCE facility is challenging. Expectedly, TCE TTR was slightly less than in LTC patients managed long-term.

First Professional Year Performance of Students Who Attended Partner and Non-Partner Schools. Jennifer M. Toth, Northeast Ohio Medical University, Seth P. Brownlee, Northeast Ohio Medical University, Madison Ivan, Northeast Ohio Medical University, Mate Soric, Northeast Ohio Medical University. Objectives: To compare students who attended partner schools of Northeast Ohio Medical University (NEOMED) and other institutions. The primary objective is to determine if pre-pharmacy institution attended is a predictor of first-year (P1) academic success. Secondary objectives are to determine other pre-pharmacy variables that predict P1 performance. Method: Pre-pharmacy institution, pre-pharmacy science grade-point averages (GPAs), overall pre-pharmacy GPAs, prior degree status, demographic characteristics, and P1 courses pass/fail rates were collected on students who matriculated between fall 2013 and 2016 from PharmCAS and NEOMED. Results: 286 students were included in the analysis. 144 students attended partner schools, and 219 obtained a bachelor's degree or higher. 54 students had at least one failure in their P1 year. Chi-square analysis revealed there was not a significant difference between partner and non-partner schools and first-year success (chi-square $(4)=3.763, \mathrm{p}=0.439)$, nor was there a relationship between degree attainment and first-year success (chi-square(1) = $0.054, p=0.817$ ). Further analysis did not reveal any relationship between partner and non-partner schools and individual P1 courses. Age, pre-pharmacy science GPA, and overall pre-pharmacy GPA were significant predictors of success in P1 year. Implications: NEOMED's partner school agreements are similar to early assurance pathways found at other pharmacy schools. Results have shown students who attend partner schools do equally well $\mathrm{P} 1$ year. This research establishes a baseline for NEOMED comparison between partner institutions. Prior degree attainment was not a significant predictor, which differs with some previous research. This research could help inform admission decisions in a rapidly changing environment.
From Catch Up to Head Start: Impact of Data-Driven Course Adjustments Accommodating Peer Tutoring. Mohammed Nur Ahmed, Appalachian College of Pharmacy, Harish S. Parihar, Philadelphia College of Osteopathic Medicine, Ajay Singh, South University, Caterina M. Hernandez, Appalachian College of Pharmacy, Ingo H. Engels, Appalachian College of Pharmacy. Objectives: An internal analysis had confirmed our students' surveyed perception, that peer tutoring is useful, specifically during the transition into an accelerated PharmD program. Tutored students with a poor initial performance in our introductory science course improved their standing by course conclusion more than their non-tutored counterparts. The course directly started with a review that is to ensure minimum core knowledge in chemistry validated in a quiz. The course schedule was subsequently modified to provide more time for extracurricular intervention addressing individual deficits ahead of the quiz. Method: Two consecutive incoming classes received the same 18-hour chemistry review, followed by a quiz comprising a subset of 15 identical questions for comparison. For the second class, both the course start was delayed by six days and the chemistry review was introduced five days later into the course. Results: Neither the classes of 2019 and 2020 nor their tutored subpopulations differed in their admission scores among each other, yet the tutored students of 2019 trended towards underperforming by $9 \%(\mathrm{p}=0.12, \mathrm{n}=77)$ relative to their non-tutored classmates. More students of 2020 (33\%) embraced the opportunity of being tutored prior to the quiz than 2019 (14\%) $(\mathrm{X} 2=7.6, \mathrm{p}=0.0059, \mathrm{n}=146) .83 \%$ of the tutored students of 2020 surpassed an average of $70 \%$ compared to $45 \%$ the previous year $(p=0.04, n=35)$. The tutored students of 2020 reached the competence level of their non-tutored peers $(\mathrm{p}=0.88, \mathrm{n}=69)$. Implications: Accommodating an early onset of peer tutoring eases the transition into an accelerated PharmD program.

Healthcare Costs Associated With the Treatment of Bipolar Disorder: A Systematic Review. Alexander Maciejewski, Drake University, Prashant Sakharkar, Roosevelt University. Objectives: The objective of this study was to review and analyze the direct and indirect costs associated with BD. Method: A systematic literature search was conducted through PubMed, PsychInfo, COCHRANE, and CINAHL to identify studies assessing healthcare costs with the treatment of BD from 2001 to 2017. Search was conducted with the title followed by MeSH terms: BD, cost of illness, cost of treatment, cost analysis. Data was abstracted for country of study origin, direct and indirect costs, perspective, time horizon and data sources used. Articles were excluded if the direct healthcare cost was not reported and cannot be derived. Reviews, commentaries or editorials, case reports or conference abstracts and articles in other than English language were excluded. Results: A total of 29 full text articles were included for final review. Majority (45\%) of the studies were conducted in the United States. Equal numbers of studies $(41 \%)$ were conducted from payers and societal perspective. Majority $(83 \%)$ of these studies included direct cost ranging from $\$ 7000$ $\$ 30.7$ Billion, whereas the indirect costs ranged between $\$ 35,000$ - $\$ 120$ Billion representing the extent of BD's economic burden. Cost data varied greatly from commercial to national databases as well the patient population. Implications: Our analyses explored BD as a major health problem with a substantial economic burden on healthcare systems and society suggesting that better disease management is warranted to decrease the economic burden of $\mathrm{BD}$ on patients and the society.

Healthcare Provider Perceptions of the Causes and Consequences of the Opioid Crisis in Northeastern Minnesota. Kelsey Lee Melgaard, University of Minnesota, Heather Blue, University of Minnesota, Dahly Ashley, University of Minnesota, Laura C. Palombi, University 


\section{American Journal of Pharmaceutical Education 2018; 82 (5) Article 7158.}

of Minnesota. Objectives: This study sought to learn more about health care providers' perceptions on the causes of and solutions to the opioid crisis. Method: One hundred and one healthcare professionals from four northern Minnesota cities attended a continuing medical education (CME) session that focused on use of naloxone, legislation pertaining to naloxone, and myths surrounding naloxone. Attendees were asked to complete a survey following the presentation regarding their perceptions of opioid use disorder and their observations of attitudes towards addiction within the healthcare community. A qualitative analysis of the collected surveys was conducted using a consensual qualitative research (CQR) method. Results: This study found that healthcare providers have widely variable perceptions, attitudes, and levels of support related to their care of patients with opioid use disorder. Also notable was the fact that the majority of survey responses were provider-centric even though the questions were patient-focused. Implications: Results of this survey may be useful in the construction of a model for healthcare providers and systems to identify and address negative attitudes towards opioid use disorder. Future work will expand education in the pharmacy core curriculum and future CME events, and will explore interprofessional interventions to reduce negative attitudes and improve health outcomes.

Health Literacy Among College Students. Briana Balsamo, Northeastern University, Jillian Egan, Northeastern University. Objectives: Health literacy is the ability to obtain, communicate, process, and understand basic health information/services necessary for medical decisions, contributing to improved health outcomes. We aim to assess health literacy levels and the perceived importance of healthy literacy of college students in order to foster core curriculum modifications to improve education and health. Method: Prospective survey to university students utilizing the validated METER health literacy assessment tool. METER contains a mix of 70 medical and false words and requires participants to identify actual medical words. Participant demographics, major, history of health-related coursework, health literacy attitudes and healthcare access were also collected. Results: Respondents completed 553 surveys (female $=80 \%$ ). Representation of each college (76 majors) and all years of study obtained. Most respondents $(55.5 \%)$ had not completed a health-related course (range:0$7+)$, stated confidence in understanding health-related information $(82.3 \%)$ and considered health literacy important (92\%). Two validated scoring methods were utilized; the first calculated correct choices from 40 actual health words (mean:35.4 \pm 7.32 ), the second subtracted for incorrectly selected words (mean:33.68 \pm 7.72 ). Most participants had functional health literacy (score: 35-40; score $2=65.5 \%$ ), followed by marginal (score: $21-34 ; 27.3 \%$ ) and low health literacy (score: 0-20; 7.2\%). College of Engineering students scored lower than those in the Bouve' College of Health Sciences $(p<0.05)$. Variations in scores also existed between different majors and demographics. Implications: While most college students appear to have moderate to functional health literacy, variation exists across colleges/ majors, highlighting need for additional education in this area.

Health Report Card: Evaluating Health Outcomes Through Student-Driven Comprehensive Service-Based Health Fairs Targeting Medicare Beneficiaries. David G. Carranza, University of the Pacific, Donna J. Cruz, University of the Pacific, Sheel M. Patel, University of the Pacific, Amandeep S. Raikhy, University of the Pacific, Bradley F. Ramos, University of the Pacific, Kristina M. Hoh, University of the Pacific, Olivia Page, University of the Pacific, Carly A. Ranson, University of the Pacific, Cynthia S. Valle-Oseguera, University of the Pacific, Edward L. Rogan, University of the Pacific, Rajul
A. Patel, University of the Pacific. Objectives: Provide a model for creating a comprehensive service-based health fair that enhances student learning, optimizes patient care, and assesses patient outcomes. Method: In the Fall of 2017, 14 health fairs targeting Medicare beneficiaries were held in 10 cities throughout Northern/Central California. Upon entry, patients received a card ("Healthcare Passport") containing a color-coded list of 13 available student-driven health screenings/services including Medication Therapy Management (MTM), Blood Pressure, Cholesterol, Diabetes, Immunizations, Bone Density, and several others. Each "Passport" was assigned a unique identification number to record screening/service uptake and facilitate patient tracking throughout the event. Results: In total, 910 patients utilized MTM services, with $822(90 \%)$ reporting a chronic condition for which a corresponding screening/service was offered. The most prevalent chronic conditions observed included hypertension 624 (69\%), hyperlipidemia 491(54\%), and diabetes 197(22\%). On average, MTM patients took advantage of 2 screenings/services. The uptake of hypertension, hyperlipidemia and diabetes screenings by those with the disease was $488(78 \%), 146(30 \%), 52(26 \%)$, respectively. Moreover, of those taking advantage of the service with the disease state $309(63 \%)$ blood pressure, $133(91 \%)$ cholesterol, and $43(83 \%)$ blood glucose readings were controlled according to current practice guidelines. Implications: Providing a student-driven, comprehensive service-based health fair using a "Passport" identification tool has multiple advantages, including: allowing tracking of screening/service uptake, providing insight into disease management, exposing students to a broader scope of patient care, developing practice-ready student pharmacists, and fulfilling Introductory Pharmacy Practice Experience requirements.

Identification of Barriers and Impact of Education for Herpes Zoster Vaccination Acceptance in Older Adults. Janelle Herren, MCPHS University, Paula J. Evans, MCPHS University-Worcester/ Manchester, Aileen Lemoine, Colleen Massey, MCPHS UniversityWorcester/Manchester. Objectives: Determine the primary factors that lead to herpes zoster (shingles) vaccination non-compliance and determine the impact of education and financial counseling on improving the rate of shingles vaccinations in older adults. Method: Pharmacists randomly surveyed call-in clients to the Pharmacy Outreach Program during Medicare Open Enrollment and in person during voluntary community drug education and medication counseling programs. Herpes zoster immunization status was determined and factors influencing that status were identified. Education was provided regarding shingles infection, post-herpetic neuralgia risk, financial options, and the availability of vaccines (Zostavax ${ }^{\circledR}$ and Shingrix ${ }^{\circledR}$ ). Follow-up calls are made 2-3 months after the first encounter to determine new vaccination status. Results: At this time, 220 patients aged 50 or over have been surveyed. 116 have not received any shingles vaccine; 96 have received the Zostavax ${ }^{\circledR}$ vaccine; 8 were unsure of their vaccine status. For those who had not received a shingles vaccine, the most common barriers included not knowing they needed a vaccine to prevent shingles (22.4\%), receiving no recommendation for the vaccine from their doctor or pharmacist $(22.4 \%)$, contraindications for the live vaccine (15.5\%), and lack of insurance or unacceptably high copay $(12.9 \%)$. The greatest determining factor for those already vaccinated was education and recommendation by a doctor or pharmacist on the need for the vaccine $(66.6 \%)$. Follow-up on the vaccine acceptance rate following education on available shingles vaccines is ongoing. Implications: Education by doctors and pharmacists is critical to the acceptance of the shingles vaccine in older adults. 


\section{American Journal of Pharmaceutical Education 2018; 82 (5) Article 7158.}

Identifying Novel Small Molecule Inhibitors of ERp57 Through a Medium Sized High Throughput Screen. Abigail Mae Henry, Western New England University, Megan M. Pantos, Western New England University, Christina Ann Verbetsky, Western New England University, Khaled Sahli, University of Reading, Gagan Flora, University of Reading, Sarah Ryan, University of Reading, Jonathan M. Gibbins, University of Reading, Daniel R. Kennedy, Western New England University. Objectives: Thiol isomerase enzymes such as ERp57 are thought to have polyfunctional involvement in the oncogenesis of cancer and thrombosis formation. Previous studies have examined the role of ERp57 in these processes using activity blocking antibodies or nonspecific thiol isomerase inhibitors such as rutin. In this study, we developed a high throughput screening method to identify the first specific small molecules inhibitors of ERp57. Method: 47,289 compounds were screened for ERp57 inhibitory activity using the insulin turbidity assay. The aggregation of insulin chain results in an increasing turbidity that can be measured at OD650. The reaction was scaled down into 1536-well plates and ran in a final volume of 10 ul per reaction, containing $100 \mathrm{ng}$ ERp57, $15 \mathrm{ug}$ of insulin and then $250 \mathrm{uM}$ of DTT to initiate the reaction. Results: Potential hits were identified by selecting those compounds which resulted in an OD reading that was below the average reading of the 48 control wells per plate. 46 compounds were cherry picked for follow-up studies, which rank ordered the compounds by potency and eliminated non-specific inhibitors. Based upon potency and potential for SAR studies, two main scaffolds were selected as leads. Implications: In this study we have identified potent and selective small molecule inhibitors of ERp57. The identification of small molecule inhibitors provides an opportunity to further explore the role of ERp57 in thrombosis as well as cancer development and could be useful in developing future novel therapies.

Impact of Accountability on Healthy Habits and Academic Performance in Pharmacy Students. Kelly A. Kempa, Wilkes University, Abby Stevens, Wilkes University, Nicole Pezzino, Wilkes University. Objectives: The objective of this project was to demonstrate the impact of accountability to exercise and the effect consistent exercise has on academic performance. The workload on pharmacy students is likely enough to produce stress. Exercise was found to be the fourth most popular stress reliever and additional studies show that performing vigorous exercise leads to reduced levels of perceived stress within college students. The time and dedication required to exercise routinely is significant, especially while maintaining good academic standing in pharmacy school. Method: A 25-item pre- and post-race questionnaire addressed demographic information, healthy habits (i.e.: sleep hygiene, running frequency), perceived benefit and study habits. Grade point averages (GPA) were also collected for statistical analysis. Study investigators utilized a paired t-test to analyze impact on GPA pre- and post- half marathon and a Spearman's correlation coefficient to compare the healthy habits on GPA. Results: Twenty-two (100\%) students responded to the survey. Grade point averages remained the same, or increased when students were training for the half marathon, without a significant change in the number of hours spent studying each week $(\mathrm{r}=0.662 ; \mathrm{p}<0.001$ and $\mathrm{r}=0.541$, $\mathrm{p}<0.014)$. Student perceived time management skills improved on days they incorporated running $(r=0.661 ; \mathrm{p}<0.02)$. Implications: Incorporating an exercise routine during pharmacy school, with accountability from classmates, led to similar or improved academic performance. Additionally, this did not result in changes in study or work habits and lead to a student-perceived improvement in time management. Future research could consider a more formal integration of an exercise regimen into the demanding curriculum.
Impact of a Video Adaptation of Noblesse Oblige on Rho Chi Membership Perceptions and Involvement. Shilvi Shah, Tracy Zhang, Helen Wang, Lucio Volino, Rutgers, The State University of New Jersey. Objectives: To evaluate the impact of a video adaptation of a noblesse oblige article on chapter members' perceptions regarding lifelong commitment to and moral obligations of Rho Chi involvement. Method: The Class of 2019 Rho Chi members were given an 8-question survey before (pre-survey) and 11-question survey (postsurvey) after viewing a video adaptation of an American Journal of Health-System Pharmacy's article on noblesse oblige and moral commitment (intervention). Paper-based pre- and post-surveys distributed during a general interest meeting collected members' self-perceptions regarding membership expectations. Pre-surveys also captured demographic information while post-surveys identified potential future video utility. Results: A total of 28 out of $35(80 \%)$ members completed both pre- and post-surveys. A majority $(85 \%)$ indicated the video had a positive impact on their perception of being in The Society. After viewing the video, $18 \%$ of respondents became interested in being more involved with Rho Chi initiatives while $25 \%$ felt a stronger obligation to the pharmacy profession. An additional 21\% identified using academic gifts to serve one's community as a minimum expectation for members. Most respondents (96\%) recommended showing the video to new members, preferably during or after the induction ceremony (88\%). Approximately half (53.6\%) felt the video could have a positive impact on non-members. Implications: Video adaptation of an article on noblesse oblige changed student members' perceptions regarding expectations for involvement in and a commitment towards Rho Chi. Utilization of a video to showcase noblesse oblige and moral commitment may provide value and assistance to other chapters and potentially non-members.

Impact of Prescription Drug Abuse Education in Health Professional Curriculum. Alexandria Marie Rivera, Lake Erie College of Osteopathic Medicine, Madison Ann Saxton, Amanda Craven, Lake Erie College of Osteopathic Medicine, Sean Gage, University of South Florida, Teri Runo, Lake Erie College of Osteopathic Medicine, Timothy Novak, Lake Erie College of Osteopathic Medicine. Objectives: To evaluate the current level of knowledge retained by medical, pharmacy and dental students at Lake Erie College of Osteopathic Medicine (LECOM) Bradenton on the topics of pain management and prescription drug abuse prevention using the existing curriculum. Also, to assess the impact of a new online curriculum on the health professional students' knowledge regarding these topics. Method: Students in the Doctor of Osteopathic Medicine (DO), Doctor of Pharmacy (PharmD) and Doctor of Dental Medicine (DMD) programs at LECOM Bradenton completed an online course on pain management and prescription drug abuse. As part of the course, the students provided demographic information, completed a current knowledge pre-assessment, viewed a presentation, and completed a post-exposure assessment. Results of the pre- and post- assessments were compared, as well as the data between the professional programs. Results: Aggregate data shows an overall 15\% average increase in assessment scores among all groups after exposure to the curriculum. Performance increased $7.2 \%$ among medical students, $12.5 \%$ for dental students and $31 \%$ for pharmacy students between pre- and postexposure assessments. Implications: Exposure to pain management and prescription drug abuse education among three Doctoral programs (Medical, Pharmacy and Dental) showed an overall increase in knowledge. These findings suggest that the level of understanding of pain management and prescription drug abuse from current education is improved upon implementation of this online course. Further investigation is needed 


\section{American Journal of Pharmaceutical Education 2018; 82 (5) Article 7158.}

to explain why pharmacy students demonstrated the largest increase in performance.

Implementing the Interprofessional Socialization and Valuing Scale for Health Students in Programs for the Underserved. Benjamin A. August, Justine S. Gortney, Wayne State University, Jennifer Mendez, Wayne State University. Objectives: Few instruments exist measuring socialization processes underlying interprofessional (IP) education. King et al. created a 24-item Interprofessional Socialization and Valuing Scale (ISVS) that evaluates a student's selfperceived ability, value, and comfort in working with others. Little information exists evaluating the ISVS in pharmacy and medical students. We incorporated ISVS into our student perception evaluations and measured IP socialization surrounding a student-run clinic serving individuals experiencing homelessness. Method: A Qualtrics link with the ISVS was emailed to pharmacy, medicine, social work, and physical therapy students 48 hours before and after participating in the clinic. Pre and post surveys were linked by unique identifiers. Attitudes regarding IP socialization were analyzed by discipline and overall. Power analysis was conducted using G-power to determine sufficient sample size using alpha of 0.05 , power of 0.8 , and effect of 0.7 ; the desired sample size was 15 students. IRB gave exemption. Results: Thirty-nine student pre/post matches from March 2017 to January 2018 were evaluated. Significance was met for all 3 survey domains (ability $\mathrm{p}=0.000$, value $\mathrm{p}=0.002$, comfort $\mathrm{p}=0.000$ ). After repeated clinic exposure, no difference was seen. Cronbach's alpha was 0.922 for initial responses. All pharmacy and social work students met the significance threshold for at least one domain. Implications: Establishing IP identities helps individuals become effective team members and understand their value to a healthcare team. The ISVS is a tool that can be used in student-run clinic evaluations to help students understand and reflect on their IP experiences.

Important Aspects Payers Consider When Making Formulary Decisions in the United States. Kelvin Pensuwan, St. John's University, Monica Hwang, St. John's University. Objectives: To identify aspects the United States (U.S.) payers look for when deciding to add or remove drugs from their formularies. Method: A literature search in two electronic databases (Embase and PubMed) was used to extract publications from 2015 to 2017. Search terms "health insurance", "reimbursement', “decision", “decision making", "payer", "formularies", and "United States" were used in identifying relevant articles. Out of the 196 publications extracted for abstract review, 20 publications were deemed relevant for this study objective and were selected for analysis. Publications were included if they mentioned any decision-making concepts or tools used by payers, publically and privately in the U.S., in their process of adding or removing drugs to their formularies. Publications that were not deemed relevant were excluded mainly due to lack of pertinent information along with lack of relevance to the U.S. payer system. Results: Four major aspects were discussed in the literature: economic, clinical, regulatory, and patient. The majority of the publications (14 publications) mentioned two combinations of the aspects (clinical and economic: 8, clinical and patient: 3 , regulatory and economic: 2 , or clinical and regulatory: 1 ). Only six publications mentioned one aspect per publication (economic: 4 or clinical: 2). Implications: Four main aspects (economic, clinical, regulatory, and patient) were identified as what US payers look for when making formulary decisions. As America progresses into the 21 st century, more studies are needed to examine if these four aspects will remain of an importance to the U.S. payer system and to what extent.
Improving Patient Educational Materials Regarding Generic Drugs: A Qualitative Study. Natalie Hohmann, Auburn University, Dan Surry, Auburn University, Kimberly B. Garza, Auburn University, Lindsey Hohmann, Auburn University, Ahmed U. Mishuk, Auburn University, Richard A. Hansen, Auburn University, Ilene Harris, IMPAQ International, LLC, Zippora Kiptanui, IMPAQ International, $L L C$, Jingjing Qian, Auburn University. Objectives: While nearly 9 in 10 prescriptions filled in the U.S. are for generics, consumers still have informational needs about generic drugs. To help educate patients about the FDA's rigorous review process, economics of pricing, and related issues about generic drugs, the FDA offers a range of educational materials. This study gathered patient/caregivers' input on the optimal content, format, channel, and level of satisfaction for FDAdeveloped educational materials about generic drugs. Method: Two focus groups were conducted with patients/caregivers at one public university in the Southeastern U.S.: 1) undergraduate students $(n=5)$, and 2) university employees $(n=6)$ recruited via email. Adults were eligible to participate if they self-identified as a patient/caregiver who managed prescription medications for themselves or friends/family. Each group discussed two generic drug educational materials (one brochure and one infographic). Focus groups were audio recorded, transcribed verbatim, and assessed via thematic analysis using Atlas. ti. Final codes were used to generate themes describing participants' opinions on the materials. Inter-coder reliability was high (Krippendorf's alpha 0.819). Results: A total of 13 codes were formed across 4 categories (material format, content, channel, and satisfaction). Six themes emerged from each group. Both groups stated a need for the materials to be visually appealing, emphasize generic drug cost savings to the consumer, reduce scare tactics when discussing adverse effects and safety, and be available from their medical providers. Implications: Tailoring these educational materials regarding generic drugs to the needs of patients/caregivers may help to improve patient/caregivers' acceptance and utilization of cost-saving generic drugs.

Improving Pharmacy Student Knowledge and Medicare Beneficiary Cost-Savings: A Community Partnership Over 5 Years. Lindsey Hohmann, Auburn University, Tessa J. Hastings, Auburn University, Salisa C. Westrick, Auburn University. Objectives: The Medicare Outreach Program was formed via a partnership between a school of pharmacy and State Health Insurance and Assistance Program (SHIP) in Alabama, with the goal of assisting beneficiaries in Medicare Part D plan selection and increasing pharmacy students' capacity to serve Medicare beneficiaries. Method: Pharmacy students were trained via an online quiz module integrated into the PharmD curriculum and student volunteers were certified as SHIP counselors. Measures were collected from 2013-2017 using online learning management software and an interviewer-assisted paper survey at community-based Program events. Outcomes included pharmacy students' training quiz scores, students' self-reported changes in knowledge, and Medicare beneficiaries' plan cost savings and satisfaction. Outcomes were analyzed using descriptive statistics. Results: On average, 114 pharmacy students were trained and 96 were certified each year, with 125 beneficiaries served at 12 events annually. Students' training quiz scores averaged (SD) $92.89 \%$ (1.12) per year. Across all years, over $87 \%$ of pharmacy students reported increased or greatly increased knowledge about Medicare prescription drug coverage and over $75 \%$ reported increased or greatly increased ability to help beneficiaries select the most appropriate Medicare Part D plan. After enrolling in a new plan, Medicare beneficiaries' average projected annual cost savings ranged from $\$ 278.71$ to $\$ 1,496.19$, and over 


\section{American Journal of Pharmaceutical Education 2018; 82 (5) Article 7158.}

$96 \%$ agreed or strongly agreed that they were satisfied with the service they received. Implications: Pharmacy student knowledge and beneficiary cost-savings were positively impacted by the Program. Community partnerships can be replicated between other schools of pharmacy and state agencies to prepare future pharmacists to assist Medicare beneficiaries.

Incorporating a Teaching Electronic Medical Record to Answer Patient-Specific Drug Information Questions in the Didactic Setting. Jacqueline Wasynczuk, Purdue University, Amy H. Sheehan, Purdue University. Objectives: Pharmacy graduates are expected to be practice-ready to deliver quality patient care. Development of pharmacy practice includes having a comprehensive knowledge of health informatics, including electronic medical records (EMRs). The objective of this study was to incorporate an EMR within a drug information (DI) assignment and to assess student perceptions of EMR use in the didactic setting. Method: Second-professional year students received a patient-specific DI question and were required to use an EMR to obtain information to provide an accurate response. Following completion of this assignment, students were invited to complete a 23 -item, retrospective pre-post survey instrument. The survey instrument was designed to collect student perceptions before and after the assignment regarding the importance of EMR access when answering patientspecific questions and the impact of didactic EMR use on their future performance and confidence. Results: A total of 132 students completed the survey instrument, resulting in a response rate of $88 \%$. Only $28.8 \%$ of respondents reported prior experience using an EMR, $45.8 \%$ of these respondents had experience from paid employment. There was a significant increase from baseline in respondents who agreed that didactic use of an EMR increased their knowledge of $(61.4 \%$ to $89.2 \%$ ), and confidence in use of EMR systems (20.5\% to $82.8 \%$ ). Implications: Implementation of an EMR within didactic instruction may serve as the first exposure to health informatics tools for students and may also positively impact students' knowledge and confidence of these tools prior to entry into pharmacy practice.

Incorporating Telepharmacy in Pharmacy Practice Experiences. Autumn Peck, Wilkes University, Kristina Powers, Wilkes University, Michelle Holt-Macey, Wilkes University. Objectives: To provide an introductory pharmacy practice experience (IPPE) in clinical telepharmacy for all third year professional pharmacy (P3) students allowing for real-world application of the Pharmacists' Patient Care Process (PPCP), with an emphasis on communication skills. Method: After orientation, P3 students are fully integrated into the telepharmacy system at the Geisinger Call Center. Activities include responding to clinical inquiries from patients and physicians, placing proactive refill calls, assessing adherence, reviewing patient profiles, and recommending monitoring, using Geisinger collaborative practice agreements. A faculty preceptor and Geisinger pharmacists provide oversight and feedback. A peer-mentor program was established, under a layered learning model, for first year professional pharmacy (P1) students for a 4 hour experience with P3 students. P3s complete a course evaluation at the conclusion of every IPPE. Results: In a cutting-edge pharmacy setting, students carry out practical applications of clinical, communication, and decision-making skills. Incorporation of layered learning, PPCP, use of collaborative practice agreements, and patient advocacy make this a unique IPPE. P3 students agree or strongly agree that the experience is worthwhile $(97.6 \%)$ and they would recommend it to other students (95.1\%). Implications: Incorporating an IPPE in telepharmacy is a method of educating students about new roles for pharmacists positively impacting patient care in the community. This model empowers students to apply classroom knowledge and skills in a unique patient care environment. In the future APPE students may be incorporated to the site and the layered learning model. Further research into the benefits of this IPPE setting is warranted.

Inhibitory Effect of Bioactive Stilbenes on MTA1/ETS2 Axis in Prostate Cancer. Kshiti Hemant Dholakia, Long Island University, Avinash Kumar, Long Island University, Anait S. Levenson, Long Island University. Objectives: Using ChIP-seq analysis, we have previously identified ETS2 (erythroblastosis E26 transformation-specific 2) as a downstream target of metastasis-associated protein 1 (MTA1), which is overexpressed in aggressive prostate cancer. Accordingly, we observed co-localization and direct interaction between MTA1 and ETS2 in prostate cancer cells. Further, we found that prostate tissues from prostate-specific Pten heterozygous mice, which express elevated levels of MTA1, also express increased ETS2 levels. Moreover, prostate-specific MTA1 transgenic mice also display significantly increased ETS2 protein levels in the prostate tissues. While elucidating the mechanisms of "oncogenic co-operation" between MTA1 and ETS2 to promote prostate cancer are in progress, we addressed targeting of MTA1/ETS2 in prostate cancer cells. Method: In this study, we show that MTA1 knockdown LNCaP, DU145, and PC3M prostate cancer cells exhibit downregulation of ETS2 on protein and mRNA levels. Furthermore, pharmacological inhibition of MTA1 by dietary polyphenols, i.e. resveratrol, double resveratrol (Gnetin C) and pterostilbene, was associated with downregulation of ETS2 on protein and mRNA levels. Results: We observed dose-dependent downregulation of MTA1 in DU145 and PC3M prostate cancer cells by all stilbenes tested. Interestingly, Gnetin $\mathrm{C}$ showed more significant downregulation of ETS2 along with MTA1 compared to resveratrol and pterostilbene. Implications: Our study suggests that natural stilbenes, particularly Gnetin $\mathrm{C}$ through its robust ability to target the MTA1-ETS2 network, may become novel chemopreventive and therapeutic strategies for clinical development in prostate cancer.

Integrating Pharmaceutics With Medication Safety: A Novel Curricular Team Activity to Promote Student Learning. Joe Su, Pacific University Oregon, Jeff Fortner, Pacific University Oregon, Reza Karimi, Pacific University Oregon, Deepa Rao, Pacific University Oregon. Objectives: We generated a curricular activity, Integrating Pharmaceutics with Medication Safety (IPMS), to enhance learning in pharmaceutics and compounding, to highlight the importance of patient safety, and to promote critical thinking and presentation skills. Method: The IPMS activity was implemented over three days during pharmaceutics class for first-year pharmacy students. Teams of 6-7 members were assigned a dosage form (e.g. tablet, ointment) and were instructed to find a related drug product that was linked to an authentic medication error. Each team was responsible for preparing a report and 15-minute presentation that included composition and compounding procedure of drug product, indication(s), mechanism of action, error incident, and error prevention. A voluntary student survey assessed the activity's effectiveness in the context of Bloom's taxonomy. Results: The survey received a $72 \%$ response rate $(n=102)$ with $93 \%$ of the students agreeing the IPMS activity enhanced awareness of the devastating effects a medication error can cause to patients and the healthcare community. 92\% agreed that the IPMS activity created an environment that promoted knowledge, comprehension, application, analysis, synthesis, and evaluation of medication safety concepts. In addition, 90\% agreed that IPMS maximized the knowledge integration of pharmaceutics coursework and 86\% agreed IPMS strengthened their learning of compounding procedures. Implications: The IPMS activity promoted student learning of pharmaceutics and compounding 


\section{American Journal of Pharmaceutical Education 2018; 82 (5) Article 7158.}

principles critical to Domain 1 foundational knowledge as outlined in CAPE 2013 and ACPE Standards 2016.

JOAB Series as Novel Pro-Inflammatory Cytokine Suppressants: Synthesis and In Vitro Pharmacological Evaluation. Ashley A. Bill, University of Saint Joseph, Christine Agudosi, University of Saint Joseph, Doreen E. Szollosi, University of Saint Joseph, Ivan O. Edafiogho, University of Saint Joseph, Ola A. Ghoneim, University of Saint Joseph. Objectives: Tumor Necrosis Factor alpha (TNF-alpha) and Interleukin-6 (IL-6) are pro-inflammatory cytokines playing a major role in the immune response. These cytokines are released from immune cells, when an infection is present. However, overproduction can cause the development of tissue/organ injury seen in sepsis, severe asthma, and autoimmunity. In our laboratory, enaminone E121 suppressed TNF-alpha and IL-6 in macrophages stimulated with lipopolysaccharide (LPS). E121 performance encouraged us to examine its involvement in suppressing inflammation, therefore we designed the JODI series where $\mathrm{N}$-arylpiperazine motif was incorporated into the aromatic side of the enaminone to examine its effect on inflammation suppression. JODI's positive results encouraged further exploration of these piperazino-enaminones, hence JOAB series was developed to identify the key structural components that are linked to proinflammatory cytokine suppression. Method: The JOAB series explored four possible modifications done methodically to determine the key pharmacophoric elements of this series. Chemical synthesis was accomplished by amination of the corresponding methyl/ethyl 2,4-dioxo-6-cyclohexanecarboxylate with the desired substituted piperazine in ethanol. The in-vitro biological activity was tested in a J774 macrophage cell line stimulated with LPS. The release of cytokines was measured after 24 hours using enzyme-linked immunosorbent assay (ELISA). Results: Synthesis of sixteen novel compounds from the JOAB series was accomplished. When examining their ability to reduce TNF-alpha or IL-6, four compounds have shown to significantly suppress TNF-alpha and IL-6 in a dose-dependent manner, and superior to E121. Implications: JOAB series and piperazinoenaminones represent a potential novel approach for inflammatory disorders as pro-inflammatory cytokine suppressants.

Knowledge and Attitude of Antibiotic Use Amongst College Students. Lameesa Z. Dhanani, St. John's University, Shireen Farzadeh, St. John's University, Yuman Lee, St. John's University. Objectives: The objectives of this research were to evaluate the knowledge and attitude of antibiotic use amongst college students at St. John's University and if students from various colleges or ethnicities may have implications to the differences in the results. Method: A 10-item, voluntary, anonymous survey was administered to assess baseline demographics, knowledge of antibiotics (true, false, don't know), and attitude towards antibiotic use (5-point Likert scale strongly agree to strongly disagree). Results: Out of 200 respondents, the average score for the knowledge-based questions was $75.1 \%$ (58.3\%-100\%). For the attitude-based questions, $24.5 \%$ (49/200) strongly agree/agree to expecting a doctor to prescribe antibiotics for a cold; $37.5 \%$ (75/ 200) strongly agree/agree to stopping antibiotics when they feel better; $10 \%(20 / 200)$ strongly agree/agree to giving antibiotics to someone who is sick; $8.5 \%$ (17/200) strongly agree/agree to acceptable to share antibiotics. In regards to knowledge, the College of Pharmacy \& Health Sciences scored the highest (84.6\%) while the College of Professional Studies scored the lowest (58.3\%). Asians/SE Asians scored the highest $(83.33 \%)$ while Hispanics/Latinos scored the lowest (51.78\%). In regards to attitude, the Peter J. Tobin College of Business and Black/African American/Caribbean ethnicity had the most strongly agree/agree to the statements above. Implications: Survey results revealed a $24.9 \%$ deficit in knowledge and a $34.9 \%$ inappropriate attitude of antibiotic use amongst college students. These results were more prominent in non-health sciences related colleges and nonAsian ethnicities. Education should be targeted to this population in order to effectively combat antimicrobial resistance.

Legal Issues Affecting Approved Biosimilars in the US. Gregory A. Phelps, The University of Tennessee, Junling Wang, The University of Tennessee, Carol Schwab, The University of Tennessee, Minghui Sam Li, The University of Tennessee. Objectives: The Biologic Price Competition and Innovation (BPCI) Act encourages marketing of biosimilars by shortening the FDA approval process. Nine biosimilars were approved by the FDA since the implementation of the BPCI act. This study aims to analyze nine FDA-approved biosimilars in the US and compare the obstacles hindering their availability. Method: We reviewed and summarized approval dates for biosimilars, patent expiration dates for originator biologics, and market available dates for biosimilars. We also examined obstacles hindering availability of certain biosimilars in the US. Results: Among nine FDA-approved biosimilars, only three (filgrastim-sndz, infliximab-dyyb, and infliximab-abda) are currently available on the US market. Other six biosimilars (etanercept-szzs, adalimumab-atta, adalimumab-adbm, bevacizumab-awwb, trastuzumab-dkst, and infliximab-qbtx) are not available yet. Etanercept-szzs has yet to hit the market due to legal issues, as the manufacturer of etanercept has sued the manufacturer of etanercept-szzs over patent issues. Adalimumab-atto is set to release in January 2023 after a settlement was made between manufacturers of adalimumab and adalimumab-atto. Similarly, another adalimumab biosimilar, adalimumab-adbm, will not be available before 2023. Bevacizumab still has patent exclusivity until July 2019 so the market launch of bevacizumab-awwb is not to be expected soon. An agreement between manufacturers of trastuzumab and trastuzumab-dkst delays the launch of trastuzumab-dkst until at least 2019. The manufacturer of infliximab-qbtx has one infliximab biosimilar on the market (infliximab-dyyb) so it does not plan to make infliximab-qbtx available soon. Implications: Legal disputes, settlements between manufacturers, and patent protections are the main hindrance for market availability of the FDA-approved biosimilars.

Manikins or Standardized Patients: Is There a Difference? Nicole M. Perea, Washington State University, Taylor G. Bertsch, Washington State University, Kimberly C. McKeirnan, Washington State University. Objectives: To assess the differences in using standardized patients and manikins in the training method and application of physical exam techniques of student pharmacists. Method: Five physical exam techniques were presented to student pharmacists by practicing pharmacists in a team based setting. Students will practice the physical exam techniques on both live patients and a manikin. A final exam will then be given in a team based setting which will include one physical exam. Student preference, cost and pass rate will be assessed in comparing training methods. Student preference will be assessed by a student survey given after the final exam. Cost will be assessed using an incremental cost effectiveness ratio analyzing the overall cost and maintenance of a manikin and the cost of training and employing a standardized patient. Student pass rate will be based on the final exam. Results: This is an ongoing analysis during the Spring 2018 semester that will be completed in May of 2018. Implications: As the scope of pharmacy practice increases, it becomes increasingly necessary to add physical assessment and point of care techniques into the doctor of pharmacy curriculum. With the introduction of these services, the cost and methods of their implementation need to be assessed as well. At the conclusion of this assessment, it should provide a multi 


\section{American Journal of Pharmaceutical Education 2018; 82 (5) Article 7158.}

factorial perspective on the training methods and application of physical exam techniques in the training of student pharmacists by practicing pharmacists.

Mentoring Undergraduates in Academic Research Laboratories: What to Know/Do/Expect as a Graduate Student and Faculty Mentor. Chad Richard Johnson, University of Maryland, Andrew Coop, University of Maryland, Shannon R. Tucker, University of Maryland. Objectives: To assess the necessary tools needed to properly introduce undergraduate students to graduate level research and the challenges that face a graduate student and faculty mentor. Method: The University of Maryland School of Pharmacy partners with Stevenson University to mentor summer undergraduate interns majoring in Chemistry. I have been responsible for mentoring and supervising two students in the following ways: introducing them to research, teaching them essential laboratory skills, assessing progress and understanding of the project, and assisting them with drafting a final report and poster to present their work. These experiences have allowed us to develop an effective plan to assist in the undergraduate's adjustment to a graduate laboratory setting and help them prepare for a research-focused professional degree. Results: Four elements were important in the success of the summer intern: (1) Introduction to the project he/she will be working on and assigned readings for the first week. (2) Introduction to basic laboratory procedures, safety protocols, essentials for handling chemicals, laboratory expectations, laboratory notebook protocols, and the review of knowledge from courses. (3) Conduct monthly assessments to review performance and reinforce project understanding. (4) Advise/assist intern in preparing their final report and poster. Implications: By utilizing this simple four-step plan undergraduate students can integrate into a graduate research laboratory and build confidence in the pursuit of a research focused professional degree. Mentoring requires a significant time commitment from the graduate student and faculty mentor, but serves as a valuable experience for the undergraduate in preparation for a future graduate education.

Mindful Traits and Metacognitive Awareness in Pharmacy Students. David Anthony DePalma, Northeastern University, Amanda Kim, Northeastern University, Monica Marie Patterson, Northeastern University, Margarita V. DiVall, Northeastern University, Jenny A. Van Amburgh, Northeastern University. Objectives: Recent literature has identified the role of metacognition in learning and decision making. Metacognition is of particular interest to the health professions, including pharmacy education and practice. Recent studies of mindfulness practice have suggested a relationship to metacognition. We sought to further define the role of mindfulness in metacognitive function in a cohort of pharmacy students at Northeastern University. Method: Two validated instruments were used as part of a web-based survey administered to P2 and P3 pharmacy students: The Metacognitive Awareness Inventory (MAI) and the Cognitive and Affective Mindfulness Scale - Revised (CAMS-R). Additional questions were asked to identify demographic characteristics, ethnic/racial background, religious and mindfulness practices. Pearson correlation coefficient was calculated for total CAMS-R and total and subscale MAI scores for entire cohort and sub-groups. Results: Among respondents $(n=196$, 72\%), 148 (54\%) completed all questions and were used for analysis. Total MAI and CAMS-R scores correlated with moderate strength $(\mathrm{r}=0.35)$ across the cohort. Correlation was moderate to high $(\mathrm{r}=0.3$ 0.74 ) among all sub-groups analyzed, with the highest correlation among male respondents $(n=44, r=0.52)$ and African-American respondents $(n=8, r=0.74)$. Across the whole cohort, CAMS-R scoring correlated most highly with the "procedural knowledge" MAI subscale $(\mathrm{r}=0.33)$. Implications: Metacognition influences planning, cognitive monitoring and performance. In practice, metacognition impacts a pharmacist's performance in critical situations. The detected correlation suggests a positive relationship between mindfulness and metacognition. Future research is warranted to investigate whether mindfulness training influences metacognition.

Modulation of Adipogenesis in 3T3-L1 Cells Using Natural Products: A Strategy to Prevent Obesity. Joe Su, Pacific University Oregon, Brianna M. Cote, Oregon State University, Karen Seo, Pacific University Oregon, Deepa Rao, Pacific University Oregon. Objectives: Obesity is one of the leading causes of preventable death, and options for its prevention are limited. Many natural products (NP) are purported to have anti-obesity effects. We hypothesize that NP such as resveratrol (RES), curcumin (CUR), glycyrrhetic acid (18GA), epigallocatechin gallate (EGCG), and hydroxyl citric acid (HCA) individually and in combination will prevent the maturation of pre-adipocytes (3T3-L1) as an obesity prevention strategy. These NP have demonstrated the ability to directly or indirectly affect PPAR $\gamma$, the master regulator for adipogenesis. Method: Cell viability of NP is assessed in 3T3-L1 cells seeded in 96 well plates post $48 \mathrm{hr}$ treatment with $0.01-1000 \mu \mathrm{M}$ NP individually or in combination. Cell viability is assessed by fluorescence using CellTiter Blue ${ }^{\circledR}$. Combination effects are assessed using CompuSyn software. Inhibition of pre-adipocyte differentiation is assessed using Nile Red post maturation of 3T3-L1 cells. Cells are treated with NP at IC50, 0.5*IC50, and $0.1 *$ IC50 for $2 \mathrm{hr}$, followed by Nile Red addition for $10 \mathrm{~min}$ and fluorescence assessed at 485nmEX/590nmEM. Results: Pre-adipocyte cell viability IC50 values were calculated for RES $(189.7 \pm 10.7 \mu \mathrm{M})$, QUE $(186.0 \pm 36.8 \mu \mathrm{M})$, CUR $(43.3 \pm 2.6 \mu \mathrm{M}), 18 \mathrm{GA}(75.1 \pm 21.7 \mu \mathrm{M})$, and EGCG $(239.3 \pm 22.6 \mu \mathrm{M})$. All NP showed a concentration dependent decrease in intracellular lipids with the highest effects seen at IC50 values. CUR and EGCG demonstrated the strongest effects at $0.5^{*} \mathrm{IC} 50$ on intracellular lipid accumulation. Implications: Co-administration of combinations of NP individually or in combination may be a viable strategy to prevent maturation of pre-adipocytes. Future studies are needed to formulate bioavailable forms of this combination for oral delivery.

Motivational Interviewing-Trained Pharmacists: Clinical and Humanistic Outcomes in a Diabetes Worksite Wellness Program. Gladys Ekong, Auburn University, Jan Kavookjian, Auburn University. Objectives: Motivational Interviewing (MI) has emerged as an evidence-based, patient-centered skills set but little is reported about MI-trained pharmacist impact on humanistic outcomes. The aim of this study was to evaluate the effectiveness of MI-trained pharmacist on clinical and humanistic outcomes among diabetes patients in a hospital-based Worksite Wellness Program (WWP). Method: A quasiexperimental study design was implemented among 170 eligible participants $\geq 19$ years, taking hypoglycemic medications and/or insulin. The 3-month intervention was delivered by MI-trained clinical pharmacists $(n=2)$ and PGY-1 resident. Data collected pre and post-intervention included clinical (hemoglobin A1C, blood pressure, BMI, and depressive symptoms via PHQ-9), and humanistic outcomes (quality of life via SF-12). The PHQ- 9 score was categorized by severity (minimal ( $\leq 4)$ and mild-moderate depression (5-14)). Data analyses included descriptive statistics and t-test; significance level was $p<0.05$. Results: There were 53 participants at baseline and 35 post-intervention; 53\% female and $\sim 54$ years old. For clinical outcomes, diastolic blood pressure improved significantly ( $\mathrm{p}=0.0002)$. The SF-12 Physical Component Summary (PCS) and Mental Component Summary (MCS) improved from 47.0 to $47.7(+0.7)$ and 50.9 to $51.1(+0.2)$ respectively, but 


\section{American Journal of Pharmaceutical Education 2018; 82 (5) Article 7158.}

change was not statistically significant. Analyses between the $\mathrm{SF}=12$ and PHQ-9 severity categories revealed statistically significant associations; participants reporting lower depressive symptoms also reported higher quality of life for MCS $(p=0.006)$ and PCS ( $p=0.047)$. Implications: Pharmacists play an important role on interprofessional disease management teams. Future research can explore student trainings where a team-based approach to diabetes management could benefit patients with depressive symptoms.

Operation Naloxone: Opioid Overdose Prevention Service Learning for Health Professions Students. Lubna Mazin, The University of Texas at Austin, Lucas G. Hill, The University of Texas at Austin, Kirk Evoy, The University of Texas at Austin, Kenneth A. Lawson, The University of Texas at Austin, Lubna Mazin, The University of Texas at Austin. Objectives: To prepare health professions students to train community members to respond to opioid overdoses with naloxone, and to determine the effect of program participation on overdoserelated knowledge, self-efficacy, and attitudes regarding harm reduction. Method: Faculty and student directors led several 90-minute train-the-trainer seminars for health professions students in Austin, San Antonio, and Houston. The seminars provided foundational knowledge regarding opioid overdose epidemiology, risk factors, symptoms, evidence-based response, naloxone formulations, case scenarios, and questions from community members. Pre- and post-training assessments evaluating overdose-related knowledge, self-efficacy, and attitudes regarding harm reduction were embedded within the seminar. Participants were invited to volunteer in a series of outreach events to provide overdose prevention education to community members. A follow-up assessment was administered electronically approximately three months after the initial seminars. Results: 344 health professions students were trained by Operation Naloxone. Subjects demonstrated significantly improved overdose-related knowledge $(4.23 / 9$ vs $6.90 / 9, \mathrm{p}<.001)$, self-efficacy $(5.22 / 10$ vs $8.82 / 10$, $\mathrm{p}<.001$ ), and more positive attitudes regarding harm reduction $(16.03 / 20$ vs $17.89 / 20, p<.001)$ when comparing the difference between pre vs post assessments. The same was found in pre vs follow-up assessment. While these scores declined slightly after three months, they continued to be significantly higher than baseline. Participation in community outreach events did not affect results of the follow-up assessment. Implications: The service learning program model is effective in impacting students' knowledge, self-efficacy, and attitudes through initial training. Brief community outreach is likely insufficient to further enhance these outcomes.

Palbociclib Synergistically Enhances the Anticancer Activity of Cisplatin in P53-Mutant Model of Upper Gastrointestinal Cancers. Dhvanir Nikunj Kansara, Long Island University, Amruta S. Samant, Long Island University, Priya Pancholi, Dana Farber Cancer Institute, Tanvi Visal, Dana-Farber Cancer Institute, Shraddha Patel, Long Island University, Vikas Sehdev, Presbyterian College. Objectives: Upper Gastrointestinal Cancers (UGCs) respond poorly to conventional chemotherapy due to overactive intrinsic mechanisms that mediate cellular proliferation. Increased activity of cyclin-dependent kinases 4/6 (CDK4/6) is one such mechanism that can be specifically targeted with Palbociclib, a second generation CDK4/6 specific inhibitor. Since Cisplatin (CDDP) is a first line therapeutic agent frequently used for treatment of UGCs we hypothesized that Palbociclib would complement the DNA-damaging activity of CDDP. Method: To test this hypothesis, we evaluated the effect of Palbociclib treatment alone and/or in combination with CDDP on FLO-1 (P53-mutant) UGC cell viability, survival, and cell cycle progression, respectively. The MTT cell viability assay and Compusyn mediated median effect plot analysis (MEPA)(Chou and Talaly) were used to determine synergistic drug combinations of Palbociclib and CDDP in FLO-1 UGC cells. Results: The cell viability data, cell survival data, and MEPA indicated that Palbociclib and CDDP show significant synergistic anticancer activity in FLO-1 UGC cells at a ratio of $1: 2$ and 1:3, respectively. In addition, the Combination Index (CI) values of 0.66 and 0.71 for the aforementioned combination ratios suggests a strong synergism between the two drugs. Furthermore, the cell cycle data exhibited a marked increase $(p<0.05)$ in the percentage of FLO- 1 cells in the sub-G1-phase (cell death) after treatment with Palbocicb and CDDP combinations for $72 \mathrm{hrs}$., respectively. Implications: The observed synergism between Palbociclib and CDDP can potentially translate into reduced individual drug dosages and toxicity for Palbociclib and/or CDDP when used in combination.

Patient Characteristics Associated With the ADE Action Plan Targets. Fatimah M. Sherbeny, Nova Southeastern University, Julie Lamoureux, Silvia Rabionet, Nova Southeastern University, Leanne Lai, Nova Southeastern University, Barry A. Bleidt, Nova Southeastern University. Objectives: In 2014, the Office of Disease Prevention and Health Promotion released the National Action Plan for Adverse Drug Event Prevention (ADE Action Plan). This research focused on the three medication classes targeted by this Plan: anticoagulants, diabetes agents, and opioids. The objective of this study was to explore the association between patient characteristics and the national incidence rate of adverse drug events during hospitalization. Method: This study was retrospective exploratory secondary data analysis. The National Inpatient Sample, 2009-2014, datafiles were used to explore the national incidence rates of ADEs. ICD-9 codes were used to identify the study population. Patient characteristics associated with ADEs included age, gender, racelethnicity, comorbidities, and type of payer. Results: The average national rate of ADEs during 2009-2014 was 44,824/year for anticoagulants, 8,493/year for diabetes agents, and $31,545 /$ year for opioids. However, this rate varied based on patient demographics, payer type and comorbidities. For instance, ADE rates were highly associated with White, older patients, and Medicare beneficiaries. Gender differences were only significant in opioid-related ADES. Also, there was no association between comorbidities and ADE rates except for opioids. Implications: The national incidence rate of hospital ADEs was highly associated with patient characteristics. ADE prevention strategies in hospital setting should be tailored based on patient's factors along with medication-related factors.

Perception of Breastfeeding Support Amongst Women Faculty at Colleges of Pharmacy in the United States. Kadyn Pace, Harding University, Jazmin Culpepper, Harding University, Gabriella Douglass, Harding University, Mendy McClelland, Harding University, Kaci A. Bohn, Harding University. Objectives: Breastfeeding reduces the risk of cancer and diabetes in nursing mothers and the incidence of obesity and asthma in infants. Currently, more than $60 \%$ of pharmacists are women and breastfeeding in the work place is becoming more relevant. Unfortunately, studies show a decline in breastfeeding due, in part, to lack of support in the work place. Our survey was designed to assess the perception of breastfeeding support amongst female faculty at colleges of pharmacy in the United States. Method: A cross-sectional study surveyed female faculty who were currently pumping or had previously pumped breast milk while working at a college of pharmacy. Results: $55.7 \%$ of women stated the amount of time spent pumping was influenced by support in the work place (facilities, administration, schedule), of which, $41.8 \%$ said it decreased their time pumping. $91.6 \%$ said space, such as a private office $(60.8 \%)$ or a dedicated breastfeeding space $(24.0 \%)$, was available on 


\section{American Journal of Pharmaceutical Education 2018; 82 (5) Article 7158.}

campus. However, the space was either unsecured or dirty $(13.1 \%$ and $6.23 \%$ respectively) and $1.77 \%$ used a restroom. Sixty three percent responded being unaware of any state legislation to protect nursing mothers and only $31.4 \% \%$ stated they were comfortable asking their administration for support. Implications: Breastfeeding is a critical component of women's health and more women are entering the pharmacy profession each year, including academic pharmacy. Women faculty should be encouraged to communicate with administration to foster a breastfeeding friendly working atmosphere. And as colleges of pharmacy, we should ensure women faculty are fully supported in their endeavors to breastfeed.

Perceptions of a Tobacco Cessation Train-the-Trainer Program for Pharmacy Faculty Members: A Qualitative Assessment. Nervana Elkhadragy, Purdue University, Robin L. Corelli, University of California, San Francisco, Alissa Russ, Purdue University, Margie E. Snyder, Purdue University, Karen S. Hudmon, Purdue University. Objectives: Between 2003-2005, pharmacy faculty members $(n=191)$ participated in a national train-the-trainer program to disseminate a shared tobacco cessation curriculum (Rx for Change; rxforchange.ucsf.edu) for pharmacy schools across the United States. The objective of the current study was to characterize faculty perceptions of the program, subsequent efforts for curricular implementation, and impact of training on their career development. Method: A subset of faculty members who participated in the train-the-trainer workshops were randomly selected allowing for snowball sampling, to participate in a semi-structured telephone interview. The qualitative data were analyzed inductively in four iterative steps: data immersion, coding, categorizing, and identifying themes. Results: Of the invited faculty members, $18(60 \%)$ participated. All participants reported having implemented components of the Rx for Change materials at their institution. Qualitative analyses yielded a descriptive model describing the use of skills learned from the training to benefit the participant's teaching, practice, networking, and research. The following major themes emerged: (1) the curriculum provided high quality, evidencebased tools that supported a hybrid approach to teaching; (2) participants appreciated opportunities for professional networking; (3) the training established the faculty member's credibility; (4) the training enhanced their capacity for conducting tobacco-related research and; (5) the training assisted them in integrating tobacco cessation counseling in clinical practice. Implications: Results from this study will inform the development of a subsequent quantitative survey to be administered to the entire population of faculty participants, to estimate the impact of a shared national curriculum disseminated via a faculty training program over a period of nearly 15 years.

Pharmacological Characterization of Nicotinic Acetylcholine Receptors Positive Allosteric Modulators: LY2087101 and CMPI. Abisola Tairu, Texas A\&M University, Ayman K. Hamouda, The University of Texas at Tyler. Objectives: Positive Allosteric Modulators (PAMs) have potential therapeutic application in neurological disorders involving nAChRs. PAMs enhance the potency and efficacy of acetylcholine (ACh) by binding to sites different from the agonist (ACh) binding site. LY2087101 and CMPI have been identified in previous studies as PAMs of $\alpha 4 \beta 2 \mathrm{nAChRs}$ and shown to potentiate responses of $(\alpha 4) 3(\beta 2) 2$ isoform of $n A C h R$. This selectivity highlights the importance of $(\alpha 4) 3(\beta 2) 2$ isoform of $n A C h R$ for drug targeting, however, it is impractical to design PAMs without knowing their binding site on the receptor. In this study, we characterized these PAM compounds and their interaction with $\alpha 4 \beta 2 \mathrm{nAChR}$ to identify their binding sites within the $\alpha 4 \beta 2$ nAChRs. Method: Points mutations were introduced into pSP6polyA plasmid with cDNA encoding for human alpha4 nAChR subunit. Xenopus oocytes were injected with nAChR subunits cRNA. Two electrode clamp recording of Xenopus oocytes were performed, and each recording included 10seconds of ACh with or without LY2087101 or CMPI. Results: Binding sites of LY2087101 was identified within the $\alpha 4 \beta 2$ nAChRs - within the $\alpha 4$ subunit helix bundle at the level of $\alpha 4 \mathrm{~L} 260 \mathrm{M}$ and $\alpha 4 \mathrm{~T} 261 \mathrm{D}$, and the inter-subunit site at the $\alpha 4: \alpha 4$ subunit interface above the level of $\alpha 4 \mathrm{~F} 316 \mathrm{~L}$. CMPI binds at the extracellular site at the $\alpha 4: \alpha 4$ subunit interface. Mutations at $\alpha 4$ Gly41, $\alpha 4$ Lys64, and $\alpha 4$ Thr66 increased potentiation of $(\alpha 4) 3(\beta 2) 2 \mathrm{nAChRs}$ by CMPI, however mutations at $\alpha 4 \mathrm{G} \ln 124$ and $\alpha 4 \mathrm{Thr} 126$ reduced potentiation by CMPI. Implications: Revealing structural information to facilitate the design of a nAChR PAM that modulate $\mathrm{nAChR}$ will provide potential therapeutic uses that have little effect on receptor desensitization.

Pharmacy Student Interest in Computer-Assisted Learning (CAL) for a Pharmaceutical Calculations Course. Taylor L. Page, Rosalind Franklin University of Medicine and Science, Jayna Sharma, Rosalind Franklin University of Medicine and Science, Francis L. Hughes, Rosalind Franklin University of Medicine and Science, Kristen Ahlschwede, Rosalind Franklin University of Medicine and Science, Danielle M. Candelario, Rosalind Franklin University of Medicine and Science. Objectives: Computer-assisted learning (CAL) is any computer-administered program that delivers interactive learning (eg. video games, computer simulation). CAL has been used to enhance and apply students' knowledge at varying levels of education and has been previously shown to be useful, valuable, and enjoyable for students. CAL through video game patient simulations may reinforce difficult concepts and provide calculations practice, improving comprehension. The Pharmaceutical Calculations course, is a required 3-credit course for first year pharmacy students. The purpose of this study is to evaluate student interest and potential usefulness of incorporating CAL into a Pharmaceutical Calculations course. Method: An IRB approved, anonymous 12-item survey was emailed to the pharmacy classes of 2019 and 2020 in the Fall of 2017. The survey consisted of 5 demographic questions and 7 Likert-type questions. Results: Response rate of $43.6 \%(n=58)$. Majority age range $23-$ 27 years (69\%), 76\% female, $52 \%$ respondents from the Class of 2020. Forty-eight percent of students cited having minimal CAL experience. Respondents' preferred learning methods were self-study $(43 \%)$ or small group discussion (35\%). The majority of students (95\%) wished to see the clinical impact of their calculations. Most agreed CAL would have increased their interest (83\%) and understanding (74\%), and better prepared them for future practice (83\%). Implications: CAL and computerized patient simulations may present a unique opportunity for students learning pharmaceutical calculations. Results suggest CAL would increase student interest, and possibly better prepare students for future practice. Subsequently, study investigators will introduce CAL into pharmaceutical calculations in the upcoming academic year.

Pharmacy Students' Perceptions of Coping Mechanisms and Resources Provided by Their Institution. Mona Desai, Midwestern University/Downers Grove, Ana C. Quinones-Boex, Midwestern University/Downers Grove. Objectives: This study examined pharmacy students' perceptions on mental health and wellness, coping mechanisms, and resources provided by Midwestern University Chicago College of Pharmacy (MWU-CCP). Method: An online questionnaire was distributed to all first, second, and third year pharmacy students on November 2017. The questionnaire included items on mental health and wellness, coping mechanisms, resources provided by MWU-CCP, and student background characteristics. The study was 


\section{American Journal of Pharmaceutical Education 2018; 82 (5) Article 7158.}

exempt from Midwestern University IRB review. Results: Response rate was $43 \%(n=250)$. Most respondents were female $(54 \%)$, Caucasian $(43 \%)$, with an average age of $24(+3)$. During their past academic quarter, $62 \%$ of students knew of a classmate who had experienced a mental health issue, $18 \%$ reported "frequently/almost always" experiencing a mental health issue themselves, and $37 \%$ reported "sometimes" experiencing a mental health issue. Almost half (48\%) of students reported "sometimes" being able to effectively cope with important changes occurring in their life. The most frequently used coping mechanisms reported were: sleeping/napping (44\%), listening to music (44\%), and having personal alone time/isolation (40\%). More than half (54\%) of students thought that the curriculum did a "poor/fair" job addressing students' mental health and wellness. Common themes among students' comments emphasized increased opportunities for discussion about mental health and coping mechanisms in the classroom or with their advisors. Implications: This study establishes baseline measures of the extent to which the current pharmacy curricula and university resources address pharmacy student needs relating to mental health and coping mechanisms.

Pharmacy Student Utilization of Electronic Devices During Inpatient Advanced Pharmacy Practice Experiences. Austin M. Kurkowski, Purdue University, Monica L. Miller, Purdue University, Alex N. Isaacs, Purdue University. Objectives: This survey aimed to assess the use and impact of electronic devices during inpatient advanced pharmacy practice experiences (APPE). Method: A 40-item questionnaire was developed within Qualtrics. All student pharmacists completing a clinical APPE within the hospital setting from July 2017 through January 2018 were eligible for study inclusion. The anonymous survey was emailed to students for voluntary participation during the final week of the APPE. Questions regarded use of smartphones and tablets for patient monitoring, provider interactions, patient care, and learning. Descriptive statistics were used to report findings. The study received exempt status from the institution's IRB. Results: Of the 111 students eligible to complete the survey, 58 responded (52\% response rate). Most students used a smartphone $(n=43)$ and 11 of those students also used a tablet. Thirty-one respondents indicated smartphones assisted them multiple times per day (72\%). Smartphones were most often used for accessing drug information resources $(n=37)$. Tablets were most often used for reading literature $(n=10)$. Two respondents indicated using either device for direct patient interaction. Tablets were mostly self-provided $(n=8)$; three respondents were given tablets. Nearly all students $(95 \%)$ that utilized a device agreed it improved their efficiency. Implications: Students found value using electronic devices and agree it improved their efficiency. They are frequently assisted by these devices, but most commonly for communication and learning purposes rather than direct patient interaction. Currently, few sites are providing tablets to students for use on inpatient APPEs. Overall, student pharmacists benefitted from using devices, but more data is needed.

Prediction of Academic Performance in Pharmacy School Based Upon Admissions Characteristics. Lindsey N. Parmelee, University of New England, Wallace Marsh, University of New England. Objectives: The objectives of the study were to determine if there is an association between obtaining a previous degree or completing prerequisite coursework at the University of New England (UNE) undergraduate campus and academic performance in pharmacy school and analyze which admission criteria is predictive for academic performance. Method: All students admitted to the college of pharmacy since its opening were included thus eight years of data were available. Linear regression and binary logistic regression analyses were used to predict the dependent variables of first and second year GPA and issuance of a warning letter. All analyses were performed with IBM SPSS v.21. For purposes of prediction, a p-value less than 0.1 was used to determine significant predictors. Results: A total of 797 student records were available. A series of linear regressions showed that degree, composite Pharmacy Curriculum Outcomes Assessment (PCOA), overall GPA, science GPA, and all components of the PCAT except for reading comprehension were found to be significant predictors of first year pharmacy GPA. Students who attended UNE for pre-requisites were found to have a significantly lower first year pharmacy GPA ( $p$ equals 0.05 ). Further analysis showed that attendance at UNE was not a significant predictor of second year pharmacy GPA (p equals 0.71). Implications: Overall GPA and composite PCAT were found to be significant predictors of academic performance in pharmacy school. Overall GPA, science GPA, composite PCAT, chemistry PCAT, and biology PCAT were significant predictors of receiving a warning letter $\mathrm{P} 1$ and $\mathrm{P} 2$ year.

Predictors of Monthly Oncology Drug Costs at Launch in the USA, 2009-2016. Wilson Haong, Western New England University, Natalia Shcherbakova, Western New England University. Objectives: To examine factors associated with the monthly costs of oncology therapeutics in the USA. Method: The FDA archive was used to extract a list of cancer drug approvals during 2009-2016. Micromedex REDBOOK was used to obtain the average wholesale price per unit for each drug. Monthly costs of therapy were calculated for adults using a standard weight of $70 \mathrm{~kg}$ and BSA of $1.73 \mathrm{~m}^{\wedge} 2$. The following variables were included: approval mechanism, novelty, indication, route of administration, orphan designation, company size, and disease prevalence. Results: A total of 53 agents were included with $68 \%$ approved under New Drug Application and the rest under Biologic License Application. The mean (SD) monthly cost of therapy was $\$ 20,068(\$ 18,194)$. Of the included agents, $53 \%$ were oral therapies with the rest being injectables. The majority received priority review (81\%) and orphan designation (72\%). Most products were indicated for the treatment of blood $(34 \%)$ and hormone-related cancers $(25 \%)$. With regards to novelty, $47 \%$ were first-in-class, $42 \%$ advance-in-class, and $11 \%$ addition-to-class agents, respectively. The mean (SD) revenue in millions for applicant companies was $\$ 30,830$ (\$26,629). Disease prevalence ranged between 650 to 245,525 patients. There was a strong significant association between BLA approval and the drug administration route with monthly cost being in the top quartile $(>=\$ 18,751)$. No significant associations between other examined variables and monthly cost were observed ( $p>0.05$ ). Implications: Our study indicates that the monthly costs of biologics and injectable cancer therapies are significantly higher. Other examined variables were not associated with higher costs.

Reporting Quality of Survey Research Articles Published in the American Journal of Pharmaceutical Education. Kiersten N. Walters, Purdue University, Ian Michael Dilley, Purdue University, Amy H. Sheehan, Purdue University, Robert D. Beckett, Manchester University. Objectives: Previous studies have indicated poor reporting quality for survey research published in the medical literature. The objective of this study is to examine the reporting quality of survey research articles published in the American Journal of Pharmaceutical Education (AJPE) in 2016. Method: Survey research articles published in AJPE from January 1, 2016 through December 31, 2016 were evaluated by two independent study investigators using a modified version of a previously-published, 35-item checklist developed by survey research methodology experts. The checklist included items from AJPE best practices for survey research, standards from the 


\section{American Journal of Pharmaceutical Education 2018; 82 (5) Article 7158.}

Enhancing the Quality and Transparency of Health Research (EQUATOR) Network, and the American Association for Public Opinion Research (AAPOR). Discrepancies were resolved by consensus. Results: Out of 94 articles assessed for eligibility, 46 (49\%) used survey research. The majority of the articles provided sufficient information regarding their questionnaire items (70\%), mode of administration $(83 \%)$, method of data analysis $(89 \%)$, response rate $(98 \%)$, and study limitations (98\%). However, few provided information regarding the validity and reliability of new instruments (17\%), definitions for complete versus partial responses $(9 \%)$, information on how non-respondents differed from respondents $(2 \%)$, and methodology for handling missing data (13\%). Implications: Survey research published in AJPE is generally consistent with published best practices. Areas for continued improvement in reporting of survey research include consideration of factors such as non-response error, handling of missing data, and reliability and validity of instruments.

Risk Attitudes and Characteristics of Student Pharmacists Across Cohorts. Kristin R. Villa, Purdue University, Kimberly S. Plake, Purdue University, Matthew M. Murawski, Purdue University. Objectives: To assess whether: 1) pre-pharmacy students and student pharmacists have different attitudes towards change and risk compared to population norms, 2) if pre-pharmacy and student pharmacists' attitudes towards change and risk differs by year in the program, and 3) if pre-pharmacy students and student pharmacists' attitudes towards change and risk differs by career intention. Method: Surveys were emailed to pre-pharmacy students and all current first professional, second professional, and third professional year student pharmacists at seven institutions in the Midwest. Demographic information and six scales assessing attitudes and personality characteristics known to reflect attitudes towards change/risk were collected. Results: One thousand seventy three students completed the survey (37.2 percent response). Compared to scale population norms, respondents had more positive attitudes towards change in general, but more negative attitudes towards workplace change and risk in general. Students favored instrumental risk decisions more than stimulating risk, were less open to new experiences, and more conscientious. Attitudes towards risk and change differ by year in the pharmacy program and Individuals with different career intentions have differing attitudes towards change and risk. Implications: Pre-pharmacy students and current student pharmacists are different than a non-pharmacy population. The personality profiles found here would be congruent with a population that is not currently comfortable with change in their professional environment. Understanding how individual personality characteristics and pharmacy culture impact the profession can help guide the profession in designing new programs to better meet the stability needs of pharmacists while stimulating evolution of new pharmacy models.

Statin-Induced Myopathy: A Comprehensive Assessment of Risk Factors. Khoa A. Nguyen, VA Indianapolis, Lang Li, The Ohio State University, Deshun Lu, Indiana University, Aida Yazdanparast, Indiana University, Rolf Kreutz, Indiana University, Elizabeth Whipple, Indiana University, Titus Schleyer, Indiana University. Objectives: To perform a comprehensive review of all known risk factors for statin-induced myopathy and provide aggregate odds ratios for these risk factors. Method: Study was done through four phases: 1. Identification of the relevant primary literature. Articles were searched from inception to May 2016 using PubMed. Search terms included: statins, myopathy, risk factors. 2. Abstract review to filter through exclusion criteria. Exclusion criteria: case reports, letters, reviews, in vitro studies, languages other than English, cerivastatin. 3. Full paper review and data extraction. Data from full articles were extracted into: study type, patient population, duration of study, clinical outcomes, risk factor(s), and statistical method(s). Clinical outcomes were classified into two categories: myopathy and rhabdomyolysis. 4 . Data synthesis and statistical analysis. All individual test statistics were calculated into incidence rate ratios for analysis, then converted to aggregate odds ratios. Results: Search results yielded 836 papers, of which 44 were analyzed and 18 different potential risk factors were collected. These included: age, gender, race, diabetes, cardiovascular diseases, renal impairment, statin drug interactions, statin doses, chronic disease score, hypertension, hypothyroidism, gout, prior elevated CK, mutations of SLCO1B1, GATM or EYE genes, low levels of CoQ10 enzyme, or Vitamin D. These potential risk factors were classified into three groups: demographic factors, clinical factors, and pharmacogenetics/biomarkers. Risk factors significant for myopathy and/or rhabdomyolysis included age, gender, diabetes, renal impairment, cardiovascular disease, certain interacting drugs, and mutations of the SLCO1B1 gene. Implications: Incorporating a comprehensive assessment of risk factors will support clinicians in reducing cases of statin-induced myopathy

Student Confidence in Hospital Pharmacist Roles After Participation in a Pharmacotherapy Skills Laboratory. Meredith L. Frey, University of Wisconsin-Madison, Andrea L. Porter, University of Wisconsin-Madison, Amanda Margolis, University of WisconsinMadison. Objectives: To assess student confidence in understanding and performing hospital pharmacist activities before and after completion of a laboratory experience. Method: Second-year doctor of pharmacy students enrolled in a required integrated pharmacotherapy skills laboratory course participated in a laboratory that followed a patient throughout a hospital stay. Students voluntarily completed a 13-question survey within one week before and after the experience over three years (2015-2017). Confidence was measured on a 10-point scale ( $1=$ no confidence; $10=$ complete confidence). Survey questions explored confidence in gathering medication information, making recommendations to providers regarding drug-related problems (DRPs), clinical skills related to anticoagulation, and discharge medication counseling. Wilcoxon Signed-Rank Test was used to determine the statistical significance of change in confidence. Results: Over three years, 363 students completed both the pre- and post-surveys (76.42\%). Confidence improved for all questions from pre- to post-survey ( $p<0.001$ ). The cumulative confidence score increased by $22.6 \%$ from baseline $(\mathrm{p}<0.001)$. Skills with the greatest gain in confidence were determining appropriate dosing for low molecular weight heparin (48.6\% increase, final score of $6.59, \mathrm{p}<0.001$ ), determining appropriate dosing for warfarin $(47.6 \%$ increase, final score of $6.31, \mathrm{p}<0.001)$, and making recommendations to providers for solutions to DRPs (29.3\% increase, final score of 6.99, p <0.001). Implications: A simulated experience on hospital pharmacy can improve student confidence in the duties of a hospital pharmacist, provide an opportunity for students to practice complex skills and topics in the curriculum, and assist in their preparation for experiential education rotations.

Student-Derived Intervention to Improve Compounded Preparation Potency Accuracy. Tanya Makhlouf, University of North Carolina at Chapel Hill, Robert P. Shrewsbury, University of North Carolina at Chapel Hill. Objectives: Analyzing student preparations for accuracy within a required compounding course has allowed for data collection of observed potency variation of many formulations. The Science of Pharmaceutical Compounding elective was created with the goal for students to identify sources of variability when compounding preparations and implementing possible solutions into the 


\section{American Journal of Pharmaceutical Education 2018; 82 (5) Article 7158.}

next iteration of the required pharmaceutical compounding course. Method: Students in the elective course conducted experiments to identify solutions for potency variability in a ketoprofen PLO emulsion preparation. Outcomes of each experiment were collectively discussed with the most effective intervention implemented into the subsequent required compounding course. Students in the subsequent course (Group 1) compounded the ketoprofen PLO emulsion preparation utilizing the intervention and their results were compared to results obtained in preparations made by students before the intervention (Group 2). Results: Ketoprofen PLO emulsions made by Group 2 $(1.29+/-0.21, \mathrm{n}=158)$ and Group $1(1.18+/-0.21, \mathrm{n}=131)$ were compared (z-test). There was a significant difference between the two groups $(\mathrm{P}=0.03)$, with less potency accuracy seen in the preparations made before implementation of the intervention. Implications: The elective course provided the opportunity for a student-derived remedy to increase accuracy in a compounded preparation. In this circular feed-back strategy, the two courses complement each other by serving as incubators of data and quality improvement. Results of these efforts may lead to a more the stringent range of $+/-5 \%$ potency in compounded preparations as opposed to current range of $+/-10 \%$.

Students' Perceptions of Successful Teamwork After Immersion in Diabetes Escape Room Team-Based Gaming Activity. Carolyn G. Marg, North Dakota State University, Brooke D. Schotters, North Dakota State University, Jeanne E. Frenzel, North Dakota State University. Objectives: In 2017, a group of NDSU faculty members designed and implemented a serious game, the Diabetes Escape Room, with third year pharmacy students in a pharmacy skills laboratory. This poster discusses elements of successful teamwork in the setting of a serious game as reported by students during focus group interviews following the gaming activity. Method: Videos of six student team interviews conducted after the game were transcribed and uploaded in NVivo(R) for qualitative analysis. Data were coded on the fly from the participants' comments during the interview. Identified codes were then analyzed and sorted into common themes that described specific aspects of successful teamwork. The data was further organized based on the game performance of each team to assess the most prevalent qualities of successful teams. Results: The results of this qualitative data analysis clearly showed two main characteristics of successful teams: team communication and shared leadership. Team communication was mentioned a total of 21 times and included 7 references to continuous feedback and 6 references to listening as essential skills. Shared leadership was coded 35 times with equal contribution (14), individual strengths and weaknesses (11), and division of tasks (6) emerging as the most referenced successful team qualities. Implications: Student focus group interviews and qualitative analysis using NVivo software helped identify attributes of successful teams competing in a serious game. The results of this analysis indicated that good teamwork is demonstrated through shared leadership and strong communication skills, and is needed for the successful completion of the educational team-based gaming activity.

Survey Driven Assessment of Drug Disposal Knowledge of Pharmacists and Consumers. Karolina J. Kwietniak, Western New England University, Arianna Bonzagni, Western New England University, Thomas Kalista, Western New England University, Melissa Mattison, Western New England University, Kam L. Capoccia, Western New England University. Objectives: The primary objective is to measure pharmacist and consumer knowledge, attitudes, and practices of safe and proper disposal of unwanted/expired drugs in the community. Method: Two surveys were distributed, one to pharmacists and one to consumers. Demographics, knowledge of location of local drug kiosks, perception of the importance of proper drug disposal, and current practices of drug disposal were ascertained. Demographic information between the two surveys will be compared using standard descriptive statistics. Differences between survey responses will be compared using chi-square or Fischer's exact testing, as appropriate. Results: Attitudes and knowledge from pharmacist and consumer perspectives will be quantified and analyzed to identify needed areas of education. The results will quantify how many people have dealt with the issue of unused medication, common drug disposal practices, and the extent of knowledge of proper drug disposal. Implications: Identifying knowledge gaps in proper drug disposal, locations of drug kiosks, drug-take-back events, and the importance of removing unwanted/expired medications will identify needed areas of education. Increased education will maximize the utilization of public drug disposal facilities and drug-take-back events, as well as aid in the removal of unwanted/expired medications in the community.

Synthesis and Evaluation of Etoposide and Podophyllotoxin Analogs Against Topoisomerase II $\alpha$ Activity and in Cancer Cells. Matthew Murphy, Lipscomb University, Priyanka Kumar, Belmont University, Amber M. Bradley, Lipscomb University, Christopher E. Barton, Belmont University, Joseph E. Deweese, Lipscomb University, Susan L. Mercer, Lipscomb University. Objectives: Etoposide is a widely-used anticancer agent that targets topoisomerase II (Top2). Evidence suggests metabolism of etoposide in myeloid progenitor cells is associated with translocations involved in leukemia development. Previous studies suggest halogenation at the C-2' position of etoposide reduces metabolism. We set out to design and synthesize etoposide analogs halogenated at the C-2' position and examine activity against Top2 and in cells. Method: Halogens were introduced into the C-2' position by electrophilic aromatic halogenation onto etoposide (ETOP), 4'demethylepipodophyllotoxin (DMEP), or podophyllotoxin (PPT). The reagents N-bromosuccinimide and N-chlorosuccinimide afforded a series of halogenated compounds (LUCOP 48-51, 53). Purified human Top2A, as well as, HCT-116 (colon) and A549 (lung) cells were used for analysis. Results: LUCOP 51 was the only analog to show concentration-dependent enhancement of Top2-mediated DNA cleavage. Furthermore, LUCOP 50, 51, and 53 all displayed variable ability to inhibit DNA relaxation. HCT116 cells were used to establish an LD50 for LUCOP 50 (20 uM), $51(120 \mathrm{uM})$, and 53 (33 uM). Assays in A549 cells with LUCOP 50 and 53 provided LD50 values of 17 and $37 \mathrm{uM}$, respectively. Flow cytometry data with LUCOP 50 and 53 in HCT-116 cells provide evidence of sub-G1 cell populations indicative of apoptosis. Preliminary flow cytometry data with A549 cells indicate LUCOP 50 and 53 may induce G1 cell cycle arrest. Implications: These results indicate halogenation of etoposide (LUCOP 51) is tolerated by Top2. Interestingly, halogenated podophyllotoxin analogs, LUCOP 50 and 53, display higher cellular activity than LUCOP 51. Further synthetic and characterization work is planned.

The Effects of Integrated Course Modules on NAPLEX ${ }^{\circledR}$ Passing Rates. Cassie Lynn Field, Western New England University, Ayeisca Enid Mateo, Western New England University, Ronny Priefer, Western New England University. Objectives: With the latest change in the NAPLEX ${ }^{\circledR}$ examination there was a noticeable drop in first-time passrates seen across the majority of PharmD programs. Our objective was to see if the drop in the NAPLEX ${ }^{\circledR}$ first-time pass-rates was correlated with the integrated course modules which some Colleges/Schools have adopted. Method: From the AACP database we initially examined the NAPLEX ${ }^{\circledR}$ first-time pass-rates of all pharmacy schools/colleges preand post-change in the examination style/format. The curriculum of all the PharmD programs were obtained either through the College/School 


\section{American Journal of Pharmaceutical Education 2018; 82 (5) Article 7158.}

webpage or via direct communication with the institutions. Correlation between integrated course modules and the changes in the NAPLEX ${ }^{\circledR}$ first-time pass-rates were analyzed. Results: The overall NAPLEX ${ }^{\circledR}$ average first-time pass-rates the year prior to the change in examination style/format was $92.3 \%$. For Colleges/Schools that had an Integrated or traditional curriculum the year prior to the change in the examination both had an average first-time pass-rates of $92.3 \%$. After the NAPLEX ${ }^{\circledR}$ change, which caused the average first-time pass-rate to drop to $86.1 \%$, no noticeable difference between the Colleges/Schools that have integrated course modules or traditional curriculum was observed. Colleges/ Schools of Pharmacy that have integrated course modules showed a change range of $33.0 \%$ whereas with traditional curriculum the fluctuation was $35.5 \%$. Implications: From this data, the effect of the change of the NAPLEX $®$ examination style/format affected the first-time pass-rate equally whether the program had integrated course modules or traditional curriculum.

The Impacts of Pre-cART Versus cART Era on HIV-Associated Neurocognitive Disorders: A Systematic Review. Farzana Shaik, Nova Southeastern University, Barry A. Bleidt, Nova Southeastern University, Silvia Rabionet, Nova Southeastern University. Objectives: The objective of this presentation is a systematic literature review to consider HIV-associated neurocognitive disorder (HAND) rates before and during the cART era. A second objective of this systematic review is to identify and summarize recent studies that examine asymptomatic neurocognitive impairment in HAND during the pre-combination Antiretroviral Therapy (pre-cART) and combination Antiretroviral Therapy (cART) era. Method: A systematic literature review was conducted to include English-language articles published from 2006 to 2016 on January 19th, 2017. A total of four electronic databases were used including MEDLINE, PUBMED, EMBASE, and PsycINFO Results: Despite enhanced research efforts and effective cART, there still is a high prevalence of HAND within the HIV-infected population. Although the most severe form of HAND, HIV associated dementia (HAD) is much less common in cART era, researchers still pose questions about any long-term benefit of cART with respect to milder forms of HAND. Preliminary findings have shown that patients with ANI will progress more quickly to more severe forms of HAND than those without ANI. Given this, the relevance of the correct diagnosis of ANI is crucial where well over $50 \%$ of all HIV-infected patients suffer from some stage of HAND mentioned above. Implications: There are various social, economic, and public health implications surrounding HIV \& neurocognitive disorders. The burden of disease for this population is increasing at an exponential rate but are still unaware of the long-term survival with chronic immune activation.Overall there is a continued need for biomarkers of HAND predisposition, detection, and monitoring

The Incorporation of CAPE Outcomes and PPCP Components in Elective Courses. Bethany A. Tabeling, University of Cincinnati, Patricia R. Wigle, University of Cincinnati. Objectives: The didactic curriculum at the University of Cincinnati James L. Winkle College of Pharmacy was mapped to the CAPE outcomes and JCPP Pharmacist Patient Care Process (PPCP). This project's objective was to determine which CAPE outcomes and PPCP components are taught in elective courses. Method: All 29 elective course syllabi were mapped to the PPCP, CAPE outcomes, and Domain subgroups. An excel spreadsheet was used to collect data and an " $\mathrm{X}$ " indicated that the elective had the Domain subgroup or JCPP process either explicitly stated or extrapolated from syllabi content. Additional information was collected to allow determination of which CAPE and PPCP components were delivered by student year, number of credit hours and faculty/non-faculty course coordination. Results: Domains 3.6 Communication, 3.1 Prob- lem Solving and 4.4 Professionalism were utilized by $76 \%, 71 \%$ and $71 \%$ of the electives, respectively. In the core didactic classes, Domains 4.4 Professionalism, 3.1 Problem Solving, and 3.6 Communication were utilized by $82 \%, 78 \%$ and $71 \%$ of the didactic courses, respectively. The least utilized was Domain 4.3 Innovation and Entrepreneurship which was in $18 \%$ of the core and $0 \%$ of the elective courses. Implications: The 3 most common CAPE Domains for both elective and didactic coursework were the same. Both elective and didactic coursework are lacking substantial involvement of Domain 4.3 and discussion is on-going regarding how to address this deficiency. This project confirmed students are getting exposure to all CAPE Domains and PPCP through our didactic and elective coursework, regardless of which elective is selected.

The Leukotriene Inhibitor Zafirlukast Inhibits Cancer Cell Growth Via Inhibition of Thiol Isomerase Activity. Christina Ann Verbetsky, Western New England University, Elizabeth Brunt, Western New England, Jamie R. Romanowski, Western New England, Daniel R. Kennedy, Western New England University. Objectives: Thiol isomerase enzymes are implicated in cancer cell growth, progression and even resistance to chemotherapeutic treatment. These enzymes, which include PDI, ERp57 and ERp5, are upregulated in many distinct cancer types, including ovarian, prostate, lung, melanoma, lymphoma and glioma. Despite their promise as therapeutic targets, no small molecule inhibitors that target thiol isomerases are FDA approved. In this study, we screened known FDA approved compounds for their ability to inhibit thiol isomerase enzymes. Method: 3641 known bioactive compounds were screened for ERp57 inhibitory activity using the insulin turbidity assay and positive hits were counterscreened against PDI, ERp5, ERp72 and thioredoxin for broad-spectrum thiol isomerase acivity. Zafirlukast was selected for further study and cancer cell growth inhibition was performed using the prestoblue assay after 24 hours. Western blot analysis also demonstrated inhibition of EGFR activation. Results: OVCar8 (human ovarian cancer cell line), HCT116 (human colon cancer), HeLa (cervical cancer cell line) and MDA-MB-231 (breast cancer cell line) cell lines were examined for their ability to inhibit cell proliferation. OVCar8 and HCT116 cells exhibited an IC50 in the 5-10 uM range. HeLa cervical cancer cells and MDA-MB231 breast cancer lines were less sensitive. Implications: In this study we have identified that zafirlukast is a broad spectrum thiol isomerase inhibitors which inhibits cancer cell growth. Considering this is an FDA approved compound, zafirlukast might be an attractive drug to repurpose as a potential oncology medication as part of a chemotherapeutic regimen in certain cancers.

The Multiple Mini Interview as an Assessment Strategy Within the First Year of a PharmD Curriculum. Michael D. Wolcott, University of North Carolina at Chapel Hill, Jacqueline M. Zeeman, University of North Carolina at Chapel Hill, Wendy C. Cox, University of North Carolina at Chapel Hill, Jacqueline McLaughlin, University of North Carolina at Chapel Hill. Objectives: To evaluate the psychometric properties of a multiple mini interview integrated within a Doctor of Pharmacy (PharmD) curriculum. Method: This study describes the integration of a 5-station end-of-year capstone MMI (cMMI) following the first year of a PharmD curriculum. The cMMI assessed professionalism constructs of students and offered formative feedback to prepare them for upcoming clinical practice experiences. Each station was designed for one rater to evaluate a single construct. A principal component analysis (PCA) was used to evaluate the structure of the model and its ability to distinguish the 5 constructs. A Multifaceted Rasch Measurement (MFRM) model assessed student performance and estimated the sources of measurement error attributed to 3 facets: 


\section{American Journal of Pharmaceutical Education 2018; 82 (5) Article 7158.}

student ability, rater stringency, and station difficulty. Students $(\mathrm{n}=$ 146) were surveyed about the cMMI experience. The university institutional review board considered the study exempt from review. Results: The PCA confirmed the MMI reliably assessed 5 unique constructs and performance on each station was not strongly correlated with one another. The MFRM analysis explained $58.79 \%$ of the total variance in student scores. Specifically, $29.98 \%$ of the variance reflected student ability, $20.25 \%$ reflected rater stringency, and $8.56 \%$ reflected station difficulty. Overall, the data demonstrated acceptable fit to the MFRM model. Most students agreed the MMI allowed them to effectively demonstrate their communication $(80.82 \%)$, critical thinking (78.77\%), and collaboration skills (70.55\%). Implications: The MMI can be a valuable assessment strategy of professionalism constructs within the PharmD curriculum. These findings suggest the MMI is well-received by students and can produce reliable results.

The Role of Pharmacists in Transitional Care Management With Respect to Medication Reconciliation. Shannon Marie Haberman, Lake Erie College of Osteopathic Medicine, Jacob C. Reaser, Lake Erie College of Osteopathic Medicine. Objectives: This research is to determine the impact pharmacists have in transitional care management (TCM) services after transition from the inpatient to outpatient setting. Success will be measured by identifying the number of medication discrepancies resolved with a pharmacist compared to regular clinic staff. TCM services assist patients transitioning back to the community setting. These services include an initial telephone contact within 2 days of discharge, an appointment within 7 to 14 days, and billing Medicare based on complexity. The Medical Associates of Erie (MAE) has been billing for this new Medicare service for over a 1 year. Previous research assessed pharmacists by measuring medication errors and adverse drug events, evaluated pharmacy-based programs with respect to hospital readmissions, and assessed interprofessional collaboration in TCM. Method: This retrospective chart-focused study includes patients from 11 outpatient offices of MAE. One of the 11 centers, The LECOM Institute for Successful Aging, has pharmacists at the clinic to assist with TCM services. The study will identify and tabulate the number of medication discrepancies for each TCM patient encounter. Then statistical analysis will be performed to determine any significant differences between the pharmacists' and the clinic staff's actions with respect to the initial telephone contact and number of medication discrepancies. Results: Preliminary data collection indicates increased amount of initial telephone contacts and medication discrepancies identified during TCM by pharmacists compared to other clinic staff. Implications: After completing the data analysis, it is expected that pharmacists will identify more medication discrepancies showing a positive impact in TCM services.

They "Like" It! Use of Web 2.0 Technologies on Advanced Pharmacy Practice Experiences. Taylor A. Imburgia, Northeastern University, Stephanie L. Sibicky, Northeastern University, Alexa A. Carlson, Northeastern University. Objectives: To investigate the impact of Web 2.0 technology in pharmacy experiential education on student engagement, student participation, and attitudes throughout the course of six-week rotations. Method: Two preceptors from Northeastern University incorporated Web 2.0 technology into their internal medicine Advanced Pharmacy Practice Experiences. Rotation requirements included daily Facebook posts on drug information topics, things they learned that day, and weekly written and video blogs. Student engagement was measured quantitatively by tracking numbers of "Likes," comments, and poll responses on Facebook and blog comments and qualitatively via a Qualtrics ${ }^{\circledR}$ survey using Likert scales and open-ended questions. Results: There were 827 "Likes,"
374 comments, and 353 poll responses on Facebook and 55 blog comments during the first two rotations. These quantitative markers of student participation did not increase steadily over time but instead fluctuated over the rotation. Nine out of twelve students completed the survey. Survey results indicate student perceptions on the technology changed from apprehension or indifference at the beginning of the rotation to more favorable opinions at completion. Most students reported that the technology encouraged them to more deeply examine pharmacy topics than they would have otherwise. Students attributed enhanced learning and communication skills to the technology and reported receiving meaningful feedback but had mixed feelings on whether all rotations should adopt this technology. Implications: Despite the small sample size, findings suggest a positive relationship between Web 2.0 technology and student engagement in learning which may be explored on a larger scale.

Unskilled \& Unaware: Assessing Metacognitive Monitoring of Student Help-Seeking Behavior. Youn Chu, University of North Carolina at Chapel Hill, Shannon Palmer, University of North Carolina at Chapel Hill, Adam M. Persky, University of North Carolina at Chapel Hill. Objectives: To develop an assessment process to help provide feedback to students on their metacognitive monitoring of help-seeking behavior. Method: This study piloted an assessment of help-seeking behavior in student pharmacists to answer: (1) does helpseeking behavior depend on content familiarity; (2) when students ask for help, does their performance and confidence differ from when they do not ask for help? One-hundred forty- three student pharmacists participated as part of their capstone assessment. Student completed 32 questions corresponding to 8 less familiar and 8 more familiar drugs on which they rated their confidence level and whether they would ask their preceptor for help. Results: Students performed significantly better on more familiar material $(\mathrm{d}=1.2)$ than less familiar, with a small perceived difference in confidence on familiar material $(\mathrm{d}=.2)$. On average, students would seek help on $71 \%$ of the questions. When students asked for help, they scored significantly lower $(d=-2.2)$ and reported lower confidence $(d=-3.7)$ compared to questions they did not ask for assistance. Students were more likely to ask for help on more familiar content than less familiar (OR 1.25, $\mathrm{p}<.05)$. Based on performance, students were divided into tertiles. The lowest performing group had lower confidence, higher bias, and lower absolute bias compared to the middle/higher performing groups. Implications: Overall, the results demonstrate the "unskilled and unaware" effect. Incorporating assessment tools early in the curriculum may help identify "unskilled and unaware" students in order to provide strategies to reverse this effect and become better learners.

Usefulness and Student Satisfaction With a Student-Led Pharmacy Curriculum Outcomes Assessment Review Session. Dareen M. Kanaan, MCPHS University-Boston, Kelsey R. O'Neil, MCPHS University-Boston, Joanna K. McCormack, MCPHS UniversityBoston, Maria D. Kostka-Rokosz, MCPHS University-Boston. Objectives: There is a paucity of data on how to prepare students for the Pharmacy Curriculum Outcomes Assessment (PCOA). We evaluated the usefulness of and student satisfaction with a studentdesigned and led PCOA review session. Method: Student leaders from two professional organizations designed and facilitated a review session one week preceding the PCOA exam. The review session targeted PCOA content domains using a game-based platform. Following the PCOA, students were surveyed to assess their satisfaction with the format, relevance of topics, knowledge of student facilitators, and helpfulness of the session when taking the PCOA. Students who did not participate were surveyed to identify barriers that prevented them 


\section{American Journal of Pharmaceutical Education 2018; 82 (5) Article 7158.}

from attending the review session. Results: One hundred thirty-four students completed the survey, of which forty attended the review session (participants). Of these forty participants, the following strongly agreed/agreed that: the format was conducive to learning (75\%), the topics covered were relevant $(60 \%)$, the student-facilitators were knowledgeable (77.5\%), the session helped them when taking the exam $(45 \%)$, and they would recommend review sessions to future classes $(72.5 \%)$. Written feedback suggested additional review sessions and questions on Drug Literature Evaluation, Pharmacology and Healthcare Delivery. Those who did not attend the session cited the evening timing (48.9\%) and scheduling conflicts $(42.5 \%)$ as the two most common reasons for not participating. Implications: Overall, the response to the review session was positive. Student feedback will be incorporated into future review session offerings.

Use of an Educational Card Game to Enhance Student Learning About Medication Adherence Tools and Strategies. Madeline G. Belk, University of South Carolina, Brianne L. Dunn, University of South Carolina, Kevin Lu, University of South Carolina, Kathy Q. Moore, University of South Carolina. Objectives: To assess the impact of an educational card game on students' knowledge of medication adherence strategies. Method: A card game was created consisting of a deck of medication adherence tools and patient barriers. Third year students $(n=101)$ were invited to participate in a pretestposttest to assess change in knowledge of medication adherence tools before and after the game. Results: Knowledge regarding medication adherence tools available for pharmacists and patients as well as knowledge regarding potential barriers to medication adherence increased significantly when compared before and after the in-class activity $(\mathrm{p}<0.001)$. Using a 5 -point Likert scale $(1=$ strongly disagree; 5 = strongly agree), students identified that "it is important for pharmacists to understand techniques to improve medication adherence" with a mean score of 4.93. Students were more likely to recommend a specific medication adherence tool based on an identified barrier to adherence (4.75) than to recommend a tool to all patients (3.77). Respondents indicated that the game was an effective way to learn about the subject (4.64) and gained a better understanding of adherence tools available for patients and pharmacists (4.64). Of the 44 adherence tools included in the game, Mango Health $(n=50)$, Med-
Reminder Watch $(n=28)$, Pill Drill $(n=26)$ and Welvista $(n=26)$ were the most common new tools learned by students. Needy Meds was identified as the most likely tool students would recommend in the future (5.00). Implications: The medication adherence card game proved to be an effective way to improve students' knowledge and confidence about medication adherence strategies.

Willingness of Community Pharmacists to Expand Cognitive Practice. Suheib A. Abdullah, University of the Incarnate Word, Christina M. Seeger, University of the Incarnate Word, Joanne Fazio-Gosser, University of the Incarnate Word, Christopher Alvarado, University of the Incarnate Word, Matthew C. Garber, University of the Incarnate Word. Objectives: Assess quantity of community locations that are offering additional services, respondent's willingness (if able) to perform additional services, and perceived barriers to entry into community collaborative practice agreements. Method: Subjects: community pharmacists in Bexar County. Survey Instrument: one-page self-administered questionnaire offered in person to all potential participants. Number of pharmacies that agreed to participate $=63$ Number of pharmacies that deferred participation $=2$ Response rate $=63 / 65=96.9 \%$. Results: Percentages of pharmacies that agreed when asked "If able, I would provide the following service(s) at my pharmacy.": - Naloxone dispensing without prescription $=70 \%$ Medication Therapy Management $=89 \%$ - Oral Contraceptives without prescription $=56 \%$ - Point-of-Care Testing $=70 \%$ - Travel Immunizations $=87 \%$ - Smoking Cessation $=55 \%$ "Pharmacists could be better utilized to improve quality of patient care." $=98 \%$ agree "Pharmacists are qualified to make drug therapy adjustments in collaboration with a physician." = 94\% agree Potential barriers if CPA implemented: - Most likely: Personal liability, Availability/Accessibility of Physician - Least likely: Educational Training/Drug Knowledge, Necessary Equipment/Physical Space. Implications: Significant legislative changes indicate a shift in focus towards pharmacist-provided patient-care services within a community pharmacy practice. Community pharmacists, within Bexar County, are willing to participate in in additional professional duties and Collaborative Practice Agreements. The basis of this methodology as well as the results may provide a platform for further research with a broader scope. 